
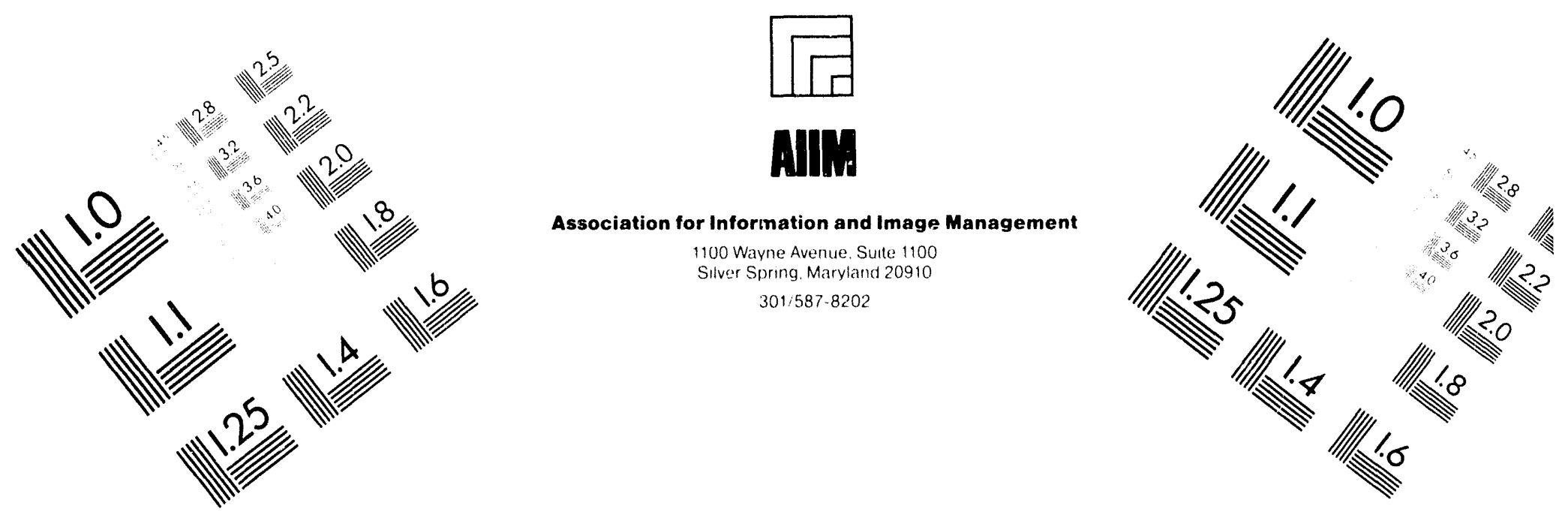

\title{
Centimeter
}

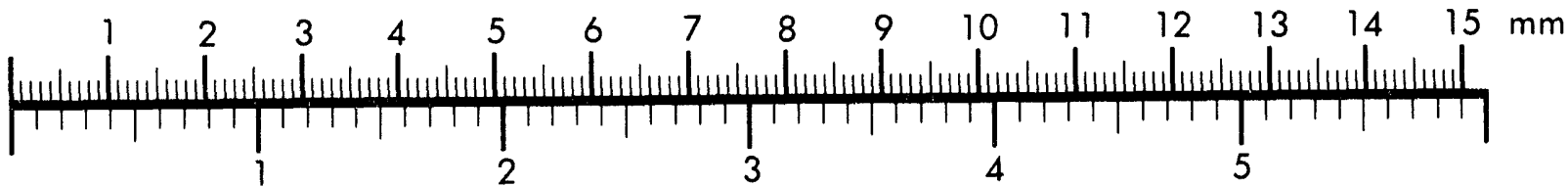
Inches
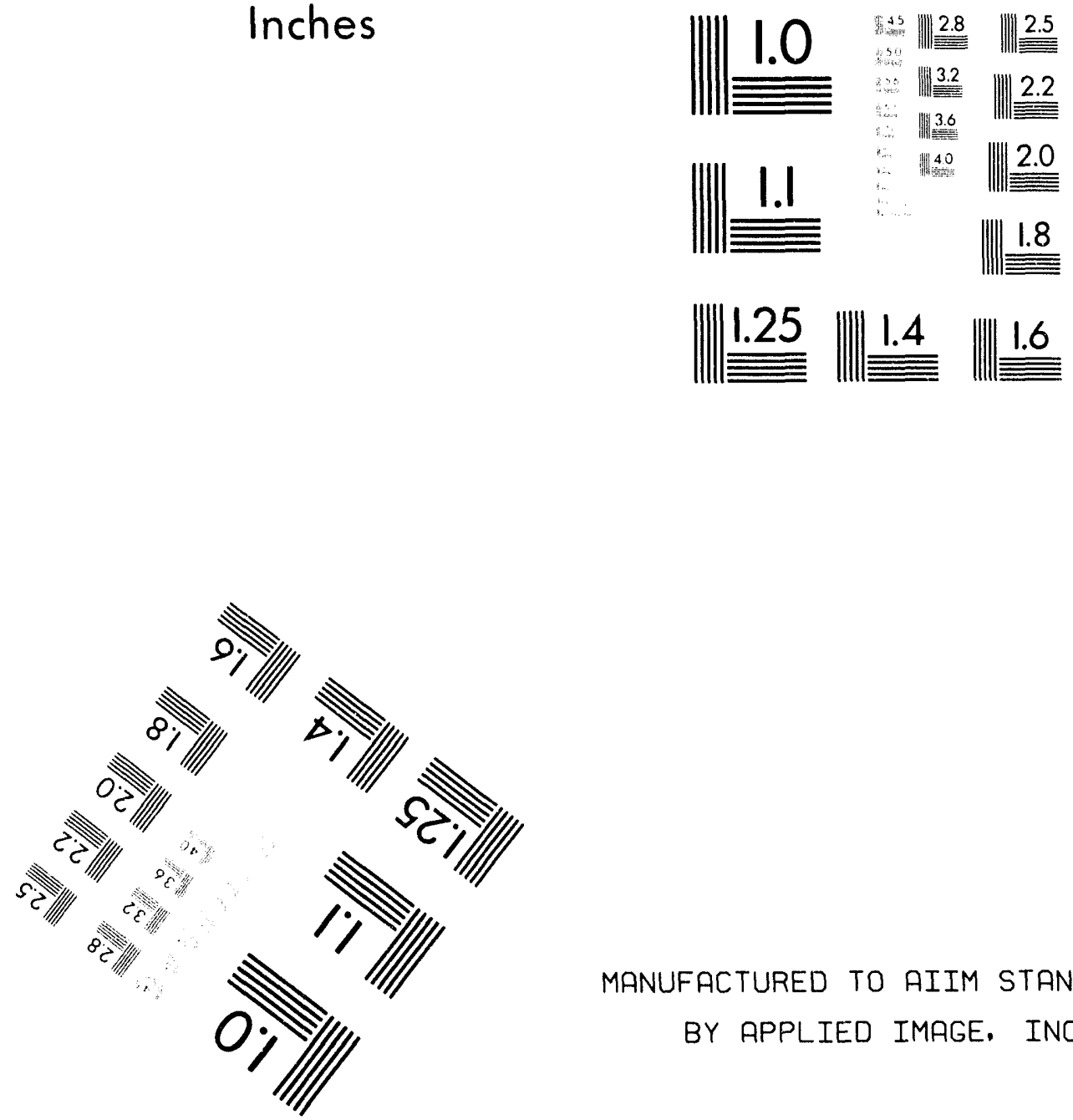

MANUFACTURED TO AIIM STANDARDS

BY APPLIED IMAGE, INC.

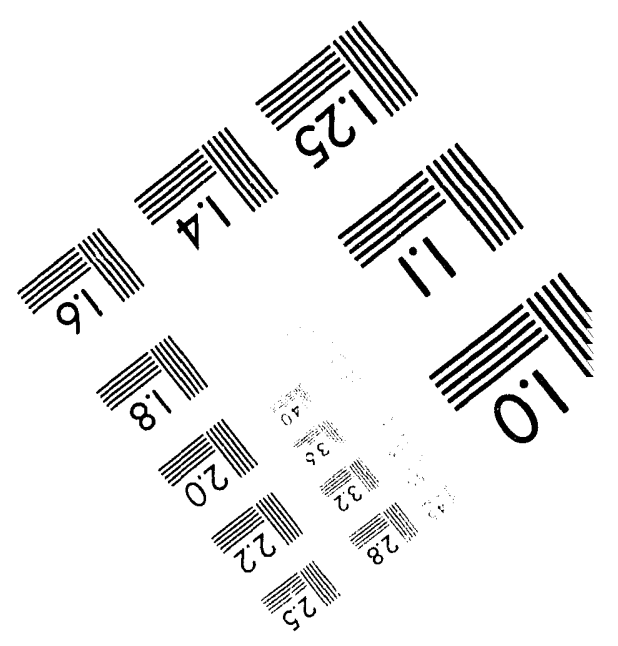



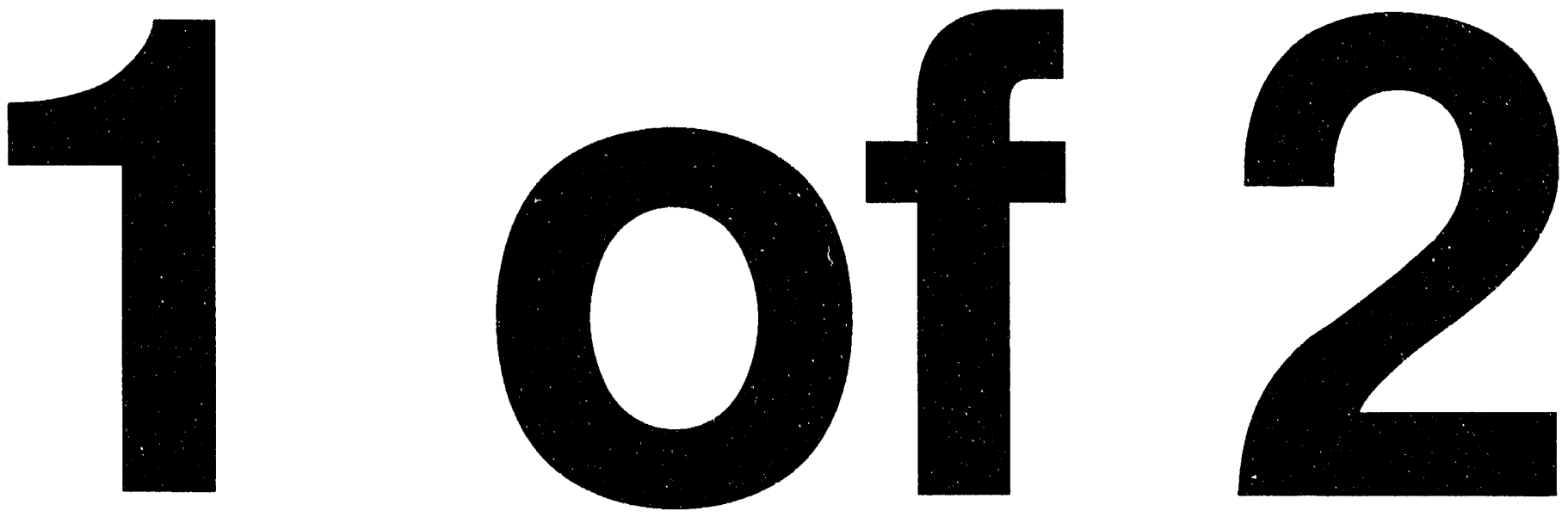


\section{Maý 1994}
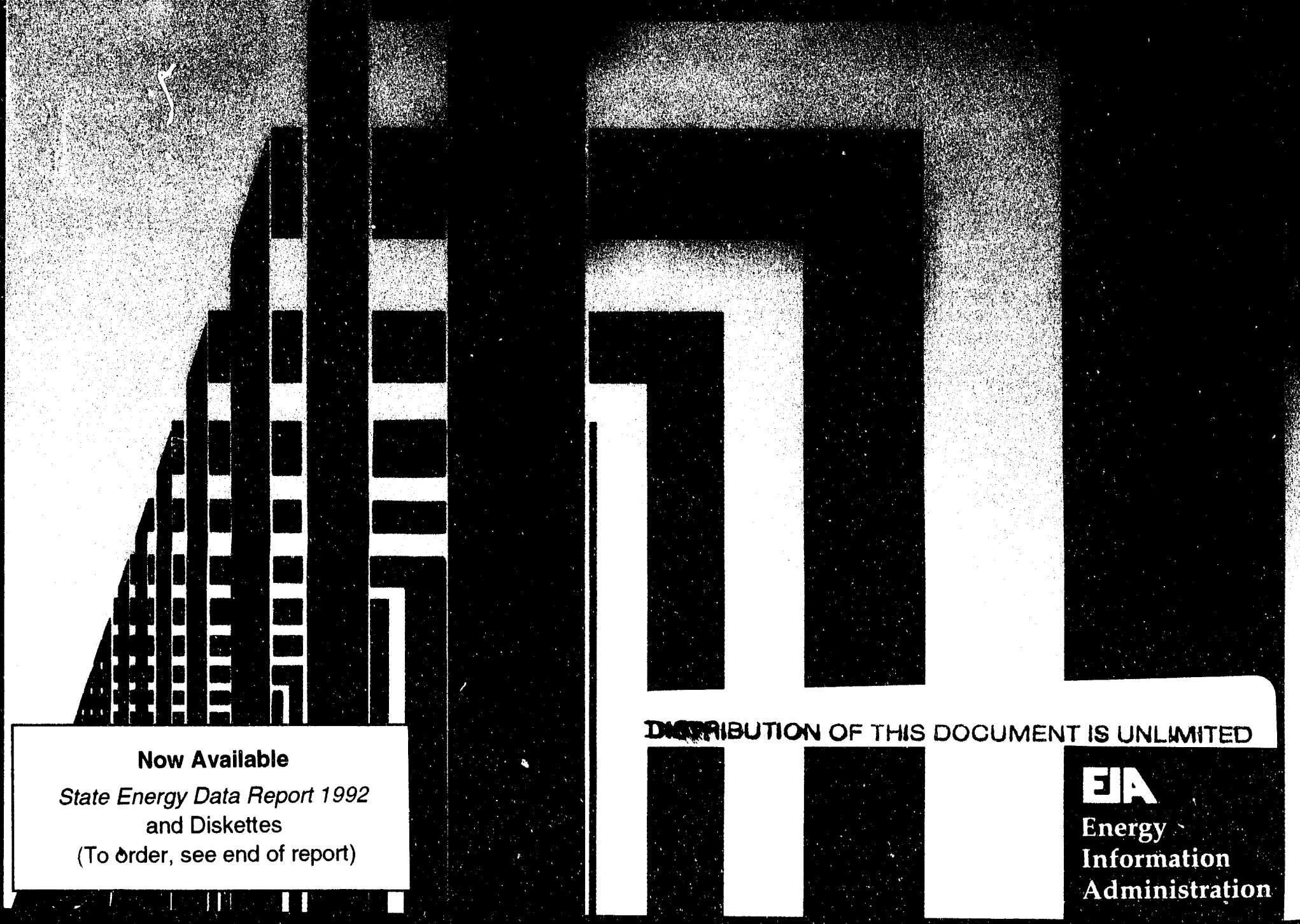


\title{
Ordering Information
}

This publication and other Energy Information Administration (EIA) publications may be purchased from the Superintendent of Documents, U.S. Government Printing Office.

\section{All telephone orders should be directed to:}

U.S. Government Printing Office

McPherson Square Bookstore

1510 H Street, N.W.

Washington, DC 20005

202-653-2050

Fax : 202-376-5055

9 a.m. to $4: 30$ p.m., eastern time, M-F
Superintendent of Documents

U.S. Government Printing Office

Washington, DC 20402

202-783-3238

Fax: 202-512-2233

8 a.m. to 4 p.m., eastern time, M-F

All mail orders should be directed to:

\author{
U.S. Government Printing Office \\ P.O. Box 371954 \\ Pittsburgh, PA 15250-7954
}

Complimentary subscriptions and single issues are available to certain groups of subscribers, such as public and academic libraries, Federal, State, local, and foreign governments, EIA survey respondents, and the media. For further information, and for answers to questions on energy statistics, please contact EIA's National Energy Information Center. Address, telephone numbers, and hours are as follows:

\author{
National Energy Information Center, EI-231 \\ Energy Information Administration \\ Forrestal Building, Room 1F-048 \\ Washington, DC 20585 \\ 202-586-8800 \\ TTY: For people who are deaf or \\ hard of hearing: 202-586-1181 \\ 9 a.m. to 5 p.m., eastern time, M-F
}

\section{Electronic Access}

Monthly Energy Review (MER) data are also available electronically. Page images of all MER tables are available via modem on the Energy Information Administration Electronic Publication System (202-586-2557) and images of selected tables are available on the U.S. Department of Commerce Electronic Bulletin Board (202-482-3870). The data shown in the tables are also available in database format via modem on the U.S. Government Printing Office (GPO) Federal Bulletin Board $(202-512-1524)$ and on personal computer diskettes by mail from the GPO $(202-52-1530)$ and from the National Technical Information Service (703-487-4650).

The Monthly Energy Review (ISSN 0095-7356) is published monthly by the Energy Information Administration, 1000 Independence Avenue, S.W., Washington, DC 20585, and sells for $\$ 77.00$ per year (price is subject to change without advance notice). Second-class postage rates are paid at Washington, DC 20066-9998, and at additional mailing offices. POSTMASTER: Send address changes to Monthly Energy Review, Energy Information Administration, EI-231, 1000 Independence Avenue, S.W., Washington, DC 20585. 


\title{
Monthly Energy Review
}

\section{May 1994}

\author{
Energy Information Administration \\ Office of Enercy Markets and End Use \\ U.S. Department of Energy \\ Washington, DC 20585
}

This report was prepared by the Energy Information Administration, the independent statistical and analytical agency within the Department of Energy. The information contained herein should not be construed as advocating or reflecting any policy position of the Department of Energy or any other organization. 


\section{Contacts}

The Monthly Energy Review is prepared by the Energy Information Administration. General information may be obtained from W. Cal vin Kilgore, Director, Office of Energy Markets and End Use, 202-586-1617; Lynda T. Carlson, Director, Energy End Use and Integrated Statistics Division, 202-586-1112; and Katherine E. Seiferlein, Chief, Integrated Statistics Branch, 202-586-5692. Questions and comments concerning the contents of the Monthly Energy Review may be directed to the Principal Analyst, Chuck Allen, 202-586-5692, or to Diane D. Perrith, 202-586-2788, Carol Swiggins, 202-586-5743, or the following subject specialists:

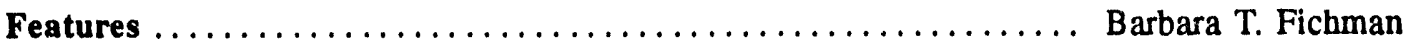

202-586-5737

Section 1. Energy Overview

Tables $1.1-1.5 \ldots \ldots \ldots \ldots \ldots \ldots \ldots \ldots \ldots$ Alethea $\mathrm{K}$. Jennings

202-586-9160

Tables 1.6-1.12 Dianne R. Dunn

202-586-2792

Section

2. Energy Consumption

Alethea K. Jennings

202-586-9160

Section 3. Petroleum

Christine D. Gray

202-586-8995

Section

4. Natural Gas

Donna Guerrina

202-586-6135

Section 5. Oil and Gas Resource Development

Herbert T. Black

202-586-4055

Section 6. Coal

Paulette Young

202-254-5481

Section

7. Electricity

Deborah Bolden

202-254-5663

Section

8. Nuclear Energy

Douglas C. Bonnar

202-254-5560

Section

9. Energy Prices

Elizabeth Scott

202-586-1258

Petroleum

Donna Guerrina

202-586-6135

Electricity

Retail Prices

Deborah Bolden

202-254-5663

Fossil-Fuel Receipts

Sandra Smith

202-254-5632

Section 10. International Energy

Petroleum

Production

Patricia Smith

202-586-6925

Consumption and Stocks

H. Vicky McLaine

202-586-9412

Nuclear Electricity Gross Generation

Douglas C. Bonnar

202-254-5560

Requests for additional information on other energy statistics available from the Energy Information Administration and questions concerning subscriptions and report distribution may be directed to the National Energy Information Center, 202-586-8800 (TTY, for people who are deaf or hard of hearing, 202-586-1181). 


\section{Contents}

Section 1. Energy Overview $\ldots \ldots \ldots \ldots \ldots \ldots \ldots \ldots \ldots \ldots, 1$

Section 2. Energy Consumption $\ldots \ldots \ldots \ldots \ldots \ldots \ldots \ldots \ldots \ldots, 21$

Section 3. Petroleum ................................ 39

Section 4. Natural Gas ........................... 69

Section 5. Oil and Gas Resource Development ............... 79

Section 6. Coal ................................... 83

Section 7. Electricity $\ldots \ldots \ldots \ldots \ldots \ldots \ldots \ldots \ldots \ldots \ldots \ldots . \ldots \ldots$

Section 8. Nuslear Energy ........................... 99

Section 9. Energy Prices ......................... 105

Section 10. International Energy $\ldots \ldots \ldots \ldots \ldots \ldots \ldots \ldots \ldots . \ldots \ldots$

Appendix A. Thermal Conversion Factors $\ldots \ldots \ldots \ldots \ldots \ldots \ldots \ldots 141$

Appendix B. Metric and Other Physical Conversion Factors ....... 151

Appendix C. List of Features .................... 155

Glossary ................................... 159 
Section

1.1

1.2

1.3

1.4

1.5

1.6

1.7

1.8

1.9

1.10

1.11

1.12

Section 2. Energy Consumption

2.1

2.2

2.3

2.4

2.5

2.6

Section

3.1

Section

4.1

4.2

4.3

4.4

4.5

Section 5. Gil and Gas Resource Development

1. Energy Overview

Energy Summary for February 1994

\section{Petroleum}

Petroleum Overview Crude Oil Supply and Disposition Petroleum Imports Imports

\section{Natural Gas}

Natural Gas Production

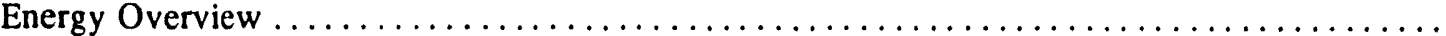

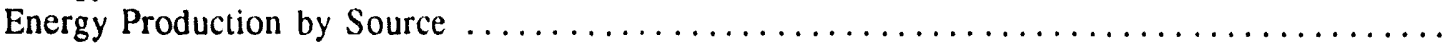

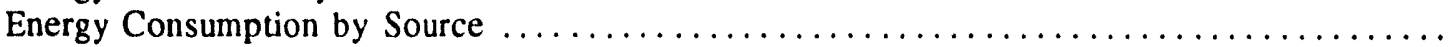

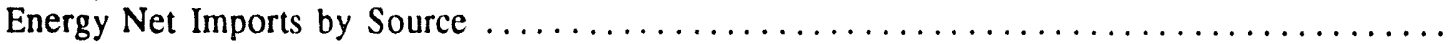

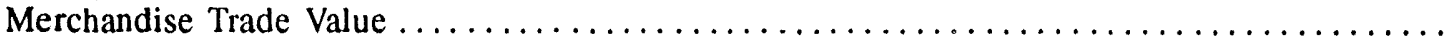

Energy Consumption per Dollar of Gross Domestic Product $\ldots \ldots \ldots \ldots \ldots \ldots \ldots \ldots \ldots \ldots$

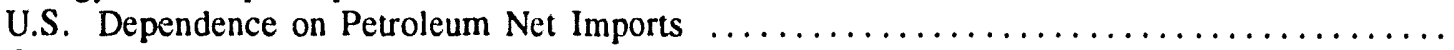

Cost of Fuels to End Users in Constant $(1982-1984)$ Dollars . . . . . . . . . . . . . . . . . . . . .

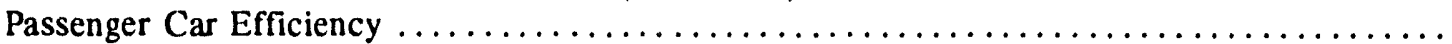

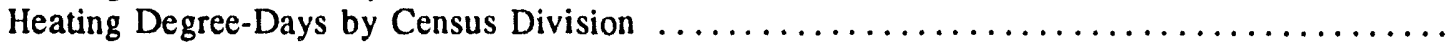

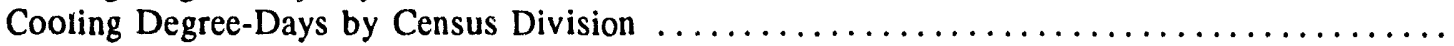

Energy Consumption Summary for February 1994

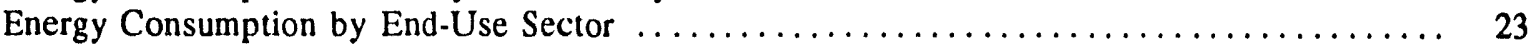

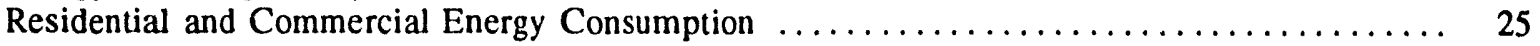

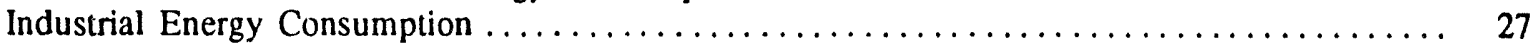

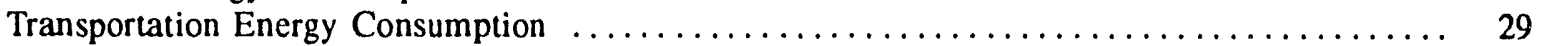

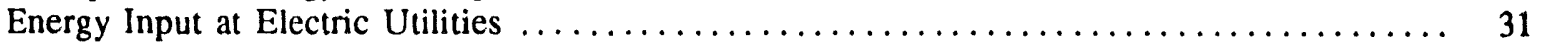

3.1a Field Production, Stock Change, Petroleum Products Supplied, and Ending Stocks ...

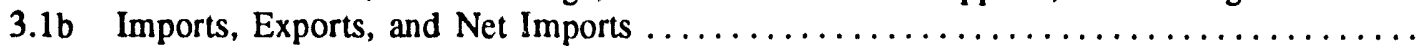

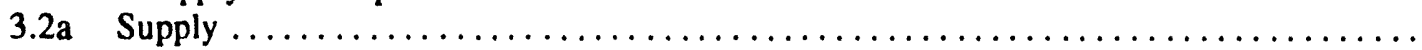

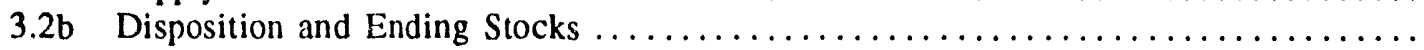

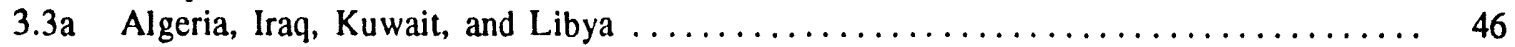

3.3b Qatar, Saudi Arabia, U.A.E., and Total Arab OPEC $\ldots \ldots \ldots \ldots \ldots \ldots \ldots \ldots \ldots \ldots \ldots$

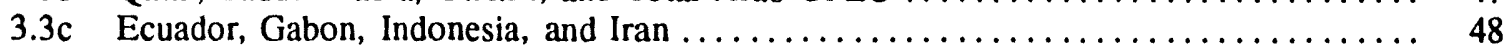

3.3d Nigeria, Venezuela, Total Non-Arab OPEC, and Total OPEC .............. 49

3.3e Angola, Australia, Bahama Islands, Brazil, Canada, and China ............... 50

3.3f Colombia, Ecuador, Italy, Malaysia, Mexico, and Netherlands .............. 51

3.3g Netherland Antilles, Norway, Puerto Rico, Russia, Spain, and Trinidad and Tobago .. 52

3.3h United Kingdom, Virgin Islands, Other Non-OPEC, Total Non-OPEC, and Total

Finished Motor Gasoline Supply and Disposition $\ldots \ldots \ldots \ldots \ldots \ldots \ldots \ldots$

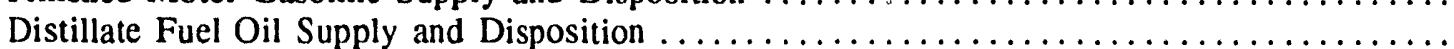

Residual Fuel Oil Supply and Disposition $\ldots \ldots \ldots \ldots \ldots \ldots \ldots \ldots \ldots \ldots \ldots \ldots \ldots \ldots \ldots$

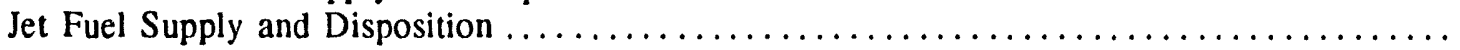

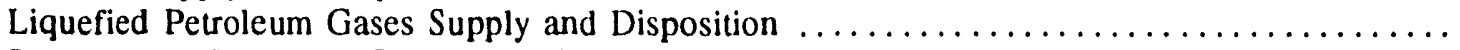

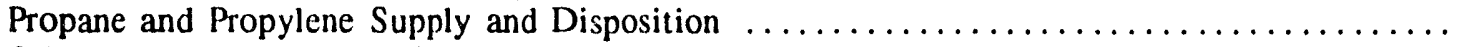

Other Petroleum Products Supply and Disposition $\ldots \ldots \ldots \ldots \ldots \ldots \ldots \ldots \ldots \ldots \ldots 6 . \ldots \ldots$

Natural Gas Supply and Disposition

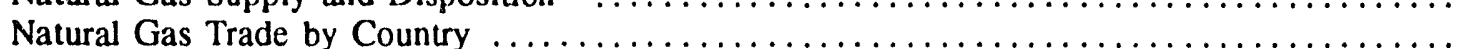

Natural Gas Consumption by End-Use Sector $\ldots \ldots \ldots \ldots \ldots \ldots \ldots \ldots \ldots \ldots \ldots \ldots \ldots \ldots \ldots \ldots$

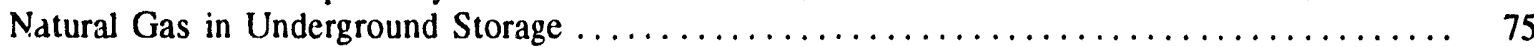

Oil and Gas Drilling Activity Measurements $\ldots \ldots \ldots \ldots \ldots \ldots \ldots \ldots \ldots \ldots \ldots \ldots \ldots \ldots$

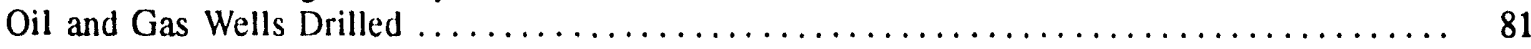




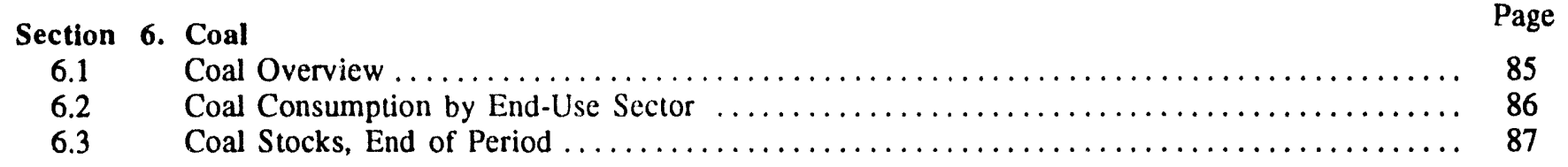

Section 7. Electricity

7.1 Electric Utility Net Generation of Electricity $\ldots \ldots \ldots \ldots \ldots \ldots \ldots \ldots \ldots \ldots \ldots \ldots \ldots$

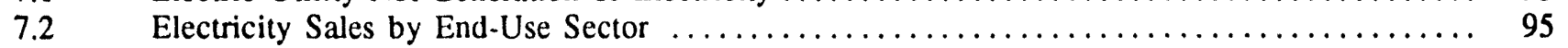

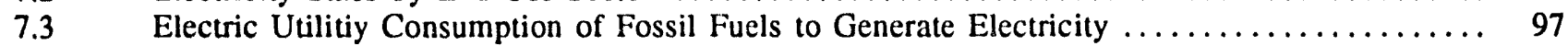

7.4 Electric Utility Stocks of Coal and Petroleum, End of Period ................... 98

$\begin{array}{crl}\text { Section } & \text { 8. } \begin{array}{l}\text { Nuclear Energy } \\ 8.1\end{array} & \text { Nuclear Power Plant Operations } \ldots \ldots \ldots \ldots \ldots \ldots \ldots \ldots \ldots \ldots \ldots \ldots \ldots \ldots \ldots \ldots \ldots \\ 8.2 & \text { Nuclear Gen } \ldots \ldots \ldots \ldots \ldots\end{array}$

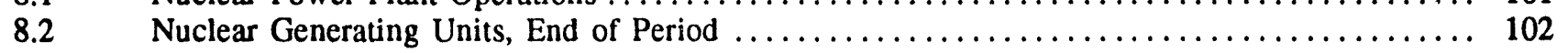

Section
9.1 $\quad \begin{aligned} & \text { Energy Prices } \\ & \text { Crude Oil Price Summary } \ldots \ldots \ldots \ldots \ldots \ldots \ldots \ldots \ldots \ldots \ldots \ldots \ldots \ldots \ldots \ldots \ldots \ldots \ldots \ldots\end{aligned}$

$9.2 \quad$ F.O.B. Cost of Crude Oil Imports from Selected Countries $\ldots \ldots \ldots \ldots \ldots \ldots \ldots \ldots \ldots \ldots \ldots$

$9.3 \quad$ Landed Cost of Crude Oil Imports from Selected Countries $\ldots \ldots \ldots \ldots \ldots \ldots \ldots \ldots \ldots \ldots \ldots \ldots$

9.4 Motor Gasoline Retail Prices, U.S. City Average $\ldots \ldots \ldots \ldots \ldots \ldots \ldots \ldots \ldots \ldots \ldots \ldots \ldots 110$

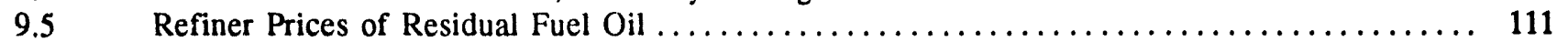

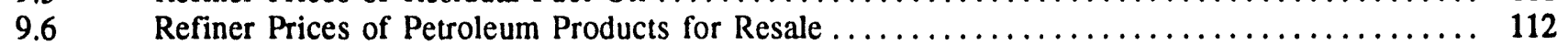

9.7 Refiner Prices of Petroleum Products to End Users $\ldots \ldots \ldots \ldots \ldots \ldots \ldots \ldots \ldots \ldots \ldots \ldots$

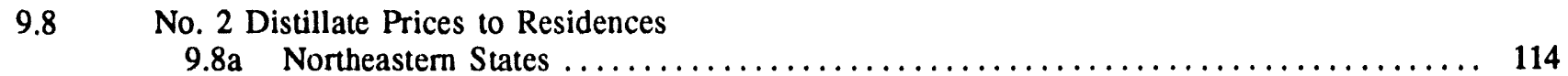

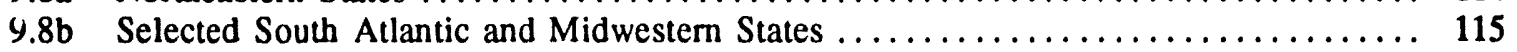

9.8c Selected Western States and U.S. Average ............................ 116

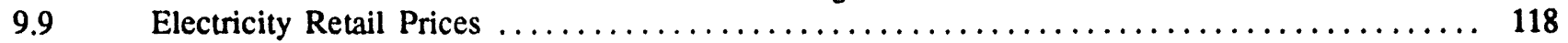

9.10 Quantity and Cost of Fossil-Fuel Receipts at Steam-Electric Utility Plants ............. 119

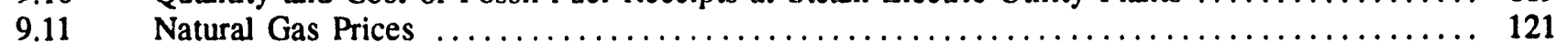

\section{Section 10. International Energy}

10.1 World Crude Oil Production

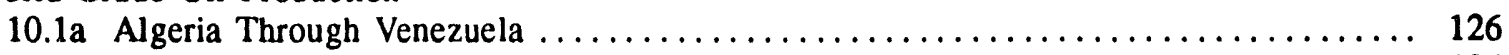

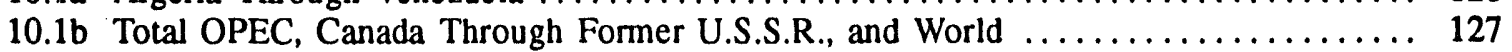

10.2 Petroleum Consumption in OECD Countries $\ldots \ldots \ldots \ldots \ldots \ldots \ldots \ldots \ldots \ldots \ldots \ldots \ldots \ldots \ldots \ldots$

10.3 Petroleum Stocks in OECD Countries, End of Period $\ldots \ldots \ldots \ldots \ldots \ldots \ldots \ldots \ldots \ldots \ldots \ldots$

Nuclear Electricity Gross Generation
$10.4 \mathrm{a}$ Regions and World $\ldots \ldots \ldots \ldots \ldots \ldots \ldots \ldots \ldots \ldots \ldots \ldots \ldots \ldots \ldots \ldots \ldots \ldots \ldots \ldots \ldots \ldots \ldots \ldots$

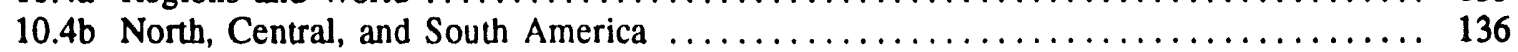

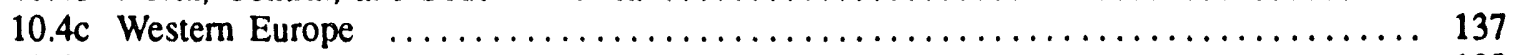

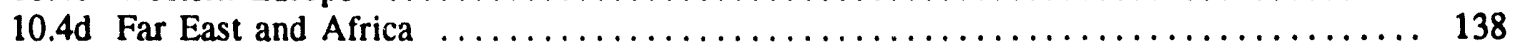

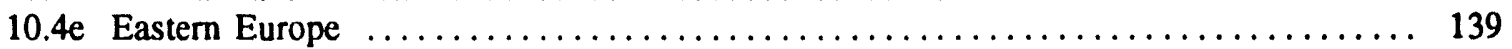

Appendix A. Thermal Conversion Factors

A1. Approximate Heat Content of Petroleum Products $\ldots \ldots \ldots \ldots \ldots \ldots \ldots \ldots \ldots \ldots \ldots 141$

A2. Approximate Heat Content of Crude Oil, Crude Oil and Products, and Natural Gas Plant Liquids 142

A3. Approximate Heat Content of Petroleum Product Weighted Averages $\ldots \ldots \ldots \ldots \ldots \ldots \ldots 142$

A4. Approximaie Heat Content of Natural Gas $\ldots \ldots \ldots \ldots \ldots \ldots \ldots \ldots \ldots \ldots \ldots \ldots \ldots \ldots$

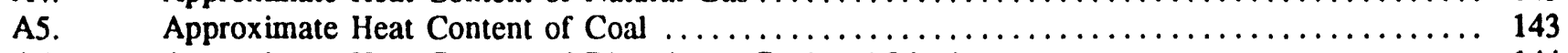

A6. Approximate Heat Content of Bituminous Coal and Lignite $\ldots \ldots \ldots \ldots \ldots \ldots \ldots \ldots \ldots \ldots 144$

A7. Approximate Heat Content of Anthracite and Coal Coke $\ldots \ldots \ldots \ldots \ldots \ldots \ldots \ldots \ldots \ldots \ldots \ldots$

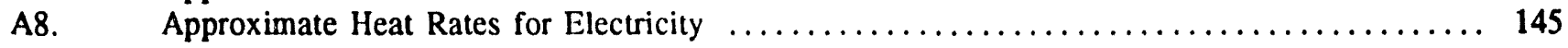

Appendix B. Metric and Other Physical Conversion Factors

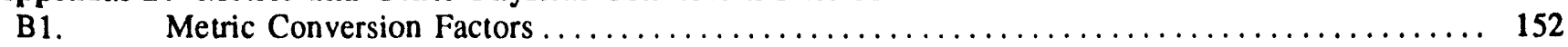

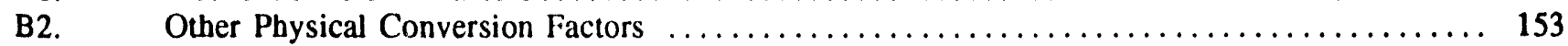

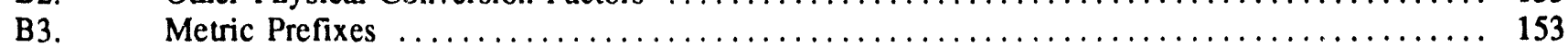


Section 1. Energy Overview

1.1 Energy Overview

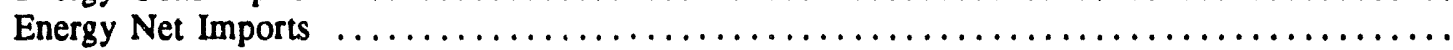

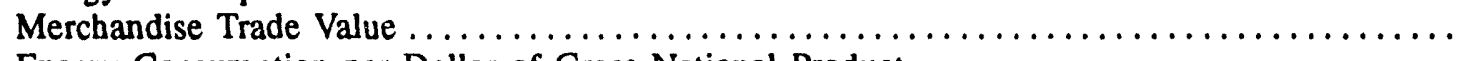

Energy Consumption per Dollar of Gross National Product $\ldots \ldots \ldots \ldots \ldots \ldots \ldots \ldots \ldots \ldots$

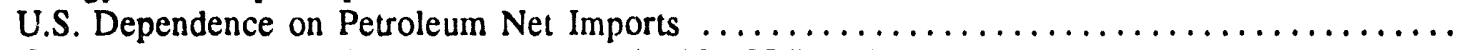

Cost of Fuels to End Users in Constant (1982-1984) Dollars ...................... 14

\section{Section 2. Energy Consumption}

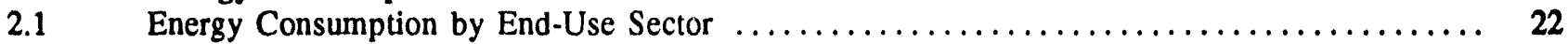

Residential and Commercial Energy Consumption .

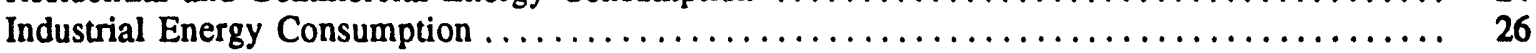

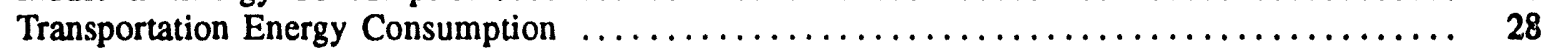

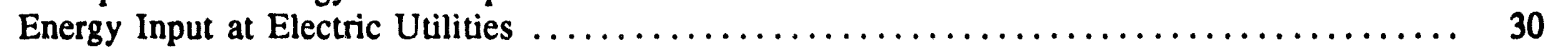

\section{Section 3. Petroleum}

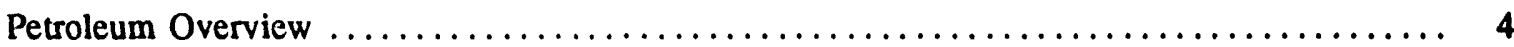

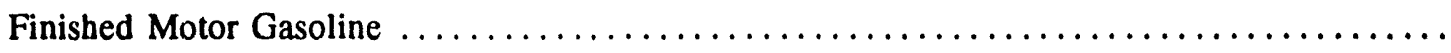

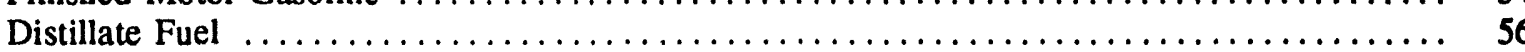

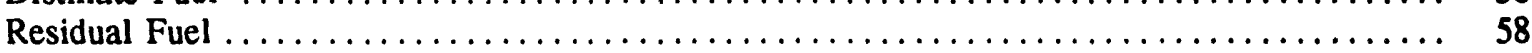

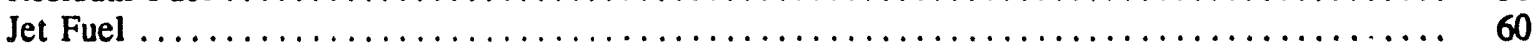

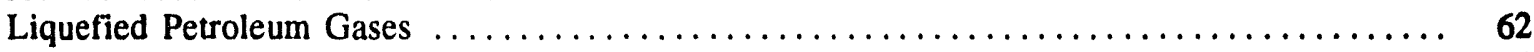

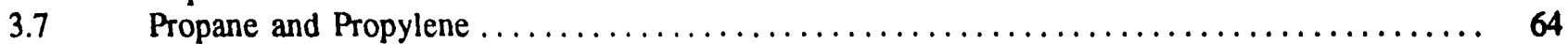

\section{Section 4. Natural Gas}

4.1 Natural Gas

Section 5. Oil and Gas Resource Development

$5.1 \quad$ Oil and Gas Resource Development Indicators $\ldots \ldots \ldots \ldots \ldots \ldots \ldots \ldots \ldots \ldots \ldots \ldots$

Section 6. Coal

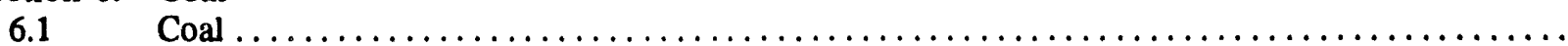

\section{Section 7. Electricity}

$7.1 \quad$ Electric Utility Net Generation of Electricity $\ldots \ldots \ldots \ldots \ldots \ldots \ldots \ldots \ldots \ldots \ldots \ldots \ldots \ldots \ldots$

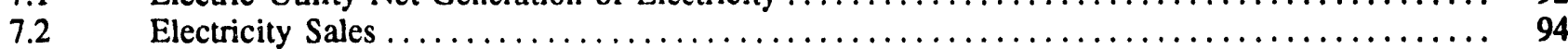

7.3 Electric Utility Consumption and Stocks of Fossil Fuels $\ldots \ldots \ldots \ldots \ldots \ldots \ldots \ldots \ldots \ldots$

Section 8. Nuclear Energy

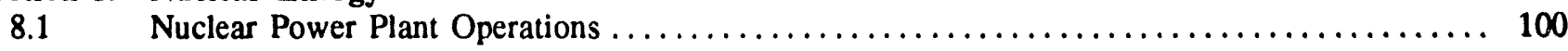

\section{Section 9. Energy Prices}

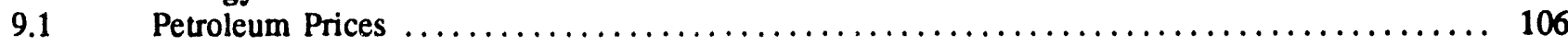

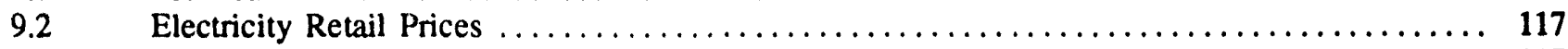

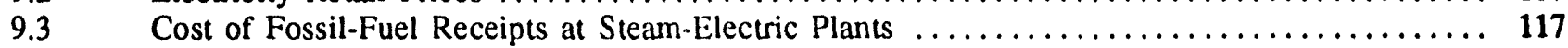

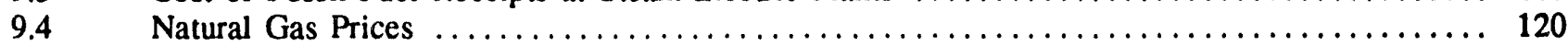

Section 10. International Energy

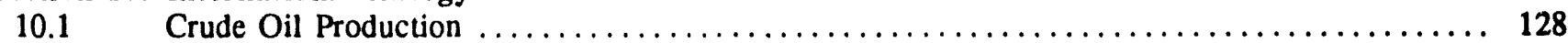

$10.2 \quad$ Crude Oil Production by Selected Country . . . . . . . . . . . . . . . . . . . . . . . . . . . . . . 129

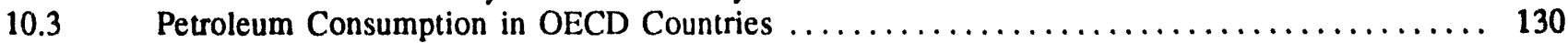

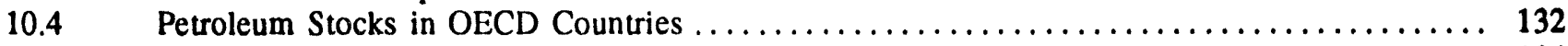

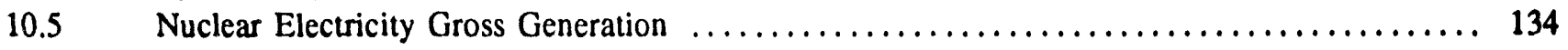




\section{Section 1. Energy Overview}

Energy production during February 1994 totaled 5.3 quadrillion Btu, a 2.2-percent increase from the level of production during February 1993. Coal production increased 9.0 percent, natural gas production rose 2.5 percent, and petroleum production decreased 3.6 percent. All other forms of energy production combined were down 3.0 percent from the level of production during February 1993.

Energy consumption during February 1994 totaled 7.5 quadrillion Btu, 4.1 percent above the level of consumption during February 1993. Natural gas consumption increased 5.8 percent, petroleum consumption rose 5.2 percent, and coal consumption was up 2.3 nercent. Consumption of all other forms of energy combined decreased 0.7 percent from the level 1 year earlier.

Net imports of energy during February 1994 totaled 1.4 quadrillion Btu, 16.9 percent above the level of net imports 1 year earlier. Net imports of petroleum increased 10.1 percent, and net imports of natural gas were down 4.9 percent. Net exports of coal fell 43.7 percent from the level in February 1993.

\section{Table 1.1 Energy Summary for February 1994} (Quadrillion Btu)

\begin{tabular}{|c|c|c|c|c|c|c|c|c|}
\hline & \multicolumn{3}{|c|}{ Fobruary } & \multicolumn{5}{|c|}{ Cumulative January Through February } \\
\hline 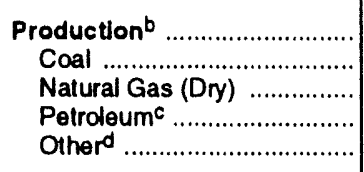 & $\begin{array}{r}5.307 \\
1.784 \\
1.506 \\
1.270 \\
.746\end{array}$ & $\begin{array}{r}5.192 \\
1.637 \\
1.470 \\
1.317 \\
.769\end{array}$ & $\begin{array}{r}2.2 \\
9.0 \\
2.5 \\
-3.6 \\
-3.0\end{array}$ & $\begin{array}{r}10.887 \\
3.463 \\
3.177 \\
2.679 \\
1.568\end{array}$ & $\begin{array}{r}0.185 \\
.059 \\
.054 \\
.045 \\
.027\end{array}$ & $\begin{array}{r}10.913 \\
3.362 \\
3.100 \\
2.781 \\
1.670\end{array}$ & $\begin{array}{r}0.185 \\
.057 \\
.053 \\
.047 \\
.028\end{array}$ & $\begin{array}{r}-0.2 \\
3.0 \\
2.5 \\
-3.6 \\
-6.1\end{array}$ \\
\hline 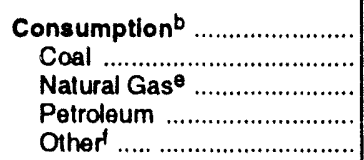 & $\begin{array}{r}7.485 \\
1.576 \\
2.367 \\
2.757 \\
.785\end{array}$ & $\begin{array}{r}7.188 \\
1.539 \\
2.237 \\
2.521 \\
.791\end{array}$ & $\begin{array}{l}4.1 \\
2.3 \\
5.8 \\
5.2 \\
-.7\end{array}$ & $\begin{array}{r}15.763 \\
3.395 \\
4.977 \\
5.747 \\
1.644\end{array}$ & $\begin{array}{l}.267 \\
.058 \\
.084 \\
.097 \\
.028\end{array}$ & $\begin{array}{r}14.857 \\
3.199 \\
4.596 \\
5.344 \\
1.719\end{array}$ & $\begin{array}{l}.252 \\
.054 \\
.078 \\
.091 \\
.029\end{array}$ & $\begin{array}{r}6.1 \\
6.1 \\
8.3 \\
7.6 \\
-4.4\end{array}$ \\
\hline $\begin{array}{l}\text { Not Imports } \\
\text { Coap } \\
\text { Natural Gas } \\
\text { Petroleum } \\
\text { Other' }\end{array}$ & $\begin{array}{r}1.363 \\
. .093 \\
.163 \\
1.253 \\
.040\end{array}$ & $\begin{array}{r}1.166 \\
-.166 \\
.172 \\
1.138 \\
.022\end{array}$ & $\begin{array}{r}16.9 \\
-43.7 \\
-4.9 \\
10.1 \\
80.3\end{array}$ & $\begin{array}{r}2.787 \\
-.204 \\
.389 \\
2.527 \\
.076\end{array}$ & $\begin{array}{l}.047 \\
.003 \\
.007 \\
.043 \\
.001\end{array}$ & $\begin{array}{r}2.461 \\
-.328 \\
.354 \\
2.387 \\
.049\end{array}$ & $\begin{array}{r}.042 \\
.006 \\
.006 \\
.040 \\
.001\end{array}$ & $\begin{array}{r}13.2 \\
-37.8 \\
9.8 \\
5.9 \\
55.3\end{array}$ \\
\hline
\end{tabular}

\footnotetext{
a Based on daily rates prior to rounding.

b Due to a lack of consistent historical data, some renewable energy sources are not included. For example, in 1991, 3.3 quadrillion Btu of renewable energy consumed by U.S. electric utilities to generate electricity for distribution is included, but an estimated 3.4 quadrillion Btu of renewable energy used by other sectors is not included.

c Includes crude oil, lease condensate, and natural gas plant liquids.

d "Other" is hydroelectric and nuclear electric power, and electricity generated for distribution from wood, waste, geothermal, wind, photovollaic, and solar thermal energy.

- Includes supplemental gaseous fuels.
}

1 "Other" is hydroelectric and nuclear electric power; electricity generated for distribution from wood, waste, geothermal, wind, photovoltaic, and solar thermal energy; and net imports of electricity and coal coke.

9 . Minus sign indicates exports are greater than imponts.

h Includes crude oil, lease condensate, petroleum products, pentanes plus, unfinished oils, gasoline blending components, and imports of crude oil for the Strategic Petroleum Reserve.

"Other" is net imports of electricity and coal coke.

Note: Totals may not equal sum of components due to independent rounding.

Sources: Tables 1.3, 1.4, and 1.5. 
Figure 1.1 Energy Overview

(Quadrillion Btu)

Consumption, Production, and Imports, 1973-1993

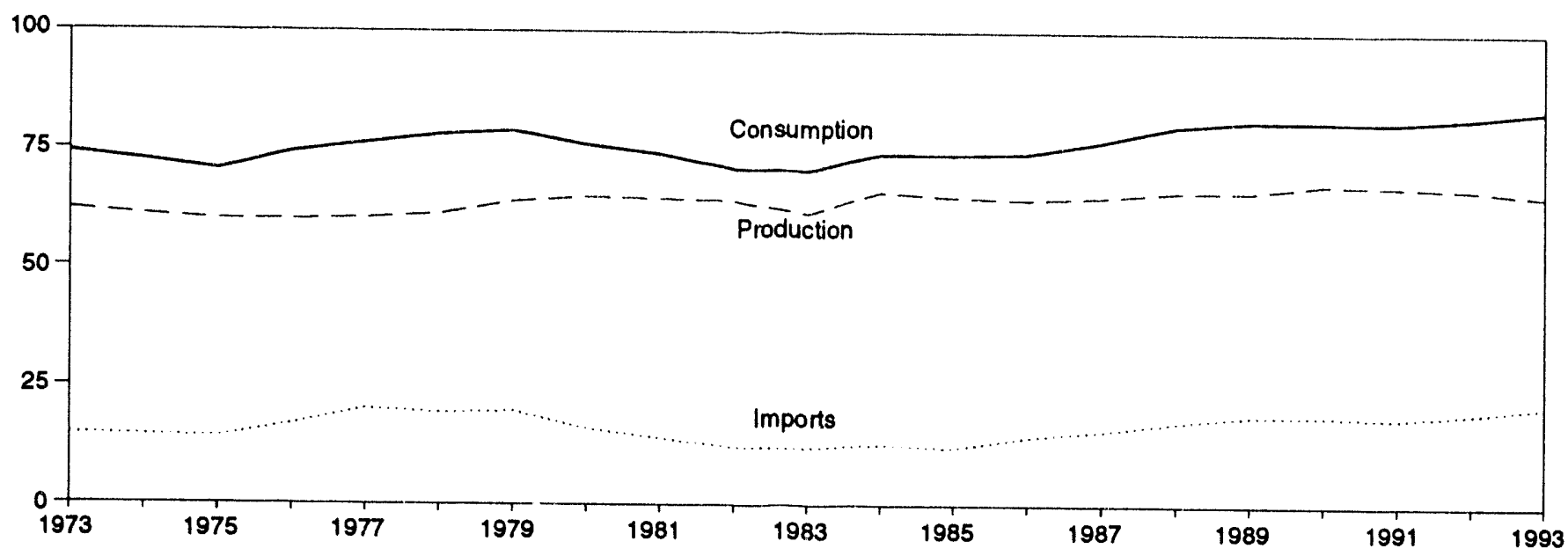

Consumption, Production, and Imports, Monthly

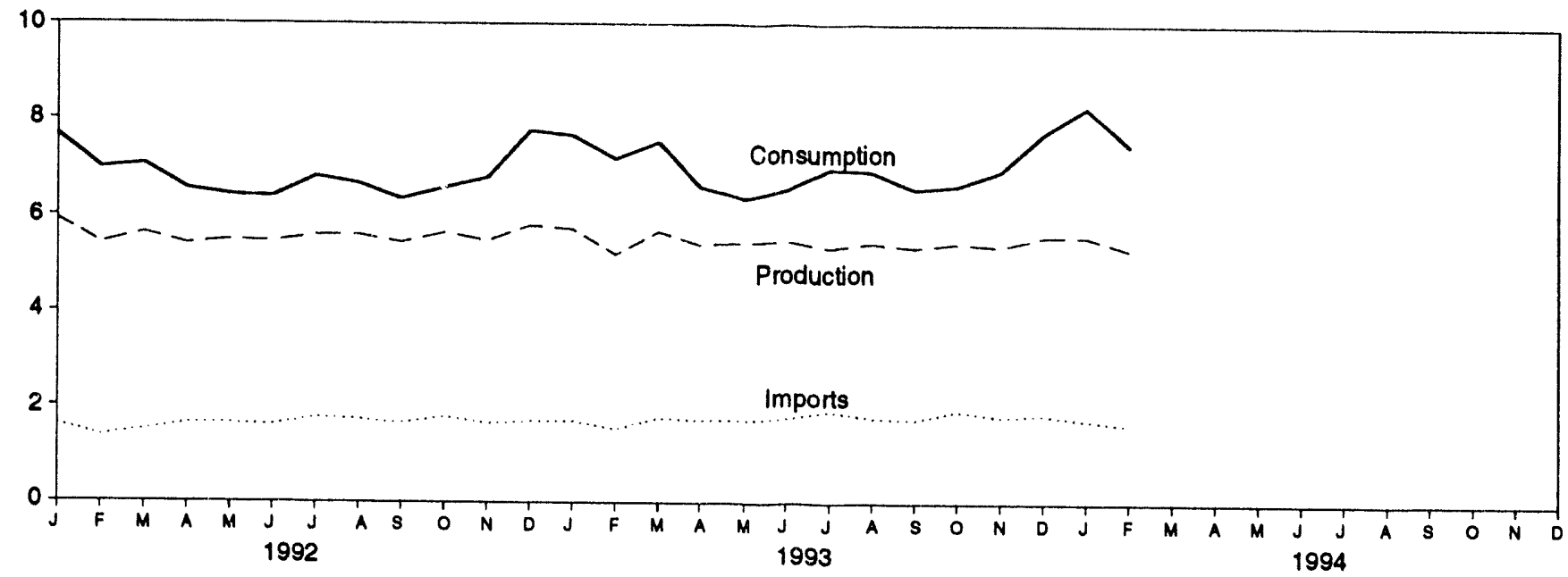

Overview, February 1994

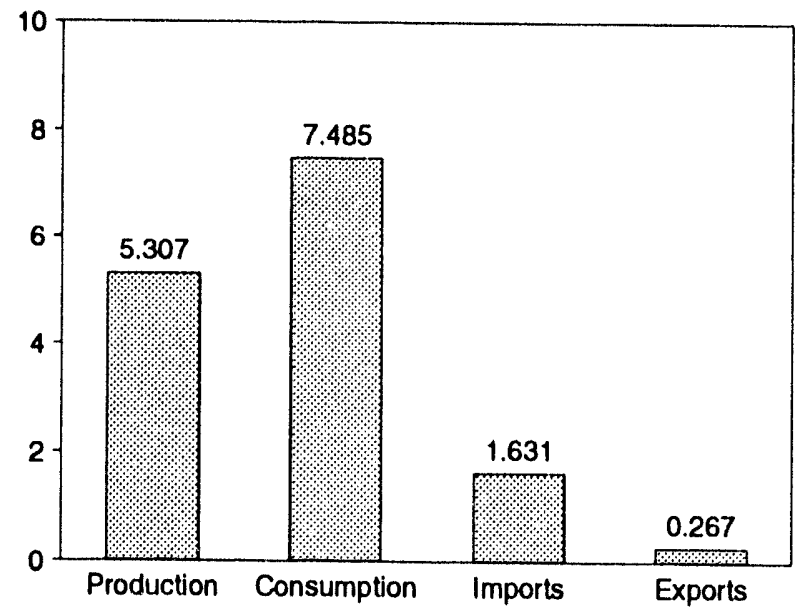

Net Imports, January and February

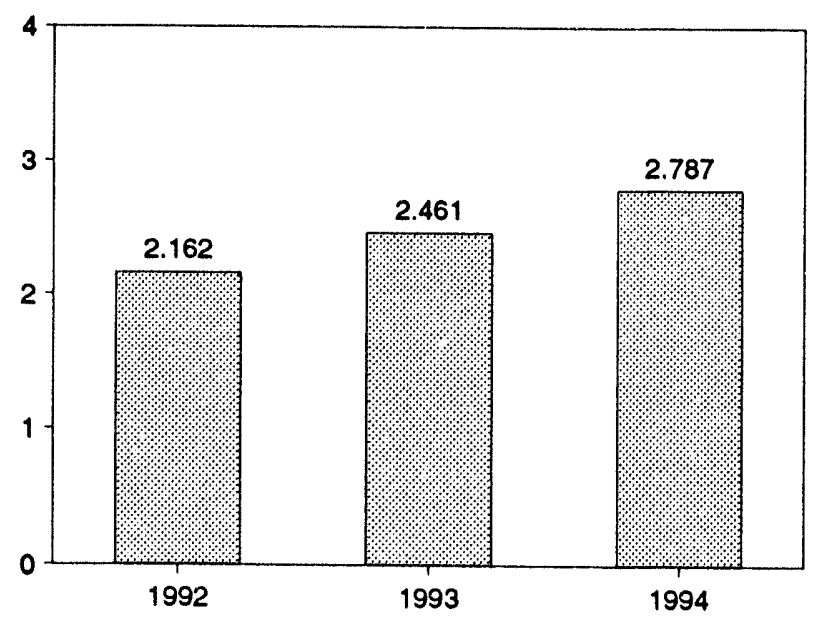

Nole: Because vertical scales differ, graphs should not be compared. Source: Table 1.2. 


\begin{tabular}{|c|c|c|c|c|c|}
\hline & Production $^{a}$ & Consumption $a, b$ & Imports & Exports & Net Imports \\
\hline 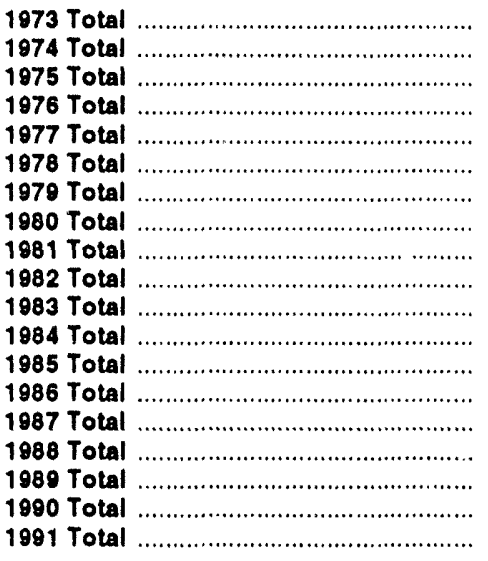 & $\begin{array}{l}62.060 \\
60.835 \\
59.860 \\
59.892 \\
60.219 \\
61.103 \\
63.801 \\
64.761 \\
64.421 \\
63.962 \\
81.279 \\
65.962 \\
64.871 \\
64.350 \\
64.952 \\
66.105 \\
66.129 \\
67.853 \\
67.484\end{array}$ & $\begin{array}{l}74.282 \\
72.543 \\
70.546 \\
74.362 \\
76.286 \\
78.089 \\
78.898 \\
75.955 \\
73.990 \\
70.848 \\
70.524 \\
74.144 \\
73.981 \\
74.297 \\
76.884 \\
80.218 \\
81.325 \\
81.265 \\
81.116\end{array}$ & $\begin{array}{l}14.731 \\
14.413 \\
14.111 \\
16.837 \\
20.090 \\
19.254 \\
18.616 \\
15.971 \\
13.975 \\
12.092 \\
12.027 \\
12.767 \\
12.103 \\
14.438 \\
15.764 \\
17.564 \\
18.947 \\
18.987 \\
18.577\end{array}$ & $\begin{array}{l}2.051 \\
2.223 \\
2.359 \\
2.188 \\
2.071 \\
1.931 \\
2.870 \\
3.723 \\
4.329 \\
4.633 \\
3.717 \\
3.804 \\
4.231 \\
4.055 \\
3.853 \\
4.415 \\
4.765 \\
4.910 \\
5.220\end{array}$ & $\begin{array}{r}12.680 \\
12.190 \\
11.752 \\
14.648 \\
18.019 \\
17.323 \\
16.746 \\
12.247 \\
9.646 \\
7.460 \\
8.310 \\
8.963 \\
7.872 \\
10.382 \\
11.811 \\
13.149 \\
14.181 \\
14.077 \\
13.357\end{array}$ \\
\hline 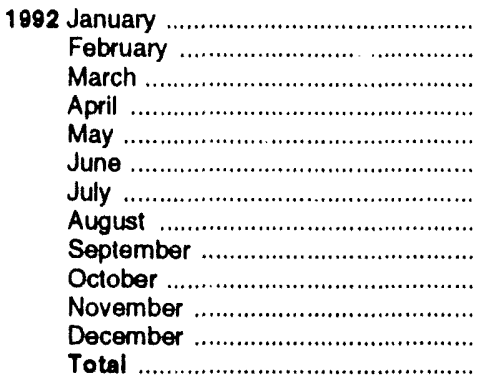 & $\begin{array}{r}5.919 \\
5.415 \\
5.630 \\
5.407 \\
5.491 \\
5.461 \\
5.587 \\
5.594 \\
5.439 \\
5.640 \\
5.479 \\
5.792 \\
66.853\end{array}$ & $\begin{array}{r}7.678 \\
6.989 \\
7.070 \\
6.565 \\
6.435 \\
6.403 \\
6.822 \\
6.673 \\
6.356 \\
6.590 \\
6.798 \\
7.765 \\
82.144\end{array}$ & $\begin{array}{r}1.615 \\
1.377 \\
1.500 \\
1.639 \\
1.641 \\
1.609 \\
1.770 \\
1.727 \\
1.654 \\
1.781 \\
1.650 \\
1.688 \\
19.650\end{array}$ & $\begin{array}{r}.458 \\
.372 \\
.416 \\
.413 \\
.434 \\
.426 \\
.441 \\
.367 \\
.417 \\
.383 \\
.428 \\
.462 \\
\mathbf{5 . 0 1 7}\end{array}$ & $\begin{array}{r}1.157 \\
1.005 \\
1.084 \\
1.226 \\
1.207 \\
1.183 \\
1.329 \\
1.360 \\
1.237 \\
1.399 \\
1.221 \\
1.226 \\
14.633\end{array}$ \\
\hline 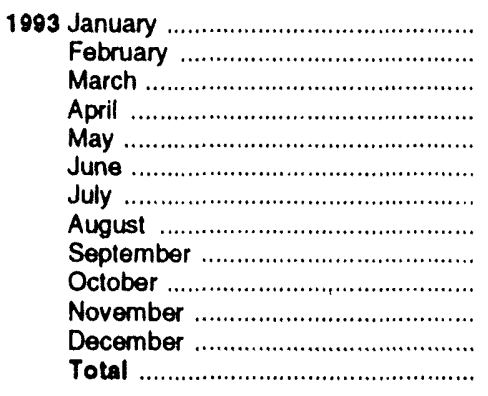 & $\begin{array}{l}R_{5} 5.720 \\
R_{5.192} \\
R_{5.669} \\
R_{5.399} \\
R_{5.447} \\
R_{5.482} \\
R_{5.337} \\
R_{5.436} \\
R_{5.351} \\
R_{5.444} \\
R_{5.371} \\
R_{5.573} \\
R_{65.423}\end{array}$ & $\begin{array}{c}{ }^{R} 7.670 \\
{ }^{R} 7.188 \\
R_{7.541} \\
R_{6.611} \\
R_{6.371} \\
{ }^{R} 6.552 \\
R_{6.990} \\
R_{6.938} \\
R_{6.575} \\
R_{6}{ }_{6.644} \\
{ }^{R} 6.948 \\
R_{7.730} \\
R_{83.759}\end{array}$ & $\begin{array}{r}1.694 \\
1.529 \\
1.762 \\
1.718 \\
1.721 \\
1.766 \\
1.913 \\
1.778 \\
1.744 \\
1.926 \\
1.805 \\
\text { R } 1.846 \\
\mathrm{R}_{21.202}\end{array}$ & 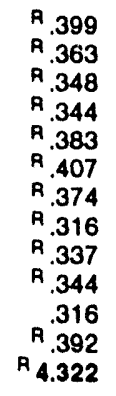 & $\begin{array}{r}R_{1} .295 \\
A_{1} .166 \\
A_{1.414} \\
A_{1} 1.374 \\
A_{1} .338 \\
A_{1} .359 \\
A_{1} .540 \\
A_{1.462} \\
A_{1.407} \\
1.582 \\
A_{1.488} \\
A_{1} 1.454 \\
R_{1} 16.880\end{array}$ \\
\hline 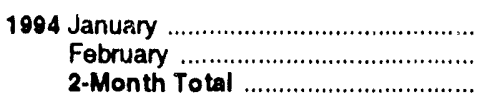 & $\begin{array}{r}A_{5.581} \\
5.307 \\
10.887\end{array}$ & $\begin{array}{r}{ }^{A} 8.278 \\
7.485 \\
15.763\end{array}$ & $\begin{array}{r}1.730 \\
1.631 \\
3.361\end{array}$ & $\begin{array}{r}\text { ค } .306 \\
.267 \\
.574\end{array}$ & $\begin{array}{r}\text { A } 1.424 \\
1.363 \\
2.787\end{array}$ \\
\hline 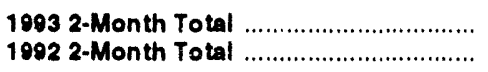 & $\begin{array}{l}10.913 \\
11.333\end{array}$ & $\begin{array}{l}14.857 \\
14.668\end{array}$ & $\begin{array}{l}3.223 \\
2.991\end{array}$ & $\begin{array}{l}.762 \\
.830\end{array}$ & $\begin{array}{l}2.461 \\
2.162\end{array}$ \\
\hline
\end{tabular}

Due to a lack of consistent historical data, some renewable energy sources are not included. For example, in 1991, 3.3 quadrillion Btu of renewable energy consumed by U.S. electric utilities to generate electricity for distribution is included, but an estimated 3.4 quadrillion Btu of renewable energy used by other sectors is not included.

The sum of domestic energy production and net imports of energy does not equal domestic energy consumption. The difference is attributed to stock changes; losses and gains in conversion, transportation, and distribution; the addition of blending compounds; shipments of anthracite to U.S. Armed
Forces in Europe; and adjustments to account for discrepancles between reporting systems.

$\mathrm{R}=$ Revised data.

Notes: - For delinitions, see Notes 1 through 4 at end of section. - Geographic coverage is the 50 States and the District of Columbia. - Totals may not equal sum of components due to independent rounding.

Sources: • Production: Table 1.3. • Consumption: Table 1.4. • Imports and Exports: Tables 3.1b, 4.2, 6.1, A2-A8, and Section 2, "Energy Consumption Notes and Sources," Notes 8 and 9. - Net Imports: Table 1.5. 
Figure 1.2 Energy Production

(Quadrillion Btu)

Total Production, 1973-1993

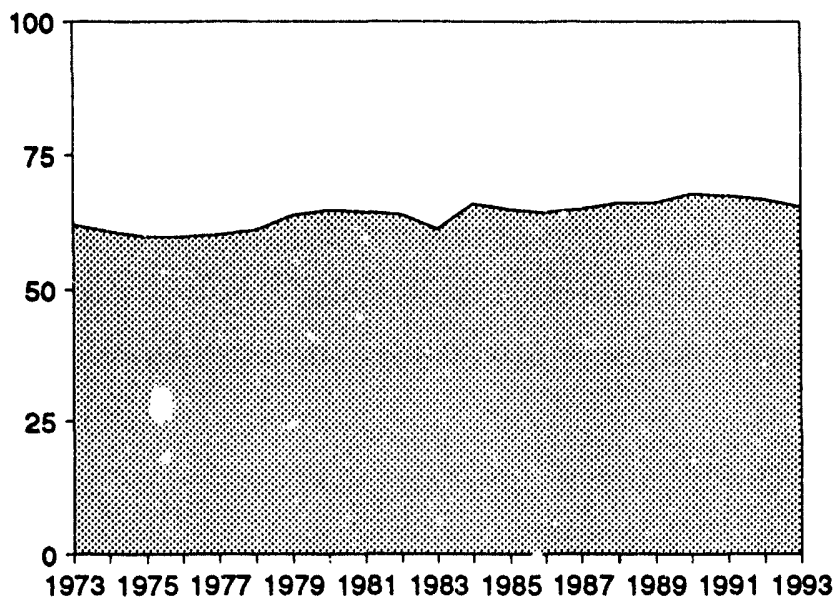

Production by Major Sources, 1973-1993

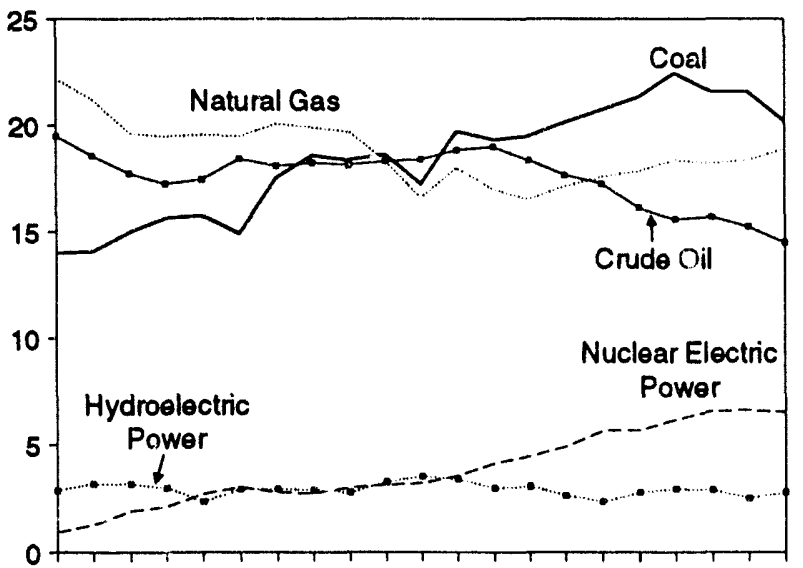

19731975197719791981198319851987198919911993

Total Production, January and February

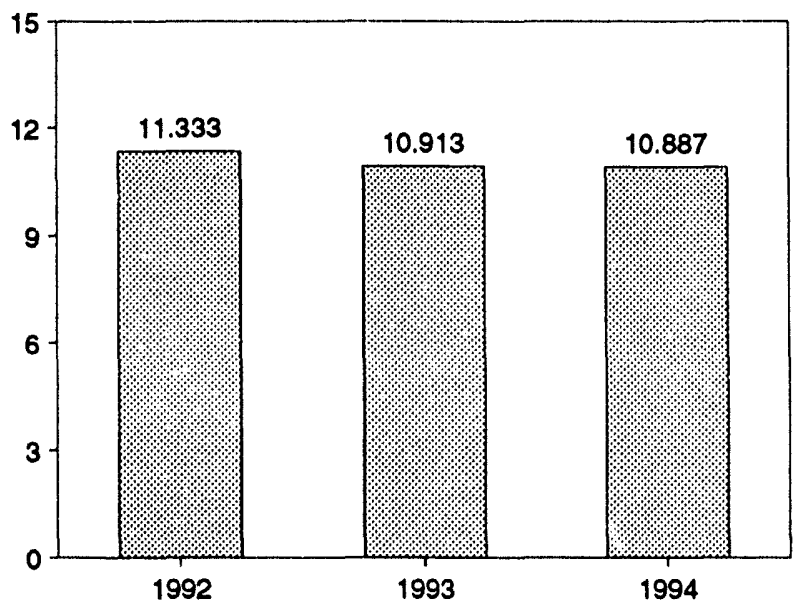

Total Production, Monthly

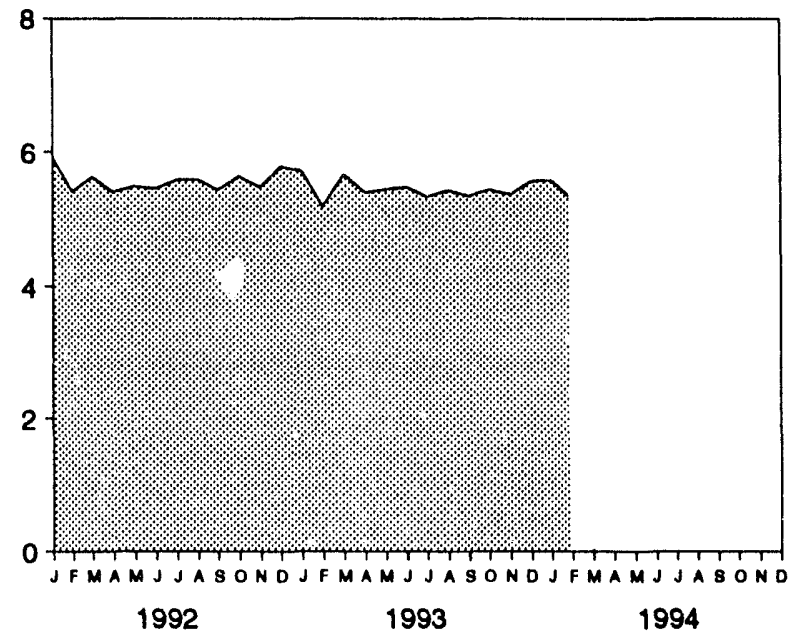

Production by Major Sources, Monthly

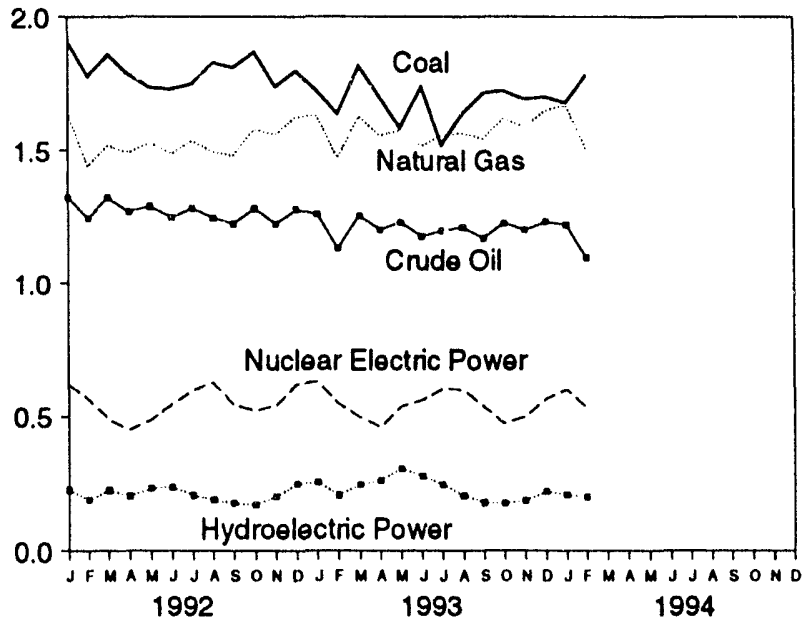

Production by Major Sources, February 1994

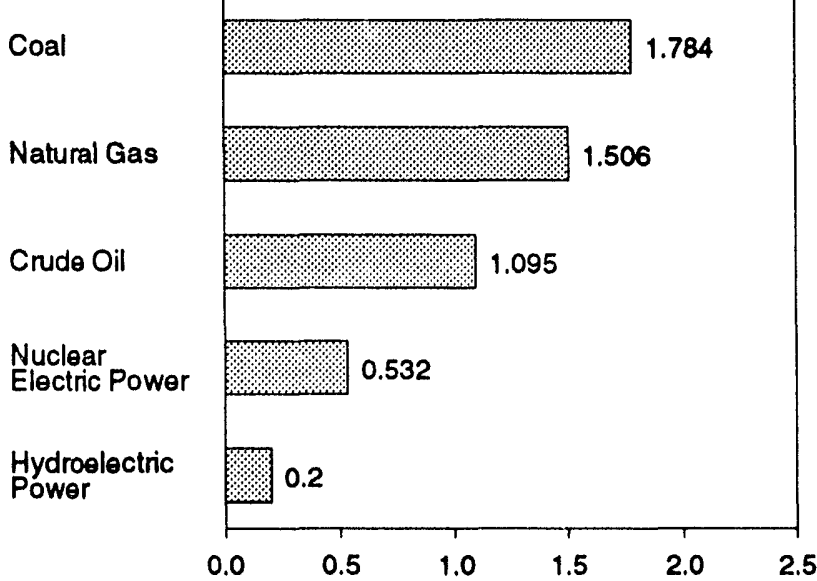

Nole: Because vertical scales difler, graphs should not be compared Source: Table 1.3. 
Table 1.3 Energy Production by Source

(Quadrillion Btu)

\begin{tabular}{|c|c|c|c|c|c|c|c|c|c|}
\hline & Coal & $\begin{array}{l}\text { Natural } \\
\text { Gas } \\
\text { (Dry) }\end{array}$ & $\begin{array}{c}\text { Crude } \\
\text { Olla }\end{array}$ & $\begin{array}{l}\text { Natural } \\
\text { Gas Plant } \\
\text { Llquids }\end{array}$ & $\begin{array}{l}\text { Nuclear } \\
\text { Electric } \\
\text { Power }\end{array}$ & $\begin{array}{l}\text { Hydro- } \\
\text { olectric } \\
\text { Poworb }\end{array}$ & $\begin{array}{c}\text { Goothermal } \\
\text { Enorgy }\end{array}$ & Otherc & Totald \\
\hline 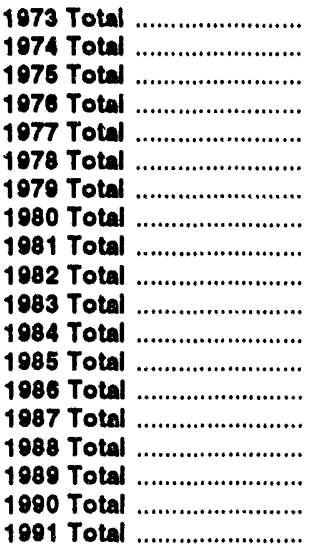 & $\begin{array}{l}13.993 \\
14.074 \\
14.090 \\
15.654 \\
15.755 \\
14.010 \\
17.530 \\
18.597 \\
18.376 \\
18.639 \\
17.246 \\
18.719 \\
19.325 \\
10.510 \\
20.142 \\
20.737 \\
21.345 \\
22.456 \\
21.594\end{array}$ & $\begin{array}{l}22.187 \\
21.210 \\
19.640 \\
19.480 \\
19.565 \\
19.485 \\
20.076 \\
19.908 \\
19.699 \\
18.319 \\
16.593 \\
18.008 \\
16.980 \\
16.541 \\
17.136 \\
17.599 \\
17.847 \\
18.362 \\
18.229\end{array}$ & $\begin{array}{l}10.493 \\
18.575 \\
17.729 \\
17.262 \\
17.454 \\
18.434 \\
18.104 \\
18.249 \\
18.146 \\
18.309 \\
18.392 \\
18.848 \\
18.992 \\
18.376 \\
17.675 \\
17.270 \\
16.117 \\
15.571 \\
15.701\end{array}$ & $\begin{array}{l}2.569 \\
2.471 \\
2.374 \\
2.327 \\
2.327 \\
2.245 \\
2.286 \\
2.254 \\
2.307 \\
2.191 \\
2.184 \\
2.274 \\
2.241 \\
2.149 \\
2.215 \\
2.260 \\
2.158 \\
2.175 \\
2.306\end{array}$ & $\begin{array}{l}0.910 \\
1.272 \\
1.900 \\
2.111 \\
2.702 \\
3.024 \\
2.776 \\
2.739 \\
3.008 \\
3.131 \\
3.203 \\
3.553 \\
4.149 \\
4.471 \\
4.906 \\
5.661 \\
5.677 \\
6.161 \\
6.579\end{array}$ & $\begin{array}{l}2.861 \\
3.177 \\
3.155 \\
2.978 \\
2.333 \\
2.937 \\
2.931 \\
2.900 \\
2.758 \\
3.266 \\
3.527 \\
3.386 \\
2.970 \\
3.071 \\
2.635 \\
2.334 \\
2.767 \\
2.926 \\
2.885\end{array}$ & $\begin{array}{l}0.043 \\
.053 \\
.070 \\
.078 \\
.077 \\
.064 \\
.084 \\
.110 \\
.123 \\
.105 \\
.129 \\
.165 \\
.198 \\
.219 \\
.220 \\
.217 \\
.197 \\
.181 \\
.170\end{array}$ & $\begin{array}{l}0.003 \\
.003 \\
.002 \\
.003 \\
.005 \\
.003 \\
.005 \\
.005 \\
.004 \\
.003 \\
.004 \\
.009 \\
.015 \\
.012 \\
.016 \\
.017 \\
.020 \\
.021 \\
.021\end{array}$ & $\begin{array}{l}62.060 \\
60.835 \\
59.860 \\
59.892 \\
60.219 \\
61.103 \\
63.801 \\
64.761 \\
64.421 \\
63.962 \\
61.279 \\
65.962 \\
64.871 \\
64.350 \\
64.952 \\
66.105 \\
66.129 \\
67.853 \\
67.484\end{array}$ \\
\hline 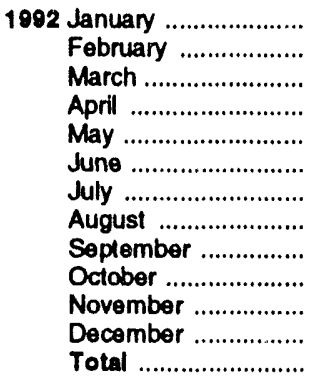 & $\begin{array}{r}1.904 \\
1.778 \\
1.859 \\
1.785 \\
1.737 \\
1.732 \\
1.750 \\
1.830 \\
1.811 \\
1.869 \\
1.739 \\
1.799 \\
21.583\end{array}$ & $\begin{array}{r}1.633 \\
1.440 \\
1.519 \\
1.491 \\
1.529 \\
1.488 \\
1.536 \\
1.495 \\
1.481 \\
1.579 \\
1.559 \\
1.626 \\
18.375\end{array}$ & $\begin{array}{r}1.323 \\
1.243 \\
1.321 \\
1.269 \\
1.289 \\
1.247 \\
1.282 \\
1.245 \\
1.223 \\
1.281 \\
1.222 \\
1.277 \\
15.223\end{array}$ & $\begin{array}{l}.199 \\
.187 \\
.200 \\
.193 \\
.200 \\
.194 \\
.198 \\
.193 \\
.189 \\
.203 \\
.200 \\
.206 \\
2.363\end{array}$ & $\begin{array}{l}.618 \\
.564 \\
.489 \\
.451 \\
.487 \\
.547 \\
.598 \\
.626 \\
.544 \\
.521 \\
.542 \\
.620 \\
6.607\end{array}$ & $\begin{array}{l}.225 \\
.188 \\
.225 \\
.203 \\
.233 \\
.237 \\
.206 \\
.189 \\
.176 \\
.171 \\
.201 \\
.248 \\
2.501\end{array}$ & $\begin{array}{l}.015 \\
.013 \\
.015 \\
.014 \\
.014 \\
.014 \\
.014 \\
.014 \\
.013 \\
.014 \\
.014 \\
.014 \\
.170\end{array}$ & $\begin{array}{l}.002 \\
.002 \\
.002 \\
.001 \\
.002 \\
.002 \\
.002 \\
.002 \\
.002 \\
.002 \\
.002 \\
.002 \\
.022\end{array}$ & $\begin{array}{r}5.919 \\
5.415 \\
5.630 \\
5.407 \\
5.491 \\
5.461 \\
5.587 \\
5.594 \\
5.439 \\
5.640 \\
5.479 \\
5.792 \\
66.853\end{array}$ \\
\hline 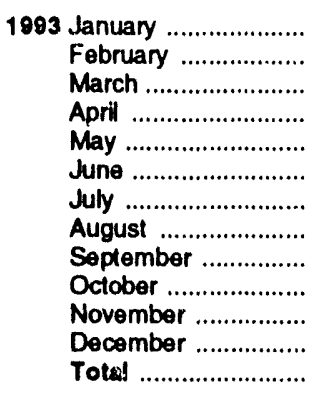 & 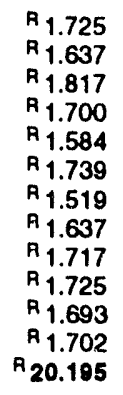 & $\begin{array}{r}1.630 \\
1.470 \\
1.627 \\
1.556 \\
R_{1.575} \\
1.516 \\
1.555 \\
1.564 \\
R_{1.544} \\
1.619 \\
R_{1} .587 \\
R_{1.650} \\
R_{18.894}\end{array}$ & $\begin{array}{r}1.260 \\
1.130 \\
1.254 \\
1.200 \\
1.229 \\
1.176 \\
1.196 \\
1.210 \\
1.168 \\
1.226 \\
1.199 \\
1.230 \\
14.476\end{array}$ & $\begin{array}{l}.204 \\
.187 \\
.212 \\
.204 \\
.203 \\
.198 \\
.203 \\
.204 \\
.196 \\
.208 \\
.191 \\
.189 \\
2.398\end{array}$ & $\begin{array}{l}.631 \\
.548 \\
.498 \\
.461 \\
.538 \\
.562 \\
.603 \\
.600 \\
.534 \\
.474 \\
.500 \\
.567 \\
6.517\end{array}$ & $\begin{array}{l}.255 \\
.206 \\
.246 \\
.262 \\
.306 \\
.277 \\
.246 \\
.205 \\
.178 \\
.176 \\
.187 \\
.220 \\
2.763\end{array}$ & $\begin{array}{l}.014 \\
.013 \\
.014 \\
.014 \\
.012 \\
.012 \\
.013 \\
.014 \\
.013 \\
.013 \\
.013 \\
.013 \\
.159\end{array}$ & $\begin{array}{l}.002 \\
.002 \\
.002 \\
.002 \\
.001 \\
.001 \\
.001 \\
.002 \\
.002 \\
.002 \\
.002 \\
.002 \\
.021\end{array}$ & $\begin{array}{r}R_{5.720} \\
R_{5.192} \\
R_{5.669} \\
R_{5.399} \\
R_{5.447} \\
R_{5.482} \\
R_{5.337} \\
A_{5.436} \\
A_{5.351} \\
R_{5.444} \\
R_{5.371} \\
R_{5.573} \\
R_{65.423}\end{array}$ \\
\hline $\begin{array}{r}1004 \text { January ..................... } \\
\text { February ................. } \\
\text { 2-Month Total .......... }\end{array}$ & $\begin{array}{r}1.679 \\
1.784 \\
\mathbf{3 . 4 6 3}\end{array}$ & $\begin{array}{r}1.670 \\
1.506 \\
3.177\end{array}$ & $\begin{array}{l}1.219 \\
1.095 \\
2.314\end{array}$ & $\begin{array}{l}.191 \\
.175 \\
.365\end{array}$ & $\begin{array}{r}.600 \\
.532 \\
1.132\end{array}$ & $\begin{array}{l}.207 \\
.200 \\
.407\end{array}$ & $\begin{array}{l}.013 \\
.012 \\
.025\end{array}$ & $\begin{array}{l}.002 \\
.002 \\
.003\end{array}$ & $\begin{array}{r}A_{5} .581 \\
5.307 \\
10.887\end{array}$ \\
\hline $\begin{array}{l}1993 \text { 2-Month Total ......... } \\
1092 \text { 2-Month Total ......... }\end{array}$ & $\begin{array}{l}3.362 \\
3.682\end{array}$ & $\begin{array}{l}3.100 \\
3.074\end{array}$ & $\begin{array}{l}2.390 \\
2.566\end{array}$ & $\begin{array}{l}.381 \\
.386\end{array}$ & $\begin{array}{l}1.179 \\
1.182\end{array}$ & $\begin{array}{l}.461 \\
.412\end{array}$ & $\begin{array}{l}.027 \\
.028\end{array}$ & $\begin{array}{l}.004 \\
.004\end{array}$ & $\begin{array}{l}10.913 \\
11.333\end{array}$ \\
\hline
\end{tabular}

- Includes lease condensale.

Electric utility and industrial generation.

c "Other" production is electricity generated for distribution from wood, waste, wind, photovoltaic, and solar thermal energy.

d Due to a lack of consistent historical data, some renewable energy sources are not included. For example, in 1991, 3.3 quadrillion Btu of renewable energy consumed by U.S. electric utilities to generate electricity for distribution is included, but an estimatea 3.4 quadrillion Btu of renewable energy used by other sectors is not included.
Noles: - See Note 1 at end of section. Geographic coverage is the 50 States and the District of Columbia. - Totals may not equal sum of components due to independent rounding.

Sources: - Coal: Tables 6.1 and A5-A7. - Natural Gas (Dry): Tables 4.1 and A4. Crude Oll and Natural Gas Plant Llquids: Tables 3.1a and A2. - Nuclear Electric Power: Tables 7.1 and A8. - Hydroolectric Power: Table 7.1; Section 2, "Energy Consumption Noles and Sources," Note 8; and Table AB. - Geothermal Energy and Other: Section 2. "Energy Consumption Notes and Sources," Note 7, and Table A8. 
Figure 1.3 Energy Consumption

(Quadrillion Btu)

Total Consumption, 1973-1993

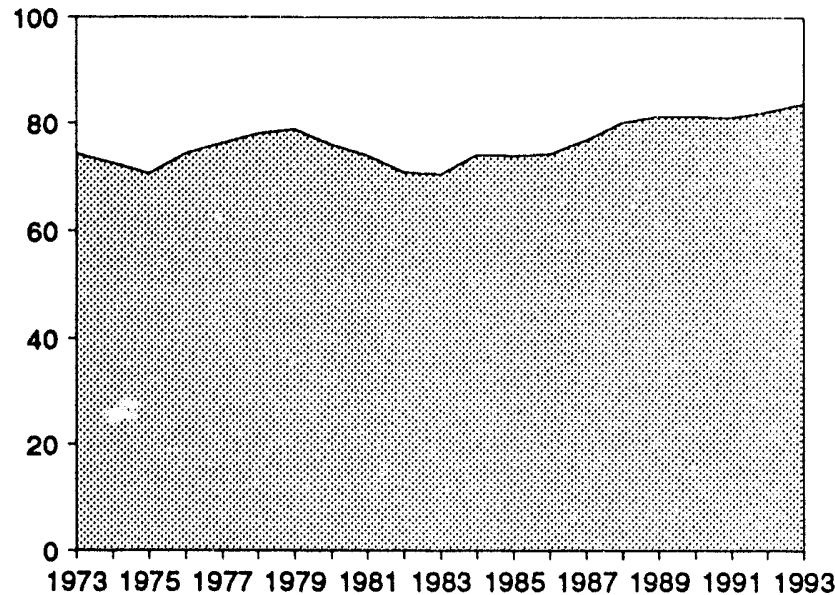

Consumption by Major Sources, 1973-1993

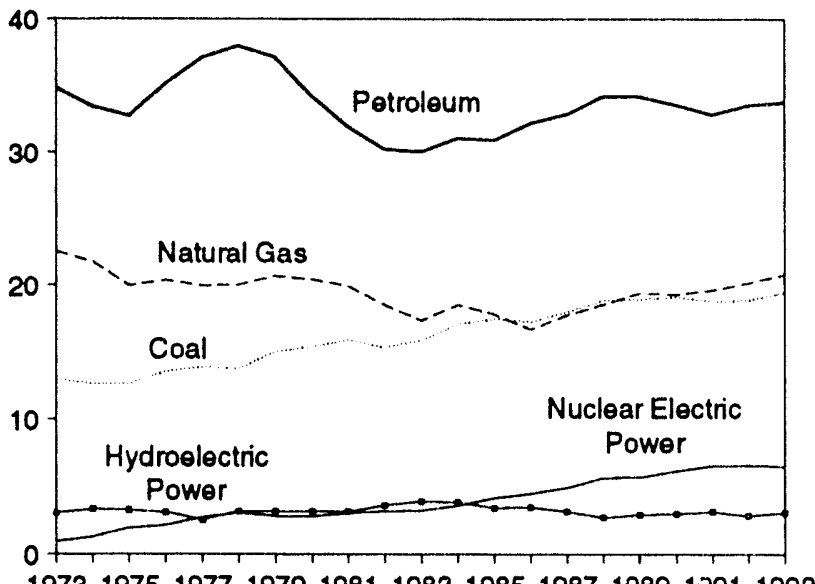

Total Consumption, January and February

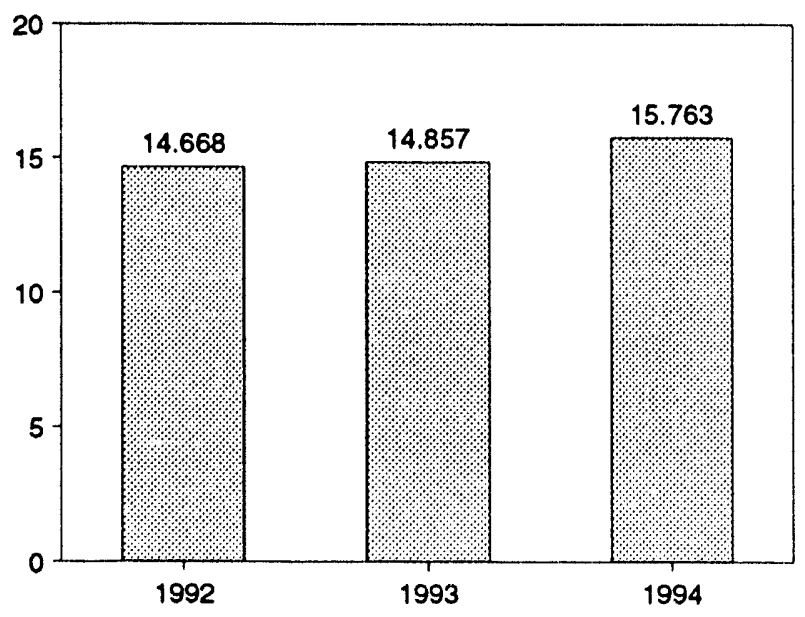

Total Consumption, Monthly

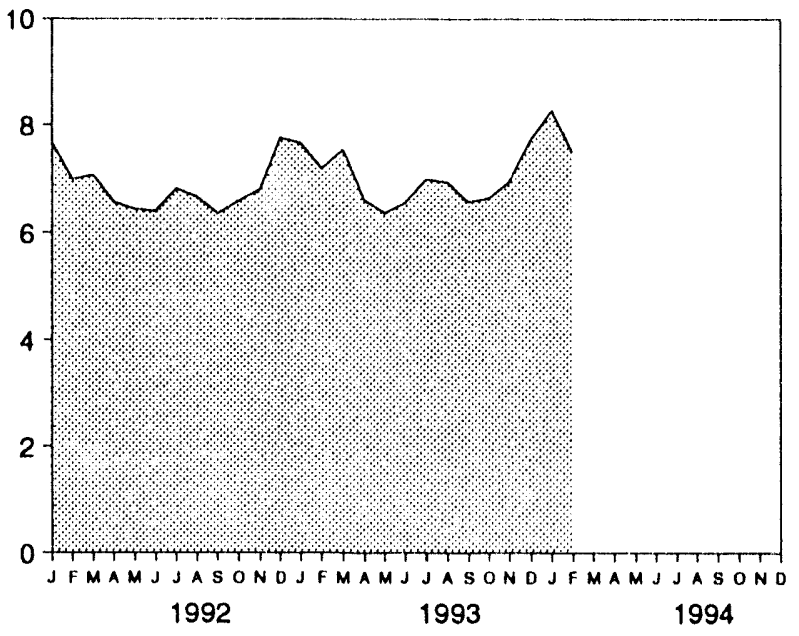

Consumption by Major Sources, Monthly

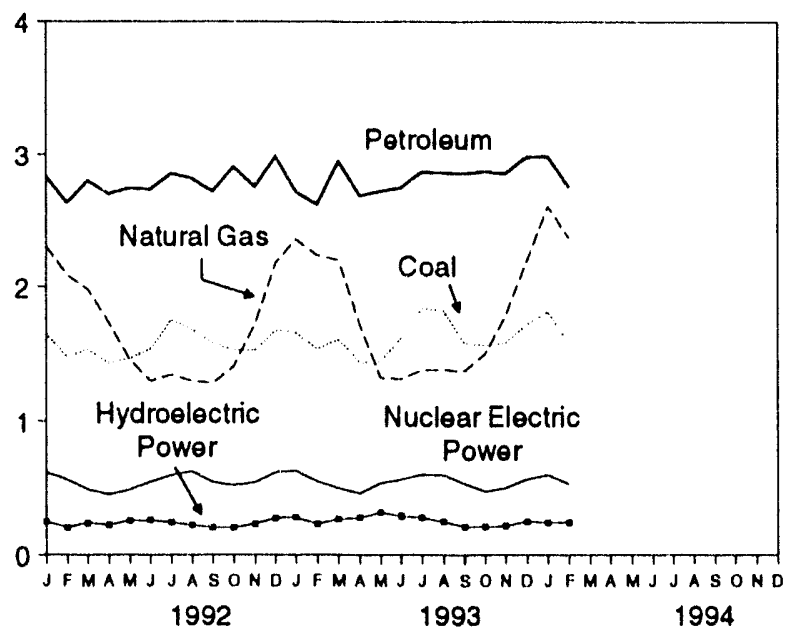

Consumption by Major Sources, February 1994

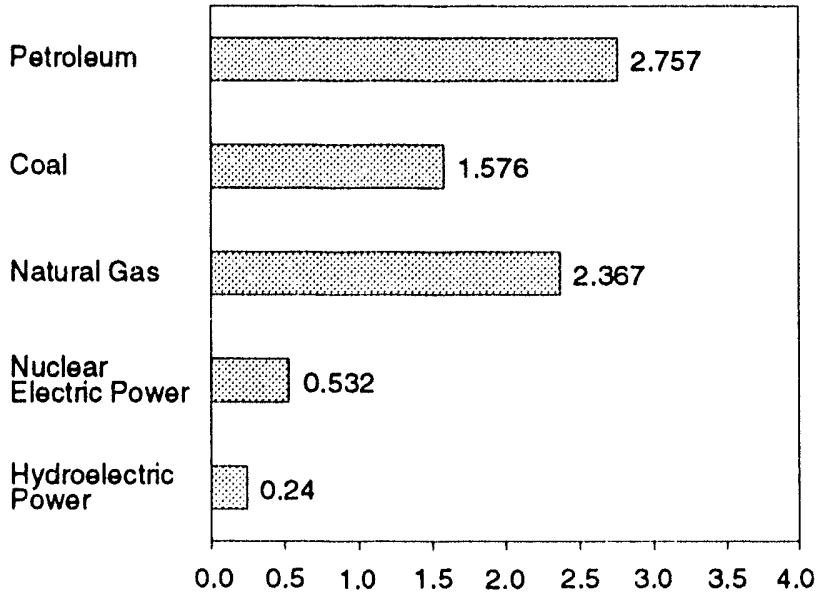

Note: Because vertical scales differ, graphs should not be compared. Source: Table 1.4. 


\begin{tabular}{|c|c|c|c|c|c|c|c|c|}
\hline & Coal & $\begin{array}{c}\text { Natural } \\
\text { Gasa }\end{array}$ & Petroleum & $\begin{array}{l}\text { Nuclear } \\
\text { Electric } \\
\text { Power }\end{array}$ & $\begin{array}{l}\text { Hydro- } \\
\text { electric } \\
\text { Powerb }\end{array}$ & $\begin{array}{l}\text { Geothermal } \\
\text { Energy }\end{array}$ & Otherc & Totald \\
\hline 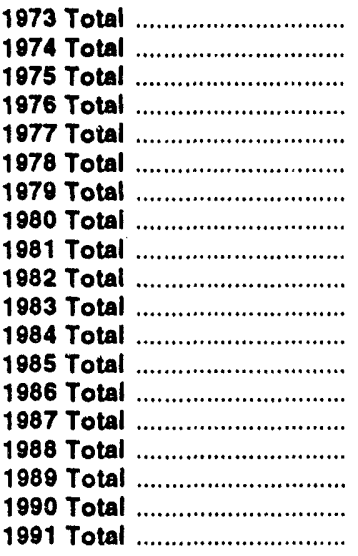 & $\begin{array}{l}12.971 \\
12.663 \\
12.663 \\
13.584 \\
13.922 \\
13.765 \\
15.039 \\
15.423 \\
15.907 \\
15.322 \\
15.894 \\
17.071 \\
17.478 \\
17.261 \\
18.008 \\
18.846 \\
18.925 \\
19.101 \\
18.770\end{array}$ & $\begin{array}{l}22.512 \\
21.732 \\
19.948 \\
20.345 \\
19.931 \\
20.000 \\
20.666 \\
20.394 \\
19.928 \\
18.505 \\
17.357 \\
18.507 \\
17.834 \\
16.708 \\
17.744 \\
18.552 \\
19.384 \\
19.296 \\
19.606\end{array}$ & $\begin{array}{l}34.840 \\
33.455 \\
32.731 \\
35.175 \\
37.122 \\
37.965 \\
37.123 \\
34.202 \\
31.931 \\
30.231 \\
30.054 \\
31.051 \\
30.922 \\
32.196 \\
32.865 \\
34.222 \\
34.211 \\
33.553 \\
32.845\end{array}$ & $\begin{array}{l}0.910 \\
1.272 \\
1.900 \\
2.111 \\
2.702 \\
3.024 \\
2.776 \\
2.739 \\
3.008 \\
3.131 \\
3.203 \\
3.553 \\
4.149 \\
4.471 \\
4.906 \\
5.661 \\
5.677 \\
6.161 \\
6.579\end{array}$ & $\begin{array}{l}3.010 \\
3.309 \\
3.219 \\
3.066 \\
2.515 \\
3.141 \\
3.141 \\
3.118 \\
3.105 \\
3.572 \\
3.899 \\
3.800 \\
3.398 \\
3.446 \\
3.117 \\
2.662 \\
2.881 \\
2.946 \\
3.115\end{array}$ & $\begin{array}{l}0.043 \\
.053 \\
.070 \\
.078 \\
.077 \\
.064 \\
.084 \\
.110 \\
.123 \\
.105 \\
.129 \\
.165 \\
.198 \\
.219 \\
.229 \\
.217 \\
.197 \\
.181 \\
.170\end{array}$ & $\begin{array}{r}-0.004 \\
.059 \\
.016 \\
.003 \\
.020 \\
.128 \\
.068 \\
. .031 \\
. .012 \\
-.018 \\
-.012 \\
. .002 \\
.001 \\
-.004 \\
.024 \\
.057 \\
.051 \\
.026 \\
.030\end{array}$ & $\begin{array}{l}74.282 \\
72.543 \\
70.546 \\
74.362 \\
76.288 \\
78.089 \\
78.898 \\
75.855 \\
73.990 \\
70.848 \\
70.524 \\
74.144 \\
73.981 \\
74.297 \\
76.894 \\
80.218 \\
81.325 \\
81.265 \\
81.116\end{array}$ \\
\hline 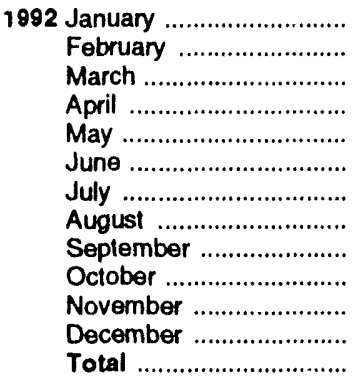 & $\begin{array}{r}1.653 \\
1.477 \\
1.535 \\
1.434 \\
1.468 \\
1.539 \\
1.756 \\
1.686 \\
1.583 \\
1.531 \\
1.529 \\
1.678 \\
18.868\end{array}$ & $\begin{array}{r}2.306 \\
2.091 \\
1.984 \\
1.735 \\
1.460 \\
1.302 \\
1.351 \\
1.302 \\
1.286 \\
1.409 \\
1.722 \\
2.182 \\
20.131\end{array}$ & $\begin{array}{r}2.836 \\
2.635 \\
2.805 \\
2.705 \\
2.748 \\
2.739 \\
2.858 \\
2.822 \\
2.723 \\
2.909 \\
2.757 \\
2.989 \\
33.527\end{array}$ & $\begin{array}{l}.618 \\
.564 \\
.489 \\
.451 \\
.487 \\
.547 \\
.598 \\
.626 \\
.544 \\
.521 \\
.542 \\
.620 \\
6.607\end{array}$ & $\begin{array}{l}.245 \\
.205 \\
.237 \\
.222 \\
.255 \\
.257 \\
.241 \\
.220 \\
.204 \\
.202 \\
.230 \\
.275 \\
2.793\end{array}$ & $\begin{array}{l}.015 \\
.013 \\
.015 \\
.014 \\
.014 \\
.014 \\
.014 \\
.014 \\
.013 \\
.014 \\
.014 \\
.014 \\
.170\end{array}$ & $\begin{array}{l}.006 \\
.004 \\
.005 \\
.005 \\
.002 \\
.005 \\
.003 \\
.003 \\
.003 \\
.004 \\
.003 \\
.007 \\
.049\end{array}$ & $\begin{array}{r}7.678 \\
6.989 \\
7.070 \\
6.565 \\
6.435 \\
6.403 \\
6.822 \\
6.673 \\
6.356 \\
6.590 \\
6.798 \\
7.765 \\
82.144\end{array}$ \\
\hline 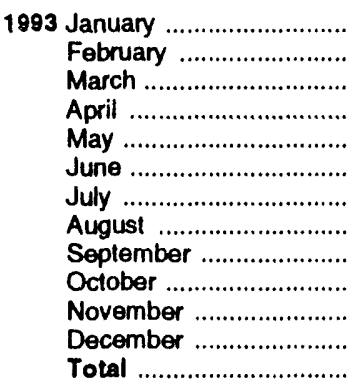 & 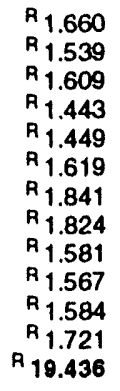 & $\begin{array}{r}2.359 \\
2.237 \\
2.201 \\
1.719 \\
1.327 \\
1.316 \\
1.385 \\
1.386 \\
1.377 \\
1.504 \\
\mathrm{R} 1.780 \\
\mathrm{R} 2.193 \\
\mathrm{R}_{20.782}\end{array}$ & $\begin{array}{r}2.722 \\
2.621 \\
2.951 \\
2.692 \\
2.726 \\
2.749 \\
2.870 \\
2.865 \\
2.859 \\
2.874 \\
2.855 \\
2.984 \\
33.768\end{array}$ & $\begin{array}{l}.631 \\
.548 \\
.498 \\
.461 \\
.538 \\
.562 \\
.603 \\
.600 \\
.534 \\
.474 \\
.500 \\
.567 \\
6.517\end{array}$ & $\begin{array}{l}.278 \\
.228 \\
.265 \\
.278 \\
.316 \\
.288 \\
.276 \\
.246 \\
.211 \\
.209 \\
.214 \\
.249 \\
3.059\end{array}$ & $\begin{array}{l}.014 \\
.013 \\
.014 \\
.014 \\
.012 \\
.012 \\
.013 \\
.014 \\
.013 \\
.013 \\
.013 \\
.013 \\
.159\end{array}$ & $\begin{array}{l}.006 \\
.001 \\
.005 \\
.004 \\
.004 \\
.004 \\
.001 \\
.004 \\
.001 \\
.003 \\
.002 \\
.004 \\
.038\end{array}$ & 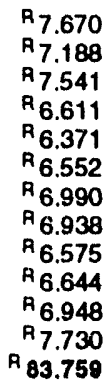 \\
\hline 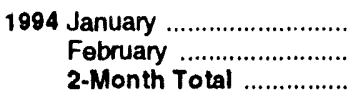 & $\begin{array}{r}\text { P }_{1.819} \\
1.576 \\
3.395\end{array}$ & $\begin{array}{r}\mathrm{A}_{2.611} \\
2.367 \\
\mathbf{4 . 9 7 7}\end{array}$ & $\begin{array}{l}2.990 \\
2.757 \\
\mathbf{5 . 7 4 7}\end{array}$ & $\begin{array}{r}.600 \\
.532 \\
1.132\end{array}$ & $\begin{array}{l}.239 \\
.240 \\
.480\end{array}$ & $\begin{array}{l}.013 \\
.012 \\
.025\end{array}$ & $\begin{array}{l}.006 \\
.001 \\
.007\end{array}$ & $\begin{array}{r}R_{8.278} \\
7.485 \\
15.763\end{array}$ \\
\hline $\begin{array}{l}1993 \text { 2-Month Total ............... } \\
1992 \text { 2-Month Total .................. }\end{array}$ & $\begin{array}{l}3.198 \\
3.129\end{array}$ & $\begin{array}{l}4.596 \\
4.397\end{array}$ & $\begin{array}{l}5.344 \\
5.471\end{array}$ & $\begin{array}{l}1.179 \\
1.182\end{array}$ & $\begin{array}{l}.506 \\
.451\end{array}$ & $\begin{array}{l}.027 \\
.028\end{array}$ & $\begin{array}{l}.008 \\
.010\end{array}$ & $\begin{array}{l}14.857 \\
14.668\end{array}$ \\
\hline
\end{tabular}

a Includes supplemental gaseous fuols.

b Electric utility and industrial generation and net imports of electricity.

c "Other" consumption is net imports of coal coke and electricity generated for distribution from wood, waste, wind, photovoltaic, and solar thermal energy.

d Due to a lack of consistent historical data, some renewable energy sources are not included. For example, in 1991, 3.3 quadrillion Blu of renewable energy consumed by U.S. electric utilities to generate electricity for distribution is included, but an estimated 3.4 quadrillion Btu of renewable energy used by other sectors is not included.
$R=$ Revised data.

Notes: - See Note 2 at end of section. - Geographic coverage is the 50 States and the District of Columbia. - Tolals may not equal sum of components due to independent rounding.

Sources: - Coal: Tables 6.1 and A5-A7. - Natural Gas: Tables 4.2 and A4. - Petroleum: Tables 3.1a and A3. - Nuclear Electric Power: Tables 7.1 and A8. - Hydroelectric Power: Table 7.1; Section 2, "Energy Consumption Notes and Sources," Note 8; and Table A8. - Geothermal Energy and Other: Section 2, "Energy Consumption Notes and Sources," Note 7, and Table A8. 
Figure 1.4 Energy Net Imports

(Quadrillion Btu, Except as Noted)

Total Net Imports, 1973-1993

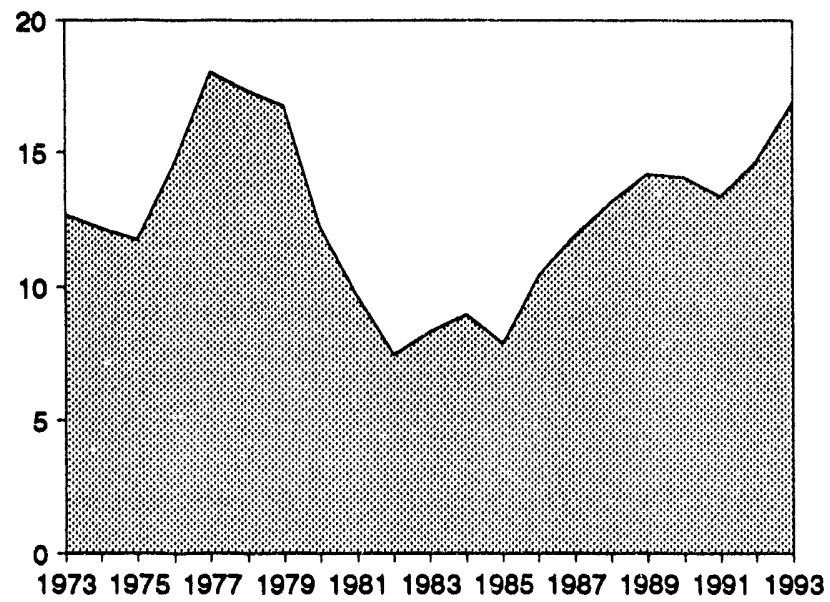

Net Imports by Major Sources, 1973-1993

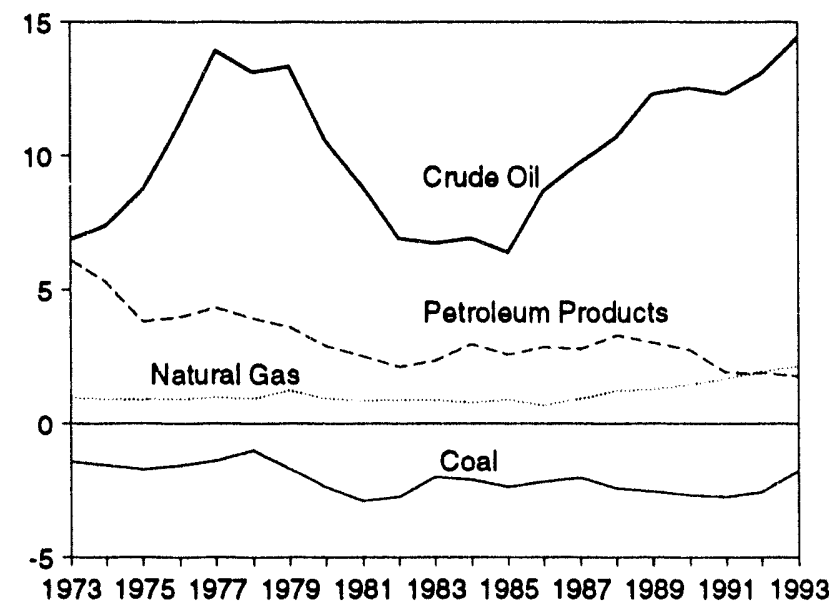

Net Imports by Major Sources,

February 1994

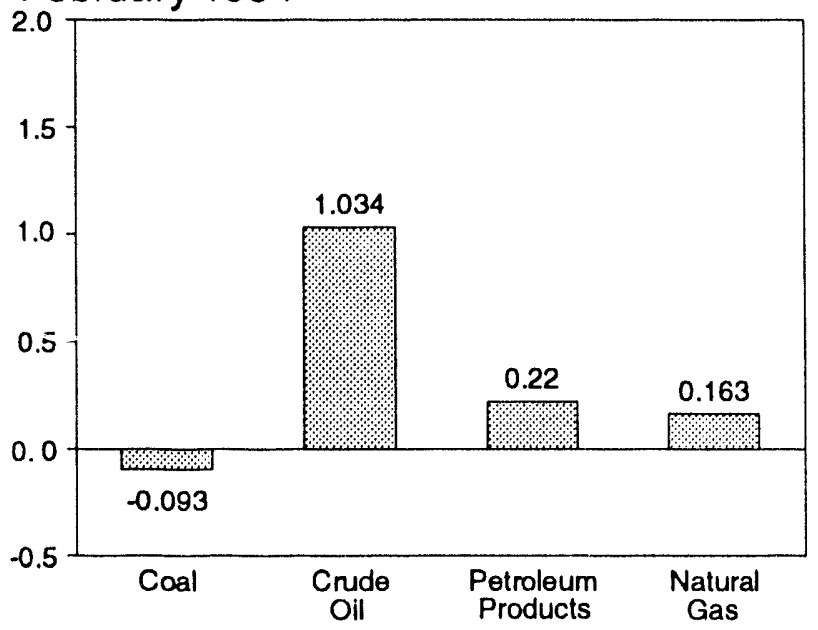

Net Imports, Monthly

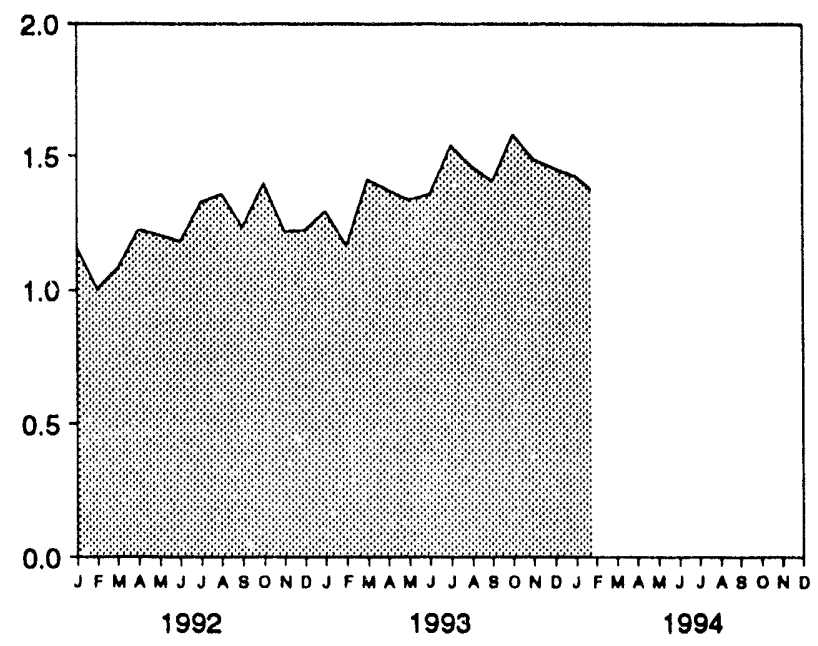

Net Imports by Major Sources, Monthly

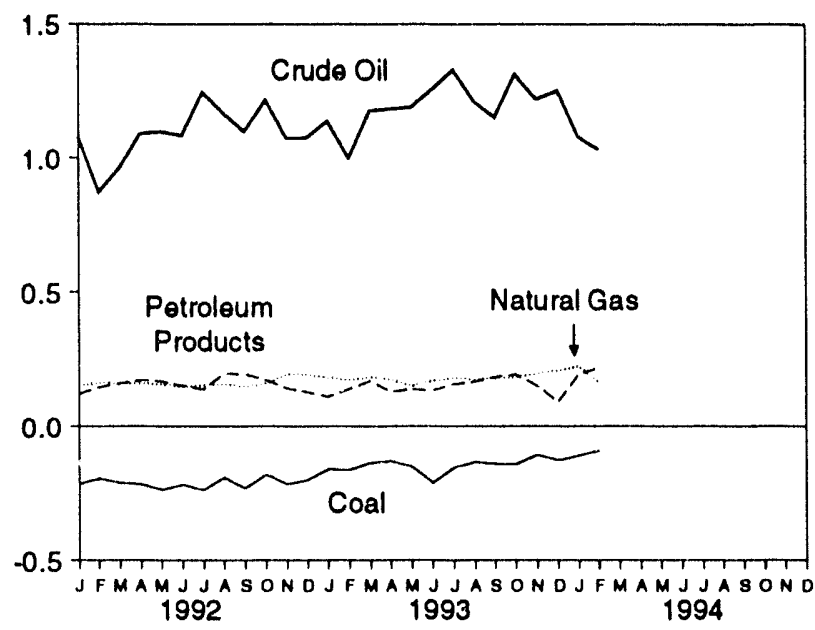

Net Imports as Share of Consumption,

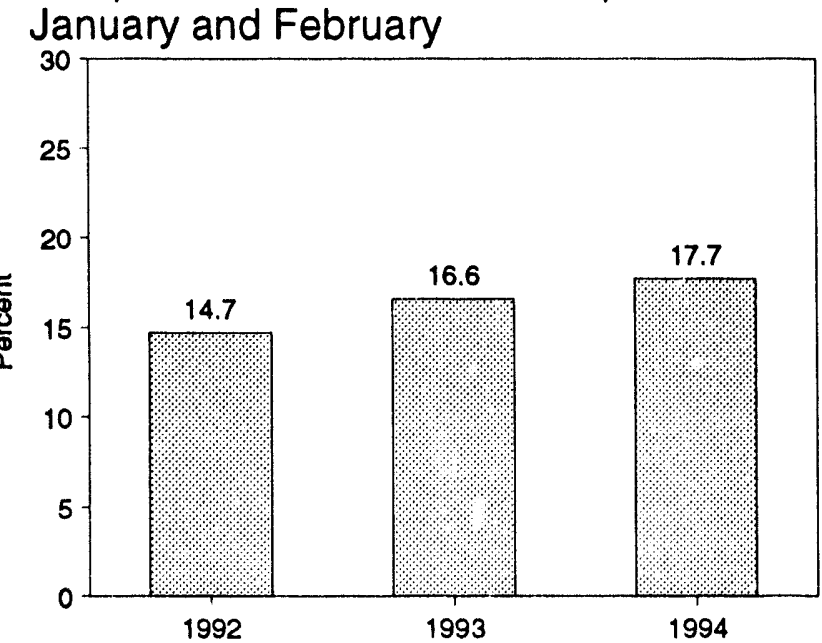

Note: Because vertical scales differ, graphs should not be compared. Sources: Tables 1.4 and 1.5. 
Table 1.5 Energy Net Imports by Source

(Quadrillion Btu)

\begin{tabular}{|c|c|c|c|c|c|c|c|}
\hline & Coal & $\begin{array}{c}\text { Natural } \\
\text { Gas }\end{array}$ & $\begin{array}{c}\text { Crude } \\
\text { Olla }\end{array}$ & $\begin{array}{l}\text { Potroleum } \\
\text { Producteb }\end{array}$ & Electricityc & $\begin{array}{l}\text { Coal } \\
\text { Coke }\end{array}$ & Total \\
\hline 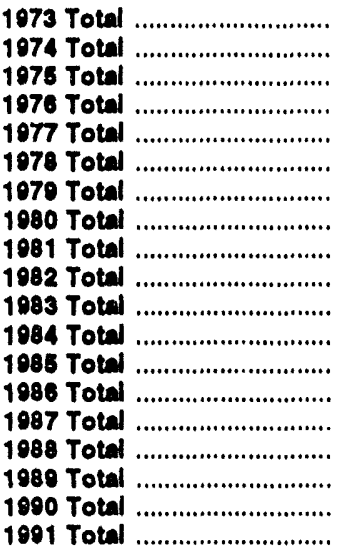 & $\begin{array}{l}-1.422 \\
-1.588 \\
-1.738 \\
-1.587 \\
-1.401 \\
-1.004 \\
-1.702 \\
-2.391 \\
-2.018 \\
-2.768 \\
-2.013 \\
-2.110 \\
-2.389 \\
-2.193 \\
-2.049 \\
-2.448 \\
-2.568 \\
-2.705 \\
-2.769\end{array}$ & $\begin{array}{r}0.081 \\
.007 \\
.004 \\
.022 \\
.081 \\
.041 \\
1.243 \\
.057 \\
.857 \\
.808 \\
.885 \\
.792 \\
.808 \\
.686 \\
.037 \\
1.221 \\
1.278 \\
1.464 \\
1.666\end{array}$ & $\begin{array}{r}6.883 \\
7.389 \\
8.708 \\
11.221 \\
13.921 \\
13.125 \\
13.328 \\
10.586 \\
8.854 \\
6.017 \\
6.731 \\
6.018 \\
6.381 \\
8.676 \\
9.748 \\
10.698 \\
12.296 \\
12.536 \\
12.308\end{array}$ & $\begin{array}{l}6.097 \\
5.273 \\
3.800 \\
3.082 \\
4.321 \\
3.032 \\
3.603 \\
2.012 \\
2.522 \\
2.128 \\
2.351 \\
2.070 \\
2.570 \\
2.855 \\
2.784 \\
3.308 \\
3.029 \\
2.757 \\
1.012\end{array}$ & $\begin{array}{l}0.148 \\
.133 \\
.084 \\
.089 \\
.182 \\
.204 \\
.211 \\
.217 \\
.347 \\
.306 \\
.372 \\
.414 \\
.428 \\
.375 \\
.483 \\
.328 \\
.113 \\
.020 \\
.231\end{array}$ & $\begin{array}{r}-0.007 \\
.056 \\
.014 \\
(8) \\
.015 \\
.125 \\
.063 \\
.035 \\
.016 \\
.0122 \\
.016 \\
.011 \\
.013 \\
.017 \\
.009 \\
.040 \\
.030 \\
.005 \\
.009\end{array}$ & $\begin{array}{r}12.680 \\
12.190 \\
11.752 \\
14.648 \\
18.010 \\
17.323 \\
16.746 \\
12.247 \\
9.846 \\
7.460 \\
8.310 \\
8.083 \\
7.872 \\
10.382 \\
11.911 \\
13.140 \\
14.181 \\
14.077 \\
13.357\end{array}$ \\
\hline 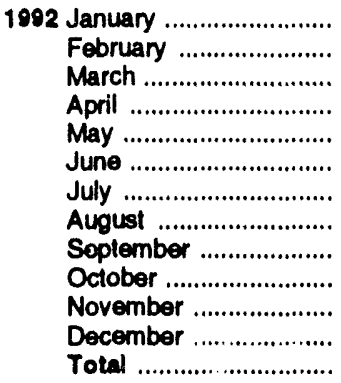 & $\begin{array}{l}-.218 \\
-.198 \\
-.214 \\
-.219 \\
. .240 \\
-.221 \\
-.241 \\
-.194 \\
-.235 \\
-.183 \\
-.219 \\
-.204 \\
-2.587\end{array}$ & $\begin{array}{l}.150 \\
.163 \\
.160 \\
.160 \\
.157 \\
.146 \\
.153 \\
.158 \\
.149 \\
.159 \\
.194 \\
.193 \\
1.941\end{array}$ & $\begin{array}{r}1.078 \\
.873 \\
.963 \\
1.090 \\
1.099 \\
1.084 \\
1.245 \\
1.168 \\
1.099 \\
1.217 \\
1.074 \\
1.076 \\
13.065\end{array}$ & $\begin{array}{l}.122 \\
.146 \\
.160 \\
.173 \\
.168 \\
152 \\
.137 \\
.197 \\
.195 \\
.173 \\
.142 \\
.129 \\
1.805\end{array}$ & $\begin{array}{l}.021 \\
.018 \\
.012 \\
.018 \\
.022 \\
.020 \\
.035 \\
.031 \\
.028 \\
.031 \\
.029 \\
.027 \\
.202\end{array}$ & $\begin{array}{l}.004 \\
.003 \\
.003 \\
.003 \\
.001 \\
.003 \\
.001 \\
.001 \\
.001 \\
.002 \\
.001 \\
.005 \\
.027\end{array}$ & $\begin{array}{r}1.157 \\
1.005 \\
1.084 \\
1.226 \\
1.207 \\
1.183 \\
1.329 \\
1.360 \\
1.237 \\
1.399 \\
1.221 \\
1.226 \\
14.633\end{array}$ \\
\hline 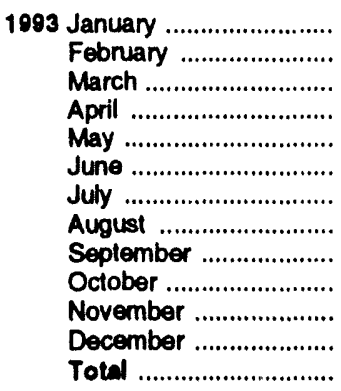 & 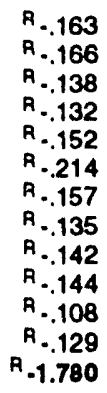 & $\begin{array}{r}.182 \\
.172 \\
.184 \\
.175 \\
.150 \\
.170 \\
.180 \\
.176 \\
.180 \\
.183 \\
.197 \\
.209 \\
\mathrm{R}_{2.157}\end{array}$ & $\begin{array}{r}1.138 \\
.999 \\
1.177 \\
1.184 \\
1.189 \\
1.256 \\
1.329 \\
1.211 \\
1.153 \\
1.314 \\
1.219 \\
1.251 \\
14.420\end{array}$ & $\begin{array}{r}.111 \\
.139 \\
.169 \\
.129 \\
.139 \\
.134 \\
.157 \\
.166 \\
.185 \\
.194 \\
.153 \\
.092 \\
1.760\end{array}$ & $\begin{array}{l}E .023 \\
E .022 \\
E .019 \\
E .016 \\
E .011 \\
E .011 \\
E .011 \\
E .031 \\
E .041 \\
E .033 \\
E .033 \\
E .027 \\
E .029 \\
E .296\end{array}$ & $\begin{array}{r}.004 \\
(s) \\
.003 \\
.002 \\
.002 \\
.003 \\
(s) \\
.002 \\
.001 \\
.001 \\
(s) \\
.002 \\
.017\end{array}$ & 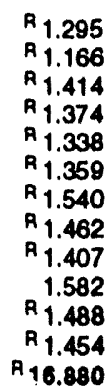 \\
\hline $\begin{array}{r}1994 \text { January .......................... } \\
\text { February ................... } \\
\text { 2-Month Total ............. }\end{array}$ & $\begin{array}{l}\text { A. } .111 \\
-.093 \\
-.204\end{array}$ & $\begin{array}{l}\text { ค } .225 \\
.163 \\
.380\end{array}$ & $\begin{array}{l}1.081 \\
1.034 \\
2.114\end{array}$ & $\begin{array}{l}.193 \\
.220 \\
.413\end{array}$ & $\begin{array}{l}E .032 \\
E .041 \\
E .072\end{array}$ & $\begin{array}{r}.004 \\
.001 \\
.003\end{array}$ & $\begin{array}{r}\text { P } 1.424 \\
1.363 \\
2.787\end{array}$ \\
\hline $\begin{array}{l}1993 \text { 2-Month Total .............. } \\
1992 \text { 2-Month Total ............. }\end{array}$ & $\begin{array}{l}-.328 \\
-.416\end{array}$ & $\begin{array}{l}.354 \\
.313\end{array}$ & $\begin{array}{l}2.138 \\
1.951\end{array}$ & $\begin{array}{l}.249 \\
.269\end{array}$ & $\begin{array}{r}E .045 \\
.038\end{array}$ & $\begin{array}{l}.004 \\
.006\end{array}$ & $\begin{array}{l}2.461 \\
2.162\end{array}$ \\
\hline
\end{tabular}

a Crude oil, lease condensate, and imports of crude oil for the Strategic Petroleum Reserve.

betroleum products, unfinished oils, pentanes plus, and gasoline blending components.

c Assumed to be hydroelectricity and estimated at the average input heat rate for lossil-fuel steam-electric power plant generation, which has ranged from 10.2 thousand Btu to 10.5 thousand Btu per kilowatthour since 1973. Actual heat rates applied in converting kilowatthours to Blu are lisled by year in Table A8.

$\mathrm{R}=$ Revised data. E=Estimate. $(\mathrm{s})=$ Less than +0.5 trillion $B t u$ and greater

than -0.5 trillion Btu.

Notes: - See Notes 3 and 4 at end of section. - Nel imports equal imports minus exports. Minus sign indicales exports are greater than imports. - Geographic coverage is the 50 States and the District of Columbla.

- Totals may nol equal sum ol components due to independent rounding.

Sources: - Coal: Tables 6.1 and A5-A7. - Natural Gas: Tables 4.2 and A4. - Crude Oll and Potroloum Products: Tables 3.1b and A2. - Electricity: Section 2, "Energy' Consumption Noles and Sources," Note 8 and Table AB. - Coal Coke: Section 2, "Energy Consumption Notes and Sources," Note 9, and Table A7. 
Figure 1.5 Merchandise Trade Value

(Billion Dollars)

Imports and Exports, 1974-1993

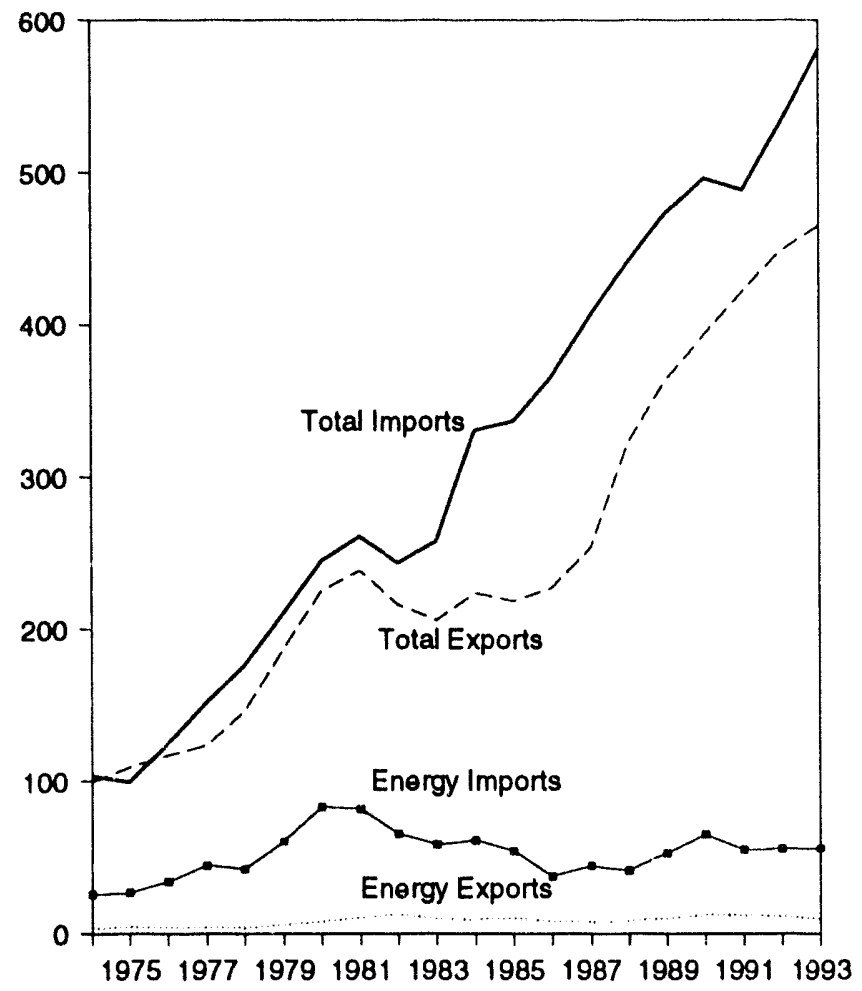

Trade Balance, 1974-1993

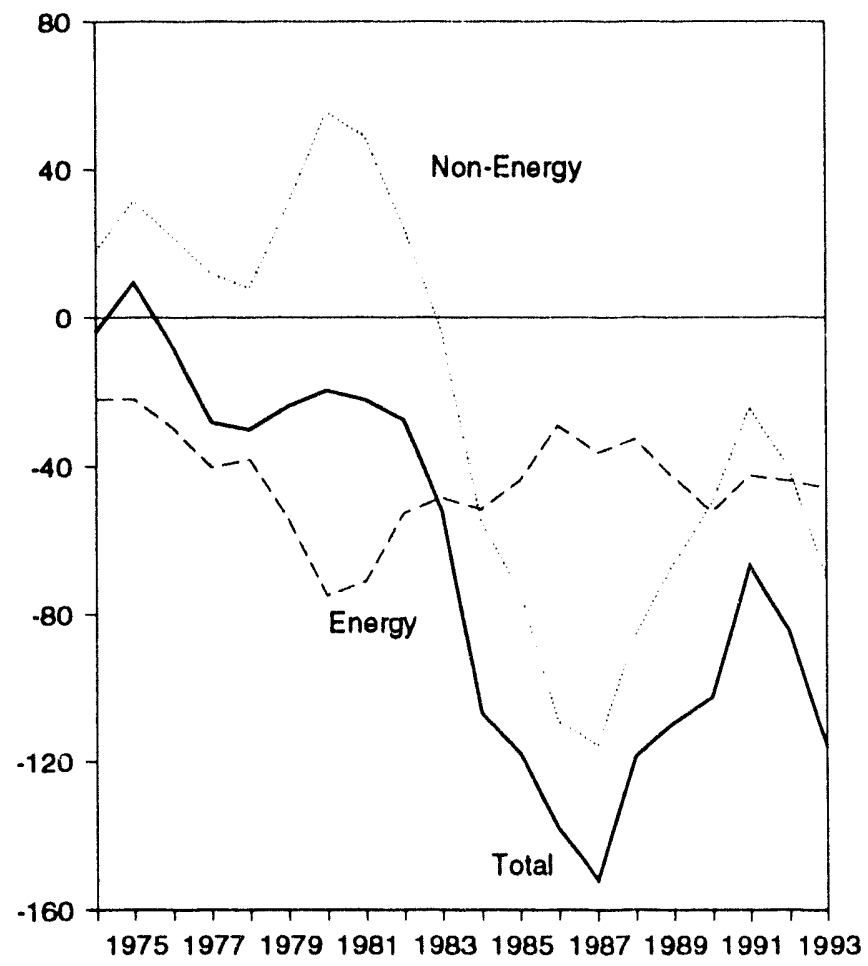

Imports and Exports, Monthly

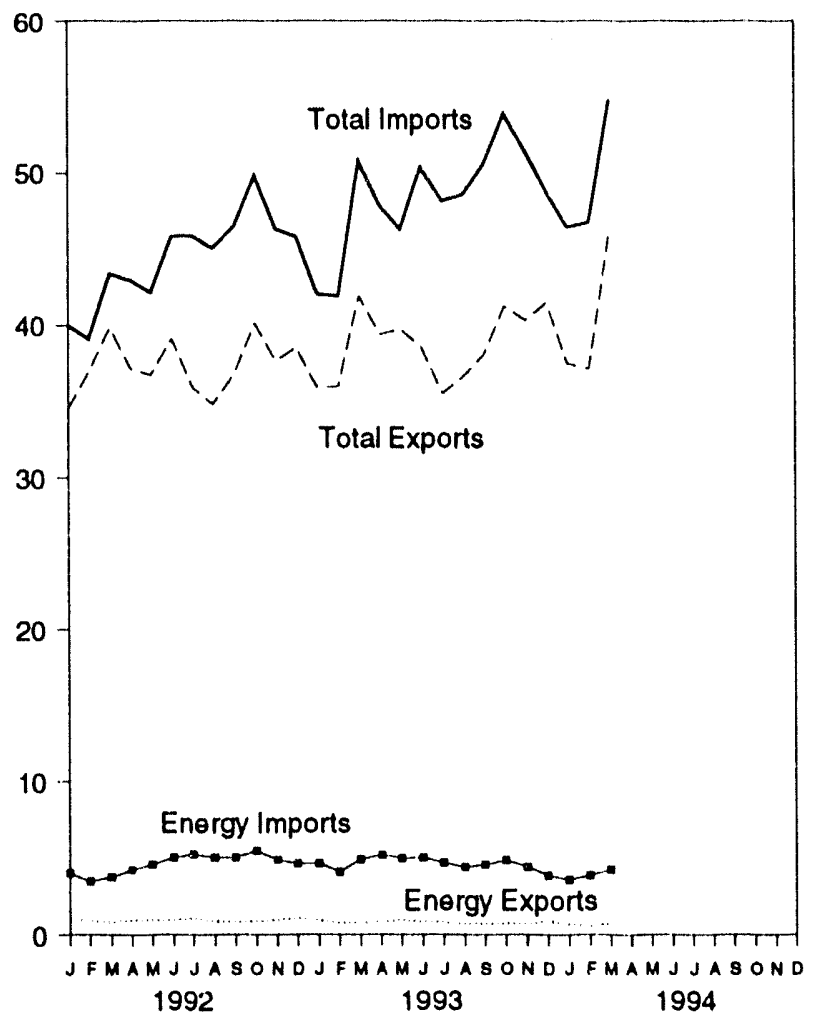

Trade Balance, Monthly

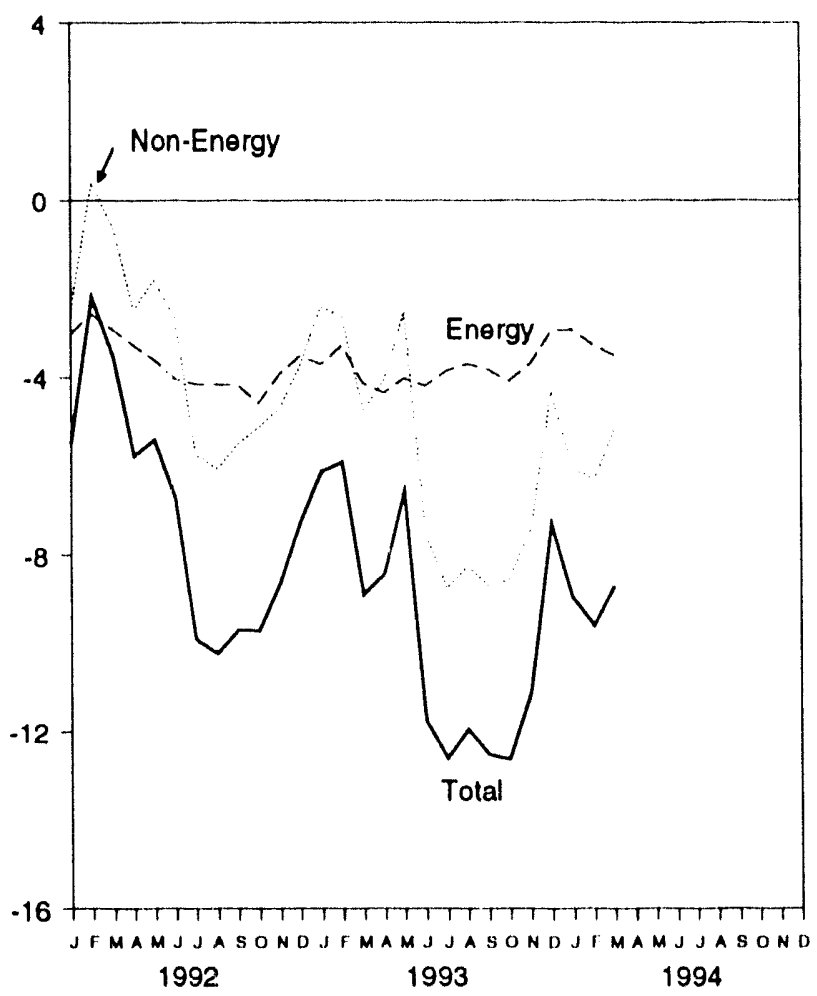

Note: Because vertical scales differ, graphs should not be compared.

Source: Table 1.6. 
Table 1.6 Merchandise Trade Value

(Million Dollars)

\begin{tabular}{|c|c|c|c|c|c|c|c|c|c|c|}
\hline & \multicolumn{3}{|c|}{ Potroloum } & \multicolumn{3}{|c|}{ Energy } & \multirow{2}{*}{$\begin{array}{l}\text { Non- } \\
\text { Energy } \\
\text { Balance }\end{array}$} & \multicolumn{3}{|c|}{ Total Merchandise } \\
\hline & Exports & Imports & Balance & Exports & Imports & Balance & & Exports & Imports & Balance \\
\hline 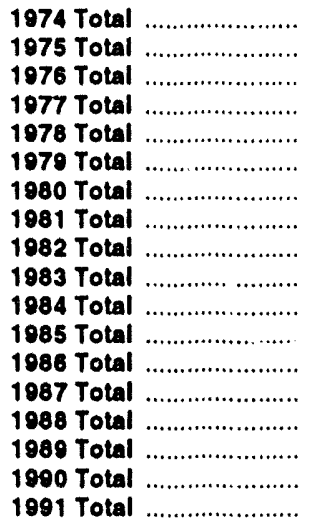 & $\begin{array}{r}782 \\
907 \\
998 \\
1,276 \\
1,561 \\
1,914 \\
2,833 \\
3,696 \\
5,947 \\
4,557 \\
4,470 \\
4,707 \\
3,640 \\
3,922 \\
3,693 \\
5,021 \\
6,901 \\
6,954\end{array}$ & $\begin{array}{l}24,668 \\
25,197 \\
32,226 \\
42,368 \\
39,526 \\
56,715 \\
78,637 \\
76,659 \\
60,458 \\
53,217 \\
56,924 \\
50,475 \\
35,142 \\
42,285 \\
38,787 \\
49,704 \\
61,583 \\
51,350\end{array}$ & $\begin{array}{l}-23,876 \\
-24,289 \\
-31,228 \\
-41,093 \\
-37,965 \\
-54,801 \\
-75,803 \\
-72,063 \\
-54,511 \\
-48,659 \\
-52,454 \\
-45,768 \\
-31,503 \\
-38,363 \\
-35,094 \\
-44,683 \\
-54,682 \\
-44,396\end{array}$ & $\begin{array}{r}3,444 \\
4,470 \\
4,226 \\
4,184 \\
3,881 \\
5,621 \\
7,982 \\
10,279 \\
12,729 \\
9,500 \\
9,311 \\
9,971 \\
8,115 \\
7,713 \\
8,235 \\
9,869 \\
12,233 \\
12,081\end{array}$ & $\begin{array}{l}25,454 \\
26,476 \\
33,996 \\
44,537 \\
42,096 \\
59,998 \\
82,924 \\
81,360 \\
65,409 \\
57,952 \\
60,980 \\
53,917 \\
37,310 \\
44,220 \\
41,042 \\
52,779 \\
64,661 \\
54,629\end{array}$ & $\begin{array}{l}-22,010 \\
-22,006 \\
-29,770 \\
-40,354 \\
-38,215 \\
-54,377 \\
-74,942 \\
-71,081 \\
-52,680 \\
-48,452 \\
-51,669 \\
-43,946 \\
-29,195 \\
-36,506 \\
-32,806 \\
-42,910 \\
-52,428 \\
-42,548\end{array}$ & $\begin{array}{r}18,126 \\
31,557 \\
21,950 \\
12,001 \\
8,010 \\
30,455 \\
55,246 \\
48,814 \\
25,170 \\
-3,957 \\
-55,033 \\
-73,765 \\
-109,084 \\
-115,613 \\
-85,720 \\
-66,490 \\
-50,068 \\
-24,175\end{array}$ & $\begin{array}{r}99,437 \\
108,856 \\
116,794 \\
123,182 \\
145,847 \\
186,363 \\
225,566 \\
238,715 \\
216,442 \\
205,639 \\
223,876 \\
218,815 \\
227,159 \\
254,122 \\
322,426 \\
363,812 \\
393,592 \\
421,730\end{array}$ & $\begin{array}{r}103,321 \\
99,305 \\
124,614 \\
151,534 \\
176,052 \\
210,285 \\
245,262 \\
260,982 \\
243,952 \\
258,048 \\
330,678 \\
336,526 \\
365,438 \\
406,241 \\
440,952 \\
473,211 \\
496,088 \\
488,453\end{array}$ & $\begin{array}{r}-3,884 \\
9,551 \\
-7,820 \\
-28,353 \\
-30,205 \\
-23,922 \\
-19,696 \\
-22,267 \\
-27,510 \\
-52,409 \\
-106,703 \\
-117,712 \\
-138,279 \\
-152,119 \\
-118,526 \\
-109,399 \\
-102,498 \\
-66,723\end{array}$ \\
\hline 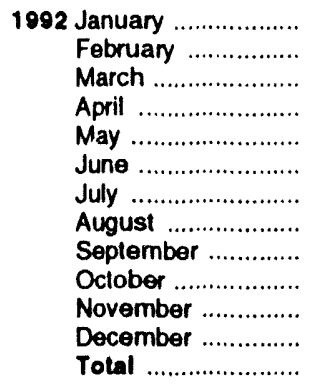 & $\begin{array}{r}602 \\
454 \\
419 \\
511 \\
535 \\
548 \\
654 \\
503 \\
428 \\
506 \\
550 \\
700 \\
6,412\end{array}$ & $\begin{array}{r}3,683 \\
3,165 \\
3,477 \\
3,931 \\
4,274 \\
4,713 \\
4,912 \\
4,702 \\
4,680 \\
5,047 \\
4,462 \\
4,172 \\
51,217\end{array}$ & $\begin{array}{l}-3,082 \\
-2,711 \\
-3,058 \\
-3,420 \\
-3,738 \\
-4,165 \\
-4,258 \\
-4,199 \\
-4,252 \\
-4,541 \\
-3,912 \\
-3,471 \\
-44,805\end{array}$ & $\begin{array}{r}1,007 \\
879 \\
831 \\
932 \\
968 \\
958 \\
1,067 \\
867 \\
839 \\
874 \\
940 \\
1,093 \\
11,254\end{array}$ & $\begin{array}{r}4,016 \\
3,452 \\
3,762 \\
4,215 \\
4,573 \\
5,007 \\
5,222 \\
5,034 \\
5,026 \\
5,456 \\
4,873 \\
4,621 \\
55,256\end{array}$ & $\begin{array}{l}-3,009 \\
-2,573 \\
-2,931 \\
-3,283 \\
-3,605 \\
-4,049 \\
-4,155 \\
-4,167 \\
-4,187 \\
-4,582 \\
-3,933 \\
-3,529 \\
-44,002\end{array}$ & $\begin{array}{r}-2,461 \\
396 \\
-596 \\
-2,489 \\
-1,804 \\
-2,669 \\
-5,738 \\
-6,051 \\
-5,506 \\
-5,124 \\
-4,711 \\
-3,747 \\
-40,500\end{array}$ & $\begin{array}{r}34,514 \\
36,898 \\
39,817 \\
37,154 \\
36,737 \\
39,094 \\
35,979 \\
34,838 \\
36,811 \\
40,115 \\
37,670 \\
38,537 \\
448,164\end{array}$ & $\begin{array}{r}39,984 \\
39,075 \\
43,344 \\
42,925 \\
42,146 \\
45,812 \\
45,872 \\
45,055 \\
46,503 \\
49,820 \\
46,314 \\
45,813 \\
532,665\end{array}$ & $\begin{array}{r}-5,470 \\
-2,178 \\
-3,527 \\
-5,772 \\
-5,409 \\
-6,718 \\
-9,893 \\
-10,218 \\
-9,693 \\
-9,706 \\
-8,644 \\
-7,276 \\
-84,501\end{array}$ \\
\hline 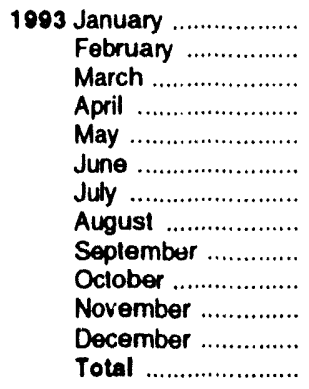 & $\begin{array}{l}617 \\
467 \\
488 \\
583 \\
647 \\
439 \\
514 \\
444 \\
436 \\
467 \\
478 \\
654 \\
6,233\end{array}$ & $\begin{array}{r}4,254 \\
3,699 \\
4,492 \\
4,845 \\
4,614 \\
4,707 \\
4,320 \\
4,031 \\
4,171 \\
4,450 \\
3,990 \\
3,443 \\
51,016\end{array}$ & $\begin{array}{r}-3,637 \\
-3,232 \\
-4,004 \\
-4,262 \\
-3,967 \\
-4,269 \\
-3,806 \\
-3,587 \\
-3,735 \\
-3,983 \\
-3,512 \\
-2,790 \\
-44,784\end{array}$ & $\begin{array}{r}936 \\
789 \\
768 \\
835 \\
944 \\
826 \\
818 \\
703 \\
723 \\
759 \\
716 \\
918 \\
9,736\end{array}$ & $\begin{array}{r}4,642 \\
4,070 \\
4,910 \\
5,191 \\
4,969 \\
5,023 \\
4,679 \\
4,404 \\
4,549 \\
4,854 \\
4,418 \\
3,875 \\
55,582\end{array}$ & $\begin{array}{r}-3,706 \\
-3,281 \\
-4,142 \\
-4,357 \\
-4,024 \\
-4,197 \\
-3,862 \\
-3,700 \\
-3,826 \\
-4,094 \\
-3,702 \\
-2,956 \\
-45,847\end{array}$ & $\begin{array}{r}-2,407 \\
-2,625 \\
-4,745 \\
-4,072 \\
-2,518 \\
-7,552 \\
-8,747 \\
-8,249 \\
-8,690 \\
-8,543 \\
-7,418 \\
-4,327 \\
-69,891\end{array}$ & $\begin{array}{r}35,922 \\
36,004 \\
41,895 \\
39,374 \\
39,751 \\
38,616 \\
35,529 \\
36,624 \\
38,052 \\
41,230 \\
40,312 \\
41,466 \\
464,773\end{array}$ & $\begin{array}{r}42,035 \\
41,909 \\
50,781 \\
47,802 \\
46,293 \\
50,365 \\
48,138 \\
48,573 \\
50,567 \\
53,867 \\
51,432 \\
48,748 \\
580,511\end{array}$ & $\begin{array}{r}-6,113 \\
-5,905 \\
-8,886 \\
-8,428 \\
-6,542 \\
-11,749 \\
-12,609 \\
-11,949 \\
-12,516 \\
-12,638 \\
-11,120 \\
-7,283 \\
-115,736\end{array}$ \\
\hline $\begin{array}{l}1904 \text { January ................ } \\
\text { February ................. } \\
\text { March ................. } \\
\text { 3-Month Total ....... }\end{array}$ & $\begin{array}{r}452 \\
366 \\
452 \\
1,270\end{array}$ & $\begin{array}{r}3,114 \\
3,298 \\
3,731 \\
10,142\end{array}$ & $\begin{array}{l}-2,662 \\
-2,932 \\
-3,279 \\
-8,872\end{array}$ & $\begin{array}{r}676 \\
573 \\
728 \\
1,977\end{array}$ & $\begin{array}{r}3,603 \\
3,860 \\
4,229 \\
11,692\end{array}$ & $\begin{array}{l}-2,927 \\
-3,287 \\
-3,501 \\
-9,715\end{array}$ & $\begin{array}{r}-6,026 \\
P_{-6,311} \\
-5,201 \\
-17,538\end{array}$ & $\begin{array}{r}37,499 \\
R_{37,118} \\
46,044 \\
120,661\end{array}$ & $\begin{array}{r}46,451 \\
\text { म6,716 } \\
54,747 \\
147,914\end{array}$ & $\begin{array}{r}\cdot 8,953 \\
R \cdot 9,598 \\
-8,702 \\
-27,253\end{array}$ \\
\hline $\begin{array}{l}1993 \text { 3-Month Total ....... } \\
1992 \text { 3-Month Total ....... }\end{array}$ & $\begin{array}{l}1,572 \\
1,475\end{array}$ & $\begin{array}{l}12,445 \\
10,325\end{array}$ & $\begin{array}{r}-10,873 \\
-8,850\end{array}$ & $\begin{array}{l}2,493 \\
2,717\end{array}$ & $\begin{array}{l}13,621 \\
11,230\end{array}$ & $\begin{array}{r}-11,128 \\
-8,513\end{array}$ & $\begin{array}{l}-9,776 \\
-2,662\end{array}$ & $\begin{array}{l}113,821 \\
111,230\end{array}$ & $\begin{array}{l}134,725 \\
122,404\end{array}$ & $\begin{array}{l}-20,905 \\
-11,174\end{array}$ \\
\hline
\end{tabular}

R=Revised data.

Notes: - Monthly data are not adjusted for seasonal variations. - The U.S. Impon statistics reflect both government and nongovernment imports of merchandise trom foreign countries into the U.S. customs territory, which comprises the 50 States, the District of Columbia, Puerto Rico, and the Virgin
Islands. - See Note 5 at end of section. - Tolals may not equal sum of components due to independent rounding.

Sources: - U.S. Department of Commerce, Bureau of the Census, Foreign Trade Division. For details, see "Sources for Table 1.6" at the end of this section. 
Figure 1.6 Energy Consumption per Dollar of Gross Domestic Product

(Thousand Btu per 1987 Dollar)

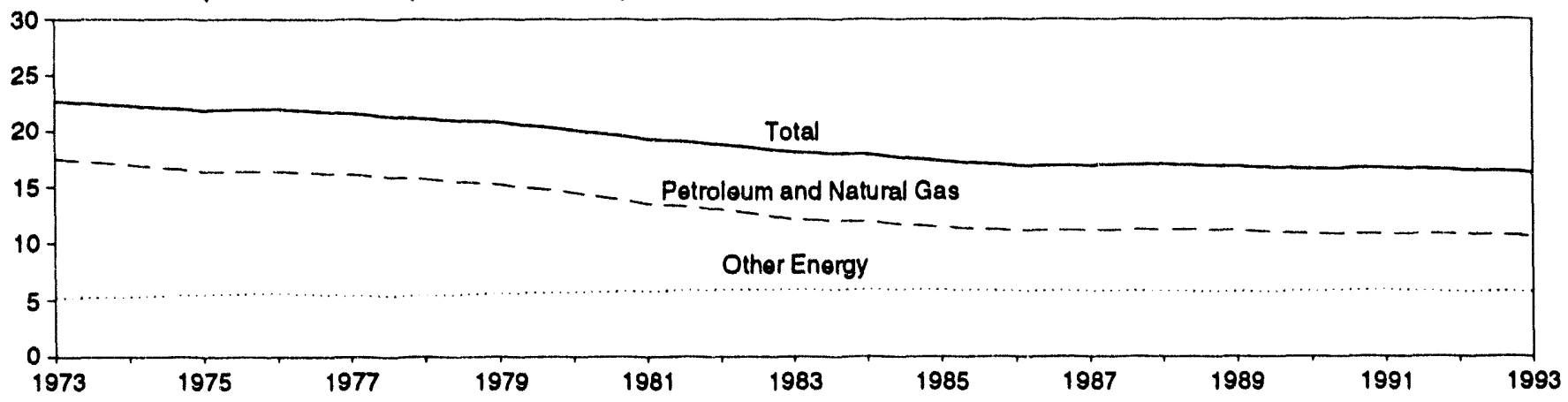

Source: Table 1.7.

Table 1.7 Energy Consumption per Dollar of Gross Domestic Product (Seasonally Adjusted at Annual Rates)

\begin{tabular}{|c|c|c|c|c|c|c|c|}
\hline & \multicolumn{3}{|c|}{ Energy Consumption } & \multirow{3}{*}{$\begin{array}{c}\begin{array}{c}\text { Groses } \\
\text { Domestle } \\
\text { Product } \\
\text { (GDP) }\end{array} \\
\begin{array}{c}\text { Trillion } \\
1987 \text { Dollars }\end{array}\end{array}$} & \multicolumn{3}{|c|}{ Enorgy Consumption per Dollar of GDP } \\
\hline & $\begin{array}{c}\text { Potroloum } \\
\text { and } \\
\text { Natural Gas }\end{array}$ & $\begin{array}{c}\text { Other } \\
\text { Energy }\end{array}$ & Totala & & $\begin{array}{c}\begin{array}{c}\text { Potroloum } \\
\text { and } \\
\text { Natural Gas }\end{array} \\
\end{array}$ & $\begin{array}{l}\text { Other } \\
\text { Energy }\end{array}$ & Total \\
\hline & \multicolumn{3}{|c|}{ Quadrillion Blu } & & \multicolumn{3}{|c|}{ Thousand Blu per 1987 Dollar } \\
\hline 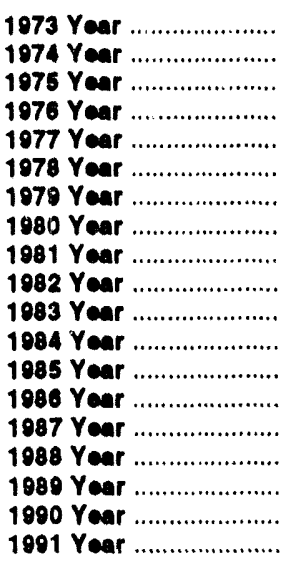 & $\begin{array}{l}\mathbf{5 7 . 3 5 2} \\
\mathbf{5 5 . 1 8 7} \\
\mathbf{5 2 . 6 7 8} \\
\mathbf{5 5 . 5 2 0} \\
\mathbf{5 7 . 0 5 3} \\
\mathbf{5 7 . 0 6 8} \\
\mathbf{5 7 . 7 8 9} \\
\mathbf{5 4 . 5 9 6} \\
\mathbf{5 1 . 8 5 9} \\
\mathbf{4 8 . 7 3 6} \\
\mathbf{4 7 . 4 1 1} \\
\mathbf{4 0 . 5 5 8} \\
\mathbf{4 8 . 7 5 6} \\
\mathbf{4 8 . 9 0 4} \\
\mathbf{5 0 . 6 0 8} \\
\mathbf{5 2 . 7 7 4} \\
\mathbf{5 3 . 5 9 5} \\
\mathbf{5 2 . 8 4 9} \\
\mathbf{5 2 . 4 5 2}\end{array}$ & $\begin{array}{l}16.030 \\
17.358 \\
17.868 \\
18.842 \\
10.235 \\
20.123 \\
21.109 \\
21.359 \\
22.131 \\
22.112 \\
23.113 \\
24.588 \\
25.225 \\
25.393 \\
26.285 \\
27.444 \\
27.730 \\
28.418 \\
28.664\end{array}$ & $\begin{array}{l}74.282 \\
72.543 \\
70.548 \\
74.362 \\
76.288 \\
78.089 \\
78.898 \\
75.955 \\
73.990 \\
70.848 \\
70.524 \\
74.144 \\
73.981 \\
74.297 \\
76.894 \\
80.218 \\
81.325 \\
81.265 \\
81.116\end{array}$ & $\begin{array}{l}3.269 \\
3.248 \\
3.222 \\
3.381 \\
3.533 \\
3.704 \\
3.797 \\
3.776 \\
3.843 \\
3.760 \\
3.007 \\
4.149 \\
4.280 \\
4.405 \\
4.540 \\
4.719 \\
4.838 \\
4.897 \\
4.861\end{array}$ & $\begin{array}{l}17.5 \\
17.0 \\
16.4 \\
16.4 \\
16.1 \\
15.7 \\
15.2 \\
14.5 \\
13.5 \\
13.0 \\
12.1 \\
11.9 \\
11.4 \\
11.1 \\
11.1 \\
11.2 \\
11.1 \\
10.8 \\
10.8\end{array}$ & $\begin{array}{l}5.2 \\
5.3 \\
5.5 \\
5.6 \\
5.4 \\
5.4 \\
5.6 \\
5.7 \\
5.8 \\
5.0 \\
5.0 \\
5.0 \\
5.0 \\
5.8 \\
5.8 \\
5.8 \\
5.7 \\
5.8 \\
5.9\end{array}$ & $\begin{array}{l}22.7 \\
22.3 \\
21.0 \\
22.0 \\
21.8 \\
21.1 \\
20.8 \\
20.1 \\
19.3 \\
18.8 \\
18.1 \\
17.9 \\
17.3 \\
16.9 \\
16.9 \\
17.0 \\
16.8 \\
16.8 \\
16.7\end{array}$ \\
\hline 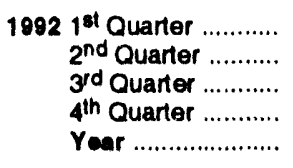 & $\begin{array}{l}\mathbf{5 3 . 7 1 4} \\
\mathbf{5 4 . 0 0 3} \\
\mathbf{5 2 . 8 3 1} \\
\mathbf{5 4 . 0 8 4} \\
\mathbf{5 3 . 6 5 7}\end{array}$ & $\begin{array}{l}28.097 \\
28.600 \\
28.274 \\
28.974 \\
28.487\end{array}$ & $\begin{array}{l}81.811 \\
82.603 \\
81.105 \\
83.058 \\
82.144\end{array}$ & $\begin{array}{l}4.922 \\
4.957 \\
4.998 \\
5.068 \\
4.986\end{array}$ & $\begin{array}{l}10.9 \\
10.9 \\
10.6 \\
10.7 \\
10.8\end{array}$ & $\begin{array}{l}5.7 \\
5.8 \\
5.7 \\
5.7 \\
5.7\end{array}$ & $\begin{array}{l}16.6 \\
16.7 \\
16.2 \\
16.4 \\
16.5\end{array}$ \\
\hline $\begin{array}{r}10931^{\text {st }} \text { Quarter ............ } \\
2^{\text {nd }} \text { Quarter ........... } \\
3^{\text {rd }} \text { Quarter ............ } \\
4^{\text {th }} \text { Quarter ............ } \\
\text { Year ....................... }\end{array}$ & $\begin{array}{r}55.837 \\
53.230 \\
54.353 \\
54.794 \\
\text { ค } \mathbf{5 4 . 5 5 0}\end{array}$ & $\begin{array}{l}A_{29.322} \\
R_{29.681} \\
R_{29.145} \\
P_{28.696} \\
R_{29.200}\end{array}$ & $\begin{array}{l}R_{85.159} \\
R_{82.911} \\
A_{83.498} \\
A_{83.490} \\
{ }^{R} 83.750\end{array}$ & $\begin{array}{l}5.078 \\
5.102 \\
5.138 \\
5.226 \\
5.136\end{array}$ & $\begin{array}{l}11.0 \\
10.4 \\
10.6 \\
10.5 \\
10.6\end{array}$ & $\begin{array}{l}5.8 \\
5.8 \\
5.7 \\
5.5 \\
5.7\end{array}$ & $\begin{array}{l}16.8 \\
16.3 \\
16.3 \\
16.0 \\
16.3\end{array}$ \\
\hline
\end{tabular}

axcludes wood, waste, geothermal, wind, photovohaic, and solar thermal energy, except for small amounts used by electric utilities to generate electricity for distribution.

$R=$ Revised data.

Notes: - Quarterty data are seasonally adjusted and shown at annual rates. Geographic coverage is the $\mathbf{5 0}$ States and the District of Columbia. - Totals may not equal sum of components due to independent rounding.
- Yearly data may not equal average of quarters due to seasonality adjustments and independent rounding.

Sources: - Eneray Consumptlon: Table 1.4. - Gross Domestic Product: 1073-1001-U.S. Department of Commerce, Bureau of Economic Analysis, Survey of Current Business, September 1993, Table 2.1902 forward-U.S. Depanment of Commerce, Bureau of Economic Analysis, United States Department of Commerce News, April 28, 1994, Table 2. 
(Net Imports as Percent of Product Supplied)

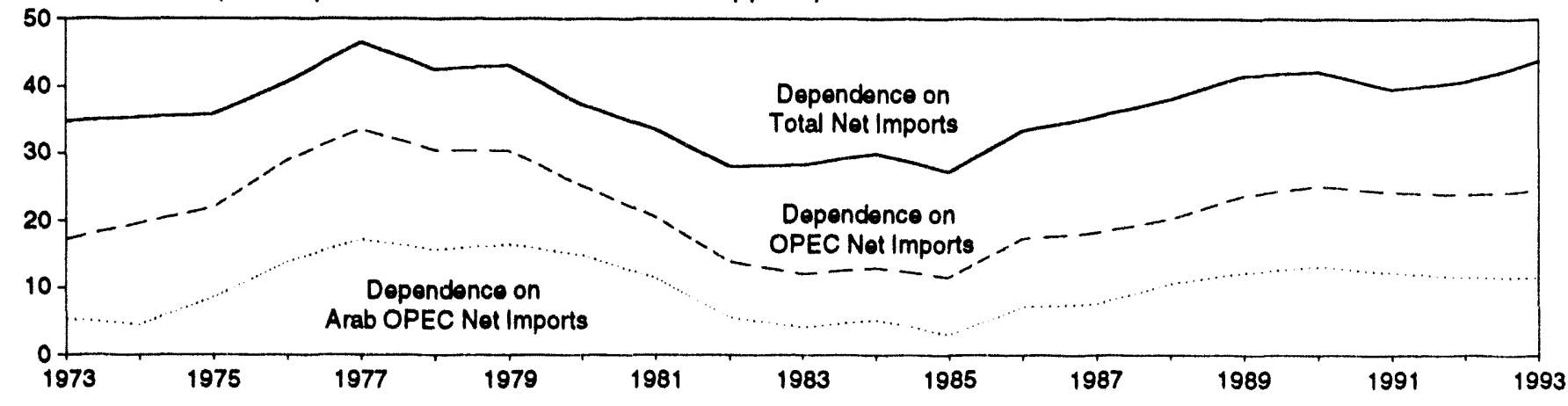

Source: Table 1.8.

Table 1.8 U.S. Dependence on Petroleum Net Imports

\begin{tabular}{|c|c|c|c|c|c|c|c|}
\hline \multirow{3}{*}{ Annual Rate } & \multicolumn{3}{|c|}{ Not Importsa } & \multirow{3}{*}{$\begin{array}{l}\text { Potroleum } \\
\text { Producte } \\
\text { Supplled }\end{array}$} & \multicolumn{3}{|c|}{$\begin{array}{l}\text { Net Imports as Porcent of } \\
\text { U.S. Potroleum Products Supplied }\end{array}$} \\
\hline & $\begin{array}{l}\text { From Arab } \\
\text { OPEC }^{b}\end{array}$ & $\begin{array}{l}\text { From } \\
\text { OPEC }\end{array}$ & $\begin{array}{l}\text { From All } \\
\text { Countrice }\end{array}$ & & $\begin{array}{l}\text { From Arab } \\
\text { OPECb }\end{array}$ & $\begin{array}{l}\text { From } \\
\text { OPEC }\end{array}$ & $\begin{array}{l}\text { From All } \\
\text { Countrice }\end{array}$ \\
\hline & \multicolumn{3}{|c|}{ Thousand Barrels per Day } & & \multicolumn{3}{|c|}{ Percent } \\
\hline 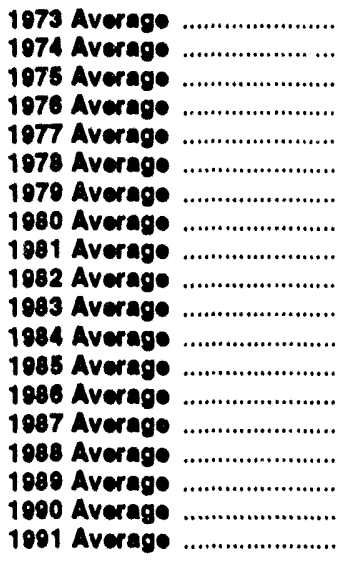 & $\begin{array}{r}914 \\
752 \\
1,392 \\
2,423 \\
3,184 \\
2,962 \\
3,054 \\
2,540 \\
1,844 \\
852 \\
630 \\
817 \\
470 \\
1,160 \\
1,272 \\
1,837 \\
2,128 \\
2,243 \\
2,057\end{array}$ & $\begin{array}{l}2,091 \\
3,277 \\
3,509 \\
5,063 \\
6,100 \\
5,747 \\
5,633 \\
4,293 \\
3,315 \\
2,136 \\
1,843 \\
2,037 \\
1,821 \\
2,828 \\
3,053 \\
3,513 \\
4,124 \\
4,285 \\
4,084\end{array}$ & $\begin{array}{l}6,025 \\
5,802 \\
5,846 \\
7,090 \\
8,565 \\
8,002 \\
7,985 \\
6,365 \\
5,401 \\
4,298 \\
4,312 \\
4,715 \\
4,286 \\
5,439 \\
5,914 \\
6,587 \\
7,202 \\
7,161 \\
6,626\end{array}$ & $\begin{array}{l}17,308 \\
16,053 \\
16,322 \\
17,461 \\
18,431 \\
18,847 \\
18,513 \\
17,056 \\
16,058 \\
15,206 \\
15,231 \\
15,726 \\
15,726 \\
16,281 \\
16,065 \\
17,283 \\
17,325 \\
16,088 \\
16,714\end{array}$ & $\begin{array}{r}5.3 \\
4.5 \\
8.5 \\
13.9 \\
17.9 \\
17.3 \\
15.7 \\
16.5 \\
14.9 \\
11.5 \\
5.6 \\
4.1 \\
4.1 \\
5.2 \\
3.0 \\
7.1 \\
7.6 \\
7.6 \\
10.6 \\
12.3 \\
13.2 \\
12.3\end{array}$ & $\begin{array}{l}17.3 \\
10.7 \\
22.0 \\
29.0 \\
33.8 \\
30.5 \\
30.4 \\
25.2 \\
20.8 \\
14.0 \\
12.1 \\
13.0 \\
11.6 \\
17.4 \\
18.3 \\
20.3 \\
23.8 \\
25.2 \\
24.3\end{array}$ & $\begin{array}{l}34.8 \\
35.4 \\
35.8 \\
40.8 \\
46.5 \\
42.5 \\
43.1 \\
37.3 \\
33.6 \\
28.1 \\
28.3 \\
30.0 \\
27.3 \\
33.4 \\
35.5 \\
38.1 \\
41.6 \\
42.2 \\
39.8\end{array}$ \\
\hline 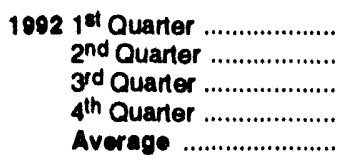 & $\begin{array}{l}2,052 \\
1,922 \\
1,910 \\
2,005 \\
1,072\end{array}$ & $\begin{array}{l}3,783 \\
4,056 \\
4,230 \\
4,210 \\
4,071\end{array}$ & $\begin{array}{l}6,239 \\
7,027 \\
7,451 \\
7,029 \\
8,030\end{array}$ & $\begin{array}{l}16,910 \\
16,740 \\
16,984 \\
17,493 \\
17,033\end{array}$ & $\begin{array}{l}12.1 \\
11.5 \\
11.2 \\
11.5 \\
11.6\end{array}$ & $\begin{array}{l}22.4 \\
24.2 \\
24.9 \\
24.1 \\
23.9\end{array}$ & $\begin{array}{l}36.9 \\
42.0 \\
43.9 \\
40.2 \\
40.7\end{array}$ \\
\hline 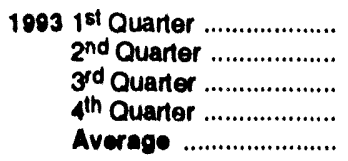 & $\begin{array}{l}2,025 \\
2,053 \\
1,907 \\
1,971 \\
1,080\end{array}$ & $\begin{array}{l}4,311 \\
4,352 \\
4,129 \\
4,134 \\
4,230\end{array}$ & $\begin{array}{l}7,038 \\
7,507 \\
7,750 \\
7,786 \\
7,523\end{array}$ & $\begin{array}{l}17,126 \\
16,678 \\
17,360 \\
17,600 \\
17,103\end{array}$ & $\begin{array}{l}11.8 \\
12.3 \\
11.0 \\
11.2 \\
11.6\end{array}$ & $\begin{array}{l}25.2 \\
26.1 \\
23.8 \\
23.5 \\
24.6\end{array}$ & $\begin{array}{l}41.1 \\
45.0 \\
44.6 \\
44.2 \\
43.8\end{array}$ \\
\hline
\end{tabular}

- "Net imports" are imports minus exports. Imports from members of the Organization of Petroleum Exporting Countries (OPEC) exclude indirect imports, which are petroleum products primarily from Caribbean and West European areas and relined from crude oil produced by OPEC.

b The Arab members of OPEC are Algerla, Iraq, Kuwail, Llbya, Qatar, Saudi Arabla, and the United Arab Emirates. Net imports from the Neutral Zone between Kuwait and Saudi Arabia are included in net imports from Arab OPEC.

c OPEC currently consists of Gabon, Indonesia, Iran, Nigeria, and Venezuela, as well as the Arab members.
Notes: - Beginning in October 1977, Strategic Petroleum Reserves are included. - Geographic coverage is the 50 States and the District of Columbia. - Annual averages may not equal average of quarters due to independent rounding.

Sources: - Imports: Tables 3.3a-3.3h. - Exports: 1973-1976-U.S. Department of the Interior, Bureau of Mines, Mineral Industry Surveys. 1977.1980-Energy Information Administration (EIA). Energy Data Reports, "Pelroloum Stalement, Annual." 1081-1092-ElA, Pelroleum Supply Annual. 1003 forward-EIA, Petroloum Supply Monthly. - Potroloum Producto Suppllod: Table 3.1a. 
Figure 1.8 Cost of Fuels to End Users in Constant (1982-84) Dollars

(Dollars per Million Btu)

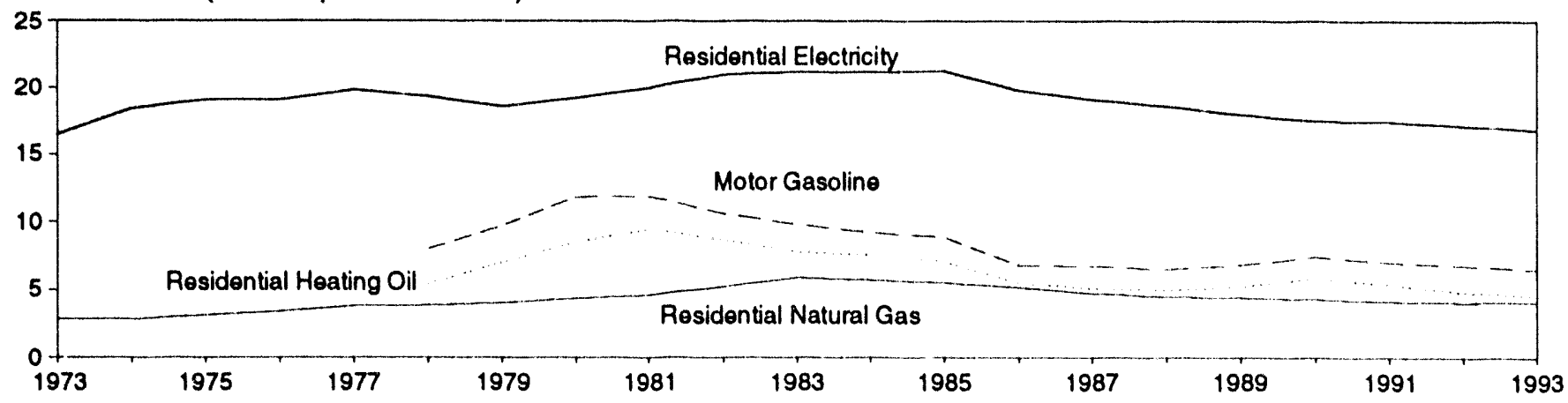

Source: Table 1.9.

Table 1.9 Cost of Fuels to End Users in Constant (1982-84) Dollars

\begin{tabular}{|c|c|c|c|c|c|c|c|c|}
\hline & \multicolumn{2}{|c|}{ Motor Gasoline } & \multicolumn{2}{|c|}{$\begin{array}{l}\text { Residentlal } \\
\text { Heating OII }\end{array}$} & \multicolumn{2}{|c|}{$\begin{array}{l}\text { Residontial } \\
\text { Natural Gas }\end{array}$} & \multicolumn{2}{|c|}{$\begin{array}{l}\text { Residontlal } \\
\text { Eloctricity }\end{array}$} \\
\hline & $\begin{array}{l}\text { Cents per } \\
\text { Gallon }\end{array}$ & $\begin{array}{l}\text { Dollars per } \\
\text { Million Btu }\end{array}$ & $\begin{array}{l}\text { Cents per } \\
\text { Gallon }\end{array}$ & $\begin{array}{l}\text { Dollars per } \\
\text { Million Btu }\end{array}$ & $\begin{array}{c}\text { Cents per } \\
\text { Thousand Cubic Foet }\end{array}$ & $\begin{array}{l}\text { Dollars per } \\
\text { Million Btu }\end{array}$ & $\begin{array}{l}\text { Conts per } \\
\text { Killowatthour }\end{array}$ & $\begin{array}{l}\text { Dollars per } \\
\text { Million Btu }\end{array}$ \\
\hline 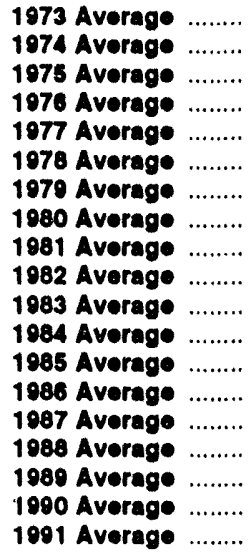 & $\begin{array}{l}\text { NA } \\
\text { NA } \\
\text { NA } \\
\text { NA } \\
\text { NA } \\
100.0 \\
121.5 \\
148.2 \\
148.8 \\
132.7 \\
123.0 \\
115.3 \\
111.2 \\
84.0 \\
84.2 \\
81.4 \\
85.5 \\
93.1 \\
87.8\end{array}$ & $\begin{array}{l}\text { NA } \\
\text { NA } \\
\text { NA } \\
\text { NA } \\
\text { NA } \\
6.00 \\
0.71 \\
11.85 \\
11.00 \\
10.61 \\
0.83 \\
0.22 \\
8.89 \\
6.79 \\
6.74 \\
6.51 \\
6.83 \\
7.44 \\
7.02\end{array}$ & $\begin{array}{l}\text { NA } \\
\text { NA } \\
\text { NA } \\
\text { NA } \\
\text { NA } \\
75.2 \\
97.0 \\
118.2 \\
131.4 \\
120.2 \\
108.2 \\
105.0 \\
97.9 \\
76.3 \\
70.7 \\
88.7 \\
72.6 \\
81.3 \\
74.8\end{array}$ & $\begin{array}{l}\text { NA } \\
\text { NA } \\
\text { NA } \\
\text { NA } \\
\text { NA } \\
5.42 \\
6.99 \\
8.52 \\
0.47 \\
6.67 \\
7.80 \\
7.57 \\
7.06 \\
5.50 \\
5.10 \\
4.96 \\
5.23 \\
5.86 \\
5.39\end{array}$ & $\begin{array}{l}290.5 \\
290.1 \\
317.8 \\
348.0 \\
387.8 \\
392.6 \\
410.5 \\
446.6 \\
471.0 \\
535.8 \\
608.4 \\
589.0 \\
568.8 \\
531.9 \\
487.7 \\
462.4 \\
454.8 \\
443.8 \\
427.3\end{array}$ & $\begin{array}{l}2.85 \\
2.83 \\
3.12 \\
3.41 \\
3.81 \\
3.86 \\
4.03 \\
4.36 \\
4.60 \\
5.22 \\
5.90 \\
5.72 \\
5.52 \\
5.17 \\
4.73 \\
4.49 \\
4.41 \\
4.31 \\
4.14\end{array}$ & $\begin{array}{l}5.6 \\
6.3 \\
6.5 \\
6.5 \\
6.8 \\
6.6 \\
6.3 \\
6.6 \\
6.8 \\
7.2 \\
7.2 \\
7.2 \\
7.2 \\
6.8 \\
6.5 \\
6.3 \\
6.1 \\
6.0 \\
5.9 \\
\end{array}$ & $\begin{array}{l}16.50 \\
18.43 \\
19.07 \\
19.06 \\
19.83 \\
19.33 \\
18.57 \\
19.21 \\
19.99 \\
20.96 \\
21.19 \\
21.16 \\
21.25 \\
19.79 \\
19.09 \\
18.58 \\
17.96 \\
17.49 \\
17.43\end{array}$ \\
\hline $\begin{array}{l}19921^{\text {st }} \text { Quarter ..... } \\
2^{\text {nd }} \text { Quarter .... } \\
3^{\text {rd }} \text { Quarter ..... } \\
4^{\text {th }} \text { Quarter .... } \\
\text { Average ........ }\end{array}$ & $\begin{array}{l}81.1 \\
85.3 \\
87.1 \\
85.6 \\
84.8\end{array}$ & $\begin{array}{l}6.49 \\
6.82 \\
6.96 \\
6.84 \\
6.78\end{array}$ & $\begin{array}{l}67.7 \\
66.0 \\
63.7 \\
66.5 \\
66.6\end{array}$ & $\begin{array}{l}4.88 \\
4.76 \\
4.59 \\
4.79 \\
4.80\end{array}$ & $\begin{array}{l}398.0 \\
443.5 \\
517.4 \\
429.2 \\
419.8\end{array}$ & $\begin{array}{l}3.86 \\
4.30 \\
5.02 \\
4.16 \\
4.07\end{array}$ & $\begin{array}{l}5.6 \\
5.9 \\
6.1 \\
5.8 \\
5.8\end{array}$ & $\begin{array}{l}16.48 \\
17.40 \\
17.89 \\
16.94 \\
17.13\end{array}$ \\
\hline $\begin{array}{r}10931^{\text {st }} \text { Quarter ..... } \\
2^{\text {nd }} \text { Quarter .... } \\
3^{\text {rd }} \text { Quarter .... } \\
4^{\text {th }} \text { Quarter ..... } \\
\text { Average ....... }\end{array}$ & $\begin{array}{l}81.9 \\
82.3 \\
80.3 \\
80.2 \\
81.2\end{array}$ & $\begin{array}{l}6.55 \\
6.58 \\
6.42 \\
6.41 \\
6.40\end{array}$ & $\begin{array}{l}66.2 \\
63.0 \\
58.7 \\
60.4 \\
63.0\end{array}$ & $\begin{array}{l}4.78 \\
4.54 \\
4.23 \\
4.35 \\
4.55\end{array}$ & $\begin{array}{l}398.3 \\
463.9 \\
544.9 \\
434.6 \\
425.6\end{array}$ & $\begin{array}{l}3.86 \\
4.50 \\
5.29 \\
4.22 \\
4.13\end{array}$ & $\begin{array}{l}5.5 \\
5.9 \\
6.0 \\
5.7 \\
5.7\end{array}$ & $\begin{array}{l}15.98 \\
17.28 \\
17.61 \\
16.68 \\
16.83\end{array}$ \\
\hline
\end{tabular}

NA=Not available.

Notes: - Fuel costs are calculated by using the Urban Consumer Price Index (CPI) developed by the Bureau of Labor Statistics. See Note 6 at end of section. - Geographic coverage is the 50 States and the District of Columbia. - Annual averages may not equal average of quarters due to independent rounding.

Sources: - Annual Data: Annual prices in Tables 9.4 (All Types), 9.8c,
9.11, and 9.9 (Monthly Series), adjusted by the CPI. - Quarterly Data: Simple averages of monthly prices in Tables 9.4 (All Types), 9.8c, 9.11, and 9.9 (Monthly Series), adjusted by the CPI. - CPI: 1973-1992-Economic Report of the President, February 1994. Taule B.59. 1993 forward-Council of Economic Advisers, Economic Indicators, April 1994, "Consumer Prices All Urban Consumers." - Conversion Factors: Tables A1, A4, and AB. 
Figure 1.9 Passenger Car Efficiency

(Index, 1973 = 100)

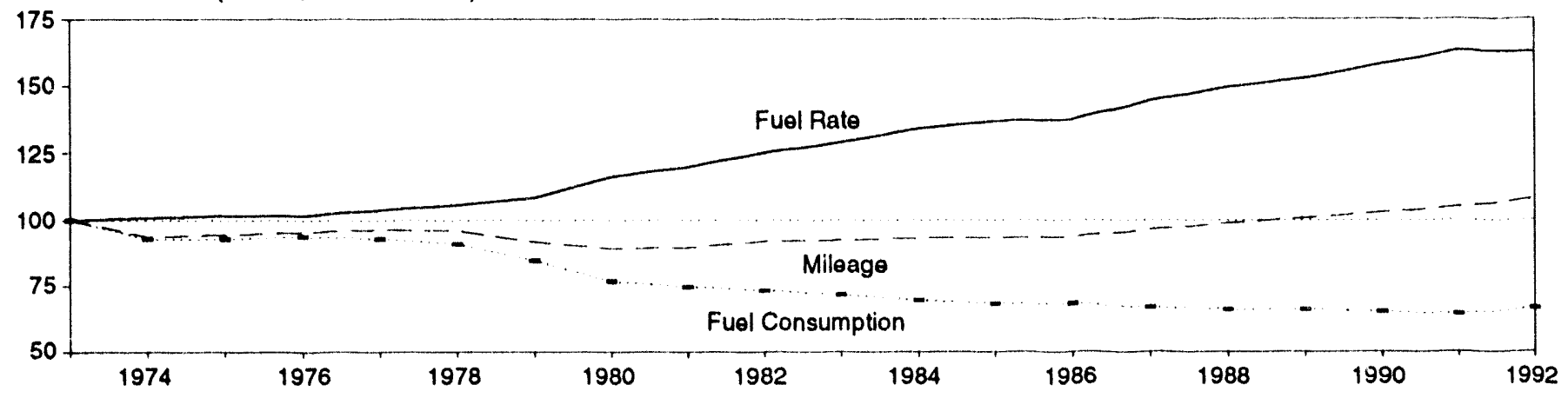

Source: Table 1.10.

Table 1.10 Passenger Car Efficiency

\begin{tabular}{|c|c|c|c|c|c|c|}
\hline & \multicolumn{2}{|c|}{ Miloago } & \multicolumn{2}{|c|}{ Fuel Consumption } & \multicolumn{2}{|c|}{ Fuel Rate } \\
\hline & $\begin{array}{l}\text { Miles } \\
\text { per Car }\end{array}$ & $\begin{array}{c}\text { Index } \\
1973=100.0\end{array}$ & $\begin{array}{l}\text { Gallons } \\
\text { per Car }\end{array}$ & $\begin{array}{c}\text { Index } \\
1973=100.0\end{array}$ & $\begin{array}{c}\text { Miles } \\
\text { per Gallon }\end{array}$ & $\begin{array}{c}\text { Index } \\
1973=100.0\end{array}$ \\
\hline $\begin{array}{l}1973 \\
1974 \\
1975 \\
1976 \\
1977 \\
1978 \\
1970\end{array}$ & $\begin{array}{r}10,256 \\
9,606 \\
9,690 \\
9,785 \\
9,879 \\
9,835 \\
9,403 \\
9,141 \\
9,186 \\
9,428 \\
9,475 \\
9,558 \\
9,560 \\
9,608 \\
9,878 \\
10,121 \\
10,332 \\
10,548 \\
10,757 \\
11,063\end{array}$ & $\begin{array}{r}100.0 \\
93.7 \\
94.5 \\
95.4 \\
96.3 \\
95.9 \\
91.7 \\
89.1 \\
69.6 \\
91.9 \\
92.4 \\
93.2 \\
93.2 \\
93.7 \\
96.3 \\
98.7 \\
100.7 \\
102.8 \\
104.9 \\
107.9\end{array}$ & $\begin{array}{l}771 \\
716 \\
716 \\
723 \\
716 \\
701 \\
653 \\
591 \\
576 \\
568 \\
553 \\
536 \\
525 \\
526 \\
514 \\
509 \\
509 \\
502 \\
496 \\
512\end{array}$ & $\begin{array}{r}100.0 \\
92.9 \\
92.9 \\
93.8 \\
92.9 \\
90.9 \\
64.7 \\
76.7 \\
74.7 \\
73.4 \\
71.7 \\
69.5 \\
68.1 \\
68.2 \\
66.7 \\
66.0 \\
66.0 \\
65.1 \\
64.3 \\
66.4\end{array}$ & $\begin{array}{l}13.30 \\
13.42 \\
13.52 \\
13.53 \\
13.80 \\
14.04 \\
14.41 \\
15.46 \\
15.94 \\
16.65 \\
17.14 \\
17.83 \\
18.20 \\
18.27 \\
10.20 \\
19.87 \\
20.31 \\
21.02 \\
21.69 \\
21.60\end{array}$ & $\begin{array}{l}100.0 \\
100.9 \\
101.7 \\
101.7 \\
103.8 \\
105.6 \\
108.3 \\
116.2 \\
119.8 \\
125.2 \\
128.9 \\
134.4 \\
136.8 \\
137.4 \\
144.4 \\
149.4 \\
152.7 \\
158.0 \\
163.1 \\
162.4\end{array}$ \\
\hline
\end{tabular}

- Preliminary data.

Nole: Geographic coverage is the 50 States and the District of Columbia.

Sources: Indices are prepared from statistics published by the U.S.

Department of Transportation, Federal Highway Administration, Federal
Highway Statistics Division. - 1973-1985: Highway Statistics Summary to 1985, Table VM-201A. - 1986 forward: Highway Statistics, annual, Table VM-1. 


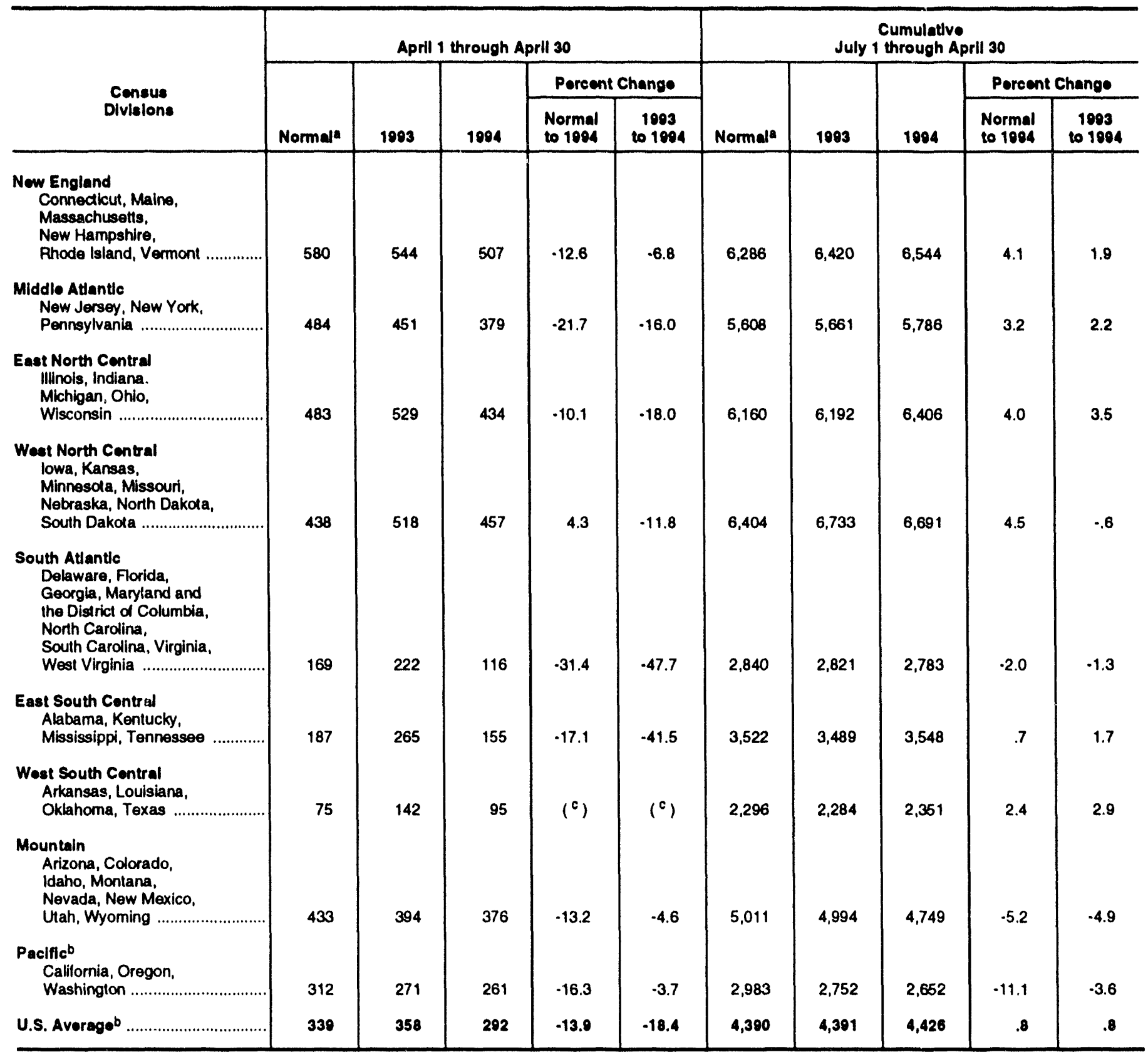

a "Normal" is based on calculations of data from 1961 through 1990.

b Excludes Alaska and Hawail.

c Percent change is not meaningful: normal is less than 100 or ratio is incalculable.

Notes: Degree-days are relative measurements of outdoor air temperature. Cooling degree-days are defined as deviations of the mean daily temperalure at a sampling station above a base temperature equal to $65^{\circ} \mathrm{F}$ by convention. Heating degree-days are deviations of the mean daily temperature below $65^{\circ} \mathrm{F}$. For example, if a weather station recorded a mean daily temperature of $78^{\circ} \mathrm{F}$, cooling degree-days for that station would be 13 (and heating degree-days, 0$)$. A weather station recording a mean daily temperature of $40^{\circ} \mathrm{F}$ would report 25 heating degreo-days (and 0 cooling degree-days).

Sources: There are several degree-day databases maintained by the

National Oceanic and Atmospheric Administration. The intomation published here is developed by the National Weather Service Climale Analysis Center, Camp Springs, MD. The data are available weekly with monthly summaries and are based on mean daily temperatures recorded at about 200 major weather stations around the country. The temperature information recorded at those weather stations is used to calculate statewide degree-day averages based on population. The State figures are then aggregated into Census Divisions and into the national average. The population weights currently used represent resident State population data estimated for 1990 by the U.S. Department of Cominerce, Bureau of the Census. The data provided here are available sooner than the Historical Climatology Series $\mathbf{5 . 1}$ (heating degree-days) and 5.2 (cooling degree-days) developed by the National Climatic Center, Asheville, NC, which compiles data from some 8,000 weather stations. 


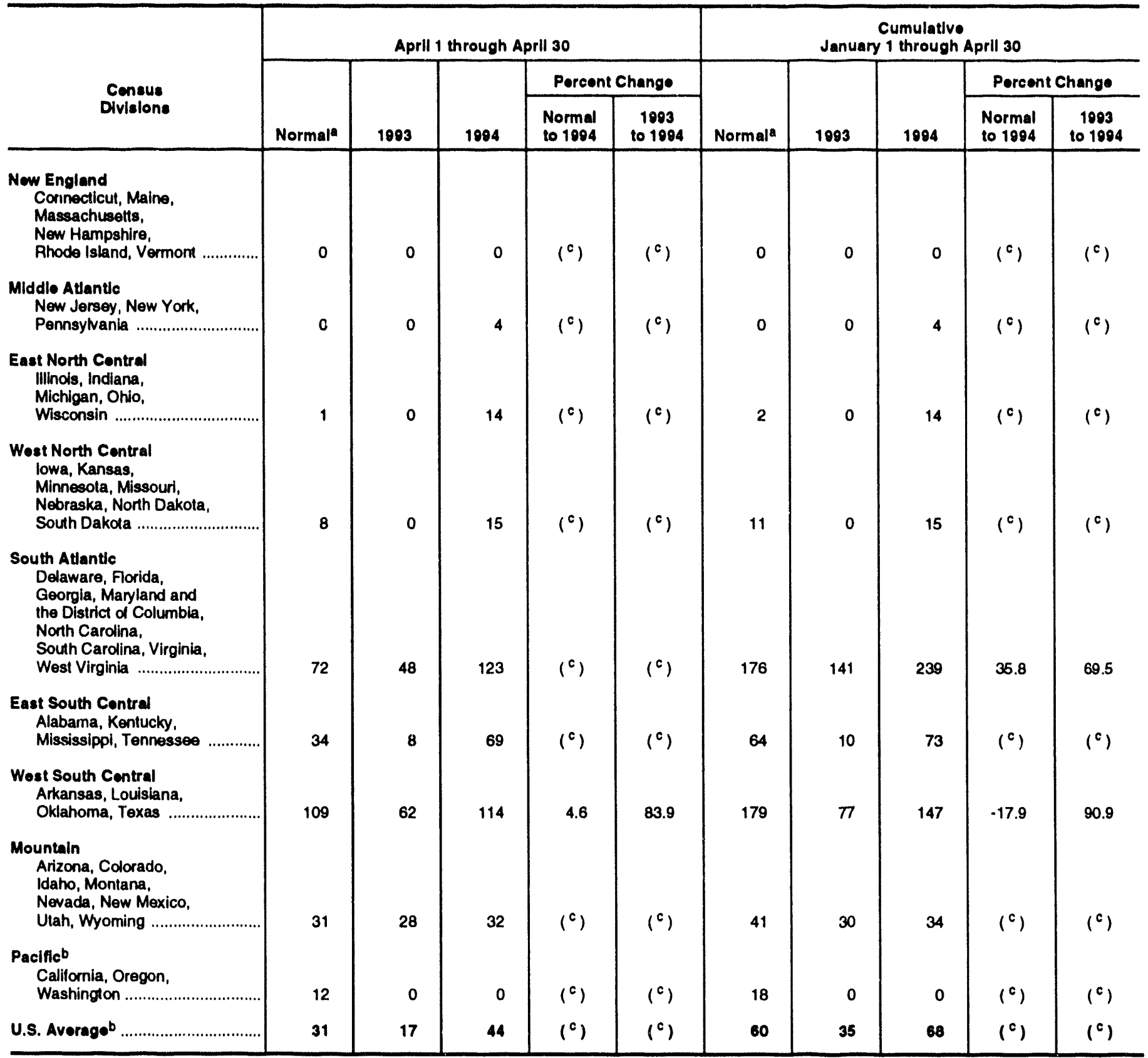

a "Normal" is based on calculations of data froril 1961 through 1990.

b Exdudes Alaska and Hawail.

c Percent change is not meaninglul: normal is less than 100 or ratio is incalculable.

Noles: Degree-days are relative measurements of outdoor air temperature. Cooling degree-days are defined as deviations of the mean daily temperature at a sampling station above a base temperature equal to $65^{\circ} \mathrm{F}$ by convention. Heating degree-days are deviations of the mean daily temperature below $65^{\circ} \mathrm{F}$. For example, a weather station recorded a mean daily temperature of $78^{\circ} \mathrm{F}$, cooling degree-days for that station would be 13 (and heating degree-days, 0). A weather station recording a mean daily temperature of $40^{\circ} \mathrm{F}$ would report 25 heating degree-days (and 0 cooling degree-days).

Sources: There are several degree-day databases maintained by the
National Oceanic and Atmospheric Administration. The information published here is developed by the National Weather Service Climate Analysis Center, Camp Springs, MD. The data are avallable weekly with monthly summaries and are based on mean daily temperatures recorded at about 200 major weather stations around the country. The temperature inlormation recorded at those weather stations is used to calculate statewide degree-day averages based on population. The State figures are then aggregated into Census Divisions and into the national average. The population weights currently used represent resident State population data estimated for 1990 by the U.S. Department of Commerce, Bureau of the Census. The data provided here are available sooner than the Historical Climalology Series 5-1 (heating degree-days) and 5-2 (cooling degree-days) developed by the National Climatic Center, Asheville, NC, which compiles data from some 8,000 weather stations. 


\section{Energy Summary Notes}

1. Energy Production: Production of energy includes production of coal, crude oil and lease condensate, natural gas plant liquids, natural gas (dry), electric utility and industrial production of hydroelectric power, and electricity generated from nuclear power. Production also includes electricity generated for distribution from wood, waste, geothermal, wind, photovoltaic, and solar thermal energy but excludes other energy obtained from those sources because consistent historical data are not available. Approximate heat contents (Btu values) are derived by using the conversion factors provided in Appendix A.

2. Energy Consumption: Consumption of energy includes consumption of coal, natural gas (including supplemental gaseous fuels), petroleum products supplied, electric utility and industrial production of hydroelectric power, net imports of electricity (assumed to be hydroelectricity), net imports of coal coke, and electricity generated from nuclear power. Consumption also includes electricity generated for distribution from wood, waste, geothermal, wind, photovoltaic, and solar thermal energy but excludes other energy obtained from those sources because consistent historical data are not available. Approximate heat contents (Btu values) are derived by using the conversion factors provided in Appendix A.

3. Energy Imports: Energy imports include imports of coal, crude oil (including crude oil imported for the Strategic Petroleum Reserve), petroleum products, natural gas, electricity (assumed to be hydroelectricity), and coal coke. Approximate heat contents (Btu values) are derived by using the conversion factors provided in Appendix A. For further information on electricity, see "Note for imports and exports of electricity" under Note 8 of the Notes and Sources for the Energy Consumption Section.

4. Energy Exports: Energy exports include coal, crude oil, petroleum products, natural gas, electricity produced from hydroelectric power, and coal coke. Approximate heat contents (Btu values) are denved by using the conversion factors provided in Appendix A. For more information on electricity, see "Note for imports and exports of electricity" under Note 8 of the Notes and Sources for the Energy Consumption Section.

5. Merchandise Trade Value: Import data presented are based on the customs value. That value does not include insurance and freight and is consequently lower than the cost, insurance, and freight (CIF) value, which is also reported by the Bureau of the Census. All export data, and import data prior to 1981, are on a free alongside ship (f.a.s.) basis.

"Balance" is exports minus imports; a positive balance indicates a surplus trade value and a negative balance indicates a deficit trade value. "Energy" includes mineral fuels, lubricants, and related material. "NonEnergy Balance" and "Total Merchandise" include foreign exports (i.e., reexports) and nonmonetary gold and Department of Defense Grant-Aid shipments. The "Non-Energy Balance" is calculated by subtracting the "Energy" from the "Total Merchandise Balance."

"Imports" consist of government and nongovernment shipments of merchandise into the 50 States, the District of Columbia, Puerto Rico, the U.S. Virgin Islands, and the U.S. Foreign Trade Zones. They reflect the total arrival from foreign countries of merchandise that immediately entered consumption channels, warehouses, the Foreign Trade Zones, or the Strategic Petroleum Reserve. They exclude shipments between the United States, Puerto Rico, and U.S. possessions, shipments to U.S. Armed Forces and diplomatic missions abroad for their own use, U.S. goods returned to the United States by its Armed Forces, and in-transit shipments.

6. The Consumer Price Index: The values for the Consumer Price Index, All Urban Consumers, All Items, $1982-84=100$, are as follows:

$\begin{array}{lrlll}1973 & 44.4 & 1991 & \text { 1st Quarter } & 134.8 \\ 1974 & 49.3 & & \text { 2nd Quarter } & 135.6 \\ 1975 & 53.8 & & \text { 3rd Quarter } & 136.7 \\ 1976 & 56.9 & & \text { 4th Quarter } & 137.7 \\ 1977 & 60.6 & & \text { Year } & 136.2 \\ 1978 & 65.2 & 1992 & \text { 1st Quarter } & 138.7 \\ 1979 & 72.6 & & \text { 2nd Quarter } & 139.8 \\ 1980 & 82.4 & & \text { 3rd Quarter } & 140.9 \\ 1981 & 90.9 & & \text { 4th Quarter } & 141.9 \\ 1982 & 96.5 & & \text { Year } & 140.3 \\ 1983 & 99.6 & 1993 & \text { 1st Quarter } & 143.1 \\ 1984 & 103.9 & & \text { 2nd Quarter } & 144.2 \\ 1985 & 107.6 & & \text { 3rd Quarter } & 144.8 \\ 1986 & 109.6 & & \text { 4th Quartcr } & 145.8 \\ 1987 & 113.6 & \text { Year } & 144.5 \\ 1988 & 118.3 & & \end{array}$

\section{Sources for Table 1.6}

U.S. Department of Commerce, Bureau of the Census, Foreign Trade Division:

- Petroleum Exports-1974-1987: "U.S. Exports," FT410, December issues. 1988: "Report on U.S. Merchandise Trade, 1988 Final Revisions." 1989: "Report on U.S. Merchandise Trade, 1989 Revisions." 1990: "U.S. Merchandise Trade, 1990 Final Report." 1991: "U.S. Merchandise Trade, 1991 Final Report," May 13, 1992. 1992: "U.S. Merchandise Trade, 1992 Final Report," May 12, 1993. 1993 and 1994: "U.S. International Trade in Goods and Services," FT900, monthly.

- Petroleum Imports-1974-1987: "U.S. Merchandise Trade," FT900, December issues, 1975-1988. 1988: "Report on U.S. Merchandise Trade, 1988 Final 
Revisions." 1989: "Report on U.S. Merchandise Trade, 1989 Revisions." 1990: "U.S. Merchandise Trade, 1990 Final Report." 1991: "U.S. Merchandise Trade, 1991 Final Report," May 13, 1992, and "U.S. Merchandise Trade, October 1992," December 17, 1992, page 3. 1992: "U.S. Merchandise Trade, 1992 Final Report," May 12, 1993. 1993 and 1994: "U.S. International Trade in Goods and Services," FT900, monthly.

- Energy Exports and Imports-1974-1987: U.S. merchandise trade press releases and database printouts for adjustments. 1988: January-July, monthly FT900 supplement, 1989 issues. August-December, monthly FT900, 1989 issues. 1989: Monthly FT900, 1990 issues. 1990: "U.S. Merchandise Trade, 1990 Final Report." 1991: "U.S. Merchandise Trade, 1991 Final Report," May 13, 1992, and "U.S. Merchandise Trade, October 1992," December 17, 1992, page 3. 1992: "U.S. Merchandise Trade, 1992 Final Report," May 12,
1993. 1993 and 1994: "U.S. International Trade in Goods and Services," FT900, monthly.

- Total Merchandise-1974-1987: U.S. merchandise trade press releases and database printouts for adjustments. 1988: "Report on U.S. Merchandise Trade, 1988 Final Revisions," August 18, 1989.1 1989: "Report on U.S. Merchandise Trade, 1989 Revisions," July 10, 1990. 1990: "U.S. Merchandise Trade, 1990 Final Report," May 10, 1991, and "U.S. Merchandise Trade, December 1992," February 18, 1993, page 3. 1991-1992: "U.S. Merchandise Trade, 1992 Final Report," May 12, 1993. 1993 and 1994: "U.S. International Trade in Goods and Services," FT900, monthly.

- Petroleum Balance, Energy Balance, and Non-Energy Balance-Calculated by the Energy Information Administration. 


\section{Section 2. Energy Consumption}

U.S. total energy consumption in February 1994 was 7.5 quadrillion Btu. Petroleum products accounted for 37 percent ${ }^{1}$ of the energy consumed in February 1994, while natural gas accounted for 32 percent and coal accounted for 21 percent.

Residential and commercial sector consumption was 3.1 quadrillion Btu in February 1994, up 6 percent from the February 1993 level. The sector accounted for 42 percent of February 1994 total consumption, up 1 percentage point from its 41 -percent share in February 1993.

Industrial sector consumption was 2.6 quadrillion Btu in February 1994, up 3 percent from the February 1993 level. The industrial sector accounted for 34 percent of February 1994 total consumption, down 1 percentage point from its 35-percent share in February 1993.

Transportation sector consumption of energy was 1.8 quadrillion Btu in February 1994, up 3 percent from the February 1993 level. The sector accounted for 24 percent of February 1994 total consumption, about the same share as share as in February 1993.

Electric utility consumption of energy totaled 2.4 quadrillion Btu in February 1994, up 2 percent from the February 1993 level. Coal contributed 57 percent of the energy consumed by electric utilities in February 1994, while nuclear electric power contributed 22 percent; hydroelectric power 10 percent; natural gas 6 percent; petroleum 4 percent; and geothermal, wood, waste, wind, photovoltaic, and solar thermal energy, about 1 percent.

\section{Table 2.1 Energy Consumption Summary for February 1994}

(Quadrillion Btu)

\begin{tabular}{|c|c|c|c|c|c|c|}
\hline \multirow[b]{2}{*}{ Energy Source } & \multicolumn{4}{|c|}{ End-Use Sectors } & \multirow[b]{2}{*}{$\begin{array}{l}\text { Electric } \\
\text { Utilties }\end{array}$} & \multirow[b]{2}{*}{ Total } \\
\hline & $\begin{array}{c}\text { and } \\
\text { Commerclal }\end{array}$ & Industrial & Transportation & Totala & & \\
\hline 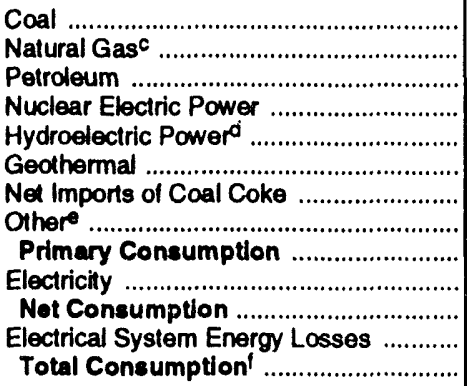 & $\begin{array}{r}016 \\
1.019 \\
.206 \\
- \\
- \\
- \\
- \\
- \\
1.540 \\
.546 \\
2.086 \\
1.059 \\
3.145\end{array}$ & $\begin{array}{r}0.209 \\
.825 \\
.757 \\
- \\
.003 \\
- \\
.001 \\
- \\
1.793 \\
.266 \\
2.059 \\
.516 \\
2.575\end{array}$ & $\begin{array}{r}\left({ }^{b}\right) \\
.071 \\
1.692 \\
- \\
- \\
- \\
- \\
- \\
1.763 \\
.001 \\
1.764 \\
.002 \\
1.766\end{array}$ & $\begin{array}{r}0.225 \\
2.214 \\
2.654 \\
- \\
.003 \\
- \\
. .001 \\
- \\
5.095 \\
.813 \\
5.807 \\
1.578 \\
7.485\end{array}$ & $\begin{array}{r}1.351 \\
.152 \\
.103 \\
.532 \\
.238 \\
.012 \\
- \\
.002 \\
2.390 \\
- \\
- \\
- \\
-\end{array}$ & $\begin{array}{r}1.576 \\
2.367 \\
2.757 \\
.532 \\
.240 \\
.012 \\
.001 \\
.002 \\
7.485 \\
- \\
- \\
- \\
-\end{array}$ \\
\hline
\end{tabular}

a Totals for coal and natural gas may not equal sum of sectors due to the use of sector-specilic conversion factors.

b Small amounts of coal consumed for transportation are reported as industrial sector consumption.

c Includes supplemental gaseous fuels. Transportation sector is pipeline fuel only.

Includes net imports of electricity.

- "Other" is electricity generated for distribution from wood, waste, wind, photovoltaic, and solar thermal energy.
1 Due to a lavi of consistent historical data, some renewable energy sources are not included. For example, in 1991, 3.3 quadrillion Btu of renewable energy consumed by U.S. electric utilities to generate electricity for distribution is included, but an estimated 3.4 quadrillion Btu of renewable energy used by other sectors is not included.

- =Not applicable.

Note: Totals may not equal sum of components due to independent rounding.

Additional Notes and Sources: See Tables 2.2-2.6 and end of section.

'Percentage changes are hasad on numbers in the following tables. 
Figure 2.1 Energy Consumption by End-Use Sector

(Quadrillion Btu)

Consumption by End-Use Sector, 1973-1993

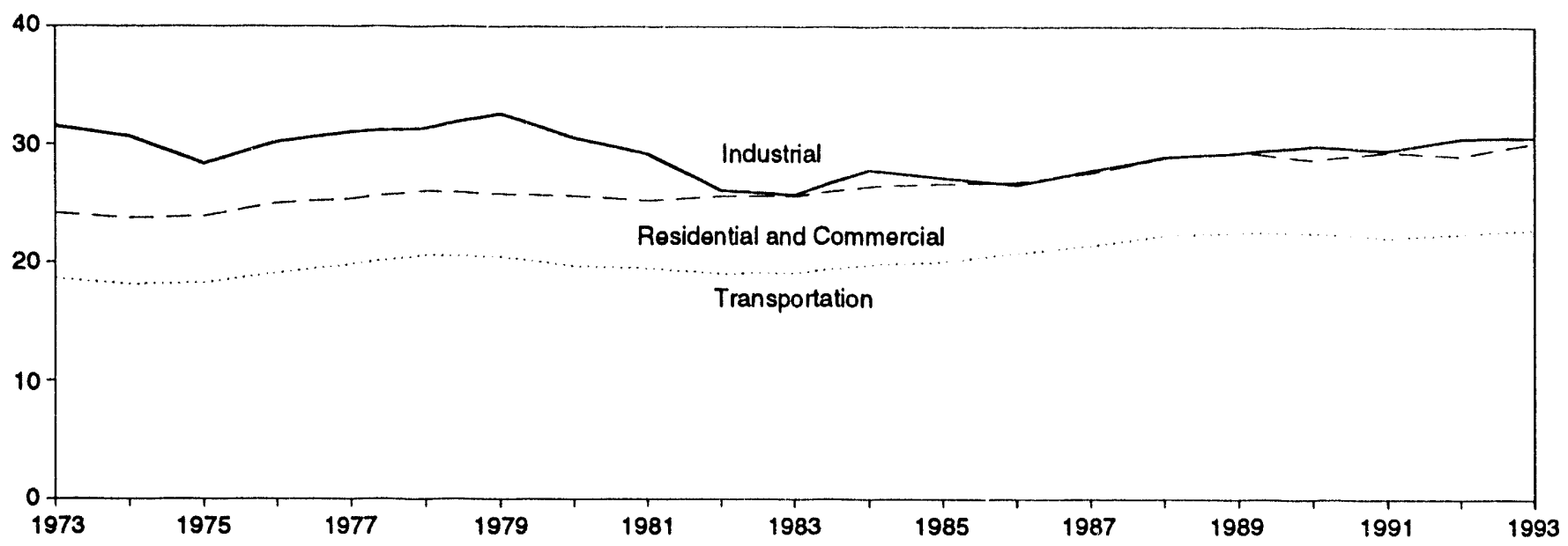

Consumption by End-Use Sector, Monthly

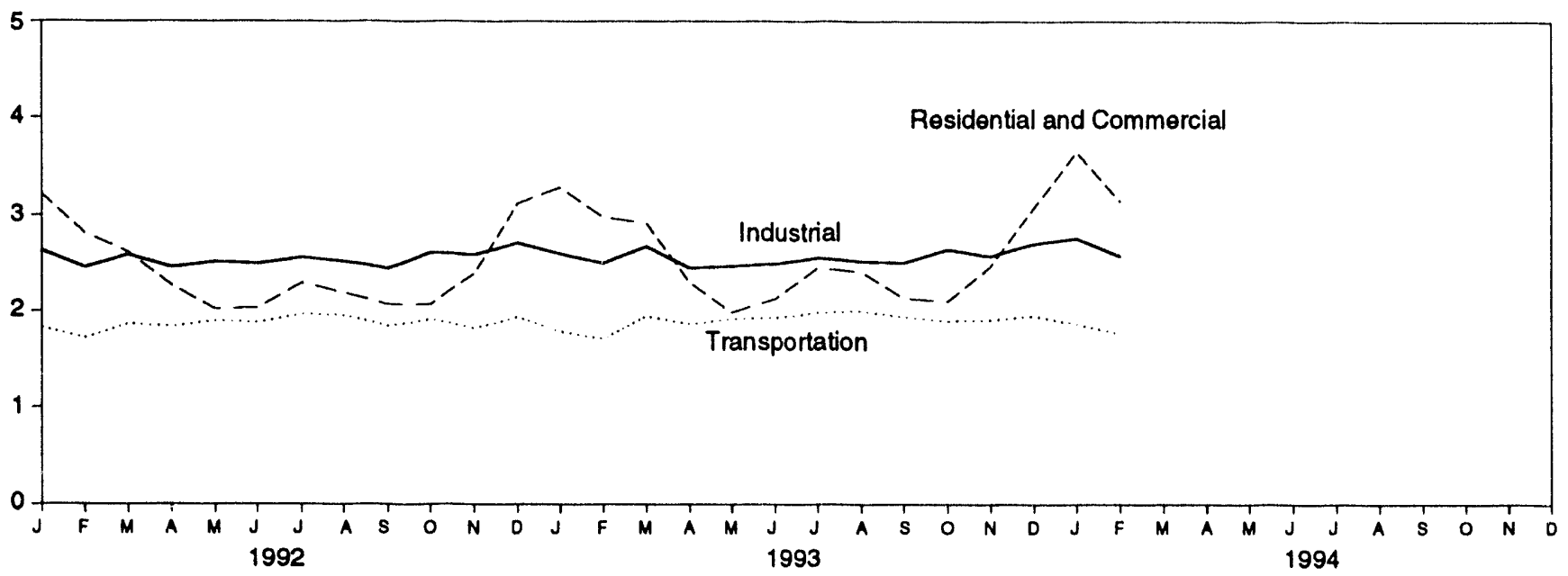

Consumption by End-Use Sector, February

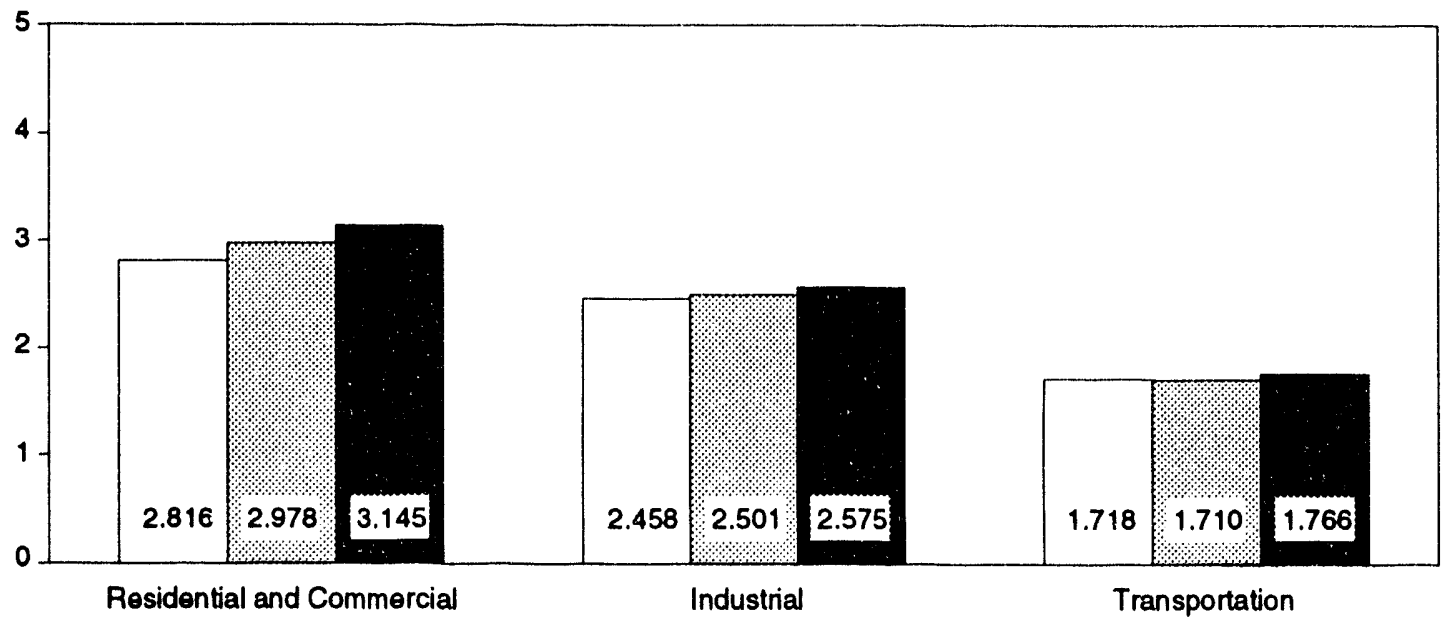

February 1992

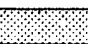

February 1993

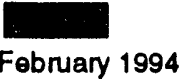

Note: Because vertical scales differ, graphs should not be compared.

Source: Table 2.2. 


\begin{tabular}{|c|c|c|c|c|c|c|c|c|}
\hline & \multicolumn{2}{|c|}{ Rosidential and Commerclal } & \multicolumn{2}{|c|}{ Industrial } & \multicolumn{2}{|c|}{ Transportation } & \multirow[b]{2}{*}{ Not } & \multirow[b]{2}{*}{ Totala } \\
\hline & Not & Total & Not & Total & Not & Total & & \\
\hline 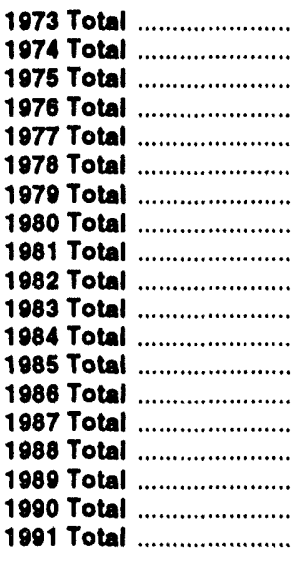 & $\begin{array}{l}15.768 \\
15.246 \\
15.200 \\
15.997 \\
15.828 \\
16.023 \\
15.709 \\
15.075 \\
14.541 \\
14.629 \\
14.395 \\
14.064 \\
14.839 \\
14.791 \\
15.146 \\
16.004 \\
16.261 \\
15.568 \\
15.986\end{array}$ & $\begin{array}{l}24.143 \\
23.725 \\
23.890 \\
25.018 \\
25.384 \\
26.084 \\
25.808 \\
25.655 \\
25.241 \\
25.629 \\
25.627 \\
26.474 \\
26.704 \\
26.852 \\
27.623 \\
28.025 \\
29.404 \\
28.786 \\
29.424\end{array}$ & $\begin{array}{l}25.917 \\
24.994 \\
22.737 \\
24.038 \\
24.593 \\
24.637 \\
25.679 \\
23.854 \\
22.533 \\
20.020 \\
19.401 \\
21.184 \\
20.520 \\
20.101 \\
21.116 \\
22.085 \\
22.272 \\
22.841 \\
22.549\end{array}$ & $\begin{array}{l}31.528 \\
30.694 \\
28.402 \\
30.236 \\
31.077 \\
31.392 \\
32.616 \\
30.606 \\
29.240 \\
26.145 \\
25.750 \\
27.867 \\
27.214 \\
26.630 \\
27.826 \\
28.986 \\
29.353 \\
29.936 \\
29.570\end{array}$ & $\begin{array}{l}18.584 \\
18.095 \\
18.219 \\
19.076 \\
19.794 \\
20.589 \\
20.447 \\
19.669 \\
19.480 \\
19.043 \\
19.100 \\
19.773 \\
20.036 \\
20.781 \\
21.419 \\
22.274 \\
22.530 \\
22.504 \\
22.090\end{array}$ & $\begin{array}{l}18.605 \\
18.117 \\
18.244 \\
19.101 \\
10.819 \\
20.611 \\
20.472 \\
19.695 \\
19.507 \\
19.069 \\
10.135 \\
10.801 \\
20.067 \\
20.812 \\
21.448 \\
22.305 \\
22.561 \\
22.535 \\
22.120\end{array}$ & $\begin{array}{l}60.274 \\
56.341 \\
56.157 \\
59.110 \\
60.223 \\
61.251 \\
61.836 \\
58.597 \\
56.556 \\
53.697 \\
52.007 \\
55.923 \\
55.391 \\
55.676 \\
57.678 \\
60.366 \\
61.070 \\
60.921 \\
60.626\end{array}$ & $\begin{array}{l}74.282 \\
72.543 \\
70.546 \\
74.362 \\
76.288 \\
78.089 \\
78.898 \\
75.955 \\
73.090 \\
70.848 \\
70.524 \\
74.144 \\
73.981 \\
74.297 \\
76.694 \\
80.218 \\
81.325 \\
81.265 \\
81.116\end{array}$ \\
\hline 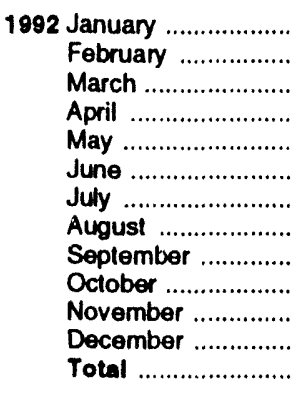 & $\begin{array}{r}2.029 \\
1.814 \\
1.596 \\
1.336 \\
1.040 \\
.941 \\
.995 \\
.974 \\
.983 \\
1.083 \\
1.381 \\
1.918 \\
16.090\end{array}$ & $\begin{array}{r}3.218 \\
2.816 \\
2.615 \\
2.272 \\
2.021 \\
2.029 \\
2.293 \\
2.195 \\
2.065 \\
2.066 \\
2.390 \\
3.118 \\
29.100\end{array}$ & $\begin{array}{r}2.062 \\
1.940 \\
2.014 \\
1.909 \\
1.917 \\
1.860 \\
1.902 \\
1.893 \\
1.862 \\
2.030 \\
1.992 \\
2.118 \\
23.498\end{array}$ & $\begin{array}{r}2.633 \\
2.458 \\
2.590 \\
2.458 \\
2.515 \\
2.494 \\
2.558 \\
2.520 \\
2.444 \\
2.610 \\
2.588 \\
2.711 \\
30.577\end{array}$ & $\begin{array}{r}1.826 \\
1.716 \\
1.864 \\
1.834 \\
1.897 \\
1.875 \\
1.963 \\
1.952 \\
1.842 \\
1.911 \\
1.818 \\
1.933 \\
22.432\end{array}$ & $\begin{array}{r}1.828 \\
1.718 \\
1.866 \\
1.837 \\
1.899 \\
1.878 \\
1.966 \\
1.954 \\
1.844 \\
1.914 \\
1.820 \\
1.936 \\
22.461\end{array}$ & $\begin{array}{r}5.916 \\
5.468 \\
5.472 \\
5.078 \\
4.853 \\
4.678 \\
4.865 \\
4.822 \\
4.689 \\
5.024 \\
5.190 \\
5.970 \\
62.025\end{array}$ & $\begin{array}{r}7.678 \\
6.989 \\
7.070 \\
6.565 \\
6.435 \\
6.403 \\
6.822 \\
6.673 \\
6.356 \\
6.590 \\
6.798 \\
7.765 \\
82.144\end{array}$ \\
\hline 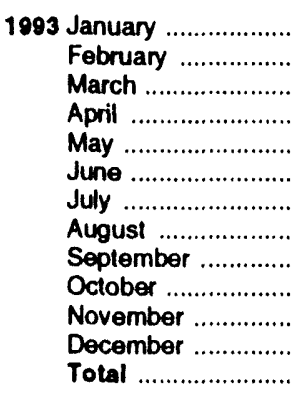 & $\begin{array}{r}2.086 \\
1.942 \\
1.836 \\
1.370 \\
1.000 \\
.973 \\
1.043 \\
1.034 \\
1.044 \\
1.104 \\
1.448 \\
1.899 \\
16.781\end{array}$ & $\begin{array}{l}A_{3.286} \\
A_{2.978} \\
A_{2.919} \\
R_{2.301} \\
A_{1.984} \\
A_{2.127} \\
A_{2.448} \\
A_{2.414} \\
A_{2.134} \\
A_{2.103} \\
A_{2.468} \\
A_{3.076} \\
A_{30.230}\end{array}$ & $\begin{array}{r}A_{2.033} \\
1.975 \\
2.108 \\
1.908 \\
R_{1.859} \\
1.851 \\
1.906 \\
1.879 \\
1.962 \\
2.069 \\
1.982 \\
2.104 \\
23.634\end{array}$ & $\begin{array}{l}A_{2.598} \\
R_{2.501} \\
R_{2.676} \\
R_{2.451} \\
A_{2.469} \\
A_{2.493} \\
A_{2.555} \\
A_{2.520} \\
A_{2.503} \\
R_{2.644} \\
A_{2.572} \\
A_{2.704} \\
A_{30.687}\end{array}$ & $\begin{array}{r}1.784 \\
1.707 \\
1.945 \\
1.858 \\
1.917 \\
1.926 \\
1.980 \\
1.996 \\
1.934 \\
1.896 \\
1.905 \\
1.947 \\
22.797\end{array}$ & $\begin{array}{r}1.787 \\
1.710 \\
1.947 \\
1.861 \\
1.919 \\
1.929 \\
1.982 \\
1.999 \\
1.937 \\
1.898 \\
1.908 \\
1.950 \\
22.826\end{array}$ & 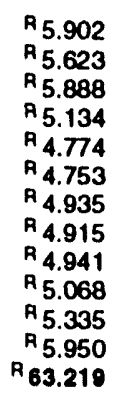 & 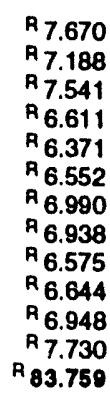 \\
\hline $\begin{array}{r}1994 \text { January ................. } \\
\text { February ............. } \\
\text { 2-Month Total ........ }\end{array}$ & $\begin{array}{r}R_{2} .364 \\
2.086 \\
4.450\end{array}$ & $\begin{array}{r}\text { P }_{3.653} \\
3.145 \\
6.799\end{array}$ & $\begin{array}{r}A_{2} .181 \\
2.059 \\
4.239\end{array}$ & $\begin{array}{r}\mathrm{A}_{2} .761 \\
2.575 \\
\mathbf{5 . 3 3 5}\end{array}$ & $\begin{array}{r}\text { A } 1.861 \\
1.764 \\
3.624\end{array}$ & $\begin{array}{r}P_{1.863} \\
1.766 \\
3.629\end{array}$ & $\begin{array}{r}R_{6.406} \\
5.907 \\
12.314\end{array}$ & $\begin{array}{r}{ }^{A} 8.278 \\
7.485 \\
15.763\end{array}$ \\
\hline $\begin{array}{l}19932 \text { Month Total ....... } \\
1092 \text { 2-Month Total ....... }\end{array}$ & $\begin{array}{l}4.028 \\
3.843\end{array}$ & $\begin{array}{l}6.264 \\
6.034\end{array}$ & $\begin{array}{l}4.008 \\
4.002\end{array}$ & $\begin{array}{l}5.099 \\
5.000\end{array}$ & $\begin{array}{l}3.491 \\
3.542\end{array}$ & $\begin{array}{l}3.496 \\
3.546\end{array}$ & $\begin{array}{l}11.525 \\
11.384\end{array}$ & $\begin{array}{l}14.857 \\
14.668\end{array}$ \\
\hline
\end{tabular}

a Due to a lack of consistent historical data, some renewable energy sources are not included. For example, in 1991, 3.3 quadrillion Blu of renewable energy consumed by U.S. electric utilities to generate electricity for distribution is included, but an estimated 3.4 quadrillion Blu of renewable energy used by other sectors is not included.
$R=$ Revised data.

Notes: • Geographic coverage is the 50 States and the District of Columbia. - Totals may not equal sum of components due to independent rounding and the use of sector-specilic conversion factors for natural gas and coal. Additional Notes and Sources: See end of section. 
Figure 2.2 Residential and Commerclal Energy Consumption

(Quadrillion Btu)

Consumption by Major Sources, 1973-1993

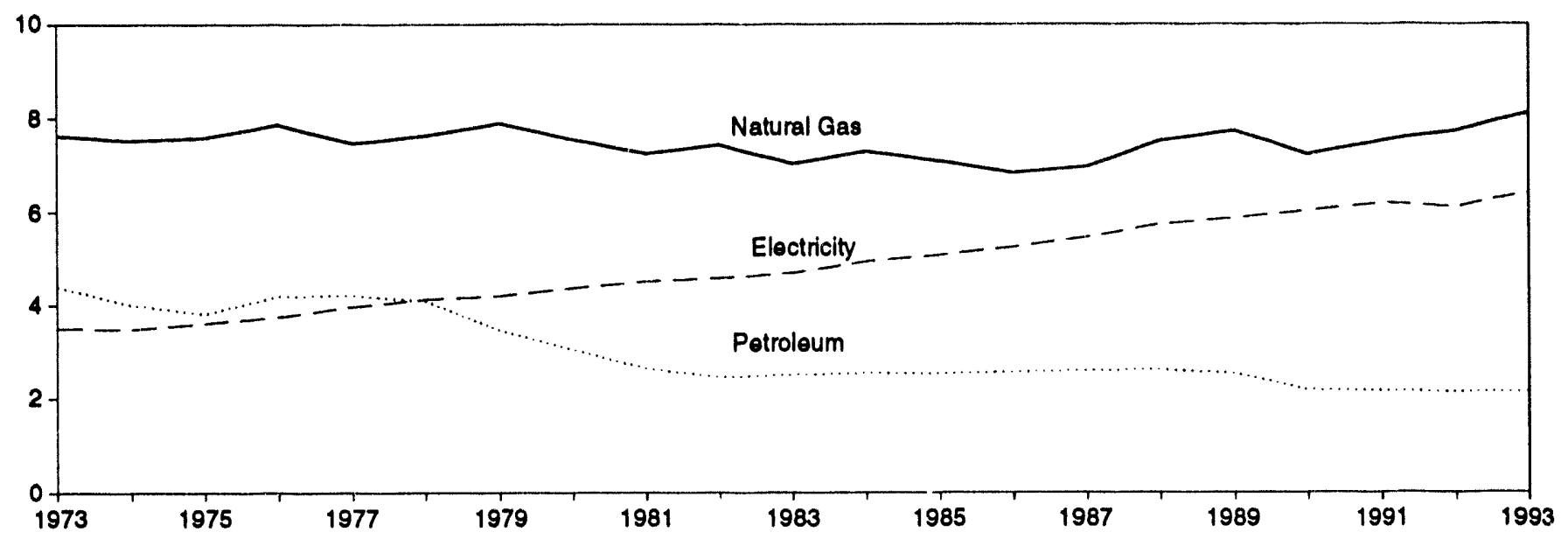

Consumption by Major Sources, Monthly

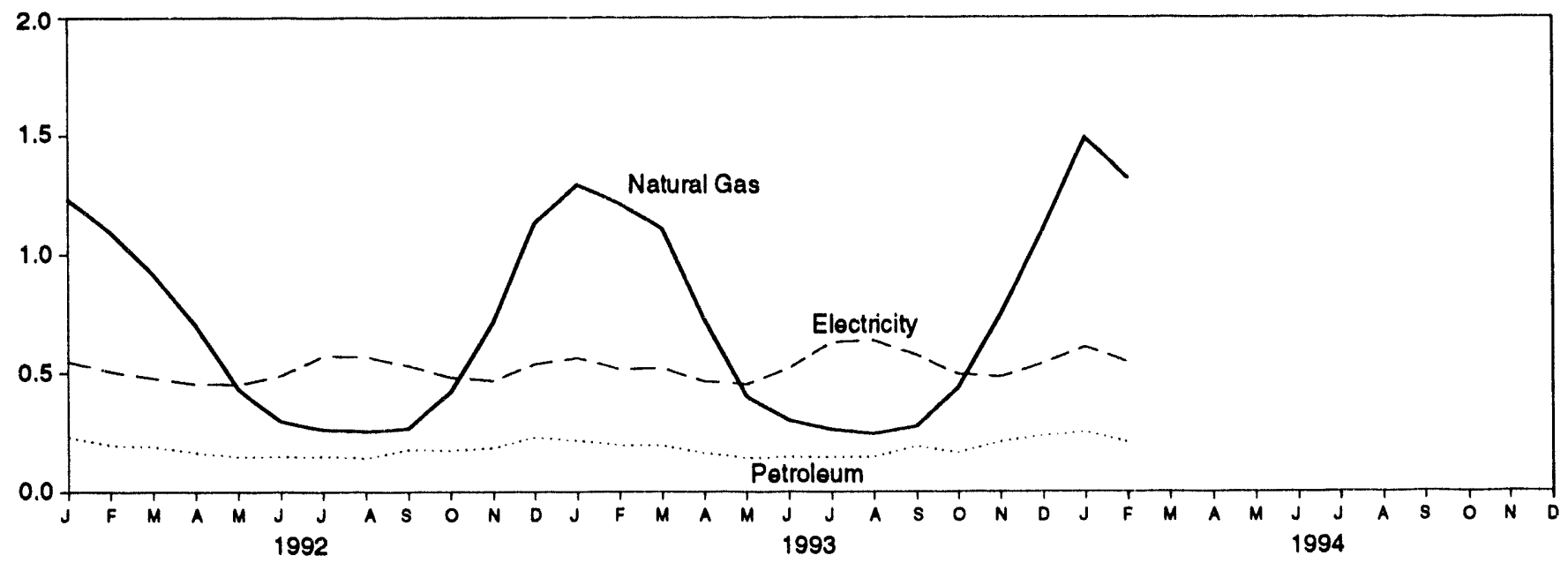

Total Consumption, January and February

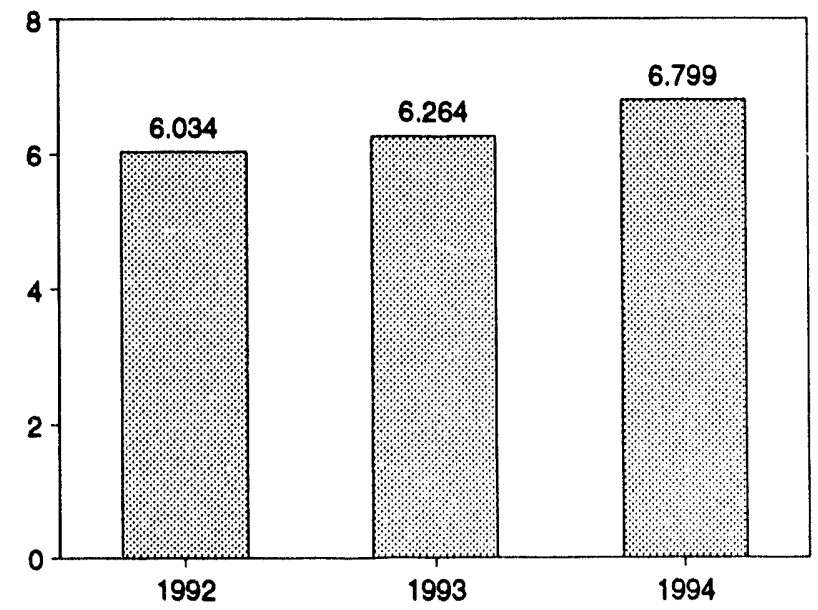

Consumption by Major Sources, February 1994

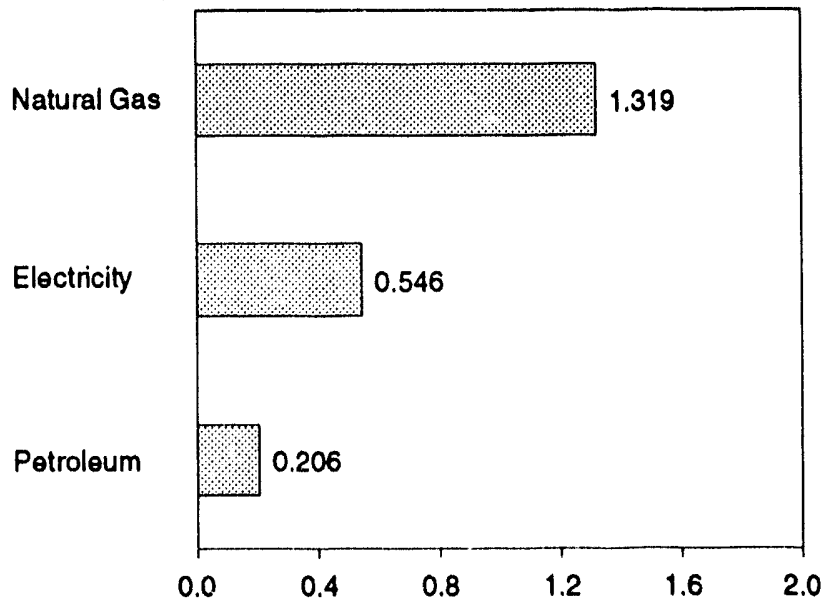

Nole: Because vertical scales differ, graphs should not be compared. Source: Table 2.3 
Table 2.3 Residential and Commerclal Energy Consumption (Quadrillion Btu)

\begin{tabular}{|c|c|c|c|c|c|c|c|c|}
\hline & Coal & $\begin{array}{l}\text { Naturd } \\
\text { Oand }\end{array}$ & Potroloum & $\begin{array}{c}\text { Primary } \\
\text { Consumption }\end{array}$ & Elcetriclty & $\begin{array}{c}\text { Net } \\
\text { Consumptlon }\end{array}$ & $\begin{array}{l}\text { Elootrical } \\
\text { Syctom } \\
\text { Energy } \\
\text { Loseses }\end{array}$ & $\begin{array}{c}\text { Total } \\
\text { Consumptionb }\end{array}$ \\
\hline 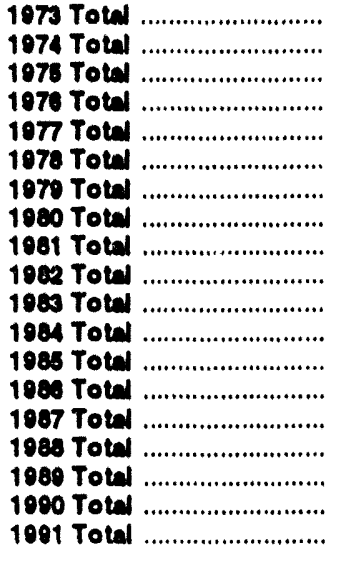 & $\begin{array}{r}0.254 \\
.287 \\
.200 \\
.203 \\
.206 \\
.214 \\
.187 \\
.148 \\
.187 \\
.187 \\
.182 \\
.200 \\
.176 \\
.176 \\
.162 \\
.168 \\
.146 \\
.156 \\
.141\end{array}$ & $\begin{array}{l}7.628 \\
\mathbf{7 . 8 1 8} \\
\mathbf{7 . 6 8 1} \\
\mathbf{7 . 8 4 6} \\
\mathbf{7 . 4 6 1} \\
\mathbf{7 . 0 2 4} \\
\mathbf{7 . 0 9 1} \\
\mathbf{7 . 6 4 0} \\
\mathbf{7 . 2 4 3} \\
\mathbf{7 . 4 2 7} \\
\mathbf{7 . 0 2 4} \\
\mathbf{7 . 2 9 2} \\
\mathbf{7 . 0 7 0} \\
\mathbf{6 . 8 2 5} \\
\mathbf{6 . 0 5 4} \\
\mathbf{7 . 5 1 3} \\
\mathbf{7 . 7 3 1} \\
\mathbf{7 . 2 2 5} \\
\mathbf{7 . 5 1 0}\end{array}$ & $\begin{array}{l}4.301 \\
3.098 \\
3.808 \\
4.181 \\
4.208 \\
4.070 \\
3.440 \\
3.038 \\
2.634 \\
2.440 \\
2.498 \\
2.538 \\
2.622 \\
2.585 \\
2.887 \\
2.800 \\
2.628 \\
2.173 \\
2.154\end{array}$ & $\begin{array}{r}12.270 \\
11.771 \\
11.808 \\
12.280 \\
11.079 \\
11.008 \\
11.826 \\
10.721 \\
10.043 \\
10.083 \\
0.715 \\
10.036 \\
0.777 \\
0.586 \\
0.703 \\
10.260 \\
10.402 \\
0.863 \\
0.805\end{array}$ & 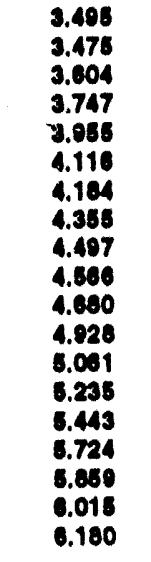 & 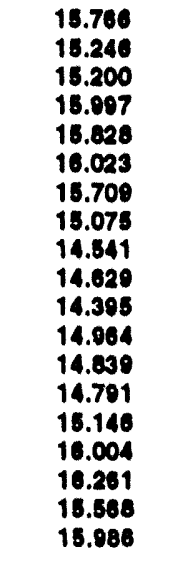 & $\begin{array}{r}8.37 \\
8.480 \\
8.700 \\
0.021 \\
0.860 \\
10.081 \\
10.100 \\
10.880 \\
10.700 \\
11.000 \\
11.232 \\
11.510 \\
11.085 \\
12.081 \\
12.477 \\
12.920 \\
13.143 \\
13.218 \\
13.430\end{array}$ & $\begin{array}{l}24.149 \\
23.728 \\
23.000 \\
25.018 \\
25.384 \\
20.084 \\
25.800 \\
28.858 \\
28.241 \\
26.020 \\
25.627 \\
28.474 \\
26.704 \\
26.052 \\
27.028 \\
28.028 \\
20.404 \\
28.786 \\
29.424\end{array}$ \\
\hline 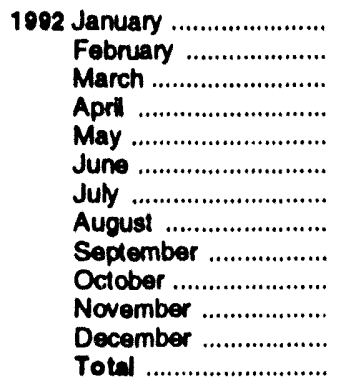 & $\begin{array}{l}.017 \\
.013 \\
.012 \\
.012 \\
.007 \\
.007 \\
.011 \\
.009 \\
.009 \\
.008 \\
.015 \\
.021 \\
.142\end{array}$ & $\begin{array}{r}1.233 \\
1.096 \\
.916 \\
.703 \\
.434 \\
.286 \\
.262 \\
.254 \\
.266 \\
.419 \\
.714 \\
1.132 \\
7.726\end{array}$ & $\begin{array}{l}.229 \\
.197 \\
.189 \\
.166 \\
.146 \\
.148 \\
.149 \\
.141 \\
.177 \\
.173 \\
.184 \\
.227 \\
2.128\end{array}$ & $\begin{array}{r}1.480 \\
1.306 \\
1.117 \\
.880 \\
.587 \\
.451 \\
.422 \\
.404 \\
.451 \\
.601 \\
.913 \\
1.380 \\
0.003\end{array}$ & $\begin{array}{l}.560 \\
.508 \\
.479 \\
.455 \\
.452 \\
.489 \\
.573 \\
.570 \\
.532 \\
.482 \\
.468 \\
.538 \\
.009\end{array}$ & $\begin{array}{r}2.029 \\
1.814 \\
1.596 \\
1.336 \\
1.040 \\
.941 \\
.995 \\
.974 \\
.983 \\
1.083 \\
1.381 \\
1.918 \\
16.090\end{array}$ & $\begin{array}{r}1.189 \\
1.002 \\
1.019 \\
.936 \\
.082 \\
1.089 \\
1.298 \\
1.221 \\
1.082 \\
.983 \\
1.009 \\
1.200 \\
13.010\end{array}$ & $\begin{array}{r}3.218 \\
2.816 \\
2.615 \\
2.272 \\
2.021 \\
2.029 \\
2.293 \\
2.185 \\
2.085 \\
2.066 \\
2.390 \\
3.118 \\
20.100\end{array}$ \\
\hline 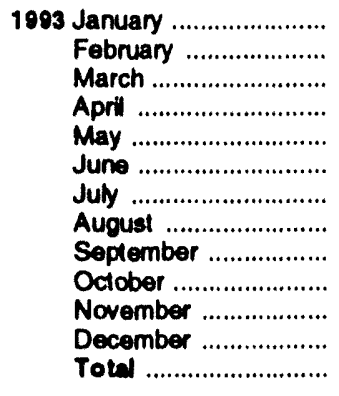 & $\begin{array}{l}.015 \\
.015 \\
.012 \\
.014 \\
.007 \\
.010 \\
.010 \\
.009 \\
.007 \\
.009 \\
.015 \\
.021 \\
.144\end{array}$ & $\begin{array}{r}1.293 \\
1.215 \\
1.110 \\
.728 \\
.401 \\
.299 \\
.261 \\
.242 \\
.273 \\
.438 \\
.744 \\
1.106 \\
8.108\end{array}$ & $\begin{array}{l}.214 \\
.196 \\
.184 \\
.162 \\
.139 \\
.145 \\
.143 \\
.145 \\
.189 \\
.163 \\
.208 \\
.234 \\
2.131\end{array}$ & $\begin{array}{r}1.522 \\
1.426 \\
1.316 \\
.905 \\
.548 \\
.453 \\
.413 \\
.396 \\
.468 \\
.610 \\
.967 \\
1.359 \\
10.383\end{array}$ & $\begin{array}{l}.564 \\
.517 \\
.521 \\
.465 \\
.452 \\
.520 \\
.630 \\
.638 \\
.576 \\
.494 \\
.482 \\
.540 \\
.308\end{array}$ & $\begin{array}{r}2.086 \\
1.842 \\
1.836 \\
1.370 \\
1.000 \\
.973 \\
1.043 \\
1.034 \\
1.044 \\
1.104 \\
1.448 \\
1.899 \\
18.781\end{array}$ & 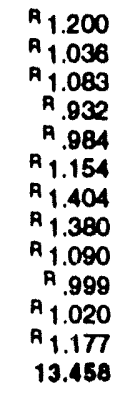 & $\begin{array}{r}A_{3.286} \\
A_{2.978} \\
A_{2.918} \\
A_{2.301} \\
A_{1.984} \\
A_{2.127} \\
A_{2.448} \\
A_{2.414} \\
A_{2.134} \\
A_{2.103} \\
A_{2.468} \\
A_{3.076} \\
A_{30.230}\end{array}$ \\
\hline 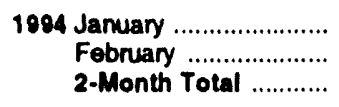 & $\begin{array}{l}.019 \\
.016 \\
.035\end{array}$ & $\begin{array}{r}\text { A } 1.488 \\
1.319 \\
2.007\end{array}$ & $\begin{array}{l}.248 \\
.206 \\
.454\end{array}$ & $\begin{array}{r}\text { A } 1.755 \\
1.540 \\
3.296\end{array}$ & $\begin{array}{r}.609 \\
.546 \\
1.156\end{array}$ & $\begin{array}{r}A_{2.364} \\
2.086 \\
4.480\end{array}$ & $\begin{array}{r}1.289 \\
1.059 \\
2.340\end{array}$ & $\begin{array}{r}\mathrm{A}_{3.653} \\
3.145 \\
6.790\end{array}$ \\
\hline $\begin{array}{l}1093 \text { 2-Month Total ........... } \\
1092 \text { 2-Month Total .......... }\end{array}$ & $\begin{array}{l}.000 \\
.000\end{array}$ & $\begin{array}{l}2.507 \\
2.328\end{array}$ & .410 & $\begin{array}{l}2.948 \\
2.785\end{array}$ & $\begin{array}{l}1.001 \\
1.058\end{array}$ & $\begin{array}{l}1.028 \\
3.843\end{array}$ & $\begin{array}{l}2.236 \\
2.101\end{array}$ & $\begin{array}{l}6.284 \\
6.034\end{array}$ \\
\hline
\end{tabular}

- Includes supplemental gaseous fuels.

b Due to a lack of consistent historical data, some renewable energy sources are not included. For example, in 1991, an estimated 0.7 quadrillion Btu of renewable energy consumed by the U.S. residential and commercial sectors (primarily the residentlal sector) is not included.
Pepevised dala.

Notes: • Geographic coverage is the 50 States and the District of Columbia.

- Tolals may not equal sum of components due to independent rounding. Additional Noles and Sources: See end of section. 
Flgure 2.3 Industrial Energy Consumption

(Quadrillion Btu)

Consumption by Major Sources, 1973-1993

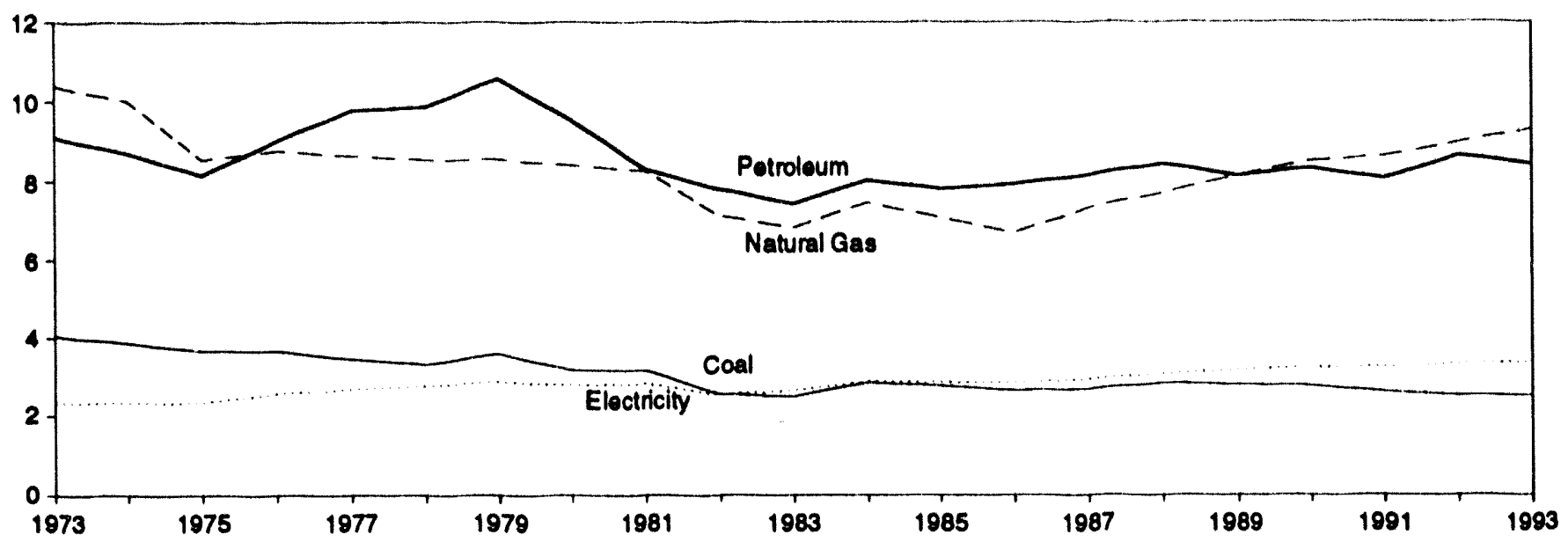

Consumption by Major Sources, Monthly

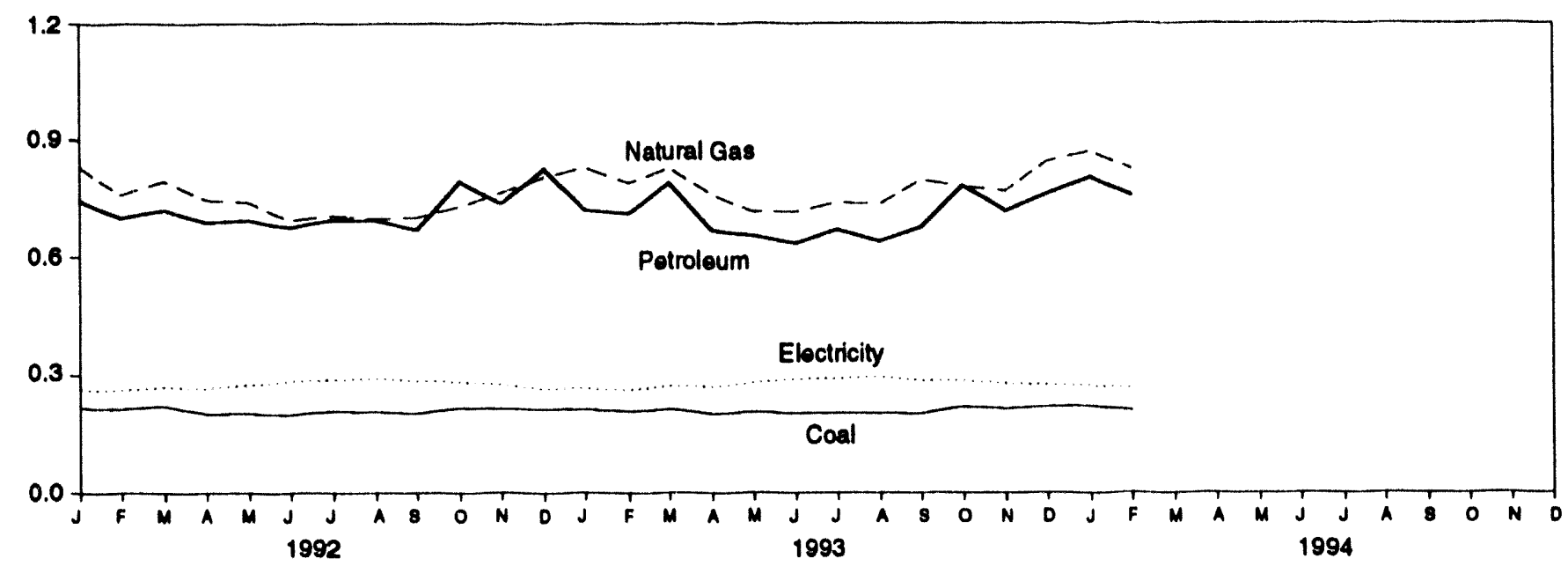

Total Consumption, January and February

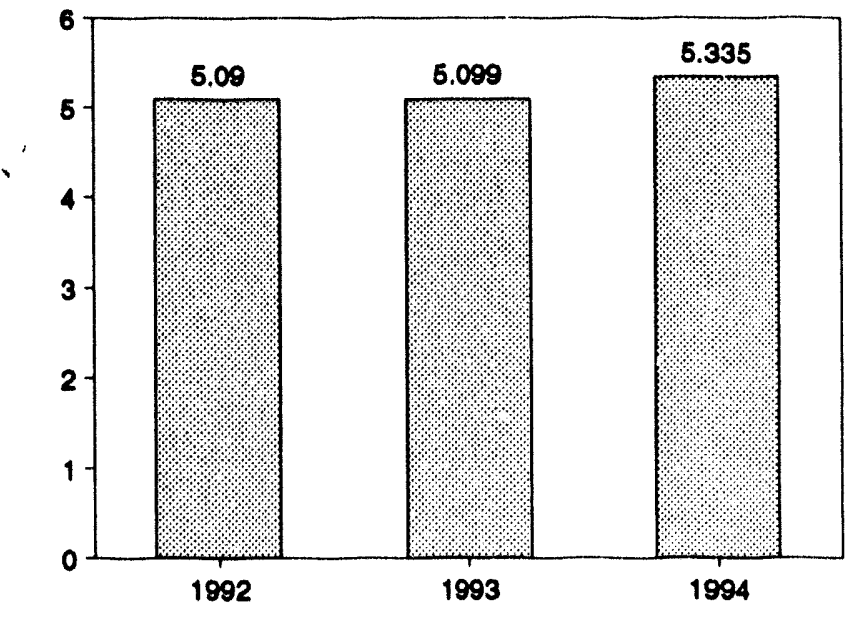

Consumption by Major Sources,

February 1994

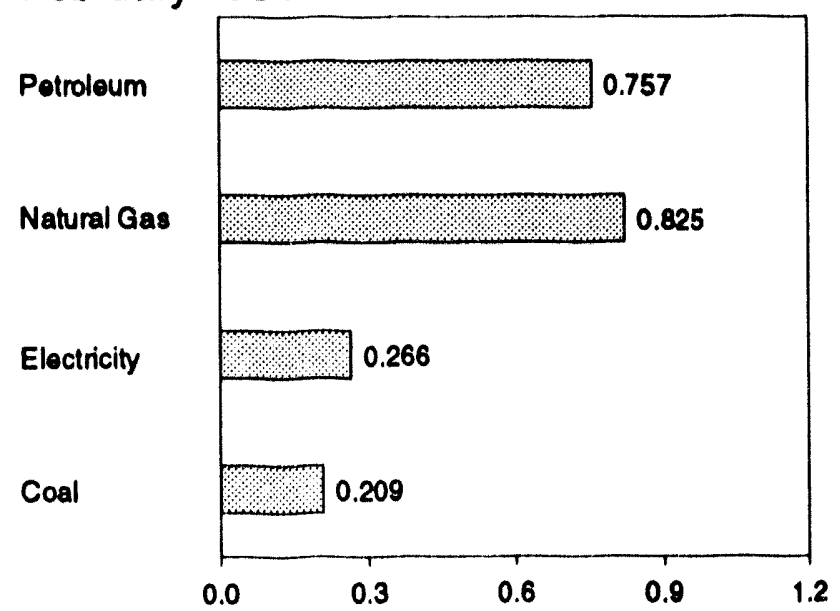

Note: Because vertical scales diller, graphs should not be compared. Source: Table 2.4. 
Table 2.4 Industrial Energy Consumption

(Quadrillion Btu)

\begin{tabular}{|c|c|c|c|c|c|c|c|c|c|c|}
\hline & Coal & $\begin{array}{c}\text { Natural } \\
\text { Gese }\end{array}$ & Potroloum & $\begin{array}{l}\text { Hydro- } \\
\text { eleetric } \\
\text { Power }\end{array}$ & $\begin{array}{l}\text { Not } \\
\text { Imports } \\
\text { of Coal } \\
\text { Coke }\end{array}$ & $\begin{array}{c}\text { Primary } \\
\text { Consumptlon }\end{array}$ & Electriclty & $\begin{array}{c}\text { Net } \\
\text { Consumption }\end{array}$ & $\begin{array}{l}\text { Electrical } \\
\text { System } \\
\text { Energy } \\
\text { Loseses }\end{array}$ & $\begin{array}{c}\text { Total } \\
\text { Consumptlonb }\end{array}$ \\
\hline 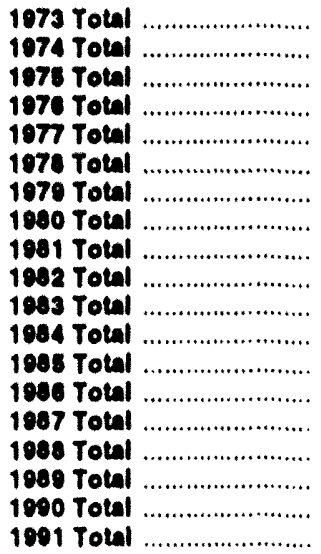 & $\begin{array}{l}4.057 \\
3.870 \\
3.697 \\
3.681 \\
3.454 \\
3.314 \\
3.803 \\
3.188 \\
3.187 \\
2.882 \\
2.490 \\
2.842 \\
2.790 \\
2.840 \\
2.673 \\
2.828 \\
2.787 \\
2.758 \\
2.801\end{array}$ & 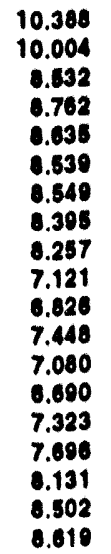 & 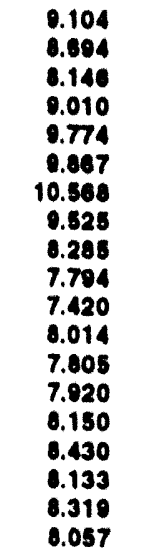 & $\begin{array}{l}0.035 \\
.033 \\
.032 \\
.033 \\
.033 \\
.032 \\
.034 \\
.033 \\
.033 \\
.033 \\
.033 \\
.033 \\
.033 \\
.033 \\
.033 \\
.033 \\
.033 \\
.033 \\
.033\end{array}$ & $\begin{array}{r}-0.007 \\
.058 \\
.014 \\
(0) \\
.015 \\
.125 \\
.083 \\
.035 \\
.016 \\
.022 \\
.016 \\
.011 \\
.013 \\
.017 \\
.009 \\
.040 \\
.030 \\
.005 \\
.009\end{array}$ & $\begin{array}{l}23.576 \\
22.887 \\
20.391 \\
21.488 \\
21.011 \\
21.876 \\
22.807 \\
21.073 \\
10.718 \\
17.470 \\
18.753 \\
18.325 \\
17.065 \\
17.267 \\
18.188 \\
19.026 \\
10.113 \\
10.615 \\
19.310\end{array}$ & $\begin{array}{l}2.341 \\
2.337 \\
2.348 \\
2.873 \\
2.682 \\
2.761 \\
2.873 \\
2.781 \\
2.817 \\
2.542 \\
2.648 \\
2.850 \\
2.855 \\
2.834 \\
2.028 \\
3.050 \\
3.158 \\
3.226 \\
3.230\end{array}$ & $\begin{array}{l}25.017 \\
24.094 \\
22.737 \\
24.038 \\
24.593 \\
24.037 \\
25.670 \\
23.854 \\
22.533 \\
20.020 \\
10.401 \\
21.184 \\
20.520 \\
20.101 \\
21.116 \\
22.088 \\
22.272 \\
22.841 \\
22.540\end{array}$ & $\begin{array}{l}5.611 \\
6.700 \\
5.688 \\
6.198 \\
6.484 \\
6.755 \\
6.936 \\
6.752 \\
6.707 \\
6.125 \\
6.359 \\
6.683 \\
6.694 \\
6.520 \\
6.710 \\
6.001 \\
7.082 \\
7.095 \\
7.021\end{array}$ & 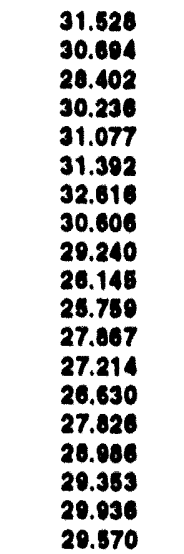 \\
\hline 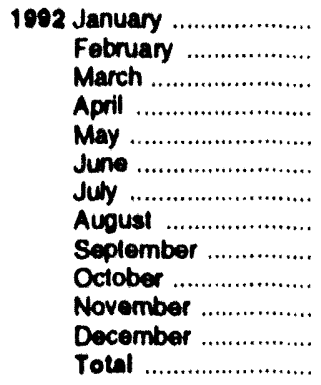 & $\begin{array}{l}.217 \\
.214 \\
.222 \\
.201 \\
.202 \\
.109 \\
.208 \\
.206 \\
.202 \\
.217 \\
.214 \\
.214 \\
2.815\end{array}$ & $\begin{array}{l}.830 \\
.759 \\
.795 \\
.746 \\
.740 \\
.694 \\
.706 \\
.698 \\
.701 \\
.730 \\
.763 \\
.805 \\
.067\end{array}$ & $\begin{array}{l}.744 \\
.700 \\
.721 \\
.689 \\
.694 \\
.676 \\
.695 \\
.694 \\
.670 \\
.794 \\
.735 \\
.826 \\
.0 .638\end{array}$ & $\begin{array}{l}.003 \\
.003 \\
.003 \\
.003 \\
.003 \\
.003 \\
.003 \\
.002 \\
.002 \\
.002 \\
.002 \\
.002 \\
.033\end{array}$ & $\begin{array}{l}.004 \\
.003 \\
.003 \\
.003 \\
.001 \\
.003 \\
.001 \\
.001 \\
.001 \\
.002 \\
.001 \\
.005 \\
.027\end{array}$ & $\begin{array}{l}1.798 \\
1.678 \\
1.744 \\
1.642 \\
1.641 \\
1.575 \\
1.613 \\
1.601 \\
1.576 \\
1.746 \\
1.715 \\
1.852 \\
20.180\end{array}$ & $\begin{array}{l}.264 \\
.262 \\
.271 \\
.267 \\
.276 \\
285 \\
.289 \\
.292 \\
.286 \\
.284 \\
.276 \\
.266 \\
3.310\end{array}$ & $\begin{array}{r}2.062 \\
1.940 \\
2.014 \\
1.909 \\
1.917 \\
1.860 \\
1.902 \\
1.893 \\
1.862 \\
2.030 \\
1.992 \\
2.118 \\
23.498\end{array}$ & $\begin{array}{l}.571 \\
.517 \\
.576 \\
.549 \\
.598 \\
634 \\
.656 \\
.627 \\
.582 \\
.580 \\
.596 \\
.593 \\
7.070\end{array}$ & $\begin{array}{r}2.633 \\
2.458 \\
2.590 \\
2.458 \\
2.515 \\
2.494 \\
2.558 \\
2.520 \\
2.444 \\
2.610 \\
2.588 \\
2.711 \\
30.577\end{array}$ \\
\hline 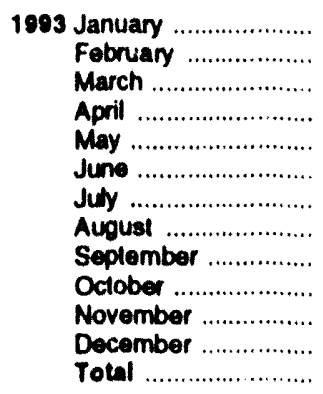 & 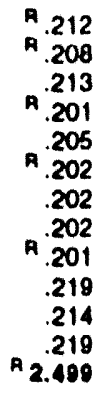 & $\begin{array}{r}.828 \\
.790 \\
.827 \\
.761 \\
\mathrm{~A} .715 \\
.716 \\
.739 \\
.735 \\
.797 \\
.780 \\
.769 \\
.844 \\
\circ .303\end{array}$ & $\begin{array}{l}.720 \\
.712 \\
.789 \\
.669 \\
.654 \\
.636 \\
.670 \\
.641 \\
.676 \\
.782 \\
.718 \\
.761 \\
8.428\end{array}$ & $\begin{array}{l}.003 \\
.003 \\
.003 \\
.003 \\
.003 \\
.003 \\
.003 \\
.002 \\
.002 \\
.002 \\
.002 \\
.002 \\
.033\end{array}$ & $\begin{array}{r}.004 \\
(\mathrm{~s}) \\
.003 \\
.002 \\
.002 \\
.003 \\
(\mathrm{~s}) \\
.002 \\
.001 \\
.001 \\
(\mathrm{~s}) \\
.002 \\
.017\end{array}$ & $\begin{array}{r}1.768 \\
1.712 \\
1.835 \\
1.637 \\
1.579 \\
1.561 \\
\text { P } 1.614 \\
1.583 \\
1.676 \\
1.784 \\
1.703 \\
1.829 \\
\text { P } 20.280\end{array}$ & $\begin{array}{l}.266 \\
.263 \\
.273 \\
.271 \\
.280 \\
.290 \\
.291 \\
.296 \\
.286 \\
.285 \\
.279 \\
.275 \\
3.354\end{array}$ & $\begin{array}{r}{ }^{\circ} 2.033 \\
1.975 \\
2.108 \\
1.908 \\
A_{1.859} \\
1.851 \\
1.906 \\
1.879 \\
1.962 \\
2.089 \\
1.982 \\
2.104 \\
{ }_{2} 23.634\end{array}$ & 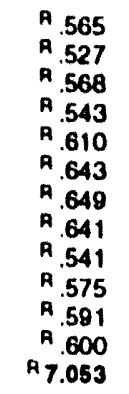 & $\begin{array}{l}A_{2.588} \\
A_{2.501} \\
A_{2.676} \\
A_{2.451} \\
A_{2.469} \\
A_{2.493} \\
A_{2.555} \\
A_{2.520} \\
A_{2.503} \\
A_{2.644} \\
A_{2.572} \\
A_{2.704} \\
\text { ค }_{30.687}\end{array}$ \\
\hline $\begin{array}{r}1094 \text { January ................... } \\
\text { Fobruary ................ } \\
\text { 24Month Total ......... }\end{array}$ & $\begin{array}{l}\text { P } .222 \\
.209 \\
.431\end{array}$ & $\begin{array}{r}.872 \\
.825 \\
1.607\end{array}$ & $\begin{array}{r}.806 \\
.757 \\
1.562\end{array}$ & $\begin{array}{l}.003 \\
.003 \\
.006\end{array}$ & $\begin{array}{r}.004 \\
.001 \\
.003\end{array}$ & $\begin{array}{r}1.907 \\
1.793 \\
3.800\end{array}$ & $\begin{array}{l}.274 \\
.266 \\
.540\end{array}$ & $\begin{array}{r}A_{2.181} \\
2.059 \\
4.230\end{array}$ & $\begin{array}{r}\text { ค .580 } \\
.516 \\
1.006\end{array}$ & $\begin{array}{r}\text { ค }_{2.761} \\
2.575 \\
8.335\end{array}$ \\
\hline $\begin{array}{l}19932 \text {-Month Total ......... } \\
10922 \text {-Month Totd ........ }\end{array}$ & $\begin{array}{l}.421 \\
.430\end{array}$ & $\begin{array}{l}1.618 \\
1.580\end{array}$ & $\begin{array}{l}1.432 \\
1.444\end{array}$ & $\begin{array}{l}.006 \\
.006\end{array}$ & $\begin{array}{l}.004 \\
.006\end{array}$ & $\begin{array}{l}3.480 \\
3.476\end{array}$ & $\begin{array}{l}.528 \\
.526\end{array}$ & $\begin{array}{l}4.008 \\
4.002\end{array}$ & $\begin{array}{l}1.002 \\
1.088\end{array}$ & $\begin{array}{l}5.000 \\
5.090\end{array}$ \\
\hline
\end{tabular}

- Includes supplemental gaserous luels.

b Due to a lack of consistent historical data, some renewable energy sources are not included. For example, in 1991, an estimated 2.7 quadrillion Blu of renewable energy consumed by the U.S. industrial sector (primarily the pulp and paper industry) is not included.
$R=$ Revised data. (s)=Less than +0.5 trillion Btu and greater than $\mathbf{0 . 5}$ Irilion Btu.

Notes: - Geographic coverage is the 50 Statos and the District of Columbia.

- Tolals may not equal sum ol components due to independent rounding. Additional Notes and Sources: Soe end of section. 
Figure 2.4 Transportation Energy Consumption

(Quadrillion Btu)

Consumption by Major Sources, 1973-1993

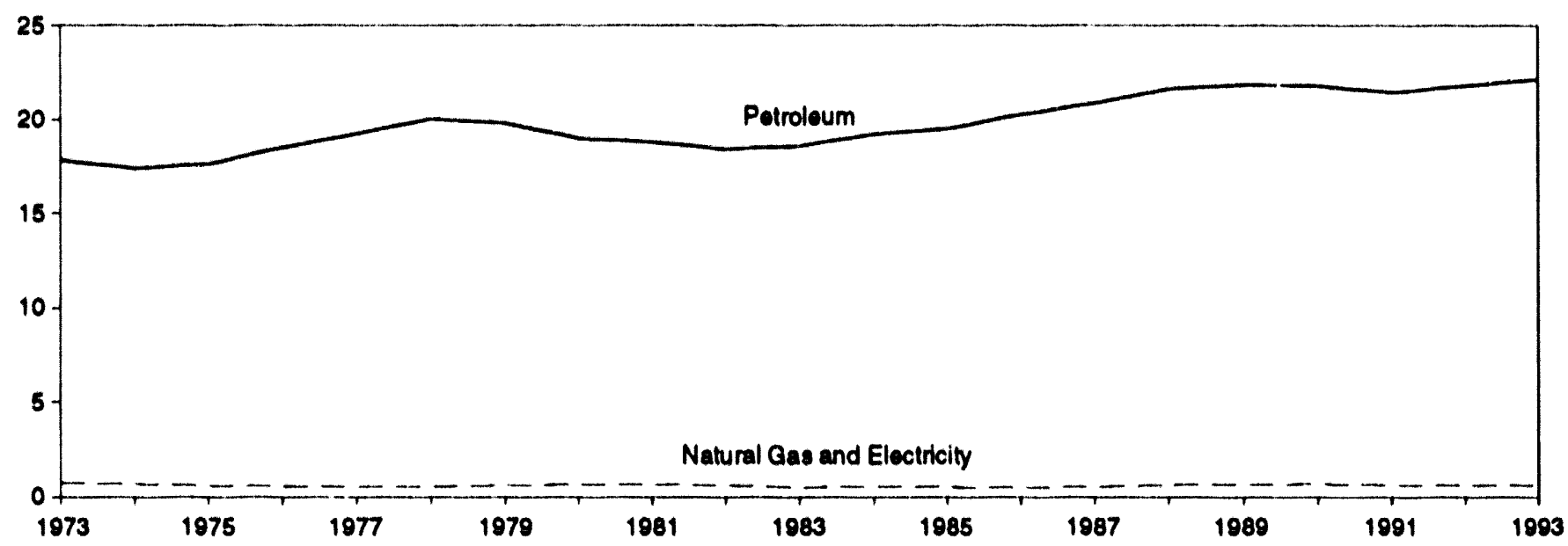

Consumption by Major Sources, Monthly

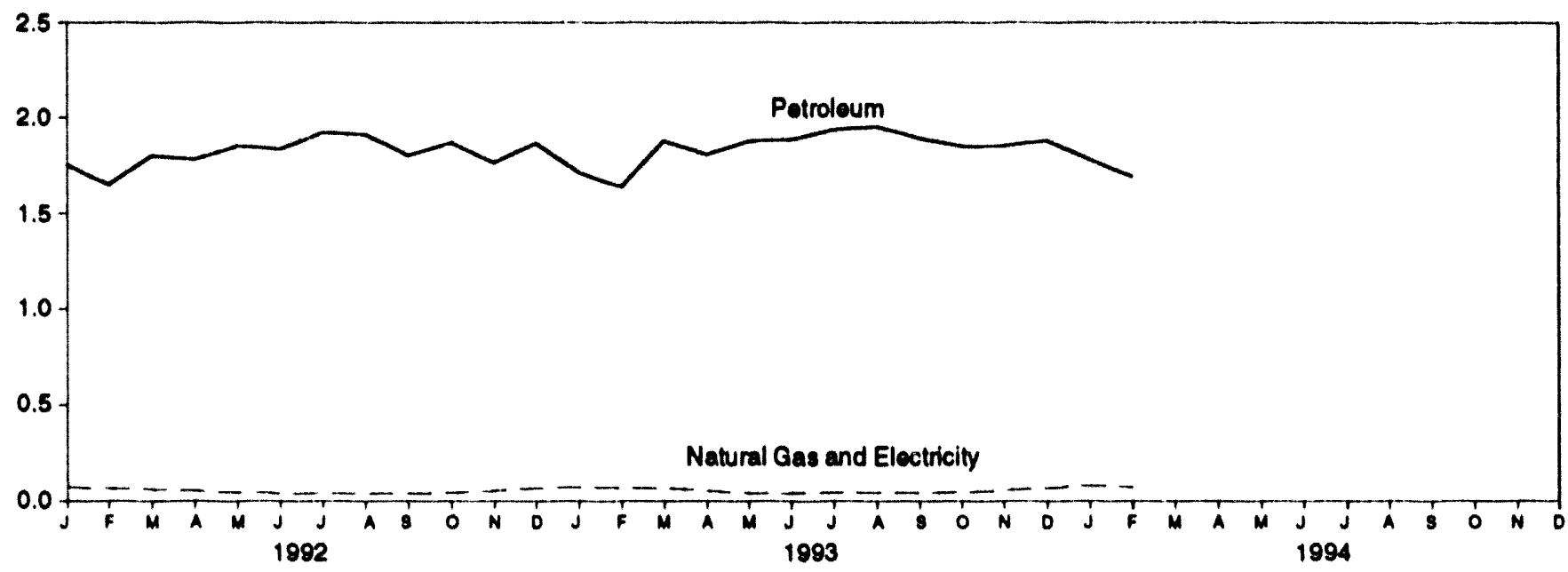

Total Consumption, January and February

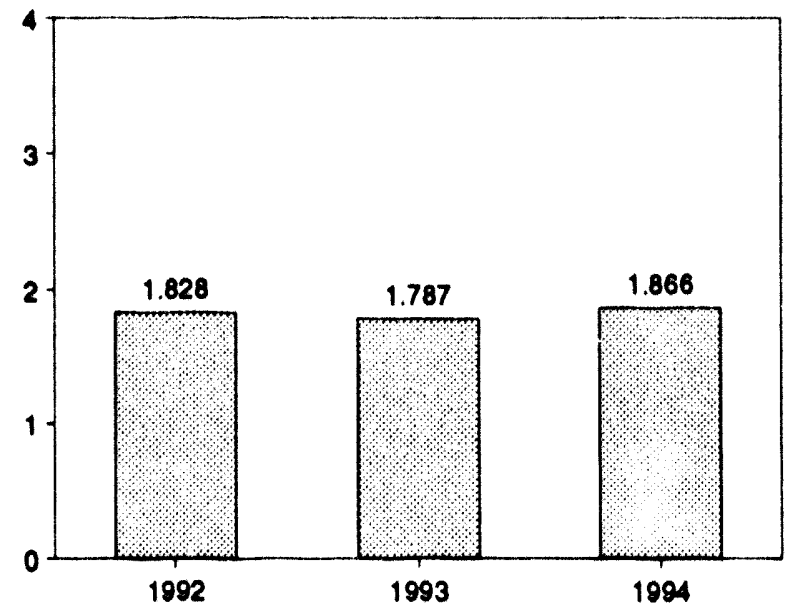

Total Consumption, Monthly

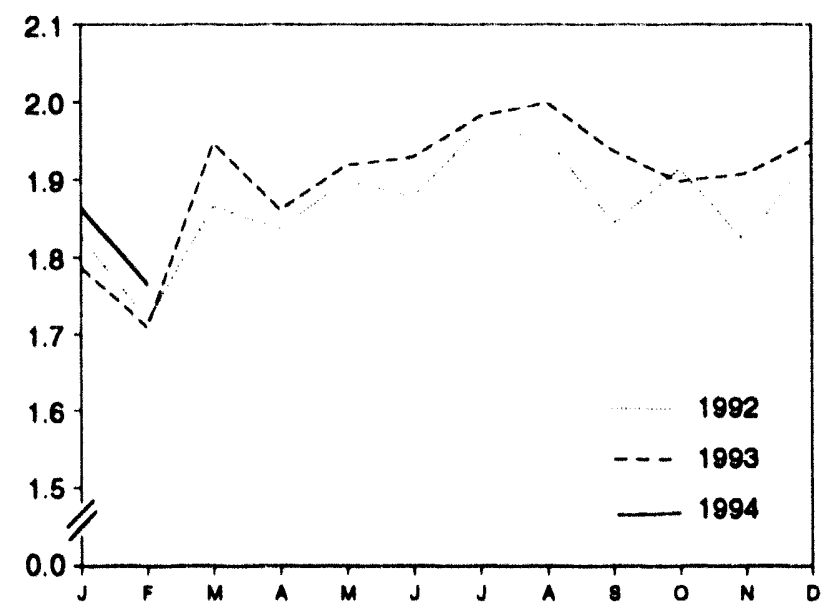

Nole: Because vertical scales ditter, graphs should not be compared.

Source: Table 2.5. 

(Quadrillion Btu)

\begin{tabular}{|c|c|c|c|c|c|c|c|c|}
\hline & Coal & $\begin{array}{c}\text { Newral } \\
\text { aese }\end{array}$ & Potroleum & $\begin{array}{c}\text { Primary } \\
\text { Conoumption }\end{array}$ & Eleetrlelty & $\begin{array}{c}\text { Net } \\
\text { Consumpton }\end{array}$ & $\begin{array}{l}\text { Eloctrical } \\
\text { Eyetom } \\
\text { Enorgy } \\
\text { Loesece }\end{array}$ & $\begin{array}{c}\text { Totel } \\
\text { Coneumptlon }\end{array}$ \\
\hline 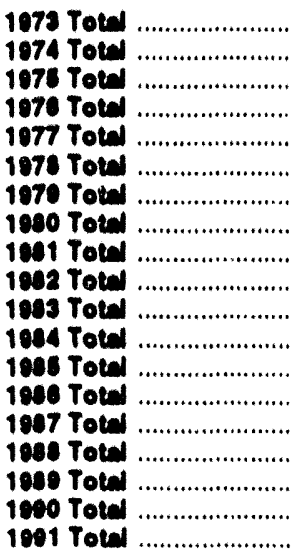 & 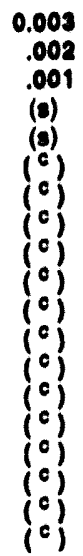 & 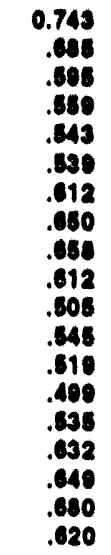 & 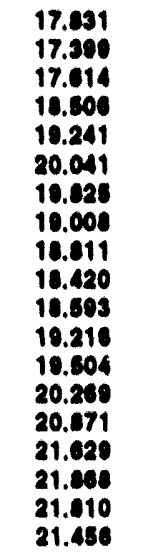 & 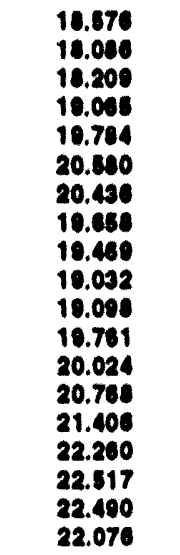 & $\begin{array}{l}0.008 \\
.000 \\
.010 \\
.010 \\
.010 \\
.000 \\
.010 \\
.011 \\
.011 \\
.011 \\
.011 \\
.012 \\
.013 \\
.013 \\
.013 \\
.014 \\
.014 \\
.014 \\
.014\end{array}$ & $\begin{array}{l}18.844 \\
10.006 \\
10.210 \\
19.070 \\
10.704 \\
20.800 \\
20.447 \\
10.680 \\
10.480 \\
10.043 \\
19.100 \\
10.773 \\
20.030 \\
20.781 \\
21.410 \\
22.274 \\
22.530 \\
22.504 \\
22.090\end{array}$ & $\begin{array}{l}0.020 \\
.022 \\
.025 \\
.028 \\
.025 \\
.022 \\
.028 \\
.028 \\
.026 \\
.029 \\
.029 \\
.028 \\
.030 \\
.031 \\
.020 \\
.031 \\
.031 \\
.031 \\
.030\end{array}$ & $\begin{array}{l}18.005 \\
18.117 \\
18.244 \\
18.101 \\
10.018 \\
20.011 \\
20.472 \\
10.008 \\
10.807 \\
10.098 \\
10.138 \\
19.801 \\
20.007 \\
20.812 \\
21.448 \\
22.308 \\
22.841 \\
22.838 \\
22.120\end{array}$ \\
\hline 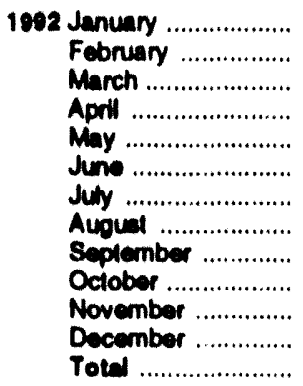 & $\left(\begin{array}{l}c \\
c \\
(c)\end{array}\right)$ & $\begin{array}{l}.070 \\
.064 \\
.060 \\
.062 \\
.044 \\
.030 \\
.040 \\
.030 \\
.038 \\
.042 \\
.062 \\
.088 \\
.000\end{array}$ & $\begin{array}{l}1.754 \\
1.651 \\
1.803 \\
1.781 \\
1.852 \\
1.835 \\
1.922 \\
1.912 \\
1.803 \\
1.868 \\
1.765 \\
1.866 \\
21.012\end{array}$ & $\begin{array}{l}1.825 \\
1.715 \\
1.863 \\
1.833 \\
1.806 \\
1.874 \\
1.862 \\
1.850 \\
1.841 \\
1.910 \\
1.817 \\
1.932 \\
22.418\end{array}$ & $\begin{array}{l}.001 \\
.001 \\
.001 \\
.001 \\
.001 \\
.001 \\
.001 \\
.001 \\
.001 \\
.001 \\
.001 \\
.001 \\
.014\end{array}$ & $\begin{array}{l}1.826 \\
1.716 \\
1.864 \\
1.834 \\
1.897 \\
1.875 \\
1.963 \\
1.952 \\
1.842 \\
1.911 \\
1.818 \\
1.933 \\
22.432\end{array}$ & $\begin{array}{l}.002 \\
.002 \\
.002 \\
.002 \\
.002 \\
.003 \\
.003 \\
.003 \\
.002 \\
.002 \\
.002 \\
.003 \\
.020\end{array}$ & $\begin{array}{l}1.828 \\
1.718 \\
1.868 \\
1.837 \\
1.899 \\
1.878 \\
1.966 \\
1.954 \\
1.844 \\
1.914 \\
1.820 \\
1.936 \\
22.461\end{array}$ \\
\hline 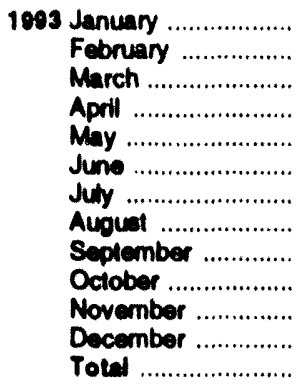 & $\left(\begin{array}{l}c \\
c\end{array}\right)$ & $\begin{array}{l}.071 \\
.067 \\
.066 \\
.052 \\
.040 \\
.040 \\
.042 \\
.042 \\
.041 \\
.045 \\
.054 \\
.066 \\
.628\end{array}$ & $\begin{array}{l}1.712 \\
1.639 \\
1.877 \\
1.806 \\
1.876 \\
1.895 \\
1.837 \\
1.053 \\
1.892 \\
1.849 \\
1.851 \\
1.880 \\
22.157\end{array}$ & $\begin{array}{l}1.783 \\
1.706 \\
1.243 \\
1.857 \\
1.916 \\
1.925 \\
1.978 \\
1.995 \\
1.033 \\
1.895 \\
1.904 \\
1.946 \\
22.783\end{array}$ & $\begin{array}{l}.001 \\
.001 \\
.001 \\
.001 \\
.001 \\
.001 \\
.001 \\
.001 \\
.001 \\
.001 \\
.001 \\
.001 \\
.014\end{array}$ & $\begin{array}{l}1.784 \\
1.707 \\
1.945 \\
1.858 \\
1.917 \\
1.926 \\
1.980 \\
1.996 \\
1.934 \\
1.896 \\
1.905 \\
1.947 \\
22.797\end{array}$ & $\begin{array}{l}.002 \\
.002 \\
.002 \\
.002 \\
.002 \\
.003 \\
.003 \\
.000 \\
.002 \\
.002 \\
.002 \\
.003 \\
.020\end{array}$ & $\begin{array}{r}1.787 \\
1.710 \\
1.947 \\
1.861 \\
1.919 \\
1.929 \\
1.982 \\
1.909 \\
1.937 \\
1.898 \\
1.908 \\
1.950 \\
22.826\end{array}$ \\
\hline $\begin{array}{l}1004 \text { January ................. } \\
\text { February .............. } \\
\text { 2-Month Total ....... }\end{array}$ & $\begin{array}{l}\left(\begin{array}{l}c \\
c\end{array}\right) \\
(c)\end{array}$ & $\begin{array}{l}\text { ค } .079 \\
.071 \\
.150\end{array}$ & $\begin{array}{l}1.781 \\
1.692 \\
3.472\end{array}$ & $\begin{array}{r}\text { A } 1.859 \\
1.763 \\
3.622\end{array}$ & $\begin{array}{l}.001 \\
.001 \\
.002\end{array}$ & $\begin{array}{r}\text { A } 1.861 \\
1.764 \\
3.824\end{array}$ & $\begin{array}{l}.003 \\
.002 \\
.005\end{array}$ & $\begin{array}{r}\text { A } 1.863 \\
1.766 \\
3.020\end{array}$ \\
\hline $\begin{array}{l}1003 \text { 2-Month Total ....... } \\
1902 \text { 2-Month Total ....... }\end{array}$ & $\left(\begin{array}{l}c \\
c\end{array}\right)$ & $\begin{array}{l}.130 \\
.134\end{array}$ & $\begin{array}{l}3.351 \\
3.408\end{array}$ & $\begin{array}{l}3.400 \\
3.530\end{array}$ & $\begin{array}{l}.002 \\
.002\end{array}$ & $\begin{array}{l}3.491 \\
3.842\end{array}$ & $\begin{array}{l}.008 \\
.005\end{array}$ & $\begin{array}{l}3.498 \\
3.546\end{array}$ \\
\hline
\end{tabular}

- Pipeline fual only, including supplemental gaseous luels.

- Dus to a lack of consistent historical data, some renewablo energy sources are not included. For example, in 1091, an estimated 0.1 quadriltion Biu of renewable energy consumed by the U.S. Iransportalion sector is not included.

- Since 1978, the small amounts of coal consumed for Iransportation are reported as industrial sector consumplion.

$R=$ Revised data. (s) =Lese than 0.5 trillion Blu.

Noles: - Geographic coverage is the 50 Stales and the District of Columbla.

- Totals may not equal sum of components due to independent rounding. Additional Noles and Sources: See end of section. 
Figure 2.5 Energy Input at Electric Utilities (Quadrillion Btu)

Total Input, 1973-1993

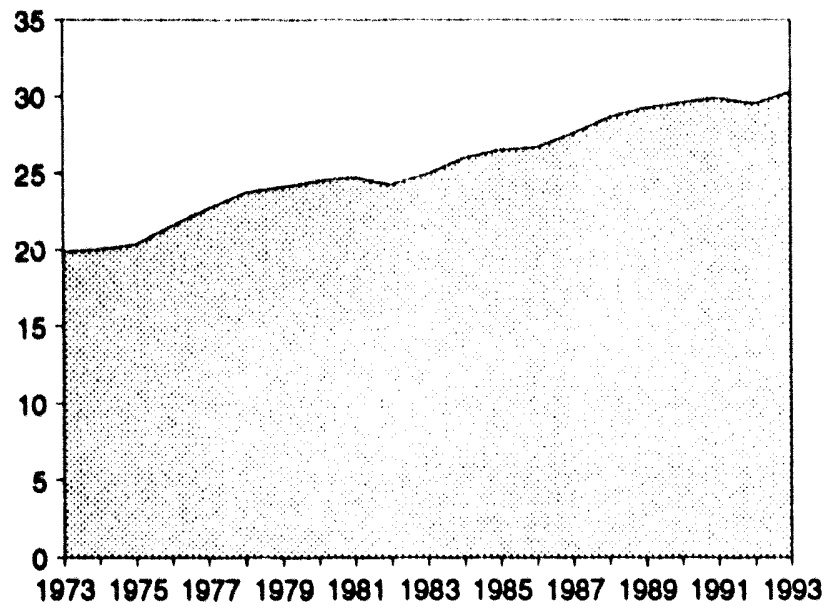

Input by Major Sources, 1973-1993

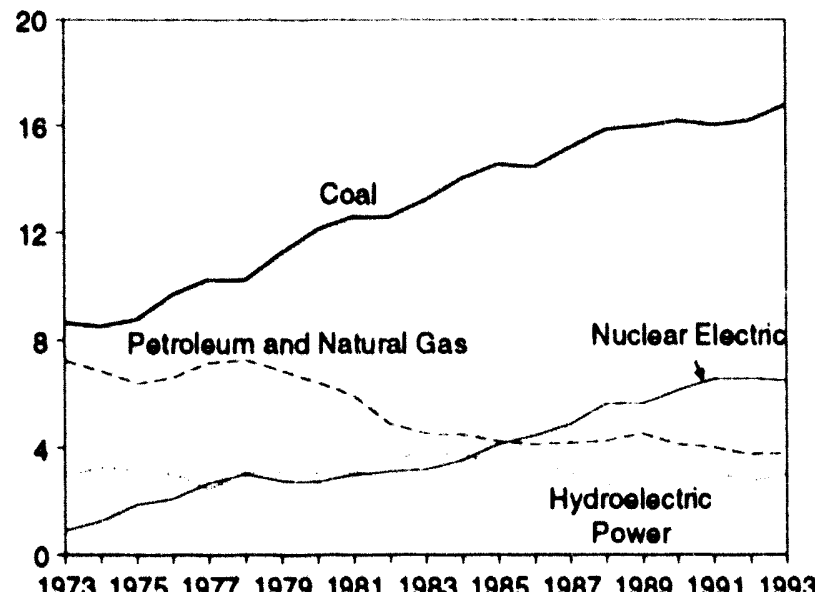

Total Input, January and February

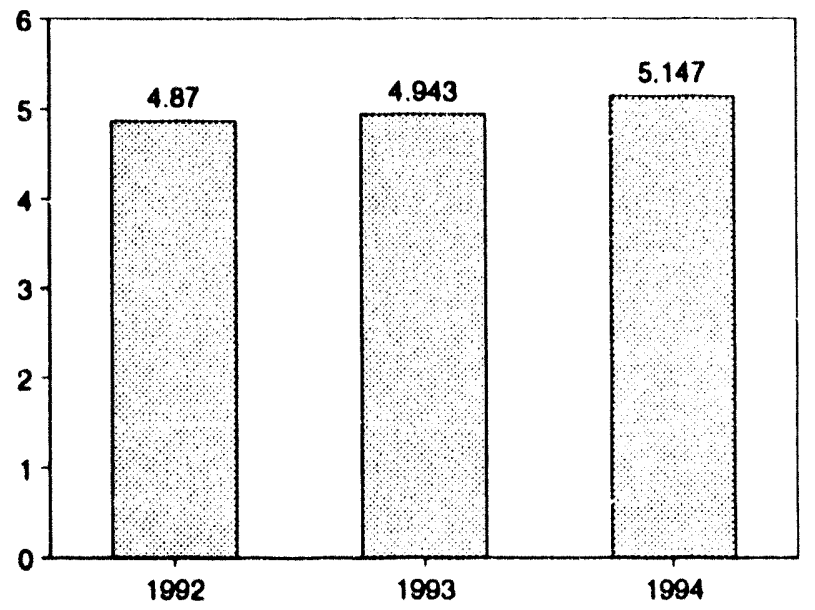

Total Input, Monthly

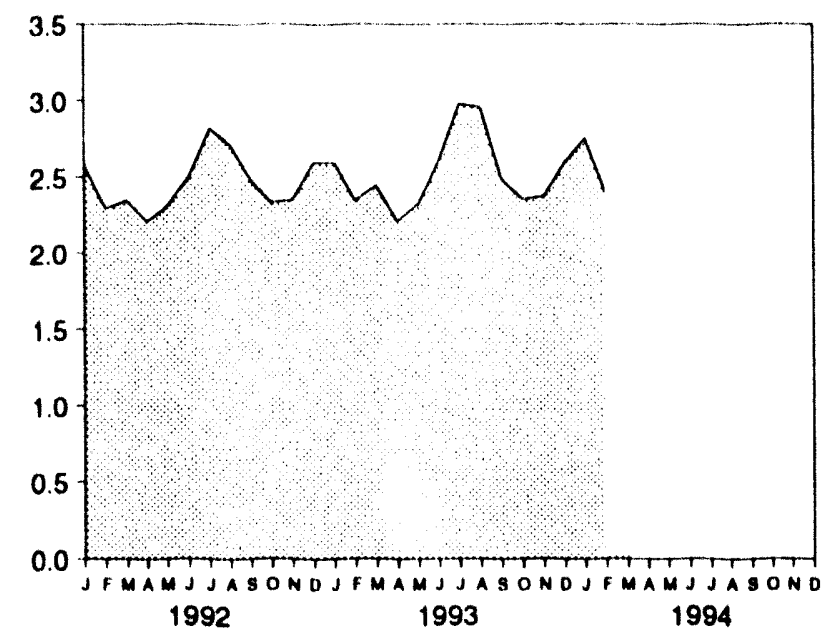

Input by Major Sources, Monthly

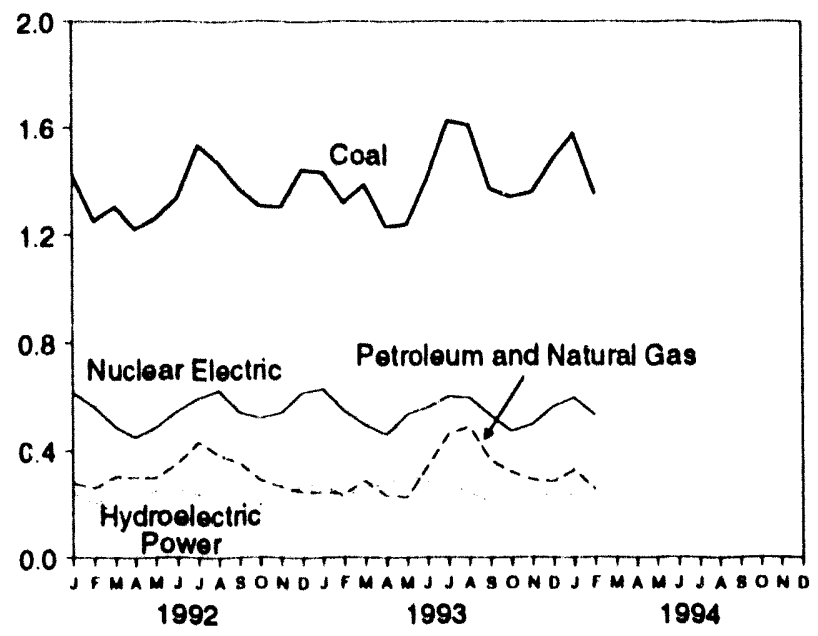

Input by Major Sources,

February 1994

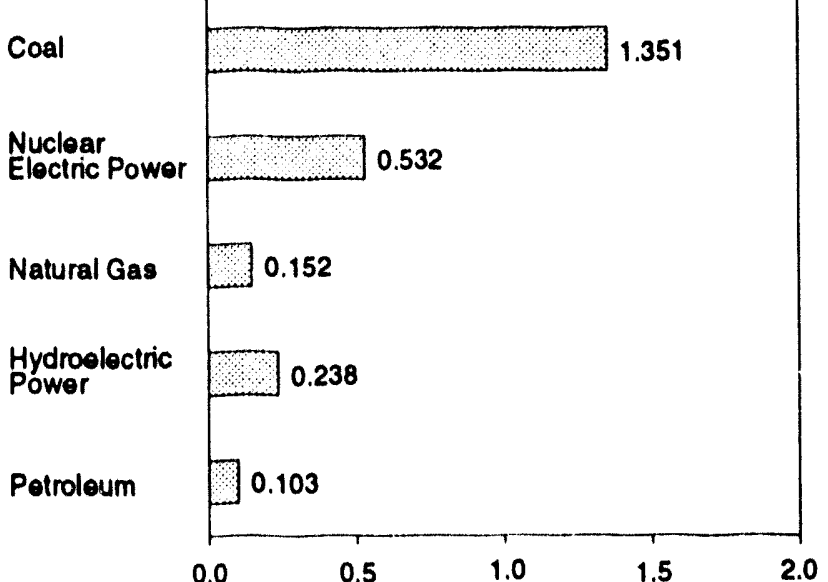

Nole: Because vertical scales difler, graphs should not be compared. Source: Table 2.6. 
Table 2.6 Energy Input at Electric Utilities (Quadrillion Btu)

\begin{tabular}{|c|c|c|c|c|c|c|c|c|}
\hline & Cod & $\begin{array}{c}\text { Natured } \\
\text { Canea }\end{array}$ & Potroloumb & $\begin{array}{l}\text { Nucloar } \\
\text { Electric } \\
\text { Power }\end{array}$ & $\begin{array}{l}\text { Hydro- } \\
\text { electrie } \\
\text { Powere }\end{array}$ & $\begin{array}{l}\text { Geothermal } \\
\text { Enorgy }\end{array}$ & Otherd & Total \\
\hline 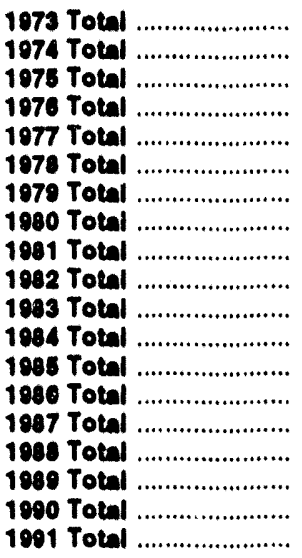 & $\begin{array}{r}8.689 \\
8.534 \\
8.786 \\
0.720 \\
10.262 \\
10.238 \\
11.260 \\
12.123 \\
12.583 \\
12.582 \\
13.213 \\
14.020 \\
14.542 \\
14.444 \\
15.173 \\
15.050 \\
18.988 \\
18.180 \\
18.028\end{array}$ & $\begin{array}{l}3.748 \\
3.510 \\
3.240 \\
3.152 \\
3.284 \\
3.297 \\
3.613 \\
3.810 \\
3.786 \\
3.342 \\
2.098 \\
3.220 \\
3.180 \\
2.691 \\
2.035 \\
2.700 \\
2.871 \\
2.882 \\
2.858\end{array}$ & $\begin{array}{l}3.815 \\
3.365 \\
3.168 \\
3.477 \\
3.001 \\
3.987 \\
3.283 \\
2.634 \\
2.202 \\
1.568 \\
1.544 \\
1.286 \\
1.090 \\
1.452 \\
1.257 \\
1.563 \\
1.685 \\
1.250 \\
1.178\end{array}$ & $\begin{array}{l}0.910 \\
1.272 \\
1.000 \\
2.111 \\
2.702 \\
3.024 \\
2.776 \\
2.739 \\
3.008 \\
3.131 \\
3.203 \\
3.553 \\
4.149 \\
4.471 \\
4.906 \\
5.861 \\
5.677 \\
6.161 \\
6.579\end{array}$ & $\begin{array}{l}2.976 \\
3.276 \\
3.187 \\
3.032 \\
2.482 \\
3.110 \\
3.107 \\
3.085 \\
3.072 \\
3.539 \\
3.866 \\
3.767 \\
3.365 \\
3.413 \\
3.084 \\
2.630 \\
2.848 \\
2.014 \\
3.083\end{array}$ & $\begin{array}{l}0.043 \\
.053 \\
.070 \\
.078 \\
.077 \\
.064 \\
.084 \\
.110 \\
.123 \\
.105 \\
.129 \\
.165 \\
.108 \\
.219 \\
.229 \\
.217 \\
.197 \\
.181 \\
.170\end{array}$ & $\begin{array}{l}0.003 \\
.003 \\
.002 \\
.003 \\
.005 \\
.003 \\
.005 \\
.005 \\
.004 \\
.003 \\
.004 \\
.009 \\
.015 \\
.012 \\
.016 \\
.017 \\
.020 \\
.021 \\
.021\end{array}$ & $\begin{array}{l}19.852 \\
20.022 \\
20.350 \\
21.574 \\
22.713 \\
23.724 \\
24.128 \\
24.505 \\
24.760 \\
24.270 \\
24.958 \\
26.020 \\
26.519 \\
26.703 \\
27.600 \\
28.648 \\
29.286 \\
29.509 \\
29.915\end{array}$ \\
\hline 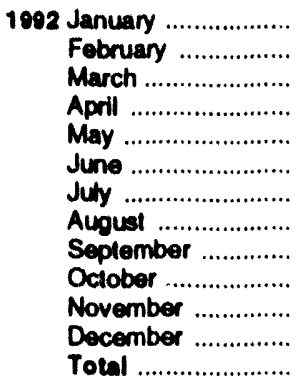 & $\begin{array}{r}1.419 \\
1.251 \\
1.303 \\
1.222 \\
1.260 \\
1.333 \\
1.534 \\
1.468 \\
1.371 \\
1.306 \\
1.302 \\
1.442 \\
16.211\end{array}$ & $\begin{array}{l}.173 \\
.174 \\
.212 \\
.234 \\
.242 \\
.272 \\
.341 \\
.309 \\
.280 \\
.217 \\
.193 \\
.179 \\
2.826\end{array}$ & $\begin{array}{l}.108 \\
.087 \\
.092 \\
.069 \\
.056 \\
.080 \\
.092 \\
.076 \\
.074 \\
.073 \\
.074 \\
.070 \\
.051\end{array}$ & $\begin{array}{l}.618 \\
.564 \\
.489 \\
.451 \\
.487 \\
.547 \\
.598 \\
.626 \\
.544 \\
.521 \\
.542 \\
.620 \\
.6 .607\end{array}$ & $\begin{array}{l}.242 \\
.203 \\
.234 \\
.219 \\
.251 \\
.254 \\
.238 \\
.217 \\
.201 \\
.200 \\
.227 \\
.272 \\
2.760\end{array}$ & $\begin{array}{l}.015 \\
.013 \\
.015 \\
.014 \\
.014 \\
.014 \\
.014 \\
.014 \\
.013 \\
.014 \\
.014 \\
.014 \\
.170\end{array}$ & $\begin{array}{l}.002 \\
.002 \\
.002 \\
.001 \\
.002 \\
.002 \\
.002 \\
.002 \\
.002 \\
.002 \\
.002 \\
.002 \\
.022\end{array}$ & $\begin{array}{r}2.577 \\
2.294 \\
2.348 \\
2.211 \\
2.311 \\
2.501 \\
2.820 \\
2.714 \\
2.485 \\
2.333 \\
2.353 \\
2.600 \\
28.547\end{array}$ \\
\hline 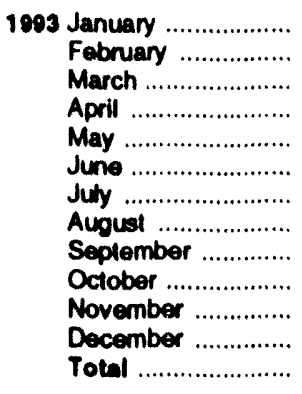 & 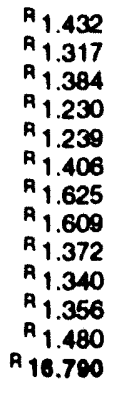 & $\begin{array}{l}.168 \\
.166 \\
.188 \\
.178 \\
.171 \\
.260 \\
.341 \\
.365 \\
.264 \\
.240 \\
.213 \\
.178 \\
2.741\end{array}$ & $\begin{array}{l}.077 \\
.074 \\
.090 \\
.055 \\
.056 \\
.083 \\
.121 \\
.126 \\
.102 \\
.080 \\
.079 \\
.108 \\
1.052\end{array}$ & $\begin{array}{l}.631 \\
.548 \\
.498 \\
.461 \\
.538 \\
.562 \\
.603 \\
.600 \\
.534 \\
.474 \\
.500 \\
.567 \\
6.517\end{array}$ & $\begin{array}{r}.275 \\
.225 \\
.262 \\
.275 \\
.313 \\
.285 \\
.274 \\
.244 \\
.209 \\
.207 \\
.211 \\
.247 \\
3.027\end{array}$ & $\begin{array}{l}.014 \\
.013 \\
.014 \\
.014 \\
.012 \\
.012 \\
.013 \\
.014 \\
.013 \\
.013 \\
.013 \\
.013 \\
.150\end{array}$ & $\begin{array}{l}.002 \\
.002 \\
.002 \\
.002 \\
.001 \\
.001 \\
.001 \\
.002 \\
.002 \\
.002 \\
.002 \\
.002 \\
.021\end{array}$ & $\begin{array}{l}A_{2.598} \\
A_{2.345} \\
A_{2} .448 \\
A_{2.214} \\
A_{2} .330 \\
A_{2} .610 \\
A_{2.979} \\
R_{2} .959 \\
A_{2} .497 \\
A_{2} .357 \\
A_{2} .375 \\
A_{2} .596 \\
A_{30.308}\end{array}$ \\
\hline $\begin{array}{l}1904 \text { January .................. } \\
\text { Fobruary .............. } \\
\text { 2-Month Total ........ }\end{array}$ & $\begin{array}{r}A_{1.576} \\
1.351 \\
2.927\end{array}$ & $\begin{array}{l}.174 \\
.152 \\
.326\end{array}$ & $\begin{array}{l}.155 \\
.103 \\
.250\end{array}$ & $\begin{array}{r}.600 \\
.532 \\
1.132\end{array}$ & $\begin{array}{l}.236 \\
.238 \\
.474\end{array}$ & $\begin{array}{l}.013 \\
.012 \\
.025\end{array}$ & $\begin{array}{l}.002 \\
.002 \\
.003\end{array}$ & $\begin{array}{r}A_{2.756} \\
2.390 \\
5.147\end{array}$ \\
\hline $\begin{array}{l}1993 \text { 2-Month Total ....... } \\
1902 \text { 2-Month Total ....... }\end{array}$ & $\begin{array}{l}2.749 \\
2.670\end{array}$ & $\begin{array}{l}.333 \\
.347\end{array}$ & $\begin{array}{l}.151 \\
.195\end{array}$ & $\begin{array}{l}1.179 \\
1.182\end{array}$ & $\begin{array}{l}.500 \\
.445\end{array}$ & $\begin{array}{l}.027 \\
.028\end{array}$ & $\begin{array}{l}.004 \\
.004\end{array}$ & $\begin{array}{l}4.943 \\
4.870\end{array}$ \\
\hline
\end{tabular}

- Includes supplemental gaseous fuels.

- Pelrobum products reported as "cil consumed in sleam plants" through 1979 and "heavy oil" from 1980 lonward, which are assumed to be residual fuel oll; petroleum products reported as "oll consumed in gas turbine and internal combustion engine plants" through 1979 and "light ofl" from 1980 lonward, which are assumed to be distillate fuel oil, kerosene, and pelroleum coke. c Includes net imports of electricity.

d "Other" is electricity generated lor distribution from wood, wasle, wind, photovollaic, and solar thermal energy.

$R=$ Revised data.

Notes: - Geographic coverage is the 50 States and the District of Columbia.

- Totals may not equal sum of components due to independent rounding. Additional Notes and Sources: See end of section. 


\section{Energy Consumption Notes and Sources}

The data in this section of the Monthly Energy Review $(M E R)$ are obtained initially from a group of energy-related surveys, typically called "supply surveys," conducted by the Energy Infornation Administration (EIA). Supply surveys are those surveys directed to suppliers and marketers of specific energy sources. They measure the quantities of specific energy sources produced, or the quantities supplied to the market, or both. The data obtained from the ElA's supply surveys are integrated to yield the summary consumption statistics published in this section (and in Section 1) of the $M E R$. Users of the EIA's energy consumption statistics should be aware of a second group of energy-related surveys, typically called "consumption surveys." Consumption surveys gather information on the types of energy consumed by end users of energy, along with the characteristics of those end users that can be associated with energy use. For example, the Manufacturing Energy Consumption Survey belongs to the consumption survey group because it collects information directly from end users (the manufacturing establishments). There are important differences between the supply and consumption surveys that need to be taken into account in any analysis that uses both data sources. For information on those differences, see Energy Consumption by End-Use Sector, A Comparison of Measures by Consumption and Supply Surveys, DOE/EIA-0533, Energy Information Administration, Washington, DC, April 6, 1990. The numbered notes that follow elaborate on essential information in Section 2.

1. Total Energy Consumed: Total energy consumed includes coal, natural gas (including supplemental gaseous fuels), petroleum products supplied, electric utility and industrial generation of bydroelectric power, net imports of electricity generated from hydroelectric power, and electricity generated from nuclear power. Total energy consumed also includes electricity generated from wood, waste, geothermal, wind, photovoltaic, and solar thermal energy but excludes other energy obtained from those sources because consistent historical data are not available.

2. Economic Sectors: Energy use is assigned to the major economic sectors according to the following guidelines as closely as possible:

- Residential-All private residences, whether occupied or vacant, owned or rented, including single-family homes, multifamily housing units, and mobile homes. Secondary homes, such as summer homes, are also included. Institutional housing, such as school dormitories, hospitals, and military barracks, generally are not included in the residential sector; they are included in the commercial sector.

- Commercial-Business establishments that are not engaged in transportation or in manufacturing or other types of industrial activity (agriculture, mining, or construction). Commercial establishments include hotels, motels, restaurants, wholesale businesses, retail stores, laundries, and other service enterprises; religious and nonprofit organizations; health, social, and educational institutions; and Federal, State, and local governments. Street lights, pumps, bridges, and public services are also included if the establishment operating them is considered commercial.

- Industrial-Manufacturing industries, which make up the largest part of the sector, along with mining, construction, agriculture, fisheries, and forestry. Establishments in this sector range from steel mills to small farms to companies assembling electronic components.

- Transportation-Private and public vehicles that move people and commodities. Included are automobiles, trucks, buses, motorcycles, railroads and railways (including streetcars), aircraft, ships, barges, and natural gas pipelines.

- Electric Utility-Privately and publicly owned establishments that generate, transmit, distribute, and sell electricity primarily for use by the public and meet the definition of an electric utility. Nonutility power producers are not included in the electric utility sector.

Although the end-use allocations are made according to these aggregations as closely as possible, some data are collected by using different classifications. For example, data on agricultural use of natural gas are collected and reported in the commercial sector, rather than in the industrial sector. Since agricultural use of natural gas cannot be identified separately, it is included in the commercial sector in this report. Another example is master-metered condominiums and apartments, and buildings with a combination of residential and commercial units. In many cases, the metering and billing practices cause residential energy usage of electricity, natural gas, or fuel oil to be included in the commercial sector. No adjustments for these discrepancies were made.

3. Conversion Factors: See the conversion factors listed in Appendix A.

4. Coal: Coal is anthracite, bituminous coal (including subbituminous coal), and lignite. Sources:

- 1973-September 1977: U.S. Department of the Interior (DOI), Bureau of Mines (BOM), Minerals Yearbook and Minerals Industry Surveys.

- Electric Utilities-October 1977 forward: Energy Information Administration (EIA), Form EIA-759 (formerly Form FPC-4), "Monthly Power Plant Report."

- Other Industrial-October 1977-December 1979: EIA, Form EIA-3, "Monthly Coal Consumption Report - Manufacturing Plants"; January 1980 for- 
ward: EIA, Form EIA-3, "Quarterly Coal Consumption Report - Manufacturing Plants," and Form EIA-6, "Coal Distribution Report."

- Coke Plants-October 1977-December 1980: EIA, Form EIA-5/5A, "Coke and Coal Chemicals - Monthly/Annual"; January 1981-December 1984: EIA, Form EIA-5/5A, "Coke Plant Report Quarterly/Annual Supplement"; January 1985 forward: EIA, Form EIA-5/5A, "Coke Plant Report," quarterly.

- Residential and Commercial-October 1977 December 1979: EIA, Form EIA-2, "Monthly Coal Report, Retail Dealers - Upper Lake Docks"; January 1980 forward: EIA, Form EIA-6, "Coal Distribution Report."

5. Natural Gas: Natural gas consumption by end use is based on data presented in Table 4.4 of this report. For Section 2 calculations, lease and plant fuel consumption are added to industrial deliveries, and pipeline fuel represents transportation use of natural gas. Values in Btu are derived by using the conversion factors provided in Appendix A. Sources:

- 1973-1975: DOI, BOM, Minerals Yearbook, "Natural Gas" chapter.

- 1976-1978: EIA, "Energy Data Reports," Natural Gas, Annual.

- 1979: EIA, Natural Gas Production and Consumption 1979.

- 1980-1992: EIA, Natural Gas Annual.

- 1993: EIA, Natural Gas Monthly.

- Electric Utilities-1973-1976: Form FPC-4, "Monthly Power Plant Report"; 1977-1981: Federal Energy Regulatory Commission (FERC), Form FPC-4, "Monthly Power Plant Report"; 1982 forward: EIA, Form EIA-759, "Monthly Power Plant Report."

- American Gas Association, "Monthly Gas Utility Statistical Report," residential and commercial monthly sales data for 1973-1979, which are used to estimate monthly consumption values from EIA annual consumption values.

6. Petroleum: Petroleum consumption by end use is the sum of all individual petroleum products estimated to be consumed in each end-use sector. First, total consumption by product is determined. Petroleum consumption in this section of the Monthly Energy Review (MER) is the series called "petroleum products supplied" in Section 3. Sources for petroleum products supplied by individual products are:

- 1973-1975: DOI, BOM, Mineral Industry Surveys, "Petroleum Statement, Annual."

- 1976-1980: EIA, Energy Data Reports, "Petroleum Statement, Annual."

- 1981-1992: EIA, Petroleum Supply Annual.

- 1993 and 1994: EIA, Petroleum Supply Monthly.
Specific petroleum products' end-use allocation procedures follow:

- Aviation Gasoline-All product supplied is assigned to the transportation sector.

- Asphalt-All product supplied is assigned to the industrial sector.

- Distillate Fuel-Product supplied is assigned to electric utilities and non-electric utilities as follows:

\section{Electric Utilities, All Periods.}

Monthly and annual consumption for 1973-1979 is assumed to be the amount of oil (minus small amounts of kerosene and kerosene-type jet fuel deliveries) reported as consumed in internal combustion and gas turbine engine plants. From January 1980, electric utility consumption of distillate fuel is assumed to $b$, the petroleum products reported as "light oil" (minus small amounts of kerosene deliveries through 1982) consumed at electric utilities.

Sources: 1973-September 1977: FPC, Form FPC4, "Monthly Power Plant Report"; October 19771981: FERC, Form FPC-4, "Monthly Power Plant Report"; 1982 forward: EIA, Form EIA-759, "Monthly Power Plant Report."

Sectors Other Than Electric Utilities, Annual Estimates Through 1992.

The aggregate non-electric utility use of distillate fuel is total distillate fuel supplied minus the electric utility consumption. The non-electric utility annual consumption totals are allocated to the individual non-electric utility sectors (residential, commercial, industrial, and transportation) in proportion to the share of "adjusted sales" of each end-use sector, as reported in EIA's Fuel Oil and Kerosene Sales (Sales) report series (DOE/EIA0535), which is based primarily on data collected by Form EIA-821, previously Form EIA-172. "Adjusted sales" are sales that have been adjusted at the PAD district level to equal EIA volume estimates of petroleum products supplied in the U.S. market. Following are notes on the individual sector groupings:

- Since 1979, the residential sector adjusted sales total is directly from the Sales reports. Prior to 1979, each year's sales subtotal of the heating plus industrial category is split into residential, commercial, and industrial (including farm) in proportion to the 1979 shares.

- Since 1979, the commercial sector adjusted sales total is directly from the Sales reports. Prior to 1979, each year's sales subtotal of the heating plus industrial category is split into residential, commer- 
cial, and industrial (including farm) in proportion to the 1979 shares.

- Since 1979, the industrial sector adjusted sales total is the sum of the adjusted sales for industrial, farm, oil company, off-highway, diesel, and all other uses. Prior to 1979, each year's sales subtotal of the heating plus industrial category is split into residential, commercial, and industrial (including farm) in proportion to the 1979 shares, and this estimated industrial portion is added to oil company, off-highway diesel, and all other uses.

- The transportation sector adjusted sales total is the sum of the adjusted sales for railroad, vessel bunkering, on-highway diesel, and military uses for all years.

Sectors Other Than Electric Utilities, Monthly Estimates Through 1992.

- Residential and commercial monthly consumption is estimated by allocating the annual estimates described above into months in proportion to each month's share of the year's sales of No. 2 heating oil as reported in the "Monthly Report of Heating Oil Sales" by the Ethyl Corporation from 19731980, the American Petroleum Institute for 1981 and 1982, and the EIA, Form EIA-782A, "Refiners'/Gas Plant Operators' Monthly Petroleum Product Sales Report," No. 2 Fuel Oil Sales to End Users and for Resale, since 1983.

- The transportation highway use portion is allocated into the months in proportion to each month's share of the year's total sales for highway use as reported by the Federal Highway Administration's Table MF-25, "Private and Commercial Highway Use of Special Fuels by Months." The remaining transportation use of distillate fuel (i.e., for railroads, vessel bunkering, and military use) is evenly distributed over the months, adjusted for the number of days per month.

- Industrial monthly estimates are made by subtracting the residential and commercial, transportation, and electric utility sector estimates from each month's total distillate fuel supplied.

\section{Sectors Other Than Electric Utilities, 1993 and 1994}

Each month's non-electric utility consumption subtotal is disaggregated into the major end-use sectors in proportion to the shares each sector held of the non-electric utility subtotal in the same month in 1992.

- Jet Fuel-Through 1982, small amounts of kerosene-type jet fuel were consumed by electric utilities. Kerosene-type jet fuel deliveries to electric utilities as reported on the Form FERC423 (formerly Form FPC-423) were used as estimates of this consumption. All remaining jet fuel (kerosene-type and naphtha-type) is consumed by the transportation sector.

- Kerosene-Total product supplied monthly is allocated to the major end-use sectors in proportion to annual sales grouped into end-use sectors from ElA's Fuel Oil and Kerosene Sales (Sales) reports (based primarily on data collected by Form EIA-821, previously Form EIA-172), as follows:

- Residential deliveries are directly from the Sales reports for 1979-1992. Sales for 1992 are used as estimates for succeeding periods. Prior to 1979, each year's sales category called "heating" is split into residential, commercial, and industrial in proportion to the 1979 shares.

- Commercial sales are directly from the Sales reports for 1979-1992. Sales for 1992 are used as estimates for succeeding periods. Prior to 1979 . each year's sales category called "heating" is split into residential, commercial, and industrial in proportion to the 1979 shares.

- Industrial sales are directly from the Sales reports for 1979-1992. Sales for 1992 are used as estimates for succeeding periods. Prior to 1979 , each year's sales category called "heating" is split into residential, commercial and industrial in proportion to the 1979 shares, and this estimated industrial (including farm) portion is added to all other uses.

- Liquefied Petroleum Gases (LPG)-The annual shares of LPG's total consumption that are estimated to be consumed by each end-use sector are applied to each month's total LPG consumption (i.e., product supplied) to create monthly end-use consumption estimates. The annual enduse shares are calculated in the following manner:

- Sales of LPG to the residential and commercial sector are converted from thousand gallons per year to thousand barrels per year and are assumed to be the annual consumption of LPG by the sector.

- The quantity of LPG sold each year for consumption in internal combustion engines is allocated between the transportation and industrial sectors on the basis of data for special fuels used on highways published by the U.S. Department of Transportation, Federal Highway Administration, in Highway Statistics. The allocations of LPG sold for internal combustion engine use to the transportation sector range from a high of 67 percent in 1981 to a low of 37 percent in 1987.

- LPG consumed annually by the industrial sector is estimated as the difference between LPG total supplied and the estimated consumption of LPG by the sum of the residential and commercial sector and the transportation sector. The industrial sector includes LPG used by chemical plants as raw materials or solvents and used in the production of 
synthetic rubber; refinery fuel use; use as synthetic natural gas feedstock and use in secondary recovery projects; all farm use; LPG sold to gas utility companies for distribution through the mains; and a portion of the use of LPG as an internal combustion engine fuel.

The sources of the annual sales data for creating annual end-use shares are:

- 1973-1982: EIA's "Sales of Liquefied Petroleum Gases and Ethane" reports, based primarily on data collected by Form EIA-174.

- 1983: End-use consumption estimates for 1983 are based on 1982 end-use consumption because the collection of data under Form EIA-174 was discontinued after data year 1982 .

- 1984-1992: American Petroleum Institute (API), "Sales of Natural Gas Liquids and Liquefied Refinery Gases," which is based on an LPG sales survey jointly sponsored by API, the Gas Processors Association, and the National Liquefied Petroleum Gas Association.

- 1993 and 1994: The 1992 source is used to estimate succeeding periods.

- Lubricants-Total product supplied is allocated to the industrial and transportation sectors for all months according to proportions developed from annual sales of lubricants to the two sectors from U.S. Department of Commerce, Bureau of the Census, Current Industrial Reports, "Sales of Lubri: ating and Industrial Oils and Greases." The 1973 shares are applied to 1973 and 1974; the 1975 shares are applied to 1975 and 1976; and the 1977 shares are applied to 1977 forward.

- Motor Gasoline-Total product supplied monthly is allocated to the major end-use sectors in proportion to aggregations of annual sales categories created on the basis of the U.S. Department of Transportation, Federal Highway Administration, Highway Statistics, Tables MF-21, MF-24, and MF-25, as follows:

- Commercial sales are the sum of sales for public non-highway use and miscellaneous and unclassified uses.

- Industrial sales are the sum of sales for agriculture, construction, and industrial and commercial use as classified in the Highway Statistics.

- Transportation sales are the sum of sales for highway use (minus the sales of special fuels, which are primarily diesel fuel and are accounted for in the transportation sector of distillate fuel) and sales for marine use.

- Petroleum Coke-The portion consumed by electric utilities is from Form EIA-759, "Monthly
Power Plant Report" (formerly Form FPC-4). The remaining petroleum coke is assigned to the industrial sector.

- Residual Fuel-Product supplied is assigned to electric utilities and non-electric utilities as follows:

\section{Electric Utilities, All Periods.}

Monthly and annual consumption for 1973-1979 is assumed to be the amount of oil reported as consumed in steam-electric power plants. From January 1980 forward, electric utility consumption of residual fuel is assumed to be the petroleum products reported as heavy oil consumed at electric utilities.

Sources: 1973-September 1977: Form FPC-4, "Monthly Power Plant Report"; October 19771981: FERC, Form FPC-4, "Monthly Power Plant Report"; 1982 forward: EIA, Form EIA-759, "Monthly Power Plant Report."

Sectors Other Than Electric Utilities, Annual Estimates Through 1992.

The aggregate non-electric utility use of residual fuel is total residual fuel supplied minus the electric utility consumption. The non-electric utility annual totals are allocated into the individual non-electric utility sectors in proportion to the amount of residual fuel sold to end users, grouped into sectors from EIA's Fuel Oil and Kerosene Sales (Sales) reports (based primarily on data collected by Form EIA-821, previously Form EIA-172), as follows:

- Since 1979, commercial sales data are directly from the Sales reports. Prior to 1979, each year's sales subtotal of the heating plus industrial category is split into commercial and industrial in proportion to the 1979 shares.

- Since 1979, industrial sales data are the sum of sales for industrial, oil company, and all other uses. Prior to 1979, each year's sales subtotal of the heating plus industrial category is split into commercial and industrial in proportion to the 1979 shares, and this estimated industrial portion is added to oil company and all other uses.

- Transportation sales are the sum of sales for railroad, vessel bunkering, and military uses for all years.

Sectors Other Than Electric Utilities, Monthly Estimates Through 1992.

- Commercial sector monthly consumption is estimated by allocating the annual commercial sector estimates described above into months in proportion to each month's share of the year's sales of No. 2 fuel oil as reported in the "Monthly Report 
of Heating Oil Sales" by the Ethyl Corporation for 1973-1980 and the American Petroleum Institute for 1981 and 1982, and the EIA, Form EIA-782A, "Refiners'/Gas Plant Operators' Monthly Petroleum Product Sales Report," No. 2 Fuel Oil Sales to End Users and for Resale, since 1983.

- Transportation monthly estimates are made by evenly distributing the annual sector estimate over the months, adjusting for the number of days per month.

- Industrial monthly estimates are made by subtracting the commercial, transportation, and electric utility sector estimates from each month's total residual fuel supplied.

\section{Sectors Other Than Electric Utilities, 1993 and 1994}

Each month's non-electric utility consumption subtotal is disaggregated into the major end-use sectors in proportion to the shares each sector held of the non-electric utility subtotal in the same month in 1992.

- Road Oil-All product supplied is assigned to the industrial sector.

- All Other Petroleum Products-The product supplied of all remaining petroleum products is assigned to the industrial sector.

7. Nuclear Electric Power, Geothermal, and Wood, Waste, Wind, Photovoltaic, and Solar Thermal Energy Sources Connected to Electric Utility Distribution Systems: Sources:

- 1973-1976: FPC, Form FPC-4, "Monthly Power Plant Report."

- 1977-1981: FERC, Form FPC-4, "Monthly Power Plant Report."

- 1982 forward: EIA, Form EIA-759, "Monthly Power Plant Report."

8. Hydroelectric Power: Includes electricity generated by hydroelectric power at electric utilities, small amounts in the industrial sector, and net imports of electricity, which are assumed to be generated by hydroelectric power and are included in the electric utilities sector.

Sources for electric utilities sector:

- 1973-1976: FPC, Form FPC-4, "Monthly Power Plant Report."

- 1977-1981: FERC, Form FPC-4, "Monthly Power Plant Report."

- 1982 forward: EIA, Form EIA-759, "Monthly Power Plant Report."
Sources for industrial sector:

- 1973-1978: FPC, Form FPC-4, "Monthly Power Plant Report," for plants with generating capacity exceeding 10 megawatts, and FPC, Form FPC12C, "Industrial Electric Generating Capacity," for all other plants.

- 1979: FPC, Form FPC-4, "Monthly Power Plant Report," for plants with generating capacity exceeding 10 megawatts and EIA estimates for all other plants.

- 1980 forward: Annual generation estimated by EIA as the average generation over the 6-year period of 1974-1979; monthly generation estimated to be in proportion to each month's hydroelectricity generation in the electric utility industry in 1980.

Sources for imports and exports of electricity:

- 1973-September 1977: Unpublished Federal Power Commission data.

- October 1977-1980: Unpublished Economic Regulatory Administration (ERA) data.

- 1981: DOE, Office of Energy Emergency Operations, "Report on Electric Energy Exchanges with Canada and Mexico for Calendar Year 1981," April 1982 (revised June 1982).

- 1982 and 1983: DOE, ERA, Electricity Exchanges Across International Borders.

- 1984-1986: DOE, ERA, Electricity Transactions Across International Borders.

- 1987 and 1988: DOE, ERA, Form ERA-781R, "Annual Report of International Electrical Export/Import Data."

- 1989-1991: DOE, Assistant Secretary for Fossil Energy, Form FE-781-R, "Annual Report of International Electrical Export//mport Data."

- 1992 forward: EIA estimates based on preliminary data from the National Energy Board of Canada and DOE, Assistant Secretary for Fossil Energy.

9. Net Imports of Coal Coke: Net imports means imports minus exports, and a minus sign indicates that exports are greater than imports. Sources:

- 1973-1975: DOI, BOM, Minerals Yearbook, "Coke and Coal Chemicals" chapter.

- 1976-1980: EIA, Energy Data Report, "Coke and Coal Chemicals" annual.

- 1981: EIA, Energy Data Report, "Coke Plant Report," quarterly.

- 1982 forward: EIA, Quarterly Coal Report.

10. Electricity: End-use consumption of electricity is based on Table 7.2 sales data. "Other," which is primarily for use in government buildings, is added to the commercial sector, except for approximately 4 per- 
cent used by railroads and railways and attributed to the tralisportation sector. For 1973-1983 and 1993, "Montilly Series" data are used directly. For 19841992, wonthly estimates are created by dividing each month's "Monthly Series" value by the "Monthly Series" total for the year and multiplying by the "Annual Series" value for the year. Kilowatthours are converted to Btu at the rate of 3,412 Btu per kilowatthour. See Table 7.2 for sources of the electricity sales data.

11. Electrical System Energy Losses: Electrical system energy losses are calculated as the difference between total energy input at electric utilities and the total energy content of electricity sold to end-use consumers. Most of those losses occur at steam-electric power plants (conventional and nuclear) in the conversion of beat energy into mechanical energy to turn electric generators. The loss is a thermodynamically necessary feature of the steam-electric cycle. Part of the energy input-to-output losses is a result of imputing fossil energy equivalent inputs for hydroelectric and other energy sources, since there is no generally accepted practice for measuring those thermal conversion rates. In addition to conversion losses, other losses include power plant use of electricity, transmission and distribution of electricity from power plants to end-use consumers (also called "line losses"), and unaccounted for electricity. Total losses are allocated to the end-use sectors in proportion to each sector's share of total electricity sales. Overall, approximately 67 percent of total energy input is lost in conversion; of electricity generated, approximately 5 percert is lost in plant use and 9 percent is lost in transmission and distribution. Calculated electrical system energy losses may be less than actual losses, because primary consumption does not include the energy equivalent of utility purchases of electricity from non-electric utilities and from Canada and Mexico, although they are included in electricity sales. 


\section{Section 3. Petroleum}

Total petroleum imports ${ }^{2}$ averaged 8.9 million barrels per day in April 1994, 4 percent $^{3}$ higher than the previous month's rate and 5 percent higher than the April 1993 rate.

In April 1994, 17.3 million barrels per day of petroleum products were supplied for domestic use, 4 percent higher than the April 1993 rate. Motor gasoline accounted for $\mathbf{4 3}$ percent of the total; distillate fuel oil, 19 percent; and residual fuel oil, 5 percent.

Motor gasoline supplied during April 1994 averaged 7.4 million barrels per day, 1 percent higher than the previous month's rate and slightly higher than the April 1993 rate. Total motor gasoline stocks were 217 million barrels at the end of April 1994, 3 million barrels above the stock level in the previous month but 5 million barrels below the level 1 year earlier.
Distillate fuel oil supplied during April 1994 averaged 3.3 million barrels per day, 2 percent lower than the previous month's rate but 11 percent higher than the April 1993 rate. Distillate fuel oil ending stocks for April 1994 were 102 million barrels, 2 million barrels above the stock level in the previous month and 4 million barrels above the stock level 1 year earlier.

Residual fuel oil supplied in April 1994 averaged 0.9 million barrels per day, 10 percent lower than the previous month's rate and 14 percent lower than the April 1993 rate. Residual fuel oil stocks measured 38 million barrels at the end of April 1994, 3 million barrels below the stock level in both the previous month and the level 1 year earlier.

Estimates (except of crude production) for the most current month are based on Energy Information Administration (EIA) weekly data and will be revised to conform with data from the EIA Petroleum Reporting System as available. For the most recent month, crude production is an EIA estimate based on historical and provisional data through January 1994.

\footnotetext{
${ }^{2}$ Total import data include imports into the Strategic Petroleum Reserve.

${ }^{3}$ Percentage changes are based on numbers shown in the following tables.
} 
Table 3.1a Petroleum Overview: Field Production, Stock Change, Petroleum Products Supplled, and Ending Stocks

\begin{tabular}{|c|c|c|c|c|c|c|c|}
\hline & \multicolumn{3}{|c|}{ Fleld Production } & \multicolumn{2}{|c|}{ Stock Changea } & \multirow[b]{2}{*}{$\begin{array}{l}\text { Petroloum } \\
\text { Produete } \\
\text { Supplled }\end{array}$} & \multirow{2}{*}{$\begin{array}{l}\text { Ending stocket } \\
\text { Crude Olfd and } \\
\text { Potroleum } \\
\text { Produete }\end{array}$} \\
\hline & $\begin{array}{l}\text { Total } \\
\text { Domestlec }\end{array}$ & $\begin{array}{l}\text { Crude } \\
\text { Oll }\end{array}$ & $\begin{array}{c}\text { Natural } \\
\text { Cose Plant } \\
\text { Productlon }\end{array}$ & $\begin{array}{l}\text { Crude } \\
\text { olld }\end{array}$ & $\begin{array}{l}\text { Petroloum } \\
\text { Producte }\end{array}$ & & \\
\hline & \multicolumn{6}{|c|}{ Thousand Barrele per Day } & Million Barrels \\
\hline 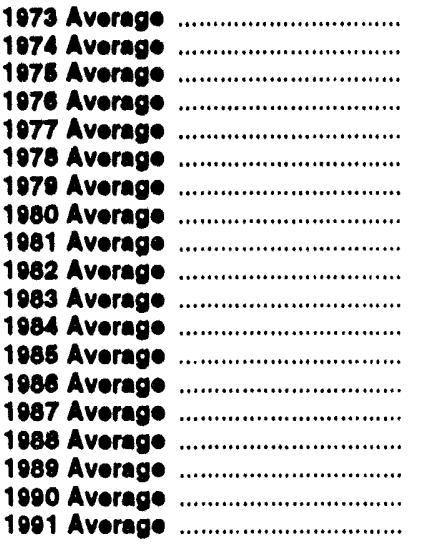 & $\begin{array}{r}10,078 \\
10,498 \\
10,048 \\
9,774 \\
9,013 \\
10,328 \\
10,179 \\
10,214 \\
10,230 \\
10,252 \\
10,290 \\
10,554 \\
10,638 \\
10,289 \\
10,008 \\
0,818 \\
9,219 \\
8,994 \\
9,168\end{array}$ & $\begin{array}{l}\mathbf{8}, 208 \\
\mathbf{8 , 7 7 4} \\
\mathbf{8 , 3 7 6} \\
\mathbf{8 , 1 3 2} \\
\mathbf{8 , 2 4 8} \\
\mathbf{8 , 7 0 7} \\
\mathbf{8 , 5 5 2} \\
\mathbf{8 , 5 9 7} \\
\mathbf{8 , 5 7 2} \\
\mathbf{8 , 6 4 9} \\
\mathbf{8 , 6 8 8} \\
\mathbf{8 , 8 7 9} \\
\mathbf{8 , 9 7 1} \\
\mathbf{8 , 6 8 0} \\
\mathbf{8 , 3 4 9} \\
\mathbf{8 , 1 4 0} \\
\mathbf{7 , 6 1 3} \\
\mathbf{7 , 3 5 5} \\
\mathbf{7 , 4 1 7}\end{array}$ & $\begin{array}{r}1,738 \\
1,688 \\
1,633 \\
1,604 \\
1,618 \\
1,687 \\
1,584 \\
1,573 \\
1,600 \\
1,550 \\
1,550 \\
1,630 \\
1,600 \\
1,551 \\
1,595 \\
1,625 \\
1,546 \\
1,560 \\
1,650\end{array}$ & $\begin{array}{r}-11 \\
62 \\
017 \\
30 \\
170 \\
78 \\
146 \\
98 \\
9290 \\
136 \\
214 \\
190 \\
50 \\
78 \\
128 \\
1 \\
86 \\
-36 \\
-42\end{array}$ & $\begin{array}{r}140 \\
117 \\
018 \\
-98 \\
378 \\
-172 \\
25 \\
42 \\
-130 \\
-283 \\
-234 \\
81 \\
-153 \\
124 \\
-07 \\
-20 \\
-120 \\
142 \\
32\end{array}$ & $\begin{array}{l}17,308 \\
18,053 \\
18,322 \\
17,481 \\
18,431 \\
18,047 \\
18,513 \\
17,056 \\
18,058 \\
15,298 \\
15,231 \\
15,728 \\
15,728 \\
16,281 \\
18,665 \\
17,283 \\
17,325 \\
18,988 \\
16,714\end{array}$ & $\begin{array}{r}1,008 \\
\bullet 1,074 \\
1,133 \\
1,112 \\
1,312 \\
1,278 \\
1,341 \\
1,302 \\
1,484 \\
1,430 \\
1,454 \\
1,686 \\
1,610 \\
1,393 \\
1,607 \\
1,697 \\
1,581 \\
1,621 \\
1,617\end{array}$ \\
\hline 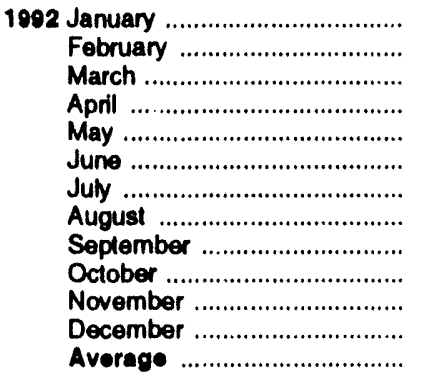 & $\begin{array}{l}9,176 \\
9,175 \\
9,123 \\
9,072 \\
8,949 \\
8,968 \\
8,961 \\
8,678 \\
8,843 \\
9,025 \\
8,975 \\
9,019 \\
8,996\end{array}$ & $\begin{array}{l}7,361 \\
7,389 \\
7,348 \\
7,293 \\
7,169 \\
7,167 \\
7,131 \\
6,922 \\
7,030 \\
7,126 \\
7,024 \\
7,103 \\
7,171\end{array}$ & $\begin{array}{l}1,688 \\
1,696 \\
1,684 \\
1,693 \\
1,695 \\
1,701 \\
1,683 \\
1,638 \\
1,660 \\
1,722 \\
1,754 \\
1,744 \\
1,697\end{array}$ & $\begin{array}{r}540 \\
171 \\
-250 \\
315 \\
-144 \\
-581 \\
244 \\
-124 \\
-160 \\
411 \\
-227 \\
-212 \\
-1\end{array}$ & $\begin{array}{r}-757 \\
.951 \\
-291 \\
92 \\
770 \\
604 \\
290 \\
161 \\
653 \\
-258 \\
77 \\
-1,203 \\
-68\end{array}$ & $\begin{array}{l}17,012 \\
16,893 \\
16,825 \\
16,764 \\
16,485 \\
16,978 \\
17,143 \\
16,929 \\
16,876 \\
17,448 \\
17,091 \\
17,928 \\
17,033\end{array}$ & $\begin{array}{l}1,610 \\
1,588 \\
1,571 \\
1,583 \\
1,602 \\
1,603 \\
1,620 \\
1,621 \\
1,636 \\
1,640 \\
1,636 \\
1,592 \\
1,692\end{array}$ \\
\hline 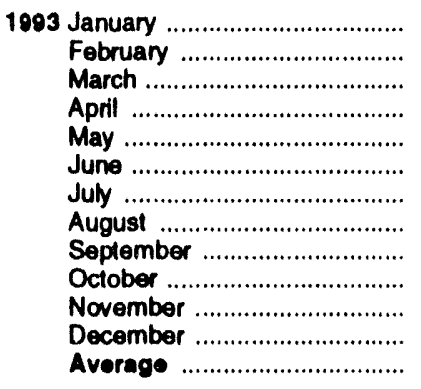 & $\begin{array}{l}E_{89,257} \\
E_{8,948} \\
E_{9,009} \\
E_{8,904} \\
E_{8,775} \\
E_{\mathbf{8}, 697} \\
E_{8,599} \\
E_{8,691} \\
E_{8,670} \\
E_{8,847} \\
E_{\mathbf{8}, 323} \\
E_{8,696} \\
E_{\mathbf{8 , 8 2 6}}\end{array}$ & $\begin{array}{l}E_{7,008} \\
E_{6,957} \\
E_{6,976} \\
E_{6,897} \\
E_{6,833} \\
E_{6,756} \\
E_{6,654} \\
E_{6,732} \\
E_{6,711} \\
E_{6,816} \\
E_{6,838} \\
E_{6,838} \\
E_{\mathbf{6 , 8 3 8}}\end{array}$ & $\begin{array}{l}1,728 \\
1,761 \\
1,799 \\
1,790 \\
1,719 \\
1,738 \\
1,723 \\
1,732 \\
1,717 \\
1,765 \\
1,674 \\
1,607 \\
1,729\end{array}$ & $\begin{array}{r}264 \\
219 \\
246 \\
537 \\
133 \\
.15 \\
41 \\
.524 \\
.439 \\
333 \\
251 \\
-58 \\
81\end{array}$ & $\begin{array}{r}0370 \\
.799 \\
.619 \\
388 \\
897 \\
586 \\
542 \\
386 \\
7 \\
420 \\
-286 \\
-1,158 \\
67\end{array}$ & $\begin{array}{l}16,320 \\
17,397 \\
17,688 \\
16,673 \\
16,340 \\
17,032 \\
17,208 \\
17,176 \\
17,709 \\
17,230 \\
17,688 \\
17,887 \\
17,193\end{array}$ & $\begin{array}{l}1,611 \\
1,595 \\
1,584 \\
1,611 \\
1,643 \\
1,680 \\
1,678 \\
1,674 \\
1,681 \\
1,685 \\
1,684 \\
1,646 \\
1,646\end{array}$ \\
\hline 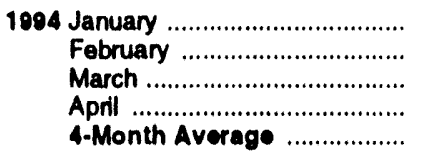 & $\begin{array}{r}E_{8,674} \\
E_{8,586} \\
F_{8,688} \\
E_{8,488} \\
E_{\mathbf{8}, 611}\end{array}$ & $\begin{array}{r}E_{6,777} \\
E_{6,745} \\
R_{6,719} \\
P_{6,607} \\
P_{6,712}\end{array}$ & $\begin{array}{r}1,619 \\
1,642 \\
A_{1,676} \\
E_{1,627} \\
\text { E }_{1,641}\end{array}$ & $\begin{array}{r}-16 \\
-164 \\
A_{339} \\
E_{125} \\
E_{76}\end{array}$ & $\begin{array}{r}-831 \\
-1.225 \\
A_{-438} \\
E .183 \\
E .568\end{array}$ & $\begin{array}{r}17,924 \\
18,302 \\
\text { R } 17,289 \\
E_{17,283} \\
E_{17,688}\end{array}$ & $\begin{array}{r}1,620 \\
1,581 \\
\text { R } 1,578 \\
\text { E } 1,591 \\
\text { E } 1,501\end{array}$ \\
\hline $\begin{array}{l}1003 \text { 4-Month Average ................ } \\
1002 \text { 4-Month Average ................ }\end{array}$ & $\begin{array}{r}E_{9,032} \\
9,138\end{array}$ & $\begin{array}{r}\mathbf{E}, 960 \\
7,347\end{array}$ & $\begin{array}{l}1,769 \\
1,603\end{array}$ & $\begin{array}{l}317 \\
193\end{array}$ & $\begin{array}{l}-154 \\
-474\end{array}$ & $\begin{array}{r}17,013 \\
16,874\end{array}$ & $\begin{array}{l}1,611 \\
1,883\end{array}$ \\
\hline
\end{tabular}

- A negative number indicates a decrease in stocks and a positive number

butyl ether) plants. indicates an increase.

b Stocks are totals as of end of period.

c Includes crude oll, natural gas plant liquids, and other liquids.

d Includes stocks located in the Strateglc Petroleum Reserve.

- See Note 4 at end of section.

1 See Note 6 at end of section.

- Beginning in 1993, includes fuel ethanol blended into finished motor gasoline and oxygenate production from merchant MTBE (methyl tertlary

$P E=$ Preliminary estimate, $\quad R=$ Revised data. $\quad N A=N o t$ available. $E=$ Estimaie

Notes: - Crude oll includes lease condensate. - Geographic coverage is the 50 States and the District of Columbia.

Sources: - 1973-1980: Energy Information Administration (EIA), Petroloum Supply Monthly, February 1993, Table S1. - 1081 fonward: EIA, Petroleum Supply Monthly, May 1994, Table S1. 
Table 3.1b Petroleum Overview: Imports, Exports, and Net Imports

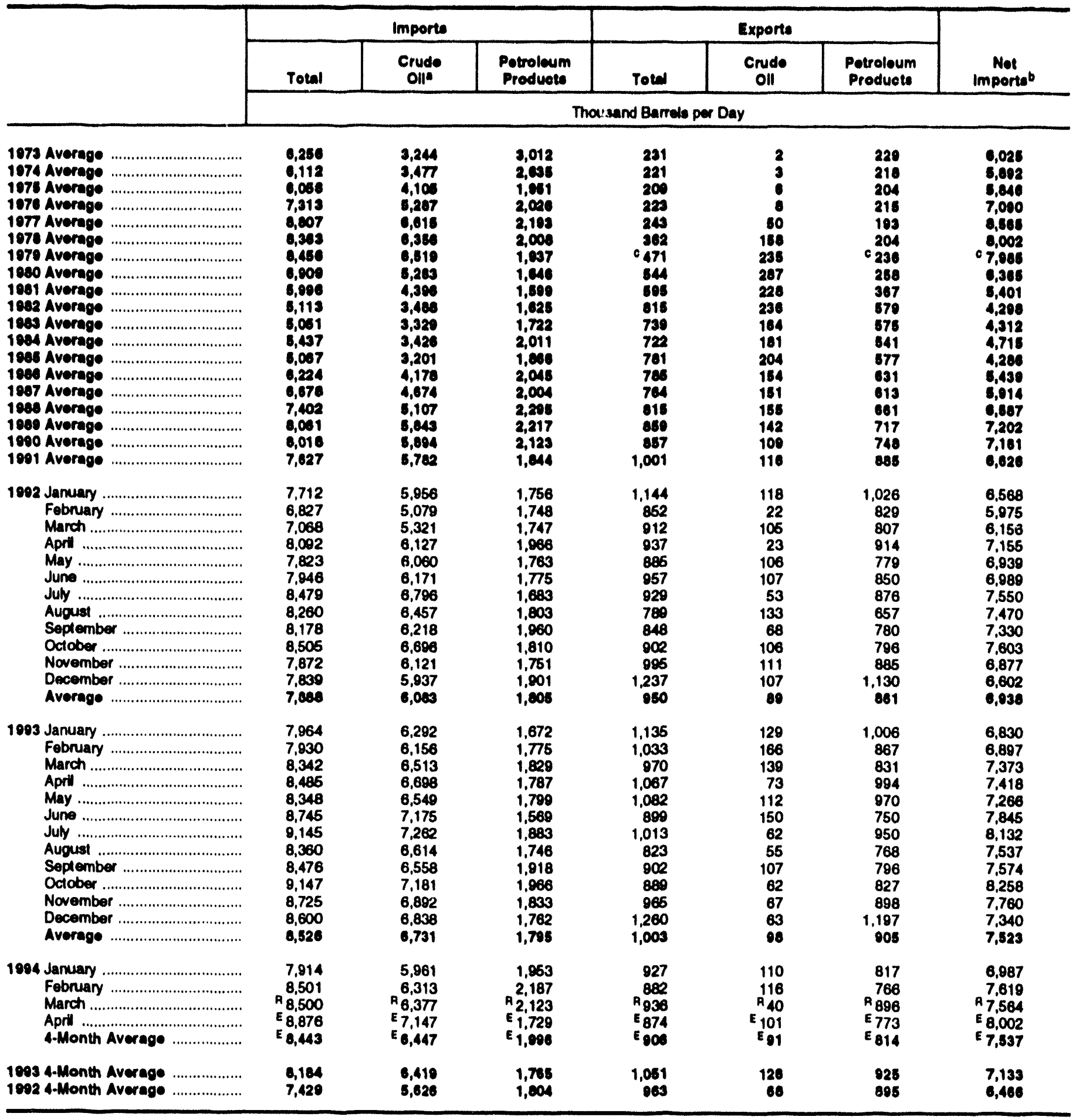

- Includes crude oil for atorage in the Strategic Petroleum Reserve.

- Totals may not equal sum of components due to independent rounding.

Sources: - 1073-1980: Energy Intormation Adminisiration (EIA).

- See Note 6 al end of section.

RaRevised data. E=Estimate.

Notes: - Geographic coverage is the 50 States and the District of Columbla.

Petroleum Supply Monthly, February 1993, Table S1. • 1981 fonwerd: EIA, Petroleum Supply Monthly, May 1894, Table S1. 


\section{Figure 3.1 Petroleum Overview}

(Million Barrels per Day)

Overview, January-April

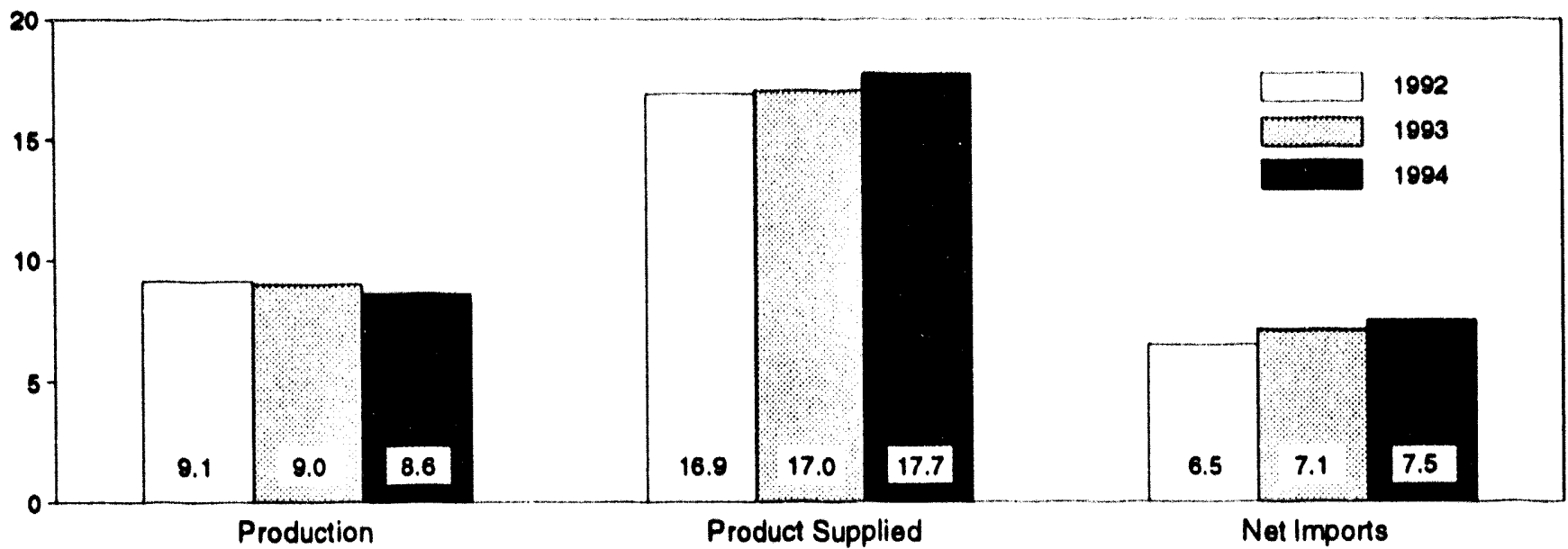

Overview, 1973-1993

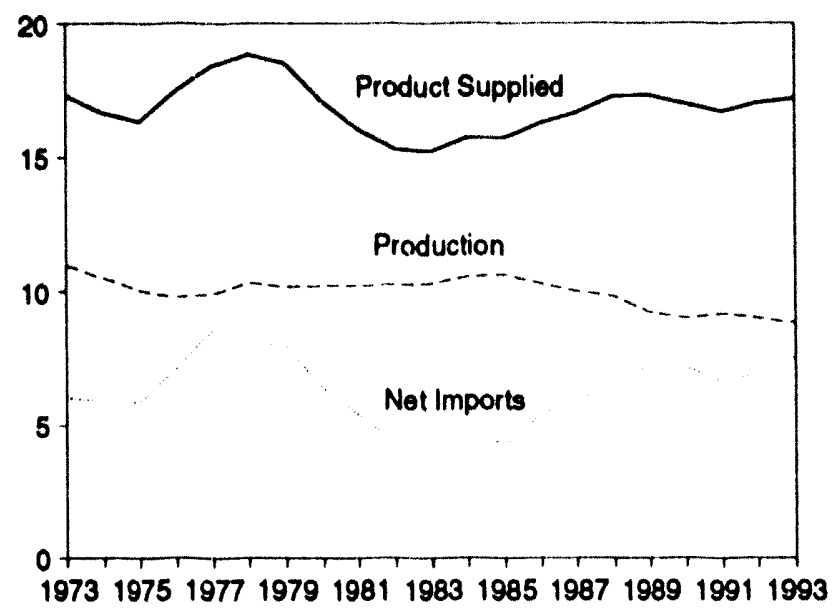

Crude Oil Production, 1973-1993

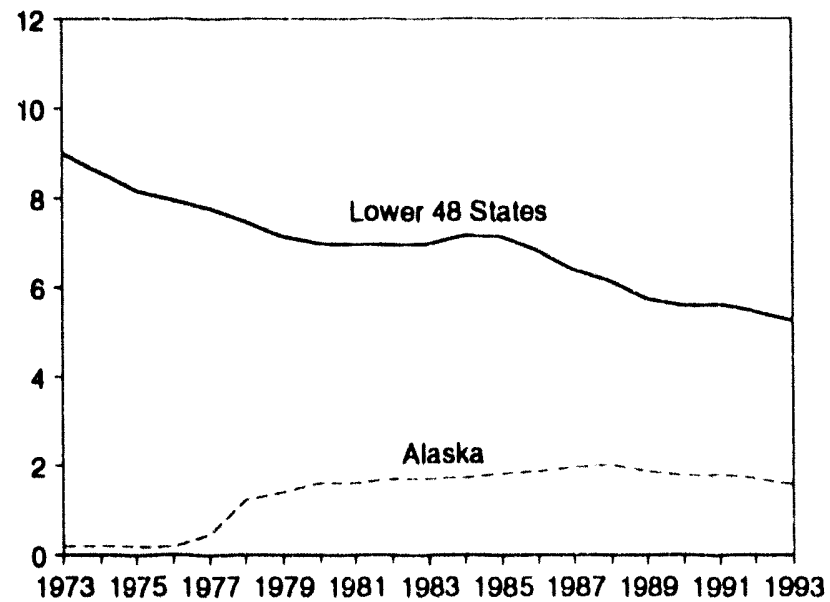

Production, 1973-1993

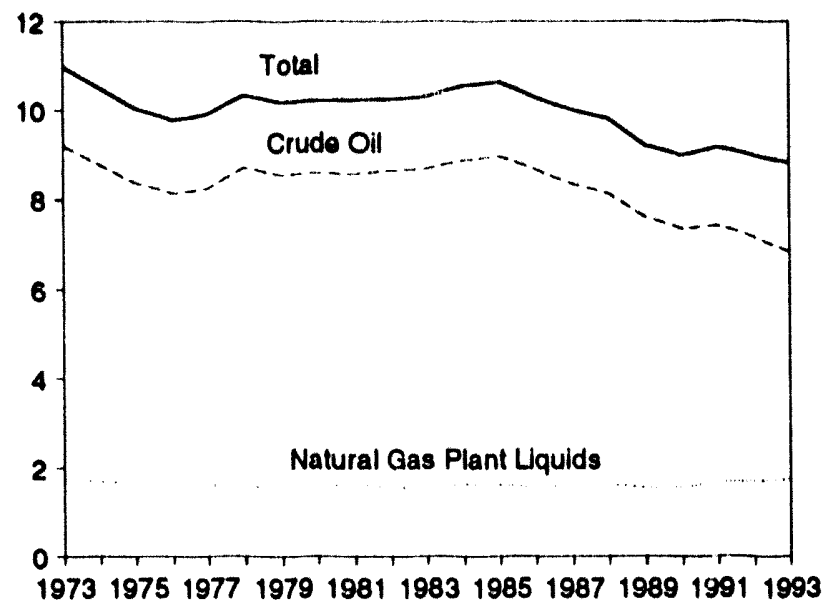

Total Production, Monthly

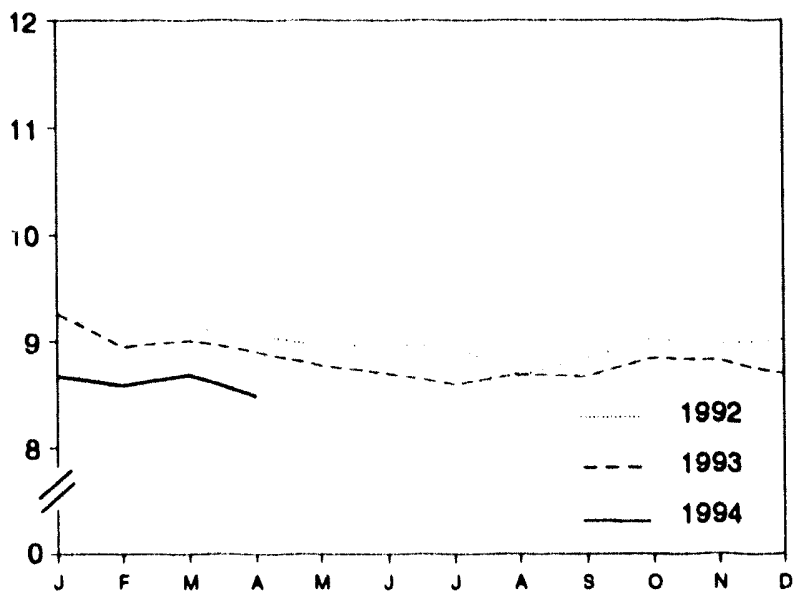

Nole: Because vertical scales difter, graphs should not be compared. Sources: Tables 3.1a, 3.1b, and 3.2a. 
Figure 3.1 Petroleum Overview (Continued) (Million Barrels per Day, Except as Noted)

Product Supplied, 1973-1993

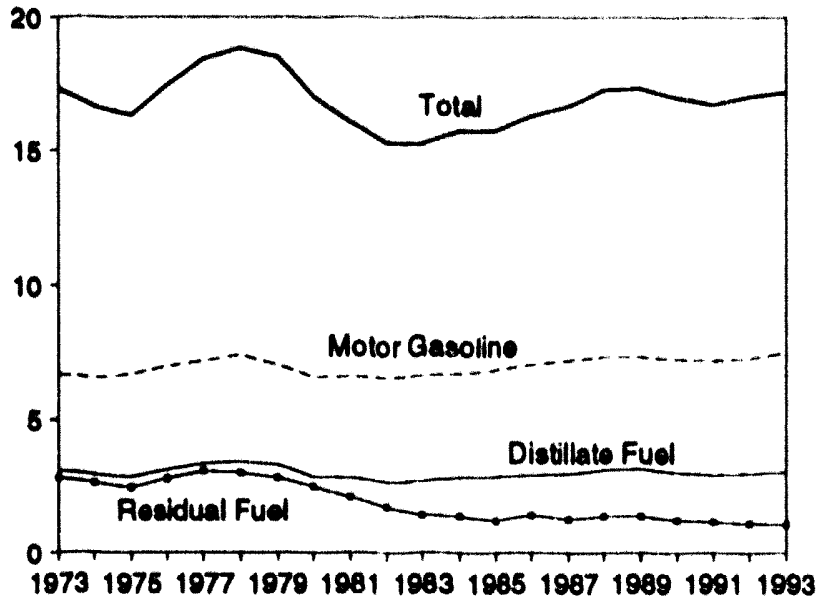

Total Product Supplied, Monthly

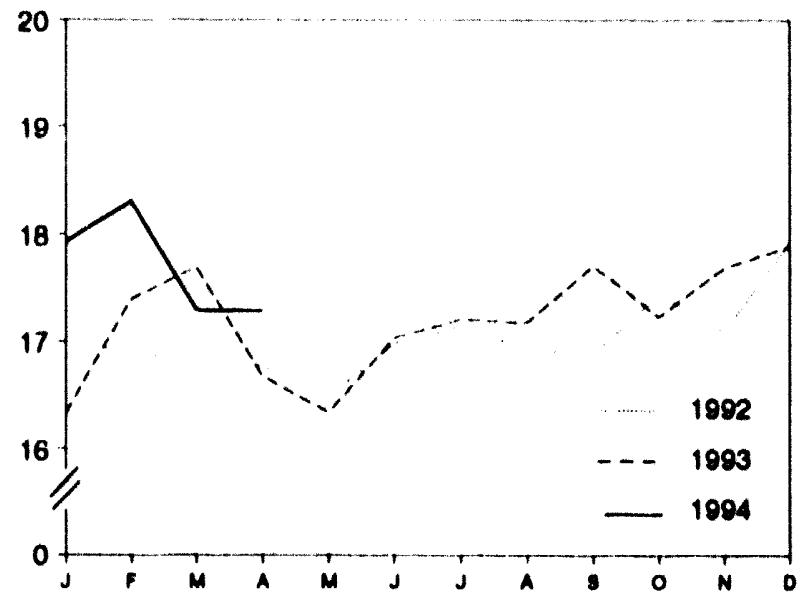

Imports from Selected Countries, March 1994

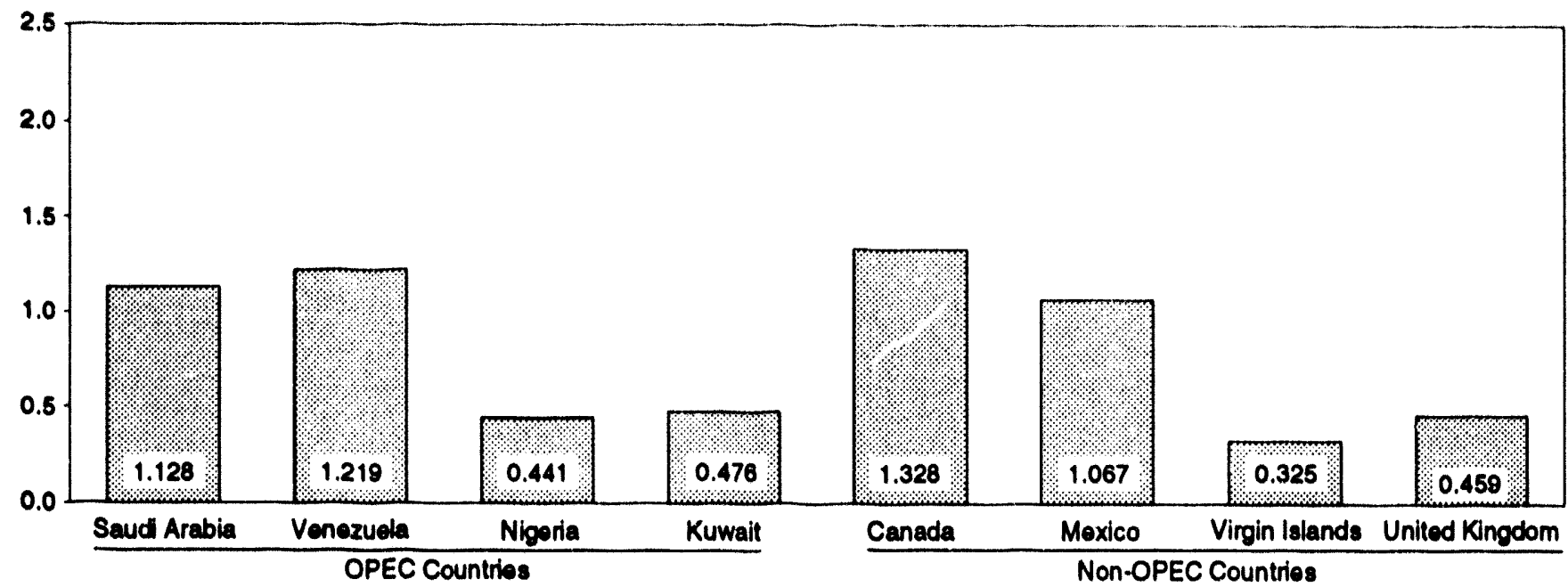

Stocks, End of Year, 1973-1993

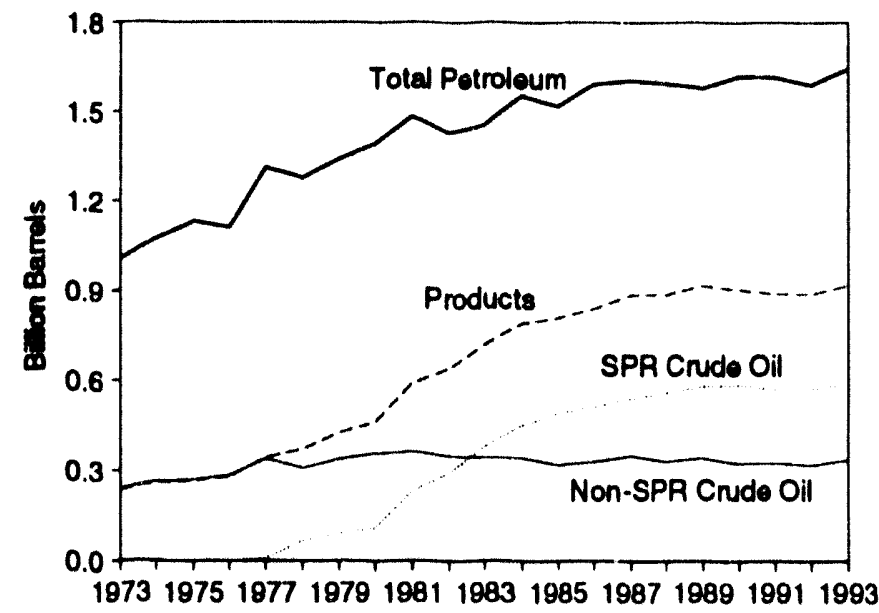

Notes: OPEC = Organizatlon of Potroleum Exponting Countries. - SPR - Sirategic Pelroleum Reserve. - Because vertical scales differ, oraphs chould not be compared.
Total Petroleum Stocks, End of Month

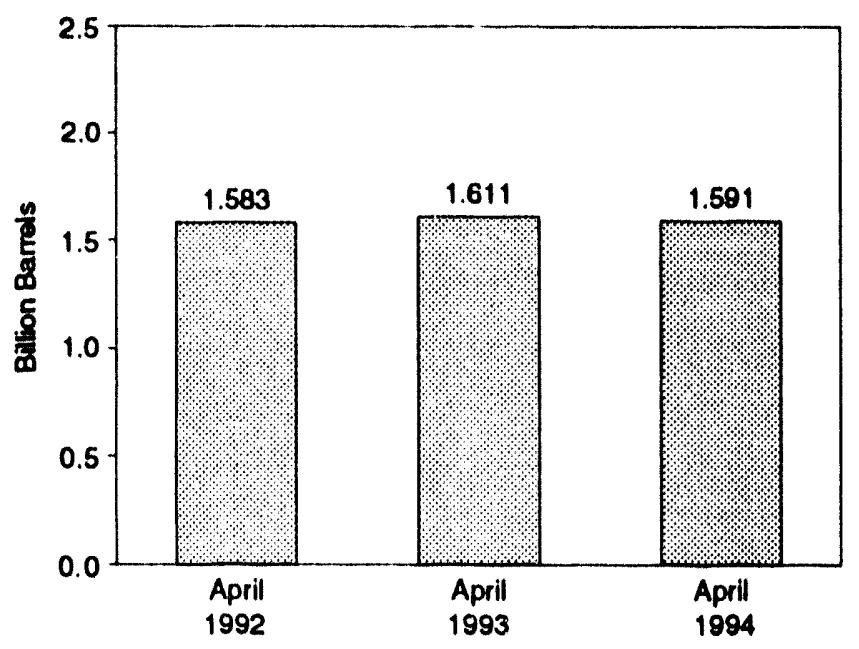

Sources: Tables 3.1a, 3.2b, 3.3a, 3.3b, 3.3d-3.3h, 3.4. 3.5, and 3.6. 
Table 3.2a Crude Oll Supply and Disposition: Supply

\begin{tabular}{|c|c|c|c|c|c|c|c|}
\hline & \multicolumn{7}{|c|}{ Supply } \\
\hline & Dotal & Alasken & Totel & spa* & Other & $\begin{array}{c}\text { Unesoounted- } \\
\text { for Crude } \\
\text { oll }\end{array}$ & $\begin{array}{l}\text { Crude oll } \\
\text { Uned } \\
\text { Oirnotye }\end{array}$ \\
\hline & \multicolumn{7}{|c|}{ Thoueand Barrels per Day } \\
\hline 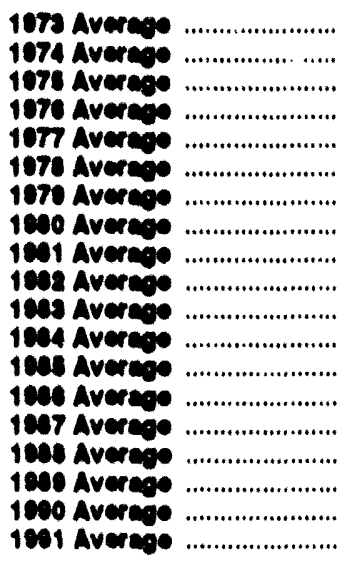 & $\begin{array}{l}0,200 \\
0,774 \\
0,370 \\
0,122 \\
0,240 \\
0,707 \\
0,602 \\
0,607 \\
0,072 \\
0,040 \\
0,060 \\
0,070 \\
0,071 \\
0,000 \\
0,340 \\
0,140 \\
7,013 \\
7,348 \\
7,417\end{array}$ & $\begin{array}{l}100 \\
109 \\
101 \\
179 \\
404 \\
1,200 \\
1,001 \\
1,017 \\
1,000 \\
1,000 \\
1,714 \\
1,720 \\
1,028 \\
1,007 \\
1,012 \\
2,017 \\
1,074 \\
1,773 \\
1,700\end{array}$ & 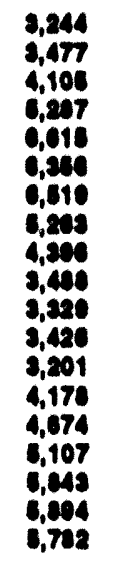 & $\begin{array}{r}- \\
- \\
\overline{81} \\
0101 \\
07 \\
41 \\
280 \\
106 \\
224 \\
197 \\
110 \\
40 \\
73 \\
61 \\
60 \\
27 \\
0\end{array}$ & 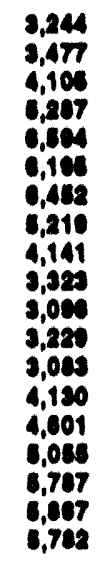 & 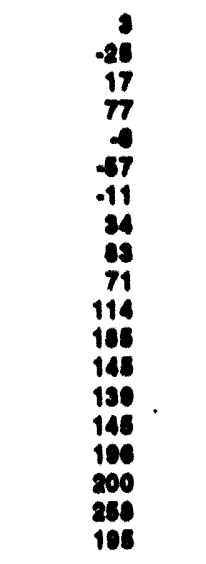 & $\begin{array}{r}.10 \\
.16 \\
.17 \\
0.10 \\
.14 \\
0.18 \\
0.14 \\
0.14 \\
+10 \\
= \\
= \\
= \\
= \\
= \\
=\end{array}$ \\
\hline 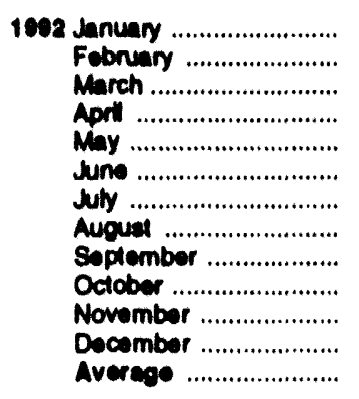 & $\begin{array}{l}7,361 \\
7,389 \\
7,349 \\
7,293 \\
7,160 \\
7,167 \\
7,131 \\
6,022 \\
7,030 \\
7.128 \\
7,024 \\
7.103 \\
7,171\end{array}$ & $\begin{array}{l}1,789 \\
1,800 \\
1,785 \\
1,741 \\
1,682 \\
1,703 \\
1,655 \\
1,636 \\
1,700 \\
1,606 \\
1,674 \\
1.706 \\
1,714\end{array}$ & $\begin{array}{l}5,056 \\
5,079 \\
5,321 \\
6,127 \\
6,000 \\
6,171 \\
6,796 \\
6,457 \\
6,218 \\
6,606 \\
6,121 \\
5,937 \\
6,043\end{array}$ & $\begin{array}{r}0 \\
0 \\
0 \\
0 \\
0 \\
34 \\
0 \\
16 \\
16 \\
49 \\
0 \\
0 \\
10\end{array}$ & $\begin{array}{l}5,066 \\
5,079 \\
5,321 \\
6,127 \\
6,060 \\
6,133 \\
6,706 \\
6,430 \\
6,202 \\
6,647 \\
6,121 \\
5,937 \\
6,073\end{array}$ & $\begin{array}{r}290 \\
229 \\
287 \\
180 \\
421 \\
259 \\
332 \\
65 \\
385 \\
290 \\
208 \\
61 \\
280\end{array}$ & $\begin{array}{l}= \\
= \\
= \\
= \\
= \\
=\end{array}$ \\
\hline 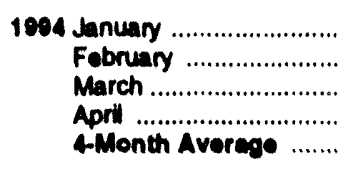 & $\begin{array}{r}6,777 \\
6,745 \\
\text { PE } 6,719 \\
\text { PE } 6,607 \\
\text { PE } 6,712\end{array}$ & $\begin{array}{l}1,658 \\
1,594 \\
\text { PE } 1,581 \\
P E \\
P E \\
1,497 \\
1,003\end{array}$ & $\begin{array}{r}\mathbf{5 , 9 8 1} \\
\mathbf{6 , 3 1 3} \\
\mathbf{R}_{\mathbf{6}, 377} \\
7,147 \\
\mathbf{6 , 4 4 7}\end{array}$ & $\begin{array}{r}0 \\
0 \\
0 \\
99 \\
67 \\
6\end{array}$ & $\begin{array}{r}5,961 \\
6,313 \\
8,278 \\
8,278 \\
7,080 \\
6,408\end{array}$ & $\begin{array}{r}651 \\
37 \\
272 \\
233 \\
330\end{array}$ & $\begin{array}{l}\bar{z} \\
\bar{z}\end{array}$ \\
\hline $\begin{array}{l}1003 \text { 4. Month Averege ....... } \\
1002 \text { 4-Month Averege ........ }\end{array}$ & $\begin{array}{r}E_{0,960} \\
7,347\end{array}$ & $\begin{array}{r}1,027 \\
1,701\end{array}$ & $\begin{array}{l}6,410 \\
6,626\end{array}$ & 30 & $\begin{array}{l}6,393 \\
8,020\end{array}$ & $\begin{array}{l}243 \\
250\end{array}$ & $\overline{-}$ \\
\hline
\end{tabular}

- Siralegic Potroloum Roserve.

- A balancing llem.

- Beginning in January 1983, crude oll used directly as fuel to shown as product supplied.

See Note 6 at end of section.

PExPreliminary ostimate. RePovbed dala. - eNol applicable. E=Estimale.

Noles: - Crude oll includes base condensale. - Geographic coverage b the 50 States and the District of Columbla. - Totals may not equal sum of components due to independert rounding.

Sources: - 1973.1080: Energy Information Adminiatration (ELA). Polrobum Supply Monthly, February 1993, Tablo S2. - 1001 forward: EM, Potroloum Supply Monthly, May 1994, Tabio S2. 


\begin{tabular}{|c|c|c|c|c|c|c|c|c|c|}
\hline & \multicolumn{6}{|c|}{ Clepodtion } & \multicolumn{3}{|c|}{ Ending stooke } \\
\hline & \multirow{2}{*}{$\begin{array}{l}\text { Crude } \\
\text { Loeces }\end{array}$} & \multicolumn{2}{|c|}{ Steok Chaneob } & \multirow{2}{*}{$\begin{array}{l}\text { Rofinory } \\
\text { Inpute }\end{array}$} & \multirow[b]{2}{*}{ Exports } & \multirow{2}{*}{$\begin{array}{l}\text { Produet } \\
\text { supplledd }\end{array}$} & \multirow[b]{2}{*}{ Total } & \multirow[b]{2}{*}{ SPAC } & \multirow{2}{*}{$\begin{array}{l}\text { Othor } \\
\text { Primary }\end{array}$} \\
\hline & & EPR० & Other & & & & & & \\
\hline & \multicolumn{6}{|c|}{ Thousand Berrels per Day } & \multicolumn{3}{|c|}{ Mullion Barrels } \\
\hline 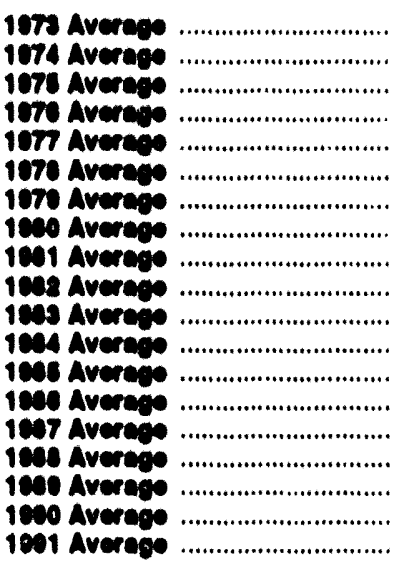 & $\begin{array}{r}13 \\
13 \\
18 \\
14 \\
10 \\
10 \\
10 \\
14 \\
6 \\
8 \\
2 \\
2 \\
1 \\
(0) \\
(0) \\
(0) \\
(0) \\
(0) \\
(0)\end{array}$ & $\begin{array}{r}- \\
- \\
- \\
20 \\
108 \\
67 \\
46 \\
290 \\
174 \\
244 \\
108 \\
117 \\
60 \\
10 \\
68 \\
60 \\
10 \\
47\end{array}$ & 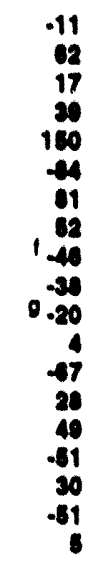 & $\begin{array}{l}12,431 \\
12,133 \\
12,442 \\
13,410 \\
14,002 \\
14,700 \\
14,040 \\
13,491 \\
12,470 \\
11,774 \\
11,098 \\
12,044 \\
12,002 \\
12,710 \\
12,094 \\
13,240 \\
13,401 \\
13,409 \\
13,301\end{array}$ & $\begin{array}{r}2 \\
3 \\
1 \\
50 \\
180 \\
238 \\
207 \\
228 \\
280 \\
104 \\
101 \\
204 \\
184 \\
181 \\
185 \\
142 \\
100 \\
110\end{array}$ & $\begin{array}{l}- \\
- \\
\overline{-} \\
- \\
- \\
- \\
- \\
- \\
- \\
10 \\
84 \\
60 \\
40 \\
34 \\
40 \\
20 \\
24 \\
10\end{array}$ & $\begin{array}{r}242 \\
208 \\
271 \\
208 \\
348 \\
370 \\
430 \\
1400 \\
804 \\
0444 \\
723 \\
700 \\
014 \\
043 \\
000 \\
090 \\
021 \\
008 \\
003\end{array}$ & $\begin{array}{c}- \\
- \\
- \\
- \\
7 \\
67 \\
01 \\
108 \\
230 \\
204 \\
370 \\
481 \\
403 \\
612 \\
841 \\
500 \\
800 \\
600 \\
880\end{array}$ & 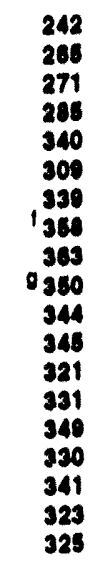 \\
\hline 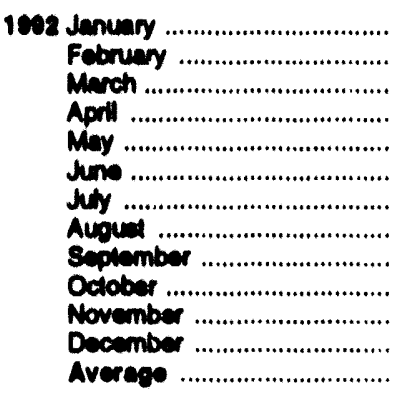 & $\begin{array}{l}0 \\
(a) \\
(0) \\
0 \\
0 \\
(0) \\
0 \\
(8) \\
0 \\
(0) \\
(a) \\
(8) \\
(0)\end{array}$ & $\begin{array}{c}(8) \\
0 \\
(0) \\
0 \\
(8) \\
34 \\
(8) \\
20 \\
43 \\
69 \\
15 \\
22 \\
17\end{array}$ & $\begin{array}{r}540 \\
171 \\
-260 \\
315 \\
.146 \\
.615 \\
244 \\
.144 \\
.204 \\
342 \\
.243 \\
.234 \\
.10\end{array}$ & $\begin{array}{l}12,023 \\
12,486 \\
13,083 \\
13,260 \\
13,679 \\
14,050 \\
13,053 \\
13,426 \\
13,714 \\
13,584 \\
13,547 \\
13,194 \\
13,411\end{array}$ & $\begin{array}{r}118 \\
22 \\
105 \\
23 \\
108 \\
107 \\
53 \\
133 \\
68 \\
106 \\
111 \\
107 \\
80\end{array}$ & $\begin{array}{r}28 \\
17 \\
18 \\
11 \\
10 \\
12 \\
9 \\
8 \\
11 \\
10 \\
10 \\
12 \\
13\end{array}$ & $\begin{array}{l}910 \\
915 \\
907 \\
917 \\
912 \\
895 \\
002 \\
898 \\
893 \\
806 \\
899 \\
603 \\
193\end{array}$ & $\begin{array}{l}560 \\
560 \\
569 \\
560 \\
569 \\
570 \\
570 \\
570 \\
571 \\
574 \\
574 \\
575 \\
578\end{array}$ & $\begin{array}{l}341 \\
346 \\
330 \\
348 \\
344 \\
325 \\
333 \\
328 \\
322 \\
333 \\
325 \\
318 \\
318\end{array}$ \\
\hline 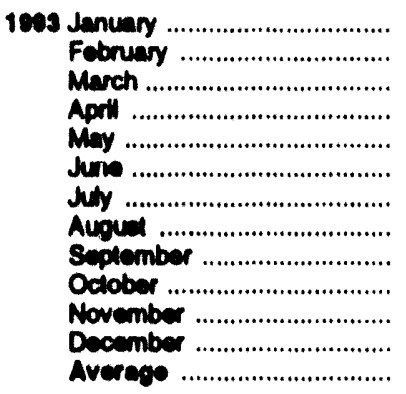 & $\begin{array}{c}(s) \\
(s) \\
0 \\
(s) \\
0 \\
0 \\
0 \\
0 \\
(0) \\
0 \\
0 \\
0 \\
(0)\end{array}$ & $\begin{array}{r}19 \\
18 \\
58 \\
138 \\
13 \\
21 \\
19 \\
24 \\
52 \\
10 \\
18 \\
0 \\
34\end{array}$ & $\begin{array}{r}245 \\
202 \\
188 \\
401 \\
120 \\
.37 \\
22 \\
.548 \\
-491 \\
314 \\
233 \\
.67 \\
47\end{array}$ & $\begin{array}{l}12,980 \\
12,923 \\
13,240 \\
13,512 \\
13,701 \\
14,125 \\
14,114 \\
13,839 \\
13,845 \\
13,733 \\
13,689 \\
13,571 \\
13,610\end{array}$ & $\begin{array}{r}129 \\
168 \\
139 \\
73 \\
112 \\
150 \\
62 \\
55 \\
107 \\
62 \\
67 \\
63 \\
00\end{array}$ & $\begin{array}{r}10 \\
10 \\
11 \\
9 \\
10 \\
8 \\
9 \\
8 \\
9 \\
12 \\
13 \\
21 \\
11\end{array}$ & $\begin{array}{l}901 \\
907 \\
915 \\
931 \\
235 \\
935 \\
936 \\
920 \\
906 \\
917 \\
924 \\
922 \\
922\end{array}$ & $\begin{array}{l}575 \\
576 \\
578 \\
582 \\
582 \\
583 \\
583 \\
584 \\
586 \\
586 \\
587 \\
587 \\
687\end{array}$ & $\begin{array}{l}326 \\
331 \\
337 \\
349 \\
353 \\
352 \\
352 \\
336 \\
321 \\
330 \\
337 \\
335 \\
335\end{array}$ \\
\hline 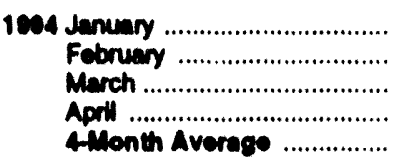 & $\begin{array}{l}0 \\
0 \\
0 \\
0 \\
0\end{array}$ & $\begin{array}{r}4 \\
(3) \\
E_{09} \\
67 \\
43\end{array}$ & $\begin{array}{r}.19 \\
-164 \\
A_{241} \\
E_{58} \\
E_{33}\end{array}$ & $\begin{array}{r}13,286 \\
13,132 \\
12,978 \\
E 13,848 \\
113,311\end{array}$ & $\begin{array}{r}110 \\
116 \\
\text { A }_{40} \\
101 \\
E_{91}\end{array}$ & $\begin{array}{r}10 \\
12 \\
10 \\
E_{11} \\
E_{11}\end{array}$ & $\begin{array}{r}922 \\
917 \\
\text { A } 928 \\
\text { E } 930 \\
E_{930}\end{array}$ & $\begin{array}{r}587 \\
587 \\
\text { A } 500 \\
E 581 \\
E 501\end{array}$ & $\begin{array}{r}336 \\
\mathbf{3 3 0} \\
A_{338} \\
E_{330} \\
E_{330}\end{array}$ \\
\hline $\begin{array}{l}10 e s \text { Henth Averege ................ } \\
\text { ive \&menth Avernes ............... }\end{array}$ & (c) & (s) & $\begin{array}{l}280 \\
193\end{array}$ & $\begin{array}{l}13,160 \\
12,043\end{array}$ & $\begin{array}{r}126 \\
68\end{array}$ & $\begin{array}{l}10 \\
18\end{array}$ & $\begin{array}{l}931 \\
917\end{array}$ & $\begin{array}{l}582 \\
580\end{array}$ & $\begin{array}{l}349 \\
340\end{array}$ \\
\hline
\end{tabular}

- Stocke are lotale as of end of period.

- A negative number indicates a decrease in atocks and a posilve number indicetes an increase.

- Sirategic Petroloum Recervo.

- Begining in January 1983, crude oll used directly as fuel is shown as product supplied.

- Sec Note 6 af end of section.

1 Siocks of Alaken crude oll in transh are included from January 1981 lonward. Sev Note 5 at end of section.
Sea Note 4 at end of section.

R-Rovised data. - =Not applicable. EmEstimate. (s)=Less than +500 barrele per day and greater than -500 barreb per day.

Notes: - Crude oil includes lease condensate. - Geographic coverage bs the 50 States and the District of Columbia. - Totals may not equal sum of components due to independent rounding.

Sources: - 1973-1080: Energy Information Administration (ELA), Petroleum Supply Monthly, February 1993, Table S2. • 1081 forward: ELA Petroloum Supply Monthly, May 1994, Table S2. 
Table 3.3a Petroleum Imports: Algeria, Iraq, Kuwait, and Libya (Thousand Barrels per Day)

\begin{tabular}{|c|c|c|c|c|c|c|c|c|}
\hline & \multicolumn{8}{|c|}{ Arab OPECa } \\
\hline & \multicolumn{2}{|c|}{ Ngeria } & \multicolumn{2}{|c|}{ Ireq } & \multicolumn{2}{|c|}{ Kuwailb } & \multicolumn{2}{|c|}{ Libye } \\
\hline 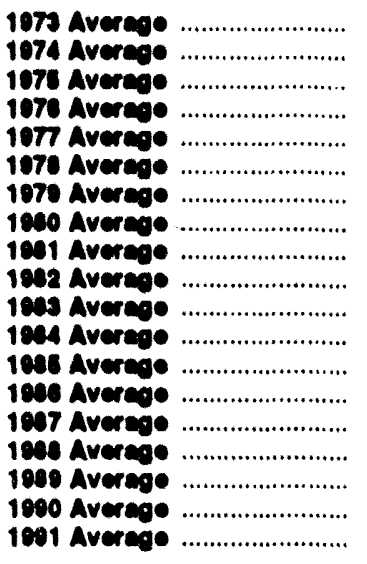 & $\begin{array}{l}130 \\
100 \\
202 \\
492 \\
860 \\
440 \\
630 \\
408 \\
311 \\
170 \\
240 \\
323 \\
107 \\
271 \\
295 \\
300 \\
260 \\
200 \\
263\end{array}$ & $\begin{array}{l}120 \\
180 \\
264 \\
100 \\
6144 \\
634 \\
600 \\
466 \\
261 \\
80 \\
170 \\
194 \\
84 \\
78 \\
118 \\
60 \\
60 \\
63 \\
44\end{array}$ & $\begin{array}{r}1 \\
0 \\
2 \\
20 \\
74 \\
62 \\
61 \\
20 \\
(1) \\
3 \\
10 \\
12 \\
40 \\
01 \\
03 \\
340 \\
140 \\
510 \\
0\end{array}$ & $\begin{array}{r}1 \\
0 \\
2 \\
20 \\
74 \\
62 \\
60 \\
20 \\
0 \\
3 \\
10 \\
12 \\
40 \\
61 \\
02 \\
343 \\
141 \\
514 \\
0\end{array}$ & $\begin{array}{r}47 \\
5 \\
16 \\
8 \\
40 \\
6 \\
27 \\
0 \\
5 \\
14 \\
36 \\
21 \\
68 \\
04 \\
157 \\
10 \\
8 \\
6\end{array}$ & $\begin{array}{r}42 \\
5 \\
1 \\
12 \\
5 \\
5 \\
27 \\
0 \\
2 \\
7 \\
24 \\
20 \\
70 \\
00 \\
155 \\
70 \\
8\end{array}$ & $\begin{array}{r}144 \\
140 \\
232 \\
463 \\
723 \\
954 \\
050 \\
584 \\
319 \\
26 \\
0 \\
1 \\
1 \\
0 \\
0 \\
0 \\
0 \\
0 \\
0\end{array}$ & $\begin{array}{r}133 \\
4 \\
293 \\
444 \\
704 \\
290 \\
242 \\
440 \\
317 \\
23 \\
0 \\
0 \\
0 \\
0 \\
0 \\
0 \\
0 \\
0 \\
0\end{array}$ \\
\hline 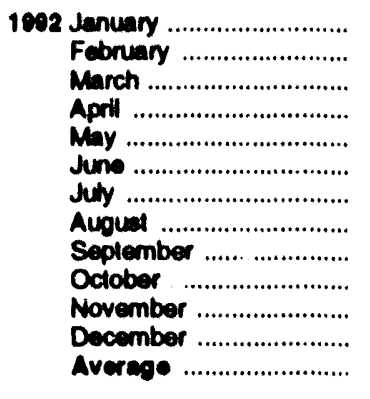 & $\begin{array}{l}206 \\
218 \\
215 \\
182 \\
202 \\
144 \\
179 \\
261 \\
184 \\
186 \\
171 \\
203 \\
106\end{array}$ & $\begin{array}{r}37 \\
57 \\
37 \\
19 \\
7 \\
12 \\
37 \\
45 \\
19 \\
8 \\
0 \\
9 \\
24\end{array}$ & $\begin{array}{l}0 \\
0 \\
0 \\
0 \\
0 \\
0 \\
0 \\
0 \\
0 \\
0 \\
0 \\
0 \\
0\end{array}$ & $\begin{array}{l}0 \\
0 \\
0 \\
0 \\
0 \\
0 \\
0 \\
0 \\
0 \\
0 \\
0 \\
0 \\
0\end{array}$ & $\begin{array}{r}0 \\
0 \\
0 \\
0 \\
0 \\
0 \\
58 \\
66 \\
70 \\
137 \\
117 \\
165 \\
51\end{array}$ & $\begin{array}{r}0 \\
0 \\
0 \\
0 \\
0 \\
0 \\
23 \\
33 \\
33 \\
109 \\
117 \\
149 \\
30\end{array}$ & $\begin{array}{l}0 \\
0 \\
0 \\
0 \\
0 \\
0 \\
0 \\
0 \\
0 \\
0 \\
0 \\
0 \\
0\end{array}$ & $\begin{array}{l}0 \\
0 \\
0 \\
0 \\
0 \\
0 \\
0 \\
0 \\
0 \\
0 \\
0 \\
0 \\
0\end{array}$ \\
\hline 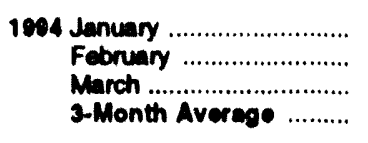 & $\begin{array}{l}233 \\
226 \\
278 \\
246\end{array}$ & $\begin{array}{l}36 \\
20 \\
22 \\
26\end{array}$ & $\begin{array}{l}0 \\
0 \\
0 \\
0\end{array}$ & $\begin{array}{l}0 \\
0 \\
0 \\
0\end{array}$ & $\begin{array}{l}300 \\
423 \\
476 \\
402\end{array}$ & $\begin{array}{l}300 \\
423 \\
476 \\
402\end{array}$ & $\begin{array}{l}0 \\
0 \\
0 \\
0\end{array}$ & $\begin{array}{l}0 \\
0 \\
0 \\
0\end{array}$ \\
\hline $\begin{array}{l}1903 \text { 3-Month Averege ......... } \\
1002 \text { 3-Month Average ......... }\end{array}$ & $\begin{array}{l}108 \\
213\end{array}$ & $\begin{array}{l}12 \\
43\end{array}$ & $\begin{array}{l}0 \\
0\end{array}$ & $\begin{array}{l}0 \\
0\end{array}$ & $\begin{array}{r}237 \\
0\end{array}$ & $\begin{array}{r}210 \\
0\end{array}$ & $\begin{array}{l}0 \\
0\end{array}$ & $\begin{array}{l}0 \\
0\end{array}$ \\
\hline
\end{tabular}

- Excludes petrobum imponted into the Uniled Stales indirectly from members of the Organization of Petrobum Exponting Countries (OPEC) primarly trom Caribbean and West European areas, as petroloum products that were rethed from crude oll produced by OPEC.

Imports trom the Neutral Zone between Kuwalt and Saudi Arabia are included in Saudl Arabla.

(s) =Leses than 500 barrels per day.
Noles: - Beginning in October 1977. Strategic Petroleum Reserve mponts are included. - Petroleum is imported into the 50 States and the District of Columbia.

Sources: - 1073-1080: Energy Iniormation Administration (ELA), Potroloum Supply Monthy, Fobruary 1993, Table S3. - 1081 forward: ELA. Petroleum Supply Monthly, May 1094, Table S3. 
Table 3.3b Petroleum Imports: Qatar, Saudi Arabia, U.A.E., and Total Arab OPEC (Thousand Barrels per Day)

\begin{tabular}{|c|c|c|c|c|c|c|c|c|}
\hline & \multicolumn{6}{|c|}{ Arab OpEca } & \multirow{2}{*}{\multicolumn{2}{|c|}{$\begin{array}{c}\text { Tolal } \\
\text { Areb OPECa }\end{array}$}} \\
\hline & \multicolumn{2}{|c|}{ Oatar } & \multicolumn{2}{|c|}{ Baudl Arablat } & \multicolumn{2}{|c|}{ United Arab Emirates } & & \\
\hline & Total & Crude Oll & Total & Crude Oll & Totil & Crude OII & Total & Crude OII \\
\hline 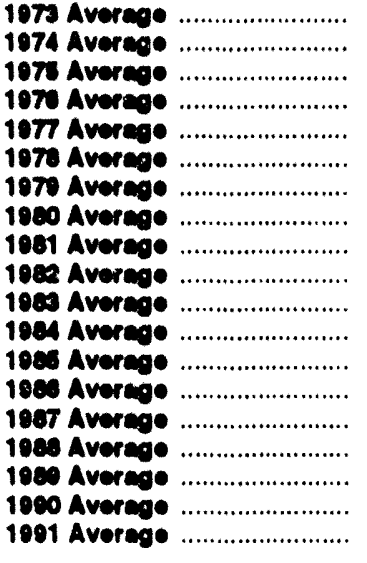 & $\begin{array}{c}7 \\
17 \\
18 \\
24 \\
67 \\
64 \\
31 \\
22 \\
7 \\
7 \\
(6) \\
5 \\
(8) \\
13 \\
0 \\
0 \\
2 \\
4 \\
0\end{array}$ & $\begin{array}{r}7 \\
17 \\
18 \\
24 \\
67 \\
44 \\
31 \\
22 \\
7 \\
7 \\
0 \\
4 \\
0 \\
12 \\
0 \\
0 \\
2 \\
4 \\
0\end{array}$ & $\begin{array}{r}480 \\
461 \\
718 \\
1,230 \\
1,380 \\
1,144 \\
1,350 \\
1,261 \\
1,120 \\
562 \\
337 \\
325 \\
160 \\
688 \\
751 \\
1,073 \\
1,224 \\
1,330 \\
1,802\end{array}$ & $\begin{array}{r}462 \\
430 \\
701 \\
1,222 \\
1,373 \\
1,142 \\
1,347 \\
1,250 \\
1,112 \\
530 \\
321 \\
300 \\
132 \\
618 \\
642 \\
011 \\
1,116 \\
1,105 \\
1,703\end{array}$ & $\begin{array}{r}71 \\
74 \\
117 \\
284 \\
338 \\
388 \\
281 \\
172 \\
81 \\
92 \\
30 \\
117 \\
48 \\
44 \\
81 \\
20 \\
28 \\
17 \\
3\end{array}$ & $\begin{array}{r}71 \\
60 \\
117 \\
284 \\
339 \\
385 \\
281 \\
172 \\
77 \\
81 \\
18 \\
90 \\
35 \\
38 \\
58 \\
23 \\
21 \\
0 \\
2\end{array}$ & $\begin{array}{r}918 \\
782 \\
1,383 \\
2,424 \\
3,184 \\
2,063 \\
3,088 \\
2,851 \\
1,049 \\
884 \\
832 \\
810 \\
472 \\
1,162 \\
1,274 \\
1,030 \\
2,130 \\
2,244 \\
2,084\end{array}$ & $\begin{array}{r}830 \\
713 \\
1,330 \\
2,376 \\
3,130 \\
2,030 \\
3,002 \\
2,503 \\
1,774 \\
730 \\
639 \\
034 \\
300 \\
884 \\
096 \\
1,415 \\
1,704 \\
1,864 \\
1,754\end{array}$ \\
\hline 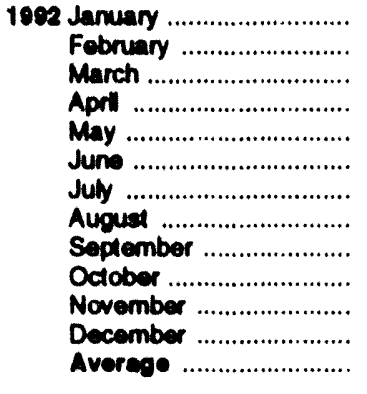 & $\begin{array}{l}0 \\
0 \\
0 \\
0 \\
0 \\
0 \\
8 \\
0 \\
0 \\
0 \\
0 \\
0 \\
1\end{array}$ & $\begin{array}{l}0 \\
0 \\
0 \\
0 \\
0 \\
0 \\
0 \\
0 \\
0 \\
0 \\
0 \\
0 \\
0\end{array}$ & $\begin{array}{l}2,017 \\
1,776 \\
1,707 \\
1,734 \\
1,764 \\
1,744 \\
1,713 \\
1,594 \\
1,593 \\
1,593 \\
1,600 \\
1,793 \\
1,720\end{array}$ & $\begin{array}{l}1,900 \\
1,687 \\
1,568 \\
1,524 \\
1,584 \\
1,610 \\
1,599 \\
1,473 \\
1,477 \\
1,482 \\
1,540 \\
1,725 \\
1,507\end{array}$ & $\begin{array}{r}18 \\
0 \\
0 \\
0 \\
0 \\
0 \\
0 \\
7 \\
0 \\
4 \\
17 \\
28 \\
0\end{array}$ & $\begin{array}{l}0 \\
0 \\
0 \\
0 \\
0 \\
0 \\
0 \\
0 \\
0 \\
0 \\
0 \\
0 \\
0\end{array}$ & $\begin{array}{l}2,241 \\
1,986 \\
1,922 \\
1,916 \\
1,966 \\
1,888 \\
1,958 \\
1,929 \\
1,847 \\
1,920 \\
1,913 \\
2,188 \\
1,974\end{array}$ & $\begin{array}{l}1,937 \\
1,746 \\
1,605 \\
1,543 \\
1,591 \\
1,621 \\
1,659 \\
1,551 \\
1,529 \\
1,590 \\
1,657 \\
1,882 \\
1,680\end{array}$ \\
\hline 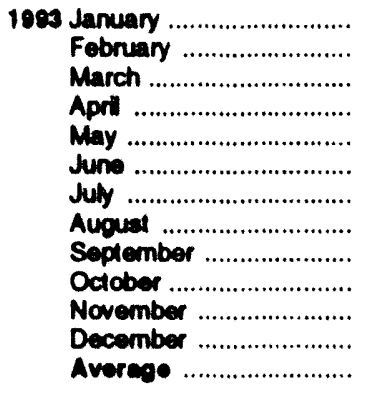 & $\begin{array}{l}0 \\
0 \\
6 \\
0 \\
0 \\
0 \\
0 \\
0 \\
0 \\
0 \\
0 \\
0 \\
1\end{array}$ & $\begin{array}{l}0 \\
0 \\
0 \\
0 \\
0 \\
0 \\
0 \\
0 \\
0 \\
0 \\
0 \\
0 \\
0\end{array}$ & $\begin{array}{l}1,687 \\
1,626 \\
1,479 \\
1,606 \\
1,524 \\
1,523 \\
1,270 \\
1,151 \\
1,329 \\
1,115 \\
1,281 \\
1,330 \\
1,400\end{array}$ & $\begin{array}{l}1,571 \\
1,480 \\
1,349 \\
1,478 \\
1,361 \\
1,396 \\
1,171 \\
1,036 \\
1,181 \\
969 \\
1,152 \\
1,205 \\
1,277\end{array}$ & $\begin{array}{r}0 \\
0 \\
0 \\
17 \\
59 \\
68 \\
19 \\
0 \\
0 \\
0 \\
1 \\
0 \\
14\end{array}$ & $\begin{array}{r}0 \\
0 \\
0 \\
17 \\
59 \\
68 \\
0 \\
0 \\
0 \\
0 \\
0 \\
0 \\
12\end{array}$ & $\begin{array}{l}1,984 \\
2,133 \\
1,987 \\
2,161 \\
2,034 \\
1,983 \\
1,904 \\
1,859 \\
1,966 \\
1,961 \\
1,984 \\
1,983 \\
1,004\end{array}$ & $\begin{array}{l}1,728 \\
1,709 \\
1,655 \\
1,783 \\
1,646 \\
1,729 \\
1,538 \\
1,515 \\
1,612 \\
1,574 \\
1,668 \\
1,713 \\
1,685\end{array}$ \\
\hline 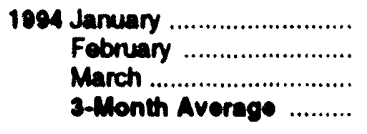 & $\begin{array}{l}0 \\
0 \\
0 \\
0\end{array}$ & $\begin{array}{l}0 \\
0 \\
0 \\
0\end{array}$ & $\begin{array}{l}1,320 \\
1,071 \\
1,128 \\
1,176\end{array}$ & $\begin{array}{l}1,175 \\
1,023 \\
1,055 \\
1,086\end{array}$ & $\begin{array}{l}0 \\
0 \\
0 \\
0\end{array}$ & $\begin{array}{l}0 \\
0 \\
0 \\
0\end{array}$ & $\begin{array}{l}1,863 \\
1,710 \\
1,883 \\
1,826\end{array}$ & $\begin{array}{l}1,520 \\
1,467 \\
1,553 \\
1,818\end{array}$ \\
\hline $\begin{array}{l}1093 \text { 3-Month Average ......... } \\
1002 \text { 3-Month Average ......... }\end{array}$ & $\begin{array}{l}2 \\
0\end{array}$ & $\begin{array}{l}\mathbf{0} \\
\mathbf{0}\end{array}$ & $\begin{array}{l}1,597 \\
1,836\end{array}$ & $\begin{array}{l}1,466 \\
1,710\end{array}$ & $\begin{array}{l}0 \\
6\end{array}$ & $\begin{array}{l}\mathbf{0} \\
\mathbf{0}\end{array}$ & $\begin{array}{l}2,032 \\
2,034\end{array}$ & $\begin{array}{l}1,007 \\
1,703\end{array}$ \\
\hline
\end{tabular}

- Excludes petroleum imported into the United States indirectly trom members of the Organization of Petroleum Exporting Countries (OPEC). primarily irom Caribbean and Weot European areas, as polroloum products that were rethed irom crude oll produced by OPEC.

importe from the Noutral Zone between Kuwait and Saudi Arabia are included in Saudi Arabia.

(8)= Lees than 500 barrels per day.
Noles: - Beginning in October 1977. Strateoic Petrobum Resenv imports are included. - Petrotoum is inported into the 50 States and the Diatrict of Columbia. - Totals may not equal sum of components duo to independent rounding.

Sources: - 1973-1980: Energy Intormation Adminbatration (EIA), Petroloum Supply Monthy, Februany 1993, Table S3. - 1091 forward: ElA, Petroloum Supply Monthly, May 1994, Table S3. 
Table 3.3c Petrolicum Imports: Ecuador, Gabon, Indonesia, and Iran (Thousand Barrels per Day)

\begin{tabular}{|c|c|c|c|c|c|c|c|c|}
\hline & \multicolumn{8}{|c|}{ Non-Arab OPECa } \\
\hline & \multicolumn{2}{|c|}{ Ecuadorb } & \multicolumn{2}{|c|}{ Gabon } & \multicolumn{2}{|c|}{ Indonesia } & \multicolumn{2}{|c|}{ Iran } \\
\hline & Total & Crude Oll & Total & Crude Oll & Total & Crude Oll & Total & Crude Oll \\
\hline 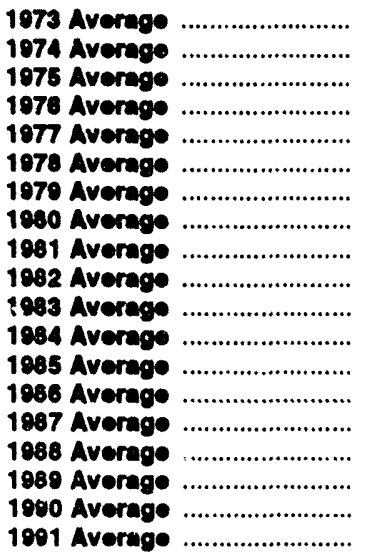 & $\begin{array}{l}48 \\
42 \\
57 \\
51 \\
57 \\
54 \\
42 \\
27 \\
48 \\
42 \\
61 \\
55 \\
67 \\
77 \\
20 \\
47 \\
89 \\
40 \\
63\end{array}$ & $\begin{array}{l}47 \\
42 \\
57 \\
51 \\
55 \\
38 \\
30 \\
17 \\
38 \\
32 \\
56 \\
47 \\
56 \\
64 \\
23 \\
33 \\
80 \\
38 \\
53\end{array}$ & $\begin{array}{l}0 \\
23 \\
27 \\
28 \\
42 \\
41 \\
42 \\
26 \\
35 \\
40 \\
50 \\
58 \\
52 \\
28 \\
35 \\
16 \\
50 \\
64 \\
24\end{array}$ & $\begin{array}{r}0 \\
23 \\
27 \\
26 \\
35 \\
38 \\
42 \\
25 \\
35 \\
40 \\
59 \\
57 \\
51 \\
25 \\
35 \\
15 \\
49 \\
64 \\
84\end{array}$ & $\begin{array}{l}213 \\
300 \\
300 \\
530 \\
541 \\
573 \\
420 \\
348 \\
368 \\
248 \\
338 \\
343 \\
314 \\
318 \\
285 \\
205 \\
183 \\
114 \\
111\end{array}$ & $\begin{array}{r}200 \\
284 \\
370 \\
537 \\
507 \\
633 \\
380 \\
314 \\
318 \\
226 \\
315 \\
304 \\
292 \\
297 \\
262 \\
186 \\
158 \\
98 \\
102\end{array}$ & $\begin{array}{r}223 \\
460 \\
280 \\
298 \\
535 \\
555 \\
304 \\
0 \\
0 \\
35 \\
48 \\
10 \\
27 \\
10 \\
98 \\
6 \\
(8) \\
0 \\
0 \\
32\end{array}$ & $\begin{array}{r}218 \\
463 \\
278 \\
208 \\
530 \\
554 \\
207 \\
8 \\
0 \\
36 \\
48 \\
10 \\
27 \\
10 \\
98 \\
\mathbf{9 8} \\
(8) \\
0 \\
0 \\
32\end{array}$ \\
\hline 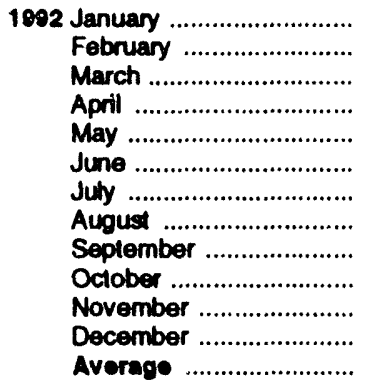 & $\begin{array}{r}56 \\
61 \\
26 \\
53 \\
51 \\
105 \\
111 \\
99 \\
97 \\
42 \\
53 \\
24 \\
65\end{array}$ & $\begin{array}{r}56 \\
48 \\
26 \\
46 \\
51 \\
101 \\
111 \\
93 \\
97 \\
36 \\
53 \\
24 \\
62\end{array}$ & $\begin{array}{r}91 \\
105 \\
25 \\
186 \\
135 \\
129 \\
143 \\
108 \\
165 \\
167 \\
114 \\
120 \\
124\end{array}$ & $\begin{array}{r}91 \\
105 \\
25 \\
186 \\
135 \\
129 \\
143 \\
108 \\
158 \\
167 \\
114 \\
120 \\
123\end{array}$ & $\begin{array}{r}125 \\
39 \\
85 \\
54 \\
155 \\
109 \\
65 \\
91 \\
57 \\
54 \\
36 \\
60 \\
78\end{array}$ & $\begin{array}{r}117 \\
39 \\
83 \\
49 \\
133 \\
102 \\
65 \\
85 \\
38 \\
43 \\
23 \\
60 \\
70\end{array}$ & $\begin{array}{l}0 \\
0 \\
0 \\
0 \\
0 \\
0 \\
0 \\
0 \\
0 \\
0 \\
0 \\
0 \\
0\end{array}$ & $\begin{array}{l}0 \\
0 \\
0 \\
0 \\
0 \\
0 \\
0 \\
0 \\
0 \\
0 \\
0 \\
0 \\
0\end{array}$ \\
\hline 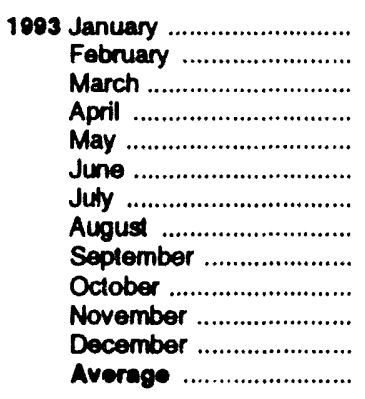 & $\begin{array}{l}\left(\begin{array}{l}b \\
b\end{array}\right) \\
\left(\begin{array}{l}b \\
b\end{array}\right) \\
\left(\begin{array}{l}b \\
b\end{array}\right) \\
\left(\begin{array}{l}b \\
b\end{array}\right) \\
\left(\begin{array}{l}b \\
b\end{array}\right)\end{array}$ & 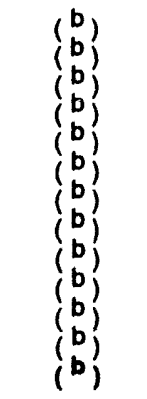 & $\begin{array}{r}90 \\
88 \\
126 \\
127 \\
169 \\
107 \\
168 \\
152 \\
211 \\
242 \\
143 \\
191 \\
152\end{array}$ & $\begin{array}{r}89 \\
88 \\
123 \\
127 \\
169 \\
107 \\
166 \\
152 \\
211 \\
242 \\
136 \\
191 \\
151\end{array}$ & $\begin{array}{r}37 \\
52 \\
67 \\
76 \\
82 \\
97 \\
55 \\
95 \\
51 \\
131 \\
74 \\
156 \\
81\end{array}$ & $\begin{array}{r}37 \\
51 \\
64 \\
76 \\
82 \\
67 \\
55 \\
80 \\
40 \\
82 \\
34 \\
114 \\
65\end{array}$ & $\begin{array}{l}0 \\
0 \\
0 \\
0 \\
0 \\
0 \\
0 \\
0 \\
0 \\
0 \\
0 \\
0 \\
0\end{array}$ & $\begin{array}{l}0 \\
0 \\
0 \\
0 \\
0 \\
0 \\
0 \\
0 \\
0 \\
0 \\
0 \\
0 \\
0\end{array}$ \\
\hline 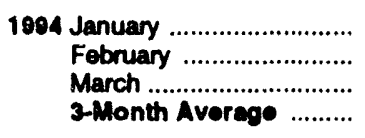 & $\left.\begin{array}{l}b \\
b \\
b \\
b \\
b\end{array}\right)$ & $\left(\begin{array}{l}b \\
b \\
b \\
b\end{array}\right)$ & $\begin{array}{r}144 \\
212 \\
91 \\
147\end{array}$ & $\begin{array}{r}144 \\
208 \\
91 \\
146\end{array}$ & $\begin{array}{l}140 \\
103 \\
112 \\
110\end{array}$ & $\begin{array}{l}81 \\
59 \\
50 \\
63\end{array}$ & $\begin{array}{l}0 \\
0 \\
0 \\
0\end{array}$ & $\begin{array}{l}0 \\
0 \\
0 \\
0\end{array}$ \\
\hline $\begin{array}{l}1903 \text { 3-Month Average ......... } \\
1902 \text { 3-Month Average ......... }\end{array}$ & $\left({ }^{b}\right)_{47}$ & $\left(\begin{array}{l}b \\
43\end{array}\right.$ & $\begin{array}{r}102 \\
73\end{array}$ & $\begin{array}{r}100 \\
73\end{array}$ & $\begin{array}{l}52 \\
84\end{array}$ & $\begin{array}{l}51 \\
81\end{array}$ & $\begin{array}{l}0 \\
0\end{array}$ & $\begin{array}{l}0 \\
0\end{array}$ \\
\hline
\end{tabular}

a Excludes petroleum imported into the United States indirectly frorn members of the Organization of Petroleum Exporting Countries (OPE(;). primarily from Caribbean and West European areas, as petroleum prodicts that were refined from crude oil produced by OPEC.

Ecuador withdrew from OPEC on December 31, 1992. As of January 1993, imports from Ecuador appear on Table 3.3 under "Non-OPEC."

c A smell amount of Iranian crude oil entered the United States in January 1988 from the Virgin Islands. The oil originated in Iran and was exported to the Virgin Islands prior to the signing of Executive Order 12613 on Odober
$29,1987$.

(s)=Less than 500 barrels per day.

Notes: - Beginning in October 1977, Strategic Petroteum Reserve imports are included. - Petroleum is imported into the 50 States and the District of Columbia.

Sources: - 1073-1080: Energy Information Administration (ElA), Petroleum Supply Monthly, February 1993, Table S3. • 1081 forwerd: EIA, Potroleum Supphy Monthly, May 1994, Table S3. 
Table 3.3d Petroleum Imports: Nigeria, Venezuela, Total Non-Arab OPEC, and Total OPEC

(Thousand Barrels per Day)

\begin{tabular}{|c|c|c|c|c|c|c|c|c|}
\hline & \multicolumn{4}{|c|}{ Non-Arab OPECa } & \multirow{2}{*}{\multicolumn{2}{|c|}{$\begin{array}{c}\text { Total } \\
\text { Non-Arab OPEca,b }\end{array}$}} & \multirow{2}{*}{\multicolumn{2}{|c|}{$\begin{array}{l}\text { Total } \\
\text { OPECa,b }\end{array}$}} \\
\hline & \multicolumn{2}{|c|}{ Nigeria } & \multicolumn{2}{|c|}{ Vonozuda } & & & & \\
\hline 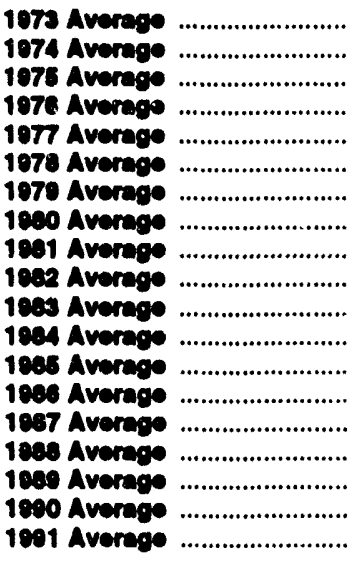 & $\begin{array}{r}460 \\
713 \\
762 \\
1,025 \\
1,149 \\
910 \\
1,080 \\
857 \\
620 \\
514 \\
302 \\
216 \\
293 \\
140 \\
536 \\
618 \\
815 \\
800 \\
703\end{array}$ & $\begin{array}{r}440 \\
697 \\
746 \\
1,014 \\
1,130 \\
910 \\
1,060 \\
841 \\
611 \\
510 \\
301 \\
207 \\
280 \\
437 \\
520 \\
607 \\
800 \\
784 \\
683\end{array}$ & $\begin{array}{r}1,135 \\
979 \\
702 \\
700 \\
690 \\
646 \\
690 \\
401 \\
406 \\
412 \\
422 \\
548 \\
605 \\
793 \\
604 \\
704 \\
673 \\
1,025 \\
1,035\end{array}$ & $\begin{array}{l}344 \\
319 \\
395 \\
241 \\
250 \\
181 \\
203 \\
156 \\
147 \\
155 \\
164 \\
253 \\
306 \\
416 \\
486 \\
430 \\
405 \\
666 \\
668\end{array}$ & $\begin{array}{l}2,078 \\
2,527 \\
2,210 \\
2,642 \\
3,008 \\
2,788 \\
2,570 \\
1,749 \\
1,476 \\
1,201 \\
1,231 \\
1,230 \\
1,358 \\
1,674 \\
1,787 \\
1,681 \\
2,010 \\
2,052 \\
2,028\end{array}$ & $\begin{array}{r}1,257 \\
1,827 \\
1,802 \\
2,167 \\
2,507 \\
2,254 \\
2,110 \\
1,361 \\
1,140 \\
998 \\
944 \\
878 \\
1,012 \\
1,250 \\
1,435 \\
1,281 \\
1,582 \\
1,650 \\
1,622\end{array}$ & $\begin{array}{l}2,093 \\
3,280 \\
3,601 \\
5,066 \\
6,103 \\
5,751 \\
5,637 \\
4,300 \\
3,323 \\
2,146 \\
1,862 \\
2,040 \\
1,830 \\
2,837 \\
3,060 \\
3,520 \\
4,140 \\
4,206 \\
4,092\end{array}$ & $\begin{array}{l}2,095 \\
2,540 \\
3,211 \\
4,545 \\
5,643 \\
5,164 \\
5,112 \\
3,864 \\
2,922 \\
1,734 \\
1,477 \\
1,512 \\
1,312 \\
2,113 \\
2,400 \\
2,696 \\
3,376 \\
3,514 \\
3,377\end{array}$ \\
\hline 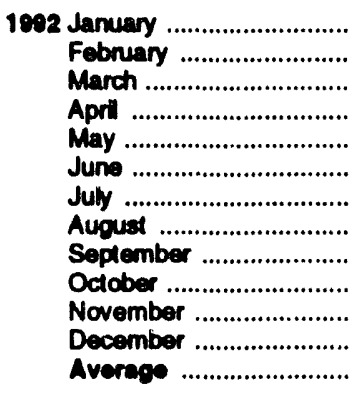 & $\begin{array}{l}593 \\
322 \\
441 \\
798 \\
773 \\
740 \\
900 \\
815 \\
774 \\
827 \\
626 \\
549 \\
681\end{array}$ & $\begin{array}{l}566 \\
303 \\
409 \\
788 \\
773 \\
740 \\
883 \\
795 \\
754 \\
813 \\
608 \\
532 \\
685\end{array}$ & $\begin{array}{l}1,119 \\
1,028 \\
1,106 \\
1,079 \\
1,038 \\
1,069 \\
1,163 \\
1,102 \\
1,333 \\
1,497 \\
1,343 \\
1,164 \\
1,170\end{array}$ & $\begin{array}{r}787 \\
655 \\
793 \\
722 \\
745 \\
738 \\
912 \\
841 \\
953 \\
1,073 \\
921 \\
763 \\
826\end{array}$ & $\begin{array}{l}1,984 \\
1,555 \\
1,684 \\
2,169 \\
2,152 \\
2,141 \\
2,382 \\
2,215 \\
2,426 \\
2,587 \\
2,173 \\
1,917 \\
2,117\end{array}$ & $\begin{array}{l}1,617 \\
1,150 \\
1,336 \\
1,791 \\
1,837 \\
1,809 \\
2,114 \\
1,922 \\
2,001 \\
2,133 \\
1,719 \\
1,499 \\
1,746\end{array}$ & $\begin{array}{l}4,224 \\
3,549 \\
3,606 \\
4,085 \\
4,118 \\
4,029 \\
4,339 \\
4,144 \\
4,274 \\
4,507 \\
4,086 \\
4,105 \\
4,002\end{array}$ & $\begin{array}{l}3,554 \\
2,895 \\
2,941 \\
3,334 \\
3,428 \\
3,430 \\
3,772 \\
3,473 \\
3,531 \\
3,732 \\
3,376 \\
3,381 \\
3,406\end{array}$ \\
\hline 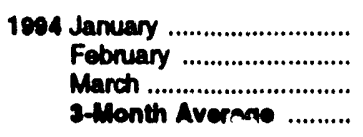 & $\begin{array}{l}310 \\
576 \\
441 \\
430\end{array}$ & $\begin{array}{l}274 \\
557 \\
402 \\
408\end{array}$ & $\begin{array}{l}1,185 \\
1,204 \\
1,219 \\
1,203\end{array}$ & $\begin{array}{l}901 \\
946 \\
915 \\
920\end{array}$ & $\begin{array}{l}1,780 \\
2,094 \\
1,862 \\
1,008\end{array}$ & $\begin{array}{l}1,400 \\
1,770 \\
1,457 \\
1,535\end{array}$ & $\begin{array}{l}3,643 \\
3,814 \\
3,745 \\
3,731\end{array}$ & $\begin{array}{l}2,920 \\
3,237 \\
3,010 \\
3,050\end{array}$ \\
\hline $\begin{array}{l}1003 \text { 3-Month Averege ......... } \\
1002 \text { 2-Month Average ......... }\end{array}$ & $\begin{array}{l}850 \\
455\end{array}$ & $\begin{array}{l}842 \\
420\end{array}$ & $\begin{array}{l}1,204 \\
1,086\end{array}$ & $\begin{array}{l}927 \\
747\end{array}$ & $\begin{array}{l}2,306 \\
1,745\end{array}$ & $\begin{array}{l}1,020 \\
1,372\end{array}$ & $\begin{array}{l}4,330 \\
3,700\end{array}$ & $\begin{array}{l}3,817 \\
3,135\end{array}$ \\
\hline
\end{tabular}

axcudes petroloum imported into the United Stales Indirectly from members of the Organization of Petroleum Exporting Countries (OPEC), primarily from Caribbean and Weet European areas, as petroloum products that were refined from crude oll produced by OPEC.

b As of January 1893, excludes petroleum imponted from Ecuador, which whidrew trom OPEC on December 31, 1992.

Notes: - Beginning in October 1977, Stralegic Petroloum Reserve imports are included. - Petroleum is imported into the 50 States and the District of Columbla. - Totals may not equal sum of components due to independent rounding.

Sources: - 1073-1980: Energy Inlormation Administration (EIA), Petroloum Supply Monthly, February 1993, Table S3. - 1981 forward: ElA Petroleum Supply Monthly, May 1994, Table S3. 
Table 3.3e Petroleum Imports: Angola, Australia, Bahama Islands, Brazil, Canada, and China

(Thousand Barrels per Day)

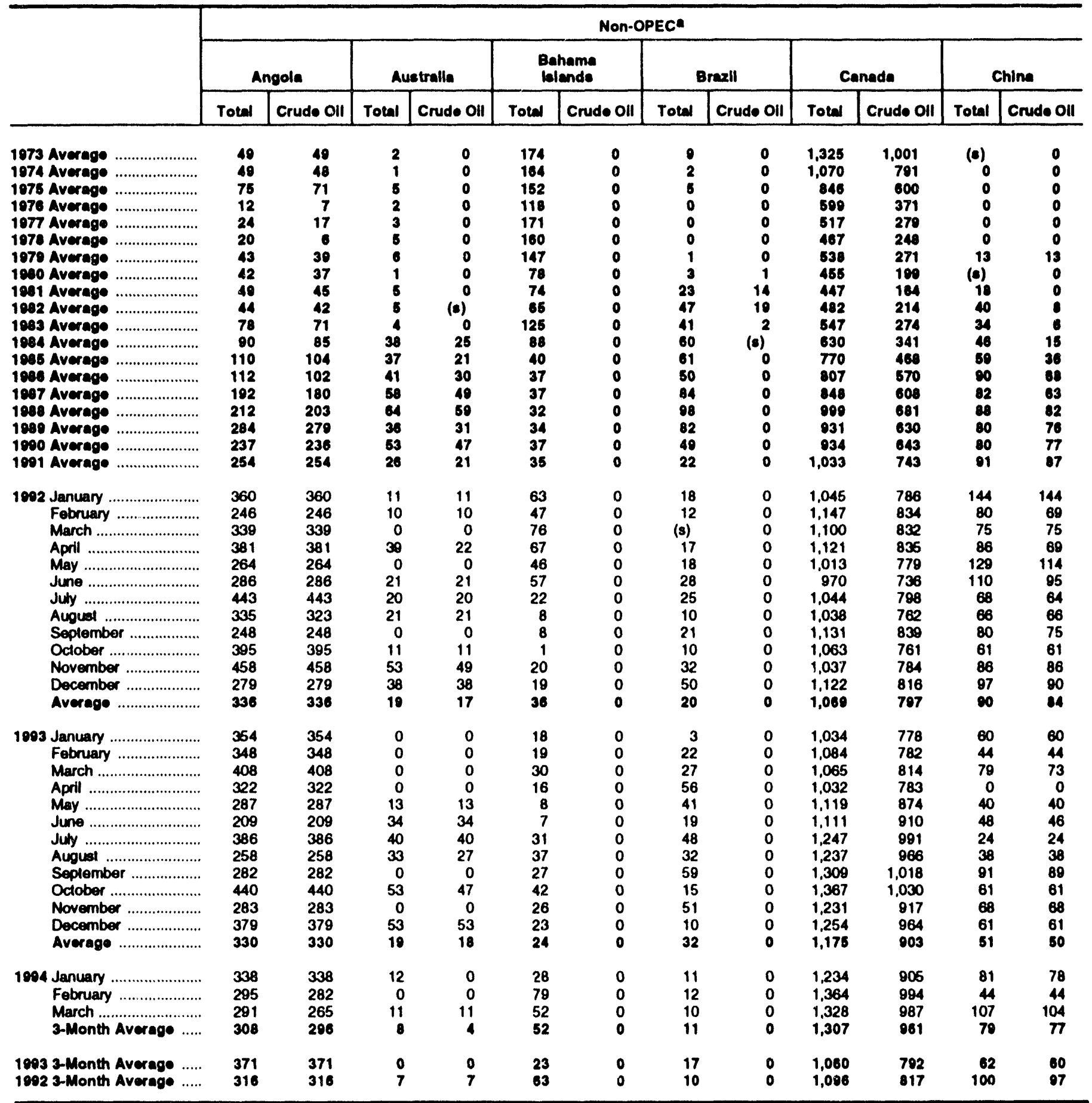

ancludes pelroleum imported into the United Stales indirectly from members of the Organization of Petroleum Exporting Countries (OPEC) primarily from Caribbean and West European areas, as petroleum products that were relined from crude oil produced by OPEC.

(s)= Less than 500 barrels per day.

Noles: - Beginning in October 1977, Strategic Petroleum Reserve imports are included. - Petroleum is imported into the 50 States and the District of Columbia.

Sources: - 1973-1980: Energy Inlormation Administration (ELA), Petroloum Supply Monthly, February 1993. Table S3. - 1981 forward: ElA, Petroloum Supply Monthly, May 1994, Table S3. 
Table 3.3f Petroleum Imports: Colombia, Ecuador, Italy, Malaysia, Mexico, and Netherlands

(Thousand Barrels per Day)

\begin{tabular}{|c|c|c|c|c|c|c|c|c|c|c|c|c|}
\hline & \multicolumn{12}{|c|}{ Non-OPECa } \\
\hline & \multicolumn{2}{|c|}{ Colombia } & \multicolumn{2}{|c|}{ Ecuador } & \multicolumn{2}{|c|}{ Italy } & \multicolumn{2}{|c|}{ Malaysia } & \multicolumn{2}{|c|}{ Moxleo } & \multicolumn{2}{|c|}{ Netherlands } \\
\hline & Total & Crude Oll & Total & Cruin SII & Total & Crude Oll & Total & Crude Oll & Total & Crude Oll & Total & Crude Oll \\
\hline 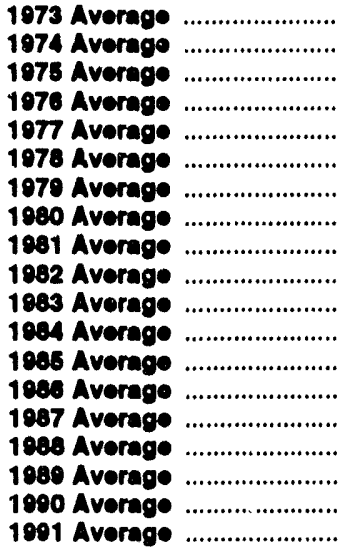 & $\begin{array}{r}9 \\
5 \\
0 \\
21 \\
17 \\
20 \\
18 \\
4 \\
1 \\
5 \\
10 \\
8 \\
23 \\
87 \\
148 \\
134 \\
172 \\
182 \\
163\end{array}$ & $\begin{array}{r}2 \\
0 \\
0 \\
6 \\
0 \\
0 \\
0 \\
0 \\
0 \\
0 \\
0 \\
0 \\
0 \\
57 \\
115 \\
108 \\
136 \\
140 \\
123\end{array}$ & $\begin{array}{l}- \\
\overline{-} \\
\overline{-} \\
\overline{-} \\
\overline{-} \\
- \\
- \\
- \\
- \\
- \\
-\end{array}$ & $\begin{array}{l}- \\
- \\
- \\
- \\
- \\
- \\
- \\
- \\
- \\
- \\
- \\
- \\
- \\
- \\
- \\
-\end{array}$ & $\begin{array}{r}125 \\
74 \\
27 \\
30 \\
51 \\
38 \\
30 \\
4 \\
11 \\
18 \\
18 \\
45 \\
60 \\
78 \\
54 \\
65 \\
34 \\
58 \\
47\end{array}$ & $\begin{array}{c}0 \\
0 \\
0 \\
0 \\
0 \\
0 \\
0 \\
0 \\
0 \\
(s) \\
(8) \\
(s) \\
(8) \\
0 \\
1 \\
5 \\
3 \\
2 \\
3\end{array}$ & $\begin{array}{r}12 \\
12 \\
8 \\
18 \\
66 \\
42 \\
68 \\
70 \\
36 \\
20 \\
4 \\
1 \\
3 \\
12 \\
13 \\
19 \\
30 \\
41 \\
24\end{array}$ & $\begin{array}{r}1 \\
1 \\
5 \\
16 \\
55 \\
37 \\
52 \\
61 \\
33 \\
18 \\
3 \\
0 \\
1 \\
11 \\
12 \\
19 \\
39 \\
40 \\
24\end{array}$ & $\begin{array}{r}16 \\
8 \\
71 \\
87 \\
179 \\
318 \\
439 \\
533 \\
522 \\
685 \\
826 \\
748 \\
816 \\
600 \\
855 \\
747 \\
767 \\
755 \\
807\end{array}$ & $\begin{array}{r}1 \\
2 \\
70 \\
87 \\
177 \\
316 \\
437 \\
507 \\
460 \\
645 \\
768 \\
650 \\
715 \\
621 \\
602 \\
674 \\
716 \\
680 \\
750\end{array}$ & $\begin{array}{r}53 \\
43 \\
10 \\
8 \\
31 \\
5 \\
23 \\
2 \\
30 \\
35 \\
65 \\
85 \\
58 \\
64 \\
60 \\
61 \\
40 \\
55 \\
20\end{array}$ & $\begin{array}{l}0 \\
0 \\
1 \\
0 \\
1 \\
2 \\
7 \\
(0) \\
(0) \\
(0) \\
3 \\
3 \\
0 \\
0 \\
0 \\
0 \\
0 \\
0 \\
0\end{array}$ \\
\hline 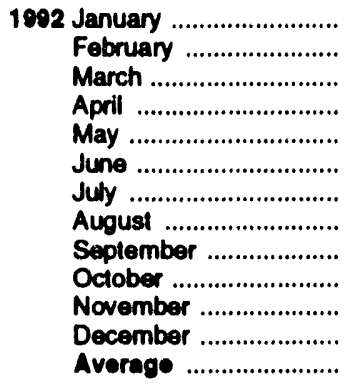 & $\begin{array}{r}158 \\
114 \\
101 \\
150 \\
57 \\
135 \\
103 \\
156 \\
190 \\
153 \\
127 \\
66 \\
126\end{array}$ & $\begin{array}{r}111 \\
92 \\
74 \\
129 \\
46 \\
414 \\
114 \\
93 \\
142 \\
179 \\
132 \\
84 \\
34 \\
102\end{array}$ & $\begin{array}{l}- \\
- \\
- \\
- \\
- \\
- \\
- \\
- \\
-\end{array}$ & $\begin{array}{l}- \\
- \\
- \\
- \\
- \\
- \\
- \\
- \\
-\end{array}$ & $\begin{array}{l}51 \\
48 \\
44 \\
75 \\
57 \\
69 \\
36 \\
94 \\
81 \\
37 \\
33 \\
37 \\
55\end{array}$ & $\begin{array}{l}0 \\
0 \\
0 \\
0 \\
0 \\
0 \\
0 \\
0 \\
0 \\
0 \\
0 \\
0 \\
0\end{array}$ & $\begin{array}{r}0 \\
0 \\
0 \\
0 \\
5 \\
8 \\
40 \\
22 \\
17 \\
17 \\
8 \\
4 \\
10\end{array}$ & $\begin{array}{r}0 \\
0 \\
0 \\
0 \\
5 \\
8 \\
40 \\
22 \\
17 \\
17 \\
8 \\
4 \\
10\end{array}$ & $\begin{array}{l}764 \\
838 \\
846 \\
857 \\
788 \\
905 \\
830 \\
857 \\
755 \\
829 \\
762 \\
830 \\
830\end{array}$ & $\begin{array}{l}721 \\
807 \\
809 \\
795 \\
764 \\
883 \\
788 \\
790 \\
720 \\
783 \\
700 \\
888 \\
787\end{array}$ & $\begin{array}{r}31 \\
9 \\
34 \\
8 \\
27 \\
25 \\
21 \\
45 \\
39 \\
18 \\
26 \\
33 \\
26\end{array}$ & $\begin{array}{l}0 \\
0 \\
0 \\
0 \\
0 \\
0 \\
0 \\
0 \\
0 \\
0 \\
0 \\
0 \\
0\end{array}$ \\
\hline 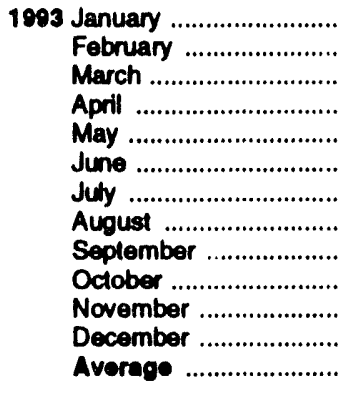 & $\begin{array}{l}188 \\
148 \\
161 \\
152 \\
147 \\
176 \\
204 \\
124 \\
224 \\
192 \\
153 \\
125 \\
168\end{array}$ & $\begin{array}{r}167 \\
137 \\
129 \\
138 \\
90 \\
143 \\
184 \\
101 \\
170 \\
182 \\
143 \\
85 \\
130\end{array}$ & $\begin{array}{r}76 \\
14 \\
59 \\
74 \\
56 \\
75 \\
85 \\
121 \\
49 \\
146 \\
115 \\
84 \\
80\end{array}$ & $\begin{array}{r}70 \\
14 \\
59 \\
62 \\
56 \\
75 \\
85 \\
121 \\
49 \\
135 \\
106 \\
84 \\
77\end{array}$ & $\begin{array}{r}48 \\
34 \\
43 \\
14 \\
18 \\
22 \\
25 \\
50 \\
32 \\
30 \\
25 \\
0 \\
28\end{array}$ & $\begin{array}{l}0 \\
0 \\
0 \\
0 \\
0 \\
0 \\
0 \\
0 \\
0 \\
0 \\
0 \\
0 \\
0\end{array}$ & $\begin{array}{r}0 \\
0 \\
11 \\
8 \\
21 \\
0 \\
11 \\
14 \\
28 \\
10 \\
0 \\
28 \\
11\end{array}$ & $\begin{array}{r}0 \\
0 \\
10 \\
8 \\
10 \\
0 \\
11 \\
14 \\
28 \\
10 \\
0 \\
28 \\
10\end{array}$ & $\begin{array}{r}858 \\
807 \\
861 \\
844 \\
907 \\
995 \\
943 \\
862 \\
929 \\
1,013 \\
1,100 \\
909 \\
910\end{array}$ & $\begin{array}{r}820 \\
748 \\
815 \\
818 \\
846 \\
977 \\
878 \\
809 \\
867 \\
851 \\
1,025 \\
837 \\
868\end{array}$ & $\begin{array}{r}11 \\
18 \\
11 \\
0 \\
10 \\
10 \\
20 \\
17 \\
22 \\
0 \\
(8) \\
6 \\
10\end{array}$ & $\begin{array}{l}0 \\
0 \\
0 \\
0 \\
0 \\
0 \\
0 \\
0 \\
0 \\
0 \\
0 \\
0 \\
0\end{array}$ \\
\hline 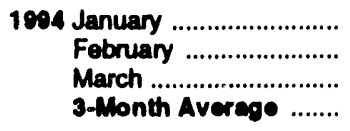 & $\begin{array}{l}182 \\
184 \\
188 \\
185\end{array}$ & $\begin{array}{l}149 \\
131 \\
167 \\
150\end{array}$ & $\begin{array}{r}128 \\
96 \\
37 \\
87\end{array}$ & $\begin{array}{r}128 \\
96 \\
37 \\
87\end{array}$ & $\begin{array}{r}8 \\
35 \\
16 \\
10\end{array}$ & $\begin{array}{l}0 \\
0 \\
0 \\
0\end{array}$ & $\begin{array}{l}11 \\
19 \\
13 \\
14\end{array}$ & $\begin{array}{r}0 \\
15 \\
0 \\
5\end{array}$ & $\begin{array}{r}971 \\
967 \\
1,067 \\
1,003\end{array}$ & $\begin{array}{r}945 \\
926 \\
1,014 \\
963\end{array}$ & $\begin{array}{l}35 \\
43 \\
33 \\
37\end{array}$ & $\begin{array}{l}0 \\
0 \\
0 \\
0\end{array}$ \\
\hline $\begin{array}{l}1093 \text { 3-Month Averege ....... } \\
1902 \text { 3-Month Averego ....... }\end{array}$ & $\begin{array}{l}168 \\
124\end{array}$ & $\begin{array}{r}146 \\
92\end{array}$ & -5 & $\begin{array}{l}49 \\
-\end{array}$ & $\begin{array}{l}42 \\
48\end{array}$ & $\begin{array}{l}0 \\
0\end{array}$ & $\mathbf{4}$ & $\mathbf{4}$ & $\begin{array}{l}843 \\
815\end{array}$ & $\begin{array}{l}706 \\
770\end{array}$ & $\begin{array}{l}13 \\
25\end{array}$ & $\begin{array}{l}\mathbf{0} \\
\mathbf{0}\end{array}$ \\
\hline
\end{tabular}

a Indudes petroleum imported into the United Stales indirectly from members of the Organization of Petroleum Exporting Countries (OPEC), primartly Irom Caribbean and West European areas, as petroleum products that were refined trom crude oll produced by OPEC.

Through 1992, Ecuador was a member of OPEC. See Table 3.3c.

- = Not applicable. (s)=Less than 500 barrels per day.
Noles: - Beginning in October 1977, Strategic Patroleun Reservo imports are included. - Petroleum is imported into the $\mathbf{5 0}$ States and the District of Columbia.

Sources: - 1973-1980: Energy Intormation Administration (EIA), Petroloum Supply Monthly, February 1993, Table S3. - 1981 forward: ElA, Perroleum Supply Monthly, May 1994, Table S3. 
Table 3.3g Petroleum Imports: Netherlands Antilles, Norway, Puerto Rico, Russia, Spain, and Trinidad and Tobago

(Thousand Barrels per Day)

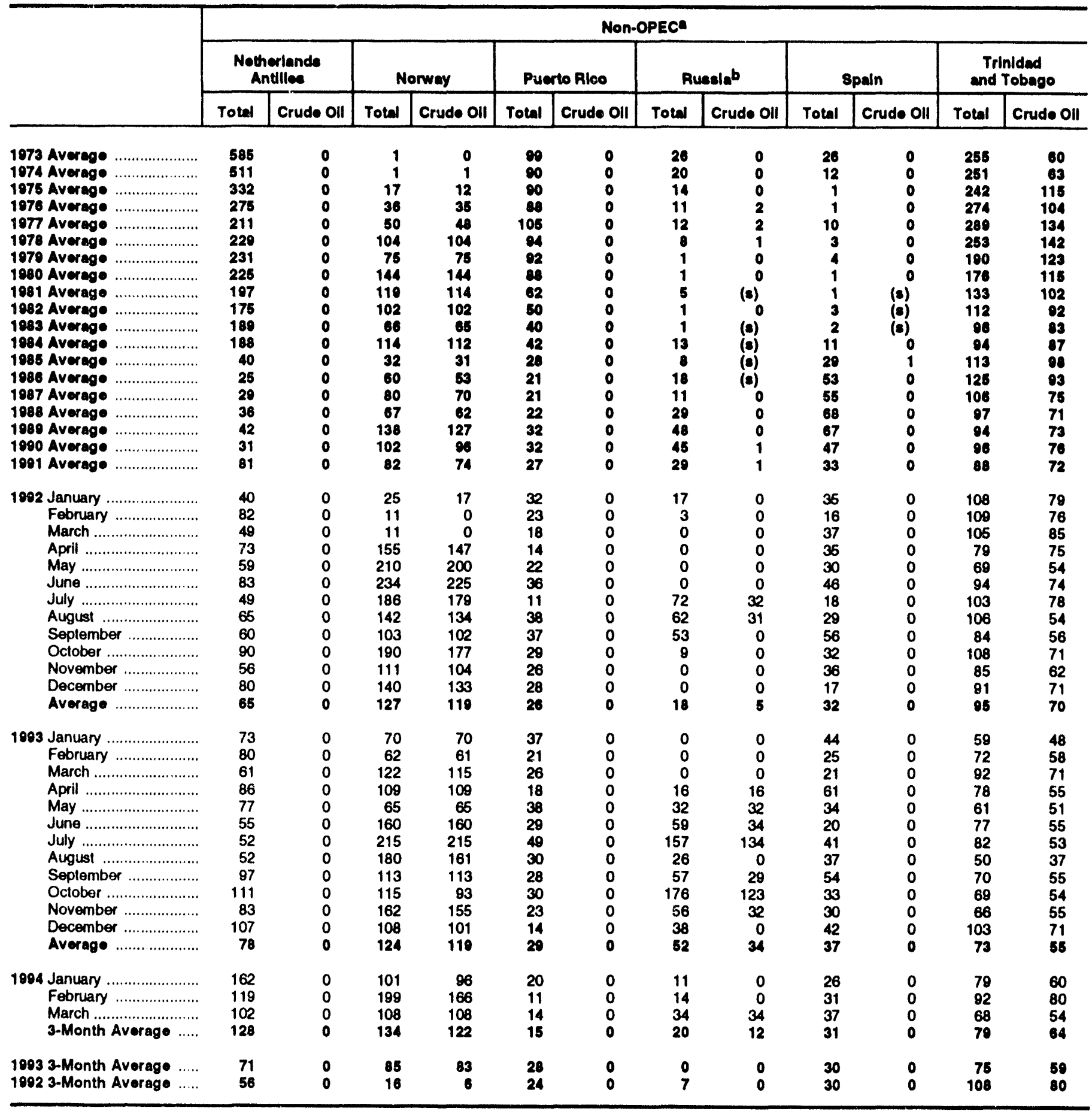

a Includes petroleum imported into the United States indirectly from members of the Organization of Petroleum Exporting Countries (OPEC), primarily from Caribbean and West European areas, as petroleum products that were relined from crude oil produced by OPEC

Imports from oher States in the former U.S.S.R. may be included in imports from Russia for the years 1973 through 1992.

(s)=Loss than 500 barrels per day.
Notes: - Beginning in October 1977, Strategic Petroleum Reserve imports are included. - Pelroleum is imported into the 50 Stales and the District of Columbia.

Sources: - 1973-1980: Energy Intormation Administration (EIA), Petroleum Supply Monthly, February 1993, Table S3. - 1981 forward: EIA, Potroleum Supply Monthly, May 1994, Table S3. 
Table 3.3h Petroleum imports: United Kingdom, Virgin Islands, Other Non-OPEC, Total Non-OPEC, and Total Imports

(Thousand Barrels per Day)

\begin{tabular}{|c|c|c|c|c|c|c|c|c|c|c|}
\hline & \multicolumn{6}{|c|}{ Non-OPEC } & \multirow{2}{*}{\multicolumn{2}{|c|}{$\begin{array}{c}\text { Total } \\
\text { Non-OPEC } a, b\end{array}$}} & \multirow{2}{*}{\multicolumn{2}{|c|}{$\begin{array}{c}\text { Total } \\
\text { Imports }\end{array}$}} \\
\hline & \multicolumn{2}{|c|}{$\begin{array}{c}\text { United } \\
\text { Kingdom }\end{array}$} & \multicolumn{2}{|c|}{ Virgin lalands } & \multicolumn{2}{|c|}{$\begin{array}{l}\text { Other } \\
\text { Non-OPEC }\end{array}$} & & & & \\
\hline & Totel & Crude Oil & Total & Crude Oll & Total & Crude Oll & Total & Crude Oll & Total & Crude OII \\
\hline 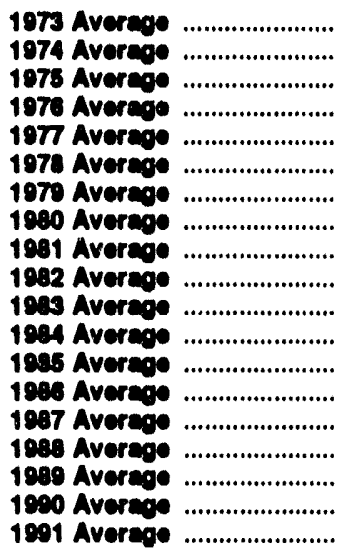 & $\begin{array}{r}15 \\
8 \\
14 \\
31 \\
126 \\
180 \\
202 \\
176 \\
375 \\
456 \\
382 \\
402 \\
310 \\
350 \\
352 \\
315 \\
215 \\
180 \\
138\end{array}$ & $\begin{array}{r}0 \\
0 \\
(0) \\
13 \\
97 \\
169 \\
197 \\
173 \\
369 \\
411 \\
365 \\
378 \\
278 \\
317 \\
304 \\
254 \\
160 \\
155 \\
106\end{array}$ & $\begin{array}{l}329 \\
391 \\
406 \\
422 \\
466 \\
428 \\
431 \\
388 \\
327 \\
316 \\
282 \\
294 \\
247 \\
244 \\
272 \\
242 \\
321 \\
282 \\
243\end{array}$ & $\begin{array}{l}0 \\
0 \\
0 \\
0 \\
0 \\
0 \\
0 \\
0 \\
0 \\
0 \\
0 \\
0 \\
0 \\
0 \\
0 \\
0 \\
0 \\
0 \\
0\end{array}$ & $\begin{array}{l}153 \\
122 \\
120 \\
203 \\
287 \\
239 \\
269 \\
219 \\
236 \\
306 \\
378 \\
411 \\
394 \\
426 \\
459 \\
487 \\
457 \\
417 \\
282\end{array}$ & $\begin{array}{r}36 \\
30 \\
14 \\
101 \\
157 \\
146 \\
192 \\
162 \\
163 \\
174 \\
215 \\
210 \\
137 \\
144 \\
196 \\
196 \\
197 \\
180 \\
137\end{array}$ & $\begin{array}{l}3,263 \\
2,832 \\
2,454 \\
2,247 \\
2,614 \\
2,612 \\
2,819 \\
2,609 \\
2,672 \\
2,968 \\
3,189 \\
3,388 \\
3,237 \\
3,387 \\
3,617 \\
3,882 \\
3,921 \\
3,721 \\
3,535\end{array}$ & $\begin{array}{r}1,149 \\
937 \\
893 \\
742 \\
971 \\
1,172 \\
1,407 \\
1,399 \\
1,474 \\
1,754 \\
1,853 \\
1,914 \\
1,888 \\
2,065 \\
2,274 \\
2,411 \\
2,467 \\
2,381 \\
2,405\end{array}$ & $\begin{array}{l}6,256 \\
6,112 \\
6,056 \\
7,313 \\
8,807 \\
8,363 \\
8,456 \\
6,909 \\
5,996 \\
5,113 \\
5,051 \\
5,437 \\
5,067 \\
6,224 \\
6,678 \\
7,402 \\
8,061 \\
8,018 \\
7,627\end{array}$ & $\begin{array}{l}3,244 \\
3,477 \\
4,105 \\
5,287 \\
6,615 \\
6,356 \\
6,519 \\
5,263 \\
4,396 \\
3,488 \\
3,329 \\
3,426 \\
3,201 \\
4,178 \\
4,674 \\
5,107 \\
5,643 \\
5,894 \\
5,782\end{array}$ \\
\hline 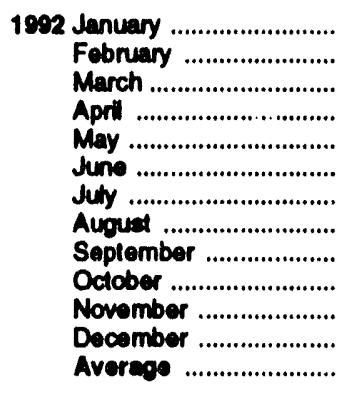 & $\begin{array}{r}129 \\
63 \\
78 \\
157 \\
198 \\
248 \\
354 \\
295 \\
341 \\
411 \\
336 \\
148 \\
230\end{array}$ & $\begin{array}{r}115 \\
0 \\
52 \\
128 \\
180 \\
206 \\
337 \\
282 \\
291 \\
411 \\
285 \\
110 \\
200\end{array}$ & $\begin{array}{l}250 \\
222 \\
202 \\
234 \\
246 \\
266 \\
280 \\
263 \\
217 \\
254 \\
274 \\
273 \\
249\end{array}$ & $\begin{array}{l}0 \\
0 \\
0 \\
0 \\
0 \\
0 \\
0 \\
0 \\
0 \\
0 \\
0 \\
0 \\
0\end{array}$ & $\begin{array}{l}208 \\
196 \\
345 \\
458 \\
467 \\
297 \\
415 \\
464 \\
382 \\
279 \\
219 \\
283 \\
335\end{array}$ & $\begin{array}{r}59 \\
50 \\
114 \\
212 \\
225 \\
95 \\
152 \\
357 \\
160 \\
144 \\
124 \\
92 \\
149\end{array}$ & $\begin{array}{l}3,488 \\
3,278 \\
3,462 \\
4,007 \\
3,705 \\
3,917 \\
4,140 \\
4,116 \\
3,904 \\
3,998 \\
3,786 \\
3,734 \\
3,796\end{array}$ & $\begin{array}{l}2,402 \\
2,184 \\
2,380 \\
2,793 \\
2,633 \\
2,741 \\
3,024 \\
2,984 \\
2,687 \\
2,964 \\
2,745 \\
2,556 \\
2,676\end{array}$ & $\begin{array}{l}7,712 \\
6,827 \\
7,068 \\
8,092 \\
7,823 \\
7,946 \\
8,479 \\
8,260 \\
8,178 \\
8,505 \\
7,872 \\
7,830 \\
7,888\end{array}$ & $\begin{array}{l}5,956 \\
5,079 \\
5,321 \\
6,127 \\
6,060 \\
6,171 \\
6,796 \\
6,457 \\
6,218 \\
6,696 \\
6,121 \\
5,937 \\
6,083\end{array}$ \\
\hline 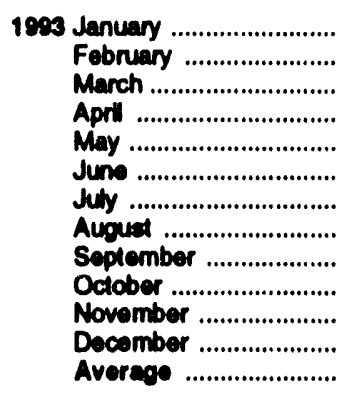 & $\begin{array}{l}228 \\
173 \\
315 \\
348 \\
486 \\
458 \\
292 \\
343 \\
286 \\
352 \\
351 \\
432 \\
340\end{array}$ & $\begin{array}{l}201 \\
127 \\
281 \\
281 \\
458 \\
408 \\
247 \\
323 \\
217 \\
338 \\
340 \\
403 \\
303\end{array}$ & $\begin{array}{l}252 \\
244 \\
244 \\
245 \\
279 \\
290 \\
202 \\
256 \\
184 \\
236 \\
330 \\
288 \\
254\end{array}$ & $\begin{array}{l}0 \\
0 \\
0 \\
0 \\
0 \\
0 \\
0 \\
0 \\
0 \\
0 \\
0 \\
0 \\
0\end{array}$ & $\begin{array}{l}325 \\
223 \\
390 \\
455 \\
356 \\
570 \\
585 \\
520 \\
551 \\
453 \\
468 \\
402 \\
443\end{array}$ & $\begin{array}{l}104 \\
151 \\
186 \\
243 \\
152 \\
405 \\
299 \\
329 \\
251 \\
233 \\
246 \\
231 \\
236\end{array}$ & $\begin{array}{r}r 3,739 \\
3,439 \\
4,026 \\
3,933 \\
4,095 \\
4,423 \\
4,741 \\
4,318 \\
4,483 \\
4,944 \\
4,621 \\
4,466 \\
4,275\end{array}$ & $\begin{array}{r}\text { b } 2,672 \\
2,471 \\
2,961 \\
2,836 \\
2,974 \\
3,454 \\
3,546 \\
3,184 \\
3,167 \\
3,698 \\
3,369 \\
3,298 \\
3,140\end{array}$ & $\begin{array}{l}7,964 \\
7,930 \\
8,342 \\
8,485 \\
8,348 \\
8,745 \\
8,145 \\
8,360 \\
8,476 \\
9,147 \\
8,725 \\
8,600 \\
8,526\end{array}$ & $\begin{array}{l}6,292 \\
6,156 \\
6,513 \\
6,698 \\
6,549 \\
7,175 \\
7,262 \\
6,614 \\
6,558 \\
7,181 \\
6,892 \\
6,838 \\
6,731\end{array}$ \\
\hline 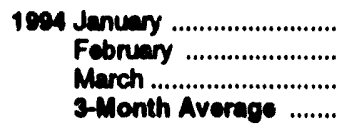 & $\begin{array}{l}205 \\
290 \\
459 \\
310\end{array}$ & $\begin{array}{l}161 \\
232 \\
304 \\
263\end{array}$ & $\begin{array}{l}276 \\
351 \\
325 \\
316\end{array}$ & $\begin{array}{l}0 \\
0 \\
0 \\
0\end{array}$ & $\begin{array}{l}353 \\
441 \\
454 \\
415\end{array}$ & $\begin{array}{l}181 \\
111 \\
181 \\
162\end{array}$ & $\begin{array}{l}4,271 \\
4,687 \\
4,755 \\
4,567\end{array}$ & $\begin{array}{l}3,041 \\
3,077 \\
3,366 \\
3,164\end{array}$ & $\begin{array}{r}7,914 \\
8,501 \\
\mathrm{R} 8,500 \\
8,298\end{array}$ & $\begin{array}{r}5,961 \\
6,313 \\
R_{6,377} \\
6,214\end{array}$ \\
\hline $\begin{array}{l}1903 \text { 3-Month Average ........ } \\
1902 \text { 3-Month Average ....... }\end{array}$ & $\begin{array}{r}241 \\
91\end{array}$ & $\begin{array}{r}205 \\
57\end{array}$ & $\begin{array}{l}247 \\
225\end{array}$ & $\begin{array}{l}0 \\
0\end{array}$ & $\begin{array}{l}316 \\
251\end{array}$ & $\begin{array}{r}146 \\
75\end{array}$ & $\begin{array}{l}3,745 \\
3,412\end{array}$ & $\begin{array}{l}2,709 \\
2,325\end{array}$ & $\begin{array}{l}8,084 \\
7,211\end{array}$ & $\begin{array}{l}6,326 \\
5,460\end{array}$ \\
\hline
\end{tabular}

a Includis petroloum imponed into the United States indirectly from members of the Organization of Petroleum Exporting Countries (OPEC), primarily Irom Carbbean and Weat European areas, as potroloum products that were relined from crude oll produced by OPEC.

As of January 1803, inchudes pelrobum imported from Ecuador, which whindrow from OPEC on December 31, 1992.

$R=$ Revieed data. (s)=Leses than 500 barrols per day.
Notes: - Beginning in October 1977, Strategic Petroloum Resenve imports are included. - Petroleum is imported into the $\mathbf{5 0}$ States and the District of Columbla. - Totals may not equal sum of components due to independent rounding.

Sources: - 1973-1980: Energy Inlormation Administration (EIA), Petroleum Supply Monthly, February 1993, Table S3. - 1981 forward: EIA Petroleum Supply Monthly, May 1994, Table S3. 
Figure 3.2 Finished Motor Gasoline

(Million Barrels per Day, Except as Noted)

Overview, 1973-1993

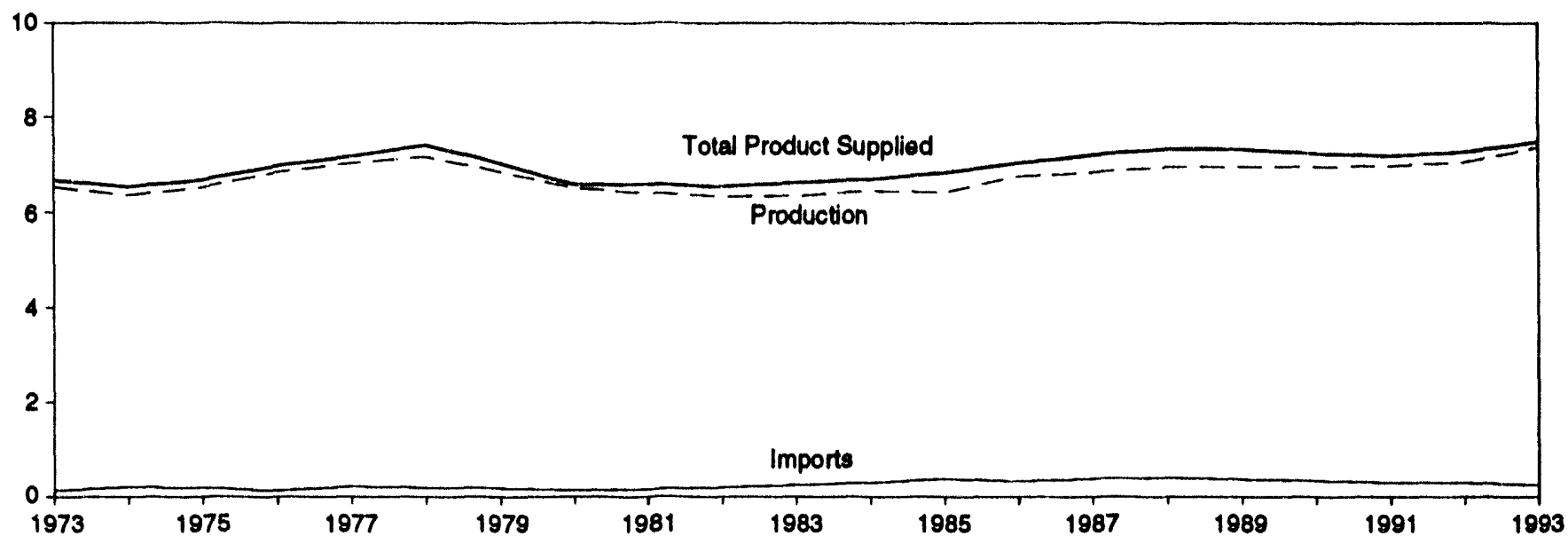

Overview, Monthly

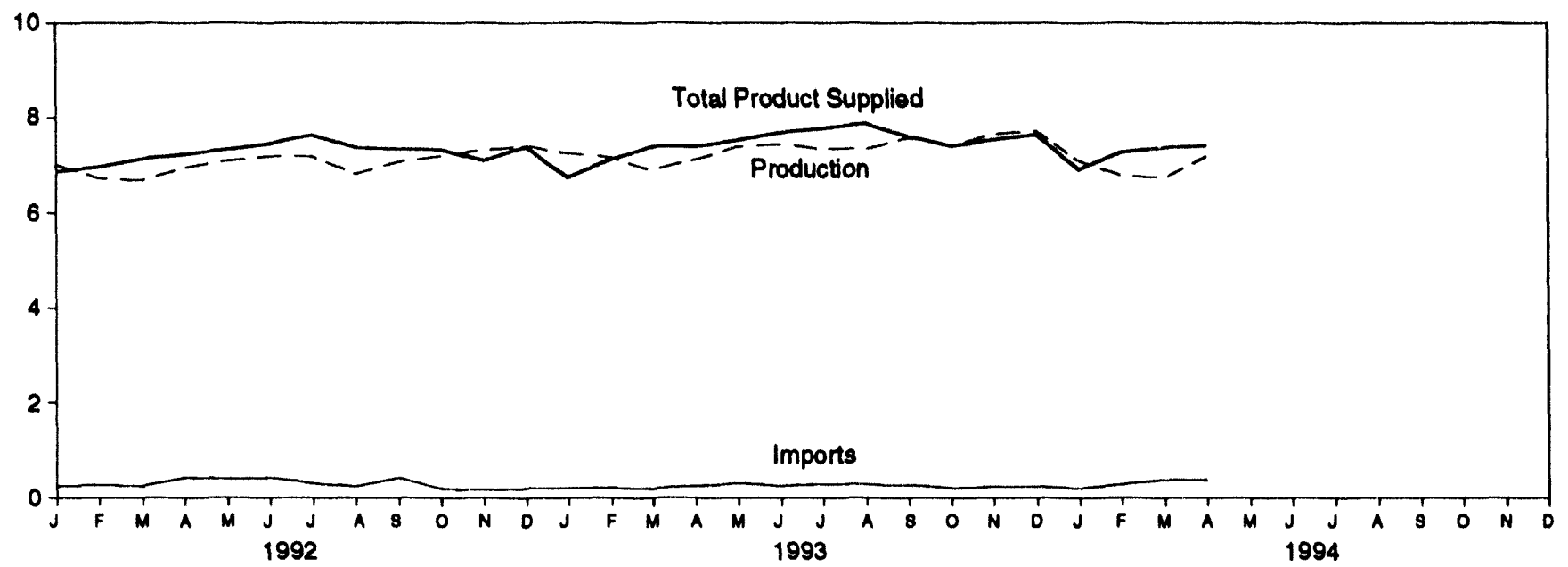

Total Product Supplied, January-April

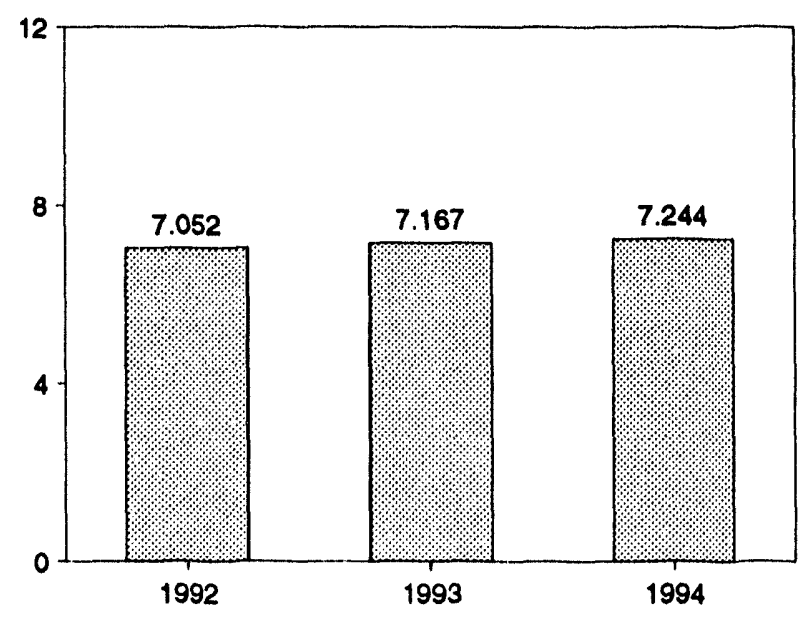

Total Stocks, End of Month

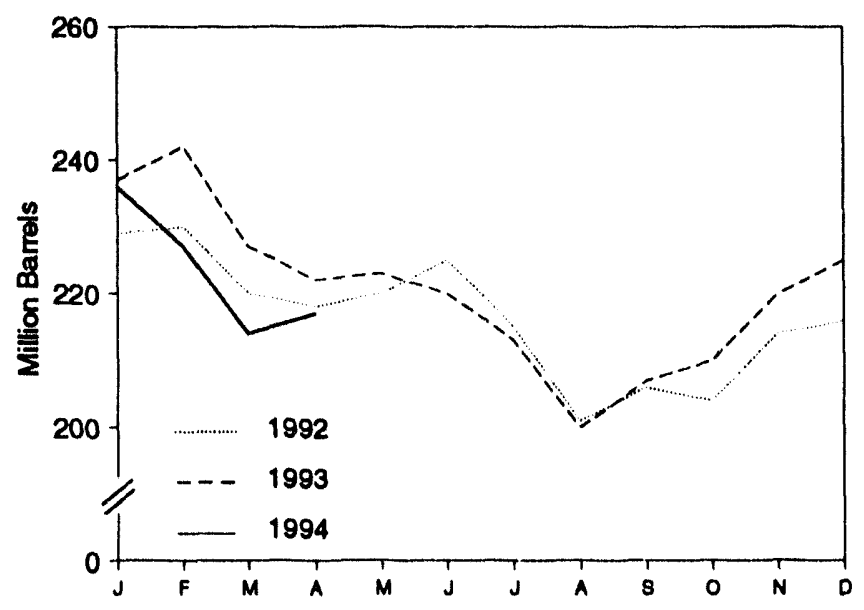

Note: Because vertical scales differ, graphs should not be compared. Source: Table 3.4. 
Table 3.4 Finished Motor Gasoline Supply and Disposition

\begin{tabular}{|c|c|c|c|c|c|c|c|c|}
\hline & \multicolumn{2}{|c|}{ supply } & \multicolumn{3}{|c|}{ Diapostition } & \multicolumn{2}{|c|}{$\begin{array}{l}\text { Motor Cesooline } \\
\text { Ending stocke* }\end{array}$} & \multirow{2}{*}{$\begin{array}{c}\text { Oxyoenutes } \\
\text { Ending } \\
\text { Slooks }\end{array}$} \\
\hline & $\begin{array}{l}\text { Total } \\
\text { Production }\end{array}$ & Importeb & $\begin{array}{l}\text { Stock } \\
\text { Changob,c }\end{array}$ & Exporte & $\begin{array}{l}\text { Product } \\
\text { Suppllod }\end{array}$ & Totald & Finished & \\
\hline & \multicolumn{5}{|c|}{ Thousand Barrels per Day } & \multicolumn{3}{|c|}{ Million Barrebs } \\
\hline 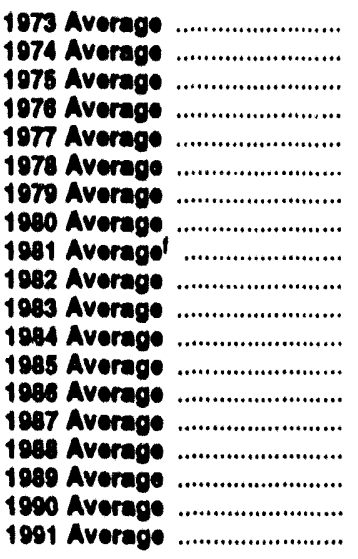 & $\begin{array}{l}6,839 \\
6,360 \\
6,820 \\
6,841 \\
7,033 \\
7,169 \\
6,852 \\
6,506 \\
6,405 \\
6,338 \\
6,340 \\
6,453 \\
6,419 \\
6,762 \\
6,841 \\
6,956 \\
6,963 \\
6,959 \\
6,075\end{array}$ & $\begin{array}{l}134 \\
204 \\
184 \\
131 \\
217 \\
100 \\
181 \\
140 \\
157 \\
107 \\
247 \\
290 \\
381 \\
326 \\
384 \\
405 \\
369 \\
342 \\
297\end{array}$ & $\begin{array}{r}-9 \\
24 \\
-28 \\
-10 \\
72 \\
-54 \\
-2 \\
68 \\
-28 \\
-25 \\
-45 \\
54 \\
-41 \\
11 \\
-15 \\
3 \\
-35 \\
10 \\
3\end{array}$ & $\begin{array}{r}4 \\
2 \\
2 \\
3 \\
2 \\
1 \\
(5) \\
1 \\
2 \\
20 \\
10 \\
6 \\
10 \\
33 \\
35 \\
22 \\
30 \\
55 \\
82\end{array}$ & $\begin{array}{l}6,674 \\
6,537 \\
6,675 \\
6,078 \\
7,177 \\
7,412 \\
7,034 \\
6,579 \\
6,586 \\
6,539 \\
6,622 \\
6,693 \\
6,831 \\
7,034 \\
7,206 \\
7,336 \\
7,328 \\
7,235 \\
7,188\end{array}$ & $\begin{array}{r}209 \\
2218 \\
235 \\
231 \\
258 \\
238 \\
237 \\
261 \\
253 \\
235 \\
222 \\
243 \\
223 \\
233 \\
226 \\
228 \\
213 \\
220 \\
210\end{array}$ & $\begin{array}{l}\text { NA } \\
\text { NA } \\
\text { NA } \\
\text { NA } \\
\text { NA } \\
\text { NA } \\
\text { NA } \\
\text { NA } \\
203 \\
0194 \\
186 \\
205 \\
190 \\
194 \\
189 \\
190 \\
177 \\
181 \\
182\end{array}$ & $\begin{array}{l}\text { NA } \\
\text { NA } \\
\text { NA } \\
\text { NA } \\
\text { NA } \\
N A \\
N A \\
N A \\
N A \\
N A \\
N A \\
N A \\
N A \\
N A \\
\text { NA } \\
\text { NA } \\
\text { NA } \\
\text { NA } \\
\text { NA }\end{array}$ \\
\hline 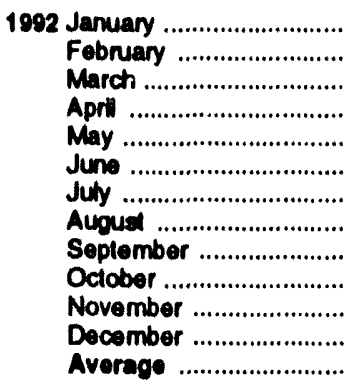 & $\begin{array}{l}7,013 \\
6,726 \\
6,683 \\
6,954 \\
7,092 \\
7,198 \\
7,195 \\
6,817 \\
7,071 \\
7,198 \\
7,323 \\
7,411 \\
7,058\end{array}$ & $\begin{array}{l}246 \\
275 \\
247 \\
428 \\
392 \\
424 \\
303 \\
240 \\
418 \\
193 \\
170 \\
202 \\
294\end{array}$ & $\begin{array}{r}304 \\
-22 \\
.278 \\
54 \\
74 \\
76 \\
.248 \\
.446 \\
60 \\
.41 \\
318 \\
32 \\
.11\end{array}$ & $\begin{array}{r}87 \\
59 \\
71 \\
80 \\
82 \\
86 \\
108 \\
123 \\
85 \\
94 \\
74 \\
184 \\
96\end{array}$ & $\begin{array}{l}6,869 \\
6,963 \\
7,137 \\
7,238 \\
7,328 \\
7,460 \\
7,639 \\
7,380 \\
7,344 \\
7,338 \\
7,102 \\
7,386 \\
7,268\end{array}$ & $\begin{array}{l}228 \\
230 \\
220 \\
218 \\
220 \\
225 \\
215 \\
201 \\
206 \\
204 \\
214 \\
216 \\
216\end{array}$ & $\begin{array}{l}191 \\
191 \\
182 \\
183 \\
186 \\
188 \\
180 \\
167 \\
168 \\
167 \\
177 \\
178 \\
178\end{array}$ & $\begin{array}{l}\text { NA } \\
\text { NA } \\
\text { NA } \\
\text { NA } \\
\text { NA } \\
\text { NA } \\
\text { NA } \\
\text { NA } \\
\text { NA } \\
\text { NA } \\
\text { NA } \\
\text { NA } \\
\text { NA }\end{array}$ \\
\hline 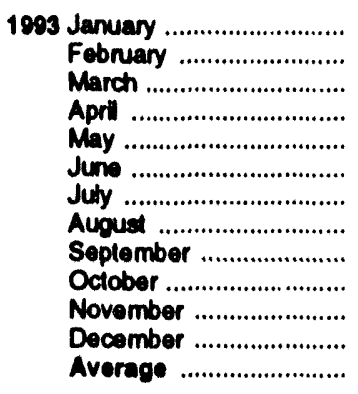 & $\begin{array}{r}97,254 \\
7,172 \\
6,897 \\
7,123 \\
7,394 \\
7,447 \\
7,344 \\
7,344 \\
7,583 \\
7,409 \\
7,664 \\
7,722 \\
7,363\end{array}$ & $\begin{array}{l}204 \\
216 \\
198 \\
253 \\
308 \\
251 \\
292 \\
283 \\
269 \\
210 \\
237 \\
238 \\
247\end{array}$ & $\begin{array}{r}571 \\
160 \\
.411 \\
.137 \\
80 \\
.75 \\
.242 \\
.336 \\
154 \\
127 \\
237 \\
152 \\
22\end{array}$ & $\begin{array}{r}142 \\
90 \\
109 \\
111 \\
90 \\
81 \\
100 \\
77 \\
85 \\
80 \\
123 \\
165 \\
105\end{array}$ & $\begin{array}{r}96,746 \\
7,129 \\
7,397 \\
7,401 \\
7,531 \\
7,692 \\
7,777 \\
7,685 \\
7,612 \\
7,411 \\
7,541 \\
7,643 \\
7,403\end{array}$ & $\begin{array}{l}237 \\
242 \\
227 \\
222 \\
223 \\
220 \\
213 \\
200 \\
207 \\
210 \\
220 \\
225 \\
225\end{array}$ & $\begin{array}{l}195 \\
200 \\
187 \\
183 \\
185 \\
183 \\
176 \\
165 \\
170 \\
174 \\
181 \\
186 \\
186\end{array}$ & $\begin{array}{l}h_{14} \\
13 \\
14 \\
15 \\
17 \\
18 \\
20 \\
21 \\
20 \\
17 \\
15 \\
13 \\
13\end{array}$ \\
\hline 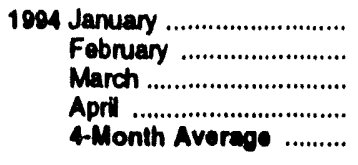 & $\begin{array}{r}7,098 \\
6,780 \\
R_{6,740} \\
E_{7,180} \\
E_{6,054}\end{array}$ & $\begin{array}{r}206 \\
281 \\
A_{387} \\
E_{373} \\
E_{312}\end{array}$ & $\begin{array}{r}291 \\
.288 \\
R .340 \\
E 67 \\
E-63\end{array}$ & $\begin{array}{r}97 \\
77 \\
\text { ค }_{88} \\
\mathrm{E}_{85}\end{array}$ & $\begin{array}{r}6,916 \\
7,272 \\
\text { R } 7,379 \\
\text { E } 7,418 \\
\text { E } 7,244\end{array}$ & $\begin{array}{l}236 \\
227 \\
214 \\
E_{217} \\
E_{217}\end{array}$ & $\begin{array}{r}185 \\
187 \\
176 \\
E_{178} \\
E_{178}\end{array}$ & $\begin{array}{l}11 \\
11 \\
13 \\
\text { NA } \\
\text { NA }\end{array}$ \\
\hline $\begin{array}{l}1993 \text { 4-Month Average ......... } \\
1992 \text { 4-Month Average ......... }\end{array}$ & $\begin{array}{l}7,110 \\
6,845\end{array}$ & $\begin{array}{l}217 \\
298\end{array}$ & $\begin{array}{l}44 \\
15\end{array}$ & $\frac{116}{77}$ & $\begin{array}{l}7,167 \\
7,052\end{array}$ & $\begin{array}{l}222 \\
218\end{array}$ & $\begin{array}{l}183 \\
183\end{array}$ & $\begin{array}{l}16 \\
\text { NA }\end{array}$ \\
\hline
\end{tabular}

- Stocks are tolals as of end of period.

b From 1881 forward, blending components are excluded.

- A negative number indicales a decrease in stocks and a positive number indicales an increase.

Includes molor gasoline blending components, but excludes oxygenates, which are reported separately.

- See Note 4 al end of section.

1 See Nole 2 al end of section.

- Beginning in 1893, motor gasoline production and product supplied include blending of fuel ethanol and an adjustment to correct for the imbalance of motor gasoline blending components. See Note 2 al end of section.

h See Note 1 at end of section.

$R=$ Revised data. NA=Nol available. ExEstimate. (s)=Less than 500 barrols per day.

Nole: Geographic coverage is the 50 States and the District of Columbla.

Sources: - 1973-1980: Energy Information Administration (ELA),

Petroloum Suppiy Monthly, Fobruary 1993, Table S4. - 1981 forward: EIA,

Petroleum Supply Monthly, May 1994, Table S4. 
(Million Barrels per Day, Except as Noted)

Overview, 1973-1993

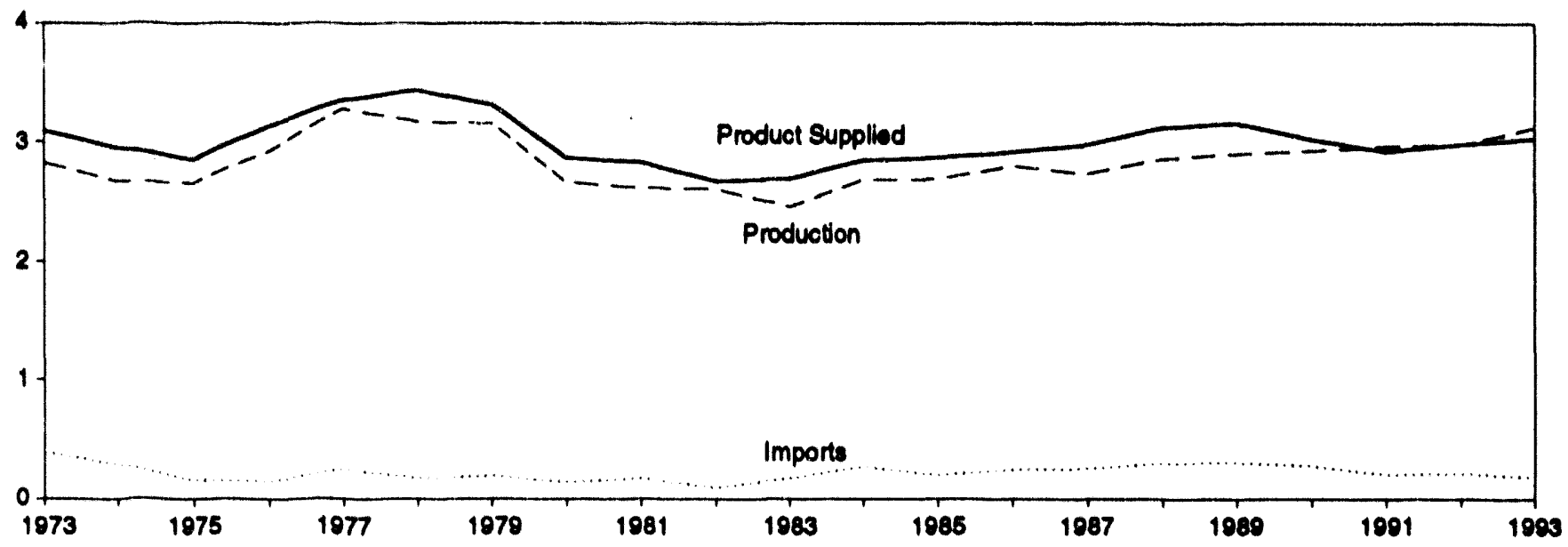

Overview, Monthly

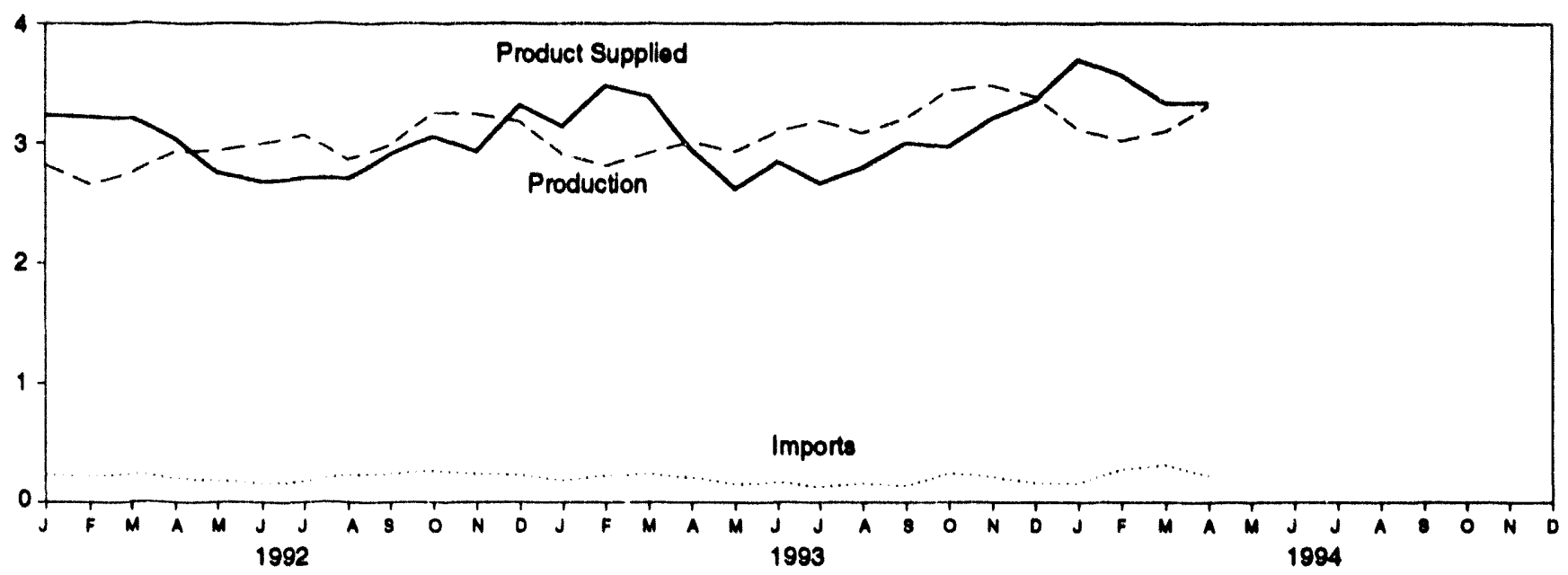

Product Supplied, January-April

Stocks, End of Month
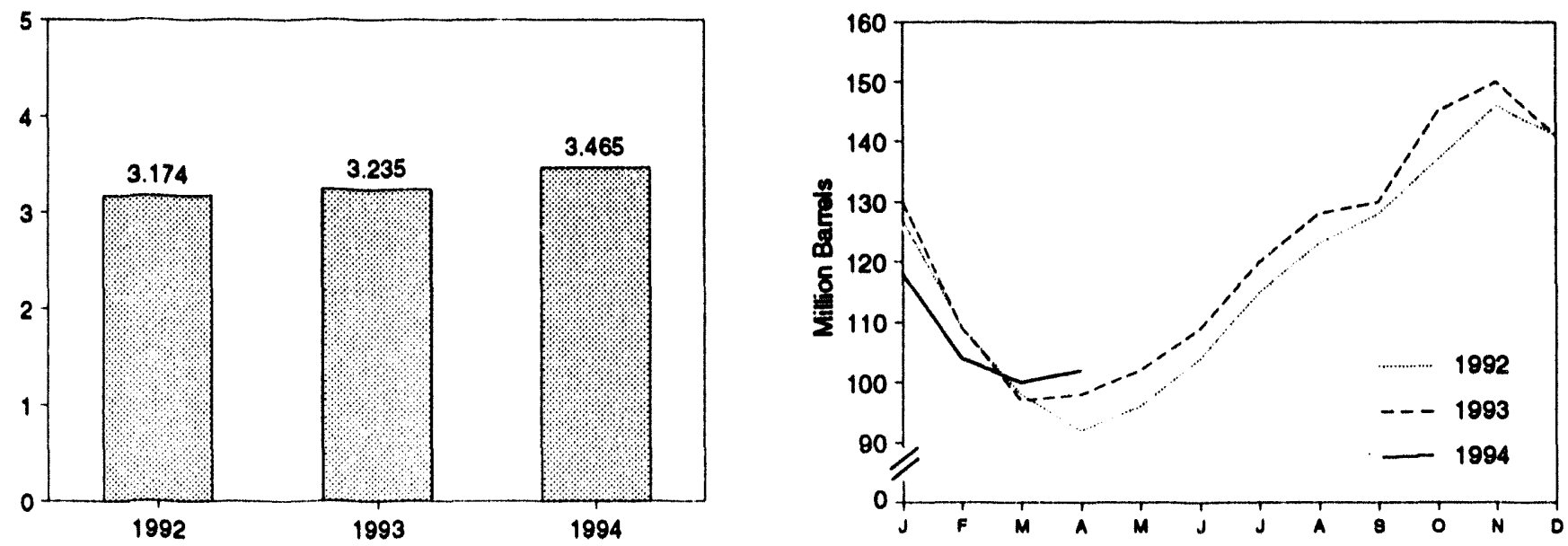

Source: Table 3.5 


\begin{tabular}{|c|c|c|c|c|c|c|c|c|c|}
\hline & \multicolumn{3}{|c|}{ supply } & \multicolumn{3}{|c|}{ Dhepostion } & \multicolumn{3}{|c|}{ Ending Stocke } \\
\hline & \multirow[b]{2}{*}{$\begin{array}{c}\text { Totel } \\
\text { Produotion }\end{array}$} & \multirow[b]{2}{*}{ Importe } & \multirow{2}{*}{$\begin{array}{l}\text { Crude Oll } \\
\text { Unesd } \\
\text { Dirvetly }\end{array}$} & \multirow[b]{2}{*}{$\begin{array}{l}\text { Etook } \\
\text { Chengo" }\end{array}$} & \multirow[b]{2}{*}{ Exporte } & \multirow[b]{2}{*}{$\begin{array}{l}\text { Produot } \\
\text { supplledo }\end{array}$} & \multirow[b]{2}{*}{ Total } & \multicolumn{2}{|c|}{ Sulfur Content } \\
\hline & & & & & & & & $\begin{array}{l}0.05 \text { Percent } \\
\text { or Lesed }\end{array}$ & $\begin{array}{l}\text { Greator Than } \\
0.05 \text { Percent }\end{array}$ \\
\hline & \multicolumn{6}{|c|}{ Thousand Barrels per Day } & \multicolumn{3}{|c|}{ Million Barrels } \\
\hline 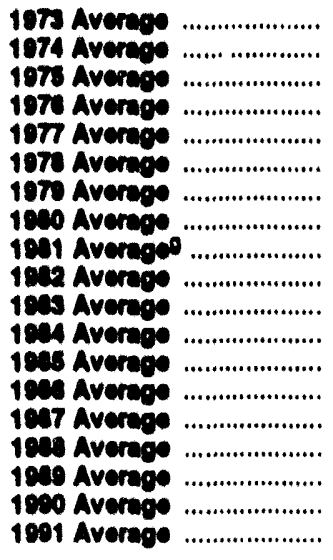 & $\begin{array}{l}2,022 \\
2,090 \\
2,044 \\
2,024 \\
3,270 \\
3,187 \\
3,183 \\
2,092 \\
2,019 \\
2,000 \\
2,490 \\
2,001 \\
2,097 \\
2,700 \\
2,731 \\
2,089 \\
2,800 \\
2,925 \\
2,082\end{array}$ & $\begin{array}{l}302 \\
200 \\
185 \\
148 \\
280 \\
173 \\
193 \\
142 \\
173 \\
93 \\
174 \\
272 \\
200 \\
247 \\
285 \\
302 \\
308 \\
270 \\
205\end{array}$ & $\begin{array}{l}2 \\
2 \\
2 \\
1 \\
1 \\
1 \\
1 \\
1 \\
10 \\
10 \\
- \\
- \\
= \\
- \\
- \\
- \\
-\end{array}$ & 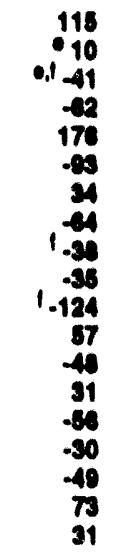 & $\begin{array}{r}9 \\
2 \\
1 \\
1 \\
1 \\
3 \\
3 \\
3 \\
5 \\
74 \\
64 \\
81 \\
67 \\
100 \\
68 \\
89 \\
97 \\
100 \\
215\end{array}$ & $\begin{array}{l}3,002 \\
2,940 \\
2,881 \\
3,133 \\
3,362 \\
3,432 \\
3,311 \\
2,868 \\
2,020 \\
2,671 \\
2,090 \\
2,848 \\
2,808 \\
2,014 \\
2,978 \\
3,122 \\
3,157 \\
3,021 \\
2,921\end{array}$ & $\begin{array}{l}108 \\
1200 \\
209 \\
186 \\
280 \\
216 \\
220 \\
1208 \\
102 \\
1179 \\
140 \\
181 \\
144 \\
185 \\
134 \\
124 \\
108 \\
132 \\
144\end{array}$ & $\begin{array}{l}\text { NA } \\
\text { NA } \\
\text { NA } \\
\text { NA } \\
\text { NA } \\
\text { NA } \\
\text { NA } \\
\text { NA } \\
N A \\
N A \\
N A \\
N A \\
N A \\
N A \\
N A \\
N A \\
\text { NA } \\
\text { NA } \\
\text { NA }\end{array}$ & $\begin{array}{l}\text { NA } \\
\text { NA } \\
\text { NA } \\
\text { NA } \\
\text { NA } \\
\text { NA } \\
\text { NA } \\
N A \\
N A \\
N A \\
N A \\
N A \\
N A \\
N A \\
N A \\
\text { NA } \\
\text { NA } \\
\text { NA } \\
\text { NA }\end{array}$ \\
\hline 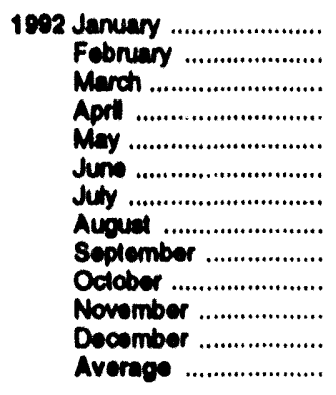 & $\begin{array}{l}2,818 \\
2,661 \\
2,749 \\
2,030 \\
2,030 \\
2,005 \\
3,067 \\
2,865 \\
2,903 \\
3,251 \\
3,240 \\
3,170 \\
2,974\end{array}$ & $\begin{array}{l}232 \\
217 \\
238 \\
202 \\
170 \\
167 \\
172 \\
229 \\
237 \\
263 \\
238 \\
229 \\
216\end{array}$ & $\begin{array}{l}- \\
- \\
- \\
- \\
- \\
- \\
- \\
- \\
-\end{array}$ & $\begin{array}{r}.541 \\
.610 \\
.368 \\
.185 \\
130 \\
268 \\
328 \\
202 \\
168 \\
290 \\
318 \\
.183 \\
-1\end{array}$ & $\begin{array}{l}360 \\
278 \\
138 \\
278 \\
222 \\
205 \\
201 \\
127 \\
145 \\
169 \\
230 \\
276 \\
219\end{array}$ & $\begin{array}{l}3,231 \\
3,219 \\
3,207 \\
3,039 \\
2,753 \\
2,679 \\
2,710 \\
2,705 \\
2,008 \\
3,066 \\
2,929 \\
3,316 \\
2,979\end{array}$ & $\begin{array}{r}127 \\
109 \\
98 \\
92 \\
96 \\
104 \\
115 \\
123 \\
128 \\
137 \\
146 \\
141 \\
141\end{array}$ & $\begin{array}{l}\text { NA } \\
\text { NA } \\
\text { NA } \\
\text { NA } \\
\text { NA } \\
\text { NA } \\
\text { NA } \\
\text { NA } \\
\text { NA } \\
\text { NA } \\
\text { NA } \\
\text { NA }\end{array}$ & $\begin{array}{l}\text { NA } \\
\text { NA } \\
\text { NA } \\
\text { NA } \\
\text { NA } \\
\text { NA } \\
\text { NA } \\
\text { NA } \\
\text { NA } \\
\text { NA } \\
\text { NA } \\
\text { NA }\end{array}$ \\
\hline 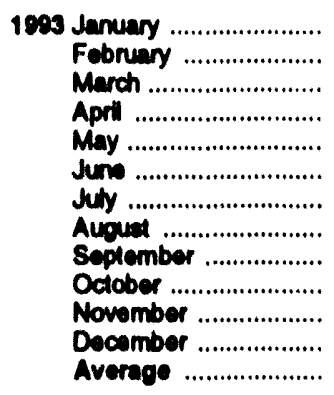 & $\begin{array}{l}2,809 \\
2,813 \\
2,818 \\
3,010 \\
2,230 \\
3,005 \\
3,185 \\
3,084 \\
3,206 \\
3,435 \\
3,478 \\
3,385 \\
3,123\end{array}$ & $\begin{array}{l}182 \\
224 \\
235 \\
209 \\
153 \\
168 \\
130 \\
159 \\
137 \\
242 \\
214 \\
160 \\
184\end{array}$ & $\begin{array}{l}- \\
- \\
- \\
- \\
- \\
- \\
- \\
- \\
- \\
- \\
- \\
-\end{array}$ & $\begin{array}{r}.336 \\
.742 \\
.388 \\
30 \\
104 \\
263 \\
348 \\
249 \\
80 \\
467 \\
158 \\
.268 \\
2\end{array}$ & $\begin{array}{l}287 \\
301 \\
154 \\
241 \\
355 \\
158 \\
298 \\
197 \\
262 \\
241 \\
330 \\
460 \\
274\end{array}$ & $\begin{array}{l}3,141 \\
3,478 \\
3,386 \\
2,949 \\
2,624 \\
2,843 \\
2,669 \\
2,797 \\
3,001 \\
2,868 \\
3,206 \\
3,351 \\
3,031\end{array}$ & $\begin{array}{r}130 \\
109 \\
97 \\
98 \\
102 \\
109 \\
120 \\
128 \\
130 \\
145 \\
150 \\
141 \\
141\end{array}$ & $\begin{array}{r}922 \\
16 \\
12 \\
13 \\
14 \\
17 \\
23 \\
46 \\
47 \\
55 \\
64 \\
63 \\
63\end{array}$ & $\begin{array}{r}9_{108} \\
94 \\
85 \\
86 \\
87 \\
92 \\
97 \\
83 \\
84 \\
90 \\
85 \\
79 \\
79\end{array}$ \\
\hline 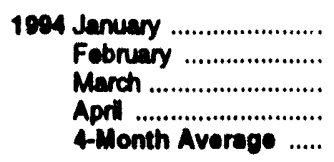 & $\begin{array}{r}\mathbf{3}, 117 \\
\mathbf{3 , 0 1 9} \\
\text { ค }_{\mathbf{3}, 095} \\
\mathrm{E}_{\mathbf{3}, \mathbf{3 0 9}} \\
\mathrm{E}_{\mathbf{3}, \mathbf{1 3 7}}\end{array}$ & $\begin{array}{r}160 \\
276 \\
A_{313} \\
E_{225} \\
E_{243}\end{array}$ & $\begin{array}{l}- \\
- \\
-\end{array}$ & $\begin{array}{r}.748 \\
.505 \\
\text { ค. } 142 \\
E .83 \\
E .327\end{array}$ & $\begin{array}{r}332 \\
235 \\
\text { A }_{220} \\
E_{173} \\
E_{241}\end{array}$ & $\begin{array}{r}3,682 \\
3,585 \\
R_{3,330} \\
E_{3,278} \\
E_{3,465}\end{array}$ & $\begin{array}{r}118 \\
104 \\
\text { A } 100 \\
E_{102} \\
E_{102}\end{array}$ & $\begin{array}{r}56 \\
49 \\
50 \\
\text { E } 55 \\
\text { E } 55\end{array}$ & $\begin{array}{r}62 \\
55 \\
A_{50} \\
E_{47} \\
E_{47}\end{array}$ \\
\hline $\begin{array}{l}1093 \text { 4-Month Average ..... } \\
1902 \text { 4-Month Average ..... }\end{array}$ & $\begin{array}{l}2,914 \\
2,790\end{array}$ & $\begin{array}{l}212 \\
222\end{array}$ & - & $\begin{array}{l}-392 \\
-426\end{array}$ & $\begin{array}{l}244 \\
263\end{array}$ & $\begin{array}{l}3,235 \\
3,174\end{array}$ & $\begin{array}{l}98 \\
92\end{array}$ & $\begin{array}{r}13 \\
\text { NA }\end{array}$ & $\begin{array}{l}86 \\
\text { NA }\end{array}$ \\
\hline
\end{tabular}

- Siocks are lotals as of end of period.

b Becinning in January 1883, cruds oll used directly as distilate fuol oll b reported conde oll product supplied on Table $3.2 \mathrm{~b}$ rather than as diatiliale fuel oll product supplied.

- A negative number indicales a decrease in stocks and a postive number Indicales en increase.

By welcht.

- See Note 6 at end of section.

1 See Nate 4 at end of section.
- Seo Note 3 at end of section.

R=Revised data. NA=Nol available. - Not applicable. $E=E$ Estimale.

Notes: - Geographic coverage the 50 States and the District of Columbla. - Totals may not equal sum of components due to independent rounding.

Scurces: - 1973-1900: Energy Information Administration (E/A), Potroloum Supply Monthy, Fobruary 1993, Table S5. - 1981 lorward: EIA, Potroloum Supply Monthly, May 1994, Table S5. 
Flgure 3.4 Residual Fuel

(Million Barrels per Day, Except as Noted)

Overview, 1973-1993

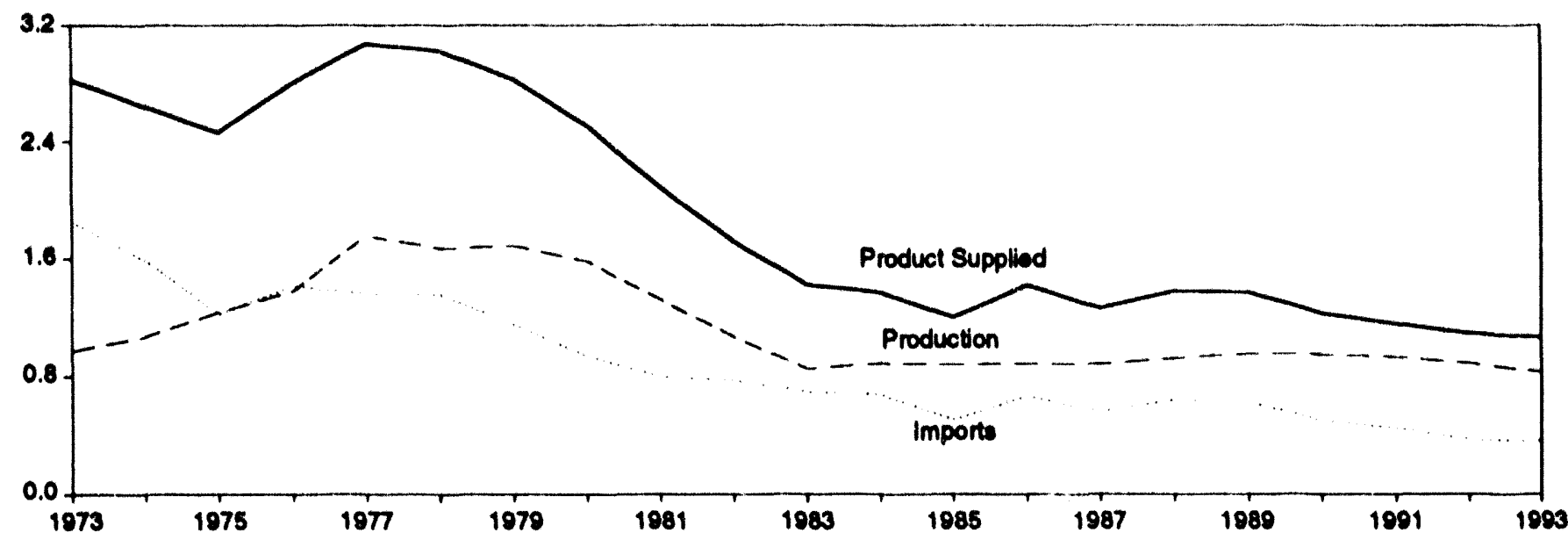

\section{Overview, Monthly}

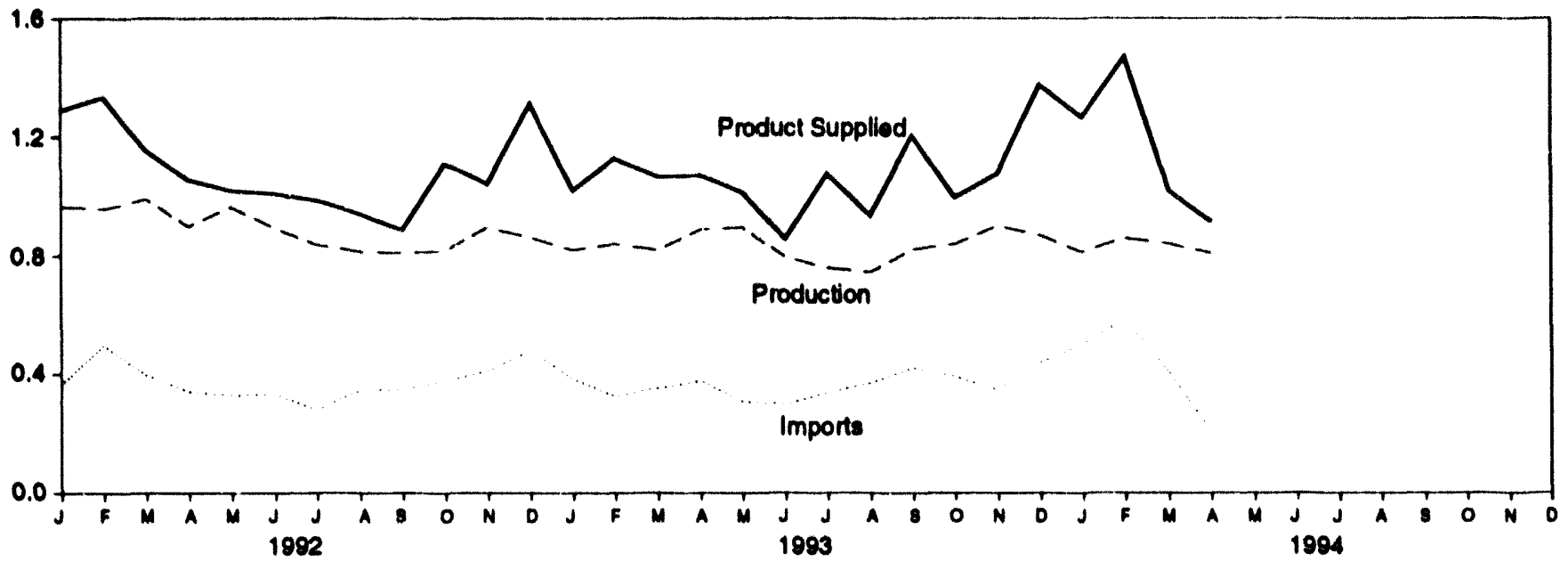

Product Supplied, January-April

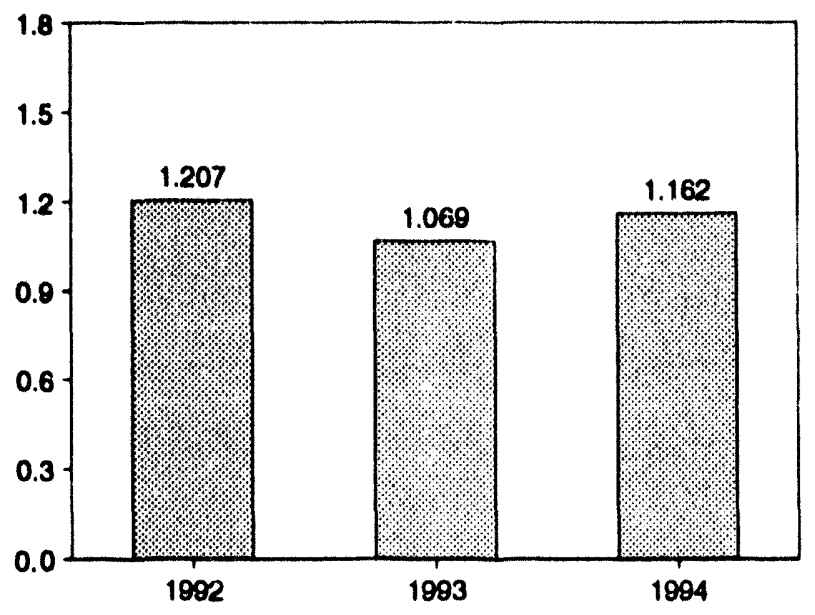

Stocks, End of Month

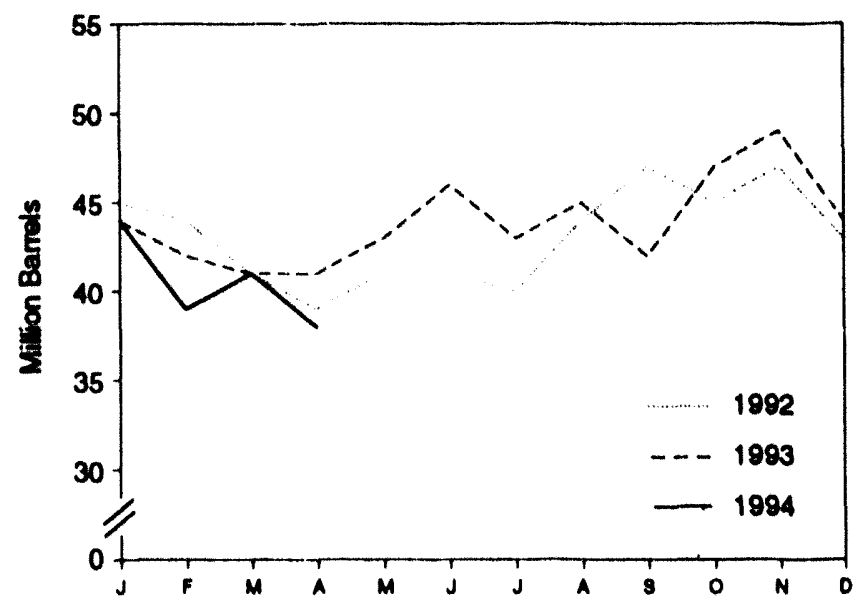

Note: Because verilcal scales dilfer, graphs should not be compared. Source: Table $\mathbf{3 . 6 .}$ 


\begin{tabular}{|c|c|c|c|c|c|c|c|}
\hline & \multicolumn{3}{|c|}{ Supply } & \multicolumn{3}{|c|}{ Diepostion } & \multirow{3}{*}{$\begin{array}{c}\text { Ending } \\
\text { stookic }\end{array}$} \\
\hline & $\begin{array}{l}\text { Toun } \\
\text { Producton }\end{array}$ & Importe & $\begin{array}{l}\text { Cnude Oll } \\
\text { Uesd } \\
\text { Directly }\end{array}$ & $\begin{array}{l}\text { stock } \\
\text { Changeb }\end{array}$ & Exports & $\begin{array}{l}\text { Product } \\
\text { supplled }\end{array}$ & \\
\hline & \multicolumn{6}{|c|}{ Thousand Barrels per Day } & \\
\hline 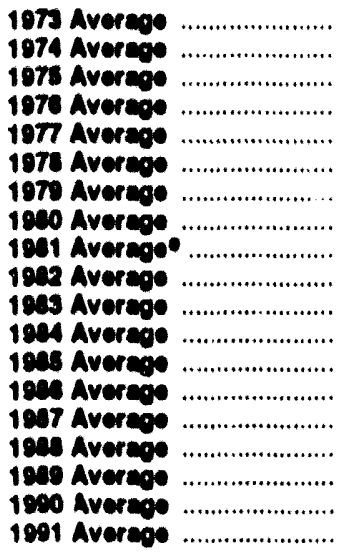 & $\begin{array}{l}971 \\
1,070 \\
1,238 \\
1,377 \\
1,794 \\
1,697 \\
1,097 \\
1,590 \\
1,321 \\
1,070 \\
852 \\
891 \\
192 \\
890 \\
895 \\
928 \\
984 \\
860 \\
934\end{array}$ & $\begin{array}{l}1,853 \\
1,507 \\
1,223 \\
1,413 \\
1,350 \\
1,385 \\
1,181 \\
930 \\
800 \\
770 \\
690 \\
801 \\
510 \\
609 \\
565 \\
644 \\
620 \\
504 \\
453\end{array}$ & $\begin{array}{l}17 \\
13 \\
15 \\
17 \\
13 \\
13 \\
12 \\
12 \\
48 \\
4 \\
- \\
- \\
- \\
- \\
- \\
- \\
-\end{array}$ & $\begin{array}{r}-5 \\
17 \\
0.2 \\
-5 \\
48 \\
1 \\
15 \\
-10 \\
0.37 \\
-32 \\
0.58 \\
12 \\
.7 \\
-1 \\
(5) \\
5 \\
-2 \\
13 \\
4\end{array}$ & $\begin{array}{r}23 \\
14 \\
15 \\
12 \\
6 \\
13 \\
9 \\
33 \\
118 \\
200 \\
185 \\
100 \\
197 \\
147 \\
106 \\
200 \\
215 \\
211 \\
226\end{array}$ & $\begin{array}{l}2,822 \\
2,639 \\
2,462 \\
2,801 \\
3,071 \\
3,023 \\
2,826 \\
2,508 \\
2,088 \\
1,716 \\
1,421 \\
1,369 \\
1,202 \\
1,418 \\
1,284 \\
1,378 \\
1,370 \\
1,229 \\
1,158\end{array}$ & $\begin{array}{r}53 \\
d 60 \\
74 \\
72 \\
90 \\
80 \\
96 \\
d 92 \\
78 \\
d 68 \\
40 \\
83 \\
50 \\
47 \\
47 \\
45 \\
44 \\
49 \\
50\end{array}$ \\
\hline 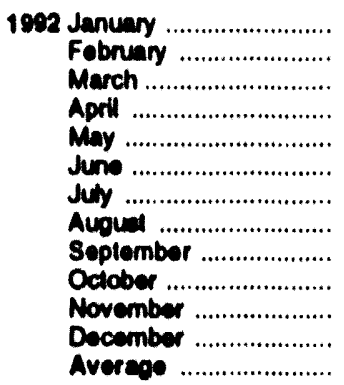 & $\begin{array}{l}965 \\
957 \\
900 \\
900 \\
904 \\
804 \\
838 \\
815 \\
810 \\
818 \\
805 \\
862 \\
092\end{array}$ & $\begin{array}{l}364 \\
488 \\
397 \\
342 \\
328 \\
334 \\
280 \\
347 \\
349 \\
376 \\
411 \\
481 \\
375\end{array}$ & $\begin{array}{l}- \\
- \\
- \\
- \\
- \\
- \\
- \\
-\end{array}$ & $\begin{array}{r}.144 \\
.55 \\
.77 \\
.78 \\
67 \\
.11 \\
.37 \\
125 \\
123 \\
.72 \\
49 \\
.127 \\
.20\end{array}$ & $\begin{array}{r}184 \\
176 \\
310 \\
265 \\
207 \\
230 \\
169 \\
96 \\
149 \\
158 \\
216 \\
159 \\
193\end{array}$ & $\begin{array}{r}1,289 \\
1,334 \\
1,154 \\
1,055 \\
1,010 \\
1,009 \\
986 \\
941 \\
887 \\
1,110 \\
1,041 \\
1,312 \\
1,094\end{array}$ & $\begin{array}{l}45 \\
44 \\
41 \\
30 \\
41 \\
41 \\
40 \\
44 \\
47 \\
45 \\
47 \\
43 \\
43\end{array}$ \\
\hline 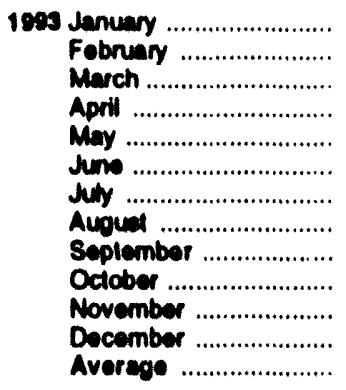 & $\begin{array}{l}820 \\
841 \\
819 \\
887 \\
898 \\
797 \\
760 \\
745 \\
822 \\
839 \\
001 \\
809 \\
833\end{array}$ & $\begin{array}{l}383 \\
325 \\
352 \\
377 \\
308 \\
299 \\
337 \\
370 \\
420 \\
391 \\
347 \\
439 \\
363\end{array}$ & $\begin{array}{l}- \\
\overline{-} \\
\overline{-} \\
\overline{-} \\
\overline{-} \\
\overline{-} \\
-\end{array}$ & $\begin{array}{r}49 \\
.75 \\
.46 \\
24 \\
53 \\
92 \\
-101 \\
61 \\
.73 \\
141 \\
85 \\
.164 \\
4\end{array}$ & $\begin{array}{r}133 \\
113 \\
152 \\
169 \\
137 \\
147 \\
122 \\
120 \\
110 \\
94 \\
88 \\
98 \\
123\end{array}$ & $\begin{array}{r}1.020 \\
1,128 \\
1.065 \\
1.070 \\
1.014 \\
857 \\
1.075 \\
235 \\
1,205 \\
905 \\
1.077 \\
1.375 \\
1,068\end{array}$ & $\begin{array}{l}44 \\
42 \\
41 \\
41 \\
43 \\
46 \\
43 \\
45 \\
42 \\
47 \\
49 \\
44 \\
44\end{array}$ \\
\hline 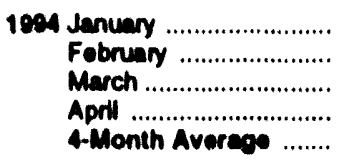 & $\begin{array}{r}813 \\
850 \\
R_{841} \\
\varepsilon_{810} \\
E_{830}\end{array}$ & $\begin{array}{r}503 \\
586 \\
\text { ค } 407 \\
207 \\
207\end{array}$ & $\begin{array}{l}- \\
- \\
-\end{array}$ & $\begin{array}{r}-16 \\
.152 \\
R_{54} \\
E_{.69}^{-69} \\
.-43\end{array}$ & $\begin{array}{r}64 \\
127 \\
P_{175} \\
E_{171} \\
134\end{array}$ & $\begin{array}{r}1,287 \\
1,470 \\
P_{1,019} \\
815 \\
1,162\end{array}$ & $\begin{array}{r}44 \\
30 \\
41 \\
39 \\
59\end{array}$ \\
\hline $\begin{array}{l}1009 \text { 4-Month Averese ....... } \\
1002 \text { 4-Month Average ....... }\end{array}$ & $\begin{array}{l}91 \\
953\end{array}$ & $\begin{array}{l}360 \\
390\end{array}$ & - & $\begin{array}{l}.11 \\
.89\end{array}$ & $\begin{array}{l}142 \\
234\end{array}$ & $\begin{array}{l}1,069 \\
1,207\end{array}$ & $\begin{array}{l}41 \\
30\end{array}$ \\
\hline
\end{tabular}

- Beginning in January 1083, crudo oll used directly as realdual fual oil is reported as crude oll product supplbd on Table $3.2 \mathrm{~b}$ rather than as residua luel oil product supplisd.

b A negaltu number indicates a dacrease in slocks and a positive number indicalcs on increase.

- Siocks are tolats as of end of period.

d See Nole 4 at end of section.
- See Nole 3 al end ol section.

Rarevibed data. - =Not applicable. Esestimate, (s)=Less than +500 barrols per day and oreater than .500 barrels per day.

Nole: Geopraphic coverage is the 50 States and the Distrid of Columbia

Sources: - 1973-1080: Energy Intormation Admintatration (ELA). Petrobum Supply Monthy. Fobnuary 1993, Table S6. - 1981 fonward: EIA, Petrobum Supply Monthy, May 1894, Table S6. 
Total Jet Fuel Overview, 1973-1993

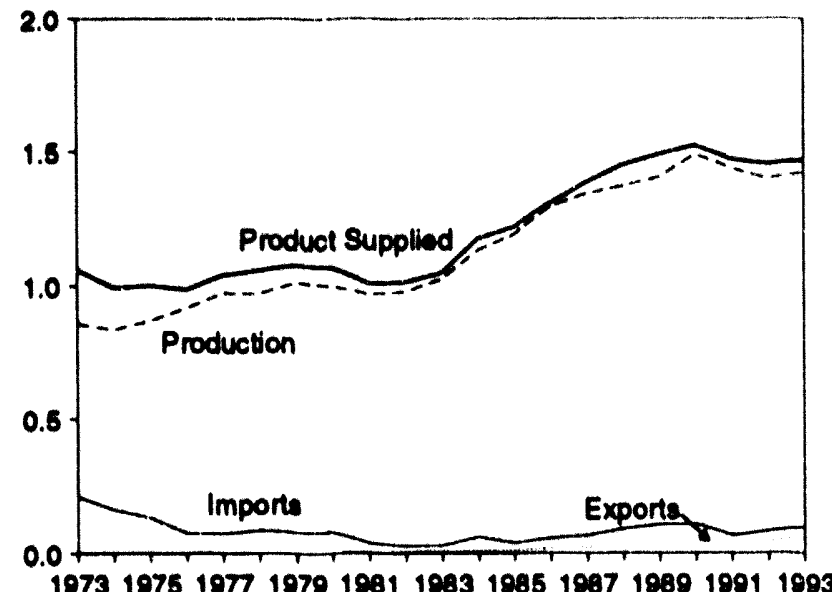

Product Supplied by Type, 1973-1993

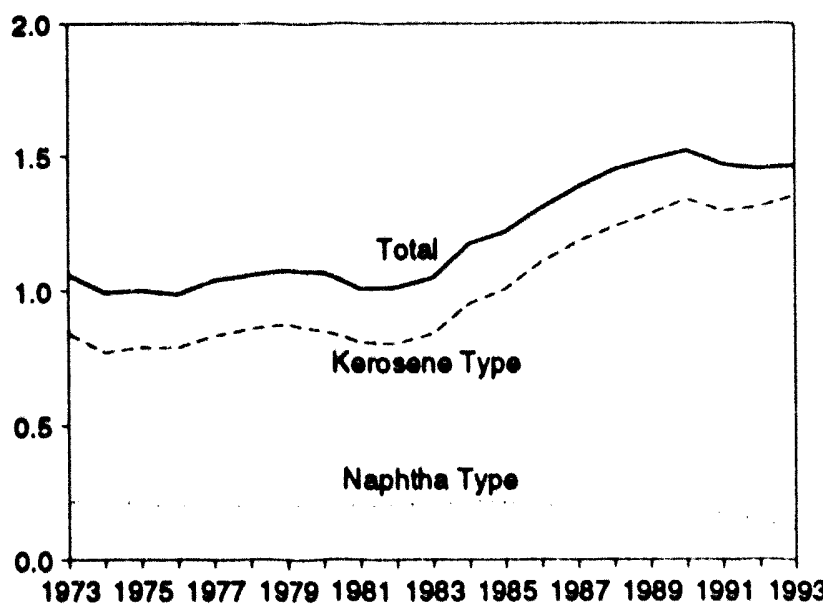

Total Product Supplied, January-April

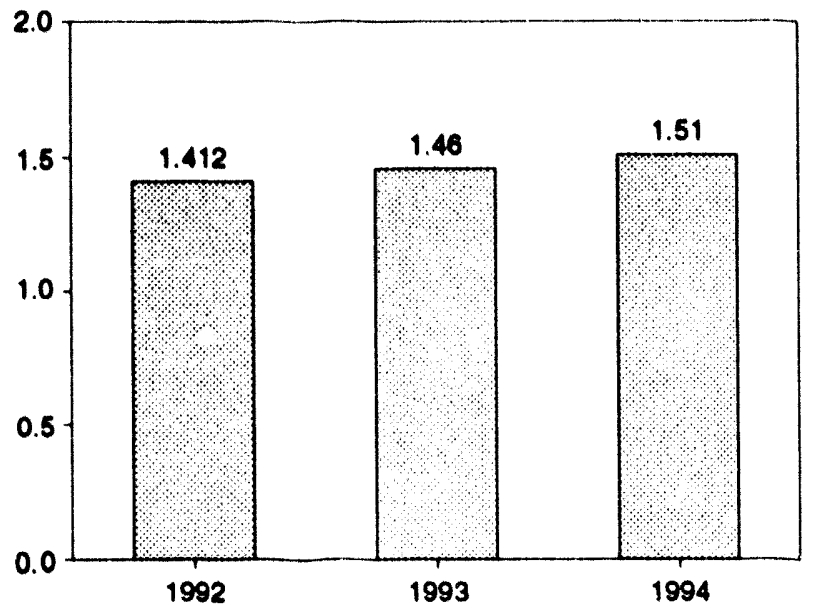

Total Jet Fuel Overview, Monthly

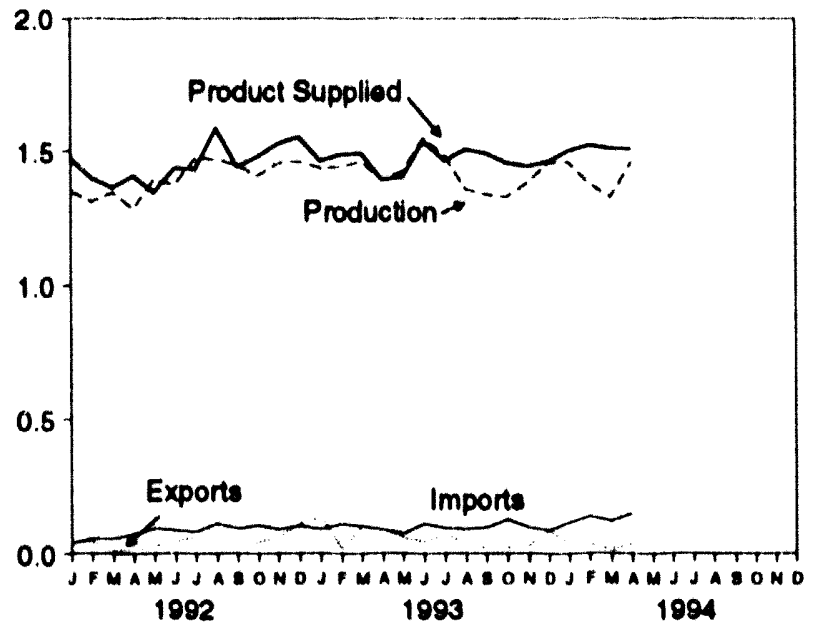

Product Supplied by Type, Monthly

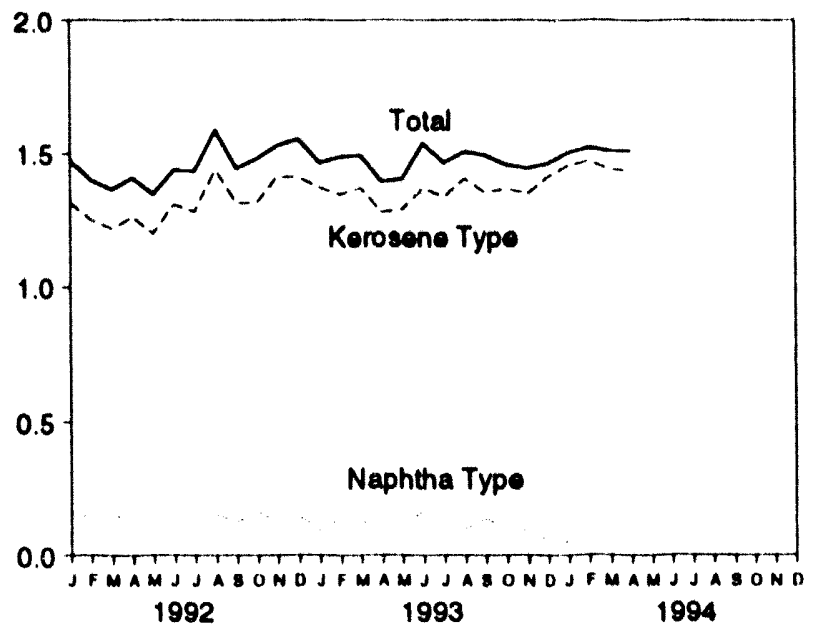

Total Stocks, End of Month

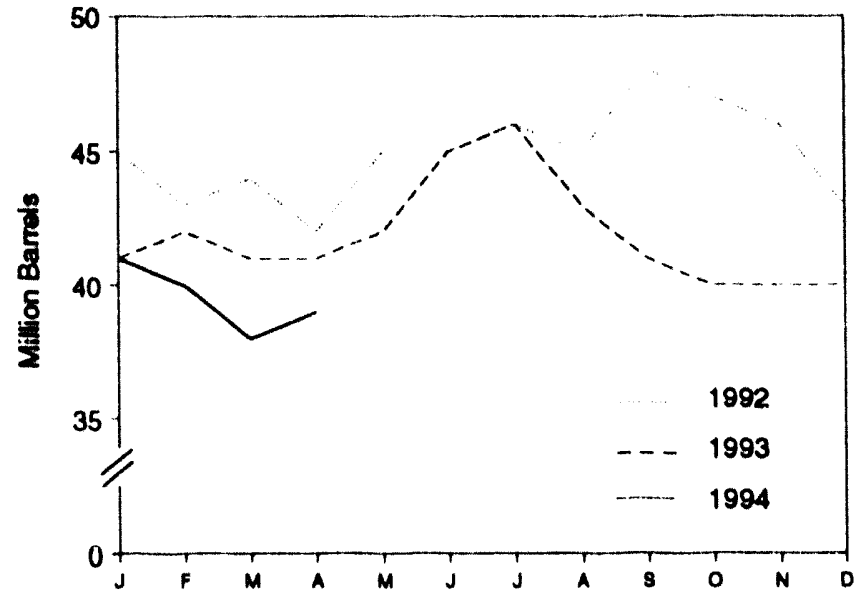

Source: Table 3.7. 
Table 3.7 Jet Fuel Supply and Disposition

\begin{tabular}{|c|c|c|c|c|c|c|c|c|c|}
\hline & \multicolumn{3}{|c|}{ Supply } & \multicolumn{4}{|c|}{ Dleposition } & & \\
\hline & \multicolumn{2}{|c|}{ Production } & \multirow[b]{2}{*}{ Importe } & \multirow{2}{*}{$\begin{array}{l}\text { stook } \\
\text { Changeb }\end{array}$} & \multirow[b]{2}{*}{ Exporte } & \multicolumn{2}{|c|}{ Product supplled } & \multicolumn{2}{|c|}{ Ending stootes } \\
\hline & Totell & Kerosene Type & & & & Toted & Kerosene Type & Total & Kerenens Typo \\
\hline & \multicolumn{7}{|c|}{ Thounand Barrels per Day } & \multicolumn{2}{|c|}{ Millon Barreb } \\
\hline 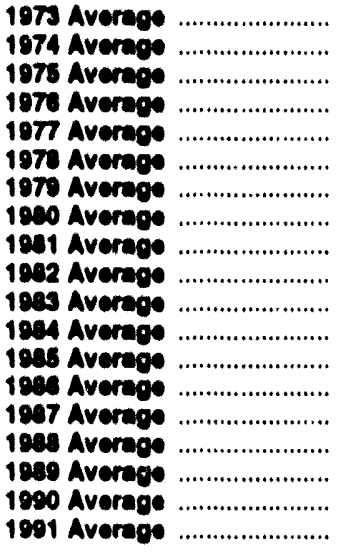 & $\begin{array}{r}260 \\
689 \\
671 \\
910 \\
973 \\
970 \\
1,012 \\
090 \\
098 \\
970 \\
1,022 \\
1,132 \\
1,100 \\
1,293 \\
1,349 \\
1,370 \\
1,403 \\
1,408 \\
1,438\end{array}$ & $\begin{array}{l}670 \\
641 \\
691 \\
731 \\
707 \\
791 \\
638 \\
811 \\
778 \\
778 \\
817 \\
910 \\
983 \\
1,007 \\
1,138 \\
1,164 \\
1,197 \\
1,311 \\
1,274\end{array}$ & $\begin{array}{r}212 \\
143 \\
133 \\
70 \\
78 \\
08 \\
76 \\
60 \\
38 \\
29 \\
20 \\
62 \\
30 \\
57 \\
67 \\
00 \\
108 \\
108 \\
87\end{array}$ & $\begin{array}{r}0 \\
2 \\
62 \\
5 \\
7 \\
-2 \\
13 \\
10 \\
0-4 \\
\cdot 12 \\
(0) \\
9 \\
-4 \\
28 \\
(0) \\
-17 \\
-9 \\
31 \\
-9\end{array}$ & $\begin{array}{r}1 \\
3 \\
2 \\
2 \\
2 \\
1 \\
1 \\
1 \\
2 \\
6 \\
6 \\
9 \\
13 \\
18 \\
24 \\
28 \\
27 \\
43 \\
43\end{array}$ & $\begin{array}{r}1,050 \\
093 \\
1,001 \\
087 \\
1,030 \\
1,057 \\
1,070 \\
1,068 \\
1,007 \\
1,013 \\
1,046 \\
1,175 \\
1,218 \\
1,307 \\
1,395 \\
1,440 \\
1,460 \\
1,522 \\
1,471\end{array}$ & $\begin{array}{r}842 \\
771 \\
791 \\
708 \\
831 \\
869 \\
870 \\
851 \\
800 \\
804 \\
839 \\
853 \\
1,005 \\
1,105 \\
1,181 \\
1,238 \\
1,284 \\
1,340 \\
1,298\end{array}$ & $\begin{array}{r}20 \\
020 \\
30 \\
32 \\
35 \\
34 \\
30 \\
642 \\
41 \\
637 \\
30 \\
42 \\
40 \\
80 \\
60 \\
44 \\
41 \\
52 \\
40\end{array}$ & $\begin{array}{r}23 \\
024 \\
28 \\
28 \\
28 \\
28 \\
33 \\
638 \\
34 \\
631 \\
32 \\
35 \\
34 \\
43 \\
42 \\
34 \\
34 \\
46 \\
44\end{array}$ \\
\hline 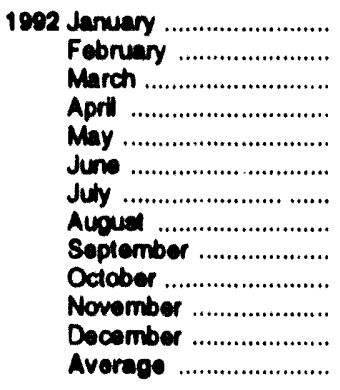 & $\begin{array}{l}1,352 \\
1,311 \\
1,347 \\
1,286 \\
1,393 \\
1,374 \\
1,473 \\
1,471 \\
1,448 \\
1,408 \\
1,466 \\
1,462 \\
1,390\end{array}$ & $\begin{array}{l}1,200 \\
1,164 \\
1,215 \\
1,131 \\
1,214 \\
1,234 \\
1,328 \\
1,339 \\
1,296 \\
1,265 \\
1,319 \\
1,336 \\
1,254\end{array}$ & $\begin{array}{r}39 \\
56 \\
56 \\
74 \\
93 \\
86 \\
81 \\
111 \\
93 \\
105 \\
90 \\
102 \\
82\end{array}$ & $\begin{array}{r}.127 \\
.73 \\
31 \\
.68 \\
114 \\
.21 \\
59 \\
.32 \\
78 \\
.12 \\
.41 \\
.101 \\
.16\end{array}$ & $\begin{array}{r}44 \\
42 \\
7 \\
18 \\
26 \\
45 \\
62 \\
28 \\
20 \\
44 \\
59 \\
112 \\
43\end{array}$ & $\begin{array}{l}1.473 \\
1,398 \\
1,385 \\
1.409 \\
1.346 \\
1.436 \\
1,433 \\
1,585 \\
1,442 \\
1.480 \\
1,528 \\
1,653 \\
1,454\end{array}$ & $\begin{array}{l}1,314 \\
1,250 \\
1,218 \\
1,262 \\
1,198 \\
1,308 \\
1,280 \\
1,438 \\
1,313 \\
1,315 \\
1,411 \\
1,410 \\
1,310\end{array}$ & $\begin{array}{l}46 \\
43 \\
44 \\
42 \\
45 \\
45 \\
46 \\
46 \\
48 \\
47 \\
46 \\
43 \\
43\end{array}$ & $\begin{array}{l}40 \\
38 \\
30 \\
37 \\
40 \\
39 \\
42 \\
41 \\
43 \\
43 \\
41 \\
30 \\
30\end{array}$ \\
\hline 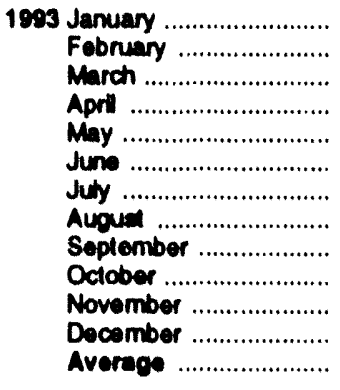 & $\begin{array}{l}1,437 \\
1,442 \\
1,463 \\
1,390 \\
1,428 \\
1,540 \\
1,485 \\
1,358 \\
1,339 \\
1,330 \\
1,383 \\
1,456 \\
1,421\end{array}$ & $\begin{array}{l}1,306 \\
1,318 \\
1,332 \\
1,262 \\
1,300 \\
1,409 \\
1,350 \\
1,257 \\
1,242 \\
1,242 \\
1,298 \\
1,370 \\
1,309\end{array}$ & $\begin{array}{r}89 \\
110 \\
102 \\
88 \\
75 \\
111 \\
94 \\
91 \\
97 \\
127 \\
96 \\
86 \\
97\end{array}$ & $\begin{array}{r}.73 \\
46 \\
.29 \\
.4 \\
37 \\
78 \\
41 \\
.91 \\
.78 \\
.24 \\
6 \\
.6 \\
-6\end{array}$ & $\begin{array}{r}134 \\
17 \\
101 \\
88 \\
60 \\
45 \\
73 \\
34 \\
21 \\
23 \\
29 \\
85 \\
60\end{array}$ & $\begin{array}{l}1,464 \\
1,468 \\
1,493 \\
1,393 \\
1,404 \\
1,538 \\
1,465 \\
1,506 \\
1,493 \\
1,467 \\
1,443 \\
1,462 \\
1,467\end{array}$ & $\begin{array}{l}1,371 \\
1,346 \\
1,371 \\
1,270 \\
1,269 \\
1,370 \\
1,337 \\
1,405 \\
1,352 \\
1,367 \\
1,347 \\
1,411 \\
1,354\end{array}$ & $\begin{array}{l}41 \\
42 \\
41 \\
41 \\
42 \\
45 \\
46 \\
43 \\
41 \\
40 \\
40 \\
40 \\
40\end{array}$ & $\begin{array}{l}38 \\
38 \\
37 \\
37 \\
38 \\
41 \\
42 \\
30 \\
38 \\
37 \\
37 \\
38 \\
38\end{array}$ \\
\hline 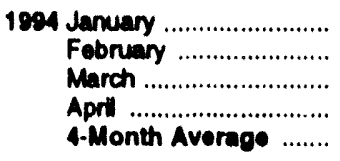 & $\begin{array}{r}1,461 \\
1,379 \\
R 1,327 \\
E_{1,462} \\
E_{1,408}\end{array}$ & $\begin{array}{l}1,384 \\
1,331 \\
A 1,271 \\
E_{1,413} \\
E_{1,352}\end{array}$ & $\begin{array}{r}116 \\
138 \\
P_{120} \\
E_{151} \\
E_{131}\end{array}$ & $\begin{array}{r}36 \\
.41 \\
.77 \\
65 \\
E_{-4}^{-4}\end{array}$ & $\begin{array}{r}40 \\
35 \\
\text { P }_{14} \\
E_{39} \\
E_{32}\end{array}$ & $\begin{array}{r}1,502 \\
1,522 \\
\text { ค } 1,509 \\
\varepsilon_{1,509} \\
E_{1,510}\end{array}$ & $\begin{array}{r}1,453 \\
1,471 \\
R_{1,440} \\
E 1,435 \\
E 1,449\end{array}$ & $\begin{array}{r}41 \\
40 \\
P_{38} \\
30 \\
39\end{array}$ & $\begin{array}{r}39 \\
38 \\
R_{36} \\
\varepsilon_{37} \\
\varepsilon_{37}\end{array}$ \\
\hline $\begin{array}{l}19934 \text { Month Average ...... } \\
1902 \text { 4-Month Average ....... }\end{array}$ & $\begin{array}{l}1,435 \\
1,324\end{array}$ & $\begin{array}{l}1,304 \\
1,178\end{array}$ & $\begin{array}{l}97 \\
56\end{array}$ & $\begin{array}{l}.18 \\
.59\end{array}$ & $\begin{array}{l}87 \\
28\end{array}$ & $\begin{array}{l}1,460 \\
1,412\end{array}$ & $\begin{array}{l}1,342 \\
1,261\end{array}$ & $\begin{array}{l}41 \\
42\end{array}$ & $\begin{array}{l}37 \\
37\end{array}$ \\
\hline
\end{tabular}

- Stocke are lotab es of end of period.

- A negative number indicates a decrease in slocks and a positive number indicates an morease.

- See Note 4 at end of section.

R=Pevieed data. ExEstimate. (s)=Less than +500 barrels per day and grealer than .500 barrels per day.

Note: Geopraphic coverage is the 50 States and the District of Coumbia.

Sources: - 1973-1980: Energy Intormation Administration (ELA), Petroleum Supply Monthy, February 1993, Table S7. - 1081 forward: ElA, Petroleum Supply Monthly, May 1994, Table S7. 
Figure 3.6 Liquefied Petroleum Gases

(Million Barrels per Day, Except as Noted)

Overview, 1973-1993

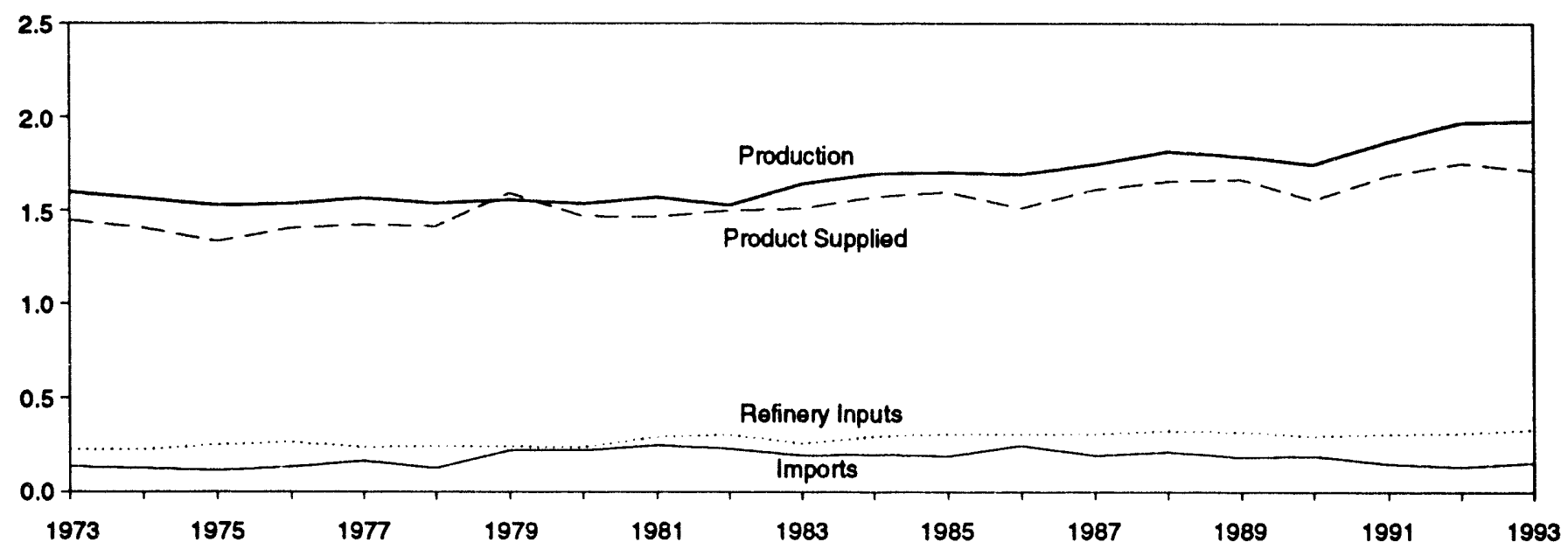

Overview, Monthly

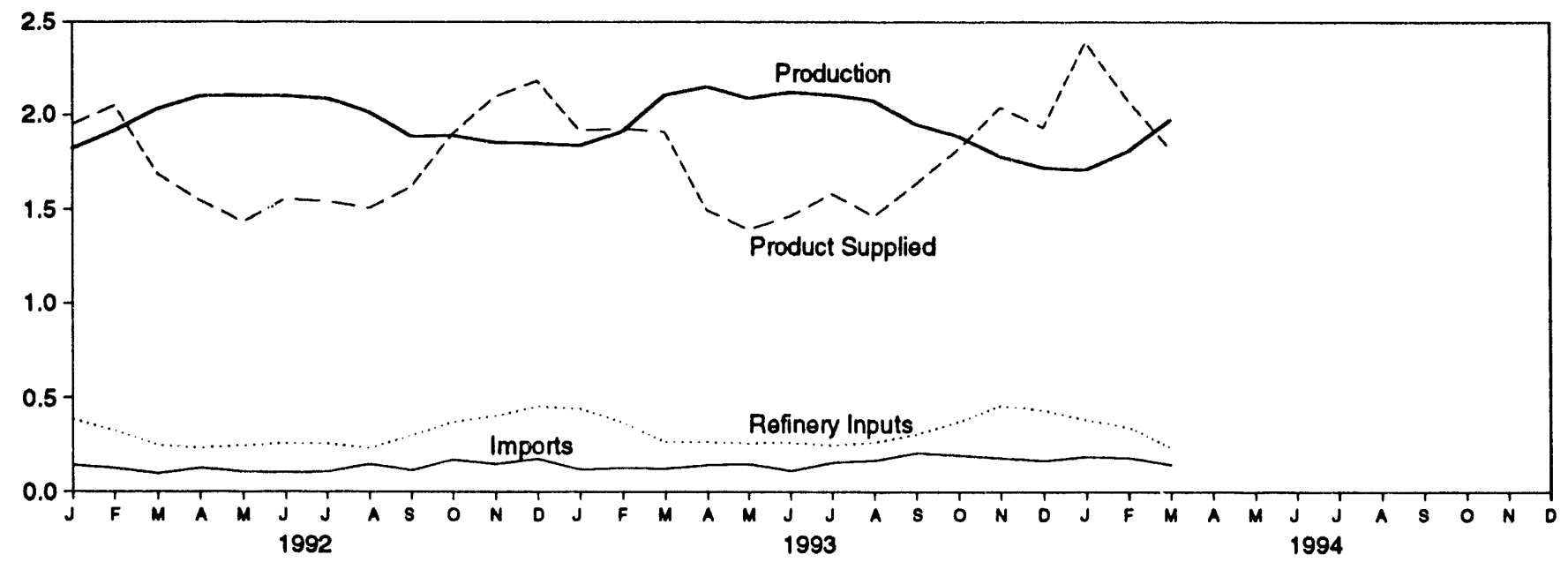

Product Supplied, January-March

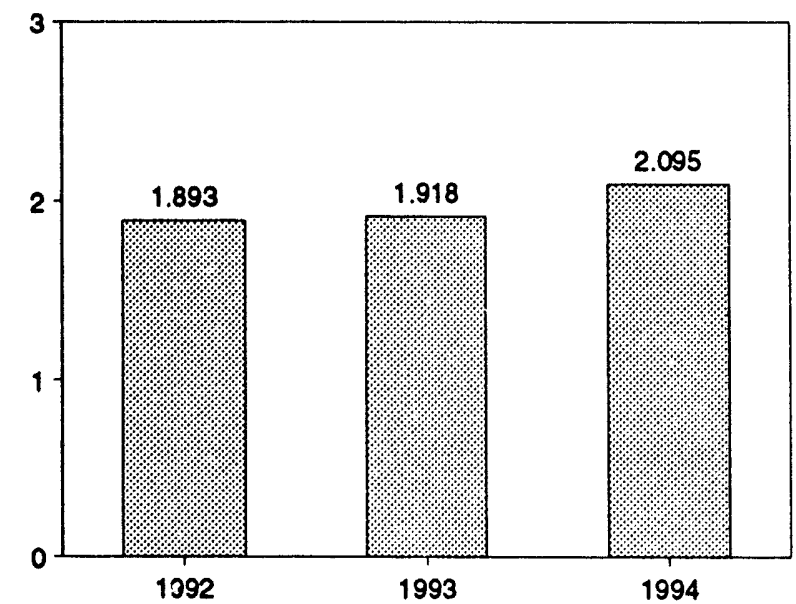

Stocks, End of Month

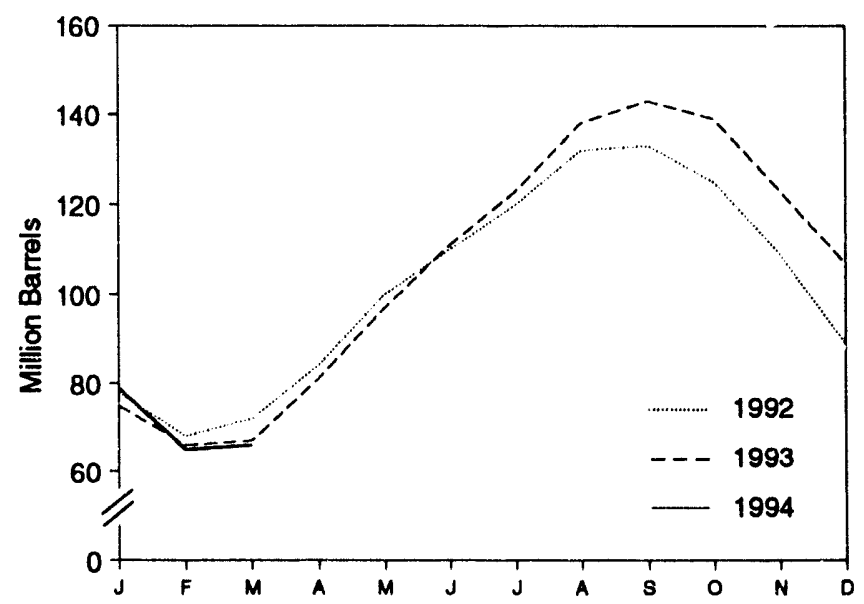

Note: Because vertical scales differ, graphs should not be compared. Source: Table $\mathbf{3 . 8}$. 
Table 3.8 Liquefied Petroleum Gases Supply and Disposition

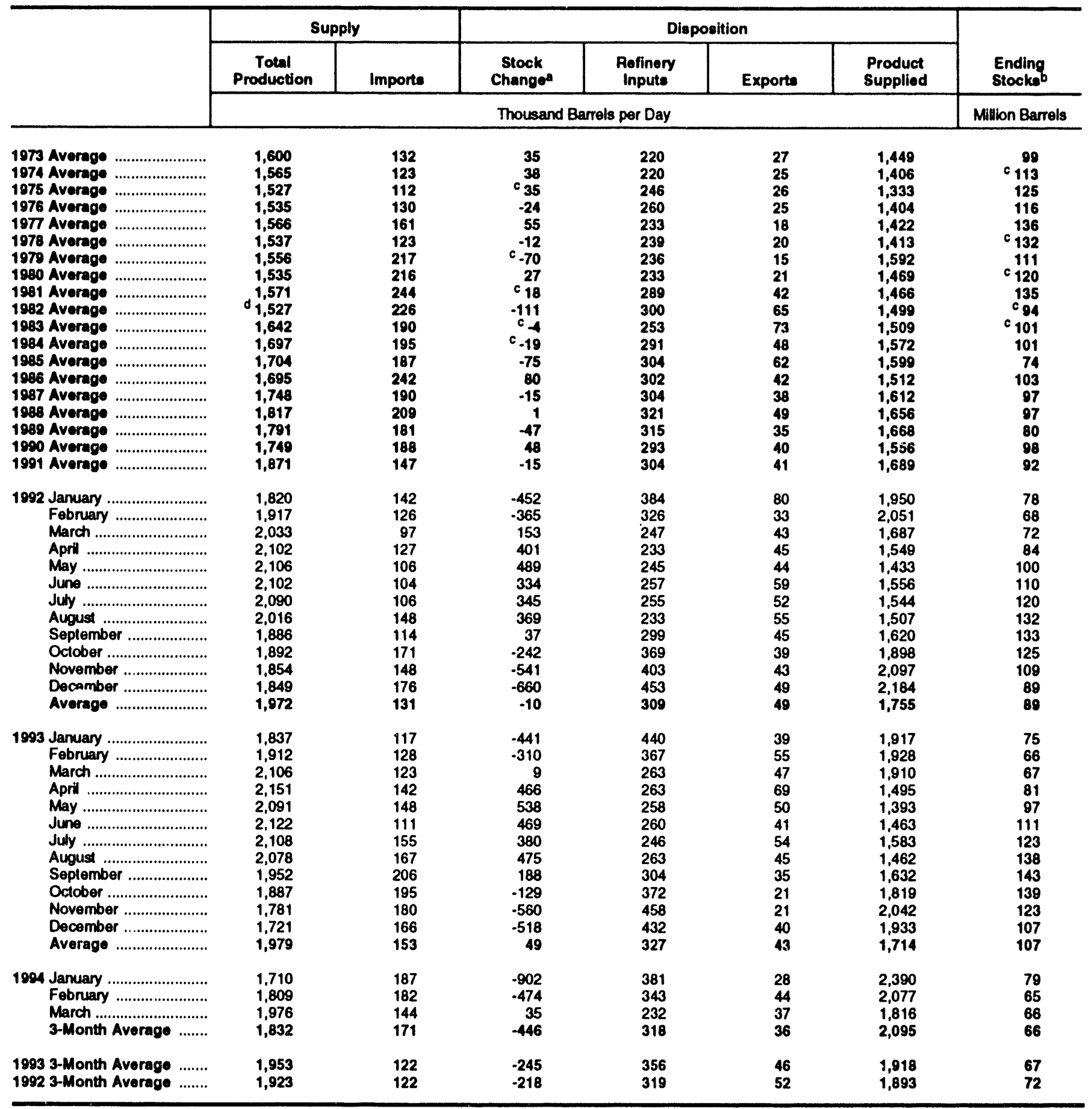

a negative number indicales a decrease in stocks and a positive number indicates an increase.

Stocks are lotals as of end of period.

c Seo Note 4 at end of section.

d See Nole 6 at end ol section.

Notes: - Liquefied petroteum gases include ethane, ethylene, propane,

propylene, normal butane, butylene, isobutane and isobutylene. - Geographic coverage is the 50 States and the District of Columbia.

Sources: - 1973-1980: Energy Information Administration (EIA). Petroleum Supply Monthly, February 1993, Table S8. - 1981 forward: EIA, Petroleum Supply Monthly, May 1994, Table S9. 
Figure 3.7 Propane and Propylene

(Million Barrels per Day, Except as Noted)

Overview, 1973-1993

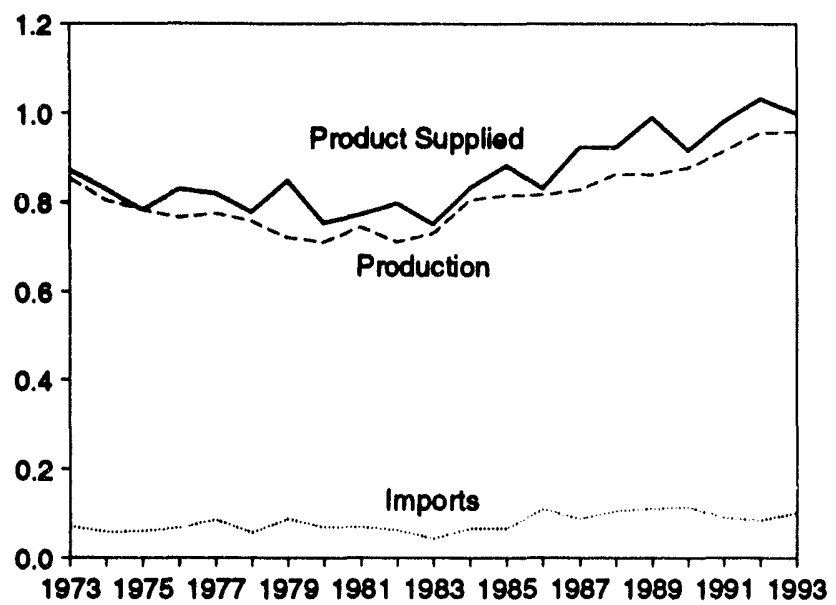

Product Supplied, Monthly

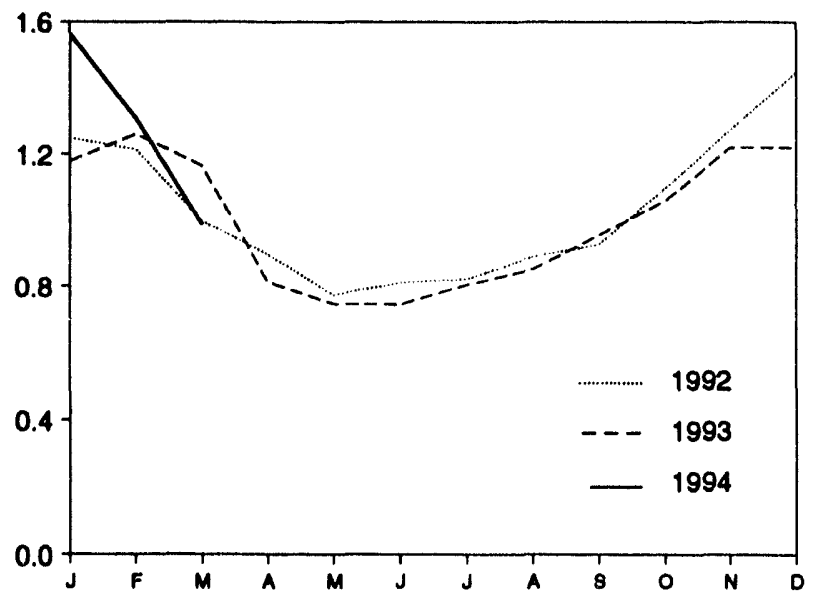

Stocks, End of Month

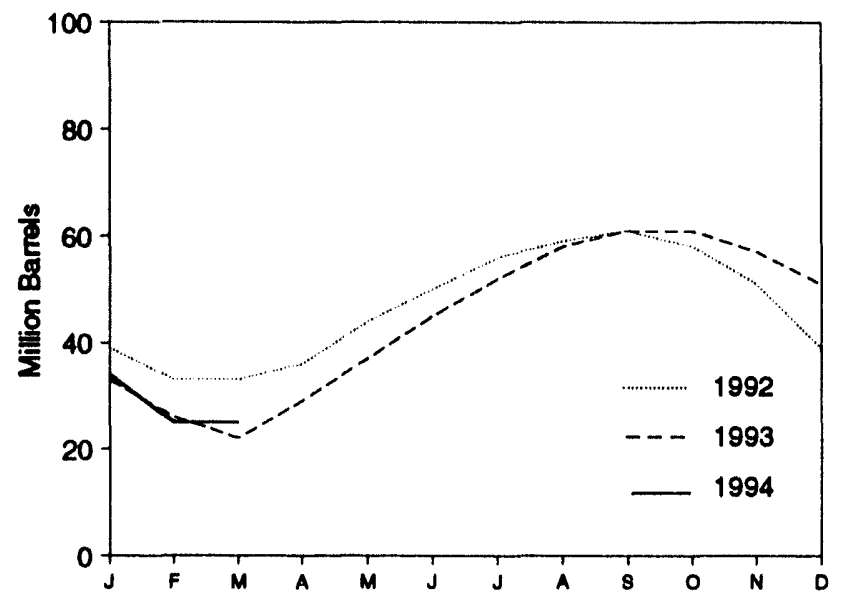

Product Supplied, January-March

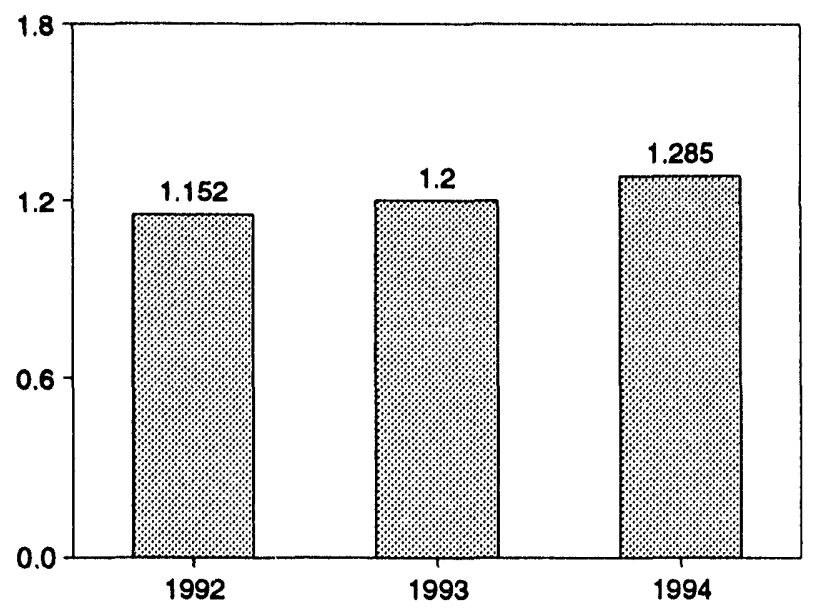

Share of Liquefied Petroleum Gases, March

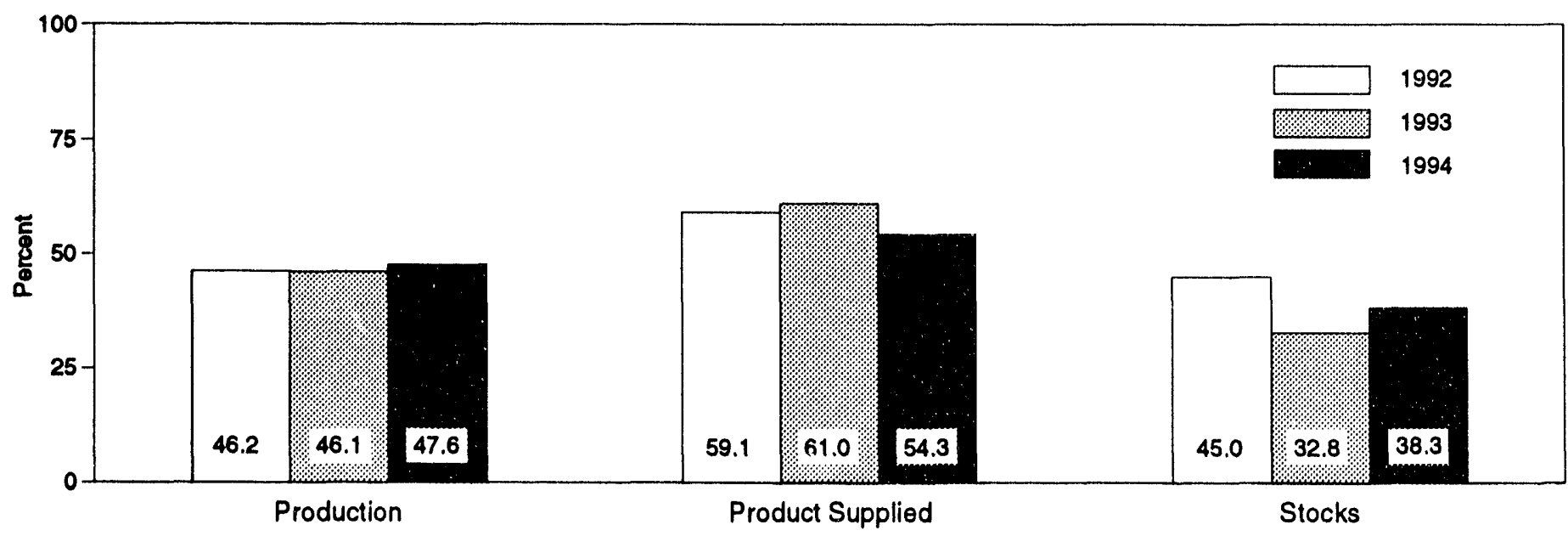

Note: Because vertical scales differ, graphs should not be compared.

Sources: Table 3.9 and, for calculation of shares, data prior to rounding for publication in Tables 3.8 and 3.9 . 
Table 3.9 Propane and Propylene Supply and Disposition (A Subset of Table 3.8)

\begin{tabular}{|c|c|c|c|c|c|c|c|}
\hline & \multicolumn{2}{|c|}{ Supply } & \multicolumn{4}{|c|}{ Disposition } & \multirow{3}{*}{$\begin{array}{c}\text { Ending } \\
\text { Stocks }\end{array}$} \\
\hline & $\begin{array}{l}\text { Total } \\
\text { Production }\end{array}$ & Imports & $\begin{array}{l}\text { Stock } \\
\text { Changea }\end{array}$ & $\begin{array}{l}\text { Refinery } \\
\text { Inputs }\end{array}$ & Exports & $\begin{array}{l}\text { Producte } \\
\text { Supplied }\end{array}$ & \\
\hline & \multicolumn{6}{|c|}{ Thousand Barrels per Day } & \\
\hline 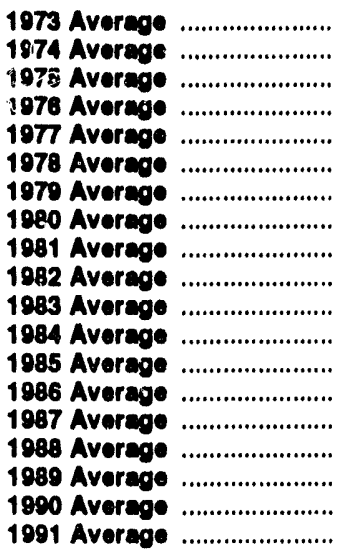 & $\begin{array}{l}854 \\
805 \\
783 \\
766 \\
775 \\
758 \\
721 \\
711 \\
745 \\
711 \\
730 \\
806 \\
816 \\
817 \\
828 \\
863 \\
862 \\
878 \\
915\end{array}$ & $\begin{array}{r}71 \\
59 \\
60 \\
68 \\
86 \\
57 \\
88 \\
69 \\
70 \\
63 \\
44 \\
67 \\
67 \\
110 \\
88 \\
106 \\
111 \\
115 \\
91\end{array}$ & $\begin{array}{r}30 \\
11 \\
36 \\
-22 \\
21 \\
15 \\
c-61 \\
4 \\
c 18 \\
-50 \\
-24 \\
c 7 \\
-50 \\
64 \\
-41 \\
7 \\
-52 \\
48 \\
-3\end{array}$ & $\begin{array}{r}8 \\
9 \\
11 \\
12 \\
10 \\
13 \\
14 \\
12 \\
5 \\
4 \\
4 \\
4 \\
3 \\
4 \\
8 \\
8 \\
11 \\
(8) \\
(8)\end{array}$ & $\begin{array}{r}15 \\
14 \\
13 \\
13 \\
10 \\
9 \\
8 \\
10 \\
18 \\
31 \\
43 \\
30 \\
48 \\
28 \\
24 \\
31 \\
24 \\
28 \\
28\end{array}$ & $\begin{array}{l}872 \\
830 \\
783 \\
830 \\
821 \\
778 \\
849 \\
754 \\
773 \\
798 \\
751 \\
833 \\
883 \\
831 \\
924 \\
923 \\
990 \\
917 \\
982\end{array}$ & $\begin{array}{r}65 \\
69 \\
82 \\
74 \\
81 \\
\circ 87 \\
64 \\
6 \quad 65 \\
76 \\
c 54 \\
648 \\
58 \\
39 \\
63 \\
48 \\
50 \\
32 \\
49 \\
48\end{array}$ \\
\hline 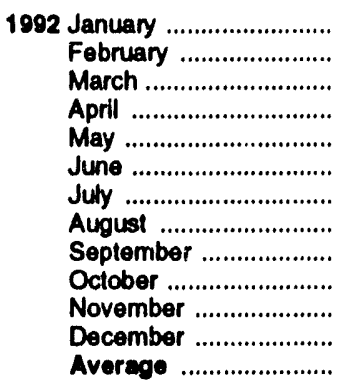 & $\begin{array}{l}949 \\
955 \\
940 \\
961 \\
977 \\
978 \\
964 \\
946 \\
931 \\
933 \\
964 \\
977 \\
956\end{array}$ & $\begin{array}{r}90 \\
86 \\
68 \\
80 \\
72 \\
66 \\
68 \\
85 \\
71 \\
104 \\
99 \\
131 \\
85\end{array}$ & $\begin{array}{r}-282 \\
-200 \\
-15 \\
120 \\
253 \\
206 \\
176 \\
117 \\
51 \\
-88 \\
-243 \\
-385 \\
-24\end{array}$ & $\begin{array}{r}(s) \\
(s) \\
(s) \\
0 \\
(s) \\
(s) \\
(s) \\
(s) \\
(s) \\
(s) \\
0 \\
0 \\
(s)\end{array}$ & $\begin{array}{l}72 \\
27 \\
26 \\
24 \\
23 \\
27 \\
35 \\
25 \\
25 \\
30 \\
33 \\
45 \\
33\end{array}$ & $\begin{array}{r}1,249 \\
1,214 \\
997 \\
896 \\
773 \\
811 \\
821 \\
889 \\
927 \\
1,095 \\
1,273 \\
1,448 \\
1,032\end{array}$ & $\begin{array}{l}39 \\
33 \\
33 \\
36 \\
44 \\
50 \\
56 \\
59 \\
61 \\
58 \\
51 \\
39 \\
39\end{array}$ \\
\hline 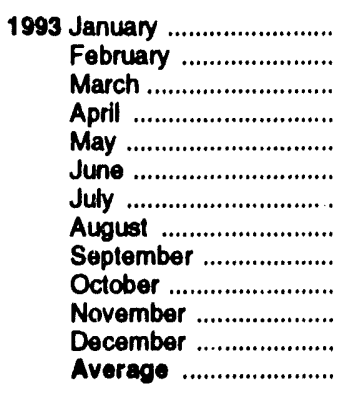 & $\begin{array}{l}965 \\
959 \\
971 \\
973 \\
942 \\
958 \\
956 \\
945 \\
956 \\
953 \\
964 \\
962 \\
959\end{array}$ & $\begin{array}{r}72 \\
78 \\
85 \\
112 \\
96 \\
75 \\
105 \\
116 \\
132 \\
107 \\
138 \\
102 \\
102\end{array}$ & $\begin{array}{r}-173 \\
-261 \\
-140 \\
233 \\
262 \\
266 \\
232 \\
184 \\
116 \\
-10 \\
-136 \\
-183 \\
34\end{array}$ & $\begin{array}{r}1 \\
(s) \\
(s) \\
(s) \\
0 \\
0 \\
0 \\
0 \\
0 \\
0 \\
0 \\
0 \\
(8)\end{array}$ & $\begin{array}{l}31 \\
37 \\
32 \\
40 \\
30 \\
23 \\
26 \\
27 \\
17 \\
13 \\
17 \\
25 \\
26\end{array}$ & $\begin{array}{r}1,179 \\
1,261 \\
1,165 \\
812 \\
746 \\
744 \\
804 \\
851 \\
955 \\
1,057 \\
1,220 \\
1,222 \\
1,000\end{array}$ & $\begin{array}{l}33 \\
26 \\
22 \\
29 \\
37 \\
45 \\
52 \\
58 \\
61 \\
61 \\
57 \\
51 \\
51\end{array}$ \\
\hline $\begin{array}{l}1994 \text { January ......................... } \\
\text { Fobruary ....................... } \\
\text { March .......................... } \\
\text { 3-Month Average ....... }\end{array}$ & $\begin{array}{l}892 \\
908 \\
941 \\
914\end{array}$ & $\begin{array}{r}134 \\
119 \\
85 \\
113\end{array}$ & $\begin{array}{r}-555 \\
-316 \\
11 \\
-286\end{array}$ & $\begin{array}{l}0 \\
6 \\
0 \\
2\end{array}$ & $\begin{array}{l}19 \\
30 \\
29 \\
26\end{array}$ & $\begin{array}{r}1,562 \\
1,308 \\
987 \\
1,285\end{array}$ & $\begin{array}{l}34 \\
25 \\
25 \\
25\end{array}$ \\
\hline $\begin{array}{l}1993 \text { 3-Month Average ....... } \\
1992 \text { 3- Month Average ....... }\end{array}$ & $\begin{array}{l}965 \\
948\end{array}$ & $\begin{array}{l}78 \\
82\end{array}$ & $\begin{array}{l}-189 \\
-165\end{array}$ & (s) & $\begin{array}{l}33 \\
42\end{array}$ & $\begin{array}{l}1,200 \\
1,152\end{array}$ & $\begin{array}{l}22 \\
33\end{array}$ \\
\hline
\end{tabular}

- A negative number indicates a decrease in stocks and a positive number

Sources: - 1973 through 1975: U.S. Department of the Interior, Bureau of Mines, Mineral Industry Surveys, "Petroleum Statement, Annual." • 1976 through 1980: Energy Information Administration (EIA), Energy Data Reports, Petroleum Statement, Annual." - 1981 forward: ElA, Petroleum Supply Monthly, May 1994, Table S8.

c Secks are lotab as of end of por

(s)=Less than 500 barrels per day.

Note: Geographic coverage is the 50 States and the District of Columbia. 
Table 3.10 Other Petroleum Products Supply and Disposition

\begin{tabular}{|c|c|c|c|c|c|c|c|}
\hline & \multicolumn{2}{|c|}{ Supply } & \multicolumn{4}{|c|}{ Dieposition } & \multirow{3}{*}{$\begin{array}{c}\begin{array}{c}\text { Ending } \\
\text { Stooks }\end{array} \\
\text { Million Barrels }\end{array}$} \\
\hline & $\begin{array}{l}\text { Totel } \\
\text { Production }\end{array}$ & Imports & $\begin{array}{l}\text { Stock } \\
\text { Change }\end{array}$ & $\begin{array}{l}\text { Rofinory } \\
\text { Inpute }\end{array}$ & Exports & $\begin{array}{l}\text { Producte } \\
\text { Supplied }\end{array}$ & \\
\hline & \multicolumn{6}{|c|}{ Thousand Barrels per Day } & \\
\hline 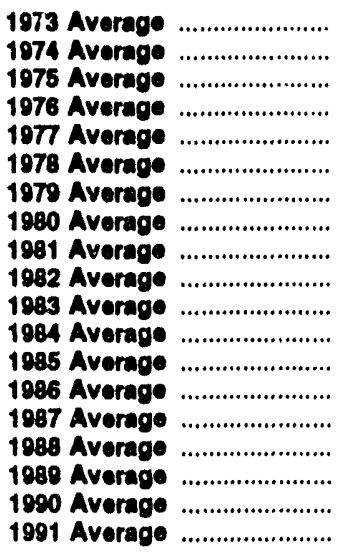 & $\begin{array}{l}2,833 \\
2,722 \\
2,547 \\
2,725 \\
2,939 \\
3,076 \\
3,141 \\
2,957 \\
2,771 \\
2,475 \\
2,437 \\
2,500 \\
2,532 \\
2,704 \\
2,737 \\
2,773 \\
2,771 \\
2,842 \\
2,826\end{array}$ & $\begin{array}{r}290 \\
269 \\
144 \\
129 \\
130 \\
80 \\
116 \\
130 \\
188 \\
305 \\
382 \\
503 \\
550 \\
504 \\
543 \\
645 \\
627 \\
705 \\
675\end{array}$ & $\begin{array}{c}1 \\
25 \\
c-6 \\
(6) \\
20 \\
-12 \\
24 \\
15 \\
c-42 \\
-68 \\
c-6 \\
c-32 \\
22 \\
-15 \\
-1 \\
22 \\
12 \\
-32 \\
18\end{array}$ & $\begin{array}{l}750 \\
665 \\
537 \\
524 \\
514 \\
492 \\
352 \\
310 \\
723 \\
787 \\
712 \\
791 \\
886 \\
888 \\
829 \\
799 \\
797 \\
887 \\
936\end{array}$ & $\begin{array}{l}162 \\
172 \\
158 \\
172 \\
164 \\
165 \\
208 \\
197 \\
197 \\
205 \\
236 \\
236 \\
227 \\
291 \\
264 \\
294 \\
305 \\
289 \\
277\end{array}$ & $\begin{array}{r}2,211 \\
2,129 \\
2,001 \\
2,158 \\
2,371 \\
2,511 \\
2,673 \\
2,566 \\
2,081 \\
d 1,857 \\
1,877 \\
2,007 \\
1,047 \\
2,045 \\
2,187 \\
2,303 \\
2,285 \\
2,402 \\
2,269\end{array}$ & $\begin{array}{r}179 \\
c^{188} \\
188 \\
188 \\
105 \\
101 \\
200 \\
c 205 \\
241 \\
c^{2} 216 \\
c 217 \\
108 \\
208 \\
201 \\
200 \\
208 \\
213 \\
201 \\
208\end{array}$ \\
\hline 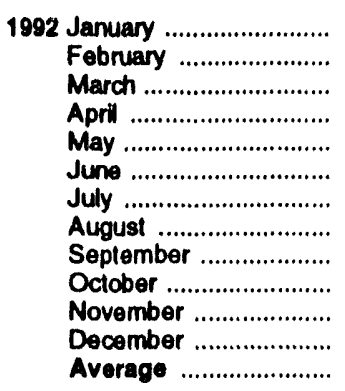 & $\begin{array}{l}2,702 \\
2,642 \\
2,752 \\
2,900 \\
2,929 \\
3,126 \\
3,207 \\
3,068 \\
3,114 \\
2,923 \\
2,915 \\
2,853 \\
2,928\end{array}$ & $\begin{array}{l}734 \\
575 \\
713 \\
793 \\
665 \\
669 \\
740 \\
729 \\
748 \\
701 \\
697 \\
711 \\
707\end{array}$ & $\begin{array}{r}203 \\
183 \\
238 \\
-31 \\
-113 \\
-42 \\
.156 \\
-116 \\
188 \\
-182 \\
-24 \\
-165 \\
-3\end{array}$ & $\begin{array}{r}787 \\
883 \\
730 \\
1,043 \\
910 \\
787 \\
996 \\
884 \\
675 \\
954 \\
989 \\
1,223 \\
906\end{array}$ & $\begin{array}{l}272 \\
240 \\
239 \\
217 \\
199 \\
225 \\
284 \\
227 \\
336 \\
295 \\
264 \\
352 \\
263\end{array}$ & $\begin{array}{l}2,175 \\
1,911 \\
2,258 \\
2,464 \\
2,598 \\
2,826 \\
2,822 \\
2,802 \\
2,663 \\
2,557 \\
2,383 \\
2,154 \\
2,470\end{array}$ & $\begin{array}{l}214 \\
219 \\
227 \\
226 \\
222 \\
221 \\
216 \\
212 \\
218 \\
212 \\
212 \\
c_{207} \\
c_{207}\end{array}$ \\
\hline 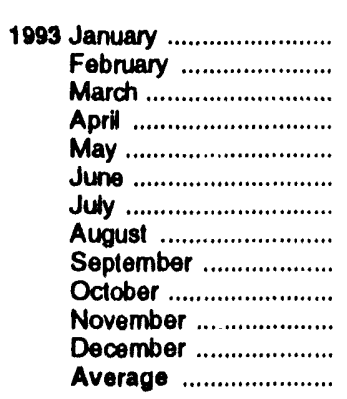 & $\begin{array}{l}{ }^{0} 3,026 \\
2,815 \\
2,866 \\
2,862 \\
2,899 \\
3,022 \\
3,116 \\
3,094 \\
3,016 \\
3,108 \\
2,978 \\
2,810 \\
2,969\end{array}$ & $\begin{array}{l}698 \\
773 \\
818 \\
719 \\
808 \\
630 \\
875 \\
676 \\
789 \\
802 \\
760 \\
674 \\
752\end{array}$ & $\begin{array}{r}{ }^{c} 600 \\
122 \\
243 \\
9 \\
85 \\
-240 \\
116 \\
27 \\
-265 \\
-164 \\
-210 \\
-356 \\
-2\end{array}$ & $\begin{array}{r}829 \\
949 \\
747 \\
900 \\
979 \\
981 \\
945 \\
865 \\
1,031 \\
1,138 \\
1,274 \\
1,389 \\
1,002\end{array}$ & $\begin{array}{l}{ }^{6} 271 \\
282 \\
269 \\
315 \\
278 \\
278 \\
302 \\
295 \\
282 \\
369 \\
309 \\
349 \\
300\end{array}$ & $\begin{array}{l}{ }^{0} 2,023 \\
2,235 \\
2,425 \\
2,357 \\
2,364 \\
2,632 \\
2,628 \\
2,583 \\
2,757 \\
2,567 \\
2,365 \\
2,101 \\
2,420\end{array}$ & $\begin{array}{l}225 \\
228 \\
236 \\
236 \\
239 \\
231 \\
235 \\
236 \\
228 \\
223 \\
217 \\
205 \\
205\end{array}$ \\
\hline 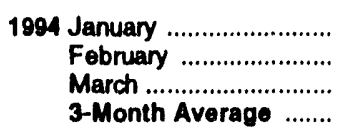 & $\begin{array}{l}2,719 \\
2,779 \\
2,805 \\
2,768\end{array}$ & $\begin{array}{l}780 \\
725 \\
753 \\
754\end{array}$ & $\begin{array}{r}507 \\
236 \\
32 \\
259\end{array}$ & $\begin{array}{l}590 \\
638 \\
939 \\
725\end{array}$ & $\begin{array}{l}256 \\
248 \\
361 \\
289\end{array}$ & $\begin{array}{l}2,147 \\
2,383 \\
2,226 \\
2,248\end{array}$ & $\begin{array}{l}221 \\
228 \\
229 \\
229\end{array}$ \\
\hline $\begin{array}{l}1993 \text { 3-Month Average ....... } \\
1992 \text { 3-Month Averago ....... }\end{array}$ & $\begin{array}{l}2,905 \\
2,700\end{array}$ & $\begin{array}{l}763 \\
676\end{array}$ & $\begin{array}{l}328 \\
208\end{array}$ & $\begin{array}{l}838 \\
798\end{array}$ & $\begin{array}{l}274 \\
250\end{array}$ & $\begin{array}{l}2,227 \\
2,119\end{array}$ & $\begin{array}{l}236 \\
227\end{array}$ \\
\hline
\end{tabular}

- A negative number indicates a decrease in stocks and a positive number indicates an increase.

b Stocks are tolals as of end of period.

c See Nole 4 at end of section.

dee Note 6 at end of section.

- Beginning in 1993, other petroleum products production, exports, and products supplied include an adjustment to oxygenates and motor gasoline blending components.

$(\mathrm{s})=$ Less than +500 barrels per day and greater than -500 barrels per day.
Notes: - Other petroleum products include pentanes plus, other hydrocarbons and oxygenates, unfinished oils, gasoline blending components, and all finished petroleum products except finished motor gasoline, distillate fuel oil, residual fuel oll, jet fuel, and liquelied petroleum gases. - Geographic coverage is the 50 Stales and the District of Columbla.

Sources: - 1973-1980: Energy Information Adminisiration (EIA), Petroleum Supply Monthly, February 1993, Table S9. - 1981 forward: EIA, Petroloum Supphy Monthly, May 1994, Table S10. 


\section{Petroleum Notes}

1. The Energy Information Administration (EIA) uses a number of sources and methods to maintain the survey respondent lists. On a regular basis, survey managers review such industry publications as the Oil and Gas Journal and Oil Daily for information on facilities or companies starting up or closing down operations. Those sources are augmented by articles in newspapers, letters from respondents indicating changes in status, and information received from survey systems.

To supplement routine frames maintenance and to provide more thorough coverage, a comprehensive frames investigation is conducted every 3 years. This investigation results in the reassessment and recompilation of the complete frame for each survey. The effort also includes the evaluation of the impact of potential frame changes on the historical time series of data published from these respondents. The results of this frame study are usually implemented in January to provide a full year under the same frame.

In 1991, the EIA conducted a frame identifier survey of companies that produce, blend, store, or import oxygenates. A summary of the results from the identification survey was published in the Weekly Petroleum Status Report dated February 12, 1992, and in the February 1992 issue of the Petroleum Supply Monthly. In order to continue to provide relevant information about U.S. and regional gasoline supply, the EIA conducted a second frame identifier survey of those companies during 1992. As a result, numerous respondents were added to the monthly surveys effective in January 1993. See Explanatory Note 7 in the Petroleum Supply Monthly.

2. Motor Gasoline: Beginning in January 1981, the EiA expanded its universe to include non-refinery blenders and separated blending components from finished motor gasoline as a reporting category. Also, survey forms were modified to describe refinery operations more accurately.

Beginning with the reporting of January 1993 data, the EIA made adjustments to the product supplied series for finished motor gasoline. It was recognized that motor gasoline statistics published by the EIA through 1992 were underreported because the reporting system was (1) not collecting all fuel ethanol blending, and (2) there was a misreporting of motor gasoline blending components that were blended into finished gasoline. The adjustments are incorporated into EIA's data beginning in January 1993. To facilitate data analysis across the 1992-1993 period, EIA has prepared a table of 1992 data adjusted according to the 1993 basis. See Petroleum Supply Monthly, March 1993, Table H3.

3. Distillate and Residual Fuel Oils: The requirement to report crude oil in pipelines or burned on leases as either distillate or residual fuel oil has been eliminated.
Prior to January 1981 , the refinery input of unfinished oils typically exceeded the available supply of unfinished oils. That discrepancy was assumed to be due to the redesignation of distillate and residual fuel oils received as such but used as unfinished oil inputs by the receiving refinery. The imbalance between supply and disposition of unfinished oils would then be subtracted from the production of distillate and residual fuel oils. Two-thirds of that difference was subtracted from distillate and one-third from residual. Beginning in January 1981, the EIA modified its survey forms to account for redesignated product and discontinued the above-mentioned adjustment.

Beginning in January 1993, the end-of-month stocks of distillate fuel oil are split into two sulfur categories $(0.05$ percent sulfur or less and greater than 0.05 percent sulfur) to meet Environmental Protection Agency requirements effective in October 1992. For further details, see the EIA, Petroleum Supply Monthly.

4. New Stock Basis: In January $1975,1979,1981$, and 1983, numerous respondents were added to bulk terminal and pipeline surveys, affecting subsequent stocks reported and stock change calculations. Using the expanded coverage (new basis), the end-of-year stocks, in million barrels, would have been:

- Crude Oil: 1982-645 (Total) and 351 (Other Primary).

- Crude Oil and Petroleum Products: 1974-1,121; 1980-1,425; and 1982-1,461.

- Motor Gasoline: 1974-225; 1980-263; 1982244 (Total) and 202 (Finished).

- Distillate Fuel Oil: 1974-224; 1980-205; and 1982-186.

- Residual Fuel Oil: 1974-75; 1980-91; and 1982-69.

- Jet Fuel: 1974-30 (Total) and 24 (Kerosene Type); 1980-42 (Total) and 36 (Kerosene Type); and 1982-39 (Total) and 32 (Kerosene Type).

- Liquefied Petroleum Gases: 1974 -113; 1978136; 1980-128; and 1982-102.

- Propane and Propylene: 1978-86; 1980-69; and 1982-57.

- Other Petroleum Products: 1974-190; 1980207; and 1982-219.

Stock change calculations beginning in 1975,1979 , 1981 , and 1983 were made by using new basis stock levels.

In January 1984, changes were made in the reporting of natural gas liquids. As a result, unfractionated stream, which was formerly included in the "Other Petroleum Products Supply and Disposition" table, is now reported on a component basis (ethane, propane, normal butane, isobutane, and pentanes plus). Most of these stocks now appear in the "Liquefied Petroleum Gases Supply and 
Disposition" table. This change affects stocks reported and stock change calculations in each table. Under the new basis, end-of-year 1983 stocks, in million barrels, would have been:

- Liquefied Petroleum Gases: 1983-108.

- Propane and Propylene: 1983-55.

- Other Petroleum Products: 1983-210.

In January 1993, changes were made in the monthly surveys to begin collecting bulk terminal and pipeline stocks of oxygenates. This change affected stocks reported and stock change calculations. However, a new basis stock level was not calculated for 1992 end-of-year stocks.
5. Stocks of Alaskan Crude Oll: Stocks of Alaskan Crude oil in transit were included for the first time in January 1981. The major impact of this change is on the reporting of stock change calculations. Using the expanded coverage (new basis), 1980 end-of-year stocks, in million barrels, would have been 488 (Total) and 380 (Other Primary).

6. Data Discrepancies: Due to differences internal to EIA data processing systems, some small discrepancies exist between data in the Monthly Energy Review (MER) and the Petroleum Supply Annual (PSA) and Petroleum Supply Monthly (PSM). The data that have discrepancies are footnoted in Section 3 tables and summarized here.

\begin{tabular}{llcrr} 
Table & \multicolumn{1}{c}{ Data Series } & $\begin{array}{c}\text { Year } \\
\text { Average }\end{array}$ & $\begin{array}{c}\text { MER } \\
\text { Data }\end{array}$ & $\begin{array}{c}\text { PSA and } P S M \\
\text { Data }\end{array}$ \\
\hline $3.1 \mathrm{a}$ & Natural Gas Plant Production & 1976 & 1,604 & 1,603 \\
$3.1 \mathrm{~b}$ & Exports, Total & 1979 & 471 & 472 \\
$3.1 \mathrm{~b}$ & Exports, Petroleum Products & 1979 & 236 & 237 \\
$3.1 \mathrm{~b}$ & Net Imports & 1979 & 7,985 & 7,984 \\
$3.2 \mathrm{a}$ & Crude Used Directly & 1976 & -19 & -18 \\
$3.2 \mathrm{a}$ & Imports, SPR & 1978 & 161 & 162 \\
$3.2 \mathrm{a}$ & Crude Used Directly & 1978 & -15 & -14 \\
$3.2 \mathrm{a}$ & Crude Used Directly & 1979 & -14 & -13 \\
$3.2 \mathrm{a}$ & Crude Used Directly & 1980 & -14 & -13 \\
$3.2 \mathrm{~b}$ & Crude Losses & 1976 & 14 & 15 \\
$3.2 \mathrm{~b}$ & Crude Losses & 1980 & 14 & 15 \\
3.5 & Stock Change & 1974 & 10 & 9 \\
3.5 & Stock Change & 1975 & -41 & -40 \\
3.8 & Total Production & 1982 & 1,527 & 1,525 \\
3.10 & Products Supplied & 1982 & 1,857 & 1,856 \\
\hline
\end{tabular}




\section{Section 4. Natural Gas}

Total dry natural gas production in the United States during March 1994 was an estimated 1.6 trillion cubic feet, 1 percent lower than production during the previous March. Dry natural gas production during the first quarter of 1994 was 4.7 trillion cubic feet, 1 percent higher than during the first quarter of 1993.

Consumption of natural and supplemental gas in March 1994 was 2.2 billion cubic feet, 4 percent above the level in March 1993. Consumption of natural and supplemental gas during the first quarter of 1994 was 7.1 trillion cubic feet, 7 percent higher than the first quarter of 1993.

Deliveries to residential consumers in February 1994 (latest date for which data are available) were 838 billion cubic feet, 9 percent above the previous February's deliveries. Total deliveries to industrial consumers during February 1994 were 704 billion cubic feet, 5 percent higher than the previous February's level.

Imports of natural gas in March 1994 were 221 billion cubic feet, 11 percent higher than imports in the previous March. Imports of natural gas during the first quarter of 1994 were an estimated 623 billion cubic feet, 7 percent higher than imports during the first quarter of 1993.

Stocks of working $\mathrm{gas}^{5}$ in underground natural gas storage reservoirs at the end of March 1994 totaled 1.0 trillion cubic feet, 7 percent below the level of stocks available 1 year earlier. Net withdrawals from storage during March 1994 were 133 billion cubic feet, 56 percent below the amount of withdrawals during the previous March.

${ }^{4}$ Percentage changes are based on unrounded data.

${ }^{3}$ Gas available for withdrawal. 
Flgure 4.1 Natural Gas

(Trillion Cubic Feet)

Overview, 1973-1993

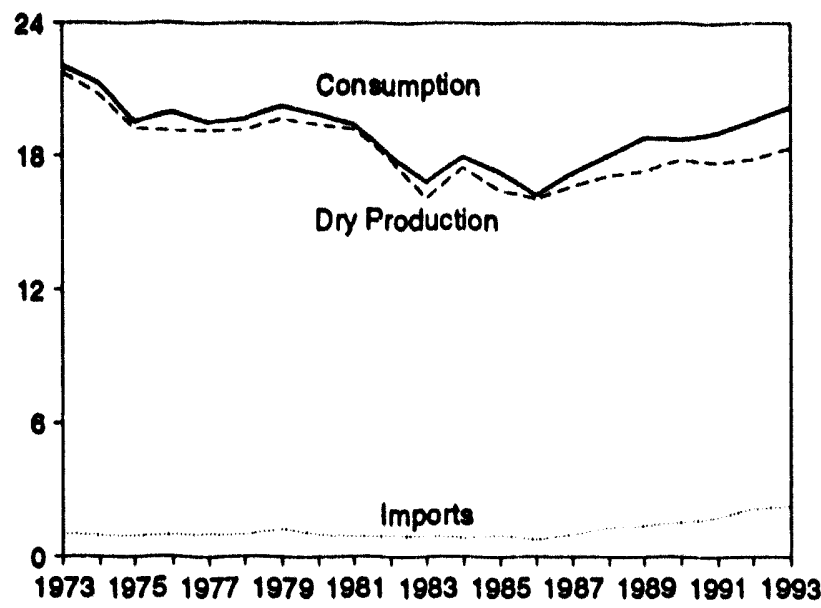

Consumption by Sector, $1973-1993$

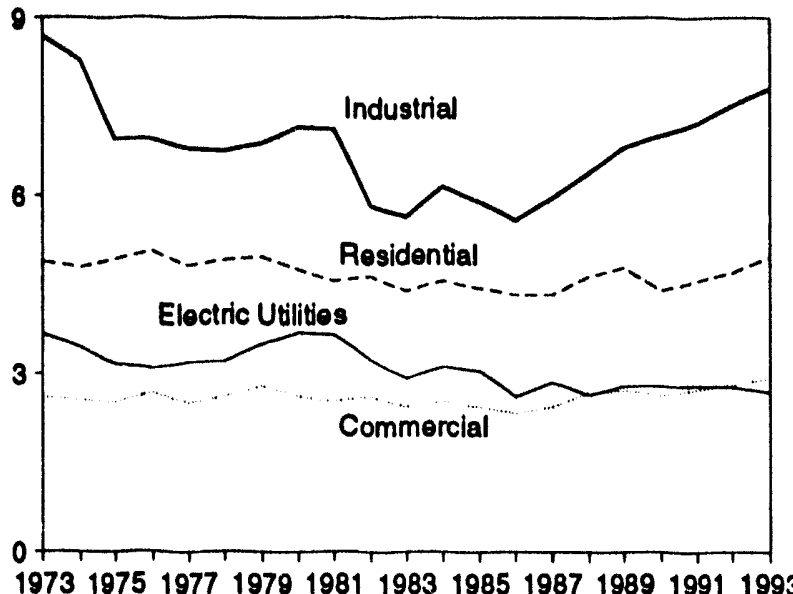

Underground Storage, End of Year, 1973-1993

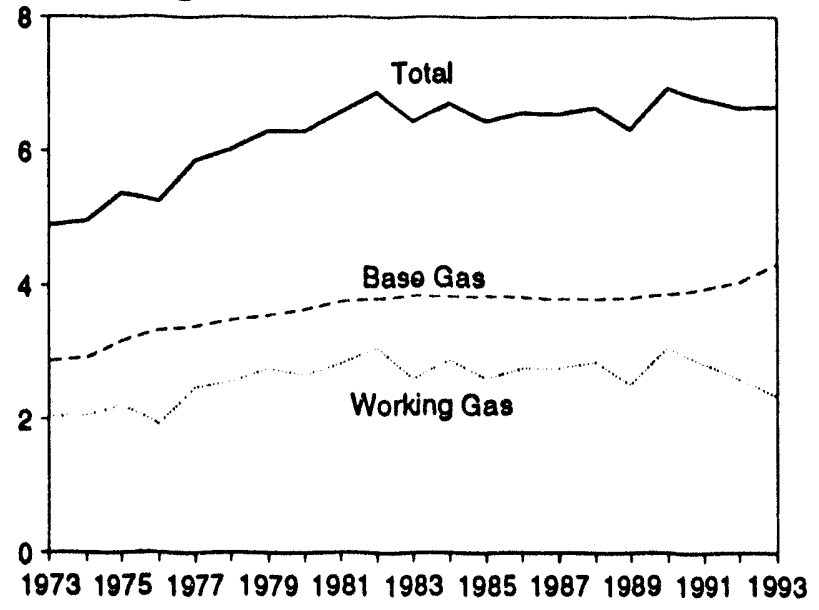

Overview, Monthly

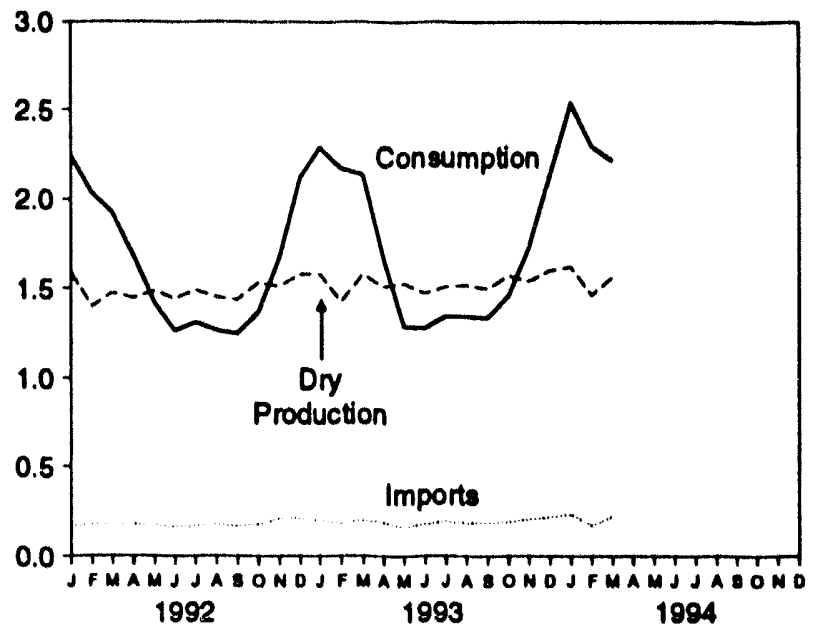

Consumption by Sector, Monthly

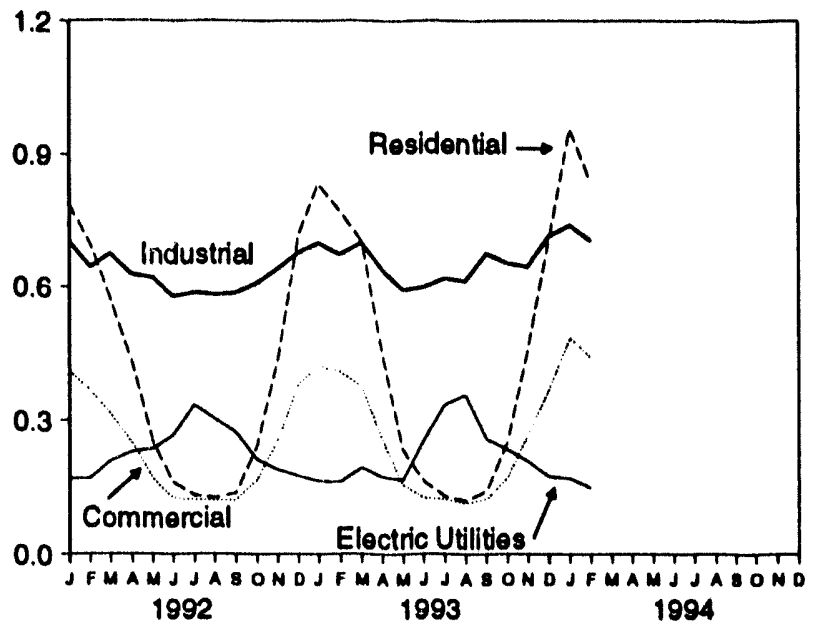

Underground Storage, End of Month

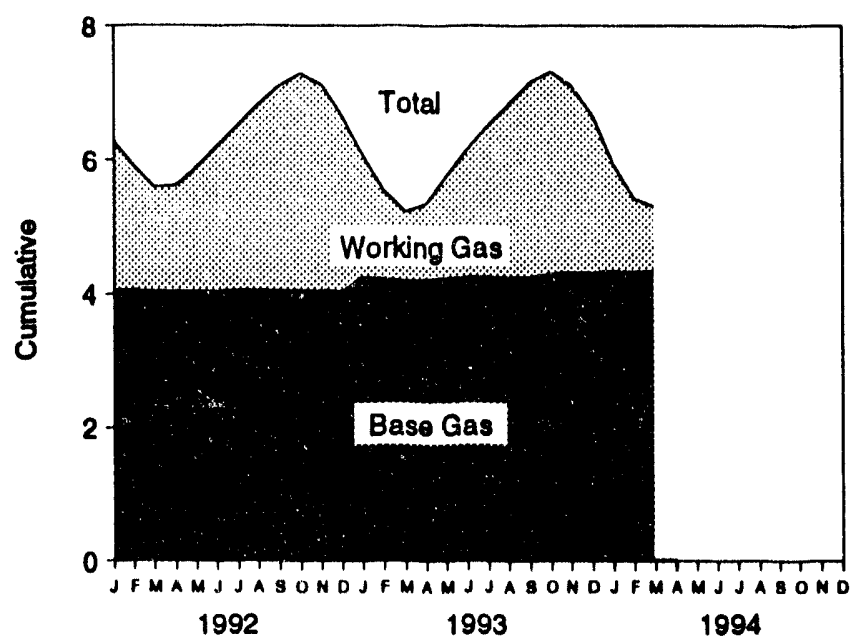

Nole: Because vertical scales differ, graphs should not be compared. Eources: Tables 4.2, 4.4, and 4.5. 
Table 4.1 Natural Gas Production

(Billion Cublc Feet)

\begin{tabular}{|c|c|c|c|c|c|c|c|}
\hline & $\begin{array}{l}\text { Croses } \\
\text { Withdrawala" }\end{array}$ & Aopreasouring & $\begin{array}{l}\text { Nonhydro- } \\
\text { carbon aceece } \\
\text { Ramovede }\end{array}$ & $\begin{array}{l}\text { Ventad } \\
\text { and } \\
\text { Flaredd }\end{array}$ & $\begin{array}{l}\text { Markoted } \\
\text { Produetion } \\
\text { (Wot) }\end{array}$ & $\begin{array}{l}\text { Extraetion } \\
\text { Loed }\end{array}$ & $\begin{array}{c}\text { Toted } \\
\text { Ory aas } \\
\text { Production: }\end{array}$ \\
\hline 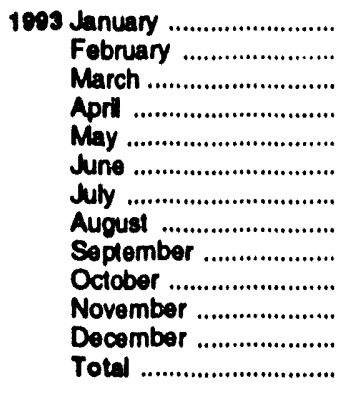 & $\begin{array}{r}1,962 \\
1,781 \\
1,961 \\
1,875 \\
A_{1,903} \\
1,822 \\
1,864 \\
1,890 \\
A_{1,860} \\
1,970 \\
A_{1} 1,946 \\
A_{2,027} \\
A_{22,861}\end{array}$ & $\begin{array}{r}264 \\
247 \\
268 \\
252 \\
261 \\
240 \\
242 \\
259 \\
250 \\
283 \\
293 \\
308 \\
3,167\end{array}$ & $\begin{array}{r}24 \\
21 \\
21 \\
22 \\
22 \\
21 \\
23 \\
22 \\
22 \\
22 \\
21 \\
22 \\
264\end{array}$ & $\begin{array}{r}14 \\
15 \\
16 \\
15 \\
16 \\
17 \\
17 \\
16 \\
16 \\
16 \\
15 \\
17 \\
P_{100}\end{array}$ & $\begin{array}{r}1,660 \\
1,497 \\
1,657 \\
1,585 \\
A_{1,604} \\
1,544 \\
1,583 \\
1,593 \\
1,572 \\
P_{1,649} \\
A_{1,616} \\
A_{1,680} \\
P_{10,240}\end{array}$ & $\begin{array}{r}77 \\
70 \\
77 \\
74 \\
75 \\
72 \\
74 \\
74 \\
73 \\
77 \\
75 \\
78 \\
907\end{array}$ & $\begin{array}{r}1,583 \\
1,427 \\
1,579 \\
1,511 \\
n_{1,520} \\
1,472 \\
1,600 \\
1,510 \\
n_{1,100} \\
1,572 \\
n_{1,541} \\
n_{1,602} \\
n_{10,343}\end{array}$ \\
\hline 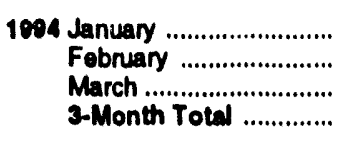 & $\begin{array}{l}R_{2,049} \\
E_{1,844} \\
E_{1,977} \\
E_{3,870}\end{array}$ & $\begin{array}{l}A_{309} \\
E_{275} \\
E_{298} \\
E_{882}\end{array}$ & $\begin{array}{l}R_{22} \\
E_{20} \\
E_{22} \\
E_{64}\end{array}$ & $\begin{array}{l}16 \\
E_{15} \\
E_{16}\end{array}$ & $\begin{array}{l}A_{1}, 701 \\
E_{1,534} \\
E 1,642 \\
E \quad 4,87\end{array}$ & $\begin{array}{r}\text { R } 70 \\
871 \\
877 \\
E_{227}\end{array}$ & $\begin{array}{l}A_{1,622} \\
1,463 \\
1,565 \\
1,080\end{array}$ \\
\hline $\begin{array}{l}1993 \text { 3-Month Total ................ } \\
1992 \text { 3-Month Total ................. }\end{array}$ & $\begin{array}{l}5,704 \\
5,537\end{array}$ & $\begin{array}{l}779 \\
752\end{array}$ & $\begin{array}{l}66 \\
68\end{array}$ & $\begin{array}{l}46 \\
40\end{array}$ & $\begin{array}{l}4,814 \\
4,677\end{array}$ & $\begin{array}{l}204 \\
210\end{array}$ & $\begin{array}{l}4,580 \\
4,450\end{array}$ \\
\hline
\end{tabular}

- Gas withdrawn trom gas and oil wells.

b The injection of natural gas into oil and gas lomations for pressure maintenance and cycling purposes.

c See Note 1 at end of section.

derted: Nalural gas released into the air on the base site $\alpha$ at processing plants. Flared: Natural gas burned in flares on the base site or at gas processing plants.

- "Gross Whindrawals" minus "Repressuring," "Nontwdrocarbon Gases Removed," and "Vented and Flared." See Nole 2 at end. Jction.
1 See Nole 3 at end of section.

- "Marketed Production (Wet)" minus "Extraction Loas."

h May include unknown quantilies of nonhydrocarbon gases.

$R=$ Revised data. NA=Not avallable. Enestimale.

Noles: - Geographic coverage is the 50 States and the Diturict of Columbla. - Tolals may not equal sum of components dus to hdependent rounding.

Sources: - 1973-1086: Energy Intormation Adminiatration (ELA), Nature Gas Annual 1991, Table 95. - 1087 fonward: ELA, Natural Gas Monthy, May 1994, Table 1. 
Table 4.2 Natural Gas Supply and Disposition

(Blllion Cublc Feet)

\begin{tabular}{|c|c|c|c|c|c|c|c|c|c|}
\hline & \multicolumn{5}{|c|}{ supply } & $\begin{array}{c}\text { Total } \\
\text { eupplyl } \\
\text { Diepositiond }\end{array}$ & \multicolumn{3}{|c|}{ Dloposition } \\
\hline 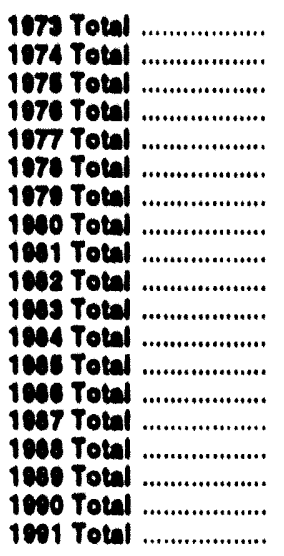 & 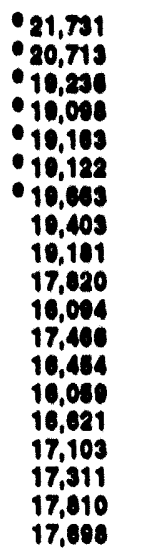 & $\begin{array}{l}1,633 \\
1,701 \\
1,700 \\
1,021 \\
1,760 \\
2,160 \\
2,047 \\
1,072 \\
1,030 \\
2,164 \\
2,270 \\
2,000 \\
2,307 \\
1,097 \\
1,006 \\
2,270 \\
2,894 \\
1,006 \\
2,762\end{array}$ & $\begin{array}{l}\text { NA } \\
\text { NA } \\
\text { NA } \\
\text { NA } \\
\text { NA } \\
\text { NA } \\
\text { NA } \\
186 \\
170 \\
148 \\
132 \\
110 \\
120 \\
113 \\
101 \\
101 \\
107 \\
123 \\
113\end{array}$ & 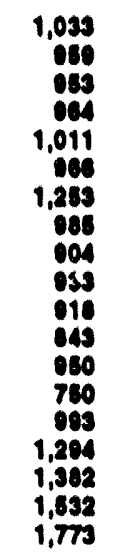 & 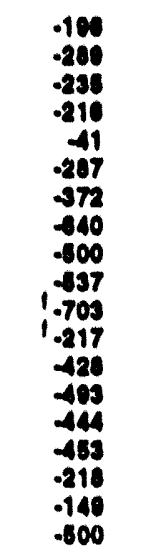 & 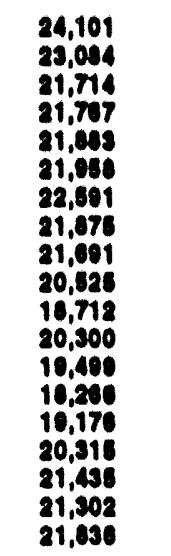 & $\begin{array}{l}1,074 \\
1,704 \\
2,104 \\
1,760 \\
2,207 \\
2,270 \\
2,290 \\
1,040 \\
2,220 \\
2,472 \\
1,020 \\
2,206 \\
2,169 \\
1,084 \\
1,011 \\
2,211 \\
2,020 \\
2,490 \\
2,072\end{array}$ & 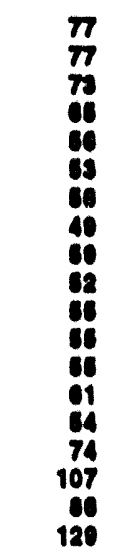 & 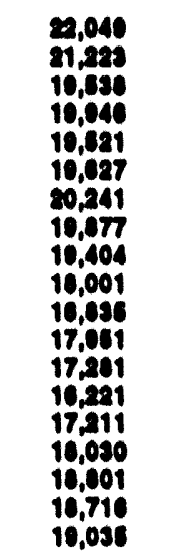 \\
\hline 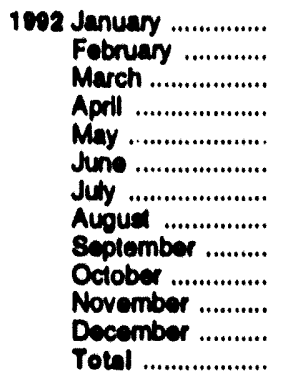 & $\begin{array}{r}1,586 \\
1,308 \\
1,475 \\
1,447 \\
1,485 \\
1,444 \\
1,491 \\
1,451 \\
1,437 \\
1,533 \\
1,514 \\
1,579 \\
17,840\end{array}$ & $\begin{array}{r}624 \\
463 \\
397 \\
142 \\
44 \\
36 \\
42 \\
46 \\
40 \\
70 \\
282 \\
687 \\
2,772\end{array}$ & $\begin{array}{r}12 \\
11 \\
11 \\
10 \\
9 \\
8 \\
8 \\
8 \\
8 \\
8 \\
10 \\
11 \\
12 \\
118\end{array}$ & $\begin{array}{l}166 \\
175 \\
180 \\
176 \\
174 \\
162 \\
167 \\
176 \\
166 \\
178 \\
210 \\
200 \\
2,130\end{array}$ & $\begin{array}{r}.71 \\
42 \\
.12 \\
80 \\
69 \\
16 \\
.8 \\
.10 \\
.24 \\
.130 \\
.230 \\
.191 \\
.600\end{array}$ & $\begin{array}{r}2,315 \\
2,089 \\
2,022 \\
1,804 \\
1,780 \\
1,068 \\
1,700 \\
1,682 \\
1,609 \\
1,060 \\
1,778 \\
2,195 \\
22,300\end{array}$ & $\begin{array}{r}60 \\
46 \\
74 \\
161 \\
344 \\
384 \\
373 \\
380 \\
362 \\
271 \\
88 \\
58 \\
2,800\end{array}$ & $\begin{array}{l}16 \\
14 \\
23 \\
18 \\
18 \\
10 \\
18 \\
16 \\
18 \\
18 \\
19 \\
10 \\
10 \\
19 \\
210\end{array}$ & $\begin{array}{r}2,230 \\
2,031 \\
1,026 \\
1,686 \\
1,418 \\
1,264 \\
1,311 \\
1,264 \\
1,240 \\
1,368 \\
1,672 \\
2,119 \\
10,644\end{array}$ \\
\hline $\begin{array}{l}1994 \text { January .............. } \\
\text { Fobruary ............ } \\
\text { March ................ } \\
\text { S-Month Total ... }\end{array}$ & $\begin{array}{l}A, 1,622 \\
\therefore 1,463 \\
E 1,565 \\
C 4,050\end{array}$ & $\begin{array}{r}A_{756} \\
A_{542} \\
230 \\
1,537\end{array}$ & $\begin{array}{l}14 \\
12 \\
11 \\
37\end{array}$ & $\begin{array}{r}A_{232} \\
A_{170} \\
221 \\
623\end{array}$ & $\begin{array}{r}R .48 \\
R_{168} \\
296 \\
418\end{array}$ & $\begin{array}{r}A_{2,577} \\
{ }^{2} 2,354 \\
2,331 \\
7,263\end{array}$ & $\begin{array}{l}A_{33} \\
A_{48} \\
105 \\
188\end{array}$ & $\begin{array}{r}9 \\
0 \\
27\end{array}$ & $\begin{array}{r}A_{2,535} \\
A_{2,298} \\
2,217 \\
7,050\end{array}$ \\
\hline $\begin{array}{l}1003 \text { 3-Month Total ... } \\
1002 \text { 3-Month Total ... }\end{array}$ & $\begin{array}{l}4,880 \\
4,450\end{array}$ & $\begin{array}{l}1,583 \\
1,483\end{array}$ & $\begin{array}{l}37 \\
34\end{array}$ & $\begin{array}{l}580 \\
820\end{array}$ & $\begin{array}{r}20 \\
.70\end{array}$ & $\begin{array}{l}6,788 \\
6,427\end{array}$ & $\begin{array}{l}142 \\
170\end{array}$ & $\begin{array}{l}47 \\
83\end{array}$ & $\begin{array}{l}0,500 \\
0,108\end{array}$ \\
\hline
\end{tabular}

- Data for 1980-1992 include underground storage and llquefled natural gas storage. All other data include underground storage only. Computation procectures are discussed in Note 8 at end of section.

See Notea at end of section.

- See Table 4.3.

- Data lor 1878 forward do nol include in-Iransit recelpts and deliverles.

- May include unknown quamtities of nontydrocarbon gases.

I See Note 7 at end of section.

$R=$ Revised data. NA=Nol avaltable. E=Estimale.

Noles: - Geographic coverage is the 50 States and the District of Columbia. - Totals may not equal sum of components due to independent rounding.
Sources: - 1973-1980: Total Dry Cas Produetton-Eneroy Intormation Administration (ELA), Natura/ Gas Annual 1991, Tablo 95. Withdrawale from Storage, 1973-1975 and 1080-1986-ElA, Natural Gas Annual 1991. Table 96. Withdrawals from storage, 1976-1 fito-EIA, Natural Gas Production and Consumption 1979, Table i. Supplemental Geseous Fuole, 1980-1086-ElA, Natural Gas Annual 1990, Volume 2, Tablo 12. Imports, Additions to storage, Exports, and Consumption-EIA, Natural Gas Annual 1991, Table 98. Total supply/Diapositton-sum of dispoestion Mems. Balaneing Itom-Total supply/dbposition minus all other supply Mems. - 1987 forward: ELA, Nafural Gas Monthly, May 1994, Table 2. 
Table 4.3 Natural Gas Trade by Country (Blllion Cublc Foet)

\begin{tabular}{|c|c|c|c|c|c|c|c|c|}
\hline & \multicolumn{4}{|c|}{ Imports } & \multicolumn{4}{|c|}{ Exports } \\
\hline & Canedn & Aleorla & Othere & Total & Coneder & Mariee & Jepen & Toty \\
\hline 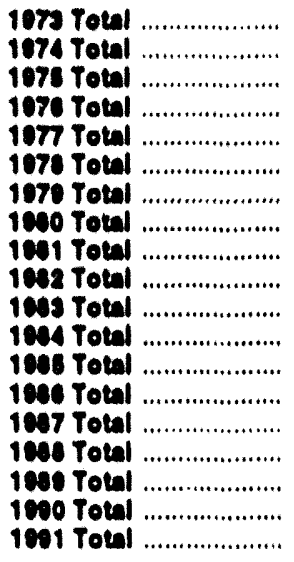 & $\begin{array}{r}1,028 \\
080 \\
048 \\
964 \\
097 \\
081 \\
1,001 \\
707 \\
702 \\
703 \\
712 \\
788 \\
928 \\
740 \\
903 \\
1,270 \\
1,330 \\
1,440 \\
1,710\end{array}$ & $\begin{array}{r}3 \\
0 \\
10 \\
11 \\
14 \\
283 \\
09 \\
37 \\
84 \\
131 \\
38 \\
24 \\
0 \\
0 \\
17 \\
42 \\
04 \\
4\end{array}$ & $\begin{array}{r}2 \\
(5) \\
0 \\
0 \\
2 \\
0 \\
0 \\
102 \\
100 \\
0 \\
7 \\
0 \\
0 \\
2 \\
0 \\
0 \\
0 \\
0 \\
0\end{array}$ & 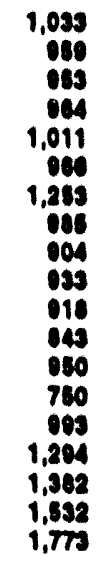 & 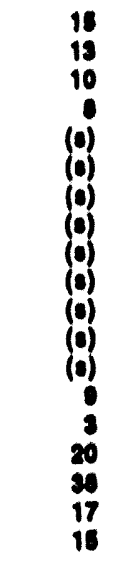 & $\begin{array}{r}14 \\
19 \\
6 \\
7 \\
4 \\
4 \\
4 \\
8 \\
2 \\
8 \\
2 \\
8 \\
2 \\
2 \\
2 \\
17 \\
10 \\
00\end{array}$ & $\begin{array}{l}40 \\
60 \\
68 \\
60 \\
18 \\
4 \\
61 \\
48 \\
60 \\
60 \\
68 \\
69 \\
68 \\
60 \\
40 \\
68 \\
61 \\
63 \\
64\end{array}$ & $\begin{array}{r}01 \\
4 \\
71 \\
107 \\
19\end{array}$ \\
\hline 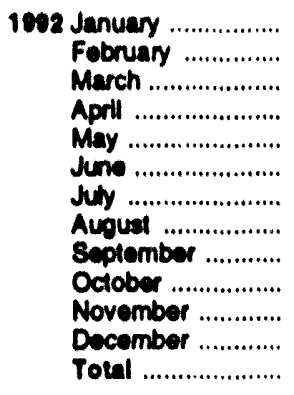 & $\begin{array}{l}157 \\
170 \\
178 \\
174 \\
174 \\
160 \\
167 \\
172 \\
164 \\
174 \\
203 \\
202 \\
2,094\end{array}$ & $\begin{array}{r}8 \\
5 \\
3 \\
3 \\
0 \\
3 \\
0 \\
2 \\
3 \\
3 \\
8 \\
8 \\
4\end{array}$ & $\begin{array}{l}0 \\
0 \\
0 \\
0 \\
0 \\
0 \\
0 \\
0 \\
0 \\
0 \\
0 \\
0 \\
0\end{array}$ & $\begin{array}{l}165 \\
175 \\
180 \\
176 \\
174 \\
160 \\
167 \\
175 \\
168 \\
176 \\
210 \\
200 \\
2,138\end{array}$ & $\begin{array}{r}2 \\
4 \\
11 \\
6 \\
6 \\
6 \\
6 \\
5 \\
6 \\
6 \\
3 \\
7 \\
0\end{array}$ & $\begin{array}{r}10 \\
6 \\
7 \\
7 \\
7 \\
7 \\
6 \\
9 \\
8 \\
10 \\
11 \\
8 \\
0\end{array}$ & $\begin{array}{l}4 \\
4 \\
4 \\
4 \\
4 \\
4 \\
3 \\
4 \\
0\end{array}$ & $\begin{array}{l}18 \\
14 \\
23 \\
18 \\
10 \\
18 \\
18 \\
18 \\
18 \\
10 \\
10 \\
10 \\
210\end{array}$ \\
\hline 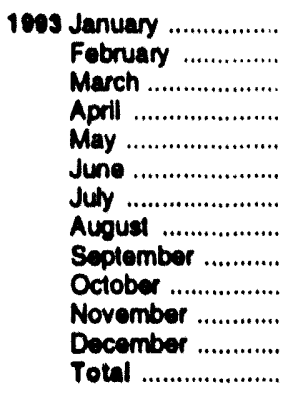 & $\begin{array}{l}193 \\
175 \\
194 \\
178 \\
155 \\
171 \\
183 \\
170 \\
178 \\
184 \\
197 \\
208 \\
2,194\end{array}$ & $\begin{array}{r}5 \\
8 \\
5 \\
8 \\
5 \\
8 \\
8 \\
5 \\
10 \\
5 \\
8 \\
8 \\
82\end{array}$ & $\begin{array}{l}0 \\
0 \\
0 \\
0 \\
0 \\
0 \\
0 \\
0 \\
0 \\
0 \\
0 \\
0 \\
1\end{array}$ & $\begin{array}{r}108 \\
183 \\
100 \\
185 \\
160 \\
178 \\
100 \\
184 \\
188 \\
189 \\
204 \\
217 \\
A_{2,277}\end{array}$ & $\begin{array}{r}6 \\
6 \\
8 \\
5 \\
4 \\
4 \\
4 \\
2 \\
3 \\
3 \\
3 \\
\text { ค }_{40}\end{array}$ & $\begin{array}{l}8 \\
2 \\
3 \\
3 \\
3 \\
4 \\
4 \\
3 \\
2 \\
2 \\
2 \\
1 \\
37\end{array}$ & $\begin{array}{r}4 \\
6 \\
4 \\
4 \\
3 \\
5 \\
5 \\
5 \\
3 \\
5 \\
7 \\
80\end{array}$ & $\begin{array}{r}18 \\
13 \\
17 \\
12 \\
12 \\
11 \\
13 \\
10 \\
10 \\
8 \\
10 \\
11 \\
149\end{array}$ \\
\hline $\begin{array}{l}1904 \text { January ................. } \\
\text { February .............. } \\
\text { March ................... } \\
\text { 3-Nonth Totel ..... }\end{array}$ & $\begin{array}{r}A_{221} \\
A_{165} \\
211 \\
597\end{array}$ & $\begin{array}{r}10 \\
5 \\
8 \\
23\end{array}$ & $\begin{array}{l}1 \\
1 \\
2 \\
4\end{array}$ & $\begin{array}{l}A_{232} \\
A_{170} \\
221 \\
623\end{array}$ & $\begin{array}{l}3 \\
4 \\
2 \\
0\end{array}$ & $\begin{array}{l}1 \\
1 \\
1 \\
3\end{array}$ & $\begin{array}{r}5 \\
4 \\
18\end{array}$ & $\begin{array}{r}0 \\
9 \\
87\end{array}$ \\
\hline $\begin{array}{l}1003 \text { 3-Month Total .... } \\
10923 \text { Month Total ..... }\end{array}$ & $\begin{array}{l}582 \\
505\end{array}$ & $\begin{array}{l}18 \\
18\end{array}$ & $\begin{array}{l}0 \\
0\end{array}$ & $\begin{array}{l}880 \\
520\end{array}$ & $\begin{array}{l}20 \\
17\end{array}$ & $\begin{array}{l}13 \\
22\end{array}$ & $\begin{array}{l}18 \\
13\end{array}$ & 67 \\
\hline
\end{tabular}

- By pipeline, except tor very small amounts of liquelied natural gas imported from Canada in 1973, 1977 and 1981. See Nole 5 at end of section. As lquefled natural gas.

C Oher imports are from Mexico, except for 1986, when they came from Indoneala.

R=Revised data. (s)=Less than 500 million cublc leet.
Notes: - See Note 5 at end of section. - Geographic coverape bs the 50 States and the District of Cotumbla. - Tolals may not equal um of components due to independent rounding.

Source: Energy Intormation Adminiatration, Naturd Ges Monthy, May 1904, Tablos 5 and 6. 
Table 4.4 Natural Gas Consesmption by End-Use Sector (Billion Cublc Feet)

\begin{tabular}{|c|c|c|c|c|c|c|c|c|}
\hline & \multirow[b]{2}{*}{$\begin{array}{l}\text { Lenee and } \\
\text { Plant Fuel }\end{array}$} & \multirow[b]{2}{*}{$\begin{array}{l}\text { Plpoline } \\
\text { Fucle }\end{array}$} & \multicolumn{5}{|c|}{ Dellivered to Consumer } & \multirow[b]{2}{*}{$\begin{array}{c}\text { Total } \\
\text { Conoumption }\end{array}$} \\
\hline & & & Acoldentlal & Commerclal & Induetrial & $\begin{array}{l}\text { Electite } \\
\text { Utilites }\end{array}$ & Total & \\
\hline 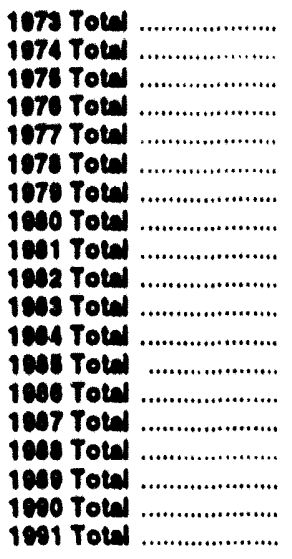 & 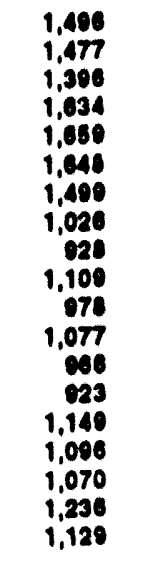 & $\begin{array}{l}726 \\
680 \\
603 \\
848 \\
633 \\
630 \\
601 \\
638 \\
642 \\
600 \\
490 \\
820 \\
604 \\
495 \\
810 \\
614 \\
620 \\
600 \\
601\end{array}$ & $\begin{array}{l}4,070 \\
4,700 \\
4,024 \\
8,081 \\
4,821 \\
4,903 \\
4,988 \\
4,732 \\
4,846 \\
4,633 \\
4,381 \\
4,385 \\
4,433 \\
4,314 \\
4,315 \\
4,030 \\
4,781 \\
4,301 \\
4,350\end{array}$ & $\begin{array}{l}2,807 \\
2,856 \\
2,808 \\
2,608 \\
2,801 \\
2,601 \\
2,706 \\
2,011 \\
2,820 \\
2,000 \\
2,433 \\
2,324 \\
2,432 \\
2,310 \\
2,430 \\
2,670 \\
2,710 \\
2,023 \\
2,720\end{array}$ & 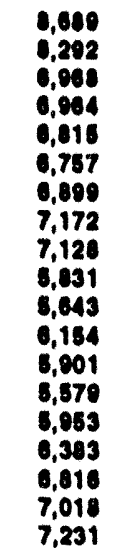 & $\begin{array}{l}3,680 \\
3,443 \\
3,180 \\
3,001 \\
3,101 \\
3,1184 \\
3,401 \\
3,082 \\
3,040 \\
3,220 \\
2,911 \\
3,111 \\
3,044 \\
2,002 \\
2,044 \\
2,038 \\
2,787 \\
2,787 \\
2,780\end{array}$ & $\begin{array}{l}10,028 \\
19,077 \\
17,860 \\
17,794 \\
17,320 \\
17,440 \\
18,141 \\
19,210 \\
17,834 \\
16,208 \\
16,387 \\
18,348 \\
18,011 \\
14,814 \\
18,842 \\
18,320 \\
17,102 \\
18,020 \\
17,305\end{array}$ & $\begin{array}{l}22,010 \\
21,201 \\
10,030 \\
18,040 \\
10,021 \\
10,027 \\
20,241 \\
19,077 \\
10,104 \\
10,001 \\
10,038 \\
17,011 \\
17,281 \\
10,221 \\
17,211 \\
10,030 \\
10,001 \\
10,710 \\
10,038\end{array}$ \\
\hline 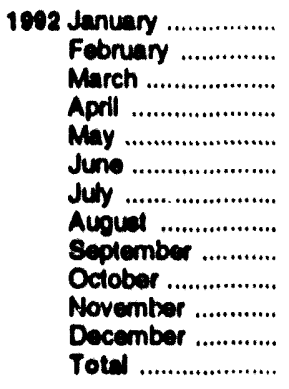 & $\begin{array}{r}104 \\
92 \\
97 \\
95 \\
97 \\
95 \\
98 \\
95 \\
94 \\
101 \\
99 \\
104 \\
1,171\end{array}$ & $\begin{array}{r}68 \\
62 \\
58 \\
51 \\
42 \\
37 \\
39 \\
37 \\
37 \\
41 \\
50 \\
64 \\
888\end{array}$ & $\begin{array}{l}786 \\
686 \\
574 \\
431 \\
251 \\
162 \\
132 \\
126 \\
137 \\
241 \\
437 \\
717 \\
4,090\end{array}$ & $\begin{array}{r}410 \\
366 \\
315 \\
250 \\
170 \\
125 \\
122 \\
121 \\
121 \\
168 \\
256 \\
381 \\
2,803 \\
\end{array}$ & $\begin{array}{r}701 \\
644 \\
674 \\
628 \\
620 \\
578 \\
587 \\
582 \\
586 \\
608 \\
641 \\
677 \\
7,527\end{array}$ & $\begin{array}{l}169 \\
170 \\
208 \\
229 \\
238 \\
266 \\
334 \\
303 \\
274 \\
213 \\
189 \\
176 \\
2,768\end{array}$ & $\begin{array}{l}2,067 \\
1,876 \\
1,770 \\
1,539 \\
1,278 \\
1,132 \\
1,175 \\
1,131 \\
1,117 \\
1,227 \\
1,523 \\
1,051 \\
17,786\end{array}$ & $\begin{array}{r}2,230 \\
2,031 \\
1,026 \\
1,686 \\
1,418 \\
1,264 \\
1,311 \\
1,264 \\
1,249 \\
1,369 \\
1,672 \\
2,110 \\
10,844\end{array}$ \\
\hline 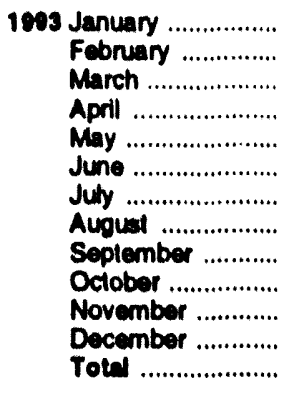 & $\begin{array}{r}104 \\
94 \\
104 \\
99 \\
100 \\
97 \\
99 \\
100 \\
98 \\
103 \\
101 \\
105 \\
1,204\end{array}$ & $\begin{array}{r}69 \\
65 \\
64 \\
50 \\
39 \\
39 \\
40 \\
40 \\
40 \\
44 \\
52 \\
64 \\
607\end{array}$ & $\begin{array}{l}833 \\
770 \\
702 \\
449 \\
233 \\
163 \\
130 \\
120 \\
142 \\
252 \\
457 \\
704 \\
4,956\end{array}$ & $\begin{array}{r}421 \\
408 \\
374 \\
257 \\
156 \\
126 \\
123 \\
115 \\
123 \\
172 \\
265 \\
368 \\
2,908\end{array}$ & $\begin{array}{l}699 \\
672 \\
699 \\
639 \\
593 \\
598 \\
618 \\
613 \\
675 \\
653 \\
645 \\
714 \\
7,819\end{array}$ & $\begin{array}{r}164 \\
162 \\
194 \\
174 \\
167 \\
255 \\
334 \\
357 \\
258 \\
235 \\
208 \\
174 \\
2,682\end{array}$ & $\begin{array}{r}2,118 \\
2,012 \\
1,969 \\
1,519 \\
1,149 \\
1,143 \\
1,206 \\
1,205 \\
1,198 \\
1,313 \\
1,575 \\
1,960 \\
18,385\end{array}$ & $\begin{array}{r}2,290 \\
2,171 \\
2,137 \\
1,660 \\
1,288 \\
1,278 \\
1,344 \\
1,345 \\
1,337 \\
1,460 \\
1,728 \\
2,120 \\
A_{20,177}\end{array}$ \\
\hline $\begin{array}{l}1004 \text { January ................ } \\
\text { February .............. } \\
\text { 2-Month Total ..... }\end{array}$ & $\begin{array}{r}P_{106} \\
96 \\
203\end{array}$ & $\begin{array}{r}A_{76} \\
\text { ค }_{69} \\
145\end{array}$ & $\begin{array}{r}{ }^{A} 958 \\
838 \\
1,700\end{array}$ & $\begin{array}{r}P_{485} \\
441 \\
926\end{array}$ & $\begin{array}{r}739 \\
704 \\
1,443\end{array}$ & $\begin{array}{l}170 \\
149 \\
319\end{array}$ & $\begin{array}{r}\text { ค }_{2,352} \\
2,133 \\
4,485\end{array}$ & $\begin{array}{r}A_{2,535} \\
A_{2,298} \\
4,833\end{array}$ \\
\hline $\begin{array}{l}1003 \text { 2-Month Total ..... } \\
1092 \text { 2-Month Total ..... }\end{array}$ & $\begin{array}{l}198 \\
198\end{array}$ & $\begin{array}{l}134 \\
130\end{array}$ & $\begin{array}{l}1,603 \\
1,482\end{array}$ & $\frac{829}{776}$ & $\begin{array}{l}1,372 \\
1,345\end{array}$ & $\begin{array}{l}320 \\
339\end{array}$ & $\begin{array}{l}4,130 \\
3,843\end{array}$ & $\begin{array}{l}4,462 \\
4,280\end{array}$ \\
\hline
\end{tabular}

- Nalural gas consumed in the operation of pipelines, primarity in compressors.

AmRevised dala.

Notes: - Natural gas includes supplemental gaseous fuels. - Geographic coverage is the 50 States and the District of Columbia. - Totals may not equal sum of components due to independent rounding.

Sources: - 1973-1988: Energy Intormation Administration (ELA), Natura Gas Annual 1991, Table 87. - 1087 forward: ELA, Naturai Gas Monthy, May 1994, Table 3. 


\begin{tabular}{|c|c|c|c|c|c|c|c|c|}
\hline & \multicolumn{3}{|c|}{$\begin{array}{l}\text { Naturat Qee in } \\
\text { Underground Storage, } \\
\text { End of Perlod }\end{array}$} & \multicolumn{2}{|c|}{$\begin{array}{c}\text { Change In Working aes } \\
\text { from same Perlod } \\
\text { Provious Year }\end{array}$} & \multicolumn{3}{|c|}{ storage Aetivity } \\
\hline & Base Ces & Working Gas & Total & Volume & Percent & Injectenob & Whitrawelob & Nop \\
\hline 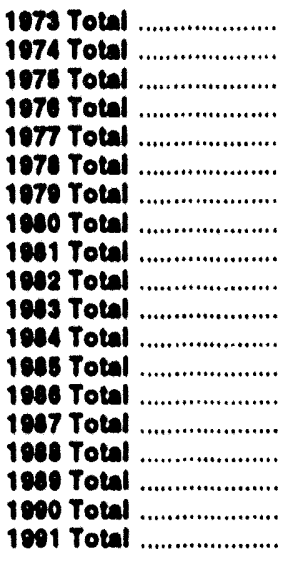 & $\begin{array}{l}2,084 \\
2,012 \\
\mathbf{3}, 182 \\
3,323 \\
3,301 \\
3,473 \\
3,863 \\
\mathbf{3 , 0 4 2} \\
\mathbf{3 , 7 8 2} \\
\mathbf{3 , 0 0 8} \\
\mathbf{3 , 0 4 7} \\
\mathbf{3 , 0 3 0} \\
\mathbf{3 , 8 4 2} \\
\mathbf{3 , 0 1 0} \\
\mathbf{3 , 7 0 2} \\
\mathbf{3 , 8 0 0} \\
\mathbf{3 , 0 1 2} \\
\mathbf{3 , 0 8 8} \\
\mathbf{3 , 9 5 4}\end{array}$ & $\begin{array}{l}2,034 \\
2,050 \\
2,212 \\
1,020 \\
2,475 \\
2,947 \\
2,753 \\
2,058 \\
2,017 \\
3,071 \\
2,898 \\
2,070 \\
2,007 \\
2,740 \\
2,750 \\
2,850 \\
2,513 \\
3,080 \\
2,024\end{array}$ & 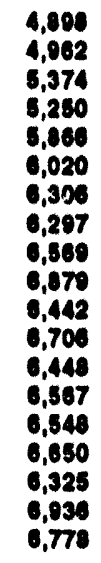 & $\begin{array}{r}305 \\
10 \\
182 \\
-288 \\
440 \\
72 \\
207 \\
.98 \\
102 \\
288 \\
478 \\
281 \\
-270 \\
142 \\
7 \\
94 \\
337 \\
865 \\
-244\end{array}$ & 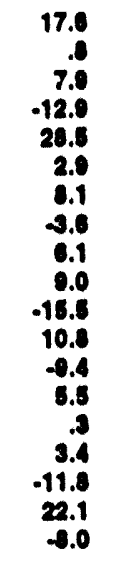 & $\begin{array}{l}1,070 \\
1,784 \\
2,104 \\
1,768 \\
2,307 \\
2,270 \\
2,208 \\
1,009 \\
2,180 \\
2,389 \\
1,700 \\
2,288 \\
2,128 \\
1,082 \\
1,087 \\
2,174 \\
2,401 \\
2,433 \\
2,608\end{array}$ & $\begin{array}{l}1,633 \\
1,701 \\
1,700 \\
1,021 \\
1,760 \\
2,180 \\
2,047 \\
1,010 \\
1,087 \\
2,004 \\
2,142 \\
2,084 \\
2,380 \\
1,012 \\
1,081 \\
2,244 \\
2,804 \\
1,034 \\
2,000\end{array}$ & 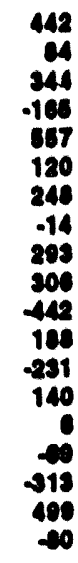 \\
\hline 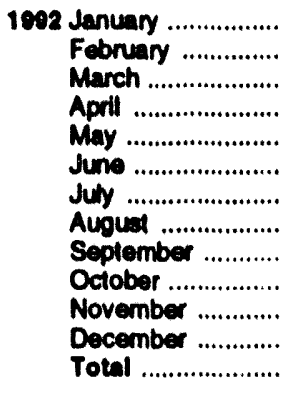 & $\begin{array}{l}4,061 \\
4,057 \\
4,046 \\
4,038 \\
4,044 \\
4,050 \\
4,064 \\
4,062 \\
4,061 \\
4,065 \\
4,061 \\
4,044 \\
4,044\end{array}$ & $\begin{array}{l}2,216 \\
1,837 \\
1,545 \\
1,573 \\
1,848 \\
2,153 \\
2,460 \\
2,761 \\
3,044 \\
3,223 \\
3,054 \\
2,597 \\
2,597\end{array}$ & $\begin{array}{l}6,277 \\
5,894 \\
5,591 \\
5,611 \\
5,892 \\
6,203 \\
6,524 \\
6,823 \\
7,105 \\
7,288 \\
7,115 \\
6,641 \\
6,641\end{array}$ & $\begin{array}{l}.146 \\
.226 \\
.387 \\
.463 \\
.425 \\
.400 \\
.311 \\
.217 \\
.157 \\
.146 \\
.94 \\
.227 \\
.227\end{array}$ & $\begin{array}{l}-6.2 \\
-10.0 \\
-19.2 \\
-22.8 \\
-18.7 \\
-15.7 \\
-11.2 \\
-7.3 \\
-4.9 \\
-4.3 \\
-3.0 \\
-8.0 \\
-4.0\end{array}$ & $\begin{array}{r}68 \\
52 \\
81 \\
167 \\
330 \\
368 \\
357 \\
364 \\
346 \\
264 \\
95 \\
65 \\
2,558 \\
\end{array}$ & $\begin{array}{r}591 \\
441 \\
381 \\
150 \\
53 \\
40 \\
50 \\
54 \\
48 \\
78 \\
276 \\
557 \\
2,724\end{array}$ & $\begin{array}{r}-524 \\
-380 \\
-301 \\
18 \\
277 \\
323 \\
307 \\
300 \\
208 \\
188 \\
.181 \\
.401 \\
-100\end{array}$ \\
\hline 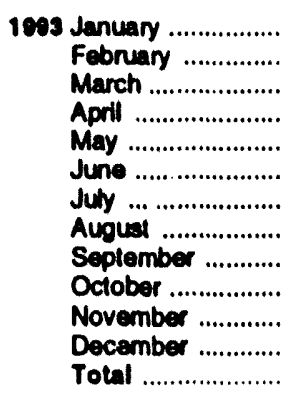 & $\begin{array}{l}P_{4,268} \\
A_{4,230} \\
A_{4,203} \\
A_{4,210} \\
A_{4,243} \\
A_{4,256} \\
A_{4,256} \\
4,263 \\
4,255 \\
4,314 \\
4,325 \\
4,325 \\
4,325\end{array}$ & $\begin{array}{l}A_{1,020} \\
A_{1}, 304 \\
A_{1,028} \\
A_{1,122} \\
A_{1,527} \\
A_{1,901} \\
A_{2,254} \\
2,572 \\
2,904 \\
2,998 \\
2,781 \\
A_{2,338} \\
2,338\end{array}$ & $\begin{array}{r}\mathbf{6 , 0 8 7} \\
5,534 \\
\mathbf{5 , 2 3 2} \\
5,340 \\
5,771 \\
6,157 \\
\mathbf{6 , 5 1 0} \\
\mathbf{6 , 8 3 6} \\
7,159 \\
7,312 \\
7,106 \\
{ }^{0,663} \\
6,663\end{array}$ & 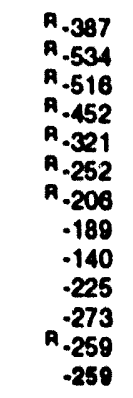 & $\begin{array}{r}\text { A.17.5 } \\
\text { ค. } 29.1 \\
\text { ค. }-33.4 \\
\text { ค. } 28.7 \\
\text { ค. }-17.4 \\
\text { A.11.7 } \\
\text { A. } 8.4 \\
-6.8 \\
-4.6 \\
-7.0 \\
-8.0 \\
\text { A. } 10.0 \\
-10.0\end{array}$ & $\begin{array}{r}A_{41} \\
A_{21} \\
A_{80} \\
A_{215} \\
A_{462} \\
A_{111} \\
A_{388} \\
A_{387} \\
382 \\
255 \\
112 \\
A_{60} \\
2,704\end{array}$ & $\begin{array}{r}A_{597} \\
A_{572} \\
A_{383} \\
A_{104} \\
A_{30} \\
A_{37} \\
A_{38} \\
A_{46} \\
28 \\
102 \\
316 \\
A_{500} \\
A_{2,754}\end{array}$ & $\begin{array}{r}.658 \\
.551 \\
.303 \\
112 \\
432 \\
A_{373} \\
360 \\
321 \\
354 \\
154 \\
-204 \\
A^{-440} \\
41\end{array}$ \\
\hline 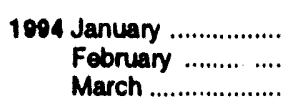 & $\begin{array}{r}A_{4,347} \\
A_{4,336} \\
4,342\end{array}$ & $\begin{array}{r}A_{1,578} \\
\text { A }_{1,089} \\
957\end{array}$ & $\begin{array}{r}A_{\mathbf{5 , 9 2 5}} \\
A_{\mathbf{5 , 4 2 6}} \\
\mathbf{5 , 2 9 9}\end{array}$ & $\begin{array}{r}\text { A. } 251 \\
\text { A. } 214 \\
.71\end{array}$ & $\begin{array}{r}\text { ค. } 13.7 \\
\text { ค. } 16.4 \\
\cdot 6.9\end{array}$ & $\begin{array}{r}A_{30} \\
A_{48} \\
105\end{array}$ & $\begin{array}{r}A_{758} \\
A_{542} \\
239\end{array}$ & $\begin{array}{r}\text { R.724 } \\
\text { A.404 } \\
.138\end{array}$ \\
\hline
\end{tabular}

- For total underground storage capacily al the end of each calendar year. seg Note 8 at end of section.

b For 1980-1992, data ditter from those shown on Table 4.2, which includes lquefled natural gas storage for that period.

- Posilve numbers incicale injections are greater than withdrawab. Negative numbers indicate withdrawab are oreater than injections. Not Injection or withdrawats may not equal the diflerence between applicable ending stocks. See Note 8 at end of section.

ReRevised data.

Notes: - Geographic coverage is the 50 States and the Distric: of Columbla. - Totals may not equal sum of components due to independent rounding.

Sources: - Storege Activity: 1973-1075-Energy Informalion Administration (ELA), Natural Gas Annual 1990, Volume?, Table 9. 1976-1979-EIA, Natural Gas Production and Consumption 15;9. Table 1.
1080-1988-ElA, Natural Gas Annual 1990, Volume 2, Table 11. 1 cat forward-EX, Natural Gas Monthy, May 1994, Table 13. - Other Onta: 1973 and 1074_American Gas Associalion (ACA), Gas Facto, 1972 Dara, Table 57, Gas Facts, 1973 Disa, Table 57, and Ges Fact, 1974 Data, Table 40. 1978 and 1078-Federal Energy Administralion (FEA), Fom FEA-G318-M-0, "Undercround Gas Storage Report," and Federd Power Commission (FPC), Form FPC-8, "Underground Cas Storage Repont." 107 and 1978-EIA, Form FEA-G318-M-0, "Undarground Gas Staras Peport," and Federal Energy Regulatory Commiasion (FERC), Fom FERC-8, "Underground Gas Siorage Report." 1070-1086-EL, Form EIA-101, "Underground Gas Storage Report," and FERC, Form FERC-8, Underground Gas Storage Repon." 1087 torward-EIA, Natural Gas Monthy, May 1904, Table 13. 


\section{Natural Gas Notes}

1. Nonhydrocarbon Gases Removed: Annual data on nonhydrocarbon gases removed from marketed production-carbon dioxide, helium, hydrogen sulfide, and nitrogen-are from the Energy Information Administration (EIA) Natural Gas Annual (NGA) 1991. Data are not available for periods prior to 1980. Monthly data are reported by three States and computed for six States. Monthly data are preliminary until after publication of the EIA NGA. Differences between annual data published in the EIA NGA and the sum of the preliminary monthly data (January-December) are allocated proportionally to the months to create final monthly data. For further information on methods of estimating preliminary monthly data, see the EIA Naural Gas Monthly (NGM).

\section{Production.}

- Annual data: Final annual data are from the EIA NGA.

- Estimated monthly data: Data for the two most recent months presented are estimated. Some of the data for earlier months are also estimated or computed. For a discussion of computation and estimation procedures, see the EIA NGM.

- Preliminary monthly data: Monthly data are considered preliminary until after publication of the EIA NGA. Preliminary monthly data are gathered from reports to the Interstate Oil Compact Commission and the U.S. Minerals Management Service. Volumetric data are converted, as necessary, to a standard $14.73 \mathrm{psi}$ pressure base. Unless there are major changes, data are not revised until after publication of the EIA NGA.

- Final monthly data: Differences between aniuual data in the EIA $N G A$ and the sum of preliminary monthly data (January-December) are allocated proportionally to the months to create final monthly data.

3. Extraction Loss: Extraction loss is the reduction in volume of natural gas resulting from the removal of natural gas liquid constituents at natural gas processing plants.

Annual data for extraction loss are from the EIA $N G A$, where they are estimated on the basis of the type and quantity of liquid products extracted from the gas stream and the calculated volume of such products at standard conditions. For a detailed explanation of the calculations used to derive estimated extraction losses, see the EIA NGA.

Preliminary monthly data are estimated on the basis of extraction loss as an annual percentage of marketed production. This percentage is applied to each month's marketed production to estimate monthly extraction loss.

Monthly data are revised and considered final after the publication of the EIA NGA. Final monthly data are estimated by allocating annual extraction loss data to the months on the basis of total natural gas marketed production data from the EIA NGA.

4. Supplemental Gaseous Fuels: Any gaseous substance that, introduced into or commingled with natural gas, increases the volume available for disposition. Such substances include, but are not limited to, propane-air, refinery gas, coke oven gas, still gas, manufactured gas, biomass gas, or air or inert gases added for Btu stabilization.

Annual data beginning with 1980 are from the EIA $N G A$. Unknown quantities of supplemental gaseous fuels are included in consumption data for 1979 and earlier years.

Monthly data are considered preliminary until after the publication of the EIA NGA. Monthly estimates are based on the annual ratio of supplemental gaseous fuels to the sum of dry gas production, net imports, and net withdrawals from storage. The ratio is applied to the monthly sum of the three elements to compute a monthly supplemental gaseous fuels figure.

5. Im $\mathrm{m}_{\mathrm{r}}$ orts and Exports: The United States imports natural gas via pipeline from Canada. Prior to 1985 , it also imported natural gas via pipeline from Mexico. Liquefied natural gas (LNG) arrives via tanker from Algeria. One shipment of LNG was received from Indonesia in December 1986. Very small amounts of LNG arrived from Canada in 1973 (667 million cubic feet), 1977 (572 million cubic feet), and 1981 (6 million cubic feet). The United States exports natural gas via pipeline to Canada and Mexico and LNG via tanker to Japan.

Annual and final monthly data are from the annual Form FPC-14, "Annual Report for Importers and Exporters of Natural Gas," which requires data to be reported by month for the calendar year.

Preliminary monthly data are EIA estimates. For a discussion of estimation procedures, see the EIA NGM. Preliminary data are revised after the publication of the EIA U.S. Imports and Exports of Natural Gas.

6. Consumption: Consumption includes pipeline fuel use, lease and plant fuel use, and deliveries to consuming sectors.

Final data are from the EIA NGA. Monthly data are considered preliminary until after publication of the EIA $N G A$. For more detailed information on the methods of 
estimating preliminary and final monthly data, see the EIA NGM.

7. Balancing Item: The balancing item for natural gas represents the difference between the sum of the components of natural gas supply and the sum of components of natural gas disposition. The differences may be due to quantities lost or to the effects of data reporting problems. Reporting problems include differences due to the net result of conversions of flow data metered at varying temperature and pressure bases and converted to a standard temperature and pressure base; the effect of variations in company accounting and billing practices; differences between billing cycle and calendar period time frames; and imbalances resulting from the merger of data reporting systems which vary in scope, format, definitions, and type of respondents.

The increase of 0.2 trillion cubic feet (Tcf) in the "Balancing Item" category in 1983, followed by a decline of $0.5 \mathrm{Tcf}$ in 1984 , reflected unusually large differences resulting from the use of the annual billing cycle (essentially December 15 through the following December 14) consumption data in conjunction with calendar year supply data. Record cold temperatures during the last half of December 1983 resulted in a reported $0.3 \mathrm{Tcf}$ increase in net withdrawals from underground storage for peak shaving as compared with the same period in 1982, but the effect of this cold weather was reflected primarily in 1984 consumption data. For underground storage data, see Table F2 in the May 1985 NGM, which was published in July 1985.

8. Natural Gas Storage: Gas in storage at the end of a reporting period may not equal the quantity derived by adding or subtracting net injections or withdrawals from the quantity in storage at the end of the previous period. The difference is due to changes in the quantity of native gas included in the base gas and/or losses in base gas due to migration from storage reservoirs.

Monthly underground storage data are collected from the Forms FERC-8 (interstate data) and EIA-191 (intrastate data). Beginning in January 1991, all data are collected on the revised Form EIA-191. Injection and withdrawal data from the FERC-8/EIA-191 survey are adjusted to correspond to data from Form EIA-176 following publication of the EIA NGA.

The final monthly and annual storage and withdrawal data for 1980-1989 include both underground and liquefied natural gas (LNG) storage. Annual data on LNG additions and withdrawals are from Form EIA176. Monthly data are estimated by computing the ratio of each month's underground storage additions and withdrawals to annual underground storage additions and withdrawals and applying the ratio to the annual LNG data.

Total underground storage capacity at the end of each calendar year since 1975 (first year available), in billion cubic feet, was:

$\begin{array}{llll}1975 & 6,280 & 1984 & 8,043 \\ 1976 & 6,544 & 1985 & 8,067 \\ 1977 & 6,678 & 1986 & 8,145 \\ 1978 & 6,890 & 1987 & 8,124 \\ 1979 & 6,929 & 1988 & 8,124 \\ 1980 & 7,434 & 1989 & 8,124 \\ 1981 & 7,805 & 1990 & 8,125 \\ 1982 & 7,915 & 1991 & 7,993 \\ 1983 & 7,985 & 1992 & 7,932\end{array}$

Current capacity is 7,932 billion cubic feet. 


\section{Section 5. Oil and Gas Resource Development}

A total of 88 seismic exploration crews were active in April 1994, 11 more crews than were active a year earlier. Of the total, 68 were land crews and 20 were aboard marine vessels. The number of land crews increased by 5 and the number of operating marine vessels increased by 6 vessels from the April 1993 count.

The April 1994 rotary rig count of 723 was 2 percent lower than the count in the previous month but 18 percent higher than the count in April 1993. Of the total number of rigs in operation, 617 were onshore and 106 were offshore. The number of onshore rigs was up 14 percent from the number in April 1993, and the number of offshore rigs was up 54 percent.
Total footage drilled in April 1994 was 9.2 million feet down 6 percent from footage drilled in March 1994 and down 16 percent from that drilled in April 1993.

The estimated number of exploratory and development oil and gas wells drilled during April 1994 was 1,103, 15 percent lower than the number drilled in March 1994 and 12 percent lower than the number drilled in April 1993. The estimated number of oil wells drilled was 677 and the estimated number of gas wells was 426, 12 percent higher and 34 percent lower, respectively, from the April 1993 levels. The estimated number of dry holes drilled in April 1994 was 503, 9 percent higher than the number drilled in March 1994 but 10 percent lower than the number drilled in April 1993.

\section{Figure 5.1 Oll and Gas Resource Development Indicators}

\section{Crews Engaged in Seismic Exploration}

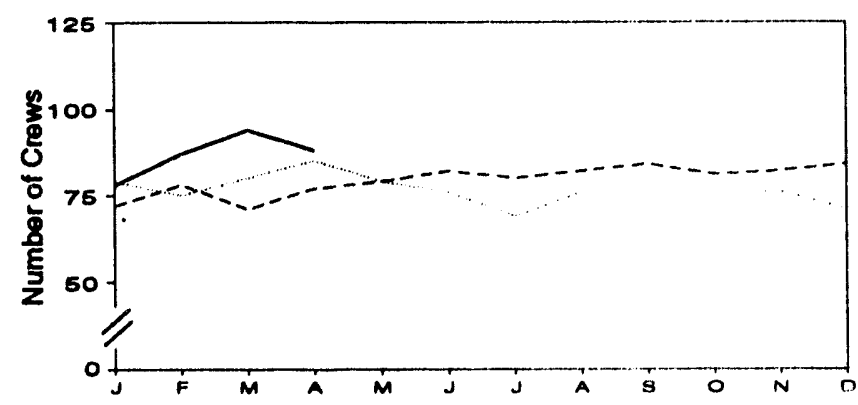

Wells Drilled

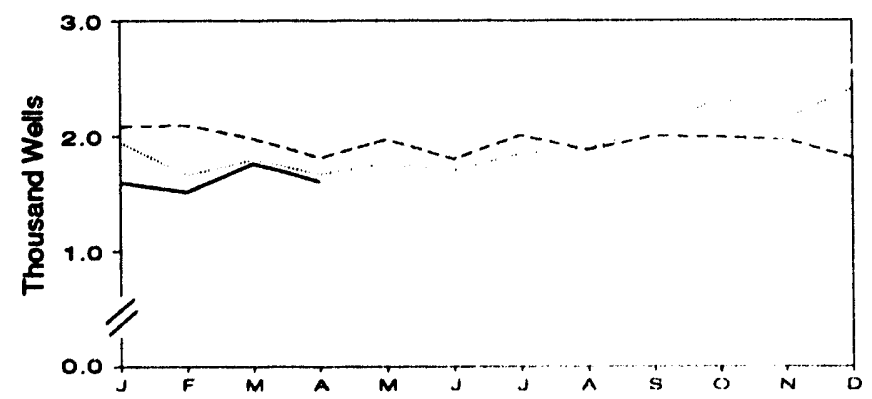

Rotary Rigs in Operation

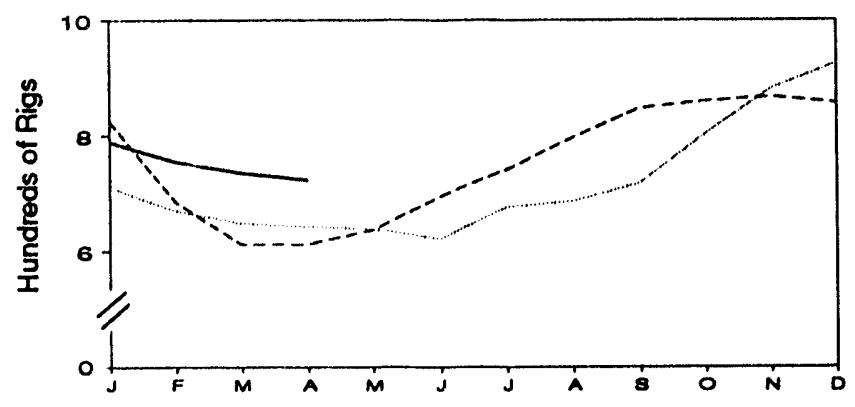

Footage Drilled

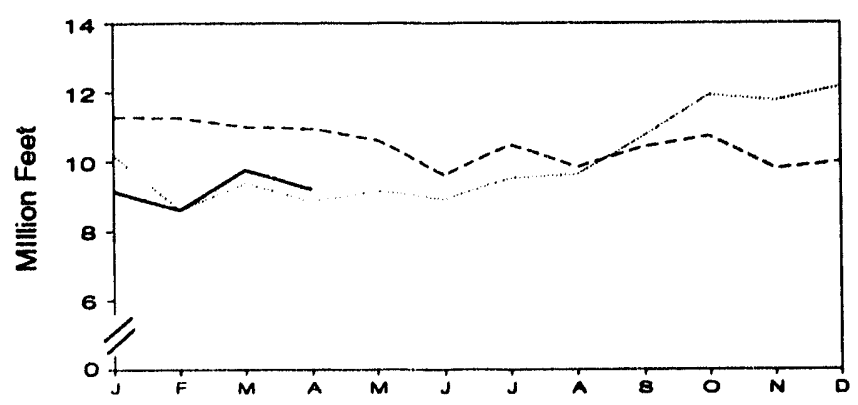




\begin{tabular}{|c|c|c|c|c|c|c|c|c|c|c|}
\hline & \multicolumn{3}{|c|}{$\begin{array}{l}\text { Crows Engaged In } \\
\text { Solsmic Exploration }\end{array}$} & \multicolumn{5}{|c|}{ Rotary Rlge in Operation } & \multirow{3}{*}{$\begin{array}{l}\text { Total } \\
\text { Footage } \\
\text { Drllledo }\end{array}$} & \multirow{3}{*}{$\begin{array}{l}\text { Actve } \\
\text { Well Bervicing } \\
\text { Unitsd }\end{array}$} \\
\hline & & & & \multicolumn{2}{|c|}{ By sito } & \multicolumn{2}{|c|}{ Bу Type } & \multirow[b]{2}{*}{ Totalb } & & \\
\hline & Offshore & Onshore & Total & Offehore & Onshore & Oll & Oas & & & \\
\hline & \multicolumn{3}{|c|}{ Monthiy Average } & \multicolumn{5}{|c|}{ Weokly Averago } & Thousend Feot & Number \\
\hline 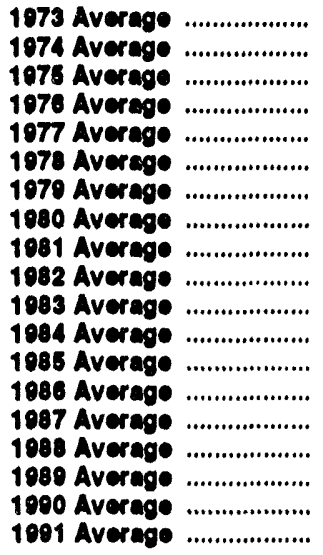 & $\begin{array}{l}23 \\
31 \\
30 \\
25 \\
27 \\
25 \\
30 \\
37 \\
44 \\
57 \\
47 \\
40 \\
45 \\
24 \\
24 \\
29 \\
23 \\
23 \\
19\end{array}$ & $\begin{array}{r}227 \\
274 \\
284 \\
237 \\
281 \\
327 \\
370 \\
493 \\
837 \\
531 \\
428 \\
445 \\
333 \\
176 \\
153 \\
153 \\
109 \\
102 \\
85\end{array}$ & $\begin{array}{l}280 \\
305 \\
284 \\
202 \\
308 \\
352 \\
400 \\
630 \\
681 \\
688 \\
473 \\
494 \\
378 \\
200 \\
177 \\
182 \\
132 \\
125 \\
104\end{array}$ & $\begin{array}{r}84 \\
94 \\
108 \\
120 \\
167 \\
185 \\
207 \\
231 \\
286 \\
243 \\
190 \\
213 \\
208 \\
99 \\
95 \\
123 \\
105 \\
108 \\
81\end{array}$ & $\begin{array}{r}1,110 \\
1,378 \\
1,654 \\
1,829 \\
1,834 \\
2,074 \\
1,070 \\
2,678 \\
3,714 \\
2,862 \\
2,033 \\
2,215 \\
1,774 \\
885 \\
841 \\
813 \\
764 \\
802 \\
779\end{array}$ & $\begin{array}{l}\text { NA } \\
N A \\
N A \\
N A \\
N A \\
N A \\
N A \\
N A \\
N A \\
N A \\
N A \\
N A \\
N A \\
N A \\
N A \\
554 \\
453 \\
532 \\
482\end{array}$ & $\begin{array}{l}\text { NA } \\
\text { NA } \\
\text { NA } \\
\text { NA } \\
\text { NA } \\
\text { NA } \\
\text { NA } \\
\text { NA } \\
\text { NA } \\
\text { NA } \\
\text { NA } \\
\text { NA } \\
\text { NA } \\
\text { NA } \\
\text { NA } \\
\mathbf{3 5 4} \\
\mathbf{4 0 1} \\
\mathbf{4 6 4} \\
\mathbf{3 5 1}\end{array}$ & $\begin{array}{r}1,104 \\
1,472 \\
1,080 \\
1,068 \\
2,001 \\
2,250 \\
2,177 \\
2,000 \\
3,070 \\
3,108 \\
2,232 \\
2,428 \\
1,080 \\
964 \\
938 \\
938 \\
880 \\
1,010 \\
860\end{array}$ & 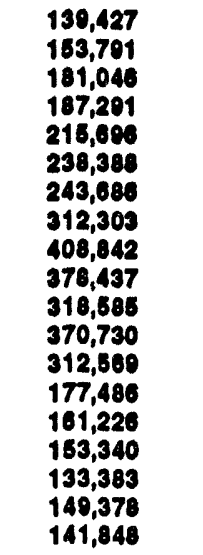 & $\begin{array}{c}N A \\
N A \\
N A \\
2,601 \\
2,820 \\
2,088 \\
3,300 \\
4,080 \\
4,050 \\
4,248 \\
3,732 \\
4,663 \\
1,710 \\
3,030 \\
3,060 \\
3,341 \\
3,301 \\
3,058 \\
3,331\end{array}$ \\
\hline 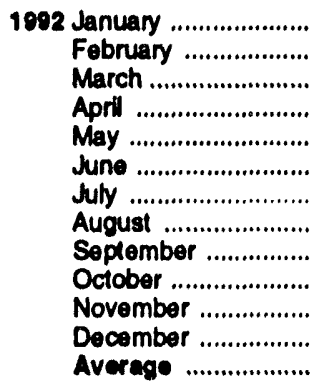 & $\begin{array}{r}18 \\
13 \\
13 \\
13 \\
13 \\
12 \\
9 \\
9 \\
10 \\
10 \\
10 \\
15 \\
13 \\
12\end{array}$ & $\begin{array}{l}61 \\
62 \\
67 \\
72 \\
68 \\
64 \\
60 \\
67 \\
68 \\
66 \\
61 \\
58 \\
64\end{array}$ & $\begin{array}{l}79 \\
75 \\
80 \\
85 \\
79 \\
76 \\
69 \\
76 \\
76 \\
76 \\
76 \\
71 \\
76\end{array}$ & $\begin{array}{l}56 \\
51 \\
54 \\
55 \\
47 \\
44 \\
48 \\
51 \\
45 \\
53 \\
60 \\
59 \\
52\end{array}$ & $\begin{array}{l}654 \\
618 \\
594 \\
587 \\
591 \\
577 \\
628 \\
635 \\
672 \\
750 \\
822 \\
867 \\
689\end{array}$ & $\begin{array}{l}400 \\
378 \\
381 \\
370 \\
358 \\
343 \\
349 \\
334 \\
345 \\
392 \\
418 \\
397 \\
373\end{array}$ & $\begin{array}{l}294 \\
277 \\
250 \\
251 \\
260 \\
260 \\
310 \\
331 \\
358 \\
399 \\
451 \\
509 \\
331\end{array}$ & $\begin{array}{l}710 \\
669 \\
648 \\
642 \\
638 \\
621 \\
676 \\
686 \\
717 \\
803 \\
882 \\
926 \\
721\end{array}$ & $\begin{array}{r}10,186 \\
8,610 \\
9,381 \\
R_{8,860} \\
9,158 \\
8,915 \\
9,529 \\
9,635 \\
10,748 \\
11,925 \\
11,764 \\
12,167 \\
R_{120,888}\end{array}$ & $\begin{array}{l}2,912 \\
2,704 \\
2,582 \\
2,727 \\
2,264 \\
2,369 \\
2,492 \\
2,630 \\
2,825 \\
3,076 \\
2,977 \\
3,218 \\
2,732 \\
\end{array}$ \\
\hline 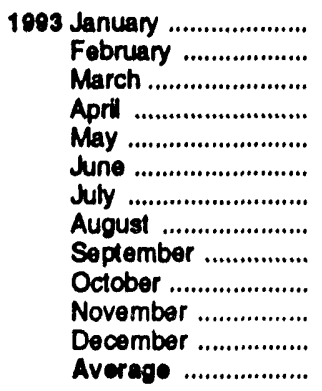 & $\begin{array}{l}17 \\
15 \\
16 \\
14 \\
15 \\
17 \\
15 \\
15 \\
16 \\
18 \\
15 \\
17 \\
18 \\
16\end{array}$ & $\begin{array}{l}55 \\
63 \\
65 \\
63 \\
64 \\
65 \\
65 \\
66 \\
66 \\
66 \\
65 \\
66 \\
63\end{array}$ & $\begin{array}{l}72 \\
78 \\
71 \\
77 \\
79 \\
82 \\
80 \\
82 \\
84 \\
81 \\
82 \\
84 \\
79\end{array}$ & $\begin{array}{r}72 \\
69 \\
62 \\
69 \\
73 \\
83 \\
85 \\
87 \\
89 \\
93 \\
99 \\
103 \\
82\end{array}$ & $\begin{array}{l}752 \\
615 \\
549 \\
543 \\
564 \\
612 \\
656 \\
710 \\
758 \\
767 \\
769 \\
754 \\
672\end{array}$ & $\begin{array}{l}335 \\
311 \\
315 \\
320 \\
323 \\
350 \\
368 \\
397 \\
418 \\
441 \\
453 \\
425 \\
373\end{array}$ & $\begin{array}{l}454 \\
334 \\
268 \\
270 \\
294 \\
327 \\
360 \\
390 \\
421 \\
411 \\
408 \\
426 \\
364\end{array}$ & $\begin{array}{l}824 \\
684 \\
611 \\
612 \\
637 \\
695 \\
741 \\
797 \\
848 \\
860 \\
868 \\
857 \\
754\end{array}$ & $\begin{array}{r}11,302 \\
11,272 \\
11,018 \\
R 10,965 \\
10,644 \\
9,620 \\
10,504 \\
9,852 \\
10,437 \\
A 10,746 \\
9,810 \\
9,985 \\
R_{126,165}\end{array}$ & $\begin{array}{l}2,807 \\
2,890 \\
2,820 \\
2,703 \\
2,848 \\
3,087 \\
3,178 \\
3,423 \\
3,341 \\
3,519 \\
3,604 \\
3,662 \\
3,158\end{array}$ \\
\hline 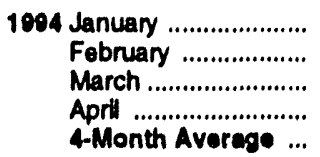 & $\begin{array}{l}18 \\
18 \\
19 \\
20 \\
10\end{array}$ & $\begin{array}{l}60 \\
69 \\
75 \\
68 \\
68\end{array}$ & $\begin{array}{l}78 \\
87 \\
94 \\
88 \\
87\end{array}$ & $\begin{array}{r}99 \\
95 \\
99 \\
106 \\
90\end{array}$ & $\begin{array}{l}690 \\
659 \\
636 \\
617 \\
650\end{array}$ & $\begin{array}{l}356 \\
337 \\
323 \\
314 \\
332\end{array}$ & $\begin{array}{l}425 \\
405 \\
403 \\
398 \\
407\end{array}$ & $\begin{array}{l}789 \\
754 \\
735 \\
723 \\
740\end{array}$ & $\begin{array}{r}9,152 \\
8,612 \\
9,769 \\
9,217 \\
36,750\end{array}$ & $\begin{array}{r}3,386 \\
3,063 \\
A_{2,977} \\
E_{3,000} \\
E_{3,148}\end{array}$ \\
\hline $\begin{array}{l}1903 \text { 4-Month Average ... } \\
1902 \text { 4-Month Average ... }\end{array}$ & $\begin{array}{l}16 \\
14\end{array}$ & $\begin{array}{l}80 \\
68\end{array}$ & $\begin{array}{l}75 \\
80\end{array}$ & $\begin{array}{l}60 \\
64\end{array}$ & $\begin{array}{l}610 \\
616\end{array}$ & $\begin{array}{l}320 \\
382\end{array}$ & $\begin{array}{l}328 \\
260\end{array}$ & $\begin{array}{l}670 \\
670\end{array}$ & $\begin{array}{l}44,667 \\
37,047\end{array}$ & $\begin{array}{l}2,810 \\
2,734\end{array}$ \\
\hline
\end{tabular}

- Monthly data are averages of 4 or 5-woek reporting periods, not calendar months. Annual data are averages of 52- or 53-weok reporting periods, not calondar yoars.

bum of oil, gas, and miscellaneous other rigs, which is not shown.

c Values shown are totals.

d Seo Glossary.

$R=$ Revised data. NA=Nol avallable. $E=E s t i m a t e$.

Note: Geographic coverage is the 50 States and the District of Columbia.
Sources: - Crewe Engeged in 8olemle Exploratton: Socloty of Exploration Geoptysicists, Tulsa, Oklahoma, Monthly Solemic Crow Count. - Rotary Rige in Operatton: Baker Hughes, Inc., Houston, Texas, Rotany Rigs Running-by State. - Total Footege Drlled: Energy Information Administration computations, which are based on well reports submitted to the American Petroleum Institute by the Potroleum Information Corporation, Denver, Colorado. - Active Well Servicing Units: American Association of Oilwell Servicing Contractors, Dallas, Texas, Well Servicing. 


\begin{tabular}{|c|c|c|c|c|c|c|c|c|c|c|c|c|}
\hline & \multicolumn{4}{|c|}{ Exploratory } & \multicolumn{4}{|c|}{ Devolopment } & \multicolumn{4}{|c|}{ Totel } \\
\hline & 이 & Qans & Dry & Total & Oll & Ges & Dry & Total & Oll & ans & Dry & Total \\
\hline 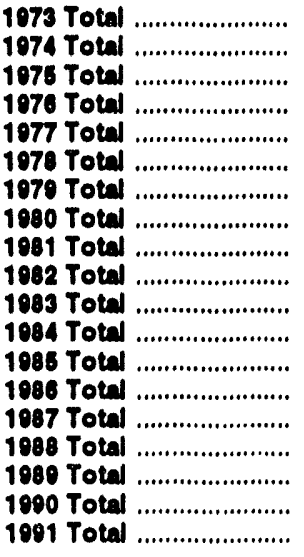 & $\begin{array}{r}664 \\
870 \\
091 \\
1,100 \\
1,183 \\
1,191 \\
1,386 \\
1,781 \\
2,867 \\
2,470 \\
2,113 \\
2,335 \\
1,870 \\
988 \\
859 \\
702 \\
580 \\
817 \\
545\end{array}$ & $\begin{array}{l}1,070 \\
1,205 \\
1,263 \\
1,382 \\
1,562 \\
1,702 \\
1,920 \\
2,004 \\
2,833 \\
2,188 \\
1,680 \\
1,590 \\
1,282 \\
733 \\
673 \\
683 \\
654 \\
584 \\
464\end{array}$ & $\begin{array}{r}6,038 \\
6,894 \\
7,207 \\
6,854 \\
7,402 \\
8,054 \\
7,478 \\
9,038 \\
12,207 \\
11,348 \\
10,271 \\
11,482 \\
0,445 \\
5,611 \\
5,179 \\
4,768 \\
4,001 \\
3,782 \\
3,303\end{array}$ & $\begin{array}{r}7,771 \\
8,060 \\
9,481 \\
9,316 \\
10,147 \\
11,037 \\
10,733 \\
12,910 \\
17,497 \\
15,984 \\
14,044 \\
15,416 \\
12,008 \\
7,232 \\
6,711 \\
6,221 \\
6,235 \\
4,983 \\
4,312\end{array}$ & $\begin{array}{r}0,697 \\
12,794 \\
18,988 \\
18,507 \\
17,517 \\
17,874 \\
10,388 \\
30,497 \\
40,176 \\
36,072 \\
35,086 \\
40,250 \\
33,142 \\
17,713 \\
15,327 \\
12,530 \\
0,750 \\
11,533 \\
11,363\end{array}$ & $\begin{array}{r}6,806 \\
8,065 \\
8,907 \\
8,076 \\
10,567 \\
12,613 \\
13,250 \\
18,129 \\
17,374 \\
18,776 \\
12,806 \\
16,413 \\
12,970 \\
7,402 \\
7,084 \\
7,575 \\
8,571 \\
9,856 \\
R 8,702\end{array}$ & $\begin{array}{r}4,428 \\
6,311 \\
6,520 \\
6,961 \\
7,634 \\
8,537 \\
8,660 \\
11,302 \\
14,087 \\
16,038 \\
14,066 \\
14,316 \\
11,763 \\
7,255 \\
6,302 \\
6,476 \\
4,490 \\
4,832 \\
4,527\end{array}$ & $\begin{array}{r}19,021 \\
24,070 \\
29,424 \\
31,024 \\
38,708 \\
39,024 \\
41,176 \\
58,028 \\
72,537 \\
68,484 \\
62,047 \\
60,078 \\
57,876 \\
32,370 \\
28,713 \\
25,581 \\
22,820 \\
26,221 \\
R_{24,592}\end{array}$ & $\begin{array}{l}10,251 \\
13,664 \\
18,079 \\
17,607 \\
16,700 \\
10,065 \\
20,703 \\
32,278 \\
42,843 \\
30,142 \\
37,100 \\
42,586 \\
36,021 \\
18,701 \\
18,186 \\
13,322 \\
10,339 \\
12,150 \\
11,008\end{array}$ & $\begin{array}{r}0,075 \\
7,170 \\
8,170 \\
0,438 \\
12,110 \\
14,408 \\
16,170 \\
17,229 \\
10,007 \\
18,944 \\
14,858 \\
17,012 \\
14,252 \\
8,436 \\
7,787 \\
8,238 \\
0,225 \\
10,440 \\
A_{0,168}\end{array}$ & $\begin{array}{r}10,486 \\
12,206 \\
13,738 \\
13,808 \\
18,036 \\
18,601 \\
16,038 \\
20,337 \\
27,284 \\
28,382 \\
24,386 \\
26,707 \\
21,208 \\
12,786 \\
11,481 \\
10,242 \\
8,491 \\
8,614 \\
7,830\end{array}$ & 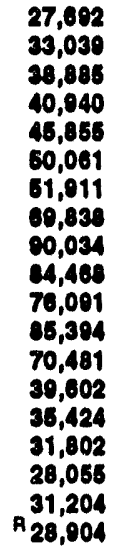 \\
\hline 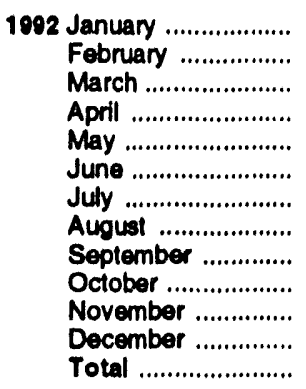 & $\begin{array}{r}46 \\
34 \\
38 \\
32 \\
35 \\
41 \\
43 \\
42 \\
R_{38} \\
P_{30} \\
38 \\
43 \\
P_{460}\end{array}$ & $\begin{array}{r}33 \\
30 \\
30 \\
22 \\
23 \\
32 \\
30 \\
31 \\
22 \\
34 \\
\text { R } 33 \\
\text { R } 33 \\
\text { R } 353\end{array}$ & $\begin{array}{r}218 \\
167 \\
205 \\
233 \\
225 \\
209 \\
256 \\
241 \\
222 \\
205 \\
165 \\
225 \\
2,571\end{array}$ & $\begin{array}{r}287 \\
231 \\
273 \\
287 \\
283 \\
282 \\
329 \\
314 \\
R_{282} \\
A_{269} \\
A_{236} \\
P_{301} \\
R_{3,384}\end{array}$ & $\begin{array}{r}741 \\
590 \\
721 \\
R 665 \\
636 \\
626 \\
664 \\
617 \\
R 783 \\
\text { ค } 748 \\
690 \\
743 \\
P_{8,224}\end{array}$ & $\begin{array}{r}587 \\
564 \\
482 \\
R_{420} \\
469 \\
462 \\
543 \\
601 \\
660 \\
949 \\
\text { P }_{890} \\
\text { P }_{973} \\
\text { P }_{7,600}\end{array}$ & $\begin{array}{l}321 \\
277 \\
319 \\
297 \\
374 \\
330 \\
312 \\
357 \\
339 \\
358 \\
331 \\
391 \\
4,006\end{array}$ & $\begin{array}{r}1,649 \\
1,431 \\
1,522 \\
R_{1,382} \\
1,479 \\
1,418 \\
1,519 \\
1,575 \\
R_{1}, 782 \\
A_{2,055} \\
R_{1}, 911 \\
R_{2,107} \\
A_{18,830}\end{array}$ & $\begin{array}{r}787 \\
624 \\
759 \\
R_{697} \\
671 \\
667 \\
707 \\
659 \\
821 \\
778 \\
728 \\
786 \\
P_{8,684}\end{array}$ & $\begin{array}{r}620 \\
594 \\
512 \\
P_{442} \\
492 \\
494 \\
573 \\
632 \\
682 \\
983 \\
923 \\
1,006 \\
\text { ค } 7,053\end{array}$ & $\begin{array}{r}639 \\
444 \\
524 \\
530 \\
599 \\
539 \\
568 \\
598 \\
561 \\
563 \\
496 \\
616 \\
6,577\end{array}$ & $\begin{array}{r}1,946 \\
1,662 \\
1,795 \\
R_{1,669} \\
1,762 \\
1,7 \times 0 \\
1,848 \\
1,889 \\
2,064 \\
2,324 \\
2,147 \\
2,408 \\
R_{23,214}\end{array}$ \\
\hline 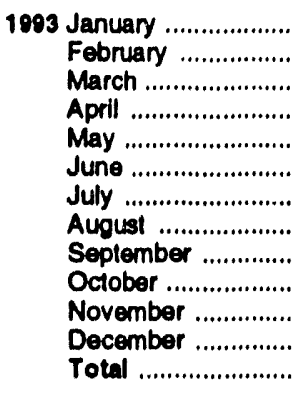 & $\begin{array}{r}41 \\
32 \\
23 \\
41 \\
36 \\
35 \\
34 \\
20 \\
28 \\
32 \\
28 \\
25 \\
375\end{array}$ & $\begin{array}{r}35 \\
42 \\
R_{25} \\
28 \\
33 \\
31 \\
26 \\
36 \\
29 \\
38 \\
36 \\
29 \\
{ }^{2} 386\end{array}$ & $\begin{array}{r}162 \\
171 \\
186 \\
205 \\
176 \\
193 \\
256 \\
226 \\
221 \\
R_{186} \\
194 \\
161 \\
R_{2,337}\end{array}$ & $\begin{array}{r}238 \\
245 \\
R_{234} \\
274 \\
245 \\
259 \\
316 \\
282 \\
278 \\
R_{254} \\
258 \\
215 \\
R_{3,098}\end{array}$ & $\begin{array}{r}627 \\
586 \\
627 \\
562 \\
588 \\
611 \\
676 \\
673 \\
675 \\
A 720 \\
651 \\
536 \\
A_{7,532}\end{array}$ & $\begin{array}{r}929 \\
919 \\
\text { P }_{867} \\
\text { ค } 622 \\
679 \\
558 \\
521 \\
565 \\
634 \\
\text { ค } 693^{6} \\
642 \\
673 \\
\text { ค }_{8,302}\end{array}$ & $\begin{array}{r}290 \\
346 \\
252 \\
355 \\
462 \\
374 \\
498 \\
359 \\
414 \\
P^{3} 324 \\
417 \\
385 \\
P_{4,476}\end{array}$ & $\begin{array}{r}1,846 \\
1,851 \\
A_{1,746} \\
A_{1,539} \\
1,729 \\
1,543 \\
1,695 \\
1,597 \\
1,723 \\
A_{1,737} \\
1,710 \\
1,594 \\
R_{20,310}\end{array}$ & $\begin{array}{r}668 \\
618 \\
650 \\
603 \\
624 \\
646 \\
710 \\
693 \\
703 \\
\text { ค } 752 \\
679 \\
561 \\
\text { R }_{7,007}\end{array}$ & $\begin{array}{r}964 \\
961 \\
892 \\
R_{650} \\
712 \\
589 \\
547 \\
601 \\
663 \\
P^{729} \\
678 \\
702 \\
P_{8,688}\end{array}$ & $\begin{array}{r}452 \\
517 \\
438 \\
560 \\
638 \\
567 \\
754 \\
585 \\
635 \\
510 \\
611 \\
546 \\
6,813\end{array}$ & $\begin{array}{r}2,084 \\
2,096 \\
1,980 \\
R_{1,813} \\
1,074 \\
1,802 \\
2,011 \\
1,879 \\
2,001 \\
\text { P }_{1,901} \\
1,968 \\
1,809 \\
R_{23,408}\end{array}$ \\
\hline $\begin{array}{l}1894 \text { January .................. } \\
\text { February .................. } \\
\text { March .................... } \\
\text { April ..................... } \\
\text { 4-Month Total ....... }\end{array}$ & $\begin{array}{r}R_{51} \\
26 \\
34 \\
40 \\
151\end{array}$ & $\begin{array}{r}P_{41} \\
29 \\
26 \\
27 \\
123\end{array}$ & $\begin{array}{r}P_{171} \\
121 \\
164 \\
193 \\
649\end{array}$ & $\begin{array}{r}R_{263} \\
176 \\
224 \\
260 \\
923\end{array}$ & $\begin{array}{r}{ }^{R} 526 \\
547 \\
693 \\
637 \\
2,403\end{array}$ & $\begin{array}{r}R 564 \\
526 \\
548 \\
399 \\
2,037\end{array}$ & $\begin{array}{r}R_{244} \\
267 \\
298 \\
310 \\
1,119\end{array}$ & $\begin{array}{r}R_{1,334} \\
1,340 \\
1,539 \\
1,346 \\
\mathbf{6 , 5 5 9}\end{array}$ & $\begin{array}{r}577 \\
573 \\
727 \\
677 \\
2,554\end{array}$ & $\begin{array}{r}605 \\
555 \\
574 \\
426 \\
2,160\end{array}$ & $\begin{array}{r}415 \\
388 \\
462 \\
503 \\
1,768\end{array}$ & $\begin{array}{l}1,597 \\
1,516 \\
1,763 \\
1,608 \\
6,482\end{array}$ \\
\hline $\begin{array}{l}1003 \text { 4-Month Total ....... } \\
1902 \text { 4-Month Total ....... }\end{array}$ & $\begin{array}{l}137 \\
150\end{array}$ & $\begin{array}{l}130 \\
115\end{array}$ & $\begin{array}{l}724 \\
823\end{array}$ & $\begin{array}{r}991 \\
1,088\end{array}$ & $\begin{array}{l}2,402 \\
2,717\end{array}$ & $\begin{array}{l}3,337 \\
2,053\end{array}$ & $\begin{array}{l}1,243 \\
1,214\end{array}$ & $\begin{array}{l}6,082 \\
5,084\end{array}$ & $\begin{array}{l}2,530 \\
2,867\end{array}$ & $\begin{array}{l}3,467 \\
2,168\end{array}$ & $\begin{array}{l}1,067 \\
2,037\end{array}$ & $\begin{array}{l}7,073 \\
7,072\end{array}$ \\
\hline
\end{tabular}

ReRevised data.

Notes: - Service wells, stratigraphic tests, and core lests are excluded. - Geographic coverage is the 50 States and the District of Columbla. - Due to the method of estimation, data shown on this page are frequently revised.
See end of section.

Sources: Energy Information Administration computations, which are based on well reports submitted to the American Petroleum Institute by the Petroleum Information Corporation, Denver, Colorado. 


\section{Oil and Gas Resource Development Notes}

Three well types are considered in the Monthly Energy Review (MER) drilling statistics: "completed for oil," "completed for gas," and "dry hole." Wells that productively encounter both crude oil and natural gas are categorized as "completed for oil." Both development wells and exploratory wells (new field wildcats, new pool tests, and extension tests) are included in the statistics. All other classes of wells drilled in connection with the search for producible hydrocarbons are excluded.

Prior to the March $1985 M E R$, drilling statistics consisted of completion data for the above types and classes of wells as reported to the American Petroleum Institute (API) during a given month. Due to time lags between the date of well completion and the date of completion reporting to the API, as-reported well completions proved to be an inaccurate indicator of drilling activity.
During 1982, for example, as-reported well completions rose, while the number of actual completions fell. Consequently, the drilling statistics published since the March 1985 MER are Energy Information Administration-generated (EIA) estimates produced by statistically imputing well counts and footage based on the partial data available from the API.

Estimates for a given month are first published in the $M E R$ for that month. Revisions of the "oil," "gas," and "dry" components are made in the 6th, 12th, and 24th subsequent months, as newly reported data allow refinement of the estimates. Unscheduled revisions may also occur when the latest estimate differs by more than 15 percent during the first 5 months, more than 10 percent during the next 6 months, or more than 2 percent thereafter through 5 years. After 5 years, the reported API data are published in lieu of EIA-generated estimates. Additional information about the EIA estimation methodology may be found in "Estimating Well Completions," the feature article published in the March 1985 MER. 


\section{Section 6. Coal}

Coal production in March 1994 totaled 98 million short tons, 16 percent $^{6}$ higher than coal production in March 1993. Coal production during the first 3 months of 1994 totaled 260 million short tons, 7 percent higher than coal production during the first 3 months of 1993.

Electric utility coal consumption in February 1994 totaled 65 million short tons, 3 percent higher than the consumption level in February 1993.
Electric utility coal stocks were 98 thousand short tons at the end of February 1994, down from 147 million short tons at the end of February 1993.

Coal exports in February 1994 totaled 4 million short tons, 37 percent lower than exports in February 1993. Coal imports in February 1994 totaled 753 thousand short tons, 66 percent higher than imports in February 1993.

${ }^{6}$ Percentage changes are based on unrounded data. 
Figure 6.1 Coal

(Million Short Tons)

Ovenlew, 1973-1993

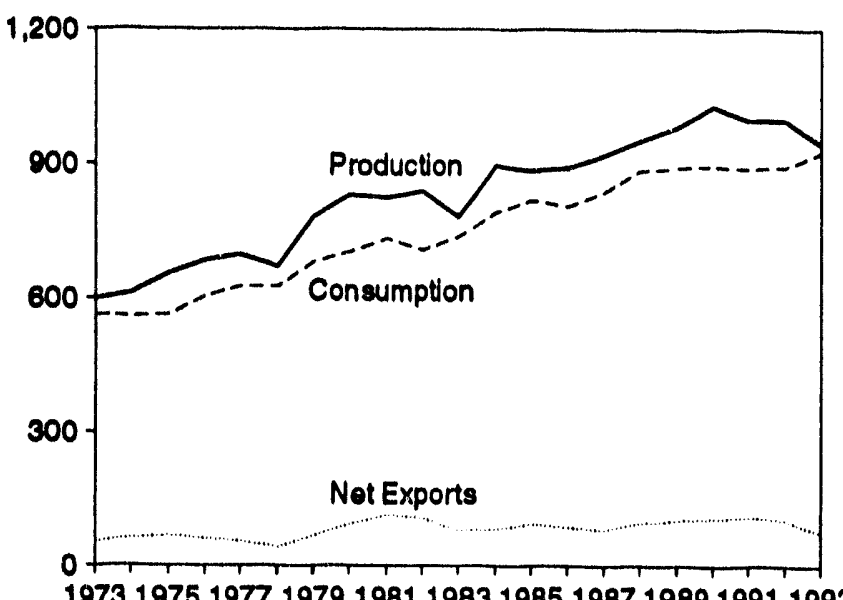

Consumption by Sector, 1973-1993

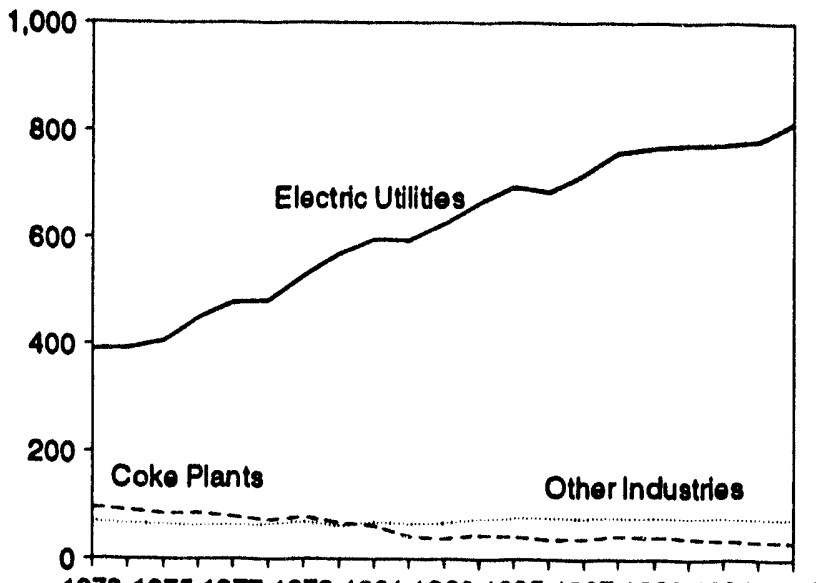

19731975197719791981198319851987198919911993

Stocks, End of Year, 1973-1993

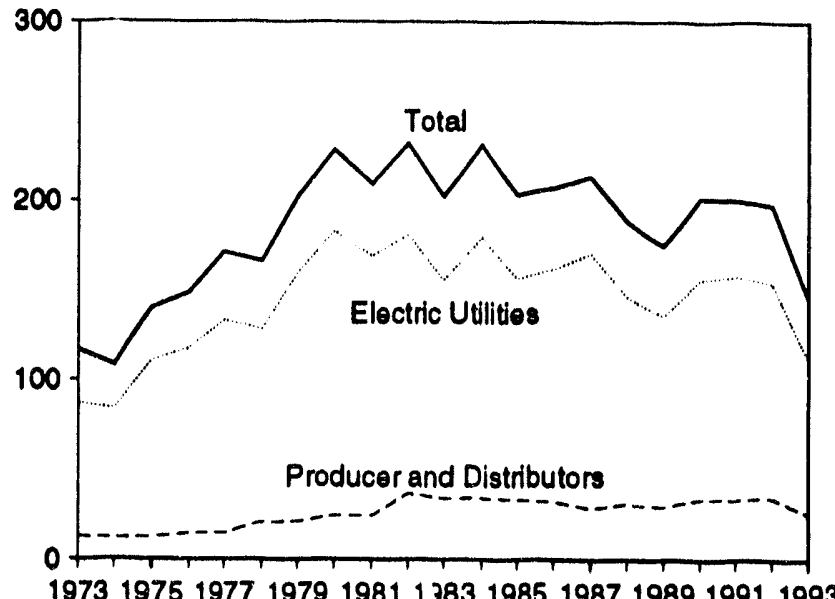

19731975197719791981178319851987198919911993
Overview, Monthly

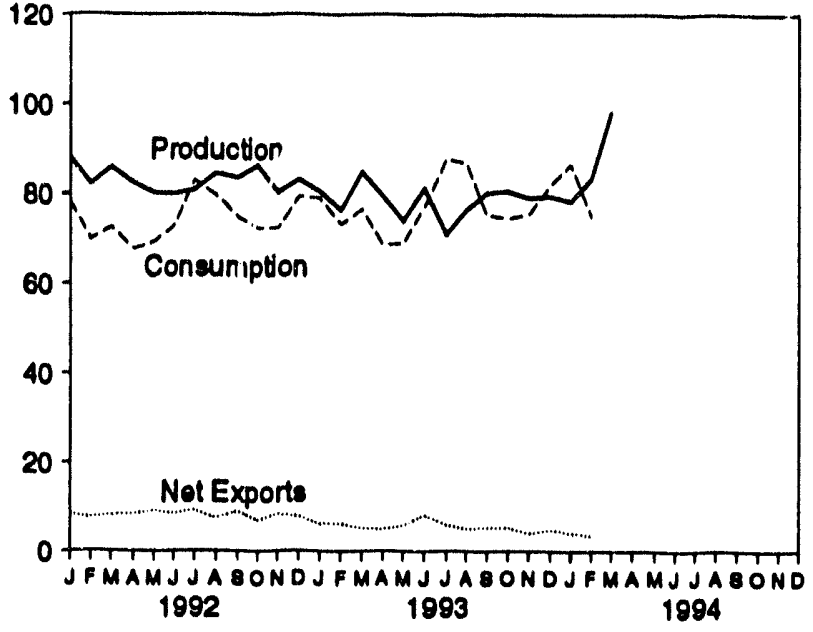

Consumption by Electric Utilities, Monthly

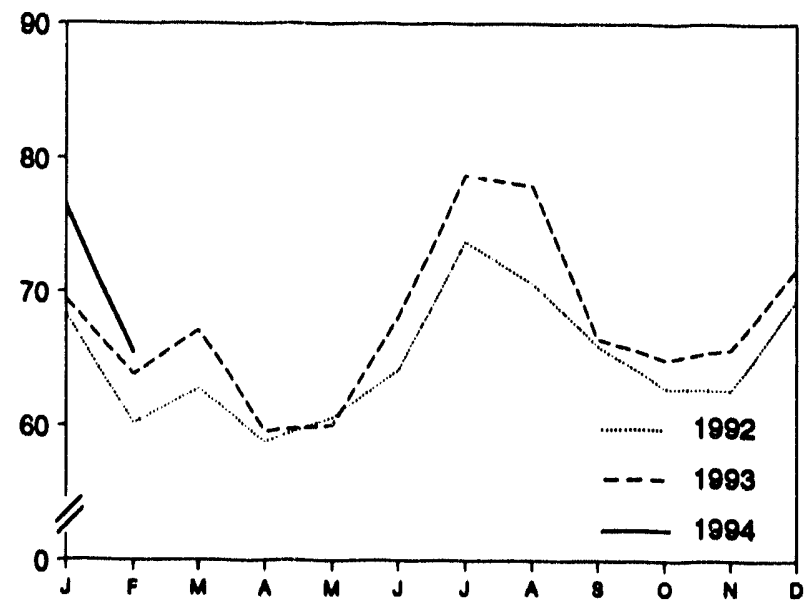

Stocks at Electric Utilities, End of Month

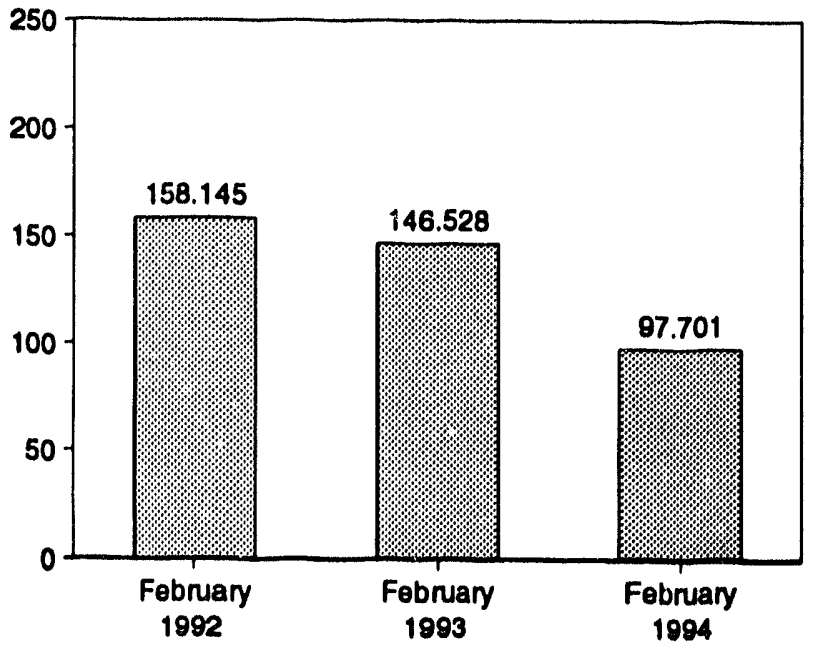

Note: Because vertical scales difler, graphs should not be compared. Sources: Tables 6.1, 8.2, and 6.3. 
Table 6.1 Coal Overview

(Thousand Short Tons)

\begin{tabular}{|c|c|c|c|c|c|}
\hline & Produetion & Consumption & Importa & Export & 8tookab \\
\hline 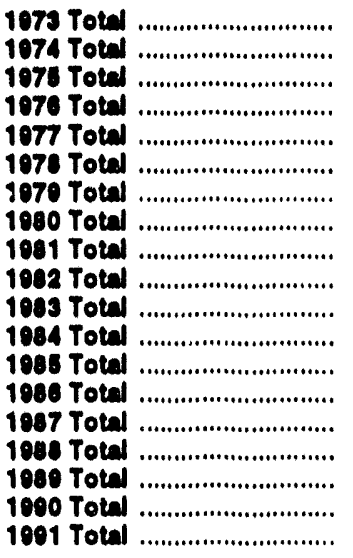 & 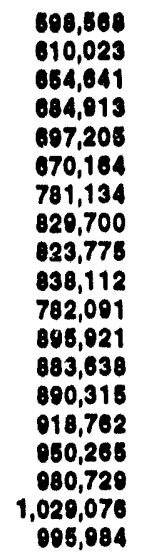 & 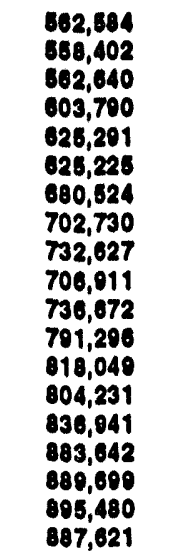 & $\begin{array}{r}127 \\
2,080 \\
840 \\
1,203 \\
1,847 \\
2,963 \\
2,080 \\
1,104 \\
1,043 \\
742 \\
1,271 \\
1,286 \\
1,962 \\
2,212 \\
1,747 \\
2,134 \\
2,851 \\
2,680 \\
3,300\end{array}$ & $\begin{array}{r}63,887 \\
60,861 \\
66,300 \\
60,021 \\
84,312 \\
40,714 \\
86,042 \\
91,742 \\
112,541 \\
106,277 \\
77,772 \\
81,483 \\
92,080 \\
85,818 \\
70,807 \\
95,023 \\
100,818 \\
105,804 \\
108,909\end{array}$ & $\begin{array}{l}116,806 \\
107,087 \\
140,168 \\
148,060 \\
171,323 \\
168,246 \\
202,472 \\
228,407 \\
200,423 \\
232,038 \\
202,684 \\
231,300 \\
203,367 \\
207,310 \\
213,780 \\
188,031 \\
176,087 \\
201,029 \\
200,082\end{array}$ \\
\hline 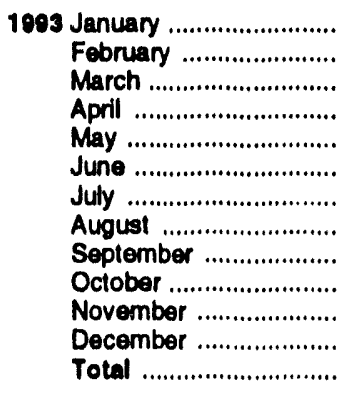 & $\begin{array}{l}80,618 \\
76,484 \\
84,919 \\
79,466 \\
74,034 \\
81,273 \\
71,000 \\
76,492 \\
80,245 \\
80,631 \\
79,124 \\
79,538 \\
943,824\end{array}$ & $\begin{array}{l}79,073 \\
73,329 \\
78,634 \\
68,742 \\
69,021 \\
77,125 \\
87,693 \\
86,868 \\
75,303 \\
74,622 \\
75,458 \\
81,968 \\
925,834\end{array}$ & $\begin{array}{r}344 \\
454 \\
415 \\
281 \\
298 \\
514 \\
643 \\
747 \\
753 \\
1,054 \\
970 \\
836 \\
7,300\end{array}$ & $\begin{array}{r}6,506 \\
6,716 \\
5,648 \\
6,268 \\
6,060 \\
8,619 \\
6,573 \\
5,830 \\
6,120 \\
6,485 \\
5,019 \\
5,677 \\
74,519\end{array}$ & $\begin{array}{l}195,005 \\
192,379 \\
190,977 \\
194,143 \\
195,609 \\
189,648 \\
168,148 \\
152,748 \\
149,040 \\
150,710 \\
151,098 \\
145,742 \\
145,742\end{array}$ \\
\hline 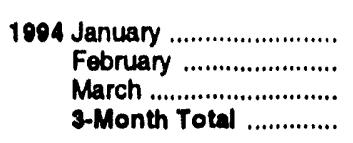 & $\begin{array}{r}78,456 \\
83,395 \\
98,228 \\
280,070\end{array}$ & $\begin{array}{c}E_{86,646} \\
E_{75,047} \\
\text { NA } \\
\text { NA }\end{array}$ & $\begin{array}{l}540 \\
753 \\
\text { NA } \\
\text { NA }\end{array}$ & $\begin{array}{c}4,731 \\
4,252 \\
\text { NA } \\
\text { NA }\end{array}$ & $\begin{array}{c}\text { E } 133,956 \\
\text { E } 133,621 \\
\text { NA } \\
\text { NA }\end{array}$ \\
\hline $\begin{array}{l}1903 \text { 3-Month Toral .............. } \\
1092 \text { 3-Month Total ................. }\end{array}$ & $\begin{array}{l}242,020 \\
255,058\end{array}$ & $\begin{array}{l}229,035 \\
220,504\end{array}$ & $\begin{array}{r}1,213 \\
670\end{array}$ & $\begin{array}{l}18,870 \\
24,731\end{array}$ & $\begin{array}{l}190,977 \\
208,486\end{array}$ \\
\hline
\end{tabular}

- Includes Puerto Rico.

- Stocks held by electric unillties, coke plants, general indusiry, and coa producers and distributors at end of period. Stocks held at retail dealers lor consumption by the residentlal and commercial sector are excluded.

$N A=$ Not available. Ex=Estimate.

Notes: - Geographic coverage is the 50 States and the District of Columbia. - Data through 1992 are final. Subsequent data are preliminary.

- Totals may not equal sum ol components due to independent rounding
- For methodology used to calculate production, consumption, and stocks, 800 Notes 1, 2, and 3 at end of section.

Sources: - Production: 1973-Soptember 1977-U.S. Department of the Interior, Bureau of Mines, Minerals Yearbook and Minerals Industry Sunveys. October 1977 forward-Energy Information Administration, Wookly Coal Production. - Consumption: Table 6.2. - Importe and Exports: U.S. Department of Commerce, Bureau of the Census, Monthly Reports IM-145 (Imports) and EM-522 (Exports). - Stocks: Table 6.3. 


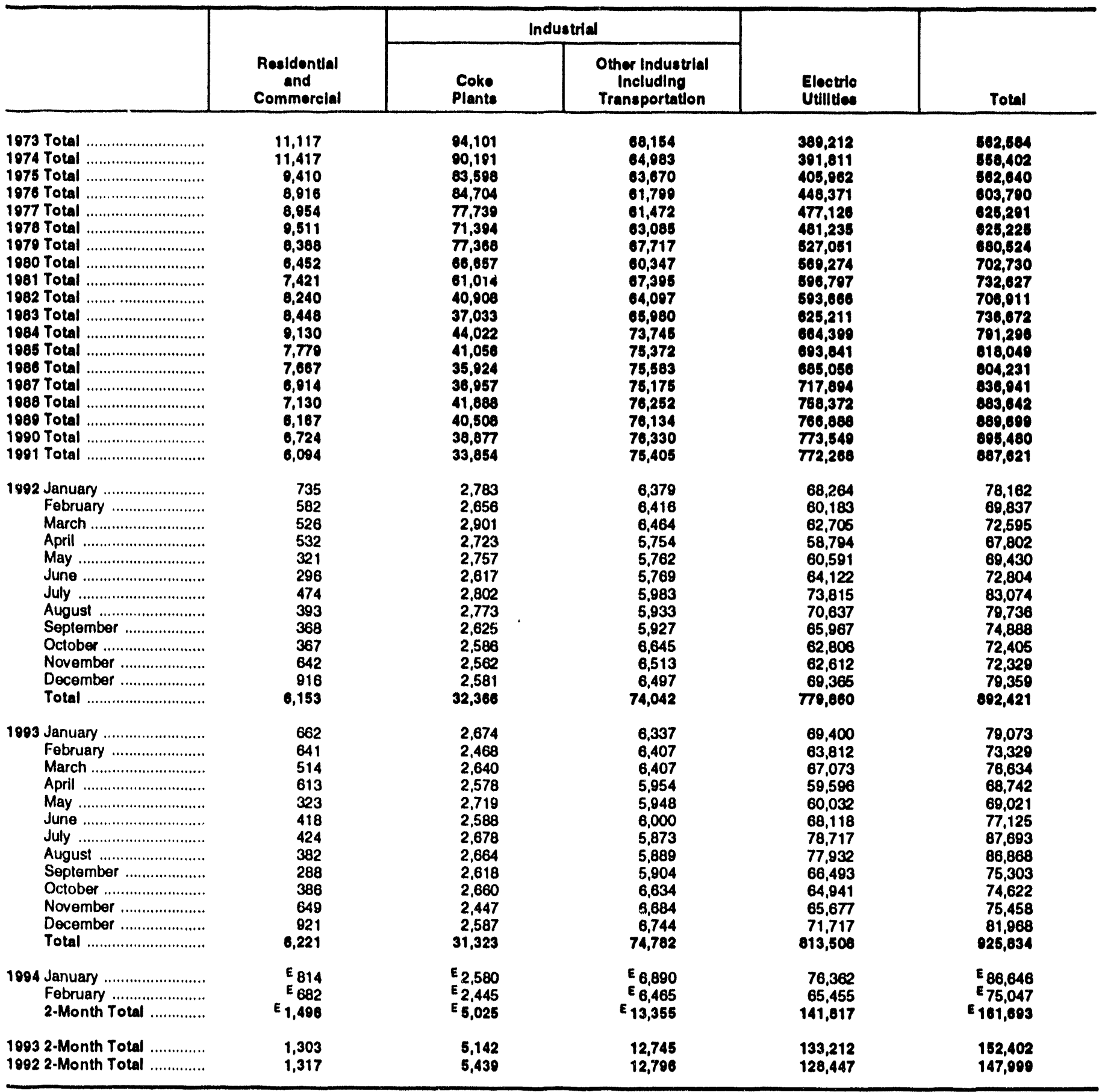

Eastimate.

Noles: - For sector-specific reporting and estimating information, see Nole 2 at end of section. - Geographic coverage is the 50 States and the District of Columbla. - Data through 1992 are final. Subsequent data are preliminary. - Totals may not equal sum of components due to independent rounding.

Sources: - Rosidential and Commorclal: 1973-1970-U.S. Department of the Interior (DOI), Bureau of Mine (BOM), Minerals Yearbook. January-September 1077-DOI, BOM, Fol ; 6-1400, "Monthly Coal Report, Retall Dealers-Upper Lake Docks." Octobor 1077-1079-Energy Intormation Administration (EIA), Form EIA-2, "Monthly Coal Report, Rotal Dealers-Upper Lake Docks." 1980 forward-EIA, Form EIA-6, "Coa Distribution Report, quarterly." • Coke Plants: 1973-September 1977-DOI,
BOM, Minerals Yearbook and Minerals Indusiny Surveys. Octobor 1077-1980-EIA, Form EIA-5/5A, "Coke and Coal ChemicabMonthly/Annual." 1981-1984-EIA, Form ELA-5/5A, "Coke Plant Report-Quarterty/Annual Supplement. 1086 fonwerd-ELA, Form EIA-6, "Coke Plant Repon," quarterty. - Other Industrial: 1073-September 1077-DOI, BOM. Minerals Yearbook and Minerals Industry Surveys. Oetober 1977-1070-ELA, Form ELA-3, Monthly Coal Consumption Report-Manufacturing Plants." 1080 forward-ELA, Form EIA-3, "Quarterty Coal Consumption Report-Manufacturing Plants," and Form ElA-6, "Coal Distribution Repon, quarterty." - Electrle Utilites: 1073-September 1077-DOI, BOM, Minerals Yearbook and Minerals Industry Surveys. Oetober 1077 forward-EIA, Form ELA-759 (formerty Form FPC-4), "Monthly Power Plant Repont." 


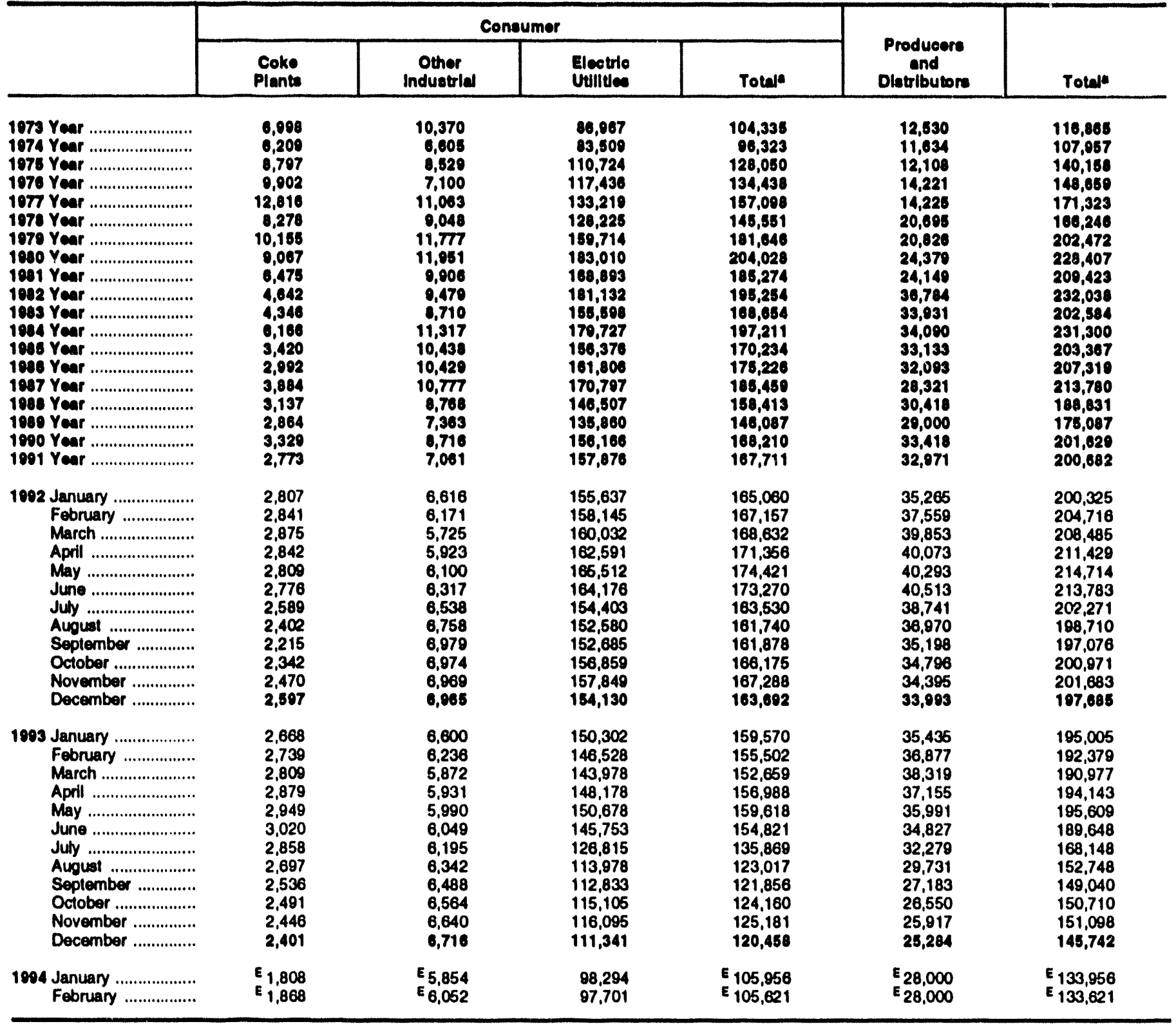

- Excludes stocks held at retail dealers for consumption by the residential and commercial sector.

Ex=stimale.

Notes: - For sector-specific reporting and estimating information, see Nole 3 at end of section. - Geographic coverage is the 50 Stales and the District of Columbia. - Data through 1992 are final. Subsequent data are preliminary. - Totals may not equal sum of components due to independent rounding.

Sources: - Coke Plantw: 1973-Soptember 1077-U.S. Department of the Interior (DOI), Bureau of Mines (BOM). Minerals Yearbook and Minerals Industry Surveys. October 1977-1080-Energy Intormation Administration (EIA), Form ELA-5/5A, "Coke and Coal Chemicals-Monthly/Annual."
1981-1984-ELA, Form EIA-5/5A "Coke Plant Report-Quarterty/Annual Supplement:" 1985 forward-EIA, Form EIA-5, "Coke Plant Repont," quarterly. - Other Industrial: 1973-Soptomber 1077-DOI, BOM, Minerals Yearbook and Minerals Industry Surveys. October 1977-1070-ELA, Form EIA-3, "Morithly Coal Consumption Report-Manufacturing Plants." 1080 forward-EIA, Form EIA-3, "Quartert' Coal Consumplion ReportManufacturing Plants," and Form ElA-6, "Co.l Distribution Report" quarterty. - Electrle Utilitios: 1973-Soptomber 1977-DOI, BOM, Minerals Yearbook and Minerals Industry Surveys. October 1977 foi ward-ELA, Form EIA.759 (formerty Form FPC-4), "Monthly Power Plant Fepont." - Producere and Dlstrlbutore: EIA, Form EIA-6, "Coal Distribution Report," quarterly. 


\section{Coal Notes}

1. Production: Preliminary monthly estimates of national coal production are the sum of weekly estimates developed by the Energy Information Administration(EIA) and published in the Weekly Coal Production report. When a week extends into a new month, production is allocated on a daily basis and added to the appropriate month. Weekly estimates are based on Association of American Rallroads data showing the number of railcars loaded with coal during the week by Class I and certain other railroads. This number is converted into tons of coal by EIA by using the average number of tons of coal per railcar loaded reported in the most recent "Quarterly Freight Commodity Statistics" from the Interstate Commerce Commission. If an average coal tonnage per railcar loaded is not available for a specific railroad, the national average is used. To derive the estimate of total weekly production, the total rail tonnage for the week is divided by the ratio of quarterly production shipped by rail and total quarterly production. Data for the corresponding quarter of previous years are used to derive this ratio. This method insures that the seasonal variations are preserved in the production estimates.

When preliminary quarterly data become available, the monthly and weekly estimates are adjusted to conform to the quarterly figure. The adjustment procedure uses State-level production data and is explained in EIA's Quarterly Coal Report. Initial estimates of annual production published in January of the following year are based on preliminary production data covering the first 9 months (three quarters) and weekly/monthly estimates for the fourth quarter. The fourth quarter estimates may or may not be revised when preliminary data become available in March of the following year, depending on the magnitude of the difference between the estimates and the preliminary data. In any event, all quarterly, monthly, and weekly production figures are adjusted to conform to the final annual production data published in the Monthly Energy Review in the fall of the following year.

2. Consumption: Coal consumption data are reported by major end-use sector. Estimated data for the most recent months (designated by an " $E$ ") are derived from forecasted values shown in the EIA Short-Term Energy Outlook (DOE/EIA .0202) table titled "Supply and Disposition of Coal: Mid World Oil Price Case." The monthly estimates are one-third of the quarterly values shown in the then current issue of the publication, regularly released in February, May, August, and November. The estimates are revised quarterly as collected data become available from the data sources. Sector-specific information follows.

- Residential and Commercial-Prior to 1980. monthly consumption estimates for the residential and commercial sector were derived by using reported data to modify baseline figures developed by the Bureau of Mines. From 1980-1987, month- ly estimates were derived by proportioning reported quarterly data by using the ratios of monthly-10-quarterly consumption data in 1979 , the last year in which montbly data were reported on Form ElA-2. During 1981 and 1982, the estimates were also modified to reflect air temperature degree-days. Quarterly consumption data were taken directly from reported data and were defined as distribution to the residential and commercial sector as reported by coal producers and distributors on Form EIA-6. Beginning in January 1988, monthly residential and commercial consumption estimates are derived from reported quarterly data by using monthly national average population weighted heating/cooling degree-days obtained from the National Oceanic and Atmospheric Administration. The monthly ratios are the monthly national sum of beating and cooling degree-days as a proportion of the quarterly national sum. Quarterly consumption data are taken directly from reported data.

- Coke Plants-Prior to 1980, monthly coke plant consumption data were taken directly from reported data. From 1980-1987, coke plant consumption estimates were derived by proportioning reported quarterly data by using the ratios of monthly-to-quarterly consumption data in 1979 , the last year in which monthly data were reported. Beginning in January 1988, monthly coke plant consumption estimates are derived from the reported quarterly data by using monthly ratios of raw steel production data from the American Iron and Steel Institute. The ratios are the monthly raw steel production from open bearth and basic oxygen process furnaces as a proportion of the quarterly production from those kinds of furnaces.

- Other Industrial-Prior to 1978, monthly consumption data for the other industrial sector (i.e., all industrial users minus coke plants) were derived by using reported data to modify baseline consumption figures from the most recent Bureau of the Census Annual Survey of Manufactures or Census of Manufactures. For 1978 and 1979. monthly estimates were derived from data reported on Forms EIA-3 and EIA-6. From 1980-1987, monthly figures were estimated by proportioning quarterly data by using the ratios of monthly-toquarterly consumption data in 1979 , the last year in which monthly data were reported on Form EIA-3. Quarterly consumption data were derived by adding beginning stocks at manufacturing plants to current receipts and subtracting ending stocks at manufacturing plants. In this calculation, current receipts were the greater of either reported receipts from manufacturing plants (Form EIA-3) or reported shipments to the other industrial sector (Form EIA-6), thereby ensuring that agriculture, forestry, fishing, mining, and construction consumption data were included where appropriate. Starting in January 1988, monthly consumption for the other industrial sector is estimated from reported quarterly data by using 
ratios derived from industrial production indices published by the Board of Governors of the Federal Reserve System. Indices for six major industry groups are used as the basis for calculatIng the ratios: foods, Standard Industrial Classification (SIC) 20; paper and products, SIC 26; chemicals and products, SIC 28; petroleum products, SIC 29; clay, glass, and stone products, SIC 32; and primary metals, SIC 33. The monthly ratios are computed as the monthly sum of the weighted indices as a proportion of the quarterly sum of the weighted indices by using the 1977 proportion as the weights.

- Electric Utilities-Monthly consumption data for electric utility plants are taken directly from reported data.

3. Stocks: Coal stocks data are reported by major enduse sector. Estimated data for the most recent months (designated by an " $E$ ") are derived from forecasted values shown in the EIA Short-Term Energy Outlook (DOE/EIA-0202) table titled "Supply and Disposition of Coal: Mid World Oil Price Case." The monthly estimates are one-third of the quarterly values shown in the then current issue of the publication, regularly released in February, May, August, and November. The estimates are revised quarterly as collected data become available from the data sources. Sector-specific information follows.

- Coke Plants-Prior to 1980, monthly stocks at coke plants were taken directly from reported data. From 1980 forward, coke plant stocks are estimated by using one-third of the current quarterly change to indicate the montbly change in stocks. Quarterly stocks are directly from data reported on Form ElA-S.

- Other Industrial-Prior to 1978 , stocks for the other industrial sector were derived by using reported data to modify baseline figures from a one-time Bureau of Mines survey of consumers. For 1978-1982, monthly estimates were derived by judgmentally proportioning reported quarterly data based on representative seasonal patterns of supply and demand. From 1983 forward, other industrial coal stocks are estimated as indicated above for coke plants. Quarterly stocks are taken directly from data reported on Form EIA-3 and therefore include only manufacturing industries; data for agriculture, forestry, fishing, mining, and construction stocks are not available.

- Electric Utilities-Monthly stocks data at electric utility plants are taken directly from reported data.

- Producers and Distributors-Quarterly stocks at producers and distributors are taken directly from reported data. Monthly data are estimated by using one-third of the current quarterly change to indicate the monthly change in stocks.

4. Imports and Exports: All coal import and export figures are taken directly from data reported monthly by the Bureau of the Census.

5. Additional Information: EIA's Quarterly Coal Report provides additional information about coal data and estimation procedures. 


\section{Section 7. Electricity}

During February 1994, electric utilities generated 225 billion kilowatthours of electricity, slightly more than in February 1993. Coal-fired generation totaled 131 billion kilowatthours, 1 percent ${ }^{7}$ more than in February 1993. Nuclear generation totaled 50 billion kilowatthours, 3 percent below the level 1 year earlier. Natural gas-fired generation was 15 billion kilowatthours, 8 percent lower than the February 1993 level. Hydroelectric generation totaled 19 billion kilowatthours, 3 percent below the February 1993 level. Petroleum-fired generation totaled 10 billion kilowatthours, 39 percent above the level 1 year earlier.

Sales of electricity to all ultimate consumers in the United States in February 1994 were 238 billion kilowatthours, 4 percent more than sales during February 1993. Sales to residential consumers during February 1994 were 89 billion kilowatthours, 7 percent above the level of sales during the previous year. Sales to industrial consumers totaled 78 billion kilowatthours in February 1994, 1 percent above the level a year ago. Commercial sales were 63 billion kilo- watthours, 4 percent above the level of commercial sales 1 year earlier. In February 1994, other sales totaled 8 billion kilowatthours, slightly higher than the February 1993 level.

Electric utility consumption of coal during February 1994 was 65 million short ions, 3 percent above consumption in February 1993. Petroleum consumption (excluding petroleum coke) during February 1994 was 16 million barrels, 40 percent above the level of consumption in February 1993. During February 1994, electric utilities consumed 149 billion cubic feet of natural gas, 8 percent below the February 1993 consumption level.

On February 28, 1994, electric utility stocks of all types of coal totaled 98 million short tons, 33 percent below the level on February 28, 1993. Stocks of petroleum (excluding petroleum coke) on February 28, 1994, totaled 60 million barrels, 8 percent below the level on February 28, 1993.

'Percentage changes are based on aumbers shown in the following tables. 

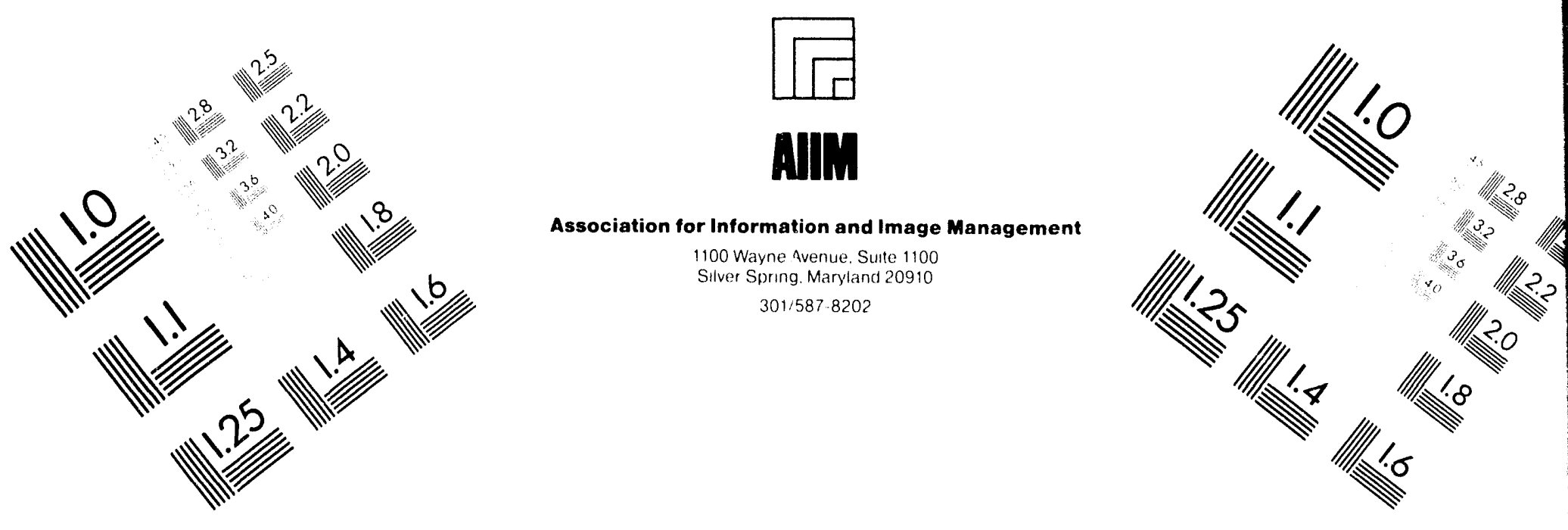

\section{Centimeter}

${ }_{1}$

Inches
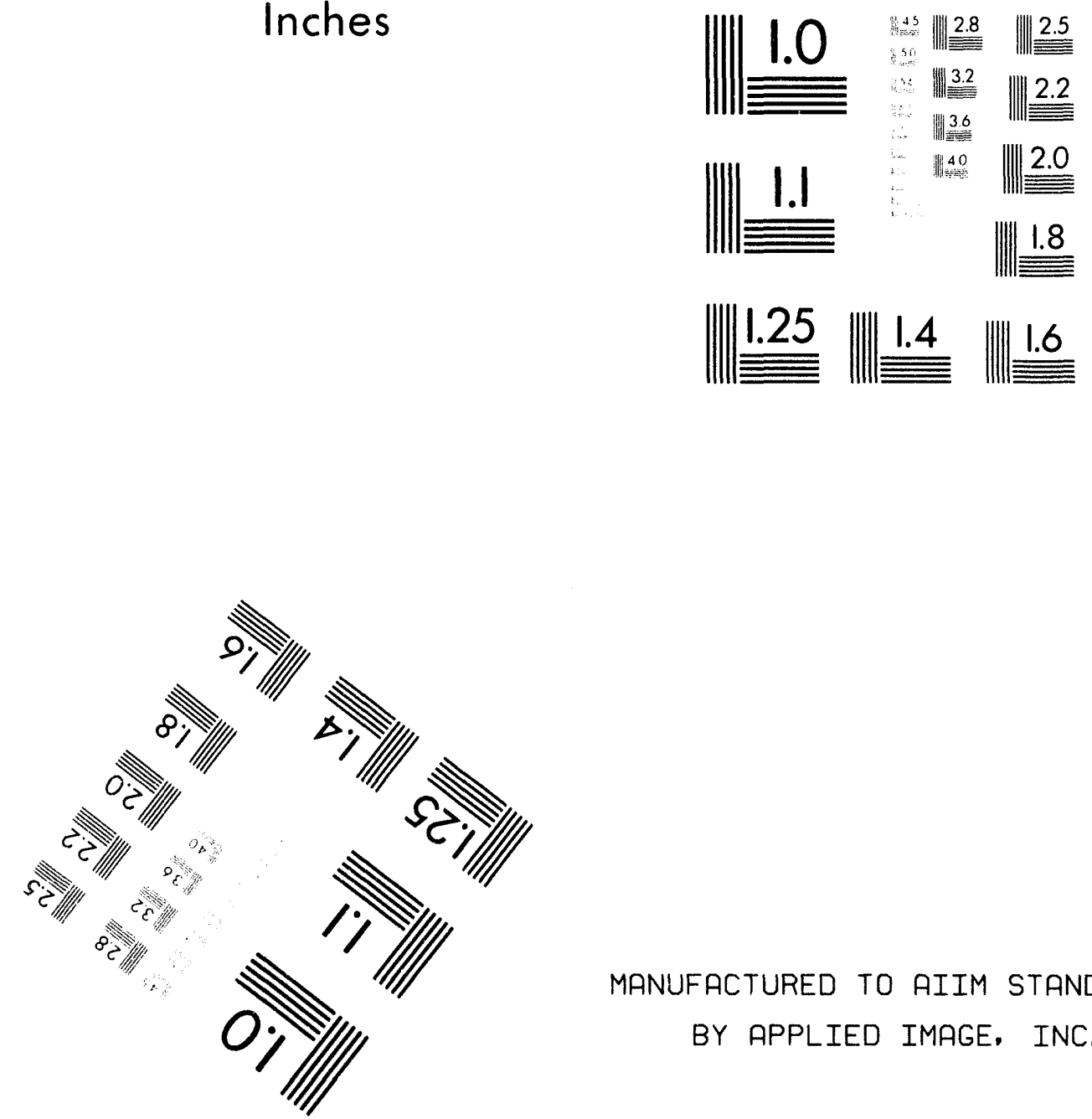

MANUFACTURED TO AIIM STANDARDS

BY APPLIED IMAGE, INC.

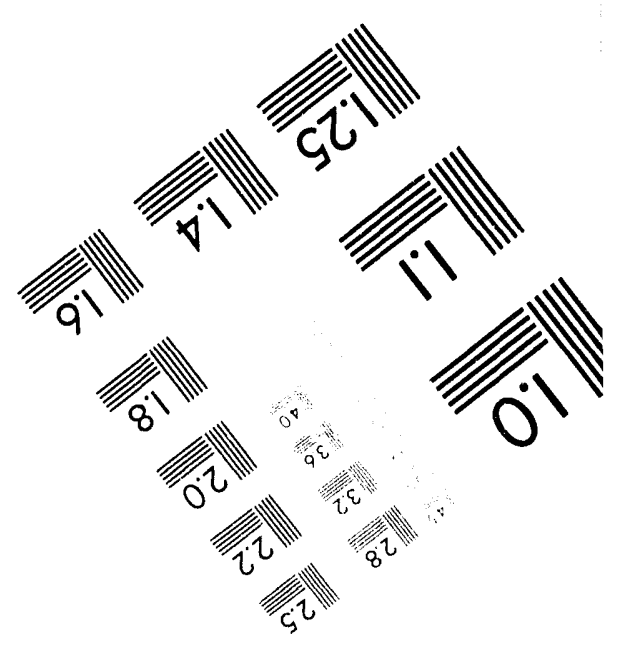



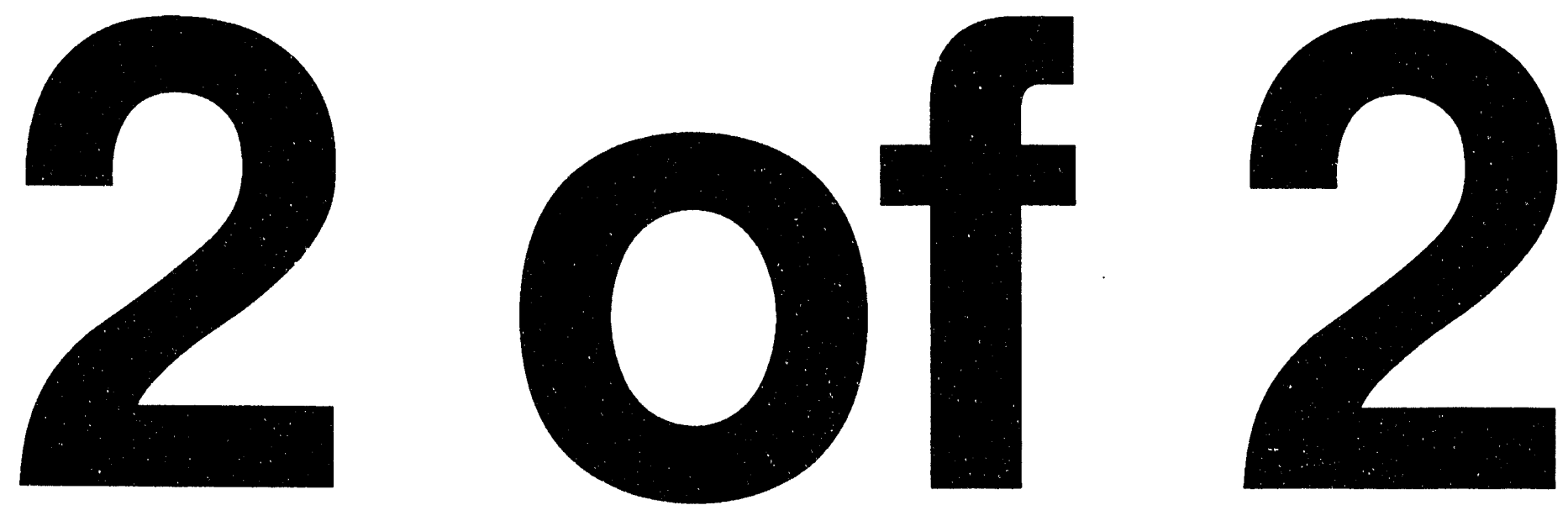
Figure 7.1 Electric Utility Net Generation of Electricity

(Billion Kilowatthours)

Net Generation by Source, 1973-1993

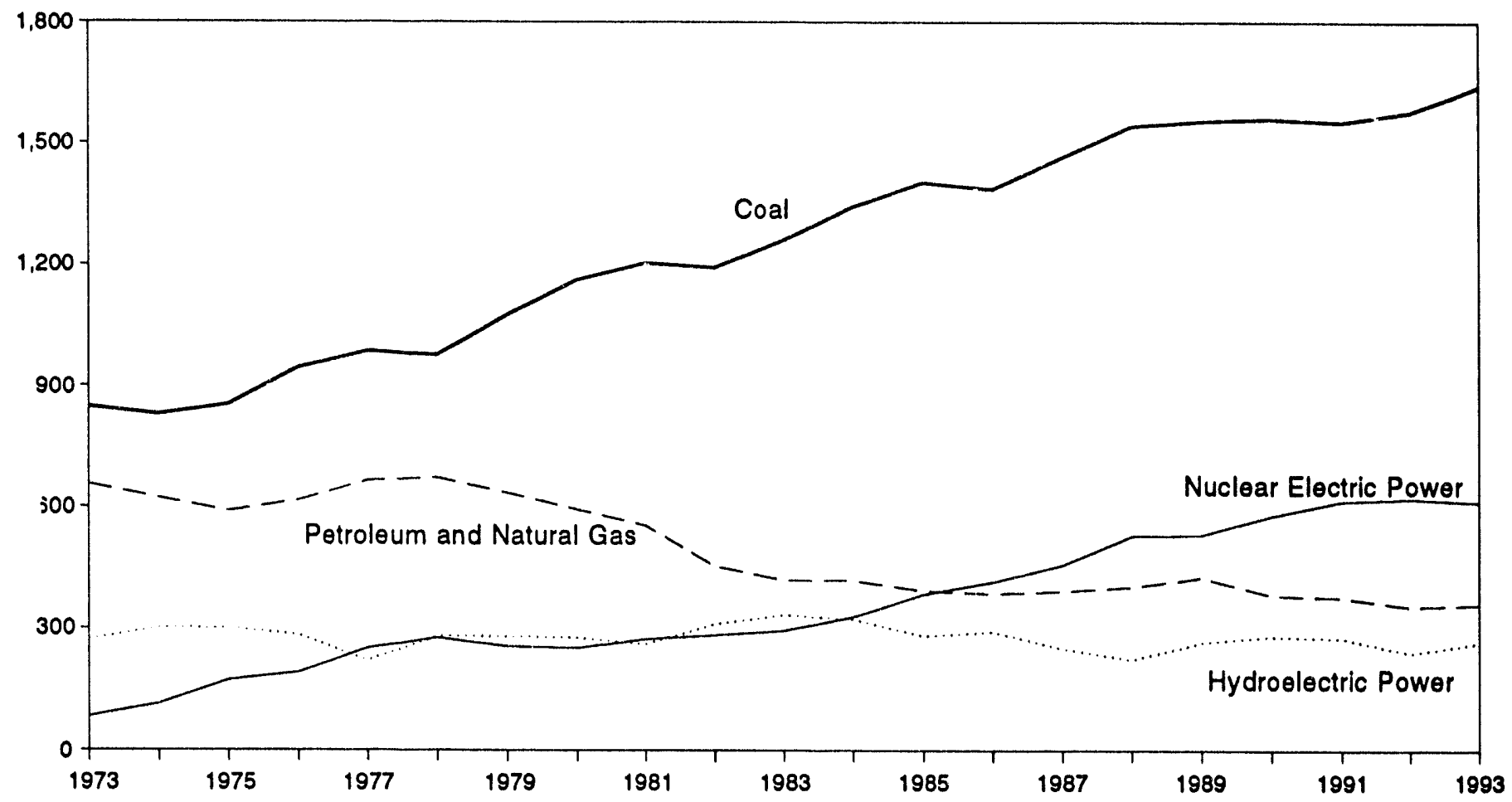

Net Generation, January and February

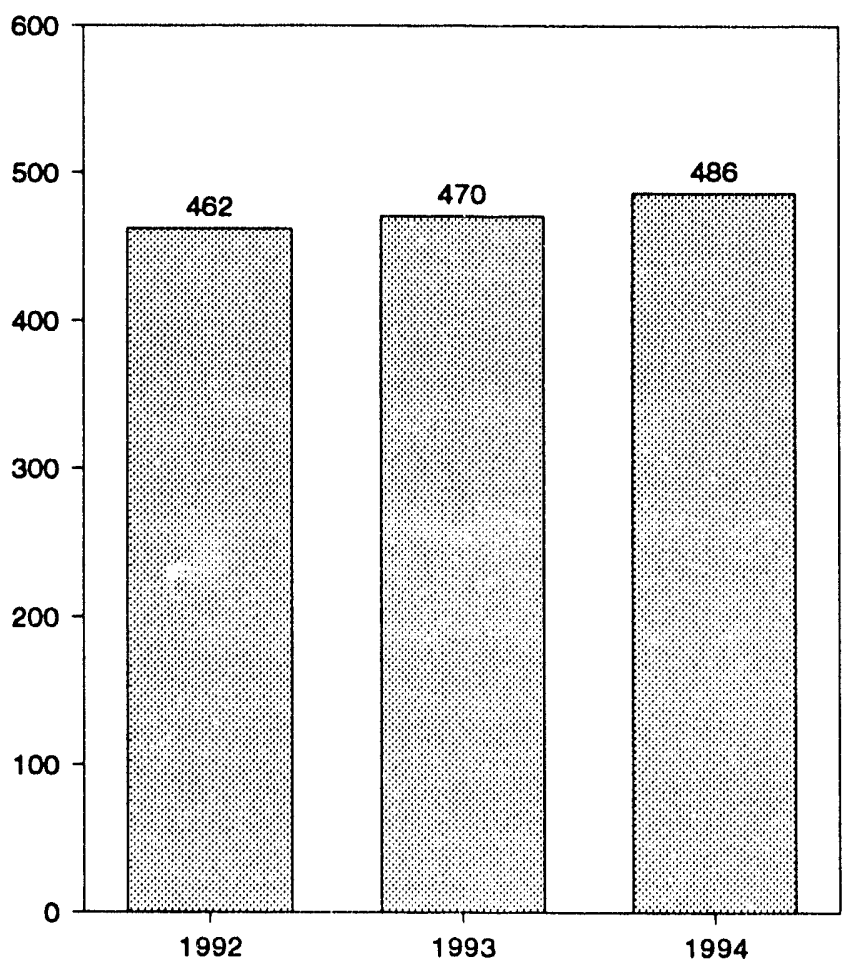

Net Generation by Source, February 1994

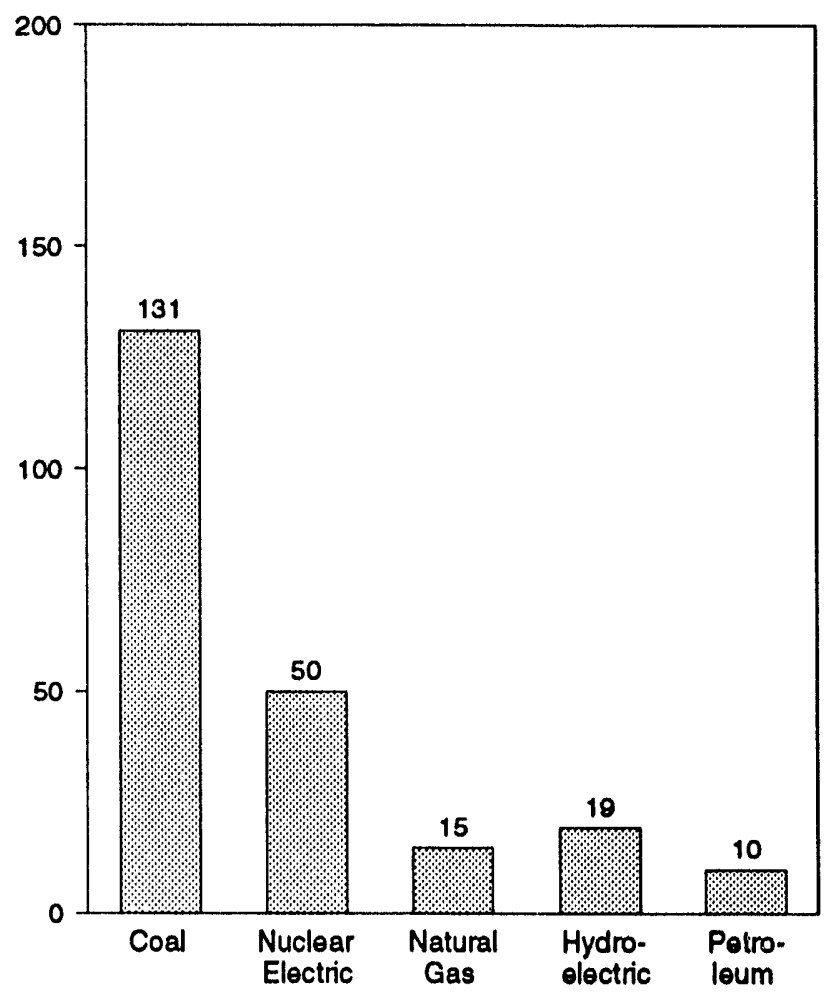

Note: Because vertical scales differ, graphs should not be compared. Source: Table 7.1 . 
Table 7.1 Electric Utility Net Generation of Electricity

(Million Kilowatthours)

\begin{tabular}{|c|c|c|c|c|c|c|c|c|}
\hline & Coal & $\begin{array}{c}\text { Natural } \\
\text { Gas }^{a}\end{array}$ & Potroloumb & $\begin{array}{l}\text { Nucloar } \\
\text { Eloctric } \\
\text { Power }\end{array}$ & $\begin{array}{l}\text { Hydro- } \\
\text { Eloctric } \\
\text { Power }\end{array}$ & $\begin{array}{l}\text { Goothormal } \\
\text { Enorgy }\end{array}$ & Othere & Total \\
\hline 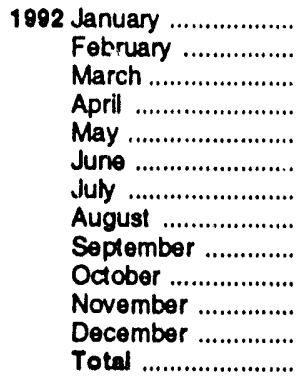 & $\begin{array}{r}137,327 \\
121,732 \\
127,678 \\
119,909 \\
123,768 \\
129,607 \\
149,028 \\
141,900 \\
133,239 \\
127,940 \\
125,535 \\
138,234 \\
1,575,895\end{array}$ & $\begin{array}{r}16,178 \\
16,165 \\
19,906 \\
21,913 \\
22,689 \\
24,997 \\
31,950 \\
28,778 \\
26,099 \\
20,420 \\
18,031 \\
16,744 \\
263,872\end{array}$ & $\begin{array}{r}10,202 \\
8,296 \\
8,809 \\
6,505 \\
5,156 \\
7,508 \\
8,540 \\
6,923 \\
6,841 \\
6,908 \\
6,838 \\
6,390 \\
88,016\end{array}$ & $\begin{array}{r}57,849 \\
52,804 \\
45,835 \\
42,268 \\
45,627 \\
51,185 \\
56,049 \\
58,656 \\
50,919 \\
48,784 \\
50,726 \\
58,075 \\
618,776\end{array}$ & $\begin{array}{r}21,502 \\
17,966 \\
21,566 \\
19,454 \\
22,285 \\
22,698 \\
19,711 \\
18,062 \\
16,839 \\
16,375 \\
19,294 \\
23,808 \\
239,559\end{array}$ & $\begin{array}{r}711 \\
626 \\
713 \\
645 \\
683 \\
675 \\
685 \\
690 \\
642 \\
677 \\
675 \\
682 \\
8,104\end{array}$ & $\begin{array}{r}202 \\
172 \\
158 \\
143 \\
147 \\
170 \\
184 \\
195 \\
183 \\
185 \\
165 \\
192 \\
2,096\end{array}$ & $\begin{array}{r}243,970 \\
217,761 \\
224,665 \\
210,837 \\
220,355 \\
236,842 \\
266,148 \\
255,203 \\
234,760 \\
221,289 \\
221,263 \\
244,126 \\
2,707,219\end{array}$ \\
\hline 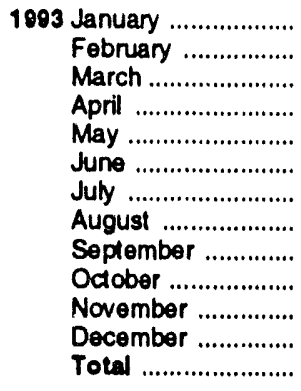 & $\begin{array}{r}138,354 \\
130,069 \\
136,404 \\
120,325 \\
120,878 \\
137,485 \\
158,400 \\
156,197 \\
134,001 \\
130,926 \\
132,288 \\
143,824 \\
1,639,151\end{array}$ & $\begin{array}{r}15,807 \\
15,768 \\
18,783 \\
16,684 \\
15,845 \\
24,393 \\
31,705 \\
34,263 \\
24,978 \\
22,912 \\
20,535 \\
17,242 \\
258,015\end{array}$ & $\begin{array}{r}7,239 \\
6,939 \\
8,569 \\
5,205 \\
5,267 \\
7,809 \\
11,341 \\
11,975 \\
9,759 \\
7,659 \\
7,479 \\
10,299 \\
90,530\end{array}$ & $\begin{array}{r}59,076 \\
51,319 \\
46,606 \\
43,199 \\
50,367 \\
52,620 \\
56,502 \\
56,209 \\
49,989 \\
44,434 \\
46,862 \\
53,108 \\
610,201\end{array}$ & $\begin{array}{r}24,453 \\
19,722 \\
23,587 \\
25,160 \\
29,323 \\
26,600 \\
23,556 \\
19,667 \\
17,073 \\
16,899 \\
17,898 \\
21,125 \\
265,063\end{array}$ & $\begin{array}{r}651 \\
633 \\
659 \\
654 \\
582 \\
586 \\
643 \\
653 \\
630 \\
625 \\
618 \\
637 \\
7,571\end{array}$ & $\begin{array}{r}202 \\
167 \\
193 \\
148 \\
135 \\
139 \\
144 \\
167 \\
173 \\
174 \\
174 \\
178 \\
1,094\end{array}$ & $\begin{array}{r}245,782 \\
224,617 \\
234,801 \\
211,374 \\
222,396 \\
249,633 \\
282,292 \\
279,132 \\
236,603 \\
223,629 \\
225,855 \\
246,412 \\
2,882,626\end{array}$ \\
\hline 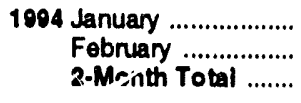 & $\begin{array}{l}152,752 \\
131,138 \\
283,890\end{array}$ & $\begin{array}{l}16,847 \\
14,526 \\
31,373\end{array}$ & $\begin{array}{r}14,600 \\
9,655 \\
24,256\end{array}$ & $\begin{array}{r}56,184 \\
49,857 \\
106,042\end{array}$ & $\begin{array}{l}19,843 \\
19,146 \\
38,080\end{array}$ & $\begin{array}{r}631 \\
574 \\
1,205\end{array}$ & $\begin{array}{l}177 \\
154 \\
330\end{array}$ & $\begin{array}{l}261,035 \\
225,051 \\
488,085\end{array}$ \\
\hline $\begin{array}{l}1993 \text { 2-Month Total ....... } \\
1992 \text { 2-Month Total ....... }\end{array}$ & $\begin{array}{l}268,423 \\
259,058\end{array}$ & $\begin{array}{l}31,575 \\
32,344\end{array}$ & $\begin{array}{l}14,178 \\
18,490\end{array}$ & $\begin{array}{l}110,395 \\
110,652\end{array}$ & $\begin{array}{l}44,175 \\
39,468\end{array}$ & $\begin{array}{l}1,283 \\
1,337\end{array}$ & $\begin{array}{l}360 \\
374\end{array}$ & $\begin{array}{l}\$ 70,300 \\
461,732\end{array}$ \\
\hline
\end{tabular}

ancludes supplemental gaseous luel.

Includes fuel oil nos. 1, 2, 4, 5, and 6, crude oil, kerosene, and petroleum coke.

c "Other" is electrictly produced from wood, waste, wind, photovoltak, and solar thermal energy sources connected to electric utility distribution systems.

Noles: - Geographic coverage is the 50 States and the District of Columbia.

- Totals may not equal sum of components due to indeperident rounding.

Sources: - 1073-Soptember 1977: Federal Power Commission, Form

FPC-4, "Monthly Power Plant Report." October 1977-1970: Federa Energy Regulatory Commission (FERC), Form FPC-4, "Monthly Power Plant
Report." - 1980: Energy Information Administration (ELA), Eloctric Power Monthly, March 1991, Table 4, and (for geothermal energy and other) FERC, Form FPC-4, "Monthly Power Plant Report:" - 1981: ElA, Electric Power Monthly, March 1992, Table 4, and (for geothermal energy and other) FERC, Form FPC.4, "Monthly Power Plant Report." - 1082 and 1901 monthly data: EIA, Electric Power Monthy, March 1993, Table 4, and (for geothermal energy and other) EIA, Form ElA-759, "Monthly Power Plant Report:" 1983 forward (oxcept 1901 monthly data): ElA, Electric Power Monthy, May 1994, Table 4, and (for goothermal energy and other) EIA, Form EIA-759, "Monthly Power Plant Report." 
Figure 7.2 Electricity Sales

(Billion Kilowatthours)

Total Sales, January and February

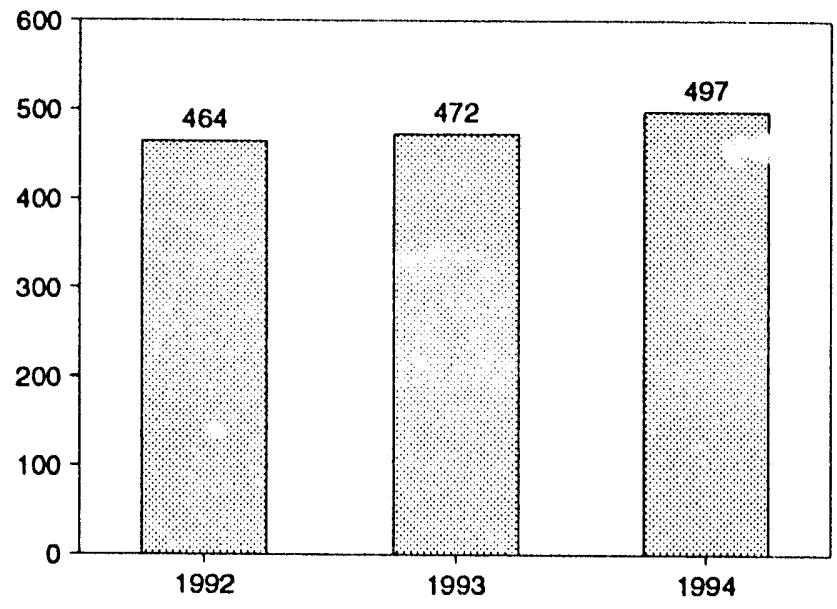

Total Sales, Monthly

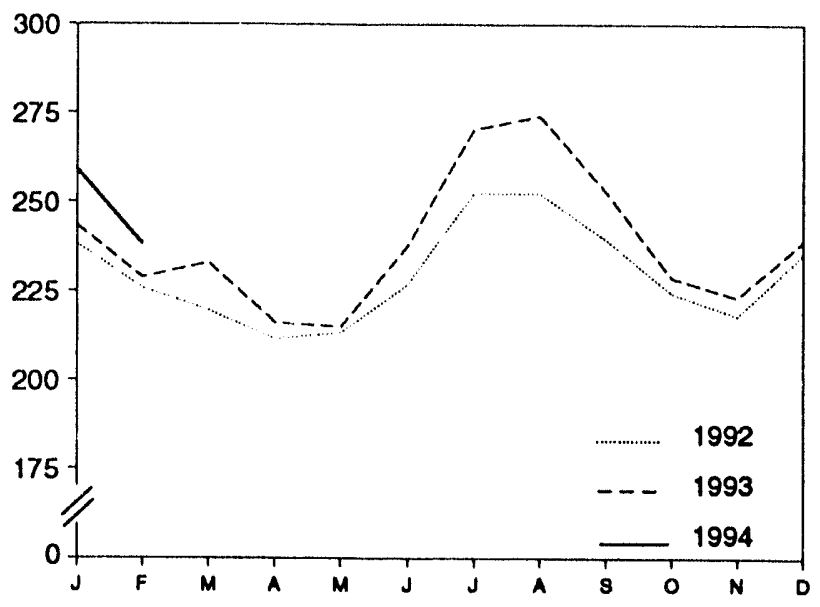

Sales by Sector, Monthly

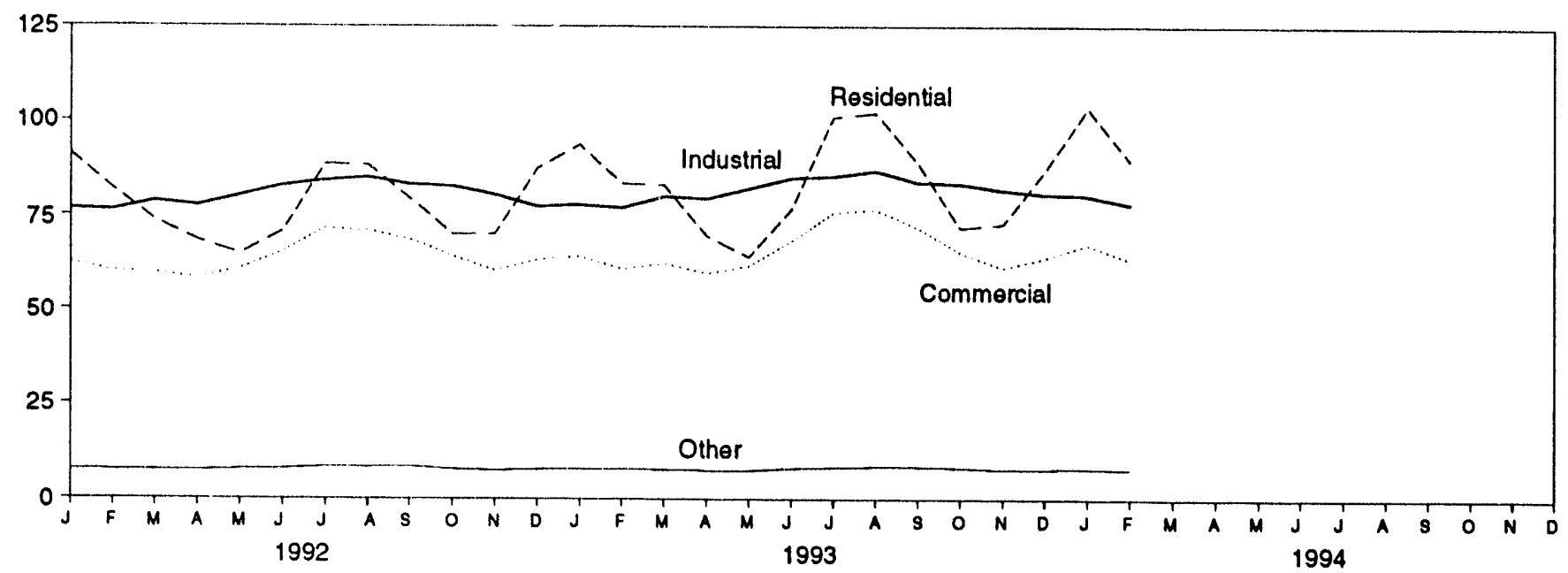

Sales by Sector, 1973-1993

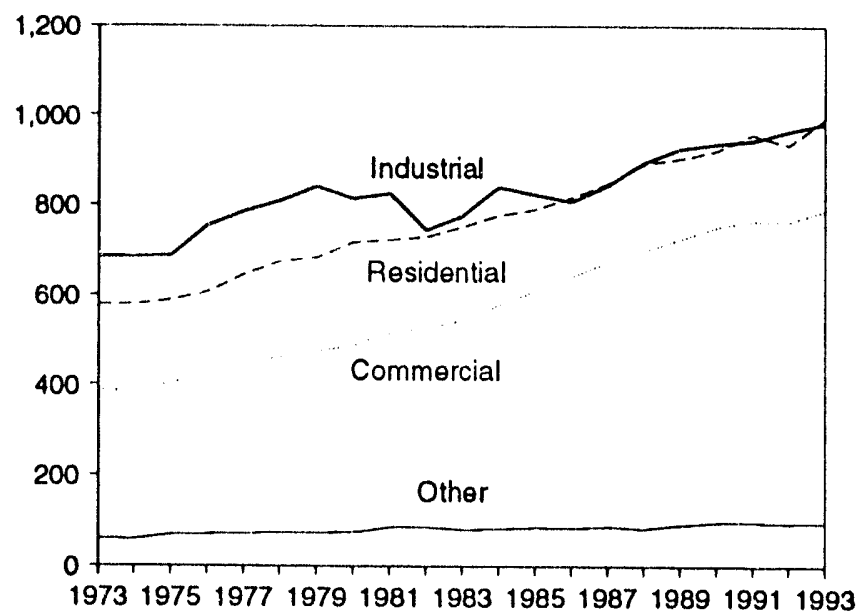

Sales by Sector, February 1994

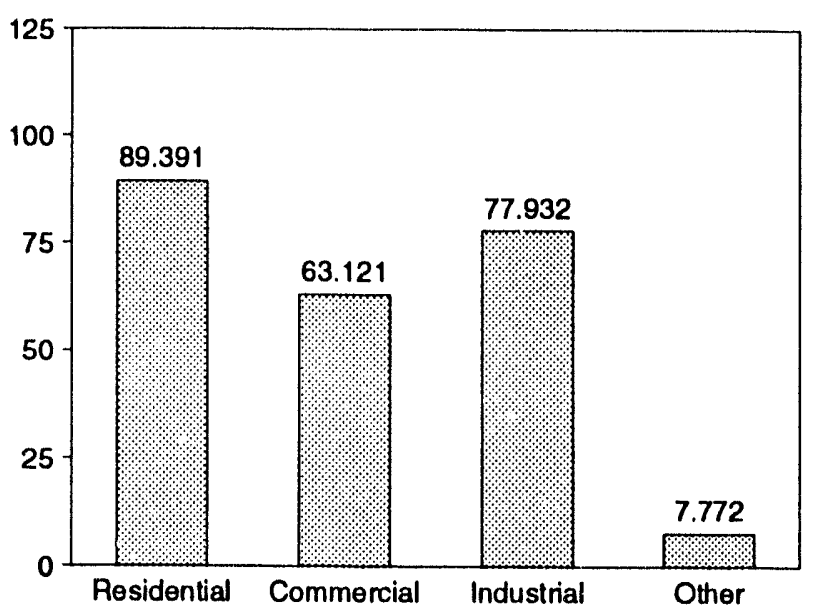

Note: Because vertical scales differ, graphs should not be compared. Source: Table 7.2, Monthly Serles. 
Table 7.2 Electricity Sales by End-Use Sector

(Million Kilowatthours)

\begin{tabular}{|c|c|c|c|c|c|c|c|c|c|c|}
\hline & \multicolumn{2}{|c|}{ Residential } & \multicolumn{2}{|c|}{ Commercial } & \multicolumn{2}{|c|}{ Industrial } & \multicolumn{2}{|c|}{ Other ${ }^{\mathrm{a}}$} & \multicolumn{2}{|c|}{ Total } \\
\hline & $\begin{array}{l}\text { Monthly } \\
\text { Series }\end{array}$ & $\begin{array}{c}\text { Annual } \\
\text { Series }\end{array}$ & $\begin{array}{l}\text { Monthly } \\
\text { Series }\end{array}$ & $\begin{array}{l}\text { Annual } \\
\text { Series }\end{array}$ & $\begin{array}{l}\text { Monthly } \\
\text { Series }\end{array}$ & $\begin{array}{l}\text { Annual } \\
\text { Series }\end{array}$ & $\begin{array}{l}\text { Monthly } \\
\text { Series }\end{array}$ & $\begin{array}{l}\text { Annual } \\
\text { Series }\end{array}$ & $\begin{array}{l}\text { Monthly } \\
\text { Series }\end{array}$ & $\begin{array}{c}\text { Annual } \\
\text { Series }\end{array}$ \\
\hline 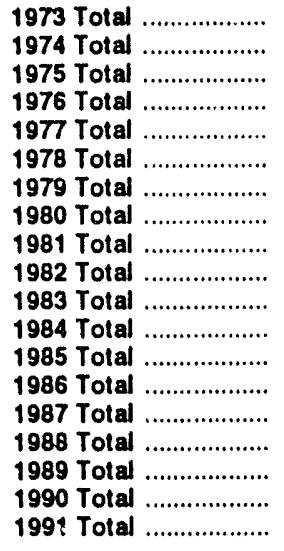 & $\begin{array}{l}579,231 \\
578,184 \\
588,140 \\
606,452 \\
645,239 \\
674,466 \\
682,819 \\
717,495 \\
722,265 \\
729,520 \\
750,948 \\
777,654 \\
790,977 \\
817,663 \\
849,613 \\
892,125 \\
903,979 \\
921,473 \\
957,801\end{array}$ & $\begin{array}{c}\text { NA } \\
\text { NA } \\
\text { NA } \\
\text { NA } \\
\text { NA } \\
\text { NA } \\
\text { NA } \\
\text { NA } \\
\text { NA } \\
\text { NA } \\
\text { NA } \\
780,092 \\
793,934 \\
819,088 \\
850,410 \\
892,866 \\
905,525 \\
924,019 \\
955,417\end{array}$ & $\begin{array}{l}388,266 \\
384,826 \\
403,049 \\
425,094 \\
446,514 \\
461,163 \\
473,307 \\
488,155 \\
514,338 \\
526,397 \\
543,788 \\
578,281 \\
608,968 \\
641,469 \\
673,707 \\
697,711 \\
725,229 \\
750,835 \\
765,476\end{array}$ & $\begin{array}{c}\text { NA } \\
\text { NA } \\
\text { NA } \\
\text { NA } \\
\text { NA } \\
\text { NA } \\
\text { NA } \\
\text { NA } \\
\text { NA } \\
\text { NA } \\
\text { NA } \\
582,621 \\
605,989 \\
630,520 \\
660,433 \\
699,100 \\
725,861 \\
751,027 \\
765,664\end{array}$ & $\begin{array}{l}686,085 \\
684,875 \\
687,680 \\
754,069 \\
786,037 \\
809,078 \\
841,903 \\
815,067 \\
825,743 \\
744,949 \\
775,999 \\
840,588 \\
824,523 \\
808,292 \\
845,266 \\
895,751 \\
926,376 \\
936,428 \\
944,684\end{array}$ & $\begin{array}{l}\text { NA } \\
\text { NA } \\
\text { NA } \\
\text { NA } \\
\text { NA } \\
\text { NA } \\
\text { NA } \\
\text { NA } \\
\text { NA } \\
\text { NA } \\
\text { NA } \\
837,836 \\
836,772 \\
830,531 \\
858,233 \\
896,498 \\
925,659 \\
945,522 \\
946,583\end{array}$ & $\begin{array}{l}59,326 \\
58,039 \\
68,222 \\
69,631 \\
70,571 \\
73,215 \\
73,070 \\
73,732 \\
84,756 \\
85,575 \\
80,219 \\
81,849 \\
85,075 \\
83,409 \\
86,854 \\
82,362 \\
91,066 \\
95,936 \\
96,513\end{array}$ & $\begin{array}{c}\text { NA } \\
\text { NA } \\
\text { NA } \\
\text { NA } \\
\text { NA } \\
\text { NA } \\
\text { NA } \\
\text { NA } \\
\text { NA } \\
\text { NA } \\
\text { NA } \\
85,248 \\
87,279 \\
88,615 \\
88,196 \\
89,598 \\
89,765 \\
91,988 \\
94,339\end{array}$ & $\begin{array}{l}1,712,909 \\
1,705,924 \\
1,747,091 \\
1,855,246 \\
1,948,361 \\
2,017,922 \\
2,071,099 \\
2,094,449 \\
2,147,103 \\
2,086,441 \\
2,150,955 \\
2,278,372 \\
2,309,543 \\
2,350,835 \\
2,455,440 \\
2,567,949 \\
2,646,651 \\
2,704,672 \\
2,764,474\end{array}$ & $\begin{array}{c}\text { NA } \\
\text { NA } \\
\text { NA } \\
\text { NA } \\
\text { NA } \\
\text { NA } \\
\text { NA } \\
\text { NA } \\
\text { NA } \\
\text { NA } \\
\text { NA } \\
2,285,796 \\
2,323,974 \\
2,368,753 \\
2,457,272 \\
2,578,062 \\
2,646,809 \\
2,712,555 \\
2,762,003\end{array}$ \\
\hline 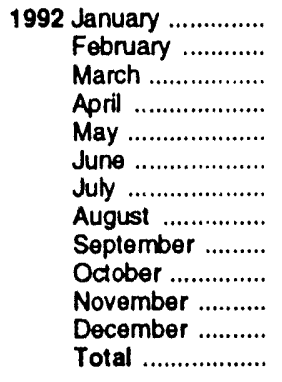 & $\begin{array}{r}91,310 \\
82,022 \\
73,635 \\
68,322 \\
64,662 \\
70,745 \\
88,510 \\
88,251 \\
79,400 \\
69,838 \\
69,970 \\
87,378 \\
934,044\end{array}$ & $\begin{array}{c}- \\
- \\
- \\
- \\
- \\
- \\
- \\
- \\
- \\
- \\
935,939\end{array}$ & $\begin{array}{l}62,441 \\
59,876 \\
59,574 \\
58,081 \\
60,559 \\
65,209 \\
71,445 \\
70,844 \\
68,437 \\
63,985 \\
60,131 \\
63,082 \\
763,664\end{array}$ & $\begin{array}{l}- \\
- \\
- \\
- \\
- \\
- \\
- \\
- \\
- \\
- \\
- \\
761,271\end{array}$ & $\begin{array}{r}76,760 \\
76,312 \\
78,741 \\
77,607 \\
80,191 \\
82,900 \\
84,195 \\
85,013 \\
83,182 \\
82,678 \\
80,421 \\
77,358 \\
965,356\end{array}$ & $\begin{array}{c}- \\
- \\
- \\
- \\
- \\
- \\
- \\
- \\
- \\
- \\
972,714\end{array}$ & $\begin{array}{r}7,725 \\
7,507 \\
7,542 \\
7,448 \\
7,767 \\
7,901 \\
8,392 \\
8,327 \\
8,441 \\
7,766 \\
7,462 \\
7,725 \\
94,003\end{array}$ & $\begin{array}{l}- \\
- \\
- \\
- \\
- \\
- \\
- \\
- \\
- \\
- \\
93,442\end{array}$ & $\begin{array}{r}238,235 \\
225,717 \\
219,491 \\
211,458 \\
213,179 \\
226,755 \\
252,541 \\
252,435 \\
239,460 \\
224,267 \\
217,984 \\
235,543 \\
2,757,067\end{array}$ & $\begin{array}{c}- \\
- \\
- \\
- \\
- \\
- \\
- \\
- \\
- \\
- \\
2,763,365\end{array}$ \\
\hline 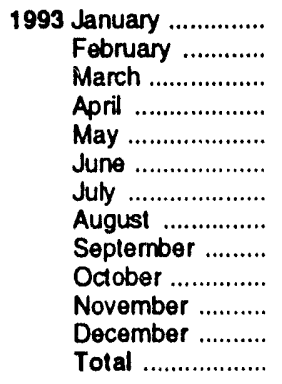 & $\begin{array}{r}93,740 \\
83,376 \\
83,023 \\
69,669 \\
63,852 \\
76,555 \\
101,026 \\
102,181 \\
88,884 \\
71,731 \\
72,687 \\
86,828 \\
993,552\end{array}$ & $\begin{array}{l}- \\
- \\
- \\
- \\
- \\
- \\
- \\
- \\
- \\
- \\
\text { NA }\end{array}$ & $\begin{array}{r}63,998 \\
60,609 \\
62,169 \\
59,479 \\
61,430 \\
68,107 \\
75,706 \\
76,533 \\
71,734 \\
65,180 \\
61,023 \\
63,740 \\
789,708\end{array}$ & $\begin{array}{l}- \\
- \\
- \\
- \\
- \\
- \\
- \\
- \\
- \\
- \\
\text { NA }\end{array}$ & $\begin{array}{r}77,832 \\
77,008 \\
80,028 \\
79,465 \\
82,090 \\
84,887 \\
85,371 \\
86,814 \\
83,804 \\
83,443 \\
81,738 \\
80,639 \\
983,118\end{array}$ & $\begin{array}{l}- \\
- \\
- \\
- \\
- \\
- \\
- \\
- \\
- \\
- \\
\text { NA }\end{array}$ & $\begin{array}{r}7,930 \\
7,752 \\
7,734 \\
7,511 \\
7,496 \\
8,088 \\
8,351 \\
8,551 \\
8,525 \\
8,271 \\
7,795 \\
7,894 \\
95,900\end{array}$ & $\begin{array}{l}- \\
- \\
- \\
- \\
- \\
- \\
- \\
- \\
- \\
- \\
\text { NA }\end{array}$ & $\begin{array}{r}243,499 \\
228,745 \\
232,954 \\
216,123 \\
214,868 \\
237,637 \\
270,454 \\
274,080 \\
252,948 \\
228,625 \\
223,244 \\
239,101 \\
2,862,279\end{array}$ & $\begin{array}{l}- \\
- \\
- \\
- \\
- \\
- \\
- \\
- \\
- \\
\overline{N A}\end{array}$ \\
\hline $\begin{array}{r}1994 \text { January .............. } \\
\text { February ............ } \\
\text { 2-Month Total ... }\end{array}$ & $\begin{array}{r}103,553 \\
89,391 \\
192,944\end{array}$ & $\begin{array}{l}- \\
-\end{array}$ & $\begin{array}{r}67,248 \\
63,121 \\
130,369\end{array}$ & $\begin{array}{l}- \\
-\end{array}$ & $\begin{array}{r}80,322 \\
77,932 \\
158,255\end{array}$ & $\begin{array}{l}- \\
-\end{array}$ & $\begin{array}{r}8,087 \\
7,772 \\
15,859\end{array}$ & - & $\begin{array}{l}259,210 \\
238,217 \\
497,427\end{array}$ & $\begin{array}{l}- \\
-\end{array}$ \\
\hline $\begin{array}{l}1993 \text { 2-Month Total ... } \\
1992 \text { 2-Month Total ... }\end{array}$ & $\begin{array}{l}177,116 \\
173,332\end{array}$ & - & $\begin{array}{l}124,607 \\
122,317\end{array}$ & - & $\begin{array}{l}154,840 \\
153,071\end{array}$ & - & $\begin{array}{l}15,682 \\
15,232\end{array}$ & $\overline{-}$ & $\begin{array}{l}472,245 \\
463,953\end{array}$ & $\overline{-}$ \\
\hline
\end{tabular}

a "Other" is public street and highway lighting, other sales to public authorities, sales to railroads and railways, and interdepartmental sales.

b Annual totals are the sums of the monthly values.

NA=Not available. $-=$ Not applicable.

Notes: - Geographic coverage is the 50 States and the District of Columbia.

- Totals may not equal sum of components due to independent rounding.

Sources: - 1973-September 1977: Federal Power Commission, Form FPC-5, "Monthly Statement of Electric Operating Revenue and Income."
October 1977-1979: Federal Energy Regulatory Commission, Form FERC-5, "Electric Operating Revenue and Income." - 1980: Energy Information Administration (EIA), Electric Power Monthy, March 1991, Table 51. 1981: ElA, Electric Power Monthly, March 1992, Table 51. 1982 and 1991 monthly data: ElA, Electric Power Monthly, March 1993, Table 51. 1983 fonward (except 1991 monthly data): EIA, Electric Power Monthy, May 1994, Table 52. 
Fuels Consumed, 1973-1993

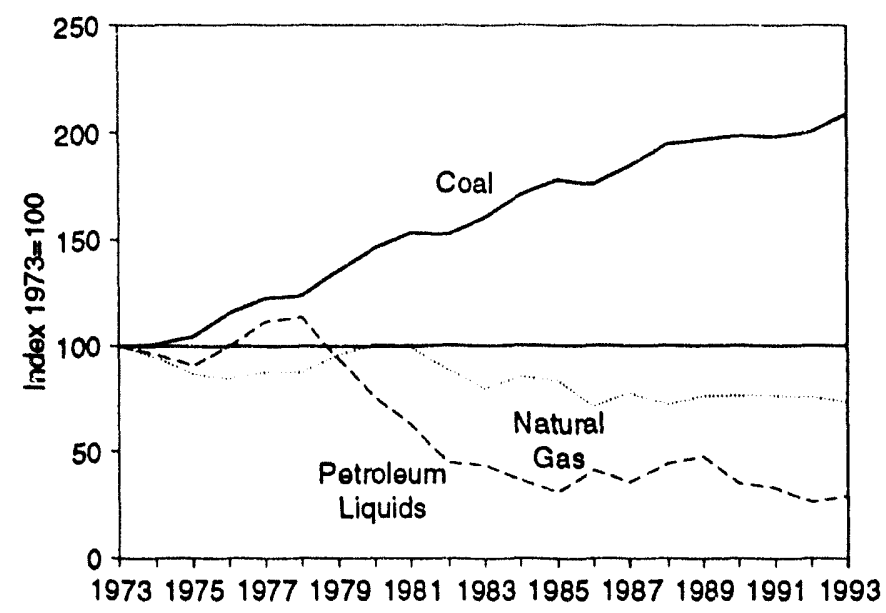

Petroleum Liquids Consumed, Monthly

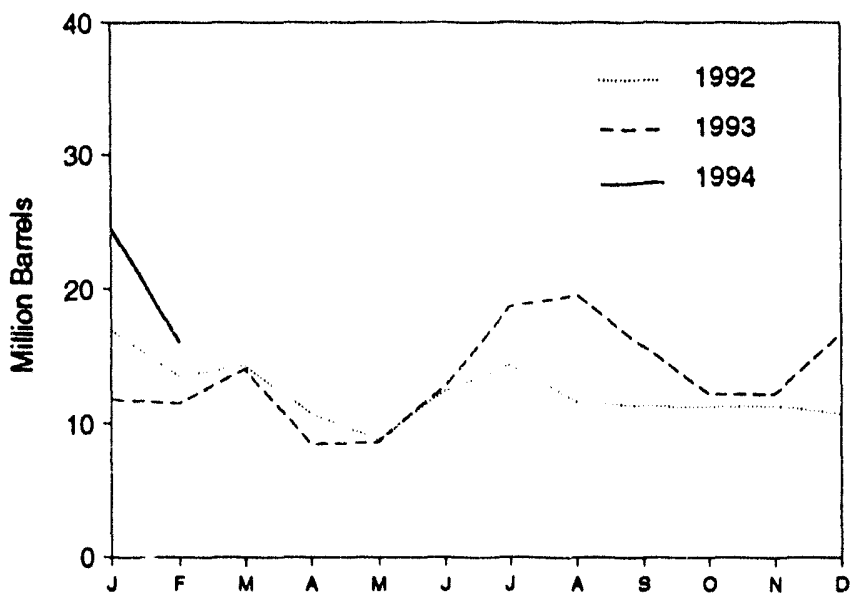

Coal Stocks, End of Month

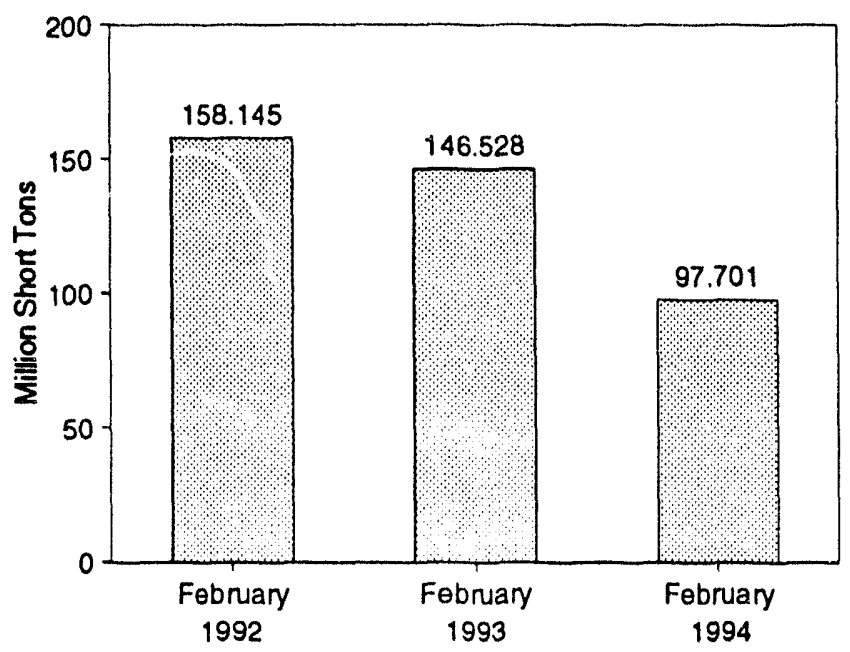

Coal Consumed, Monthly

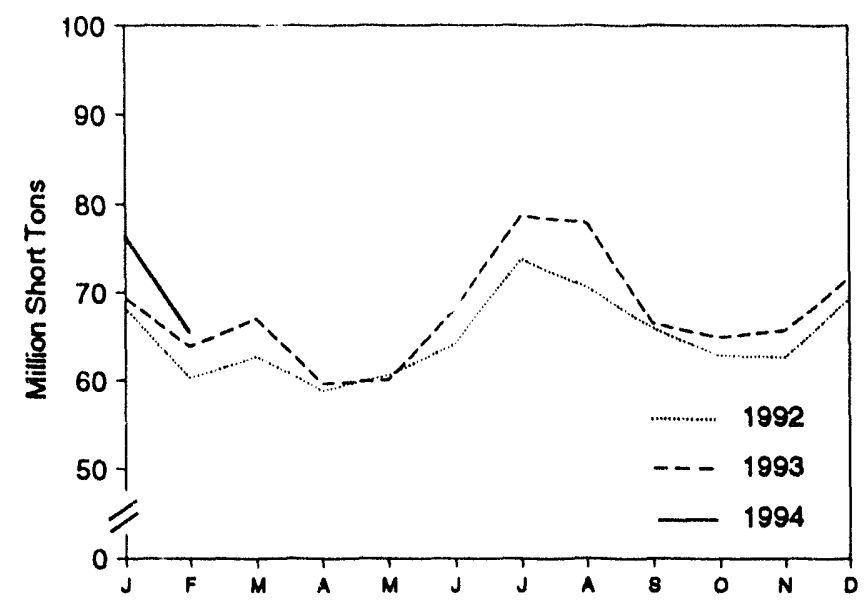

Natural Gas Consumed, Monthly

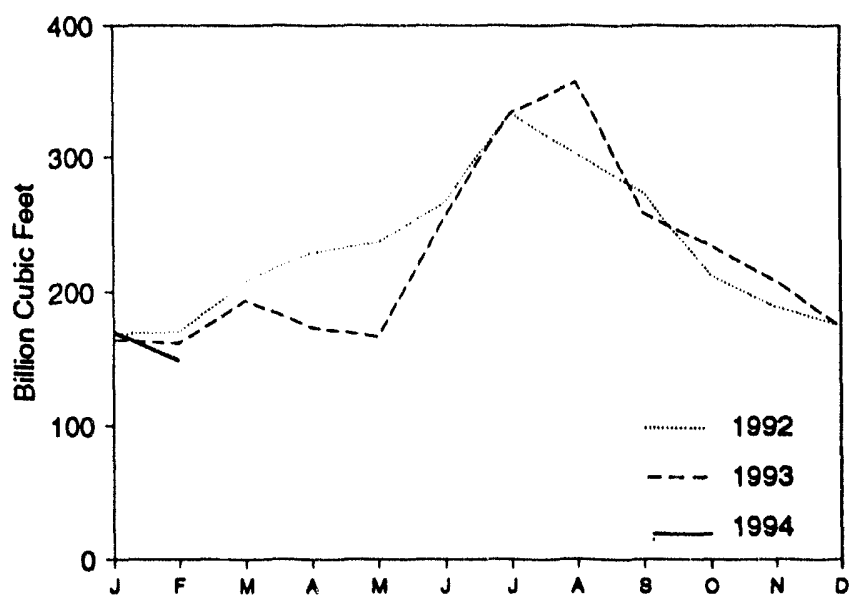

Petroleum Liquids Stocks, End of Month

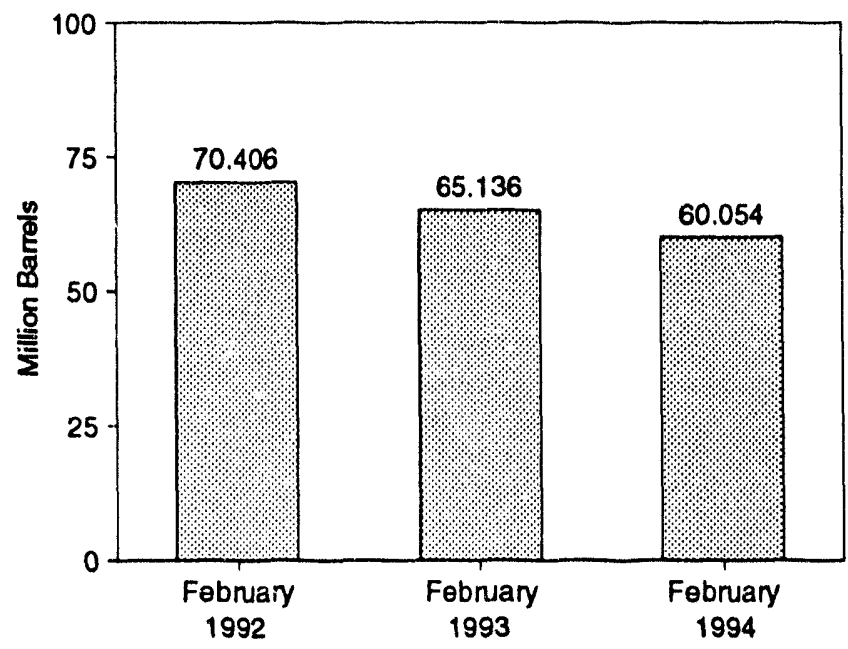

Nole: Because vertlcal scales differ, graphs should not be compared. Sources: Tables 7.3 and 7.4 . 
Table 7.3 Electric Utility Consumption of Fossil Fuels To Generate Electricity

\begin{tabular}{|c|c|c|c|c|c|c|c|c|c|c|c|}
\hline & \multicolumn{4}{|c|}{ Coal } & \multicolumn{6}{|c|}{ Potroleum } & \multirow[b]{3}{*}{$\begin{array}{c}\text { Naturd } \\
\text { Gasd }\end{array}$} \\
\hline & \multirow[b]{2}{*}{$\begin{array}{c}\text { Anthra- } \\
\text { clto }\end{array}$} & \multirow[b]{2}{*}{$\begin{array}{c}\text { Bituminous } \\
\text { Coal }\end{array}$} & \multirow[b]{2}{*}{ Lignite } & \multirow[b]{2}{*}{ Total } & \multicolumn{2}{|c|}{$\begin{array}{c}\text { By Typo } \\
\text { of Potroloum }\end{array}$} & \multicolumn{2}{|c|}{$\begin{array}{l}\text { By Prime } \\
\text { Mover Type }\end{array}$} & \multirow[b]{2}{*}{$\begin{array}{c}\text { Total } \\
\text { Liquilds }\end{array}$} & \multirow[b]{2}{*}{$\begin{array}{c}\begin{array}{c}\text { Potroloum } \\
\text { Coko }\end{array} \\
\end{array}$} & \\
\hline & & & & & $\begin{array}{c}\text { Heavy } \\
\text { Olla }\end{array}$ & $\begin{array}{l}\text { Light } \\
\text { ollo }\end{array}$ & $\begin{array}{l}\text { Steam } \\
\text { Plants }\end{array}$ & GT/AC & & & \\
\hline & \multicolumn{4}{|c|}{ Thousand Short Tons } & \multicolumn{5}{|c|}{ Thousand Barrels } & $\begin{array}{l}\text { Thousand } \\
\text { Short Tons }\end{array}$ & $\begin{array}{c}\text { Million } \\
\text { Cubic Feet }\end{array}$ \\
\hline 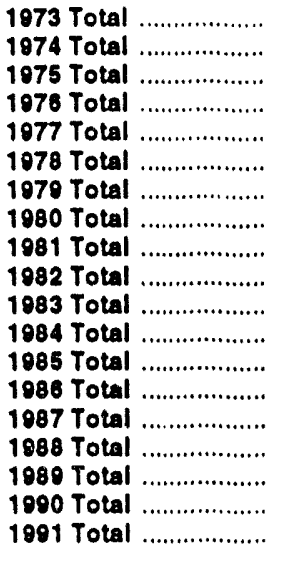 & $\begin{array}{r}1,443 \\
1,498 \\
1,480 \\
1,350 \\
1,425 \\
1,064 \\
1,046 \\
951 \\
1,221 \\
1,075 \\
1,036 \\
1,070 \\
1,033 \\
829 \\
972 \\
1,063 \\
1,049 \\
1,031 \\
994\end{array}$ & $\begin{array}{l}376,975 \\
378,643 \\
388,523 \\
425,205 \\
451,051 \\
448,763 \\
488,129 \\
526,680 \\
550,784 \\
543,346 \\
570,108 \\
606,339 \\
631,885 \\
616,134 \\
647,824 \\
631,048 \\
688,504 \\
694,317 \\
691,275\end{array}$ & $\begin{array}{l}10,794 \\
11,670 \\
15,960 \\
21,817 \\
24,650 \\
31,407 \\
37,878 \\
41,642 \\
44,792 \\
49,245 \\
54,067 \\
56,990 \\
60,923 \\
68,093 \\
69,098 \\
76,260 \\
77,335 \\
78,201 \\
70,989\end{array}$ & $\begin{array}{l}389,212 \\
391,811 \\
405,962 \\
448,371 \\
477,126 \\
481,235 \\
527,051 \\
569,274 \\
596,797 \\
593,666 \\
625,211 \\
664,399 \\
693,841 \\
685,058 \\
717,894 \\
758,372 \\
766,888 \\
773,549 \\
772,268\end{array}$ & $\begin{array}{c}\text { NA } \\
\text { NA } \\
\text { NA } \\
\text { NA } \\
\text { NA } \\
\text { NA } \\
\text { NA } \\
391,163 \\
329,798 \\
234,434 \\
228,984 \\
189,289 \\
158,779 \\
216,156 \\
184,011 \\
229,327 \\
241,960 \\
181,231 \\
171,157\end{array}$ & $\begin{array}{c}\text { NA } \\
\text { NA } \\
\text { NA } \\
\text { NA } \\
\text { NA } \\
\text { NA } \\
\text { NA } \\
29,051 \\
21,313 \\
15,337 \\
18,512 \\
15,190 \\
14,635 \\
14,328 \\
15,367 \\
18,769 \\
25,491 \\
14,823 \\
13,729\end{array}$ & $\begin{array}{l}513,190 \\
483,146 \\
467,221 \\
514,077 \\
574,869 \\
588,310 \\
492,606 \\
401,863 \\
339,680 \\
243,537 \\
237,845 \\
197,050 \\
166,842 \\
222,500 \\
190,818 \\
235,817 \\
250,315 \\
187,531 \\
177,286\end{array}$ & $\begin{array}{r}47,058 \\
53,128 \\
38,907 \\
41,843 \\
48,837 \\
47,520 \\
30,691 \\
18,351 \\
11,431 \\
6,234 \\
7,652 \\
7,420 \\
6,572 \\
7,983 \\
8,580 \\
12,270 \\
17,136 \\
8,523 \\
7,600\end{array}$ & $\begin{array}{l}560,248 \\
536,274 \\
506,128 \\
555,020 \\
623,705 \\
635,839 \\
523,297 \\
420,214 \\
351,111 \\
249,771 \\
245,497 \\
204,470 \\
173,414 \\
230,482 \\
199,378 \\
248,096 \\
267,451 \\
106,054 \\
184,886\end{array}$ & $\begin{array}{r}507 \\
625 \\
70 \\
68 \\
98 \\
398 \\
268 \\
179 \\
139 \\
149 \\
261 \\
252 \\
231 \\
313 \\
348 \\
409 \\
517 \\
810 \\
722\end{array}$ & $\begin{array}{l}3,660,172 \\
3,443,428 \\
3,157,669 \\
3,080,868 \\
3,191,200 \\
3,188,363 \\
3,490,523 \\
3,681,595 \\
3,640,154 \\
3,225,518 \\
2,010,767 \\
3,111,342 \\
3,044,083 \\
2,602,370 \\
2,844,051 \\
2,635,613 \\
2,787,012 \\
2,787,332 \\
2,789,014\end{array}$ \\
\hline 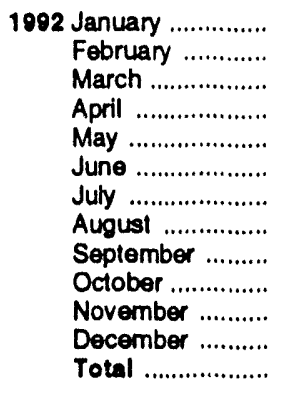 & $\begin{array}{r}80 \\
80 \\
93 \\
73 \\
69 \\
84 \\
90 \\
84 \\
83 \\
85 \\
74 \\
93 \\
986\end{array}$ & $\begin{array}{r}60,881 \\
53,687 \\
56,243 \\
53,314 \\
54,664 \\
57,179 \\
66,318 \\
62,937 \\
58,899 \\
56,366 \\
56,186 \\
61,951 \\
698,628\end{array}$ & $\begin{array}{r}7,304 \\
6,415 \\
6,368 \\
5,407 \\
5,858 \\
6,859 \\
7,407 \\
7,616 \\
6,985 \\
6,356 \\
6,352 \\
7,321 \\
80,248\end{array}$ & $\begin{array}{l}68,264 \\
60,183 \\
62,705 \\
58,794 \\
60,591 \\
64,122 \\
73,815 \\
70,637 \\
65,967 \\
62,806 \\
62,612 \\
69,365 \\
770,860\end{array}$ & $\begin{array}{r}15,811 \\
12,730 \\
13,492 \\
9,929 \\
7,910 \\
11,372 \\
12,939 \\
10,607 \\
10,456 \\
10,454 \\
10,330 \\
9,749 \\
135,770\end{array}$ & $\begin{array}{r}1,103 \\
806 \\
843 \\
811 \\
843 \\
1,077 \\
1,428 \\
1,011 \\
849 \\
792 \\
1,004 \\
989 \\
11,556\end{array}$ & $\begin{array}{r}16,332 \\
13,093 \\
13,932 \\
10,335 \\
8,385 \\
11,881 \\
13,392 \\
11,067 \\
10,820 \\
10,867 \\
10,803 \\
10,256 \\
141,163\end{array}$ & $\begin{array}{r}582 \\
444 \\
404 \\
404 \\
367 \\
568 \\
974 \\
551 \\
485 \\
379 \\
531 \\
482 \\
6,172\end{array}$ & $\begin{array}{r}16,915 \\
13,536 \\
14,336 \\
10,740 \\
8,752 \\
12,449 \\
14,367 \\
11,619 \\
11,305 \\
11,246 \\
11,333 \\
10,737 \\
147,335\end{array}$ & $\begin{array}{r}71 \\
76 \\
83 \\
66 \\
50 \\
66 \\
72 \\
116 \\
98 \\
103 \\
93 \\
105 \\
990\end{array}$ & $\begin{array}{r}169,125 \\
170,293 \\
207,656 \\
229,012 \\
236,316 \\
265,882 \\
333,567 \\
302,544 \\
273,670 \\
212,640 \\
189,296 \\
175,608 \\
2,765,808\end{array}$ \\
\hline 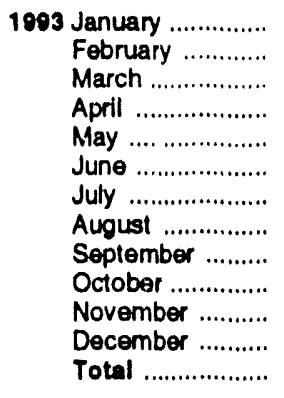 & $\begin{array}{r}79 \\
88 \\
101 \\
84 \\
81 \\
80 \\
73 \\
67 \\
60 \\
64 \\
81 \\
92 \\
951\end{array}$ & $\begin{array}{r}61,703 \\
57,293 \\
60,969 \\
53,755 \\
53,380 \\
61,090 \\
71,134 \\
70,241 \\
60,143 \\
59,125 \\
59,385 \\
64,516 \\
732,736\end{array}$ & $\begin{array}{r}7,617 \\
6,431 \\
6,002 \\
5,757 \\
6,570 \\
6,948 \\
7,511 \\
7,624 \\
6,289 \\
5,752 \\
6,211 \\
7,109 \\
70,821\end{array}$ & $\begin{array}{r}69,400 \\
63,812 \\
67,073 \\
59,596 \\
60,032 \\
68,118 \\
78,717 \\
77,932 \\
66,493 \\
64,941 \\
65,677 \\
71,717 \\
813,508\end{array}$ & $\begin{array}{r}10,804 \\
10,569 \\
12,784 \\
7,629 \\
7,722 \\
11,756 \\
16,896 \\
18,044 \\
14,730 \\
11,318 \\
11,339 \\
15,694 \\
149,287\end{array}$ & $\begin{array}{r}1,013 \\
935 \\
1,277 \\
819 \\
868 \\
1,033 \\
1,817 \\
1,566 \\
1,031 \\
897 \\
886 \\
1,027 \\
13,168\end{array}$ & $\begin{array}{r}11,265 \\
11,002 \\
13,313 \\
8,094 \\
8,198 \\
12,249 \\
17,406 \\
18,509 \\
15,111 \\
11,771 \\
11,781 \\
16,206 \\
154,905\end{array}$ & $\begin{array}{r}552 \\
503 \\
748 \\
354 \\
392 \\
540 \\
1,306 \\
1,101 \\
650 \\
444 \\
444 \\
514 \\
7,540\end{array}$ & $\begin{array}{r}11,817 \\
11,504 \\
14,061 \\
8,448 \\
8,590 \\
12,789 \\
18,713 \\
19,610 \\
15,761 \\
12,216 \\
12,225 \\
16,720 \\
162,454\end{array}$ & $\begin{array}{r}92 \\
81 \\
87 \\
79 \\
86 \\
98 \\
125 \\
112 \\
129 \\
112 \\
101 \\
120 \\
1,220\end{array}$ & $\begin{array}{r}164,374 \\
161,828 \\
193,811 \\
173,834 \\
168,840 \\
254,823 \\
334,101 \\
357,027 \\
258,325 \\
234,544 \\
208,335 \\
174,498 \\
2,682,440\end{array}$ \\
\hline $\begin{array}{l}1004 \text { January ............. } \\
\text { February ........... } \\
\text { 2-Month Total ... }\end{array}$ & $\begin{array}{r}82 \\
98 \\
180\end{array}$ & $\begin{array}{r}69,022 \\
58,843 \\
127,865\end{array}$ & $\begin{array}{r}7,257 \\
6,514 \\
13,771\end{array}$ & $\begin{array}{r}76,362 \\
65,455 \\
141,817\end{array}$ & $\begin{array}{l}20,743 \\
14,697 \\
35,440\end{array}$ & $\begin{array}{l}3,710 \\
1,397 \\
5,106\end{array}$ & $\begin{array}{l}21,602 \\
15,242 \\
38,844\end{array}$ & $\begin{array}{r}2,851 \\
851 \\
3,702\end{array}$ & $\begin{array}{l}24,453 \\
16,094 \\
40,548\end{array}$ & $\begin{array}{r}112 \\
88 \\
200\end{array}$ & $\begin{array}{l}169,995 \\
149,173 \\
319,168\end{array}$ \\
\hline $\begin{array}{l}1093 \text { 2-Month Total ... } \\
1092 \text { 2-Month Total ... }\end{array}$ & $\begin{array}{l}167 \\
159\end{array}$ & $\begin{array}{l}118,996 \\
114,568\end{array}$ & $\begin{array}{l}14,049 \\
13,719\end{array}$ & $\begin{array}{l}133,212 \\
128,447\end{array}$ & $\begin{array}{l}21,373 \\
28,542\end{array}$ & $\begin{array}{l}1,948 \\
1,900\end{array}$ & $\begin{array}{l}22,267 \\
29,425\end{array}$ & $\begin{array}{l}1,055 \\
1,026\end{array}$ & $\begin{array}{l}23,322 \\
30,451\end{array}$ & $\begin{array}{l}173 \\
147\end{array}$ & $\begin{array}{l}326,302 \\
330,418\end{array}$ \\
\hline
\end{tabular}

- Heavy oll includes fuel oil nos. 4, 5, and 6, and residual fuel sils.

b Light oil includus luel oil nos. 1 and 2, kerosene, and jet fuel.

- GT/IC = Gas lurbine and internal combustion plants.

d Includes supplemental gaseous fuels.

$\mathrm{NA}=\mathrm{Na}$ available.

Noles: - Geographic coverage is the 50 States and the Distria of Columbia.

- Tolals may not equal sum of components due to independent rounding.

Sources: - Prime Mover Type Data: 1973-September 1977-Federal

Power Commission (FPC), Form FPC-4, "Monthly Power Plant Repont."

October 1077.1081-Federal Energy Regulalory Commission (FERC), Form
FPC.4, "Monthly Power Plant Report." 1982 forward-Energy Information Administration (EIA), Form EIA-759, "Monthly Power Plant Report." - All Other Data: 1073-Soptember 1077-FPC, Form FPC-4, "Monthly Power Plant Repont: October 1977-1979-FERC, Form FPC-4, "Monthly Power Plant Repon:" 1980-EIA, Electric Power Monthly, March 1991, Table 17. 1081-EIA, Electric Power Monthy, March 1992, Table 17. 1082 and 1091 monthly data-EIA, Electric Power Monthly, March 1993, Table 17. 1083 forward (excopt 1001 monthly data)-ELA, Electilc Power Monthly, May 1994, Table 18. 
Table 7.4 Electric Utility Stocks of Coal and Petroleum, End of Period

\begin{tabular}{|c|c|c|c|c|c|c|c|c|c|c|}
\hline & \multicolumn{4}{|c|}{ Coal } & \multicolumn{6}{|c|}{ Potroloum } \\
\hline & \multirow[b]{2}{*}{ Anthracito } & \multirow[b]{2}{*}{$\begin{array}{c}\text { Bituminous } \\
\text { Coal }\end{array}$} & \multirow[b]{2}{*}{ Llgnite } & \multirow[b]{2}{*}{ Total } & \multicolumn{2}{|c|}{$\begin{array}{c}\text { By Typo } \\
\text { of Petroloum }\end{array}$} & \multicolumn{2}{|c|}{$\begin{array}{c}\text { By Prlme } \\
\text { Mover Type }\end{array}$} & \multirow[b]{2}{*}{$\begin{array}{l}\text { Total } \\
\text { Llquilds }\end{array}$} & \multirow[b]{2}{*}{$\begin{array}{c}\text { Potroloum } \\
\text { Coke }\end{array}$} \\
\hline & & & & & $\begin{array}{l}\text { Heavy } \\
\text { Oila }\end{array}$ & $\begin{array}{l}\text { Ught } \\
\text { Oll }\end{array}$ & $\begin{array}{l}\text { Stoam } \\
\text { Plants }\end{array}$ & GTACC & & \\
\hline & \multicolumn{4}{|c|}{ Thousand Short Tons } & \multicolumn{5}{|c|}{ Thousand Barrets } & $\begin{array}{l}\text { Thousand } \\
\text { Short Tons }\end{array}$ \\
\hline 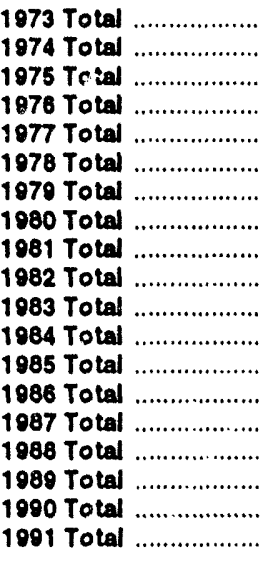 & $\begin{array}{r}1,066 \\
030 \\
982 \\
1,000 \\
2,321 \\
2,178 \\
3,274 \\
4,741 \\
5,537 \\
6,080 \\
6,507 \\
6,710 \\
7,189 \\
7,099 \\
6,940 \\
6,561 \\
6,403 \\
6,499 \\
6,513\end{array}$ & $\begin{array}{r}84,941 \\
81,712 \\
107,927 \\
114,130 \\
128,210 \\
123,020 \\
152,981 \\
174,154 \\
158,258 \\
170,480 \\
145,250 \\
167,118 \\
142,144 \\
148,665 \\
156,670 \\
133,434 \\
122,967 \\
142,650 \\
145,367\end{array}$ & $\begin{array}{r}961 \\
867 \\
1,815 \\
2,306 \\
2,688 \\
3,027 \\
3,459 \\
4,115 \\
5,098 \\
4,573 \\
3,841 \\
5,899 \\
7,043 \\
6,042 \\
7,187 \\
6,512 \\
6,490 \\
7,016 \\
5,996\end{array}$ & $\begin{array}{r}86,967 \\
83,509 \\
110,724 \\
117,436 \\
133,219 \\
128,225 \\
159,714 \\
183,010 \\
168,893 \\
181,132 \\
155,598 \\
179,727 \\
156,376 \\
181,806 \\
170,797 \\
146,507 \\
135,860 \\
156,166 \\
157,876\end{array}$ & $\begin{array}{c}\text { NA } \\
\text { NA } \\
\text { NA } \\
\text { NA } \\
\text { NA } \\
\text { NA } \\
\text { NA } \\
105,351 \\
102,042 \\
95,515 \\
70,573 \\
68,503 \\
57,304 \\
56,841 \\
55,069 \\
54,187 \\
47,446 \\
67,030 \\
58,636\end{array}$ & $\begin{array}{c}\text { NA } \\
\text { NA } \\
\text { NA } \\
\text { NA } \\
\text { NA } \\
\text { NA } \\
\text { NA } \\
30,023 \\
28,094 \\
23,389 \\
18,801 \\
12,116 \\
16,386 \\
16,269 \\
15,759 \\
15,099 \\
13,824 \\
16,471 \\
16,357\end{array}$ & $\begin{array}{r}79,121 \\
97,718 \\
108,825 \\
106,993 \\
124,750 \\
102,402 \\
111,121 \\
117,227 \\
112,380 \\
105,287 \\
78,285 \\
76,836 \\
64,704 \\
64,258 \\
61,705 \\
60,311 \\
53,309 \\
73,306 \\
65,032\end{array}$ & $\begin{array}{r}10,095 \\
15,190 \\
16,432 \\
14,703 \\
19,281 \\
16,386 \\
20,301 \\
18,147 \\
15,756 \\
13,597 \\
11,090 \\
10,784 \\
8,985 \\
8,153 \\
0,123 \\
8,974 \\
7,962 \\
10,195 \\
0,961\end{array}$ & $\begin{array}{r}89,216 \\
112,017 \\
125,257 \\
121,696 \\
144,031 \\
118,788 \\
131,422 \\
135,374 \\
128,136 \\
118,804 \\
89,375 \\
87,610 \\
73,689 \\
73,111 \\
70,827 \\
69,285 \\
61,270 \\
83,501 \\
74,093\end{array}$ & $\begin{array}{r}312 \\
35 \\
31 \\
32 \\
44 \\
108 \\
183 \\
52 \\
42 \\
41 \\
55 \\
50 \\
40 \\
40 \\
51 \\
86 \\
105 \\
94 \\
70\end{array}$ \\
\hline 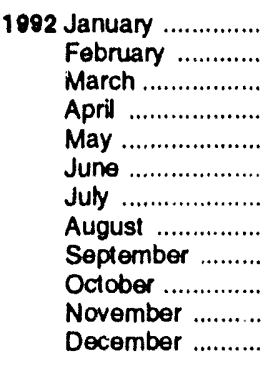 & $\begin{array}{l}6,488 \\
6,455 \\
6,398 \\
6,379 \\
6,370 \\
6,355 \\
6,341 \\
6,343 \\
6,329 \\
6,374 \\
6,273 \\
6,215\end{array}$ & $\begin{array}{l}143,466 \\
146,338 \\
147,978 \\
149,824 \\
152,275 \\
151,224 \\
141,613 \\
140,166 \\
140,409 \\
144,068 \\
145,406 \\
142,156\end{array}$ & $\begin{array}{l}5,683 \\
5,352 \\
5,656 \\
6,387 \\
6,867 \\
6,596 \\
6,4,9 \\
6,071 \\
5,946 \\
6,487 \\
6,169 \\
5,759\end{array}$ & $\begin{array}{l}155,637 \\
158,145 \\
160,032 \\
162,591 \\
165,512 \\
164,176 \\
154,403 \\
152,580 \\
152,685 \\
156,859 \\
157,849 \\
154,130\end{array}$ & $\begin{array}{l}\mathbf{5 3 , 1 3 6} \\
54,750 \\
54,513 \\
52,815 \\
55,144 \\
53,794 \\
53,445 \\
54,434 \\
52,731 \\
52,919 \\
53,632 \\
\mathbf{5 6 , 1 3 5}\end{array}$ & $\begin{array}{l}15,712 \\
15,655 \\
15,589 \\
15,371 \\
15,214 \\
15,117 \\
14,995 \\
15,456 \\
15,251 \\
15,351 \\
15,302 \\
15,714\end{array}$ & $\begin{array}{l}59,340 \\
61,085 \\
60,840 \\
59,044 \\
61,145 \\
59,648 \\
59,273 \\
60,644 \\
58,646 \\
58,869 \\
59,535 \\
62,374\end{array}$ & $\begin{array}{l}9,500 \\
9,321 \\
9,262 \\
9,143 \\
9,214 \\
9,263 \\
9,167 \\
9,246 \\
9,336 \\
9,400 \\
9,398 \\
0,475\end{array}$ & $\begin{array}{l}68,849 \\
70,406 \\
70,103 \\
68,186 \\
70,358 \\
68,910 \\
68,440 \\
69,890 \\
67,982 \\
68,269 \\
68,934 \\
71,840\end{array}$ & $\begin{array}{l}75 \\
62 \\
56 \\
47 \\
63 \\
67 \\
56 \\
46 \\
51 \\
55 \\
59 \\
67\end{array}$ \\
\hline 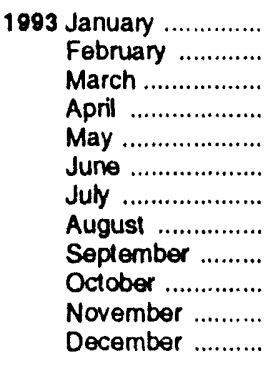 & $\begin{array}{l}6,166 \\
6,107 \\
6,036 \\
5,802 \\
5,773 \\
5,766 \\
5,755 \\
5,745 \\
5,735 \\
5,718 \\
5,693 \\
5,638\end{array}$ & $\begin{array}{r}138,615 \\
135,063 \\
132,183 \\
136,199 \\
138,668 \\
133,977 \\
115,383 \\
102,582 \\
100,951 \\
102,700 \\
103,447 \\
88,560\end{array}$ & $\begin{array}{l}5,521 \\
5,357 \\
5,758 \\
6,177 \\
6,238 \\
6,009 \\
5,677 \\
5,651 \\
6,147 \\
6,687 \\
6,955 \\
7,142\end{array}$ & $\begin{array}{l}150,302 \\
146,528 \\
143,978 \\
148,178 \\
150,678 \\
145,753 \\
126,815 \\
113,978 \\
112,833 \\
115,105 \\
116,095 \\
111,341\end{array}$ & $\begin{array}{l}53,781 \\
50,005 \\
45,313 \\
47,356 \\
50,422 \\
49,294 \\
47,401 \\
43,943 \\
45,913 \\
46,298 \\
46,603 \\
46,769\end{array}$ & $\begin{array}{l}15,840 \\
15,131 \\
14,914 \\
14,856 \\
14,669 \\
14,736 \\
14,618 \\
14,842 \\
14,774 \\
14,822 \\
14,878 \\
15,674\end{array}$ & $\begin{array}{l}60,193 \\
56,303 \\
51,528 \\
53,475 \\
56,495 \\
55,604 \\
53,639 \\
50,223 \\
52,071 \\
52,385 \\
52,812 \\
53,360\end{array}$ & $\begin{array}{l}9,428 \\
8,833 \\
8,698 \\
8,736 \\
8,596 \\
8,626 \\
8,380 \\
8,562 \\
8,617 \\
8,735 \\
8,668 \\
8,083\end{array}$ & $\begin{array}{l}69,620 \\
65,136 \\
60,227 \\
62,211 \\
65,091 \\
64,230 \\
62,019 \\
58,735 \\
60,687 \\
61,120 \\
61,481 \\
62,443\end{array}$ & $\begin{array}{l}65 \\
60 \\
66 \\
77 \\
82 \\
92 \\
90 \\
99 \\
62 \\
69 \\
84 \\
69\end{array}$ \\
\hline $\begin{array}{l}1994 \text { January .................. } \\
\text { February ............ }\end{array}$ & $\begin{array}{l}5,576 \\
5,496\end{array}$ & $\begin{array}{l}86,043 \\
85,486\end{array}$ & $\begin{array}{l}6,676 \\
6,720\end{array}$ & $\begin{array}{l}98,294 \\
97,701\end{array}$ & $\begin{array}{l}42,781 \\
44,764\end{array}$ & $\begin{array}{l}15,127 \\
15,290\end{array}$ & $\begin{array}{l}49,922 \\
51,211\end{array}$ & $\begin{array}{l}7,986 \\
8,843\end{array}$ & $\begin{array}{l}57,908 \\
60,054\end{array}$ & $\begin{array}{l}83 \\
73\end{array}$ \\
\hline
\end{tabular}

Heavy oil includes fuel oil nos. 4, 5, and 6, and residual fuel oils.

b Light oil includes fuel oil nos. 1 and 2 , kerosene, and jet fuel.

c $\mathrm{GT} / \mathrm{lC}=$ Gas turbine and internal combustion plants.

$\mathrm{NA}=\mathrm{Not}$ available.

Noles: - Geographic coverage is the 50 States and the District of Columbia.

- Totals may not equal sum of components dive to independent rounding.

Sources: - Prime Movor Typo Date: 1973-September 1977-Federal Power Commission (FPC), Form FPC-4, "Monthly Power Plant Report." October 1977-1981 -Federal Energy Regulatory Commission (FERC). Form
FPC-4, "Monthly Power Plant Repon:" 1882 forward-Energy Intormation Administration (EIA), Form EIA.759, "Monthly Power Plant Report." - All Other Data: 1973-Soptember 1977-FPC, Form FPC-4, "Monthly Power Plant Report." October 1977-1979-FERC, Form FPC-4, "Monthly Power Plant Report:" 1980-ElA, Electric Power Monthly, March 1991, Table 29. 1981-ElA, Electric Power Monthly, March 1992, Table 29. 1982 and 1091 monthly data-EIA, Electric Power Monthly, March 1993, Table 28. 1983 forward (oxcopt 1891 monthly data)-EIA, Electric Power Monthly, May 1994. Table 29. 


\section{Section 8. Nuclear Energy}

In February 1994, U.S. nuclear generating units produced a total of 50 net terawatthours (billion kilowatthours) of electricity, 3 percent $^{8}$ less than in February 1993. Nuclear units generated at an average capacity factor of 74.9 percent, 3 percentage points lower than in February 1993. Nuclear power supplied 22.2 percent of the total electric utility-generated electricity in February 1994, compared with 22.8 percent in February 1993.

No low- or full power licenses for nuclear power plants were issued by the Nuclear Regulatory Commission during February 1994.

On February 28, 1994, there were 109 operable nuclear generating units in the United States, with a collective net summer capability of 99.1 million kilowatts of electricity. Of the 109 operable units, 19 units generated at less than 25 percent of capacity because of maintenance, refueling, or repair outage, and 11 of the 19 units generated no electricity during the month.

Two operable units, Browns Ferry 1 and 3, have been shut down since March 1985 . Each unit had a capacity of 1,065 megawatts electric.

As of February 28, 1994, there were 115 domestic nuclear generating units in all stages of construction and operation (construction of the Perry-2 nuclear unit, in North Perry, Ohio, was canceled as of January 4, 1994). The aggregate net design capacity of operable units was 101.1 million kilowatts, and the design capacity of units under construction was 7.3 million kilowatts, for a total design capacity of 108.4 million kilowatts.

${ }^{8}$ Percentage changes are based on numbers shown in the following tables. 
Figure 8.1 Nuclear Power Plant Operations

Operable Units, End of Year, 1973-1993

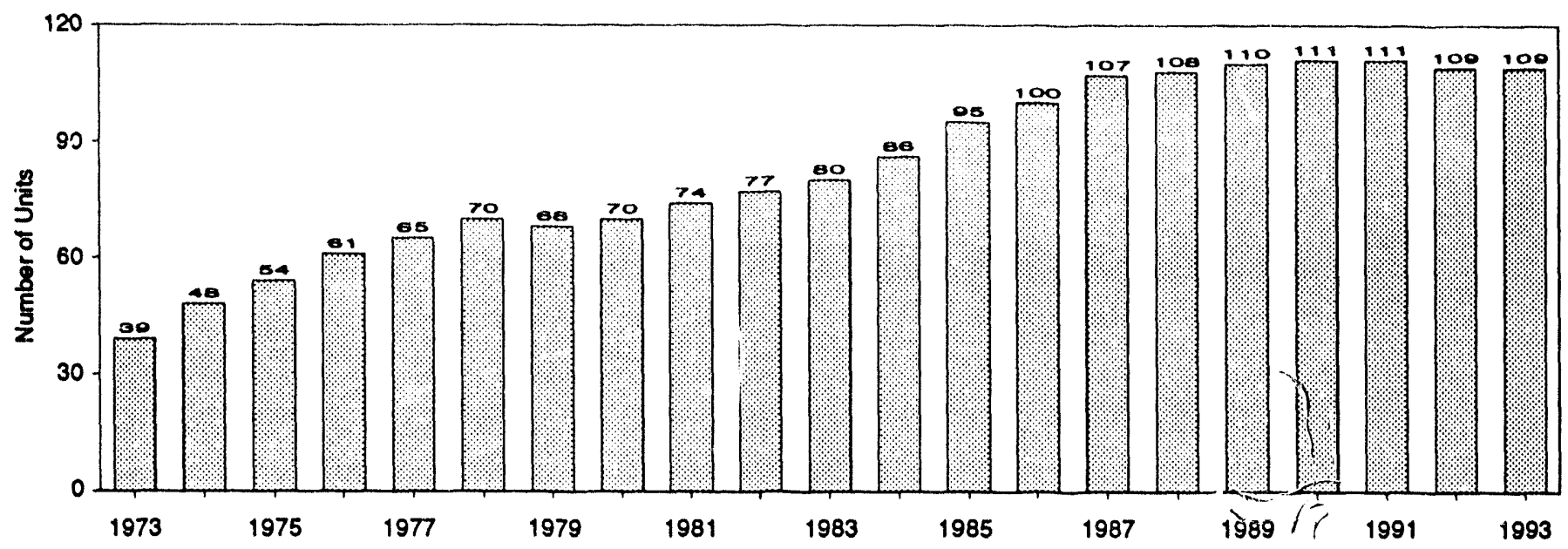

Net Generation of Electricity, 1973-1993

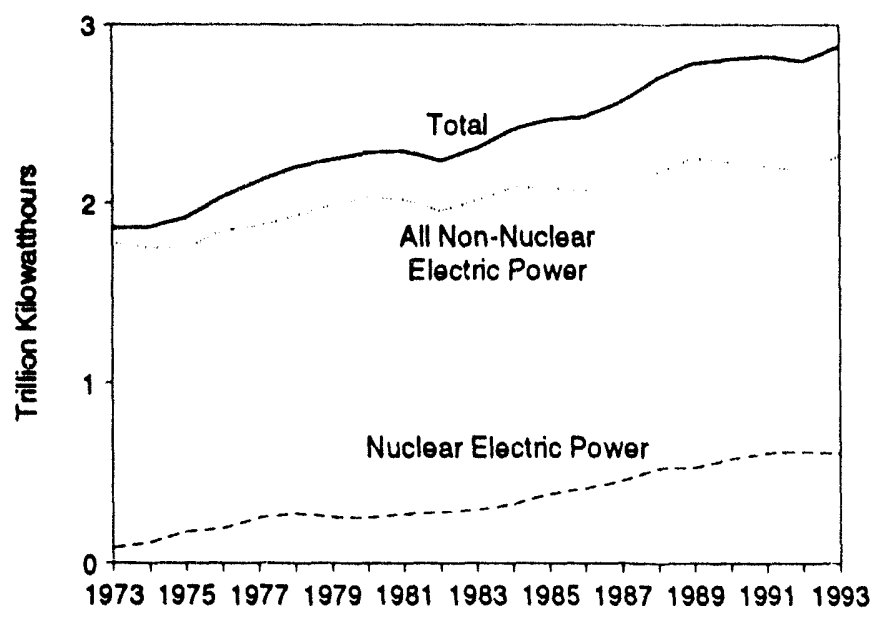

Nuclear Electricity Net Generation

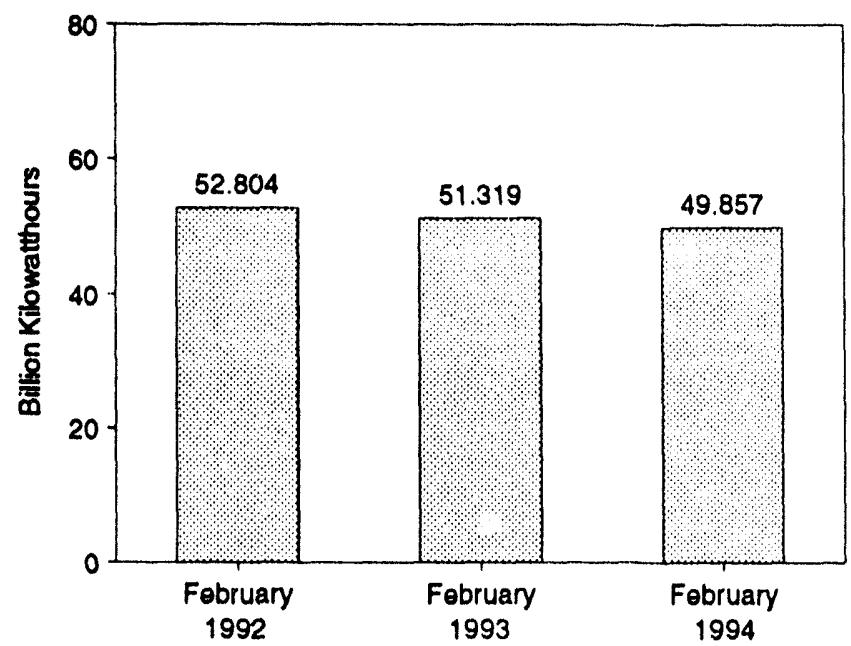

Nuclear Portion of Domestic Electricity

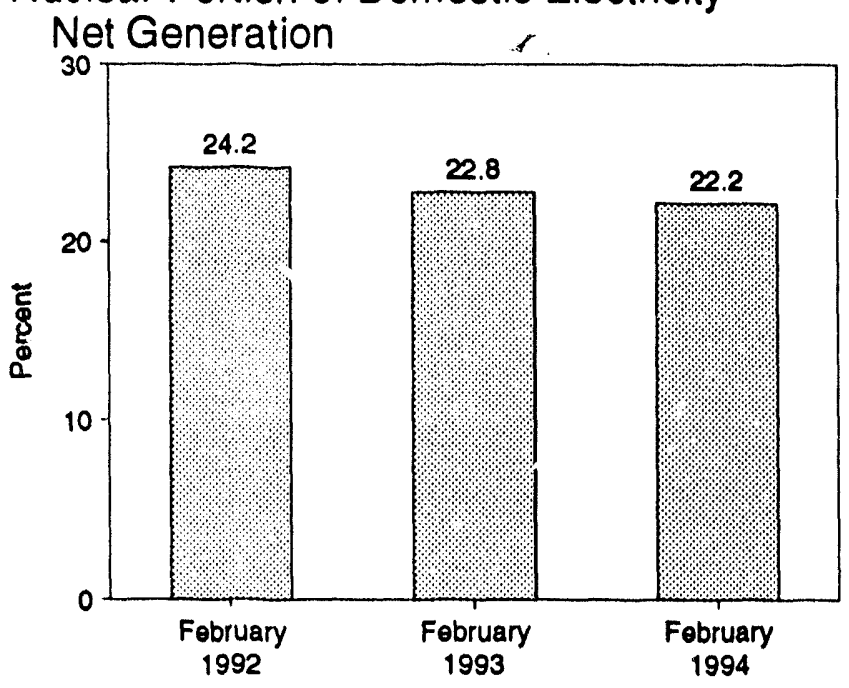

Capacity Factor, Monthly

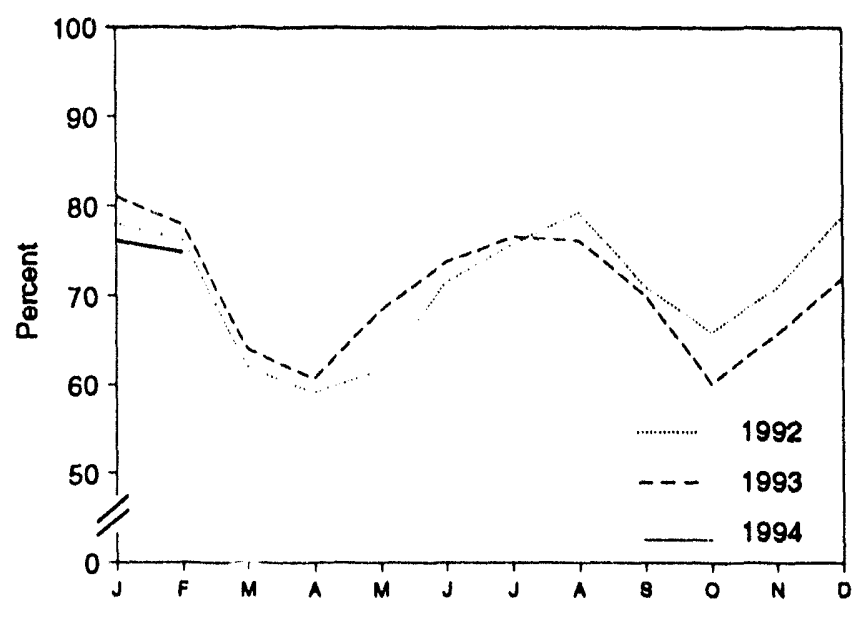

Note: Because vertical scales differ, graphs should not be compared. Sources: Tables 7.1 and 8.1 . 


\begin{tabular}{|c|c|c|c|c|c|}
\hline & $\begin{array}{l}\text { Operable } \\
\text { Units }\end{array}$ & $\begin{array}{l}\text { Nuclear } \\
\text { Electricity } \\
\text { Not } \\
\text { Generation }\end{array}$ & $\begin{array}{c}\text { Nuclear } \\
\text { Portion of } \\
\text { Domestle } \\
\text { Electricity } \\
\text { Net Generation }\end{array}$ & $\begin{array}{c}\text { Not } \\
\text { Summer } \\
\text { Capability } \\
\text { of Operable } \\
\text { Unitsa,c }\end{array}$ & $\begin{array}{l}\text { Capacity } \\
\text { Factord }\end{array}$ \\
\hline & Number & $\begin{array}{c}\text { Million } \\
\text { Kilowatthours }\end{array}$ & Percent & $\begin{array}{l}\text { Million } \\
\text { Kilowatts }\end{array}$ & Percent \\
\hline 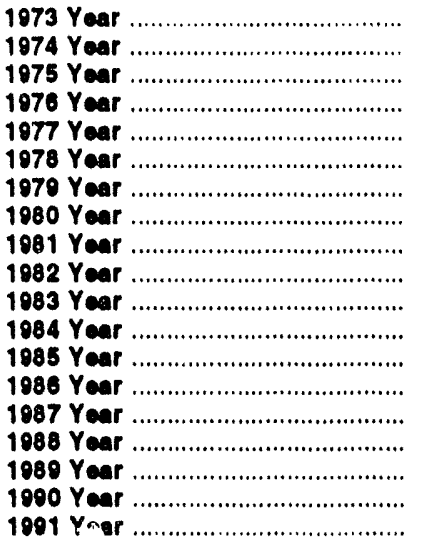 & $\begin{array}{l}39 \\
48 \\
54 \\
61 \\
65 \\
70 \\
68 \\
70 \\
74 \\
77 \\
80 \\
86 \\
95 \\
100 \\
107 \\
108 \\
110 \\
111 \\
111\end{array}$ & $\begin{array}{r}83,479 \\
113,976 \\
172,505 \\
191,104 \\
250,883 \\
276,403 \\
255,155 \\
251,116 \\
272,674 \\
282,773 \\
293,677 \\
327,634 \\
383,691 \\
414,038 \\
455,270 \\
526,073 \\
529,355 \\
578,862 \\
612,565\end{array}$ & $\begin{array}{r}4.5 \\
6.1 \\
9.0 \\
9.4 \\
11.8 \\
12.5 \\
11.4 \\
11.0 \\
11.0 \\
12.6 \\
12.7 \\
13.6 \\
15.5 \\
18.6 \\
17.7 \\
19.5 \\
19.0 \\
20.5 \\
21.7\end{array}$ & $\begin{array}{l}22.683 \\
31.867 \\
37.287 \\
43.822 \\
46.303 \\
50.824 \\
49.747 \\
51.810 \\
56.042 \\
60.035 \\
63.009 \\
69.652 \\
79.397 \\
85.241 \\
93.583 \\
94.695 \\
98.161 \\
99.624 \\
99.589\end{array}$ & $\begin{array}{l}53.5 \\
47.8 \\
55.0 \\
54.7 \\
63.3 \\
64.5 \\
58.4 \\
56.3 \\
58.2 \\
56.6 \\
54.4 \\
56.3 \\
56.0 \\
56.0 \\
57.4 \\
63.5 \\
62.2 \\
66.0 \\
70.2\end{array}$ \\
\hline 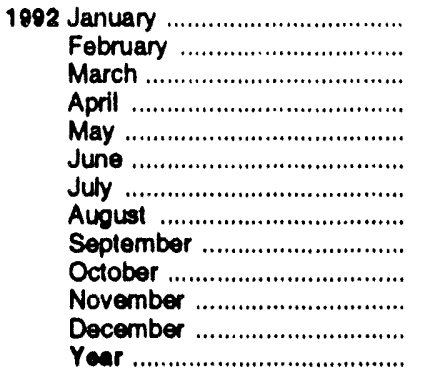 & $\begin{array}{l}111 \\
110 \\
110 \\
110 \\
110 \\
110 \\
110 \\
110 \\
110 \\
110 \\
110 \\
109 \\
100\end{array}$ & $\begin{array}{l}57,849 \\
52,804 \\
45,835 \\
42,268 \\
45,627 \\
51,185 \\
56,049 \\
58,656 \\
50,919 \\
48,784 \\
50,726 \\
58,075 \\
618,776\end{array}$ & $\begin{array}{l}2 j .7 \\
24.2 \\
20.4 \\
20.0 \\
20.7 \\
21.6 \\
21.1 \\
23.0 \\
21.7 \\
22.0 \\
22.9 \\
23.8 \\
22.1\end{array}$ & $\begin{array}{l}99.589 \\
99.421 \\
99.421 \\
99.421 \\
99.421 \\
99.421 \\
99.421 \\
99.421 \\
99.421 \\
99.421 \\
99.421 \\
98.985 \\
98.085\end{array}$ & $\begin{array}{l}78.1 \\
76.3 \\
62.0 \\
59.1 \\
61.7 \\
71.5 \\
75.8 \\
79.3 \\
71.1 \\
65.9 \\
70.9 \\
78.9 \\
70.9\end{array}$ \\
\hline 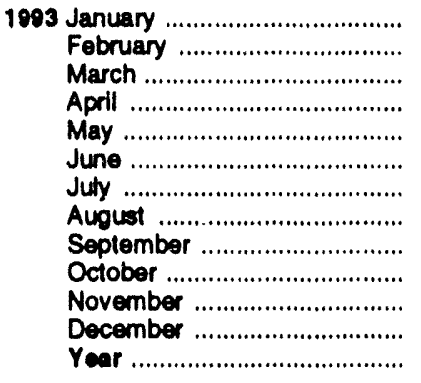 & $\begin{array}{l}108 \\
108 \\
108 \\
109 \\
109 \\
109 \\
109 \\
109 \\
109 \\
109 \\
109 \\
109 \\
109\end{array}$ & $\begin{array}{l}59,076 \\
51,319 \\
46,606 \\
43,199 \\
50,367 \\
52,620 \\
56,502 \\
56,209 \\
49,989 \\
44,434 \\
46,862 \\
53,108 \\
610,291\end{array}$ & $\begin{array}{l}24.0 \\
22.8 \\
19.8 \\
20.4 \\
22.6 \\
21.1 \\
20.0 \\
20.1 \\
21.1 \\
19.8 \\
20.7 \\
21.6 \\
21.2\end{array}$ & $\begin{array}{l}97.881 \\
97.881 \\
97.881 \\
99.031 \\
99.031 \\
99.031 \\
99.031 \\
99.031 \\
99.031 \\
99.094 \\
99.094 \\
99.094 \\
99.094\end{array}$ & $\begin{array}{l}81.1 \\
78.0 \\
64.0 \\
60.7 \\
68.4 \\
73.8 \\
76.6 \\
76.2 \\
70.1 \\
60.2 \\
65.7 \\
72.0 \\
70.5\end{array}$ \\
\hline 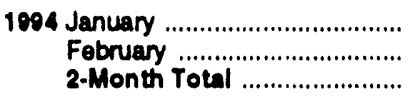 & $\begin{array}{l}109 \\
109 \\
100\end{array}$ & $\begin{array}{r}56,184 \\
49,857 \\
108,042\end{array}$ & $\begin{array}{l}21.5 \\
22.2 \\
21.8\end{array}$ & $\begin{array}{l}99.094 \\
99.094 \\
99.094\end{array}$ & $\begin{array}{l}76.2 \\
74.9 \\
75.8\end{array}$ \\
\hline 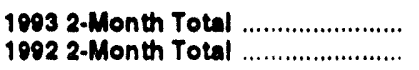 & $\begin{array}{l}108 \\
110\end{array}$ & $\begin{array}{l}110,395 \\
110,652\end{array}$ & $\begin{array}{l}23.5 \\
24.0\end{array}$ & $\begin{array}{l}\bullet 7.881 \\
90.421\end{array}$ & $\begin{array}{l}79.7 \\
77.2\end{array}$ \\
\hline
\end{tabular}

- At end of period.

- See Note 1 at end of section.

c For the definition of "Net Summer Capability." see Note 3 at end of section.

For an explanation of the method of calculating the capacity factor, seo Nole 4 at end of section.

Notes: - Geographic coverage is the 50 States and the District of Columbia. - Nuclear electricity net generation totals may not equal sum of components due 10 independent rounding.

Sources: - Operable Unite: 1973-1982-U.S. Department of Energy (DOE), Otilce of Nuclear Programs, "U.S. Central Station Nuclear Electric
Generating Units: Significant Milestones." 1983 forward-Nuclear Regulatory Commission (NRC), "Licensed Operating Reactors" (NUREG-0020). - Nuclear Electricity Not Generation: Table 7.1. - Nuclear Portion of Domestle Electriclty Not Generation: Calculated from data in Table 7.1. - Net Summor Capability of Operable Unite: 1973-1982-Complled from various sources, primarily DOE, Ot!'ce of Nuclear Reactor Programs, "U.S. Central Station Nuclear Electric Generating Unita: Signiticant Milestones." 1983 forward-Energy Intormation Administration (ElA), Form ElA-860, "Annual Eloctric Generation Report," and monthly updates as appropriate. - Capacity Factor: EIA, Otlice of Coal, Nuclear, Electric and Alternate Fuels. 
Table 8.2 Nuclear Generating Units, End of Period

\begin{tabular}{|c|c|c|c|c|c|c|c|c|}
\hline & \multicolumn{2}{|c|}{$\begin{array}{l}\text { Ljcensed } \\
\text { for Operation }\end{array}$} & \multicolumn{2}{|c|}{$\begin{array}{l}\text { Construction } \\
\text { Permits }\end{array}$} & \multirow[b]{2}{*}{ On Order } & \multirow[b]{2}{*}{ A7nounced } & \multirow[b]{2}{*}{ Total } & \multirow{3}{*}{$\frac{\begin{array}{c}\text { Total } \\
\text { Dosign } \\
\text { Capacityc }\end{array}}{\begin{array}{c}\text { Million } \\
\text { Kilowalts }\end{array}}$} \\
\hline & Operablea & In Startup b & Granted & Pending & & & & \\
\hline & \multicolumn{7}{|c|}{ Number of Units } & \\
\hline 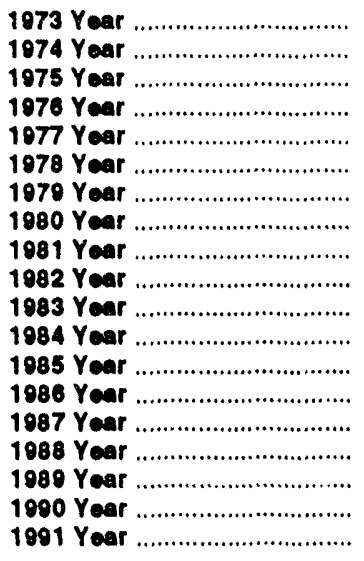 & $\begin{array}{r}39 \\
48 \\
54 \\
61 \\
65 \\
70 \\
68 \\
70 \\
74 \\
77 \\
80 \\
86 \\
95 \\
100 \\
107 \\
108 \\
110 \\
111 \\
111\end{array}$ & $\begin{array}{l}2 \\
5 \\
2 \\
1 \\
2 \\
0 \\
0 \\
1 \\
0 \\
2 \\
3 \\
6 \\
3 \\
7 \\
4 \\
3 \\
1 \\
0 \\
0\end{array}$ & $\begin{array}{r}57 \\
62 \\
69 \\
71 \\
78 \\
88 \\
90 \\
82 \\
76 \\
60 \\
53 \\
38 \\
30 \\
10 \\
14 \\
12 \\
10 \\
8 \\
8\end{array}$ & $\begin{array}{r}52 \\
75 \\
69 \\
63 \\
49 \\
32 \\
24 \\
12 \\
11 \\
3 \\
0 \\
0 \\
0 \\
0 \\
0 \\
0 \\
0 \\
0 \\
0\end{array}$ & $\begin{array}{r}49 \\
30 \\
14 \\
16 \\
13 \\
5 \\
3 \\
3 \\
2 \\
2 \\
2 \\
2 \\
2 \\
2 \\
2 \\
0 \\
0 \\
0 \\
0\end{array}$ & $\begin{array}{l}0 \\
6 \\
5 \\
2 \\
2 \\
0 \\
0 \\
0 \\
0 \\
0 \\
0 \\
0 \\
0 \\
0 \\
0 \\
0 \\
0 \\
0 \\
0\end{array}$ & $\begin{array}{l}208 \\
226 \\
213 \\
214 \\
209 \\
105 \\
185 \\
168 \\
163 \\
144 \\
138 \\
132 \\
130 \\
128 \\
127 \\
123 \\
121 \\
110 \\
110\end{array}$ & $\begin{array}{l}198 \\
223 \\
212 \\
211 \\
203 \\
101 \\
180 \\
162 \\
157 \\
134 \\
129 \\
123 \\
121 \\
110 \\
110 \\
115 \\
113 \\
111 \\
111\end{array}$ \\
\hline 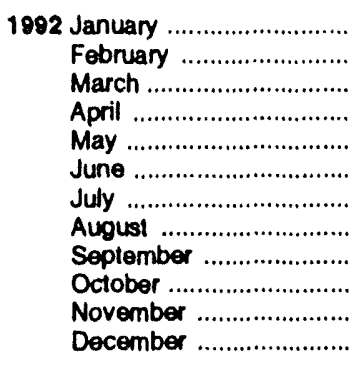 & $\begin{array}{l}111 \\
110 \\
110 \\
110 \\
110 \\
110 \\
110 \\
110 \\
110 \\
110 \\
110 \\
100\end{array}$ & $\begin{array}{l}0 \\
0 \\
0 \\
0 \\
0 \\
0 \\
0 \\
0 \\
0 \\
0 \\
0 \\
0\end{array}$ & $\begin{array}{l}8 \\
8 \\
8 \\
8 \\
8 \\
8 \\
8 \\
8 \\
8 \\
8 \\
8 \\
8\end{array}$ & $\begin{array}{l}0 \\
0 \\
0 \\
0 \\
0 \\
0 \\
0 \\
0 \\
0 \\
0 \\
0 \\
0\end{array}$ & $\begin{array}{l}0 \\
0 \\
0 \\
0 \\
0 \\
0 \\
0 \\
0 \\
0 \\
0 \\
0 \\
0\end{array}$ & $\begin{array}{l}0 \\
0 \\
0 \\
0 \\
0 \\
0 \\
0 \\
0 \\
0 \\
0 \\
0 \\
0\end{array}$ & $\begin{array}{l}119 \\
118 \\
118 \\
118 \\
118 \\
118 \\
118 \\
118 \\
118 \\
118 \\
118 \\
117\end{array}$ & $\begin{array}{l}111 \\
111 \\
111 \\
111 \\
111 \\
111 \\
111 \\
111 \\
111 \\
111 \\
111 \\
111\end{array}$ \\
\hline 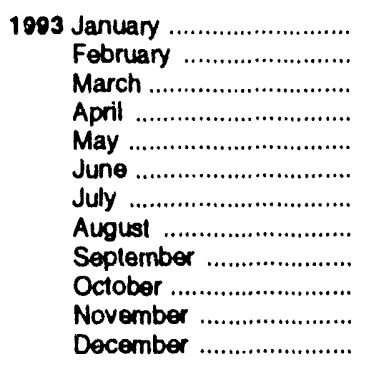 & $\begin{array}{l}108 \\
108 \\
108 \\
109 \\
109 \\
109 \\
109 \\
109 \\
109 \\
109 \\
109 \\
109\end{array}$ & $\begin{array}{l}0 \\
1 \\
1 \\
0 \\
0 \\
0 \\
0 \\
0 \\
0 \\
0 \\
0 \\
0\end{array}$ & $\begin{array}{l}8 \\
7 \\
7 \\
7 \\
7 \\
7 \\
7 \\
7 \\
7 \\
7 \\
7 \\
7\end{array}$ & $\begin{array}{l}0 \\
0 \\
0 \\
0 \\
0 \\
0 \\
0 \\
0 \\
0 \\
0 \\
0 \\
0\end{array}$ & $\begin{array}{l}0 \\
0 \\
0 \\
0 \\
0 \\
0 \\
0 \\
0 \\
0 \\
0 \\
0 \\
0\end{array}$ & $\begin{array}{l}0 \\
0 \\
0 \\
0 \\
0 \\
0 \\
0 \\
0 \\
0 \\
0 \\
0 \\
0\end{array}$ & $\begin{array}{l}116 \\
116 \\
116 \\
116 \\
116 \\
116 \\
116 \\
116 \\
116 \\
116 \\
116 \\
116\end{array}$ & $\begin{array}{l}110 \\
110 \\
110 \\
110 \\
110 \\
110 \\
110 \\
110 \\
110 \\
110 \\
110 \\
110\end{array}$ \\
\hline 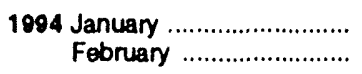 & $\begin{array}{l}109 \\
109\end{array}$ & $\begin{array}{l}0 \\
0\end{array}$ & $\begin{array}{l}6 \\
6\end{array}$ & $\begin{array}{l}0 \\
0\end{array}$ & $\begin{array}{l}0 \\
0\end{array}$ & $\begin{array}{l}0 \\
0\end{array}$ & $\begin{array}{l}115 \\
115\end{array}$ & $\begin{array}{l}108 \\
108\end{array}$ \\
\hline
\end{tabular}

- See Nole 1 at end of section.

bee Note 2 at end of section.

c Net design electrical rating (DER) is used because many of the units were canceled prior to being assigned a net summer capability. See Note 3 at end of section.

Note: Geographic coverage is the 50 States and the District of Columbia.

Sources: - Liceneed for Operation: 1073-1982-U.S. Department of Energy (DOE), Otfice of Nuclear Programs, "U.S. Central Station Nuclear Electric Generating Units: Significant Milestones." 1983 forward-Nuclear Regulatory Commission (NRC), "Licensed Operating Reactors" (NUREG-0020). - Construction Permits, On Order, and Announced: 1973-1982-Compiled from various sources, primarily DOE, Otfice of Nuclear Reactor Programs, "U.S. Central Station Nuclear Electric Generating Units: Significant Milestones"; Energy Information Administration (EIA), Olfice of Coal, Nuclear, Electric and Alternate Fuels (CNEAF), "Nuclear Sleam-Electric
Units That Have Been in Operation as of 1957-1989"; EIA, CNEAF, "Nuclear Plant Cancellations: Causes, Costs, and Consequences"; and Utility Data Institute, Inc., "U.S. Nuclear Plant Statistics, 1987." 1983 forward-NRC, "Summary Information Report" (NUREG-0871); NRC, "Licensed Operaling Reactors" (NUREG-0020); and various journals. - Total Design Capacity: 1973-1982-Compiled from various sources, primarily DOE, Otice of Nuclear Reactor Programs, "U.S. Central Station Nuclear Electric Generating Units: Signiticant Milestones"; EIA, CNEAF, "Nuclear Steam-Electric Units That Have Been in Operation as of 1957-:987": EIA, CNEAF, "Monthly Report for Electric Utilities-Power Generation": EIA, CNEAF, "Nuclear Plant Cancellations: Causes, Costs, and Consequences'; and Utility Data Instihus, Inc., "U.S. Nuclear Plant Statistics, 1987." 1983 forward-NRC, "Summary Inlormation Report" (NUREG-0871); NRC, "Licensed Operating Reactors" (NUREG-0020); and EIA, Form EIA-860, "Annual Electric Generator Report." 


\section{Nuclear Energy Notes}

1. Operable Units: Nuclear generating units that have been issued a full-power license by the Nuclear Regulatory Commission (NRC).

Exceptions: The Shippingport (60 megawatts (MW)) and the Hanford-N (840 MW) nuclear units were included in the operable units until 1982 and 1988 , respectively. The Shippingport unit was excluded from the operable category during March 1974-August 1977 due to a major core modification outage. Hanford-N, an unlicensed unit used for defense material production, was included in the operable category because power was producid as by-product and sold commercially. Three Mile 'sland 2 ( $880 \mathrm{MW}$ ) experienced a major accident in 1979 and, although that unit still retains its operating license and site cleanup continues, there is no plan to restart it. Therefore, it has not been included in the operable category since March 1979. Although Shoreham received a full-power license in April 1989, the unit is not curreistly scheduled to operate and, therefore, has not been included in the operable category. Rancho Seco $(873 \mathrm{MW})$ was shut down by the Sacramento Municipal Utility District (SMUD) in June 1989 following a referendum on its continued operation. Because there are currently no plans to operate it as a nuclear unit, it is no longer included as an operable unit but is identified as a unit shut down for an extended period. As soon as SMUD and the NRC formalize the plant's official retirement, it will be noted as such in this report. The Department of Energy-njerated Experimental Breeder Reactor 2 unit is not a commercial reactor and is therefore not included in the operable category.

In addition, nine units have been retired and therefore removed from the operable category. Those units are: Peach Bottom $1(40 \mathrm{MW})$ and Indian Point 1 (265 MW), both retired in 1974; Humboldt Bay (65 MW), officially retired in 1976; Dresden 1 (200 MW), retired in August 1979; LaCrosse (51 MW), retired in May 1987; Fort Saint Vrain (217 MW), retired in August 1989; Yankee Rowe 1 (185 MW), retired in February 1992; San Onofre 1 (436 MW), retired in December 1992; and Trojan (1,104 MW), retired in January 1993.

2. In Startup: The period of time between a nuclear generating unit's initial fuel loading date and the issuance of its full-power license. During that period, the unit is und rgoing low-power testing and the maximum level of operation is 5 percent of the unit's design thermal rating.

3. Capacity: Nuclear generating units may have more than one type of net capacity rating, including the following:

(a) Net Summer Capability-The steady hourly output that generating equipment is expected to supply to system load, exclusive of auxiliary power, as demonstrated by test at the time of summer peak demand. Auxiliary power of a typical nuclear power plant is about 5 percent of gross generation.

(b) Net Design Capacity or Net Design Electrical Rating (DER)-The nominal net electrical output of a unit, specified by the utility and used for plant design.

4. Monthly Capacity Factors: The monthly capacity factors are computed as the actual monthly generation divided by the maximum possible generation for that month. The maximum possible generation is the number of hours in the month multiplied by the net summer capability at the end of the month. That fraction is then multiplied by 100 to obtain a percentage. Annual capacity factors are averages of the monthly values for that year. 


\section{Section 9. Energy Prices}

Crude Oil. The average price of domestic crude oil purchased at the wellhead was $\$ 10.51$ per barrel in January 1994, 28 percent below the level in January 1993. The refiner acquisition cost of imported crude oil in January 1994 was \$12.97 per barrel, 23 percent below the January 1993 level. The average cost of domestic crude oil in January 1994 was \$12.72, 27 percent less than the January 1993 average.

Motor Gasoline. The national city average retail price of unleaded regular gasoline at all types of stations was $\$ 1.05$ per gallon in February 1994, 5 percent lower than the price in February 1993. The price of unleaded premium gasoline averaged $\$ 1.25$ per gallon in February 1994, 4 percent lower than the price in February 1993.

Residual Fuel Oil. The average price, excluding taxes, of residual fuel oil sold to end users in January 1994 was 33 cents per gallon, 11 percent higher than the previous month's price but 8 percent below the January 1993 average. The average resale price, excluding taxes, of residual fuel oil in January 1994 was 29 cents per gallon, 15 percent above the December 1993 average but 8 percent lower than the price 1 year earlier.

Aviation Fuel. The average price, excluding taxes, of aviation gasoline sold to end users in January 1994 was 89 cents per gallon, 3 percent lower than the previous month's price and 12 percent lower than the January 1993 price. The average price, excluding taxes, of kerosene-type jet fuel sold to end users in January 1994 was 52 cents per gallon, 1 percent higher than the previous month's average price but 12 percent lower than the January 1993 average price.

No. 2 Distillate Fuel Oil. The January 1994 national average price, excluding taxes, of heating oil sold to residential customers was 90 cents per gallon, 3 percent higher than the December 1993 price but 5 percent lower than the January 1993 price. The average price of No. 2 fuel oil sold to all end users was 60 cents per gallon in January 1994, 7 percent above the
December 1993 price but 5 percent lower than the January 1993 price.

Electricity. The average price of electricity sold to all ultimate consumers in the United States in February 1994 was 6.7 cents per kilowatthour, 2 percent above the February 1993 mean price. The price of electricity sold to residential consumers in February 1994 averaged 7.9 cents per kilowatthour, 1 percent above the February 1993 price. The price of electricity sold to commercial consumers averaged 7.5 cents per kilowatthour in February 1994, 1 percent above the February 1993 price. The price of electricity sold to other consumers was 6.7 cents per kilowatthour, 3 percent higher than the February 1993 price. The price of electricity sold to industrial users in February 1994 averaged 4.7 cents per kilowatthour, the same as the price 1 year earlier.

Beginning with January 1986, there were new series of national average price estimates based on a statistically derived sample of both publicly and privately owned electric utilities. Previously, average price estimates were derived from selected privately owned electric utilities and were not national averages.

Natural Gas. The estimated average wellhead price of natural gas for February 1994 was $\$ 2.24$ per thousand cubic feet, 29 percent above the February 1993 price.

The average price of natural gas delivered to electric utility plants was $\$ 2.67$ per thousand cubic feet in January 1994 (latest date for which data are available), 1 percent below the January 1993 price. The average price of natural gas used by residential consumers in February 1994 was $\$ 6.06$ per thousand cubic feet, 6 percent above the February 1993 price. The average price of natural gas used by commercial consumers in February 1994 was $\$ 5.54$ per thousand cubic feet, 9 percent higher than the February 1993 price. The average price of natural gas used by industrial consumers in February 1994 was $\$ 3.50$ per thousand cubic feet, 12 percent above the February 1993 price. 
Figure 9.1 Petroleum Prices

Crude Oil Prices, 1973-1993

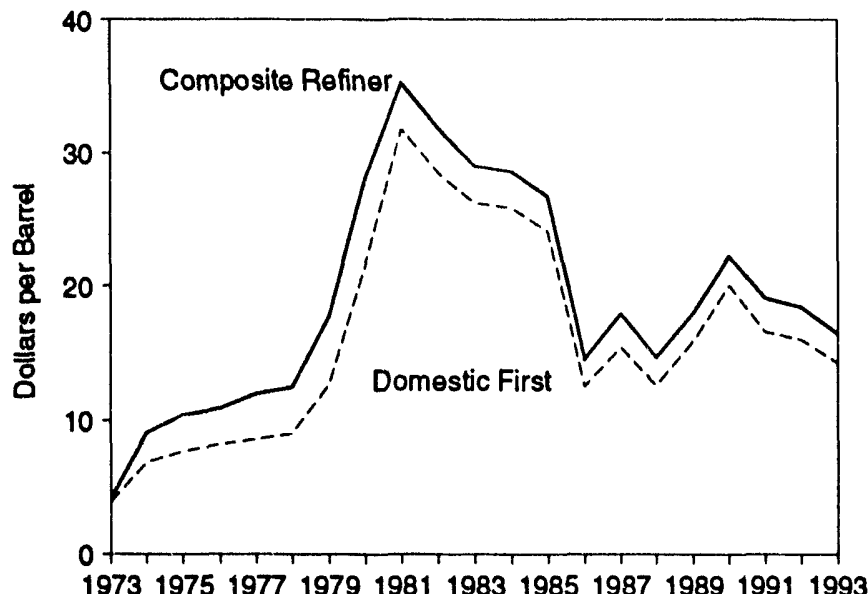

Composite Refiner Acquisition Cost, Monthly

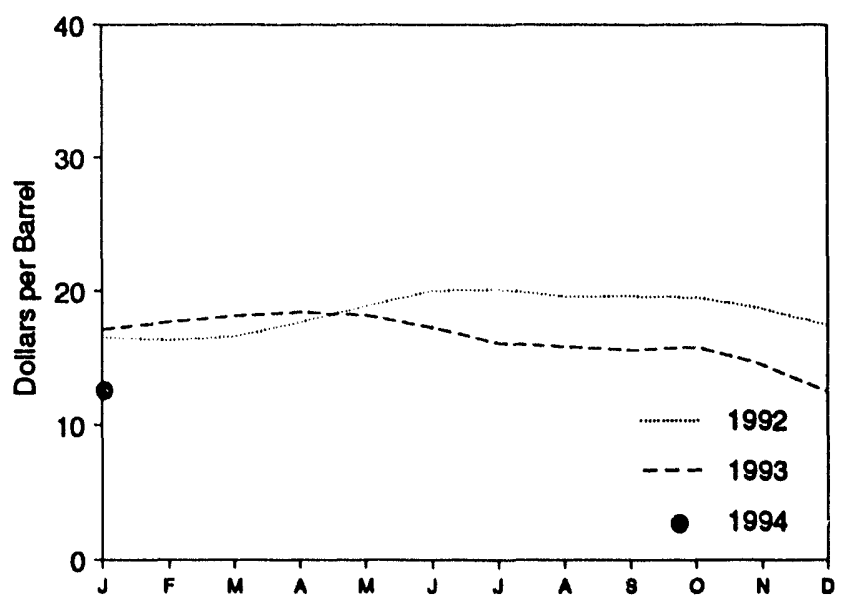

Refiner Prices to End Users: Motor Gasoline, Diesel Fuel, and Jet Fuel, Monthly

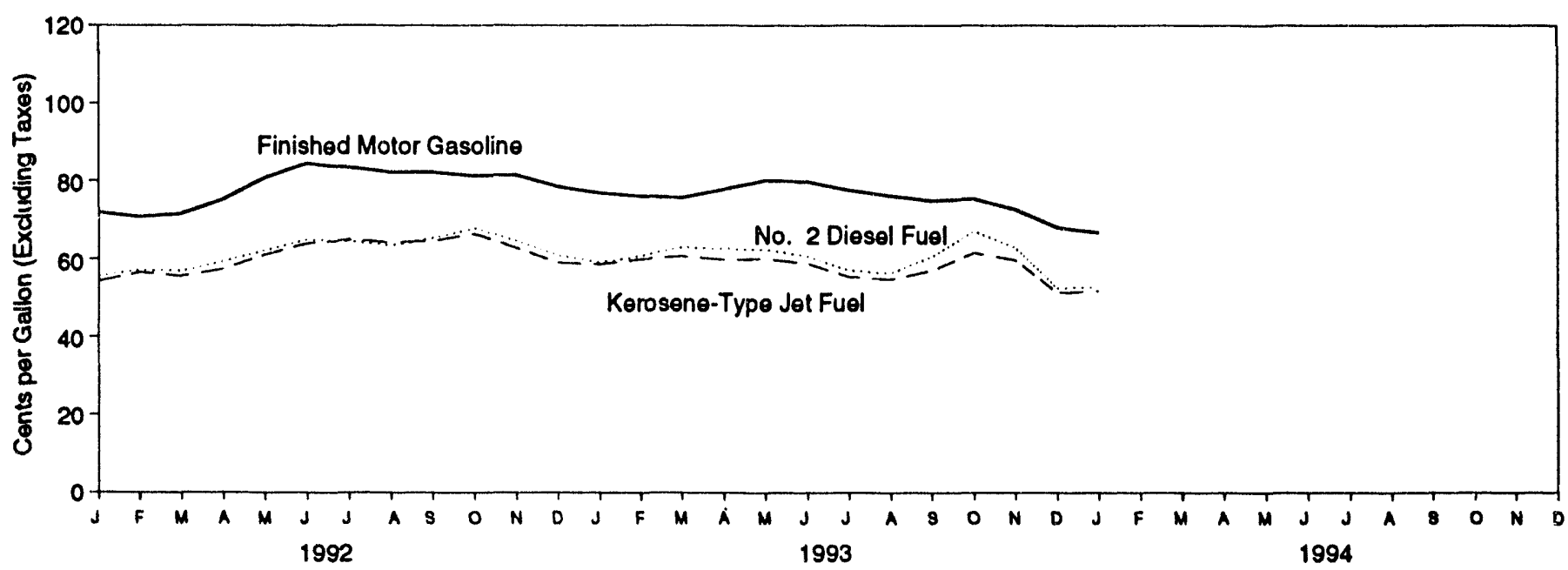

Refiner Prices to End Users: No. 2 Fuel Oil, Propane, and Residual Fuel, Monthly

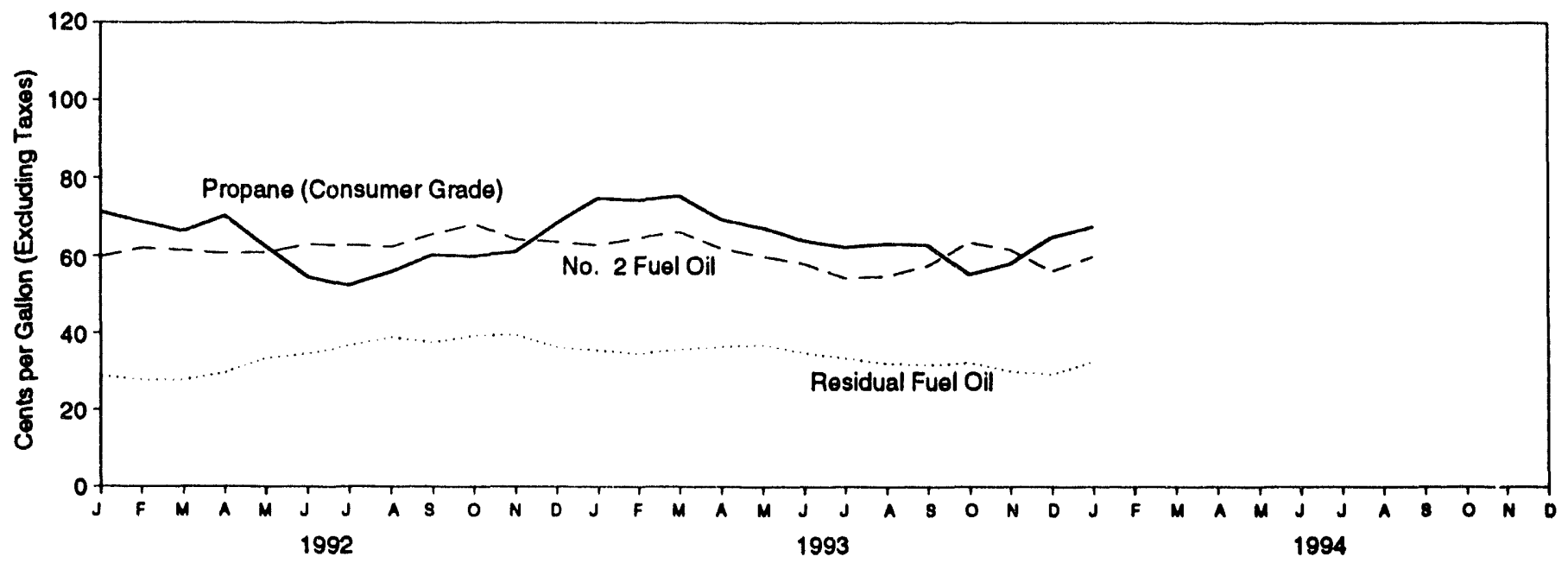

Sources: Tables 9.1, 9.5, and 9.7. 
Table 9.1 Crude Oil Price Summary

(Dollars per Barrel)

\begin{tabular}{|c|c|c|c|c|c|c|}
\hline & \multirow[b]{2}{*}{$\begin{array}{l}\text { Domestlc First } \\
\text { Purchase Priceb }\end{array}$} & \multirow[b]{2}{*}{$\begin{array}{l}\text { F.O.B. Cost } \\
\text { of Imports }\end{array}$} & \multirow[b]{2}{*}{$\begin{array}{l}\text { Landed Cost } \\
\text { of Imports }\end{array}$} & \multicolumn{3}{|c|}{ Refiner Acqulsition Costa } \\
\hline & & & & Domestic & Imported & Composite \\
\hline 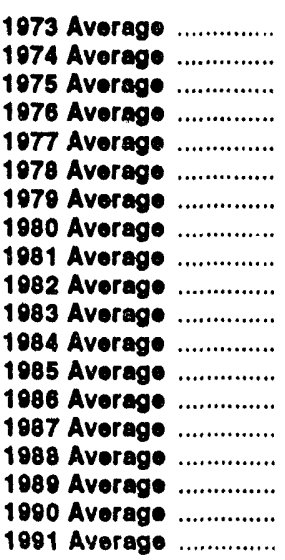 & $\begin{array}{r}3.89 \\
6.87 \\
7.67 \\
8.19 \\
8.57 \\
9.00 \\
12.64 \\
21.59 \\
31.77 \\
28.52 \\
26.19 \\
25.88 \\
24.09 \\
12.51 \\
15.40 \\
12.58 \\
15.86 \\
20.03 \\
16.54\end{array}$ & $\begin{array}{l}0.21 \\
10.81 \\
11.18 \\
12.15 \\
13.24 \\
13.29 \\
20.07 \\
32.37 \\
35.15 \\
32.02 \\
27.81 \\
27.60 \\
25.84 \\
12.52 \\
16.69 \\
13.25 \\
16.89 \\
20.37 \\
16.89\end{array}$ & $\begin{array}{l}0.41 \\
12.32 \\
12.70 \\
13.32 \\
14.36 \\
14.35 \\
21.45 \\
33.67 \\
36.47 \\
33.18 \\
28.93 \\
28.54 \\
26.67 \\
13.49 \\
17.65 \\
14.08 \\
17.68 \\
21.13 \\
18.02\end{array}$ & $\begin{array}{r}E_{4.17} \\
7.18 \\
8.39 \\
8.84 \\
8.55 \\
10.61 \\
14.27 \\
24.23 \\
34.33 \\
31.22 \\
28.87 \\
28.53 \\
26.66 \\
14.82 \\
17.76 \\
14.74 \\
17.87 \\
22.50 \\
19.33\end{array}$ & $\begin{array}{l}E_{4.08} \\
12.52 \\
13.93 \\
13.48 \\
14.53 \\
14.57 \\
21.67 \\
33.89 \\
37.05 \\
33.55 \\
29.30 \\
28.88 \\
26.99 \\
14.00 \\
18.13 \\
14.56 \\
18.08 \\
21.76 \\
18.70\end{array}$ & $\begin{array}{r}E_{4.15} \\
0.07 \\
10.38 \\
10.89 \\
11.08 \\
12.46 \\
17.72 \\
28.07 \\
35.24 \\
31.87 \\
28.09 \\
28.63 \\
26.75 \\
14.55 \\
17.90 \\
14.67 \\
17.07 \\
22.22 \\
19.06\end{array}$ \\
\hline 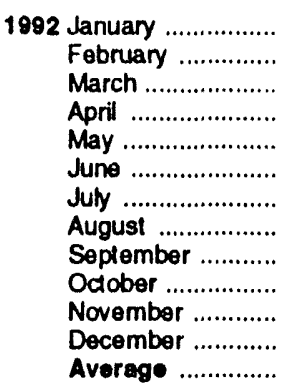 & $\begin{array}{l}13.99 \\
14.04 \\
14.12 \\
15.36 \\
16.38 \\
17.96 \\
17.80 \\
17.07 \\
17.20 \\
17.16 \\
16.00 \\
14.94 \\
15.99\end{array}$ & $\begin{array}{l}14.32 \\
14.68 \\
14.96 \\
16.57 \\
17.56 \\
18.38 \\
18.01 \\
17.65 \\
18.04 \\
17.68 \\
16.49 \\
15.62 \\
16.77\end{array}$ & $\begin{array}{l}15.28 \\
15.60 \\
16.00 \\
17.40 \\
18.38 \\
19.44 \\
19.13 \\
18.74 \\
18.90 \\
18.75 \\
17.64 \\
16.58 \\
17.75\end{array}$ & $\begin{array}{l}16.80 \\
16.54 \\
16.71 \\
17.88 \\
18.86 \\
20.13 \\
20.42 \\
19.84 \\
19.88 \\
19.64 \\
18.90 \\
17.85 \\
18.63\end{array}$ & $\begin{array}{l}16.10 \\
16.00 \\
16.36 \\
17.37 \\
18.79 \\
19.83 \\
19.74 \\
19.25 \\
19.26 \\
19.34 \\
18.40 \\
16.94 \\
18.20\end{array}$ & $\begin{array}{l}16.50 \\
16.30 \\
16.56 \\
17.66 \\
18.83 \\
19.99 \\
20.10 \\
19.56 \\
19.59 \\
19.49 \\
18.66 \\
17.43 \\
18.43\end{array}$ \\
\hline 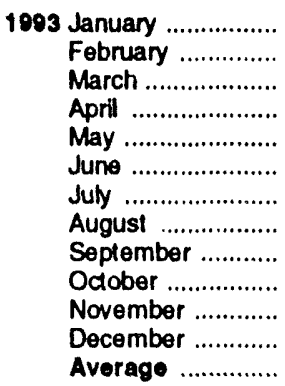 & $\begin{array}{r}14.64 \\
15.47 \\
15.88 \\
16.08 \\
15.97 \\
15.00 \\
13.78 \\
13.69 \\
13.39 \\
13.87 \\
12.65 \\
10.38 \\
14.23\end{array}$ & $\begin{array}{r}15.24 \\
16.09 \\
16.61 \\
16.39 \\
16.27 \\
15.12 \\
14.23 \\
14.21 \\
14.19 \\
14.21 \\
12.87 \\
A_{11.65} \\
\text { P } 14.75\end{array}$ & $\begin{array}{l}16.34 \\
17.12 \\
17.56 \\
17.58 \\
17.35 \\
16.31 \\
15.44 \\
15.26 \\
15.00 \\
15.07 \\
R_{13.79} \\
R_{12.29} \\
A_{15.73}\end{array}$ & $\begin{array}{l}17.40 \\
17.84 \\
18.31 \\
18.49 \\
18.43 \\
17.70 \\
16.36 \\
16.03 \\
15.82 \\
16.04 \\
14.99 \\
12.45 \\
16.66\end{array}$ & $\begin{array}{l}16.78 \\
17.41 \\
17.82 \\
18.35 \\
17.89 \\
16.80 \\
15.82 \\
15.62 \\
15.32 \\
15.59 \\
14.05 \\
12.56 \\
16.14\end{array}$ & $\begin{array}{l}17.10 \\
17.64 \\
18.08 \\
18.42 \\
18.16 \\
17.26 \\
16.10 \\
15.84 \\
15.59 \\
15.81 \\
14.51 \\
12.51 \\
16.41\end{array}$ \\
\hline 1994 January ................ & 10.51 & 12.01 & 12.61 & 12.72 & 12.97 & 12.85 \\
\hline
\end{tabular}

a See Note 4 at end of section.

b Seo Note 1 at end of section.

c See Note 2 at end of section.

d See Note 3 at end of section.

- Based on October, November, and December data only.

$R=$ Revised data. $E=$ Estimate.

Notes: - Geographic coverage is the 50 States, the District of Columbia,

Puento Rico, the Virgin Islands, and all U.S. Territories and Possessions.

- Values tor Domestic First Purchase Price and Refiner Acquisition Cost for

the current month and for F.O.B. and Landed Costs of Imports for the current

2 months are preliminary. - F.O.B. and landed costs through 1980 reflect the period of reporting; prices after 1980 reflect the period of loading Annual averages are the averages of the monthly prices, weighted by volume.

Sources: - Domestic FIrst Purchase Price: 1973-1976-U.S.

Department of the Interior (DOI), Bureau of Mines (BOM). Minerals Yearbook,

"Crude Petroleum and Petroleum Products" chapter. 1877-Federal Energy

Administration (FEA), based on Form FEA.P124, "Domestic Crude Oil
Purchaser's Monthly Report. 1978 torward-Energy Informalion Administration (ElA), Petroleum Marketing Monthly, April 1994, Table 1. - F.O.B. and Landed Cost of Imports: October 1973-September 1977-FEA, Form FEA-F701-M-0. "Transfer Pricing Report." OctoberDecember 1977-EIA, Form FEA-F701-M-0, "Transfer Pricing Report." 1078 forward-ElA, Petroleum Marketing Monthly, April 1994, Table 1. - Refiner Acquisition Cost: 1873-ElA estimates. The domestic price was derived by adding estimated transportation costs to the reported domestic first purchase price. The imported price was derived by adding an estimated ocean transport cost to the average "Free Alongside Ship" value published by the U.S. Bureau of the Census. 1974-1976-DOI, BOM, Minera/s Yearbook "Crude Petroleum and Petroleum Products" chapter. 1977-JanuarySeptember-FEA, based on Form FEA-P110-M-1, 'Refiners' Monthly Cost Allocation Report." October-December-ELA, based on Form FEA.P110-M-1, "Retiners' Monthly Cost Allocation Report." 1078 forward-EIA, Petroleum Marketing Monthly, April 1994, Table 1.

Data required to update this table were not available in time for publication. 
Table 9.2 F.O.B. Costs of Crude Oil Imports from Selected Countries

(Dollars per Barrel)

\begin{tabular}{|c|c|c|c|c|c|c|c|c|c|c|c|}
\hline & Algeria & Indonesle & Iran & Mexico & Nlgerle & $\begin{array}{l}\text { Saudl } \\
\text { Arabla }\end{array}$ & $\begin{array}{l}\text { United } \\
\text { KIngdom }\end{array}$ & Venozuola & $\begin{array}{c}\text { Other } \\
\text { Countrles }\end{array}$ & $\begin{array}{c}\text { Arab } \\
\text { OPECa }\end{array}$ & $\begin{array}{c}\text { Total } \\
\text { OPECb }\end{array}$ \\
\hline $\begin{array}{l}1073 \text { Averagec ..... } \\
1074 \text { Average ...... } \\
1078 \text { Average ...... } \\
1076 \text { Average ..... } \\
1077 \text { Average ...... } \\
1078 \text { Average ...... } \\
1070 \text { Average ...... } \\
1080 \text { Average ...... } \\
1081 \text { Average ...... } \\
1982 \text { Average ...... } \\
1083 \text { Average ...... } \\
1084 \text { Average ...... } \\
1985 \text { Average ...... } \\
1088 \text { Average ...... } \\
1987 \text { Average ...... } \\
1088 \text { Average ...... } \\
1980 \text { Average ...... } \\
1980 \text { Average ...... } \\
1091 \text { Average ...... }\end{array}$ & $\begin{array}{r}7.23 \\
13.23 \\
11.03 \\
13.05 \\
14.35 \\
14.12 \\
20.53 \\
36.67 \\
39.08 \\
34.20 \\
30.09 \\
28.34 \\
26.80 \\
13.82 \\
18.70 \\
W \\
W \\
W \\
W\end{array}$ & $\begin{array}{r}5.87 \\
11.09 \\
12.55 \\
12.76 \\
13.57 \\
13.61 \\
19.03 \\
32.17 \\
35.62 \\
35.11 \\
29.02 \\
29.13 \\
27.12 \\
13.10 \\
17.40 \\
13.81 \\
17.01 \\
21.29 \\
18.60\end{array}$ & $\begin{array}{l}4.24 \\
10.85 \\
10.81 \\
11.61 \\
12.68 \\
12.65 \\
22.93 \\
N A \\
(d) \\
30.07 \\
28.30 \\
27.42 \\
W \\
W \\
W \\
\text { d) } \\
\text { dj) } \\
(d) \\
15.58\end{array}$ & $\begin{array}{l}N A \\
W \\
11.44 \\
12.22 \\
13.42 \\
13.24 \\
20.27 \\
31.06 \\
33.01 \\
28.08 \\
25.20 \\
26.30 \\
25.33 \\
11.84 \\
16.36 \\
12.18 \\
15.96 \\
19.26 \\
15.37\end{array}$ & $\begin{array}{r}7.81 \\
12.44 \\
11.82 \\
13.08 \\
14.44 \\
14.05 \\
21.69 \\
35.93 \\
38.31 \\
35.13 \\
29.81 \\
29.51 \\
28.04 \\
14.35 \\
18.47 \\
15.16 \\
18.31 \\
22.48 \\
20.29\end{array}$ & $\begin{array}{r}3.25 \\
10.17 \\
10.87 \\
11.62 \\
12.38 \\
12.70 \\
17.28 \\
28.17 \\
32.60 \\
33.73 \\
27.53 \\
27.67 \\
22.04 \\
11.36 \\
15.12 \\
12.16 \\
16.20 \\
20.36 \\
14.62\end{array}$ & $\begin{array}{c}N A \\
N A \\
N A \\
W \\
14.11 \\
13.82 \\
21.70 \\
34.36 \\
36.06 \\
33.42 \\
29.01 \\
28.87 \\
27.64 \\
13.84 \\
18.28 \\
14.80 \\
17.89 \\
23.43 \\
20.81\end{array}$ & $\begin{array}{r}6.39 \\
10.71 \\
11.04 \\
11.30 \\
12.63 \\
12.38 \\
16.90 \\
24.81 \\
28.95 \\
23.74 \\
21.48 \\
24.23 \\
23.64 \\
10.02 \\
15.08 \\
12.06 \\
16.09 \\
10.55 \\
14.01\end{array}$ & $\begin{array}{r}4.84 \\
10.02 \\
10.86 \\
11.02 \\
13.19 \\
13.35 \\
21.10 \\
34.34 \\
36.69 \\
31.06 \\
27.96 \\
27.79 \\
26.12 \\
13.32 \\
17.11 \\
13.45 \\
17.12 \\
19.88 \\
17.79\end{array}$ & $\begin{array}{r}4.08 \\
10.98 \\
11.18 \\
12.08 \\
13.13 \\
13.28 \\
10.27 \\
31.57 \\
34.70 \\
33.84 \\
28.28 \\
27.70 \\
24.34 \\
11.50 \\
15.80 \\
12.57 \\
16.72 \\
18.84 \\
15.50\end{array}$ & $\begin{array}{r}6.43 \\
11.33 \\
11.34 \\
12.24 \\
13.20 \\
13.31 \\
19.88 \\
32.21 \\
35.17 \\
33.48 \\
28.46 \\
27.79 \\
25.67 \\
12.21 \\
16.43 \\
13.43 \\
17.06 \\
20.40 \\
16.09\end{array}$ \\
\hline $\begin{array}{l}1992 \text { January ........ } \\
\text { February ...... } \\
\text { March ........... } \\
\text { April ............. } \\
\text { May .............. } \\
\text { June ............. } \\
\text { July .............. } \\
\text { August ........ } \\
\text { Seplember ... } \\
\text { October ........ } \\
\text { November .... } \\
\text { December .... } \\
\text { Average ...... }\end{array}$ & 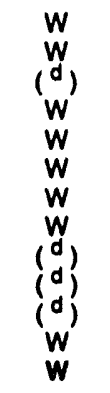 & $\begin{array}{l}W \\
W \\
W \\
16.23 \\
W \\
W \\
W \\
W \\
W \\
W \\
W \\
W \\
17.06\end{array}$ & (d) & $\begin{array}{l}12.45 \\
12.40 \\
12.68 \\
14.11 \\
16.05 \\
17.09 \\
16.88 \\
16.36 \\
16.88 \\
16.90 \\
15.78 \\
14.79 \\
15.26\end{array}$ & $\begin{array}{l}18.58 \\
18.28 \\
18.10 \\
19.59 \\
20.47 \\
21.42 \\
20.83 \\
20.33 \\
20.84 \\
20.76 \\
20.00 \\
18.42 \\
19.98\end{array}$ & $\begin{array}{c}W \\
14.61 \\
14.87 \\
W \\
17.61 \\
W \\
17.60 \\
W \\
16.69 \\
W \\
14.62 \\
15.62 \\
15.85\end{array}$ & 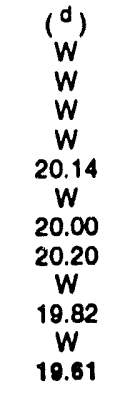 & $\begin{array}{l}12.32 \\
12.53 \\
12.45 \\
14.38 \\
15.03 \\
15.33 \\
15.10 \\
15.38 \\
16.21 \\
15.40 \\
13.82 \\
13.38 \\
14.39\end{array}$ & $\begin{array}{l}15.44 \\
16.04 \\
16.01 \\
17.10 \\
18.35 \\
19.20 \\
18.74 \\
18.43 \\
18.65 \\
18.70 \\
17.57 \\
16.13 \\
17.65\end{array}$ & $\begin{array}{l}14.07 \\
15.35 \\
15.20 \\
17.26 \\
18.13 \\
17.95 \\
18.20 \\
17.99 \\
17.11 \\
15.89 \\
15.12 \\
15.91 \\
16.50\end{array}$ & $\begin{array}{l}14.50 \\
15.04 \\
15.28 \\
17.25 \\
17.83 \\
18.44 \\
18.09 \\
17.69 \\
18.01 \\
17.42 \\
15.87 \\
15.60 \\
18.87\end{array}$ \\
\hline $\begin{array}{l}1993 \text { January ........ } \\
\text { February ....... } \\
\text { March .......... } \\
\text { April ............ } \\
\text { May ............... } \\
\text { June ............ } \\
\text { July .............. } \\
\text { August ......... } \\
\text { September .... } \\
\text { October ........ } \\
\text { November .... } \\
\text { December .... } \\
\text { Average ...... }\end{array}$ & $\begin{array}{l}w \\
(d) \\
w \\
w \\
w \\
w \\
w\end{array}$ & $\begin{array}{c}W \\
W \\
W \\
W \\
19.14 \\
W \\
16.48 \\
17.74 \\
W \\
W \\
W \\
W \\
\text { ค 17.16 }\end{array}$ & (d) & $\begin{array}{l}14.14 \\
14.64 \\
15.17 \\
15.04 \\
15.15 \\
14.06 \\
13.09 \\
13.20 \\
13.50 \\
13.76 \\
12.24 \\
A_{11.19} \\
\text { A }_{13.74}\end{array}$ & $\begin{array}{l}17.95 \\
19.06 \\
19.33 \\
19.19 \\
18.92 \\
18.01 \\
17.46 \\
17.42 \\
16.72 \\
17.02 \\
15.80 \\
14.21 \\
17.78\end{array}$ & $\begin{array}{c}15.55 \\
16.17 \\
16.45 \\
16.03 \\
14.54 \\
W \\
W \\
W \\
W \\
12.88 \\
10.58 \\
W \\
P_{14.27}\end{array}$ & $\begin{array}{l}18.29 \\
18.13 \\
18.51 \\
18.36 \\
18.29 \\
17.15 \\
16.07 \\
16.73 \\
16.06 \\
16.31 \\
15.29 \\
14.33 \\
16.62\end{array}$ & $\begin{array}{r}12.99 \\
13.68 \\
14.22 \\
14.52 \\
13.89 \\
12.47 \\
11.96 \\
12.56 \\
12.72 \\
11.87 \\
9.97 \\
P_{9.34} \\
{ }_{12.46}\end{array}$ & $\begin{array}{r}15.17 \\
16.51 \\
16.85 \\
16.90 \\
16.73 \\
15.89 \\
14.96 \\
14.68 \\
14.29 \\
14.88 \\
R_{13.87} \\
A_{11.84} \\
R_{15.20}\end{array}$ & $\begin{array}{r}15.60 \\
16.39 \\
16.83 \\
16.24 \\
15.03 \\
14.29 \\
13.56 \\
14.40 \\
13.97 \\
14.03 \\
11.87 \\
R_{11.32} \\
A_{14.62}\end{array}$ & $\begin{array}{r}15.62 \\
16.49 \\
16.92 \\
16.59 \\
16.32 \\
14.94 \\
14.18 \\
14.24 \\
14.37 \\
13.94 \\
12.37 \\
R_{11.40} \\
A_{14.84}\end{array}$ \\
\hline 1904 January ......... & $w$ & $w$ & (d) & 11.29 & 14.88 & 10.59 & W & 10.86 & 12.11 & 11.12 & 12.34 \\
\hline
\end{tabular}

- The Arab members of OPEC are Algeria, Iraq, Kuwah, Libya, Qalar, Saudi Arabia, and the United Arab Emirates.

b Current members of OPEC are Gabon, Indonesia, Iran, Nigeria, and Venezuela, as well as the Arab members. Prior to 1993, Ecuador was also a member. The cost of imports from the Neutral Zone between Kuwait and Saudi Arabia is included in the cost of imports trom "Total OPEC."

Cased on October, November, and December dala only.

d No data reported.

$R=$ Pevised data. $N A=N o t$ available. $W=$ Value withheld to avoid disclosure of individual company data.

Notes: - The Free on Board (F.O.B.) cost at the country of origin excludes all costs related to insurance and transportation. See Note 2 at end of section. - Values for the current 2 months are preliminary. - Prices through
1980 rellect the period of reponing; prices after 1980 reflect the period of loading. - Annual averages are averages of the monthly prices, including prices not published, weighted by volume. - Cargoes that are purchased on a "netback" basis, or under similar contractual arrangements whereby the actual purchase price is not established at the time the crude oll is acquired for importation into the United States, are not included in the published data until the actual prices have been determined and reported.

Sources: - October 1973-Soptember 1977: Federal Energy Administration, Form FEA-F701-M-0, "Transfer Pricing Report." - October 1977-Docombor 1977: Energy Information Administration (EIA), Form FEA-F701-M-0, "Transfer Pricing Report." - 1978 forward: EIA, Petroloum Marketing Monthly, April 1994, Table 21.

Data required to update this table were not available in time for publication. 
(Dollars per Barrel)

\begin{tabular}{|c|c|c|c|c|c|c|c|c|c|c|c|c|}
\hline & Algorla & Canada & Indonosla & Iran & Moxico & Nigoria & $\begin{array}{l}\text { Saudl } \\
\text { Arabla }\end{array}$ & $\begin{array}{c}\text { Unlted } \\
\text { Kingdom }\end{array}$ & Venezuela & $\begin{array}{l}\text { Other } \\
\text { Countries }\end{array}$ & $\begin{array}{c}\text { Arab } \\
\text { OPEC }\end{array}$ & $\begin{array}{l}\text { Total } \\
\text { OPECb }\end{array}$ \\
\hline 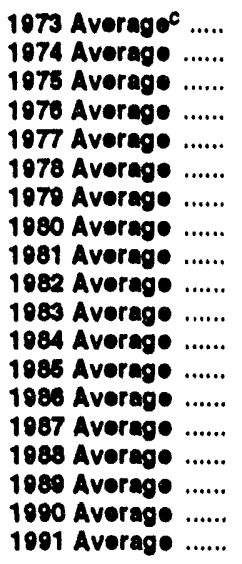 & $\begin{array}{c}8.30 \\
13.07 \\
12.86 \\
13.00 \\
15.24 \\
14.03 \\
21.88 \\
37.92 \\
40.46 \\
35.35 \\
31.26 \\
29.06 \\
27.51 \\
14.82 \\
17.87 \\
W \\
10.13 \\
W \\
W\end{array}$ & $\begin{array}{r}5.33 \\
11.46 \\
12.84 \\
13.36 \\
14.13 \\
14.41 \\
20.22 \\
30.11 \\
32.32 \\
27.15 \\
25.63 \\
26.56 \\
25.71 \\
13.43 \\
17.04 \\
13.50 \\
16.81 \\
20.48 \\
17.16\end{array}$ & $\begin{array}{r}7.22 \\
13.20 \\
13.83 \\
13.85 \\
14.65 \\
14.65 \\
20.63 \\
33.92 \\
37.31 \\
36.70 \\
31.67 \\
30.87 \\
28.67 \\
14.63 \\
18.40 \\
15.15 \\
18.35 \\
22.50 \\
20.20\end{array}$ & $\begin{array}{l}6.48 \\
12.48 \\
12.51 \\
12.86 \\
13.86 \\
13.80 \\
24.21 \\
N A \\
(d) \\
32.46 \\
29.81 \\
28.70 \\
25.70 \\
12.38 \\
18.28 \\
W \\
\text { d d } \\
\text { (d) } \\
17.54\end{array}$ & $\begin{array}{l}\text { NA } \\
W \\
12.61 \\
12.64 \\
13.82 \\
13.56 \\
20.77 \\
31.77 \\
33.70 \\
28.63 \\
25.78 \\
26.85 \\
25.63 \\
12.17 \\
16.60 \\
12.58 \\
16.35 \\
10.64 \\
15.80\end{array}$ & \begin{tabular}{r|}
9.08 \\
13.16 \\
12.70 \\
13.81 \\
15.20 \\
14.88 \\
22.97 \\
37.15 \\
39.86 \\
36.16 \\
30.85 \\
30.36 \\
28.96 \\
15.29 \\
10.32 \\
15.88 \\
19.19 \\
23.33 \\
21.30
\end{tabular} & \begin{tabular}{r|}
5.37 \\
11.63 \\
12.50 \\
13.06 \\
13.60 \\
13.94 \\
18.05 \\
29.80 \\
34.20 \\
34.09 \\
29.27 \\
29.20 \\
24.72 \\
12.84 \\
16.81 \\
13.37 \\
17.34 \\
21.82 \\
17.22 \\
\end{tabular} & $\begin{array}{l}\text { NA } \\
N A \\
N A \\
W \\
W \\
14.83 \\
14.53 \\
22.97 \\
35.68 \\
37.29 \\
34.25 \\
30.87 \\
29.45 \\
28.36 \\
14.63 \\
18.78 \\
15.82 \\
18.74 \\
22.65 \\
21.37\end{array}$ & $\begin{array}{r}8.90 \\
11.35 \\
12.36 \\
11.89 \\
13.11 \\
12.84 \\
17.85 \\
25.82 \\
29.91 \\
24.93 \\
22.94 \\
25.10 \\
24.43 \\
11.52 \\
15.76 \\
13.86 \\
16.78 \\
20.31 \\
15.92\end{array}$ & $\begin{array}{r}6.00 \\
12.03 \\
12.66 \\
13.30 \\
14.56 \\
14.58 \\
22.86 \\
38.15 \\
38.54 \\
34.03 \\
20.66 \\
29.21 \\
27.33 \\
14.25 \\
18.30 \\
14.45 \\
18.08 \\
20.52 \\
10.73\end{array}$ & $\begin{array}{r}5.02 \\
12.30 \\
12.71 \\
13.31 \\
14.30 \\
14.36 \\
20.70 \\
32.97 \\
36.22 \\
36.16 \\
29.87 \\
20.10 \\
25.90 \\
13.14 \\
17.32 \\
13.80 \\
17.41 \\
20.84 \\
17.45\end{array}$ & $\begin{array}{r}\mathbf{6 . 8 6} \\
12.40 \\
12.70 \\
13.32 \\
14.36 \\
14.34 \\
21.20 \\
33.86 \\
36.80 \\
34.81 \\
20.84 \\
20.06 \\
26.86 \\
13.46 \\
17.04 \\
14.16 \\
17.70 \\
21.23 \\
18.06\end{array}$ \\
\hline $\begin{array}{l}1003 \text { January ........ } \\
\text { February ...... } \\
\text { March ........... } \\
\text { April ............. } \\
\text { May ............. } \\
\text { June ............ } \\
\text { July ............. } \\
\text { August ........ } \\
\text { Seplember ... } \\
\text { October ........ } \\
\text { November .... } \\
\text { December .... } \\
\text { Average ...... }\end{array}$ & 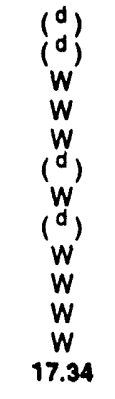 & $\begin{array}{l}15.27 \\
15.84 \\
16.48 \\
16.79 \\
16.82 \\
16.25 \\
15.30 \\
14.94 \\
14.56 \\
15.14 \\
14.28 \\
12.44 \\
15.27\end{array}$ & $\begin{array}{c}W \\
W \\
W \\
19.89 \\
20.57 \\
W \\
17.86 \\
19.28 \\
W \\
W \\
W \\
W \\
A_{15.72} \\
A_{18.47}\end{array}$ & $\begin{array}{l}\left(\begin{array}{l}d \\
d\end{array}\right) \\
\left(\begin{array}{l}d \\
d\end{array}\right) \\
\left(\begin{array}{l}d \\
d\end{array}\right) \\
\left(\begin{array}{l}d \\
d\end{array}\right. \\
\left(\begin{array}{l}d \\
d\end{array}\right. \\
\left(\begin{array}{l}d \\
d\end{array}\right) \\
\left(\begin{array}{l}d \\
d\end{array}\right) \\
\left(\begin{array}{l}d \\
\text { d }\end{array}\right.\end{array}$ & $\begin{array}{r}14.50 \\
14.98 \\
15.50 \\
15.55 \\
15.57 \\
14.50 \\
13.44 \\
13.66 \\
13.81 \\
14.11 \\
12.60 \\
A_{11.39} \\
\text { म }_{14.10}\end{array}$ & \begin{tabular}{|l|}
18.96 \\
19.92 \\
20.25 \\
20.18 \\
19.79 \\
18.93 \\
18.31 \\
18.08 \\
17.62 \\
17.96 \\
16.70 \\
15.08 \\
18.72
\end{tabular} & $\begin{array}{r}16.36 \\
17.29 \\
17.56 \\
17.56 \\
16.64 \\
15.72 \\
14.94 \\
15.11 \\
14.62 \\
14.46 \\
\text { ค }_{12.89} \\
\text { ค } 11.61 \\
\text { ค } 15.43\end{array}$ & $\begin{array}{l}19.12 \\
19.28 \\
19.43 \\
19.32 \\
19.33 \\
18.67 \\
17.51 \\
17.56 \\
17.04 \\
16.67 \\
16.57 \\
R 15.16 \\
\text { R } 17.91\end{array}$ & $\begin{array}{l}14.07 \\
14.60 \\
15.14 \\
15.54 \\
14.91 \\
13.53 \\
12.92 \\
13.32 \\
13.46 \\
12.70 \\
10.81 \\
\text { P } 10.14 \\
\text { P } 13.39\end{array}$ & \begin{tabular}{|l|}
17.21 \\
18.17 \\
18.43 \\
18.48 \\
18.41 \\
17.44 \\
16.44 \\
16.01 \\
15.56 \\
15.71 \\
$R_{14.71}$ \\
$R_{12.71}$ \\
$R_{16.45}$
\end{tabular} & $\begin{array}{l}16.39 \\
17.29 \\
17.63 \\
17.55 \\
16.79 \\
15.86 \\
14.96 \\
15.11 \\
14.56 \\
14.60 \\
R_{13.03} \\
A_{11.53} \\
\text { A }_{15.31}\end{array}$ & $\begin{array}{r}16.64 \\
17.43 \\
17.83 \\
17.77 \\
17.30 \\
16.03 \\
15.30 \\
15.24 \\
14.96 \\
14.81 \\
A_{13.25} \\
\text { P }_{11.97} \\
\text { A }_{15.00}\end{array}$ \\
\hline 1904 January ......... & $w$ & 12.00 & $w$ & (d) & 11.64 & 15.57 & 11.57 & 14.98 & 11.73 & 13.34 & 11.78 & 12.78 \\
\hline
\end{tabular}

- The Arab members of OPEC are Algeria, Iraq, Kuwall, Libya, Qatar, Saudi Arabia, and the United Arab Emirates.

b Current members of OPEC are Gabon, Indonesia, Iran, Nigeria, and Venezuela, as well as the Arab members. Prior to 1993, Ecuador was also a member. The cost of imports from the Neutral Zone between Kuwait and Saudi Arabia is included in the cost of imports from "Total OPEC."

c Based on October, November, and December data only.

No data reported.

$R=$ Revised data. NA=Not available. $W=$ Value witheld to avoid disclosure of individual company data.

Noles: - See Note 3 at end of section. - Values for the current 2 months are preliminary. - Prices through 1980 relled the period of reporting; prices since then reflect the period of loading. - Annual averages are averages of the monthly prices, including prices not published, welghted by volume. - Cargoes that are purchased on a "netback' basis, or under similar contractual arrangements whereby the actual purchase price is not established at the time the crude oil is acquired for importation into the United Siates, are not included in the published data until the actual prices have been determined and reportes.

Sources: - October 1973-September 1977: Federal Energy Administration, Form FEA-F701-M-0. "Transler Pricing Report." - October 1977.December 1977: Energy Inlormation Adminisiration (EIA). Form FEA-F701-M-0, "Transler Pricing Report." 1978 forward: EIA, Petroloum Marketing Monthly, April 1994, Table 22.

Data required to update this table were not available in time for publication. 
Table 9.4 Motor Gasoline Retail Prices, U.S. City Average

(Cents per Gallon, Including Taxes)

\begin{tabular}{|c|c|c|c|c|}
\hline & $\begin{array}{l}\text { Leaded } \\
\text { Regular }\end{array}$ & $\begin{array}{l}\text { Unleaded } \\
\text { Regular }\end{array}$ & $\begin{array}{l}\text { Unleaded } \\
\text { Premium }\end{array}$ & All Typesa \\
\hline $\begin{array}{l}1073 \text { Average } \\
1974 \text { Average } \\
1975 \text { Average } \\
1976 \text { Average } \ldots \ldots \ldots \ldots \ldots \ldots \\
1977 \text { Average } \ldots \ldots \ldots \ldots \ldots \ldots \\
1978 \text { Average } \\
1979 \text { Average } \\
1980 \text { Average } \\
1981 \text { Averege } \\
1982 \text { Average }\end{array}$ & \begin{tabular}{r|}
38.8 \\
53.2 \\
56.7 \\
59.0 \\
62.2 \\
62.6 \\
85.7 \\
119.1 \\
131.1 \\
122.2 \\
115.7 \\
112.9 \\
111.5 \\
85.7 \\
89.7 \\
89.9 \\
99.8 \\
114.9 \\
NA
\end{tabular} & $\begin{array}{r}\text { NA } \\
\text { NA } \\
\text { NA } \\
61.4 \\
65.6 \\
67.0 \\
80.3 \\
124.5 \\
137.8 \\
129.6 \\
124.1 \\
121.2 \\
120.2 \\
92.7 \\
94.8 \\
94.6 \\
102.1 \\
116.4 \\
114.0\end{array}$ & $\begin{array}{r}\text { NA } \\
\text { NA } \\
\text { NA } \\
\text { NA } \\
\text { NA } \\
\text { NA } \\
\text { NA } \\
\text { NA } \\
\text { c } 147.0 \\
141.5 \\
138.3 \\
136.6 \\
134.0 \\
108.5 \\
109.3 \\
110.7 \\
119.7 \\
134.9 \\
132.1\end{array}$ & $\begin{array}{r}\text { NA } \\
\text { NA } \\
\text { NA } \\
\text { NA } \\
\text { NA } \\
65.2 \\
88.2 \\
122.1 \\
135.3 \\
128.1 \\
122.5 \\
110.8 \\
119.6 \\
93.1 \\
95.7 \\
96.3 \\
108.0 \\
121.7 \\
110.6\end{array}$ \\
\hline 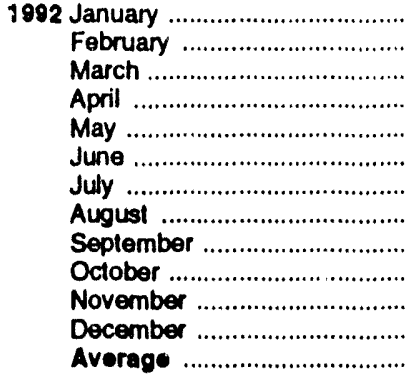 & $\begin{array}{l}\text { NA } \\
\text { NA } \\
\text { NA } \\
\text { NA } \\
\text { NA } \\
\text { NA } \\
\text { NA } \\
\text { NA } \\
\text { NA } \\
\text { NA } \\
\text { NA } \\
\text { NA } \\
\text { NA }\end{array}$ & $\begin{array}{l}107.3 \\
105.4 \\
105.8 \\
107.9 \\
113.6 \\
117.9 \\
117.5 \\
115.8 \\
115.8 \\
115.4 \\
115.9 \\
113.6 \\
112.7\end{array}$ & $\begin{array}{l}126.7 \\
124.8 \\
125.0 \\
126.8 \\
131.7 \\
135.9 \\
136.3 \\
134.8 \\
134.6 \\
134.5 \\
135.1 \\
133.0 \\
131.6\end{array}$ & $\begin{array}{l}113.5 \\
111.7 \\
112.2 \\
114.3 \\
119.7 \\
123.8 \\
123.8 \\
122.1 \\
122.2 \\
121.8 \\
122.3 \\
120.1 \\
110.0\end{array}$ \\
\hline 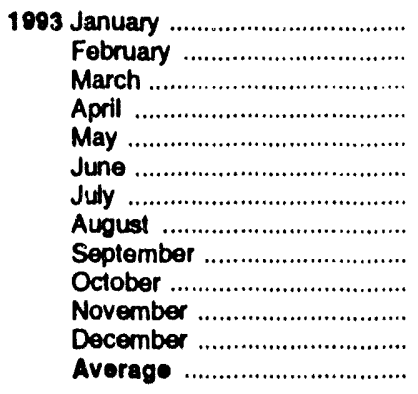 & $\begin{array}{l}\text { NA } \\
\text { NA } \\
\text { NA } \\
\text { NA } \\
\text { NA } \\
\text { NA } \\
\text { NA } \\
\text { NA } \\
\text { NA } \\
\text { NA } \\
\text { NA } \\
\text { NA } \\
\text { NA }\end{array}$ & $\begin{array}{l}111.7 \\
110.8 \\
109.8 \\
111.2 \\
112.9 \\
113.0 \\
110.9 \\
109.7 \\
108.5 \\
112.7 \\
111.3 \\
107.0 \\
110.8\end{array}$ & $\begin{array}{l}131.3 \\
130.1 \\
129.4 \\
130.4 \\
131.9 \\
132.1 \\
130.5 \\
129.4 \\
128.2 \\
132.3 \\
130.5 \\
126.8 \\
130.2\end{array}$ & $\begin{array}{l}118.2 \\
117.2 \\
116.3 \\
117.5 \\
119.3 \\
119.4 \\
117.4 \\
116.3 \\
115.1 \\
119.3 \\
117.8 \\
113.6 \\
117.3\end{array}$ \\
\hline 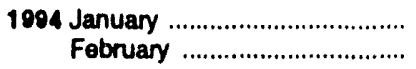 & $\begin{array}{l}\text { NA } \\
\text { NA }\end{array}$ & $\begin{array}{l}104.3 \\
105.1\end{array}$ & $\begin{array}{l}124.0 \\
124.5\end{array}$ & $\begin{array}{l}110.9 \\
111.4\end{array}$ \\
\hline
\end{tabular}

- Also includes types of motor gasoline not shown separately.

b In September 1981, the Bureau of Labor Statistics changed the weights used in the calculation of average motor gasoline prices. From September 1981 forward, gasohol is included in the average for all types, and unleaded premium is weighted more heavily.

C September through December data only.

$\mathrm{NA}=\mathrm{Not}$ avallable.

Notes: - See Nole 5 at end of section. - Geographic coverage for

1973-1977 is 56 urban areas. Geographic coverage for 1978 forward is 85 urban areas.

Sources: - Monthly Data: U.S. Department of Labor, Bureau of Labor Statistics, Consumer Prices: Energy. - Annual Data: 1073-Platt's Oil Price Handbook and Oilmanac, 1974, 51st Edition. 1974 forward-calculated by the Energy Information Administration as the simple averages of monthly data.

Data required to update this table were not available in time for publication. 
Table 9.5 Refiner Prices of Residual Fuel Oil (Cents per Gallon, Excluding iaxes)

\begin{tabular}{|c|c|c|c|c|c|c|}
\hline & \multicolumn{2}{|c|}{$\begin{array}{c}\text { Residual Fuel Oll } \\
\text { Sulfur Content Less } \\
\text { Than or Equal to } 1 \text { Percent }\end{array}$} & \multicolumn{2}{|c|}{$\begin{array}{l}\text { Residual Fuel Oll } \\
\text { Sulfur Content } \\
\text { Greater Than } 1 \text { Percent }\end{array}$} & \multicolumn{2}{|c|}{ Average } \\
\hline & $\begin{array}{c}\text { Sales for } \\
\text { Resaile }\end{array}$ & $\begin{array}{l}\text { Sales to } \\
\text { End Users }\end{array}$ & $\begin{array}{l}\text { Sales for } \\
\text { Resale }\end{array}$ & $\begin{array}{l}\text { Sales to } \\
\text { End Users }\end{array}$ & $\begin{array}{c}\text { Seles for } \\
\text { Resale }\end{array}$ & $\begin{array}{l}\text { Sales to } \\
\text { End Users }\end{array}$ \\
\hline 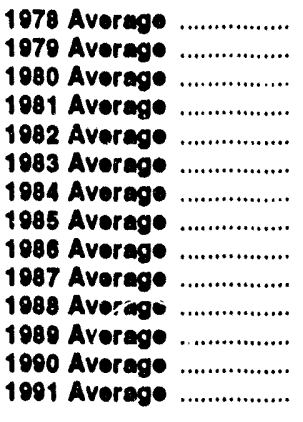 & $\begin{array}{l}29.3 \\
45.0 \\
60.8 \\
74.8 \\
68.5 \\
64.3 \\
68.5 \\
61.0 \\
32.8 \\
41.2 \\
33.3 \\
40.7 \\
47.2 \\
36.4\end{array}$ & $\begin{array}{l}31.4 \\
46.8 \\
67.5 \\
82.9 \\
74.7 \\
69.5 \\
72.0 \\
64.4 \\
37.2 \\
44.7 \\
37.2 \\
43.6 \\
60.5 \\
40.2\end{array}$ & $\begin{array}{l}24.5 \\
36.6 \\
47.9 \\
62.2 \\
57.2 \\
59.1 \\
63.9 \\
56.0 \\
28.9 \\
36.2 \\
27.1 \\
33.1 \\
37.2 \\
29.2\end{array}$ & $\begin{array}{l}27.5 \\
38.9 \\
52.3 \\
67.3 \\
61.1 \\
61.1 \\
65.9 \\
58.2 \\
31.7 \\
39.6 \\
30.0 \\
34.4 \\
40.0 \\
30.6\end{array}$ & $\begin{array}{l}26.3 \\
39.0 \\
52.8 \\
66.3 \\
61.2 \\
60.0 \\
65.4 \\
57.7 \\
30.5 \\
38.5 \\
30.0 \\
36.0 \\
41.3 \\
31.4\end{array}$ & $\begin{array}{l}29.8 \\
43.6 \\
60.7 \\
75.6 \\
67.6 \\
65.1 \\
68.7 \\
61.0 \\
34.3 \\
42.3 \\
33.4 \\
38.5 \\
44.4 \\
34.0\end{array}$ \\
\hline 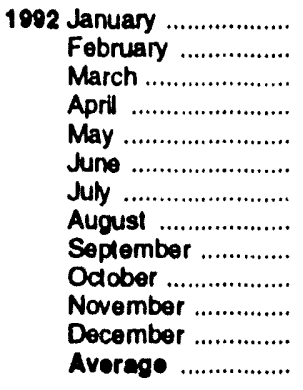 & $\begin{array}{l}30.3 \\
32.7 \\
30.8 \\
31.6 \\
33.1 \\
35.9 \\
38.0 \\
37.7 \\
37.9 \\
41.4 \\
39.2 \\
35.9 \\
35.4\end{array}$ & $\begin{array}{l}35.7 \\
36.2 \\
34.8 \\
35.3 \\
37.2 \\
38.8 \\
41.4 \\
42.1 \\
42.0 \\
44.7 \\
42.8 \\
40.2 \\
38.9\end{array}$ & $\begin{array}{l}21.1 \\
20.9 \\
21.1 \\
25.2 \\
29.1 \\
30.7 \\
33.3 \\
33.2 \\
32.9 \\
35.5 \\
33.8 \\
28.1 \\
28.4\end{array}$ & $\begin{array}{l}24.7 \\
23.6 \\
24.4 \\
27.5 \\
32.0 \\
33.1 \\
34.9 \\
37.0 \\
35.3 \\
37.3 \\
37.6 \\
33.4 \\
31.3\end{array}$ & $\begin{array}{l}24.4 \\
25.6 \\
24.6 \\
27.4 \\
30.2 \\
32.5 \\
34.7 \\
34.7 \\
34.8 \\
37.4 \\
35.9 \\
30.6 \\
30.7\end{array}$ & $\begin{array}{l}28.9 \\
27.7 \\
27.7 \\
29.6 \\
33.4 \\
34.5 \\
36.7 \\
38.8 \\
37.5 \\
39.2 \\
39.4 \\
36.2 \\
33.8\end{array}$ \\
\hline 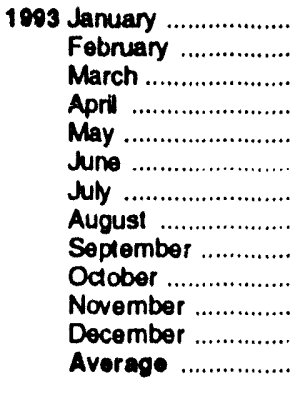 & $\begin{array}{l}36.6 \\
35.5 \\
39.0 \\
38.4 \\
34.7 \\
33.7 \\
32.7 \\
31.5 \\
31.9 \\
32.4 \\
31.0 \\
27.4 \\
33.8\end{array}$ & $\begin{array}{l}40.8 \\
40.8 \\
42.6 \\
43.6 \\
41.9 \\
40.6 \\
41.9 \\
37.2 \\
37.7 \\
38.7 \\
38.7 \\
35.6 \\
40.3\end{array}$ & $\begin{array}{r}27.2 \\
27.1 \\
27.5 \\
29.2 \\
27.8 \\
26.4 \\
24.6 \\
23.7 \\
24.0 \\
25.7 \\
23.6 \\
R_{21.9} \\
R_{25.8}\end{array}$ & $\begin{array}{l}32.4 \\
30.8 \\
31.6 \\
32.2 \\
34.1 \\
31.5 \\
28.5 \\
28.7 \\
28.6 \\
29.4 \\
27.0 \\
25.8 \\
30.2\end{array}$ & $\begin{array}{l}31.2 \\
31.1 \\
32.9 \\
33.6 \\
31.0 \\
30.0 \\
27.4 \\
26.9 \\
26.8 \\
28.7 \\
26.7 \\
R_{24.9} \\
R_{29.4}\end{array}$ & $\begin{array}{l}35.3 \\
34.4 \\
35.6 \\
36.3 \\
36.8 \\
34.7 \\
33.2 \\
31.9 \\
31.5 \\
32.0 \\
29.9 \\
29.2 \\
33.6\end{array}$ \\
\hline 1984 January ..................... & 33.7 & 39.7 & 23.6 & 27.7 & 28.7 & 32.5 \\
\hline
\end{tabular}

P=Pevised data.

Notes: - Sales for resale are those made to purchasers other than ullimate consumers. Sales to end users are those made directly to the ulimate consumer, Including bulk customers, such as agriculture, industry. and electric utilities, as well as commercial customers. - Geographic coverage is the 50 States and the District of Columbla. Values for the current month are preliminary. - Prices prior to 1983 are Energy Intormation Administration (EIA) estimates. See Note 6 at end of section.

Source: ElA, Petroleum Marketing Monthly, April 1994, Table 17.

Data required to update this table were not available in time for publication. 
Table 9.6 Refiner Prices of Petroleum Products for Resale

(Cents per Gallon, Excluding Taxes)

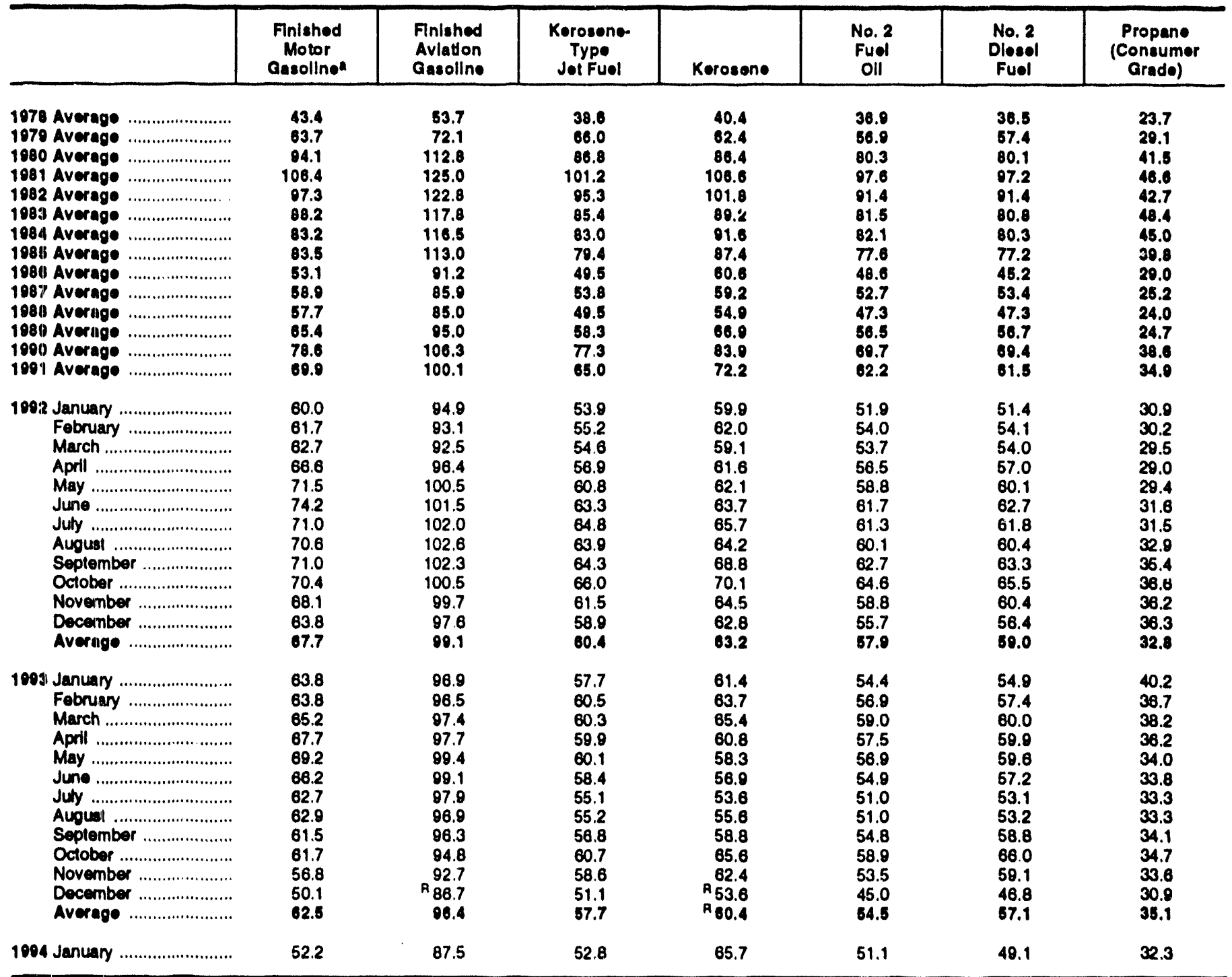

- See Nole 5 al end of section.

RaRevised data.

Notea: - Sales for resale are those made to purchasers other than ulimate consumers. Sales to end users are shown in Table 8.7; they are sales made directly to the ultimate consumer, including bulk customers, such as agricutture, industry, and electric vilities, as woll as residential and

commercial customers. - Geographic coverage is the 60 States and the District of Columbla. - Values lor the current month are prellminary. - Price prior to 1983 are Energy Information Administration (EIA) Sestimates. See Note 6 at end of section.

Source: EIA, Petroloum Marketing Monthly, April 1994, Table 4.

Data required to update this table were not available in time for publication. 
Table 9.7 Refiner Prices of Petroleum Products to End Users

(Cents per Gallon, Excluding Taxes)

\begin{tabular}{|c|c|c|c|c|c|c|c|}
\hline & $\begin{array}{c}\text { Finlshed } \\
\text { Motor } \\
\text { Gasollno }\end{array}$ & $\begin{array}{l}\text { Finlshed } \\
\text { Avlation } \\
\text { Gasollne }\end{array}$ & $\begin{array}{c}\text { Korosene- } \\
\text { Type } \\
\text { Jot Fuel }\end{array}$ & Kerosene & $\begin{array}{c}\text { No. } 2 \\
\text { Fud } \\
\text { Oll }\end{array}$ & $\begin{array}{l}\text { No. } 2 \\
\text { Dlosel } \\
\text { Fuel }\end{array}$ & $\begin{array}{c}\text { Propene } \\
\text { (Coneumer } \\
\text { Ornde) }\end{array}$ \\
\hline 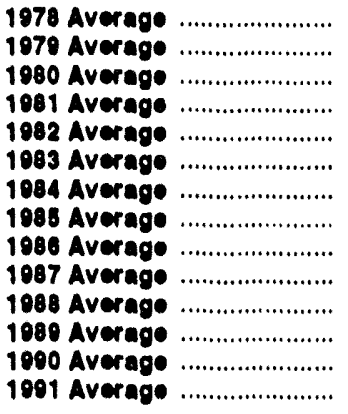 & $\begin{array}{r}48.4 \\
71.3 \\
103.5 \\
114.7 \\
108.0 \\
05.4 \\
90.7 \\
91.2 \\
62.4 \\
68.0 \\
67.3 \\
78.6 \\
88.3 \\
70.7\end{array}$ & $\begin{array}{r}51.6 \\
88.0 \\
108.4 \\
130.3 \\
131.2 \\
125.8 \\
123.4 \\
120.1 \\
101.1 \\
90.7 \\
89.1 \\
09.5 \\
112.0 \\
104.7\end{array}$ & \begin{tabular}{r|}
38.7 \\
54.7 \\
86.8 \\
102.4 \\
08.3 \\
87.8 \\
84.2 \\
70.8 \\
52.0 \\
54.3 \\
51.3 \\
59.2 \\
76.8 \\
85.2
\end{tabular} & $\begin{array}{r}42.1 \\
58.6 \\
00.2 \\
112.3 \\
108.0 \\
96.1 \\
103.6 \\
103.0 \\
70.0 \\
77.0 \\
73.8 \\
70.0 \\
92.3 \\
83.8\end{array}$ & $\begin{array}{l}40.0 \\
51.0 \\
78.8 \\
91.4 \\
90.5 \\
91.0 \\
91.0 \\
84.0 \\
58.0 \\
58.1 \\
54.4 \\
58.7 \\
73.4 \\
68.5\end{array}$ & $\begin{array}{l}37.7 \\
88.6 \\
81.8 \\
09.5 \\
94.2 \\
82.6 \\
82.3 \\
78.0 \\
47.8 \\
56.1 \\
50.0 \\
58.5 \\
72.5 \\
84.8\end{array}$ & $\begin{array}{r}33.8 \\
38.7 \\
48.2 \\
68.8 \\
60.2 \\
70.0 \\
73.7 \\
71.7 \\
74.6 \\
70.1 \\
71.4 \\
81.6 \\
74.6 \\
73.0\end{array}$ \\
\hline 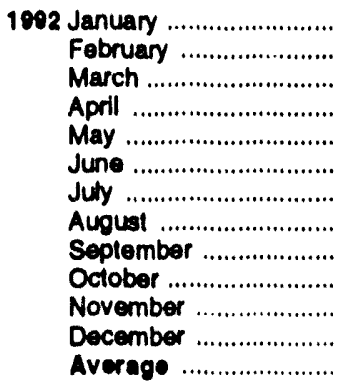 & $\begin{array}{l}71.9 \\
70.8 \\
71.6 \\
75.2 \\
80.8 \\
84.5 \\
83.5 \\
82.3 \\
82.3 \\
81.3 \\
81.5 \\
78.5 \\
78.4\end{array}$ & $\begin{array}{r}98.5 \\
88.5 \\
88.0 \\
89.1 \\
102.4 \\
106.4 \\
106.8 \\
105.7 \\
104.9 \\
104.3 \\
103.4 \\
101.3 \\
102.7\end{array}$ & $\begin{array}{l}54.2 \\
56.5 \\
65.5 \\
57.3 \\
61.0 \\
63.9 \\
64.9 \\
64.2 \\
64.6 \\
68.4 \\
62.7 \\
58.9 \\
61.0\end{array}$ & $\begin{array}{l}83.3 \\
78.3 \\
80.2 \\
78.3 \\
73.3 \\
68.7 \\
70.5 \\
69.0 \\
70.5 \\
87.2 \\
83.3 \\
84.0 \\
78.6\end{array}$ & $\begin{array}{l}69.7 \\
62.0 \\
61.4 \\
60.6 \\
60.9 \\
62.9 \\
62.8 \\
62.3 \\
65.6 \\
68.2 \\
64.3 \\
63.6 \\
62.7\end{array}$ & $\begin{array}{l}b 5.5 \\
57.1 \\
56.8 \\
59.2 \\
62.1 \\
64.9 \\
64.5 \\
63.4 \\
65.3 \\
67.8 \\
64.5 \\
60.8 \\
61.8\end{array}$ & $\begin{array}{l}71.3 \\
N A \\
68.4 \\
70.3 \\
62.5 \\
64.5 \\
52.3 \\
55.8 \\
60.3 \\
59.9 \\
61.1 \\
68.4 \\
68.2\end{array}$ \\
\hline 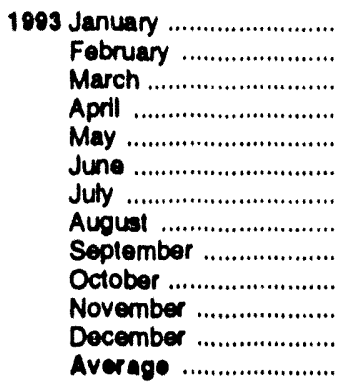 & $\begin{array}{r}76.9 \\
76.1 \\
75.7 \\
77.8 \\
80.1 \\
79.8 \\
77.6 \\
76.2 \\
74.9 \\
75.4 \\
72.6 \\
68.0 \\
76.0\end{array}$ & $\begin{array}{r}100.3 \\
99.9 \\
99.4 \\
100.7 \\
102.2 \\
102.5 \\
99.7 \\
98.8 \\
98.2 \\
98.0 \\
95.7 \\
91.2 \\
99.0\end{array}$ & $\begin{array}{l}58.5 \\
59.8 \\
60.6 \\
59.7 \\
59.9 \\
58.7 \\
55.3 \\
54.6 \\
56.9 \\
61.4 \\
59.6 \\
51.2 \\
57.0\end{array}$ & $\begin{array}{l}82.4 \\
81.3 \\
83.2 \\
77.0 \\
68.8 \\
65.3 \\
61.4 \\
61.9 \\
66.5 \\
75.5 \\
79.4 \\
72.6 \\
75.4\end{array}$ & $\begin{array}{l}62.7 \\
64.6 \\
66.2 \\
61.8 \\
59.8 \\
57.9 \\
54.1 \\
54.6 \\
57.3 \\
63.4 \\
61.5 \\
55.9 \\
60.2\end{array}$ & $\begin{array}{l}59.0 \\
60.6 \\
62.9 \\
62.5 \\
62.3 \\
60.5 \\
56.9 \\
56.2 \\
60.4 \\
67.0 \\
62.7 \\
52.4 \\
60.3\end{array}$ & 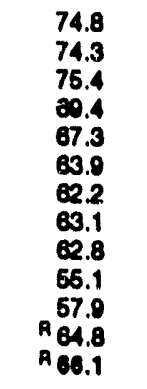 \\
\hline 1004 January ........................... & 66.7 & 88.6 & 51.6 & 79.3 & 59.7 & 52.7 & 67.5 \\
\hline
\end{tabular}

- See Nole 5 at end of section.

$R=$ Revised data. NA=Not avallable.

Notes: - Sales to end users are those made directly to the ultimate consumer, Including bulk customers, such as agriculture, industry, and electric utilitles, as well as residential and commercial customers. Sales for resale are shown in Table 9.6 ; they are sales made to purchasers other than

ultimale consumers. - Geographic coverage bs the 50 States and the Dlatrict a Columbla. - Values for the current month are prellminary. - Prices prior to 1983 are Energy Intormation Adminibtration (EIA) extimates. Seo Noto 6 at end of section.

Source: ElA, Petroleum Marketing Monthly, Aprll 1904, Table 2.

Data required to update this table were not available in time for publication. 
Table 9.8a No. 2 Distillate Prices to Residences: Northeastern States (Cents per Gallon, Excluding Taxes)

\begin{tabular}{|c|c|c|c|c|c|c|c|c|c|}
\hline & Maine & $\begin{array}{c}\text { New } \\
\text { Hampshire }\end{array}$ & Vermont & Massachusetts & $\begin{array}{l}\text { Rhode } \\
\text { Island }\end{array}$ & Connectlcut & $\begin{array}{l}\text { Now } \\
\text { York }\end{array}$ & $\begin{array}{l}\text { New } \\
\text { Jersey }\end{array}$ & Ponnsylvania \\
\hline 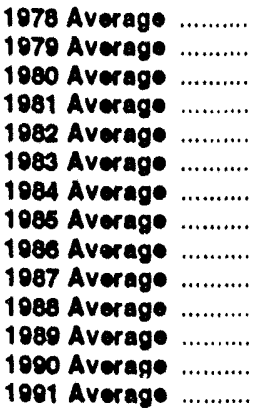 & $\begin{array}{r}48.6 \\
68.8 \\
98.3 \\
120.4 \\
115.5 \\
102.8 \\
103.9 \\
90.7 \\
74.4 \\
74.7 \\
77.7 \\
69.4 \\
08.9 \\
06.0\end{array}$ & $\begin{array}{r}50.3 \\
72.5 \\
100.4 \\
123.7 \\
117.4 \\
104.1 \\
108.4 \\
102.4 \\
75.9 \\
76.5 \\
78.2 \\
89.3 \\
102.8 \\
91.8\end{array}$ & $\begin{array}{r}50.8 \\
72.5 \\
101.5 \\
125.4 \\
120.1 \\
112.9 \\
111.9 \\
107.7 \\
86.6 \\
81.1 \\
82.6 \\
90.5 \\
107.0 \\
101.9\end{array}$ & $\begin{array}{r}48.8 \\
70.9 \\
97.8 \\
121.3 \\
117.6 \\
109.1 \\
111.6 \\
107.0 \\
82.1 \\
80.6 \\
82.1 \\
92.6 \\
108.4 \\
103.0\end{array}$ & $\begin{array}{r}50.7 \\
72.8 \\
101.1 \\
123.8 \\
120.1 \\
110.5 \\
111.4 \\
108.7 \\
82.8 \\
82.5 \\
83.6 \\
93.9 \\
108.6 \\
99.9\end{array}$ & $\begin{array}{r}50.1 \\
72.0 \\
98.3 \\
121.7 \\
118.3 \\
109.1 \\
112.1 \\
108.0 \\
89.0 \\
83.4 \\
85.3 \\
92.9 \\
109.8 \\
106.2\end{array}$ & $\begin{array}{r}50.1 \\
71.2 \\
98.2 \\
123.2 \\
120.5 \\
112.1 \\
115.5 \\
111.3 \\
91.1 \\
85.2 \\
86.3 \\
95.8 \\
112.5 \\
111.3\end{array}$ & $\begin{array}{r}49.6 \\
71.0 \\
97.0 \\
121.5 \\
117.4 \\
107.0 \\
111.0 \\
105.0 \\
80.2 \\
84.3 \\
84.8 \\
91.8 \\
108.7 \\
104.0\end{array}$ & $\begin{array}{r}48.8 \\
69.8 \\
98.4 \\
118.1 \\
113.7 \\
105.8 \\
107.0 \\
102.3 \\
81.4 \\
76.0 \\
77.8 \\
85.1 \\
102.6 \\
\infty 9.7\end{array}$ \\
\hline 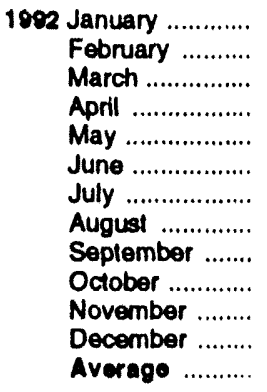 & $\begin{array}{l}87.7 \\
88.2 \\
86.4 \\
85.5 \\
85.5 \\
87.1 \\
87.7 \\
87.8 \\
86.8 \\
89.3 \\
88.3 \\
85.7 \\
87.1\end{array}$ & $\begin{array}{l}88.1 \\
86.5 \\
83.3 \\
81.8 \\
81.7 \\
82.9 \\
82.3 \\
81.8 \\
83.0 \\
87.6 \\
87.6 \\
87.7 \\
85.6\end{array}$ & $\begin{array}{l}92.4 \\
92.8 \\
92.2 \\
91.7 \\
91.5 \\
90.7 \\
89.1 \\
89.4 \\
91.6 \\
92.0 \\
92.6 \\
92.9 \\
92.2\end{array}$ & $\begin{array}{l}93.2 \\
92.5 \\
91.5 \\
91.4 \\
91.0 \\
91.3 \\
90.4 \\
89.6 \\
90.7 \\
93.5 \\
93.8 \\
93.5 \\
92.4\end{array}$ & $\begin{array}{l}90.7 \\
91.7 \\
90.9 \\
90.4 \\
90.9 \\
89.7 \\
89.9 \\
89.4 \\
89.8 \\
92.7 \\
92.5 \\
91.5 \\
91.2\end{array}$ & $\begin{array}{l}96.4 \\
95.5 \\
94.0 \\
93.3 \\
93.1 \\
91.8 \\
93.1 \\
90.5 \\
91.8 \\
94.9 \\
95.8 \\
95.2 \\
94.7\end{array}$ & $\begin{array}{r}103.4 \\
103.8 \\
102.1 \\
101.1 \\
101.1 \\
101.7 \\
100.7 \\
99.0 \\
99.7 \\
102.7 \\
104.7 \\
104.3 \\
102.8\end{array}$ & $\begin{array}{l}95.6 \\
95.1 \\
93.5 \\
92.9 \\
89.2 \\
90.4 \\
90.3 \\
88.1 \\
90.8 \\
94.0 \\
94.6 \\
95.4 \\
93.0\end{array}$ & $\begin{array}{l}91.4 \\
91.5 \\
80.1 \\
89.4 \\
88.6 \\
86.5 \\
83.0 \\
81.7 \\
84.4 \\
87.5 \\
89.6 \\
89.3 \\
88.9\end{array}$ \\
\hline 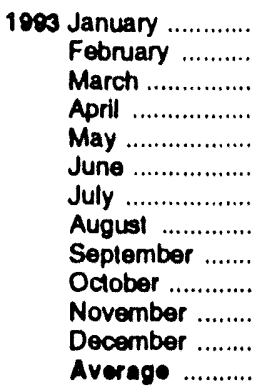 & $\begin{array}{r}85.2 \\
85.4 \\
86.5 \\
83.0 \\
81.5 \\
80.8 \\
78.2 \\
77.3 \\
78.3 \\
82.8 \\
80.6 \\
79.4 \\
R 82.5\end{array}$ & $\begin{array}{r}87.1 \\
87.0 \\
86.6 \\
85.0 \\
83.8 \\
82.5 \\
78.0 \\
76.1 \\
75.2 \\
77.2 \\
77.1 \\
77.8 \\
83.0\end{array}$ & $\begin{array}{r}93.4 \\
93.3 \\
93.7 \\
91.2 \\
91.2 \\
89.7 \\
85.5 \\
85.6 \\
86.6 \\
86.8 \\
86.1 \\
\text { ค } 87.1 \\
90.4\end{array}$ & $\begin{array}{r}94.0 \\
94.4 \\
94.8 \\
91.3 \\
90.9 \\
88.6 \\
83.9 \\
83.4 \\
83.8 \\
86.1 \\
85.9 \\
83.9 \\
89.6\end{array}$ & $\begin{array}{l}91.7 \\
91.8 \\
92.4 \\
90.3 \\
90.6 \\
87.6 \\
85.2 \\
82.7 \\
84.1 \\
86.0 \\
87.7 \\
85.9 \\
80.3\end{array}$ & $\begin{array}{r}94.9 \\
96.2 \\
96.7 \\
93.6 \\
91.7 \\
88.6 \\
86.5 \\
84.0 \\
84.3 \\
88.6 \\
88.9 \\
{ }_{88.2} \\
{ }^{8} 92.0\end{array}$ & $\begin{array}{r}104.3 \\
104.2 \\
104.2 \\
100.1 \\
99.3 \\
97.8 \\
95.2 \\
92.9 \\
93.5 \\
96.1 \\
95.9 \\
\text { R } 93.9 \\
100.1\end{array}$ & $\begin{array}{r}96.5 \\
96.7 \\
96.2 \\
95.1 \\
91.6 \\
88.0 \\
87.9 \\
85.7 \\
85.9 \\
88.7 \\
89.6 \\
\text { P }_{87.2} \\
02.5\end{array}$ & $\begin{array}{r}89.0 \\
89.1 \\
89.8 \\
89.0 \\
86.6 \\
84.0 \\
78.8 \\
77.0 \\
80.4 \\
83.2 \\
85.2 \\
R 84.3 \\
86.4\end{array}$ \\
\hline 1984 January ............. & 83.7 & 80.9 & 89.0 & 87.7 & 87.9 & 91.3 & 97.3 & 91.9 & 87.7 \\
\hline
\end{tabular}

$R=$ Revised data.

Notes: - States are grouped in Tables 9.8a, 9.8b, and 9.8c by geographic region of the country. - Values for the current month are preliminary.
- Prices prior to 1983 are Energy Inlormation Administration (E|A) estimates. See Note 6 at end of section.

Source: EIA, Petroleum Marketing Monthly, April 1994, Table 16.

Data required to update this table were not available in time for publication. 
Table 9.8b No. 2 Distillate Prices to Residences: Selected South Atlantic and Midwestern States

(Cents per Gallon, Excluding Taxes)

\begin{tabular}{|c|c|c|c|c|c|c|c|c|c|c|c|}
\hline & Delaware & $\begin{array}{c}\text { Dlstrict } \\
\text { of } \\
\text { Columbla }\end{array}$ & Maryland & Virginla & $\begin{array}{l}\text { West } \\
\text { Virginla }\end{array}$ & Ohio & Michlgan & Indlana & IIIInols & Wisconsin & Minnesoth \\
\hline 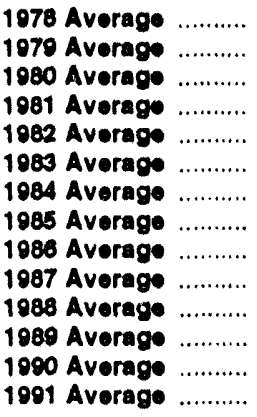 & $\begin{array}{r}47.8 \\
68.2 \\
95.4 \\
117.3 \\
111.3 \\
106.0 \\
109.6 \\
104.6 \\
85.0 \\
79.3 \\
80.1 \\
88.2 \\
105.8 \\
99.7\end{array}$ & $\begin{array}{r}50.7 \\
74.2 \\
102.8 \\
127.4 \\
124.5 \\
117.0 \\
118.7 \\
114.3 \\
93.1 \\
91.8 \\
91.6 \\
98.6 \\
107.8 \\
112.2\end{array}$ & $\begin{array}{r}49.2 \\
70.1 \\
97.0 \\
121.4 \\
117.1 \\
110.3 \\
113.5 \\
108.8 \\
91.4 \\
86.6 \\
87.0 \\
93.6 \\
111.9 \\
108.4\end{array}$ & $\begin{array}{r}49.1 \\
70.4 \\
98.5 \\
120.5 \\
117.7 \\
108.7 \\
110.5 \\
106.3 \\
86.6 \\
79.5 \\
80.5 \\
87.0 \\
110.6 \\
101.1\end{array}$ & $\begin{array}{r}46.2 \\
65.1 \\
92.2 \\
115.0 \\
109.3 \\
101.0 \\
102.1 \\
98.0 \\
74.6 \\
76.4 \\
74.2 \\
83.0 \\
99.1 \\
93.4\end{array}$ & $\begin{array}{r}47.4 \\
68.6 \\
91.9 \\
113.2 \\
110.2 \\
101.3 \\
102.1 \\
99.7 \\
77.7 \\
74.7 \\
74.7 \\
81.6 \\
98.1 \\
91.0\end{array}$ & $\begin{array}{r}47.0 \\
70.0 \\
97.8 \\
118.3 \\
113.9 \\
106.4 \\
105.0 \\
102.1 \\
81.0 \\
77.5 \\
77.5 \\
85.3 \\
100.9 \\
94.2\end{array}$ & $\begin{array}{r}48.5 \\
72.7 \\
99.6 \\
118.5 \\
114.3 \\
100.7 \\
103.1 \\
99.1 \\
74.8 \\
75.4 \\
75.4 \\
83.2 \\
99.3 \\
91.8\end{array}$ & $\begin{array}{r}46.5 \\
68.8 \\
95.8 \\
114.9 \\
110.9 \\
100.4 \\
100.1 \\
97.5 \\
\text { NA } \\
79.8 \\
77.6 \\
80.9 \\
96.1 \\
92.7\end{array}$ & $\begin{array}{r}44.7 \\
67.3 \\
91.5 \\
109.1 \\
107.8 \\
101.2 \\
101.0 \\
98.3 \\
75.6 \\
75.1 \\
73.0 \\
81.1 \\
94.2 \\
89.5\end{array}$ & $\begin{array}{r}47.8 \\
72.4 \\
00.9 \\
118.4 \\
115.1 \\
103.1 \\
104.1 \\
101.0 \\
70.2 \\
74.6 \\
73.5 \\
82.4 \\
101.4 \\
01.1\end{array}$ \\
\hline 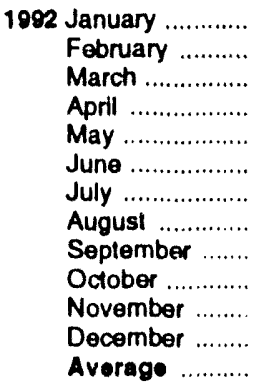 & $\begin{array}{l}94.4 \\
92.7 \\
92.4 \\
91.5 \\
90.2 \\
91.4 \\
90.6 \\
89.5 \\
90.3 \\
93.7 \\
92.8 \\
90.9 \\
92.4\end{array}$ & $\begin{array}{l}107.3 \\
107.3 \\
105.3 \\
104.7 \\
102.3 \\
102.7 \\
102.0 \\
101.9 \\
101.2 \\
104.0 \\
105.7 \\
105.4 \\
105.7\end{array}$ & $\begin{array}{r}101.6 \\
100.9 \\
100.3 \\
99.0 \\
97.2 \\
97.6 \\
95.7 \\
95.2 \\
95.7 \\
98.8 \\
100.4 \\
100.4 \\
998\end{array}$ & $\begin{array}{l}94.3 \\
93.7 \\
93.7 \\
92.6 \\
91.7 \\
89.6 \\
90.2 \\
88.4 \\
89.4 \\
91.9 \\
92.1 \\
93.3 \\
92.8\end{array}$ & $\begin{array}{l}85.5 \\
86.9 \\
86.6 \\
85.6 \\
84.2 \\
86.5 \\
82.3 \\
81.4 \\
85.4 \\
88.3 \\
88.0 \\
89.0 \\
86.4\end{array}$ & $\begin{array}{l}82.0 \\
83.0 \\
82.5 \\
82.9 \\
83.5 \\
85.3 \\
81.7 \\
82.3 \\
84.7 \\
86.4 \\
84.6 \\
84.5 \\
83.6\end{array}$ & $\begin{array}{l}86.6 \\
86.5 \\
86.6 \\
86.7 \\
86.4 \\
86.1 \\
85.0 \\
85.7 \\
88.2 \\
90.0 \\
88.2 \\
87.9 \\
87.1\end{array}$ & $\begin{array}{l}77.8 \\
78.7 \\
79.5 \\
80.2 \\
81.2 \\
79.6 \\
82.4 \\
83.1 \\
84.8 \\
85.8 \\
82.7 \\
81.8 \\
81.1\end{array}$ & $\begin{array}{l}85.2 \\
85.6 \\
88.1 \\
88.4 \\
89.0 \\
90.8 \\
87.9 \\
86.4 \\
88.9 \\
90.8 \\
90.4 \\
88.2 \\
87.6\end{array}$ & $\begin{array}{l}80.1 \\
79.8 \\
79.2 \\
80.4 \\
81.5 \\
81.9 \\
81.1 \\
80.6 \\
83.6 \\
84.1 \\
83.7 \\
84.3 \\
81.6\end{array}$ & $\begin{array}{l}79.4 \\
79.6 \\
79.7 \\
81.8 \\
83.9 \\
82.9 \\
84.5 \\
84.1 \\
85.0 \\
87.1 \\
86.0 \\
83.1 \\
82.3\end{array}$ \\
\hline 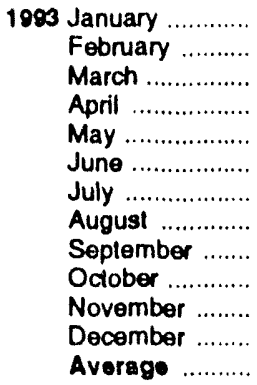 & $\begin{array}{r}90.8 \\
90.8 \\
92.4 \\
91.6 \\
89.4 \\
90.9 \\
90.2 \\
83.5 \\
85.0 \\
88.6 \\
88.3 \\
{ }^{8} 8.8 \\
\text { R }_{90.1}\end{array}$ & $\begin{array}{r}105.2 \\
106.8 \\
108.5 \\
107.1 \\
104.3 \\
100.4 \\
100.2 \\
96.1 \\
95.0 \\
102.3 \\
100.6 \\
\text { R } 100.6 \\
\text { R } 104.6\end{array}$ & $\begin{array}{r}100.5 \\
101.3 \\
101.6 \\
99.2 \\
96.2 \\
95.2 \\
92.3 \\
91.3 \\
92.6 \\
94.2 \\
95.9 \\
94.6 \\
98.1\end{array}$ & $\begin{array}{r}92.4 \\
93.5 \\
94.2 \\
90.3 \\
88.6 \\
86.0 \\
84.7 \\
84.0 \\
84.9 \\
85.0 \\
84.4 \\
86.3 \\
89.6\end{array}$ & $\begin{array}{r}88.3 \\
88.6 \\
89.9 \\
86.9 \\
84.8 \\
86.7 \\
81.2 \\
79.1 \\
79.2 \\
83.6 \\
84.0 \\
84.7 \\
85.2\end{array}$ & $\begin{array}{r}84.2 \\
85.5 \\
86.6 \\
86.9 \\
86.0 \\
85.7 \\
79.3 \\
78.6 \\
81.4 \\
85.6 \\
84.5 \\
80.9 \\
83.9\end{array}$ & $\begin{array}{l}88.3 \\
87.6 \\
90.1 \\
90.8 \\
89.8 \\
87.4 \\
83.4 \\
82.1 \\
85.5 \\
89.7 \\
86.6 \\
82.1 \\
87.2\end{array}$ & $\begin{array}{r}81.8 \\
82.3 \\
83.1 \\
84.9 \\
83.6 \\
82.1 \\
79.0 \\
76.6 \\
80.3 \\
83.1 \\
81.0 \\
76.6 \\
81.1\end{array}$ & $\begin{array}{r}87.2 \\
88.2 \\
90.0 \\
\text { NA } \\
84.8 \\
81.2 \\
79.4 \\
77.2 \\
80.9 \\
87.0 \\
82.3 \\
\text { R } 77.8 \\
\text { R }_{84.1}\end{array}$ & $\begin{array}{r}82.1 \\
83.3 \\
84.0 \\
84.7 \\
84.9 \\
84.2 \\
84.1 \\
78.7 \\
82.8 \\
82.0 \\
82.6 \\
\text { R } 7.7 \\
82.3\end{array}$ & $\begin{array}{r}82.9 \\
83.0 \\
83.9 \\
83.3 \\
84.1 \\
83.4 \\
82.0 \\
80.0 \\
83.1 \\
87.1 \\
85.6 \\
\mathrm{R}_{80.5} \\
\mathrm{R}_{83.3}\end{array}$ \\
\hline 1994 January ............ & 92.1 & 101.9 & 98.5 & 89.4 & 87.5 & 82.4 & 85.7 & 78.7 & 76.3 & 78.8 & 81.1 \\
\hline
\end{tabular}

$R=$ Revised data. NA=Not available.

Notes: - States are grouped in Tables $9.8 \mathrm{a}, 9.8 \mathrm{~b}$, and $9.8 \mathrm{c}$ by geographic region of the country. Values for the current month are preliminary.
- Prices prior to 1983 are Energy Information Administration (EIA) estimates. See Note 6 at end of section.

Source: EIA, Petroleum Marketing Monthly, April 1994, Table 16.

Data required to update this table were not available in time for publication. 
Table 9.8c No. 2 Distillate Prices to Residences: Selected Western States and U.S. Average

(Cents per Gallon, Excluding Taxes)

\begin{tabular}{|c|c|c|c|c|c|}
\hline & Idaho & Washington & Oregon & Alaska & $\begin{array}{c}\text { U.S. } \\
\text { Average }\end{array}$ \\
\hline 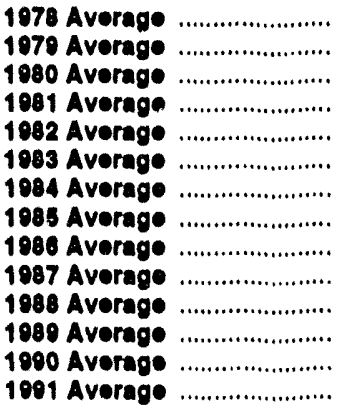 & $\begin{array}{r}43.6 \\
62.1 \\
91.6 \\
110.4 \\
110.4 \\
101.8 \\
98.8 \\
97.2 \\
73.8 \\
68.8 \\
68.8 \\
77.8 \\
97.4 \\
95.1\end{array}$ & $\begin{array}{r}48.6 \\
69.7 \\
100.8 \\
116.5 \\
117.6 \\
109.0 \\
102.8 \\
101.1 \\
77.5 \\
79.5 \\
78.5 \\
87.4 \\
102.8 \\
101.8\end{array}$ & $\begin{array}{r}45.8 \\
68.0 \\
97.3 \\
111.4 \\
111.6 \\
103.6 \\
90.3 \\
97.1 \\
70.4 \\
72.5 \\
70.0 \\
80.2 \\
97.0 \\
93.3\end{array}$ & $\begin{array}{r}63.2 \\
68.2 \\
97.8 \\
118.0 \\
117.4 \\
108.8 \\
106.9 \\
108.3 \\
94.0 \\
86.5 \\
86.9 \\
06.4 \\
110.1 \\
105.0\end{array}$ & $\begin{array}{r}40.0 \\
70.4 \\
97.4 \\
110.4 \\
116.0 \\
107.8 \\
100.1 \\
105.3 \\
83.8 \\
80.3 \\
81.3 \\
90.0 \\
108.3 \\
101.0\end{array}$ \\
\hline 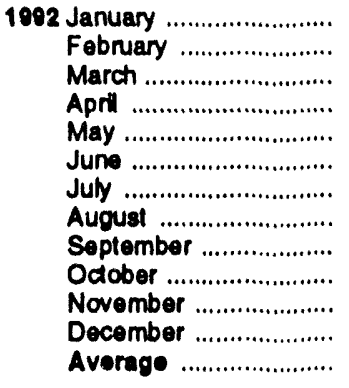 & $\begin{array}{l}86.1 \\
79.2 \\
82.2 \\
84.2 \\
86.1 \\
84.6 \\
86.1 \\
79.4 \\
88.0 \\
89.6 \\
81.7 \\
86.8 \\
85.7\end{array}$ & $\begin{array}{l}92.0 \\
90.9 \\
91.8 \\
92.0 \\
94.3 \\
90.6 \\
88.0 \\
84.0 \\
90.3 \\
94.5 \\
98.7 \\
99.7 \\
94.3\end{array}$ & $\begin{array}{l}85.3 \\
83.5 \\
82.6 \\
85.5 \\
88.9 \\
89.2 \\
87.3 \\
84.0 \\
87.6 \\
91.7 \\
82.8 \\
91.5 \\
87.8\end{array}$ & $\begin{array}{l}92.7 \\
91.1 \\
93.0 \\
92.1 \\
93.6 \\
93.9 \\
93.0 \\
96.8 \\
93.4 \\
96.8 \\
97.7 \\
95.8 \\
94.0\end{array}$ & $\begin{array}{l}94.2 \\
94.2 \\
93.2 \\
92.5 \\
92.3 \\
92.0 \\
90.4 \\
88.6 \\
90.1 \\
93.7 \\
94.8 \\
94.5 \\
93.4\end{array}$ \\
\hline 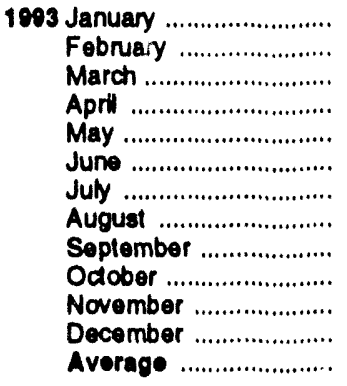 & $\begin{array}{r}84.8 \\
84.2 \\
87.8 \\
84.1 \\
82.9 \\
82.8 \\
80.0 \\
77.0 \\
85.3 \\
94.8 \\
98.2 \\
\text { P }_{81.2} \\
\text { P }_{88.1}\end{array}$ & $\begin{array}{r}100.6 \\
101.4 \\
99.7 \\
101.5 \\
100.3 \\
95.1 \\
91.3 \\
89.3 \\
97.1 \\
105.5 \\
104.3 \\
\text { P } 96.7 \\
100.2\end{array}$ & $\begin{array}{r}91.7 \\
89.9 \\
90.7 \\
82.1 \\
91.3 \\
90.2 \\
86.1 \\
83.5 \\
92.0 \\
100.2 \\
97.4 \\
\text { A }_{87.9} \\
R_{91.8}\end{array}$ & $\begin{array}{l}95.1 \\
95.1 \\
94.2 \\
94.7 \\
96.6 \\
97.1 \\
95.3 \\
95.5 \\
94.9 \\
96.7 \\
94.3 \\
91.7 \\
94.0\end{array}$ & $\begin{array}{r}94.3 \\
94.6 \\
95.4 \\
82.5 \\
81.0 \\
88.9 \\
85.6 \\
84.1 \\
85.4 \\
88.7 \\
88.6 \\
R 88.7 \\
91.1\end{array}$ \\
\hline 1094 January ........................ & 73.1 & 94.5 & 86.3 & 88.4 & 89.7 \\
\hline
\end{tabular}

$R=$ Revised data.

Notes: - Stales are grouped in Tables 9.8a, 9.8b, and 9.8c by geographic reglon of the country. - Values for the current month are preliminary.
- Prices prior to 1983 are Energy Information Administration (ELA) estimates. Seo Note 6 at end of section.

Source: EIA, Petroleum Marketing Monthly, April 1994, Table 16.

Data required to update this table were not available in time for publication. 
Figure 9.2 Electricity Retall Prices

(Cents per Kilowatthour)

Prices by Sector, 1973-1993

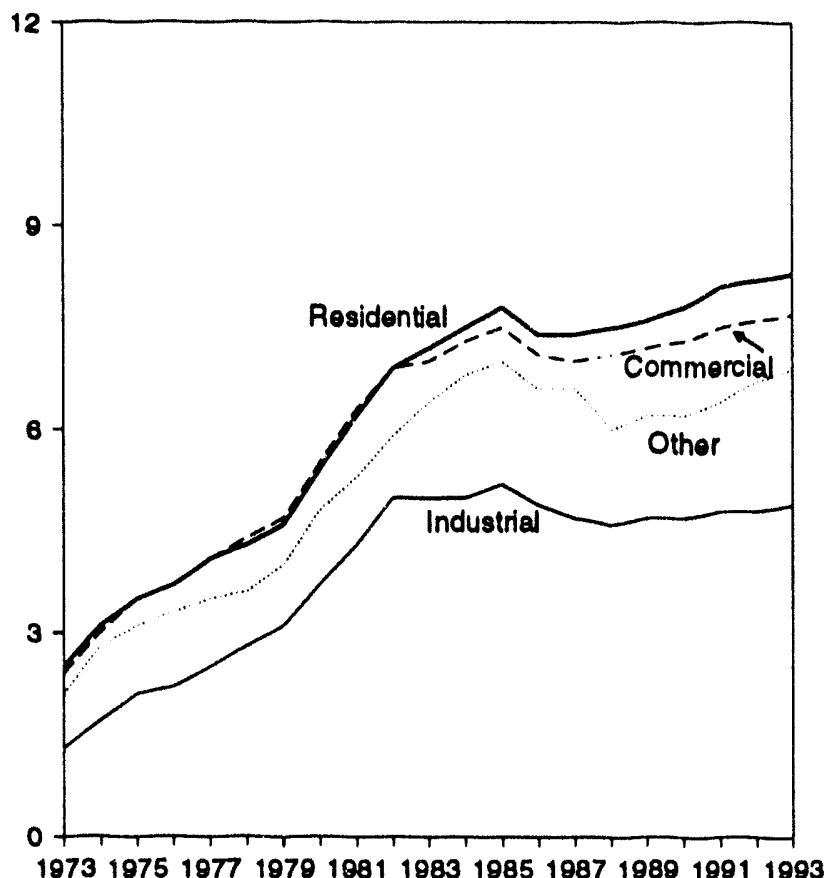

Prices by Sector, Monthly

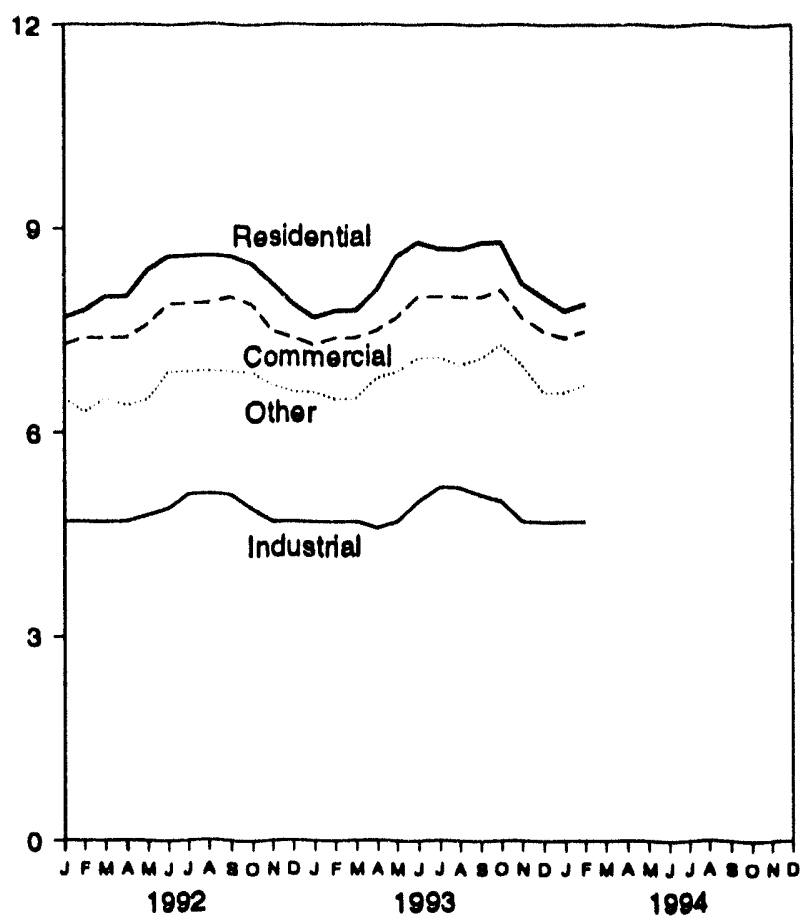

Source: Table 9.9, Monthly Serles.

Figure 9.3 Cost of Fossil-Fuel Recelpts at Steam-Electric Plants (Dollars per Million Btu)

Fossil Fuels Costs, 1973-1993

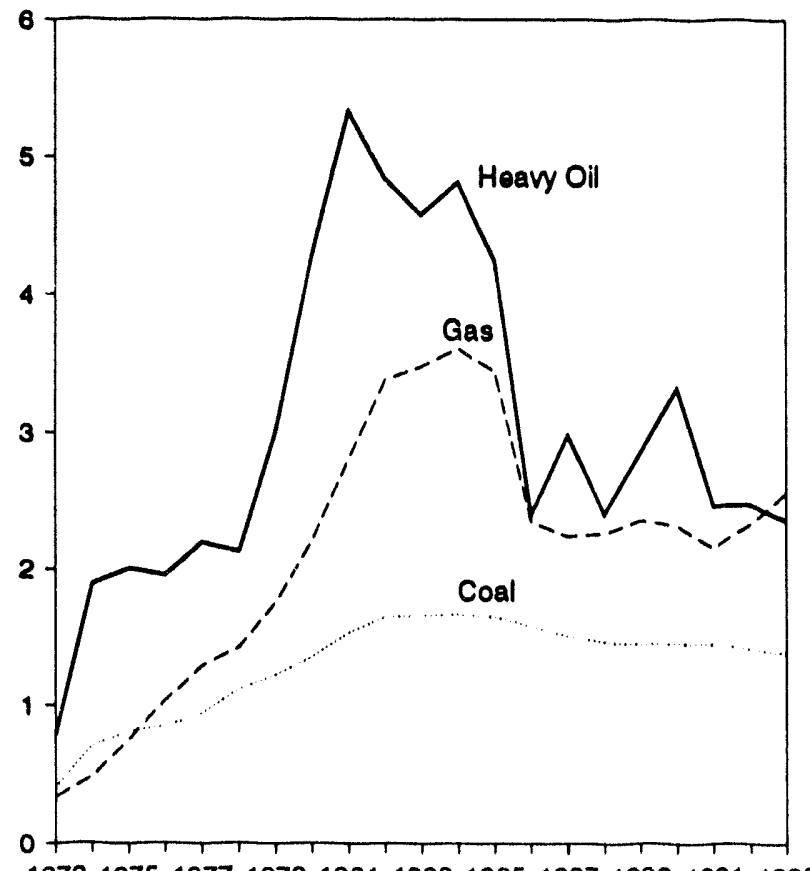

19731975197719791981198319851987198919911993
Fossil Fuel Costs, Monthly

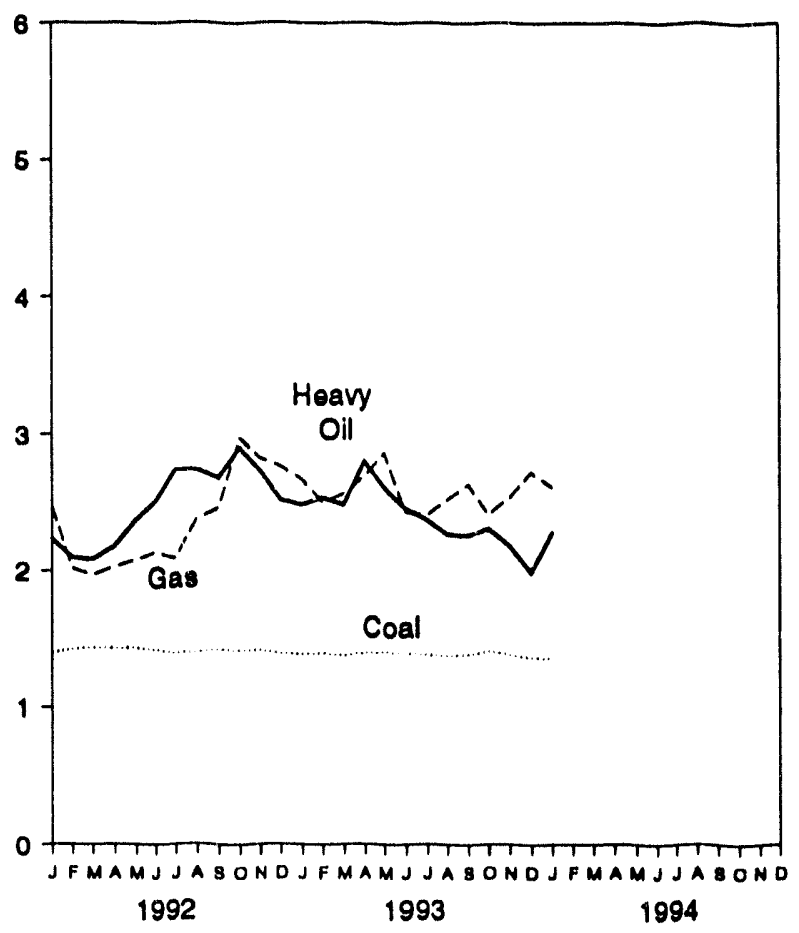

Source: Table 9.10. 
Table 9.9 Electricity Retail Prices

(Cents per Kilowatthour)

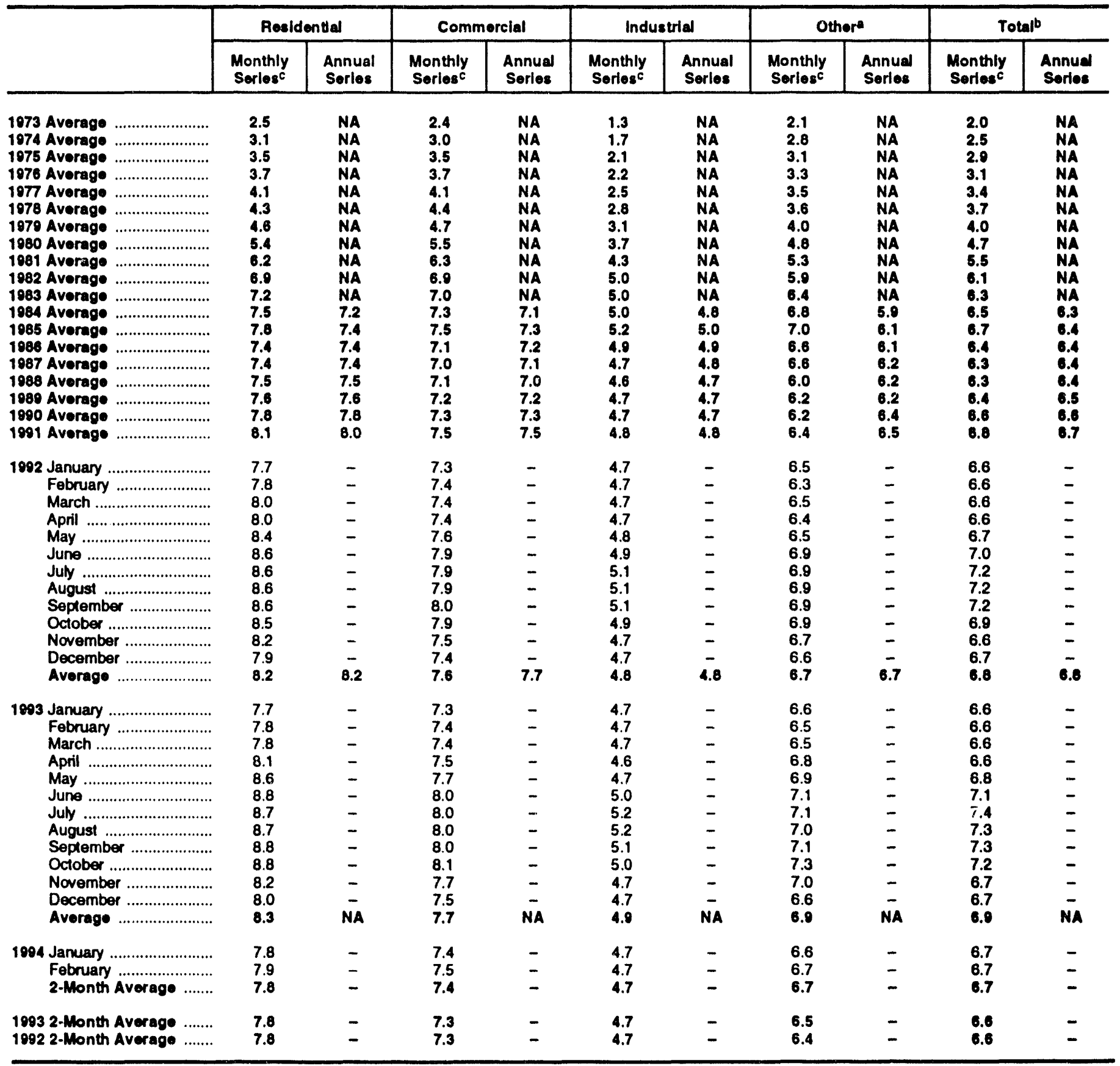

- "Other" is public street and highway lighting, other sales to public authorities, sales to railroads and railways, and interdepartmental sales.

Average price for tolal sales to utimale consumers.

c Annual values are the sum of the monthly revenue divided by the sum of the monthly sales. Dala through 1979 cover privateby owned electric utilities in Classes $A$ and 8 . Dala for 1980-1985 cover selected privalely owned electric utilities in Class A whose electric cperating revenue was $\$ 100$ million or more during the previous year. See Note 7 at end of section.

NA=Not available. - =Not applicable.

Noles: - Prices are calculated by dividing revenue by sales. Revenue may not corresponid to sales for a particular month because of electric utility billing and accounting procedures. That lack of correspondence could result in uncharaderistic increases or decreases in the monthly prices. See Note 7 at end of section. - Geographic coverage is the 50 States and the District of Columbia.

Sources: - Monthly Series: 1973-September 1977-Federal Power Commission, Form FPC-5, "Monthly Statement of Electric Operating Revenue and Income." October 1077-February 1980_Federal Energy Regulatory Commission (FERC), Form FERC-5, "Electric Operating Revenue and Income:" March 1080-December 1080-FERC, Form FERC-5, "Electric Utility Company Monthly Statement. 1081-Energy Inlormation Administration (ELA), Electric Power Monthy, March 1992, Table 59.1922 and 1991 monthly data-ELA, Electric Power Monthly, March 1993, Table 59. 1983 forward (except 1991 monthly data)-ElA, Eloctric Power Monthly, May 1994, Table 60. - Annual Sortes: ElA, Electric Powor Monthly, May 1994, Table 60. 
Table 9.10 Quantity and Cost of Fossil-Fuel Receipts at Steam-Electric Utility Plants

\begin{tabular}{|c|c|c|c|c|c|c|c|c|c|}
\hline & \multicolumn{2}{|c|}{ Coal } & \multicolumn{4}{|c|}{ Potroloum } & \multicolumn{2}{|c|}{ Gas: } & \multirow{3}{*}{$\begin{array}{c}\begin{array}{c}\text { All Fosell } \\
\text { Fusteb }\end{array} \\
\text { Cost } \\
\text { (conte per } \\
\text { million Btu) }\end{array}$} \\
\hline & \multirow[b]{2}{*}{$\begin{array}{l}\text { Quentity } \\
\text { (thousand } \\
\text { short tons) }\end{array}$} & \multirow[b]{2}{*}{$\begin{array}{c}\text { Cost } \\
\text { (conts per } \\
\text { million Btu) }\end{array}$} & \multicolumn{2}{|c|}{ Heavy Oilb } & \multicolumn{2}{|c|}{ Totajb,c } & \multirow[b]{2}{*}{$\begin{array}{l}\text { Quantity } \\
\text { (million } \\
\text { cuble foot) }\end{array}$} & \multirow[b]{2}{*}{$\begin{array}{c}\text { Cost } \\
\text { (cents por } \\
\text { million Btu) }\end{array}$} & \\
\hline & & & $\begin{array}{l}\text { Quantity } \\
\text { (thousand } \\
\text { barrels) }\end{array}$ & $\begin{array}{c}\text { Cost } \\
\text { (cents per } \\
\text { million Btu) }\end{array}$ & $\begin{array}{c}\text { Quantity } \\
\text { (thousand } \\
\text { barrols) }\end{array}$ & $\begin{array}{c}\text { Cost } \\
\text { (cents per } \\
\text { million Btu) }\end{array}$ & & & \\
\hline 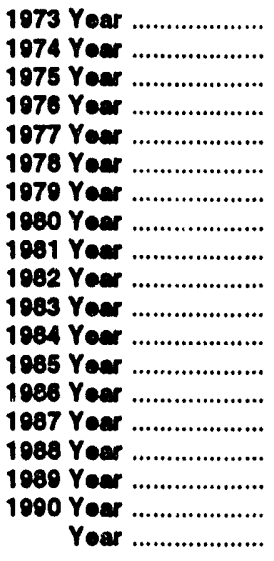 & $\begin{array}{l}374,642 \\
384,868 \\
431,527 \\
454,858 \\
490,415 \\
476,169 \\
556,558 \\
593,995 \\
679,374 \\
601,427 \\
592,728 \\
684,111 \\
686,743 \\
686,964 \\
721,298 \\
727,775 \\
753,217 \\
786,627 \\
768,923\end{array}$ & $\begin{array}{r}40.5 \\
70.9 \\
81.4 \\
84.8 \\
94.7 \\
111.6 \\
122.4 \\
135.1 \\
153.2 \\
164.7 \\
165.6 \\
166.4 \\
164.8 \\
157.9 \\
150.6 \\
146.6 \\
144.5 \\
145.5 \\
144.7\end{array}$ & $\begin{array}{l}512,650 \\
479,166 \\
457,582 \\
495,363 \\
563,685 \\
546,197 \\
479,705 \\
394,159 \\
327,477 \\
228,200 \\
211,705 \\
193,832 \\
156,410 \\
220,585 \\
187,300 \\
230,234 \\
237,668 \\
202,281 \\
163,106\end{array}$ & $\begin{array}{r}78.5 \\
189.0 \\
200.5 \\
195.2 \\
219.8 \\
212.5 \\
298.8 \\
426.7 \\
533.4 \\
483.2 \\
457.8 \\
481.2 \\
424.4 \\
240.1 \\
287.6 \\
240.5 \\
284.6 \\
331.0 \\
246.5\end{array}$ & $\begin{array}{l}535,859 \\
515,217 \\
510,352 \\
549,973 \\
635,556 \\
616,040 \\
515,695 \\
419,140 \\
345,544 \\
239,111 \\
219,652 \\
202,372 \\
164,947 \\
228,522 \\
104,578 \\
236,924 \\
246,422 \\
209,350 \\
169,625\end{array}$ & \begin{tabular}{|r|}
80.0 \\
191.0 \\
202.3 \\
199.0 \\
224.9 \\
219.1 \\
307.2 \\
435.1 \\
542.5 \\
492.2 \\
462.8 \\
486.3 \\
431.7 \\
243.7 \\
301.1 \\
243.9 \\
289.3 \\
338.4 \\
254.8
\end{tabular} & $\begin{array}{l}3,382,677 \\
3,225,203 \\
3,034,808 \\
2,962,811 \\
3,106,403 \\
3,140,654 \\
3,368,976 \\
3,588,814 \\
3,573,558 \\
3,161,348 \\
2,732,248 \\
2,878,808 \\
2,808,921 \\
2,387,622 \\
2,605,101 \\
2,362,721 \\
2,472,506 \\
2,490,970 \\
2,630,818\end{array}$ & \begin{tabular}{|r|}
33.8 \\
48.2 \\
75.2 \\
103.4 \\
129.1 \\
142.2 \\
174.9 \\
219.0 \\
280.5 \\
337.6 \\
347.4 \\
360.3 \\
344.4 \\
235.1 \\
224.0 \\
226.3 \\
235.5 \\
232.1 \\
215.3
\end{tabular} & \begin{tabular}{|r|}
47.8 \\
91.4 \\
104.4 \\
111.9 \\
129.7 \\
141.1 \\
163.9 \\
192.8 \\
225.8 \\
224.9 \\
220.8 \\
219.1 \\
209.4 \\
175.0 \\
170.6 \\
164.3 \\
167.5 \\
168.9 \\
160.3
\end{tabular} \\
\hline 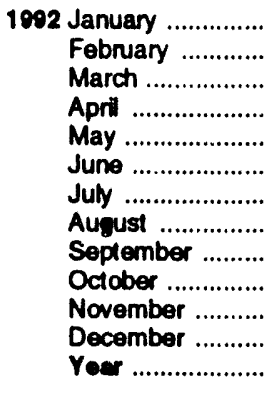 & $\begin{array}{l}64,678 \\
61,603 \\
63,857 \\
60,661 \\
63,407 \\
63,704 \\
64,400 \\
70,241 \\
66,503 \\
66,907 \\
64,005 \\
65,998 \\
775,963\end{array}$ & $\begin{array}{l}139.6 \\
142.1 \\
143.4 \\
142.7 \\
142.9 \\
141.9 \\
139.3 \\
139.6 \\
142.0 \\
141.3 \\
141.5 \\
138.6 \\
141.2\end{array}$ & $\begin{array}{r}12,039 \\
13,634 \\
12,779 \\
10,144 \\
10,079 \\
10,888 \\
12,706 \\
12,152 \\
8,883 \\
10,772 \\
11,161 \\
13,302 \\
138,537\end{array}$ & $\begin{array}{l}223.2 \\
209.8 \\
208.2 \\
217.8 \\
237.1 \\
251.4 \\
274.1 \\
274.1 \\
268.5 \\
290.5 \\
273.5 \\
252.1 \\
247.5\end{array}$ & $\begin{array}{r}12,539 \\
14,107 \\
13,186 \\
10,555 \\
10,498 \\
11,352 \\
13,217 \\
12,664 \\
9,319 \\
11,221 \\
11,636 \\
14,097 \\
144,390\end{array}$ & $\begin{array}{l}230.0 \\
216.1 \\
214.1 \\
225.7 \\
245.1 \\
260.0 \\
281.2 \\
281.2 \\
277.6 \\
297.7 \\
280.5 \\
261.9 \\
255.1\end{array}$ & $\begin{array}{r}159,815 \\
160,328 \\
198,040 \\
218,468 \\
227,857 \\
254,025 \\
315,543 \\
287,373 \\
259,771 \\
205,039 \\
182,505 \\
168,913 \\
2,637,678\end{array}$ & $\begin{array}{l}247.1 \\
201.7 \\
196.8 \\
202.6 \\
207.8 \\
213.6 \\
208.9 \\
237.3 \\
246.3 \\
297.9 \\
282.6 \\
276.5 \\
232.8\end{array}$ & $\begin{array}{l}155.2 \\
152.7 \\
153.7 \\
154.8 \\
156.4 \\
158.3 \\
159.2 \\
161.6 \\
163.0 \\
167.5 \\
164.5 \\
160.0 \\
150.0\end{array}$ \\
\hline 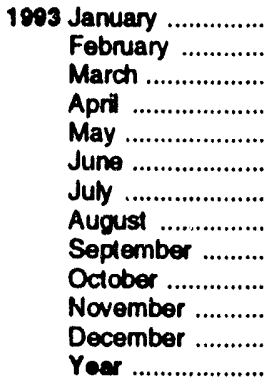 & $\begin{array}{l}65,219 \\
59,229 \\
63,694 \\
63,807 \\
62,599 \\
63,701 \\
59,859 \\
65,739 \\
65,358 \\
67,122 \\
65,927 \\
66,563 \\
769,018\end{array}$ & $\begin{array}{l}138.5 \\
139.3 \\
137.6 \\
139.3 \\
139.9 \\
139.0 \\
138.0 \\
137.4 \\
138.5 \\
140.5 \\
138.0 \\
136.2 \\
138.5\end{array}$ & $\begin{array}{r}8,437 \\
7,002 \\
8,548 \\
10,074 \\
10,392 \\
10,633 \\
15,419 \\
15,099 \\
15,324 \\
13,596 \\
10,736 \\
16,331 \\
141,590\end{array}$ & $\begin{array}{l}248.7 \\
254.1 \\
248.6 \\
280.0 \\
261.2 \\
245.8 \\
237.3 \\
227.0 \\
226.1 \\
231.0 \\
218.2 \\
198.8 \\
236.2\end{array}$ & $\begin{array}{r}9,026 \\
7,421 \\
9,022 \\
10,539 \\
10,825 \\
11,144 \\
16,040 \\
15,624 \\
15,766 \\
14,005 \\
11,272 \\
17,085 \\
147,760\end{array}$ & $\begin{array}{l}259.1 \\
263.8 \\
258.8 \\
286.6 \\
268.1 \\
254.2 \\
243.3 \\
232.2 \\
231.0 \\
236.6 \\
227.2 \\
205.5 \\
243.3\end{array}$ & $\begin{array}{r}159,318 \\
153,681 \\
18 \%, 075 \\
169,844 \\
163,925 \\
243,599 \\
312,270 \\
339,454 \\
249,708 \\
226,136 \\
201,759 \\
165,685 \\
2,571,453\end{array}$ & $\begin{array}{l}267.3 \\
250.8 \\
256.6 \\
268.9 \\
286.3 \\
243.2 \\
241.0 \\
252.5 \\
263.6 \\
241.3 \\
253.9 \\
272.4 \\
256.0\end{array}$ & $\begin{array}{l}156.2 \\
155.6 \\
156.5 \\
159.9 \\
161.6 \\
159.8 \\
164.4 \\
165.1 \\
162.9 \\
159.1 \\
156.4 \\
154.9 \\
159.5\end{array}$ \\
\hline 1904 January .............. & 62,601 & 135.8 & 16.700 & 228.5 & 17,781 & 237.9 & 160,321 & 261.5 & 156.6 \\
\hline
\end{tabular}

- Inctudes supplemental gaseous fuels.

- Heavy oil includes fuel oil nos. 4, 5, and 6, and lopped crude oil. The weighted averages for petroleum and all lossil fuels include both heavy and light oil (No. 2 fuel oil, kerosene, and jet fuel) prices. Data do not include petroleum coke.

c Data for 1973-1982 do not include small quantities of rerefined motor oil. bunker oil, and liquelied petroleum gas.

Notes: - See Note 8 at end of section. - Geographic coverage is the 50 States and the District of Columbia.

Sources: - 1073-1070: Annual data for quantity are simple sums of unrounded monthly values and for cost are averages of monthly values. weighted by quantities of Blu, from the following: 1973-May 1977-Federal Power Commission, Form FPC-423, "Monthly Report on Cost and Quality of Fuels for Electric Utility Plants." June 1077-Docember 1077-Federal Energy Regulatory Commission, Form FERC-423, "Monthly Report on Cost and Quality of Fuels for Electric Utility Plants." 1978 and 1979-Energy Information Administration (EIA), Form FERC-423, "Monthly Repont on Cost and Quality of Fuels for Electric Utility Plants." - 1980: ElA, Electric Power Monthly, April 1991, Table 33. - 1981: ElA, Electric Power Monthly, April 1992, Table 33. - 1982 and 1991 monthly data: ElA, Electric Powel Monthly, April 1993, Table 33. - 1983 forward (except 1091 monthly data): EIA, Electric Power Monthly, May 1994, Table 34. 
Figure 9.4 Natural Gas Prices

(Dollars per Thousand Cubic Feet)

Selected Prices, 1973-1993

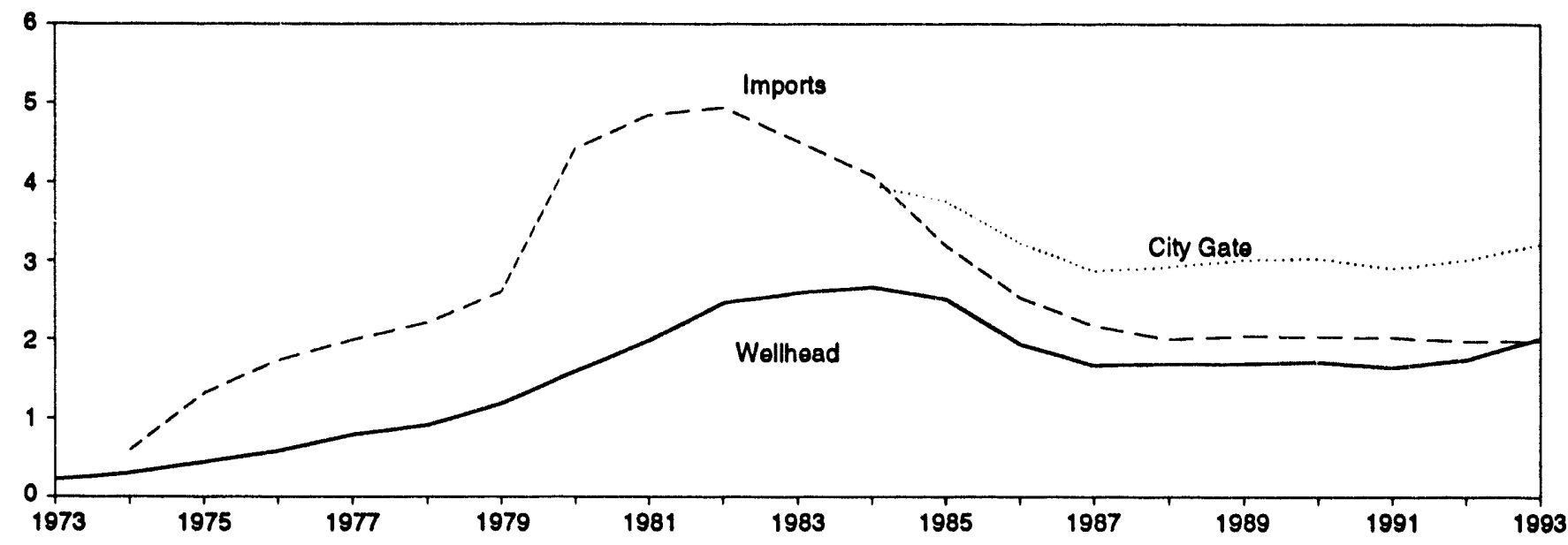

Delivered to Consumers, $1973-1993$

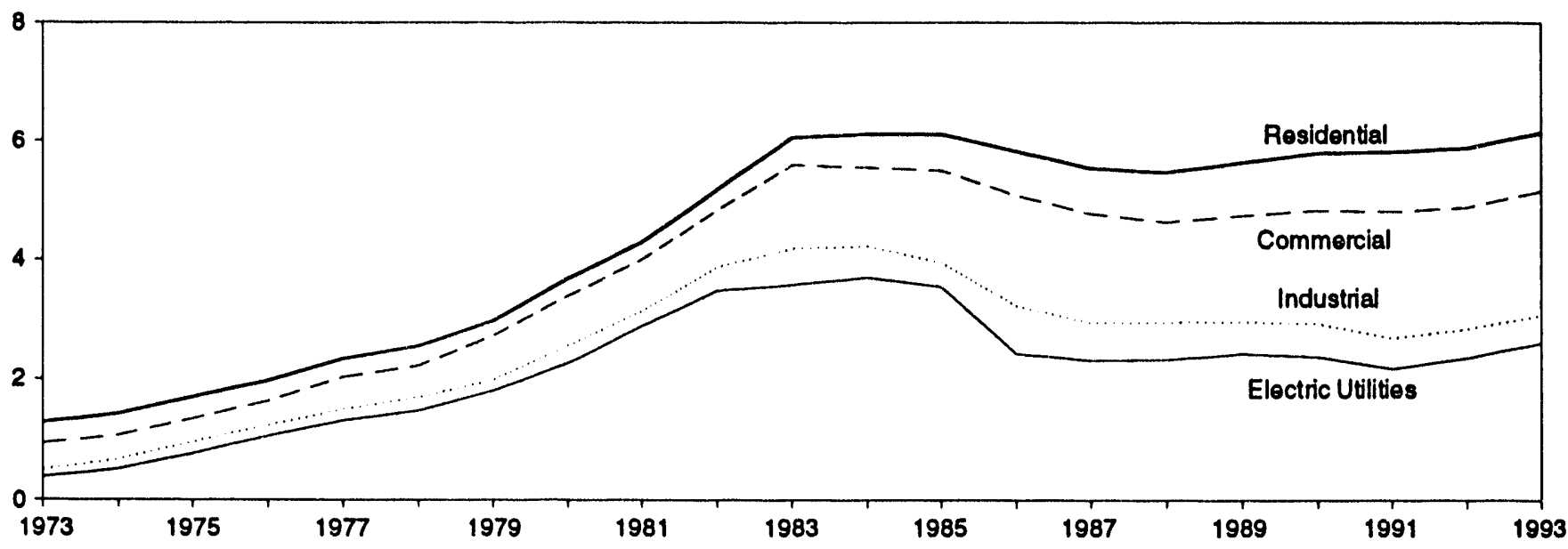

Delivered to Consumers, i lonthly

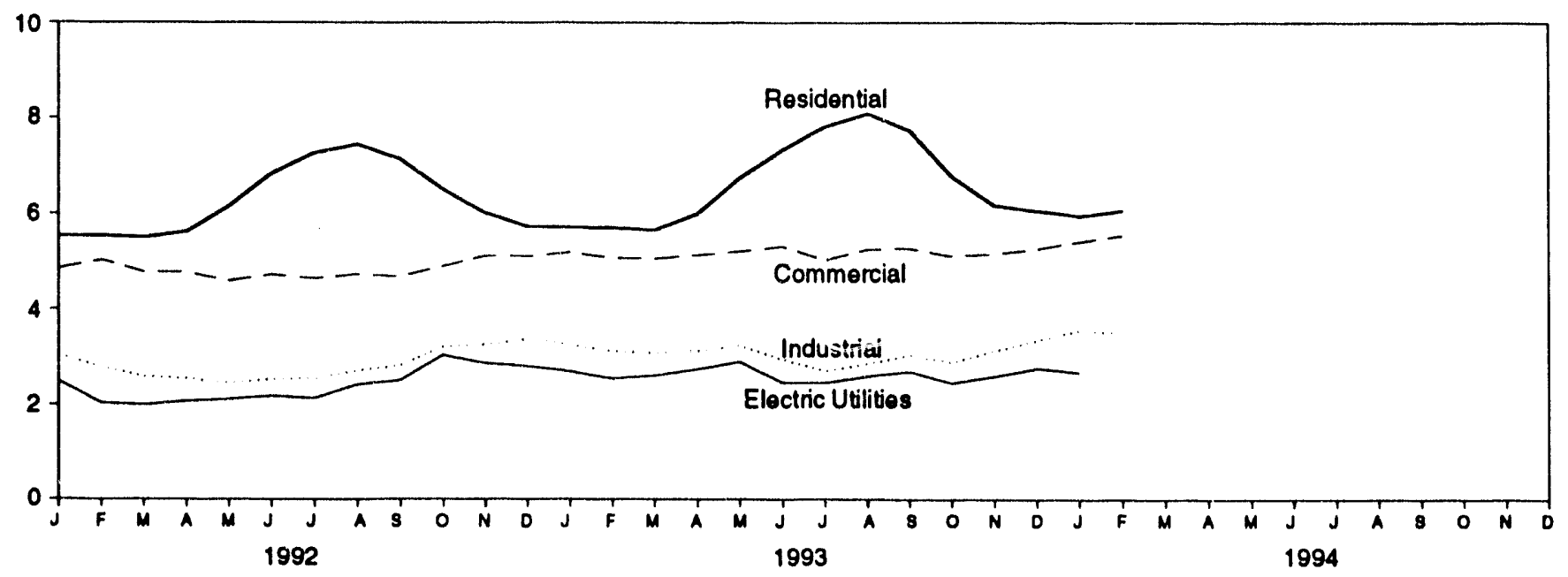

Note: Because vertical scsles differ, graphs should not be compared. Source: Table \{.11. 
Table 9.11 Natural Gas Prices

(Dollars per Thousand Cubic Feet)

\begin{tabular}{|c|c|c|c|c|c|c|c|c|}
\hline & \multirow[b]{2}{*}{ Wollhead } & \multicolumn{2}{|c|}{$\begin{array}{l}\text { Major Interstate } \\
\text { Plpelino Companles }\end{array}$} & \multirow[b]{2}{*}{$\begin{array}{l}\text { City } \\
\text { Cate }\end{array}$} & \multicolumn{4}{|c|}{ Dellvered to Consumere $a, b$} \\
\hline & & Importe & $\begin{array}{c}\text { Purchases from } \\
\text { Producers }\end{array}$ & & Residentlal & Commereld & Industrial & $\begin{array}{l}\text { Electrle } \\
\text { Utilltese }\end{array}$ \\
\hline 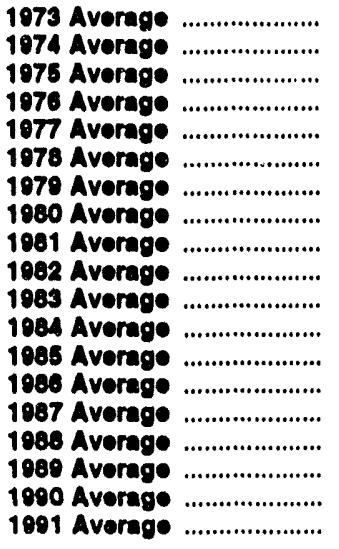 & $\begin{array}{r}0.22 \\
.35 \\
.44 \\
.51 \\
.70 \\
.01 \\
1.18 \\
1.50 \\
1.98 \\
2.40 \\
2.50 \\
2.68 \\
2.51 \\
1.04 \\
1.07 \\
1.69 \\
1.69 \\
1.71 \\
1.64\end{array}$ & $\begin{array}{l}\text { NA } \\
.80 \\
1.31 \\
1.73 \\
1.09 \\
2.21 \\
2.60 \\
4.42 \\
4.84 \\
4.94 \\
4.51 \\
4.08 \\
3.19 \\
2.53 \\
2.17 \\
2.00 \\
2.04 \\
2.03 \\
2.02\end{array}$ & $\begin{array}{l}\text { NA } \\
.27 \\
.37 \\
.48 \\
.70 \\
.83 \\
1.22 \\
1.03 \\
2.15 \\
2.72 \\
2.03 \\
2.91 \\
2.85 \\
2.30 \\
2.10 \\
2.13 \\
2.18 \\
2.10 \\
1.92\end{array}$ & $\begin{array}{l}\text { NA } \\
\text { NA } \\
\text { NA } \\
\text { NA } \\
\text { NA } \\
\text { NA } \\
\text { NA } \\
\text { NA } \\
\text { NA } \\
\text { NA } \\
3.05 \\
3.75 \\
3.22 \\
2.07 \\
2.02 \\
3.01 \\
3.03 \\
2.00\end{array}$ & $\begin{array}{l}1.20 \\
1.43 \\
1.71 \\
1.08 \\
2.35 \\
2.58 \\
2.08 \\
3.68 \\
4.20 \\
5.17 \\
6.06 \\
6.12 \\
6.12 \\
5.83 \\
5.54 \\
5.47 \\
5.64 \\
5.80 \\
5.82\end{array}$ & $\begin{array}{l}0.04 \\
1.07 \\
1.35 \\
1.64 \\
2.04 \\
2.23 \\
2.73 \\
3.39 \\
4.00 \\
4.82 \\
5.59 \\
5.55 \\
5.50 \\
5.08 \\
4.77 \\
4.63 \\
4.74 \\
4.83 \\
4.81\end{array}$ & $\begin{array}{r}0.50 \\
.07 \\
.08 \\
1.24 \\
1.50 \\
1.70 \\
1.09 \\
2.58 \\
3.14 \\
3.07 \\
4.18 \\
4.22 \\
3.95 \\
3.23 \\
2.94 \\
2.95 \\
2.98 \\
2.93 \\
2.08\end{array}$ & $\begin{array}{r}0.38 \\
.81 \\
.77 \\
1.05 \\
1.32 \\
1.46 \\
1.81 \\
2.27 \\
2.80 \\
3.46 \\
3.60 \\
3.70 \\
3.58 \\
2.43 \\
2.32 \\
2.33 \\
2.43 \\
2.38 \\
2.18\end{array}$ \\
\hline 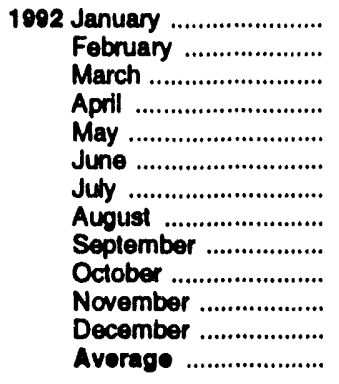 & $\begin{array}{l}1.74 \\
1.26 \\
1.35 \\
1.42 \\
1.51 \\
1.62 \\
1.55 \\
1.84 \\
1.92 \\
2.38 \\
2.13 \\
2.07 \\
1.74\end{array}$ & $\begin{array}{l}2.20 \\
1.98 \\
1.45 \\
2.01 \\
1.79 \\
2.03 \\
1.09 \\
1.85 \\
2.05 \\
2.13 \\
2.32 \\
1.92 \\
1.97\end{array}$ & $\begin{array}{l}2.10 \\
1.70 \\
1.90 \\
1.73 \\
1.99 \\
2.16 \\
1.86 \\
2.14 \\
2.13 \\
2.69 \\
2.33 \\
2.40 \\
2.09\end{array}$ & $\begin{array}{l}2.90 \\
2.70 \\
2.61 \\
2.74 \\
2.90 \\
3.00 \\
3.01 \\
3.18 \\
3.23 \\
3.50 \\
3.33 \\
3.17 \\
3.01\end{array}$ & $\begin{array}{l}\mathbf{5 . 5 3} \\
\mathbf{5 . 5 4} \\
\mathbf{5 . 5 0} \\
\mathbf{5 . 6 2} \\
\mathbf{6 . 1 5} \\
\mathbf{6 . 8 4} \\
\mathbf{7 . 2 7} \\
\mathbf{7 . 4 5} \\
\mathbf{7 . 1 5} \\
\mathbf{6 . 5 2} \\
\mathbf{6 . 0 2} \\
\mathbf{5 . 7 4} \\
\mathbf{5 . 8 0}\end{array}$ & $\begin{array}{l}4.85 \\
5.03 \\
4.77 \\
4.77 \\
4.59 \\
4.72 \\
4.64 \\
4.73 \\
4.69 \\
4.90 \\
5.12 \\
5.11 \\
4.88\end{array}$ & $\begin{array}{l}3.04 \\
2.78 \\
2.58 \\
2.54 \\
2.44 \\
2.53 \\
2.54 \\
2.71 \\
2.82 \\
3.21 \\
3.26 \\
3.38 \\
2.84\end{array}$ & $\begin{array}{l}2.49 \\
2.03 \\
1.90 \\
2.07 \\
2.11 \\
2.18 \\
2.13 \\
2.42 \\
2.51 \\
3.04 \\
2.87 \\
2.81 \\
2.38\end{array}$ \\
\hline 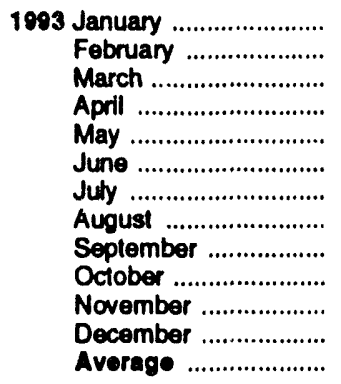 & $\begin{array}{l}A_{1} .98 \\
A_{1.74} \\
A_{1.92} \\
A_{2.06} \\
A_{2.32} \\
A_{1.89} \\
A_{1.92} \\
A_{2.02} \\
A_{2.15} \\
11.93 \\
A_{1.94} \\
A_{2.20} \\
A_{2.01}\end{array}$ & $\begin{array}{l}2.04 \\
1.91 \\
1.78 \\
2.15 \\
2.13 \\
1.95 \\
1.78 \\
2.02 \\
2.17 \\
1.97 \\
1.85 \\
2.02 \\
1.08\end{array}$ & $\begin{array}{r}A_{2} .17 \\
1.94 \\
2.20 \\
2.34 \\
2.81 \\
2.03 \\
2.02 \\
2.35 \\
2.58 \\
2.05 \\
2.32 \\
2.82 \\
2.30\end{array}$ & $\begin{array}{l}3.11 \\
2.94 \\
3.06 \\
3.24 \\
3.58 \\
3.44 \\
3.34 \\
3.35 \\
3.53 \\
3.15 \\
3.15 \\
3.26 \\
3.21\end{array}$ & $\begin{array}{l}5.72 \\
5.71 \\
5.66 \\
6.00 \\
6.74 \\
7.34 \\
7.82 \\
8.10 \\
7.74 \\
6.78 \\
6.17 \\
6.06 \\
6.15\end{array}$ & $\begin{array}{l}5.19 \\
5.08 \\
5.06 \\
5.14 \\
5.21 \\
5.32 \\
5.03 \\
5.26 \\
5.27 \\
5.11 \\
5.16 \\
5.26 \\
5.16\end{array}$ & $\begin{array}{l}3.25 \\
3.12 \\
3.08 \\
3.13 \\
3.24 \\
2.95 \\
2.71 \\
2.86 \\
3.03 \\
2.88 \\
3.12 \\
3.35 \\
3.07\end{array}$ & $\begin{array}{l}2.70 \\
2.55 \\
2.61 \\
2.75 \\
2.80 \\
2.47 \\
2.46 \\
2.60 \\
2.69 \\
2.45 \\
2.59 \\
2.76 \\
2.61\end{array}$ \\
\hline $\begin{array}{r}1094 \text { January ...................... } \\
\text { February ................... } \\
\text { 2-Month Average ...... }\end{array}$ & $\begin{array}{l}A_{1.99} \\
E_{2.24} \\
E_{2.12}\end{array}$ & $\begin{array}{l}2.08 \\
1.81 \\
1.95\end{array}$ & $\begin{array}{l}2.83 \\
3.31 \\
3.07\end{array}$ & $\begin{array}{r}\text { P }_{3.0} 0 \\
3.25 \\
3.15\end{array}$ & $\begin{array}{r}A_{5.95} \\
6.06 \\
6.00\end{array}$ & $\begin{array}{r}\mathrm{P}_{5.41} \\
5.54 \\
5.47\end{array}$ & $\begin{array}{r}\text { A }_{3.55} \\
3.50 \\
3.53\end{array}$ & $\begin{array}{c}\text { A }_{2.67} \\
\text { NA } \\
\text { NA }\end{array}$ \\
\hline $\begin{array}{l}1903 \text { 2-Month Average ..... } \\
1092 \text { 2-Month Average ..... }\end{array}$ & $\begin{array}{l}1.86 \\
1.50\end{array}$ & $\begin{array}{l}1.98 \\
2.00\end{array}$ & $\begin{array}{l}2.06 \\
1.90\end{array}$ & $\begin{array}{l}3.03 \\
2.81\end{array}$ & $\begin{array}{l}5.71 \\
5.54\end{array}$ & $\begin{array}{l}5.13 \\
4.93\end{array}$ & $\begin{array}{l}3.19 \\
2.02\end{array}$ & $\begin{array}{l}2.63 \\
2.28\end{array}$ \\
\hline
\end{tabular}

- Includes supplemental gaseous fuels.

See Nole 9 at end of section.

c See Note 8 at end of section.

$R=$ Revised data. $N A=N o t$ available. E=Estimate.

Notes: - Prices shown on this page are intended to include all taxes. See Note 9 at end of section. - Geographic coverage is the 50 States and the District of Columbia. - Wellhead annual and year-to-date prices are simple averages of the monthly prices; all other annual and year-to-date prices are volume-weighted averages of the monthly prices.

Sources: - 1973-1986: Wollhead-Energy Information Administration
(ElA), Natural Gas Annual 1991. Table 95. Major Intersthte Plpoline Companies, 1974-1977-Calculated from revenue and sales data reported to the Federal Power Commission (FPC), Form FPC.11, "Naturdl Gas Pipeline Company Monthly Statement." Major Interstate Pipeline Compenies, 1078-1083-ElA, Natural Gas Monthly, December 1984, Table 10. Major Interstate PIpeline Companles, 1084-1086-EIA, Natural Gas Monthy, December 1989. Table 4. Clty Gate, 1084-1988-ElA, Natural Gas Monthly, December 1989, Table 4. Delivered to Consumers, 1973-1088-ELA, Natural Gas Annual 1991, Table 98. - 1987 forward: ElA, Natural Gas Monthly, May 1994, Table 4. 


\section{Energy Prices Notes}

1. The average domestic first purchase price represents the average price at which all domestic crude oil is purchased. Prior to February 1976, the price represented an estimate of the average of posted prices; beginning with February 1976, the price represents an average of actual first purchase prices. The data series was previously called "Actual Domestic Wellhead Price."

2. F.O.B. literally means "Free on Board." It denotes a transaction whereby the seller makes the product available with an agreement on a given port at a given price; it is the responsibility of the buyer to arrange for the transportation and insurance.

3. The landed cost of imported crude oil from selected countries does not represent the total cost of all imported crude. Prior to March 1975, imported crude costs to U.S. company-owned refineries in the Caribbean were not included in the landed cost, and costs of crude oil from countries that export only small amounts to the United States were also excluded. Beginning in March 1975, however, coverage was expanded to include U.S. company-owned refineries in the Caribbean. Landed costs do not include supplemental fees.

4. Beginning with January 1981, refiner acquisition costs of crude oil are from data collected on Form EIA14, "Refiners' Monthly Cost Report." Those costs were previously published from data collected on Form ERA49. "Domestic Crude Oil Entitlements Program Refiners Monthly Report." Form ERA-49 was discontinued with the decontrol of crude oil on January 28, 1981. Crude oil purchases and costs are defined for Form EIA-14 in accordance with conventions used for Form ERA-49. Also, the respondents for the two forms are essentially the same. However, due to possible different interpretations of the filing requirements and a different method for handling prior period adjustments, care must be taken when comparing the data collected on the two forms.

The refiner acquisition cost of crude oil is the average price paid by refiners for crude oil booked into their refineries in accordance with accounting procedures generally accepted and consistently and historically applied by the refiners concerned. Domestic crude oil is that oil produced in the United States or from the outer continental shelf as defined in 43 USC Section 1331. Imported crude oil is either that oil reported on Form ERA-51, "Transfer Pricing Report," or any crude oil that is not domestic oil. The composite cost is the weighted average of domestic and imported crude oil costs.

Crude oil costs and volumes reported on Form ERA-49 excluded unfinished oils but included the Strategic Petroleum Reserve (SPR). Crude oil costs and volumes reported on Form FEA-P110-M-1, "Refiners' Monthly Cost Allocation Report," included unfinished oils but excluded SPR. Imported averages derived from Form
ERA-49 exclude oil purchased for SPR, whereas the composite averages derived from Form ERA-49 include SPR. None of the prices derived from Form EIA-14 include either unfinished oils or SPR.

5. Several different series of motor gasoline prices are published in this section. U.S. City Average Retail Prices of Motor Gasoline are calculated monthly by the Bureau of Labor Statistics during the development of the Consumer Price Index (CPI). These prices include all Federal, State, and local taxes paid at the time of sale. For the period 1974-1977, prices were collected in 56 urban areas. For the period 1978 forward, prices were collected from a new sample of service stations in 85 urban areas selected to represent all urban consumersabout 80 percent of the total U.S. population. The service stations are selected initially, and on a replacement basis, in such a way that they represent the purchasing habits of the CPI population. Service stations in the current sample include those providing all types of service (i.e., full-, mini-, and self-serve).

Refiner prices of finished motor gasoline for resale and to end users are determined by the Energy Information Administration (EIA) in a monthly survey of refiners and gas plant operators (Form EIA-782A). The prices do not include any Federal, State, or local taxes paid at the time of sale. Estimates of prices prior to January 1983 are based on Form FEA-P302-M-1/EIA-460, "Petroleum Industry Monthly Report for Product Prices," and also exclude all Federal, State, or local taxes paid at the time of sale. Sales for resale are those made to purchasers who are other-than-ultimate consumers. Sales to end users are sales made directly to the consumer of the product, including bulk consumers, such as agriculture, industry, and utilities, as well as residential and commercial consumers.

6. Starting in January 1983, Form EIA-782, "Monthly Petroleum Product Sales Report," replaced 10 previous surveys. Every attempt was made to continue the most important price series. However, prices published through December 1982 and those published since January 1983 do not necessarily form continuous data series due to changes in survey forms, definitions, instructions, populations, samples, processing systems, and statistical procedures. To provide historical data, continuous series were generated for annual data 1978-1982 and for monthly data 1981 and 1982 by estimating the prices that would have been published had Form ElA-782 survey and system been in operation at that time. This form of estimation was performed after detailed adjustment was made for product and sales type matching and for discontinuity due to other factors. An important difference between the previous and present prices is the distinction between wholesale and resale and between retail and end user. The resale category continues to include sales among resellers. However, bulk sales to utility, industrial, and commercial accounts previously included in the wholesale category are now counted as made to end users. The end-user category continues to include retail sales through company owned and operated 
outlets but also includes the bulk utility, industrial, and commercial sales. Additional information may be found in Estimated Historic Time Series for the EIA-782, a feature article reprinted from the December 1983 [3] Petroleum Marketing Monthly, published by EIA.

7. National average electricity prices are shown in two data series. The "Annual Series" is based on data from more than 3,000 publicly and privately owned electric utilities that report on Form EIA-861, "Annual Electric Utility Report." The "Monthly Series" is based on data from over 400 utilities statistically chosen as a stratified sample of the utilities that report on Form EIA-861. The selected utilities report monthly on Form EIA-826, "Monthly Electric Utility Sales and Revenue Report with State Distributions," formerly the "Electric Utility Company Monthly Statement." Annual values shown for the monthly series are the sum of the monthly revenue divided by the sum of the monthly sales. Prior to January 1986 , only privately owned utilities were included in the monthly survey and the sample was chosen by using cut-off, rather than stratification, techniques.

8. Data for 1973-1982 cover all electric generating plants at which the generator nameplate capacity of all steam-electric units combined totaled 25 megawats or greater. From 1974-1982, peaking units were included in the data and counted towards the 25-megawatt-or-greater total. Data for 1983-1990 cover all electric generating plants at which the generator nameplate capacity of all steam-electric units combined totaled 50 megawatts or greater. Data for 1991 forward cover all electric generating plants at which the generator nameplate capacity of all steam-electric units and combined-cycle units together totaled 50 megawatts or greater.

9. Natural gas prices are intended to include all taxes. Instructions on the data collection forms specifically direct that all Federal, State, and local taxes, surcharges, and/or adjustments billed to consumers are to be included. However, sales and other taxes itemized on consumers' bills are sometimes excluded by the reporting utilities.

Delivered-to-consumers prices for 1987 forward represent natural gas delivered and sold to residential, commercial, industral, and electric utility consumers. They do not include the price of natural gas delivered to industrial and commercial consumers on behalf of third parties. Volumes of natural gas delivered on behalf of third parties are included in the consumption data shown in Table 4.3. Additional information is available in the EIA Natural Gas Monthly, Appendix C. 


\section{Section 10. International Energy}

Crude Oll Production. World crude oil production during February 1994 was 60 million barrels per day, down 0.2 million barrels per day from the level in the previous month.

Organization of Petroleum Exporting Countries (OPEC) production during February 1994 averaged 26 million barrels per day, down slightly from the level during the previous month. Production by the Arab members of OPEC in February 1994 averaged 16 million barrels per day, up slightly from the January 1994 level. During February 1994, production increased in the United Arab Emirates by 25 thousand barrels per day and in Kuwait by 3 thousand barrels per day. Production decreased in Qatar by 15 thousand barrels per day and in Saudi Arabia by 7 thousand barrels per day. Production remained unchanged in Algeria, Iraq, and Libya. Among the non-Arab members of OPEC, production during February 1994 decreased in Iran by 50 thousand barrels per day. Production remained unchanged in Indonesia, Nigeria, and Venezuela.

Among the non-OPEC nations, production during February 1994 increased in China by 20 thousand barrels per day and in Mexico by 5 thousand barrels per day. Production decreased in the former U.S.S.R. by 210 thousand barrels per day, the United States by 32 thousand barrels per day, and the United Kingdom by 15 thousand barrels per day. Production remained unchanged in Canada.

Petroleum Consumption. In December 1993, consumption in all Organization for Economic Cooperation and Development (OECD) countries was 40.8 million barrels per day, slightly less than the December 1992 rate. The consumption rate was higher than it was 1 year ago in Italy $(+6 \text { percent })^{9}$, Germany $(+5$ percent $)$, and the United Kingdom $(+1$ percent). Consumption was lower in France ( -7 percent), Japan ( -6 percent), and Canada and the United States (both down less than 1 percent), compared with levels 1 year earlier.

Petroleum Stocks. For all OECD countries, petroleum stocks at the end of December 1993 totaled 3.6 billion barrels, 1 percent higher than the ending stock level in December 1992. Stock levels were higher than the levels 1 year ago in France $(+8$ percent), the United Kingdom ( +4 percent), the United States $(+3$ percent), and Japan $(+2$ percent). Stocks were lower in Italy ( -6 percent), Canada ( -3 percent), and Germany (down less than 1 percent) compared with levels 1 year earlier.

Nuclear Electricity Generation. Based on Nucleonics Week information for February 1994, all reporting countries with nuclear capacity generated 177.0 gross terawatthours $^{10}$ of nuclear-generated electricity.

During 1993, 9 nuclear units became operable: Comanche Peak-2 in the United States; Darlington-4 in Canada; Guangdong-1 in China; Golfech-2 in France; Shika-1, Hamaoka-4, Genkai-3, and Kashiwazaki Kariwa-4 in Japan; and Balakova-4 in Russia. However, 3 units were permanently shutdown in 1993: Trojan in the United States; and Trawsfynydd-1 and Trawsfynydd-2 in the United Kingdom.

Guangdong-2, a 984-gross megawatt pressurized lightwater reactor unit in China, became operable on February 7, 1994.

As of February 28, 1994, there were 431 operable nuclear generating units in the world.

\footnotetext{
${ }^{9}$ Percentage changes are based on unrounded data.

${ }^{10}$ One terawathour equals 1 billion kilowatthours.
} 
(Thousand Barrels per Day)

\begin{tabular}{|c|c|c|c|c|c|c|c|c|c|c|c|c|}
\hline & Algorla & Ireq & Kumait & Llbye & Oatar & $\begin{array}{c}\text { Saudl } \\
\text { Arabla }\end{array}$ & $\begin{array}{c}\text { United } \\
\text { Arab } \\
\text { Enilratos }\end{array}$ & $\begin{array}{c}\text { Arab } \\
\text { OPECb }\end{array}$ & Indonesle & Iran & Nigerla & Vencexuel \\
\hline 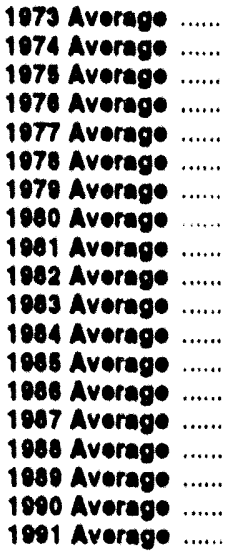 & $\begin{array}{r}1,007 \\
1,000 \\
083 \\
1,075 \\
1,182 \\
1,231 \\
1,224 \\
1,100 \\
1,002 \\
087 \\
088 \\
1,014 \\
1,037 \\
048 \\
1,046 \\
1,040 \\
1,008 \\
1,175 \\
1,230\end{array}$ & $\begin{array}{r}2,018 \\
1,071 \\
2,202 \\
2,418 \\
2,348 \\
2,803 \\
3,477 \\
2,814 \\
1,000 \\
1,012 \\
1,005 \\
1,200 \\
1,433 \\
1,600 \\
2,070 \\
2,005 \\
2,007 \\
2,040 \\
308\end{array}$ & 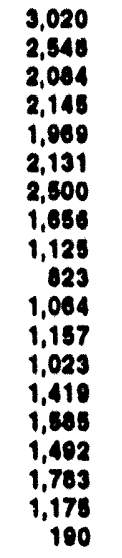 & $\begin{array}{l}2,175 \\
1,921 \\
1,480 \\
1,033 \\
2,083 \\
1,083 \\
2,092 \\
1,787 \\
1,140 \\
1,160 \\
1,105 \\
1,097 \\
1,050 \\
1,034 \\
972 \\
1,175 \\
1,150 \\
1,375 \\
1,483\end{array}$ & $\begin{array}{l}570 \\
518 \\
438 \\
407 \\
448 \\
487 \\
508 \\
472 \\
405 \\
330 \\
205 \\
394 \\
301 \\
308 \\
203 \\
340 \\
300 \\
408 \\
305\end{array}$ & 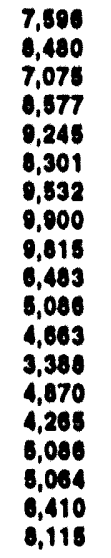 & $\begin{array}{l}1,833 \\
1,670 \\
1,684 \\
1,030 \\
1,090 \\
1,831 \\
1,831 \\
1,700 \\
1,474 \\
1,280 \\
1,149 \\
1,146 \\
1,193 \\
1,330 \\
1,841 \\
1,608 \\
1,860 \\
2,117 \\
2,386\end{array}$ & $\begin{array}{r}18,008 \\
17,724 \\
18,085 \\
18,870 \\
18,221 \\
18,628 \\
21,183 \\
10,144 \\
16,081 \\
12,038 \\
10,072 \\
10,070 \\
8,434 \\
11,908 \\
11,783 \\
13,380 \\
14,220 \\
14,008 \\
14,104\end{array}$ & $\begin{array}{l}1,330 \\
1,376 \\
1,307 \\
1,304 \\
1,606 \\
1,638 \\
1,501 \\
1,377 \\
1,805 \\
1,330 \\
1,343 \\
1,412 \\
1,326 \\
1,300 \\
1,343 \\
1,342 \\
1,400 \\
1,402 \\
1,302\end{array}$ & $\begin{array}{l}8,001 \\
6,022 \\
8,380 \\
8,083 \\
8,083 \\
8,242 \\
3,188 \\
1,082 \\
1,380 \\
2,214 \\
2,440 \\
2,174 \\
2,250 \\
2,038 \\
2,290 \\
2,240 \\
2,010 \\
3,080 \\
3,312\end{array}$ & $\begin{array}{l}2,054 \\
2,286 \\
1,703 \\
2,007 \\
2,008 \\
1,007 \\
2,302 \\
2,068 \\
1,493 \\
1,201 \\
1,241 \\
1,398 \\
1,498 \\
1,467 \\
1,341 \\
1,480 \\
1,710 \\
1,010 \\
1,002\end{array}$ & 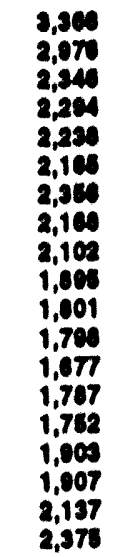 \\
\hline $\begin{array}{l}1092 \text { January ........ } \\
\text { February ...... } \\
\text { March ........... } \\
\text { April ............ } \\
\text { May .............. } \\
\text { June ........... } \\
\text { July .............. } \\
\text { Auguit ........ } \\
\text { September ... } \\
\text { October ........ } \\
\text { November .... } \\
\text { December .... } \\
\text { Average ...... }\end{array}$ & $\begin{array}{l}1,230 \\
1,230 \\
1,230 \\
1,230 \\
1,210 \\
1,210 \\
1,210 \\
1,210 \\
1,210 \\
1,210 \\
1,210 \\
1,210 \\
1,217\end{array}$ & $\begin{array}{l}450 \\
450 \\
450 \\
450 \\
450 \\
450 \\
450 \\
450 \\
450 \\
450 \\
450 \\
450 \\
450\end{array}$ & $\begin{array}{r}565 \\
630 \\
735 \\
863 \\
915 \\
1,015 \\
1,080 \\
1,130 \\
1,200 \\
1,280 \\
1,375 \\
1,550 \\
1,020\end{array}$ & $\begin{array}{l}1,550 \\
1,550 \\
1,450 \\
1,500 \\
1,450 \\
1,450 \\
1,450 \\
1,425 \\
1,475 \\
1,500 \\
1,500 \\
1,500 \\
1,403\end{array}$ & $\begin{array}{l}360 \\
325 \\
375 \\
375 \\
375 \\
375 \\
400 \\
425 \\
425 \\
440 \\
440 \\
440 \\
396\end{array}$ & $\begin{array}{l}8,790 \\
8,640 \\
8,260 \\
8,213 \\
8,265 \\
8,315 \\
8,360 \\
8,400 \\
8,450 \\
8,505 \\
8,500 \\
8,575 \\
8,438\end{array}$ & $\begin{array}{l}2,435 \\
2,425 \\
2,300 \\
2,300 \\
2,300 \\
2,275 \\
2,300 \\
2,330 \\
2,320 \\
2,310 \\
2,305 \\
2,305 \\
2,325\end{array}$ & $\begin{array}{l}15,370 \\
15,250 \\
14,800 \\
14,930 \\
14,965 \\
15,090 \\
15,240 \\
15,370 \\
15,530 \\
15,695 \\
15,780 \\
16,030 \\
18,338\end{array}$ & $\begin{array}{l}1,580 \\
1,605 \\
1,630 \\
1,605 \\
1,530 \\
1,560 \\
1,550 \\
1,540 \\
1,550 \\
1,550 \\
1,550 \\
1,550 \\
1,560\end{array}$ & $\begin{array}{l}3,500 \\
3,500 \\
3,350 \\
3,250 \\
3,250 \\
3,250 \\
3,300 \\
3,450 \\
3,450 \\
3,650 \\
3,650 \\
3,550 \\
3,420\end{array}$ & $\begin{array}{l}1,075 \\
1,025 \\
1,000 \\
1,025 \\
1,025 \\
1,025 \\
1,075 \\
2,000 \\
2,025 \\
2,060 \\
2,050 \\
2,100 \\
1,002\end{array}$ & $\begin{array}{l}2,300 \\
2,340 \\
2,190 \\
2,190 \\
2,200 \\
2,290 \\
2,290 \\
2,340 \\
2,300 \\
2,440 \\
2,440 \\
2,415 \\
2,334\end{array}$ \\
\hline $\begin{array}{l}1893 \text { January ........ } \\
\text { Fobruary ...... } \\
\text { March ........... } \\
\text { April ............ } \\
\text { May .............. } \\
\text { June ........... } \\
\text { July .............. } \\
\text { Auguet ........ } \\
\text { Seplember ... } \\
\text { October ........ } \\
\text { November ... } \\
\text { December .... } \\
\text { Average ....... }\end{array}$ & $\begin{array}{l}1,210 \\
1,210 \\
1,200 \\
1,200 \\
1,200 \\
1,200 \\
1,180 \\
1,180 \\
1,180 \\
1,180 \\
1,170 \\
1,170 \\
1,100\end{array}$ & $\begin{array}{l}500 \\
500 \\
500 \\
500 \\
500 \\
500 \\
500 \\
500 \\
530 \\
530 \\
540 \\
540 \\
512\end{array}$ & $\begin{array}{l}1,675 \\
1,865 \\
1,650 \\
1,645 \\
1,713 \\
1,775 \\
1,840 \\
2,045 \\
2,020 \\
2,045 \\
2,045 \\
2,050 \\
1,872\end{array}$ & $\begin{array}{l}1,480 \\
1,425 \\
1,360 \\
1,360 \\
1,360 \\
1,360 \\
1,360 \\
1,370 \\
1,370 \\
1,390 \\
1,370 \\
1,370 \\
1,377\end{array}$ & $\begin{array}{l}450 \\
430 \\
400 \\
400 \\
420 \\
400 \\
410 \\
410 \\
410 \\
410 \\
410 \\
410 \\
413\end{array}$ & $\begin{array}{l}8,500 \\
8,440 \\
8,300 \\
8,000 \\
8,000 \\
8,150 \\
8,240 \\
8,345 \\
8,270 \\
8,145 \\
7,995 \\
8,000 \\
8,198\end{array}$ & $\begin{array}{l}2,295 \\
2,305 \\
2,270 \\
2,270 \\
2,230 \\
2,230 \\
2,210 \\
2,210 \\
2,220 \\
2,220 \\
2,220 \\
2,220 \\
2,241\end{array}$ & $\begin{array}{l}16,110 \\
16,175 \\
15,670 \\
15,365 \\
15,413 \\
15,605 \\
15,830 \\
16,060 \\
18,000 \\
15,920 \\
15,750 \\
15,760 \\
15,803\end{array}$ & $\begin{array}{l}1,550 \\
1,530 \\
1,500 \\
1,480 \\
1,510 \\
1,510 \\
1,510 \\
1.510 \\
1,510 \\
1,480 \\
1,480 \\
1,510 \\
1,507\end{array}$ & $\begin{array}{l}3,650 \\
3,750 \\
3,700 \\
3,500 \\
3,650 \\
3,650 \\
3,800 \\
3,500 \\
3,650 \\
3,700 \\
3,550 \\
3,700 \\
3,650\end{array}$ & $\begin{array}{l}2,125 \\
2,105 \\
2,075 \\
2,025 \\
2,025 \\
1,095 \\
1,075 \\
2,025 \\
2,045 \\
2,005 \\
2,025 \\
2,175 \\
2,080\end{array}$ & $\begin{array}{l}2,410 \\
2,390 \\
2,340 \\
2,340 \\
2,340 \\
2,340 \\
2,390 \\
2,390 \\
2,340 \\
2,400 \\
2,400 \\
2,400 \\
2,377\end{array}$ \\
\hline $\begin{array}{l}1904 \text { January ........ } \\
\text { Fobruary ...... } \\
\text { 2-Mo. Avo. .. }\end{array}$ & $\begin{array}{l}1.170 \\
1.170 \\
1,170\end{array}$ & $\begin{array}{l}540 \\
540 \\
840\end{array}$ & $\begin{array}{l}1,995 \\
1,998 \\
1,908\end{array}$ & $\begin{array}{l}1.370 \\
1,370 \\
1,370\end{array}$ & $\begin{array}{l}410 \\
395 \\
403\end{array}$ & $\begin{array}{l}8,095 \\
8,088 \\
8,091\end{array}$ & $\begin{array}{l}2,220 \\
2,245 \\
2,232\end{array}$ & $\begin{array}{l}15,800 \\
15,805 \\
15,802\end{array}$ & $\begin{array}{l}1,510 \\
1,510 \\
1,510\end{array}$ & $\begin{array}{l}3,600 \\
3,550 \\
3,576\end{array}$ & $\begin{array}{l}2,175 \\
2,175 \\
2,176\end{array}$ & $\begin{array}{r}2,400 \\
2,400 \\
2,400\end{array}$ \\
\hline $\begin{array}{l}1003 \text { 2-Mo. Avg. .. } \\
1092 \text { 2-Mo. Avg. .. }\end{array}$ & $\begin{array}{l}1,210 \\
1,230\end{array}$ & $\begin{array}{l}500 \\
450\end{array}$ & $\begin{array}{r}1,785 \\
596\end{array}$ & $\begin{array}{l}1,454 \\
1,350\end{array}$ & $\begin{array}{l}441 \\
338\end{array}$ & $\begin{array}{l}8,472 \\
8,718\end{array}$ & $\begin{array}{l}2,300 \\
2,430\end{array}$ & $\begin{array}{l}16,141 \\
15,312\end{array}$ & $\begin{array}{l}1,541 \\
1,592\end{array}$ & $\begin{array}{l}3,697 \\
3,500\end{array}$ & $\begin{array}{l}2,116 \\
1,081\end{array}$ & $\begin{array}{l}2,401 \\
2,364\end{array}$ \\
\hline
\end{tabular}

- Includes about one-hall of the production in the Kuwait-Saudi Arabia Neutral Zone Irom 1973 through July 1990 and in June 1991. Kuwaili Neutral Zone output was discontinued Iollowing Iraq's invasion of Kuwait on August 2. 1990. but was resumed in June 1991. In February 1994. Neutral Zone production by both Kuwall and Saudi Arabla lotaled about 375 thousand barrels per day.

- The Arab members of the Organization of Petroleum Exporting Countries (OPEC) are Algeria, Iraq, Kuwail, Libya, Qatar, Saudi Arabia, and the Uniled Arab Emirates. Production in the Neutral Zone between Kuwail and Saudi Arabia is included in "Arab OPEC."

$\mathrm{A}=$ Pevised data.

Notes: - Crude oll includes lease condensate but excludes nalural gas plant liquids. - U.S. goographic coverage is the 50 States and the District of Columbia. - Monthly data are often preliminary figures and may nol average to the annual totals because of rounding or because updates to the preliminary monthly data are nol avallable.

Sources: - Unitod states: Table 3.1a. - Other Countrics: Annual Data-1973-1970-Energy Inlormation Administralion (ELA), Intermationa Energy Annual 1981, Table 8. 1080-EIA, International Energy Annual 1089. Table 1. 1081-EIA, International Energy Annual 1990, Table 1. 1082-EU. International Energy Annual 1991. Table 1. 1083-1002-EL, Intemationa Energy Annual 1992, Table 1. 1993-Average of monihly data. Monthly data-Petrokum Intelligence Weoks, the Oil and Gas Jouma, and other industry sources. - World: Annual date-1973-1070-ElA, Intemationa Energy Annual 1981. Table 8. 1080-EIA, International Energy Annud 1989. Table 1. 1981-EIA, International Energy Annual 1990, Table 1. 1082-EL, Internitional Energy Annual 1991. Table 1. 1083-1892-EU. Intermatione Energy Annual 1992. Table 1. 1093-Average of monthly data. Monthly data-ElA, Intemational Petroloum Statistics Report, sum of all countiles' monthly data. 
Table 10.1b World Crude Oil Production: Total OPEC, Canada Through Former U.S.S.R., and World

(Thousand Barrels per Day)

\begin{tabular}{|c|c|c|c|c|c|c|c|c|c|c|}
\hline & $\begin{array}{l}\text { Total } \\
\text { OPEC }\end{array}$ & $\begin{array}{l}\text { Persian } \\
\text { Qulf } \\
\text { Nattons }\end{array}$ & Canada & Moxlco & $\begin{array}{l}\text { Unitod } \\
\text { Kingdom }\end{array}$ & $\begin{array}{l}\text { Unlted } \\
\text { States }\end{array}$ & China & $\begin{array}{l}\text { Former } \\
\text { U.S.S.R. }\end{array}$ & Othere & World \\
\hline 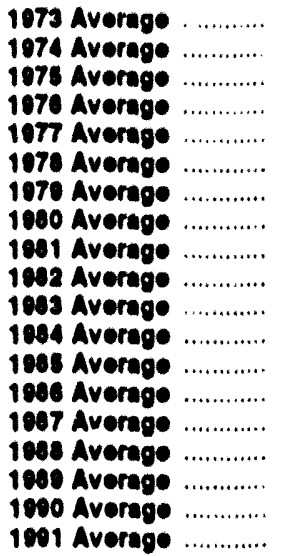 & $\begin{array}{l}30,770 \\
30,582 \\
28,094 \\
30,540 \\
31,115 \\
20,670 \\
30,784 \\
26,781 \\
20,032 \\
18,034 \\
17,054 \\
17,800 \\
18,353 \\
18,441 \\
18,072 \\
20,403 \\
22,270 \\
20,485 \\
23,860\end{array}$ & $\begin{array}{r}20,068 \\
21,292 \\
18,034 \\
21,814 \\
21,726 \\
20,804 \\
21,096 \\
17,081 \\
18,248 \\
12,186 \\
11,081 \\
10,784 \\
9,830 \\
11,696 \\
12,103 \\
13,487 \\
14,937 \\
18,270 \\
14,741\end{array}$ & $\begin{array}{l}1,708 \\
1,551 \\
1,430 \\
1,314 \\
1,321 \\
1,316 \\
1,500 \\
1,438 \\
1,283 \\
1,271 \\
1,388 \\
1,438 \\
1,471 \\
1,474 \\
1,535 \\
1,618 \\
1,880 \\
1,853 \\
1,548\end{array}$ & $\begin{array}{r}468 \\
571 \\
705 \\
831 \\
981 \\
1,200 \\
1,461 \\
1,938 \\
2,313 \\
2,748 \\
2,689 \\
2,780 \\
2,748 \\
2,438 \\
2,548 \\
2,512 \\
2,520 \\
2,563 \\
2,680\end{array}$ & $\begin{array}{r}2 \\
2 \\
12 \\
248 \\
768 \\
1,082 \\
1,868 \\
1,622 \\
1,811 \\
2,088 \\
2,291 \\
2,480 \\
2,530 \\
2,530 \\
2,408 \\
2,232 \\
1,802 \\
1,020 \\
1,707\end{array}$ & 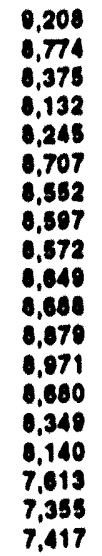 & $\begin{array}{l}1,000 \\
1,319 \\
1,490 \\
1,070 \\
1,874 \\
2,082 \\
2,182 \\
2,114 \\
2,012 \\
2,048 \\
2,120 \\
2,200 \\
2,305 \\
2,820 \\
2,090 \\
2,730 \\
2,757 \\
2,774 \\
2,835\end{array}$ & $\begin{array}{r}9,324 \\
0,012 \\
9,823 \\
10,080 \\
10,003 \\
11,108 \\
11,384 \\
11,708 \\
11,050 \\
11,012 \\
11,072 \\
11,061 \\
11,588 \\
11,808 \\
11,085 \\
11,070 \\
11,028 \\
10,080 \\
0,087\end{array}$ & $\begin{array}{r}4,013 \\
4,030 \\
4,300 \\
4,843 \\
4,700 \\
4,084 \\
6,303 \\
6,409 \\
8,601 \\
6,087 \\
, 406 \\
7,183 \\
7,021 \\
6,143 \\
0,416 \\
0,071 \\
9,017 \\
10,070 \\
10,373\end{array}$ & 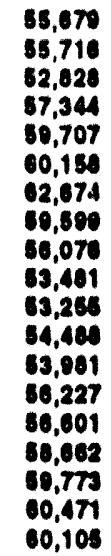 \\
\hline 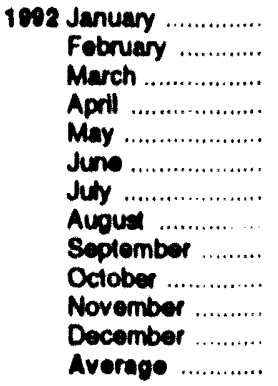 & $\begin{array}{l}25,100 \\
24,080 \\
24,170 \\
24,205 \\
24,265 \\
24,420 \\
24,660 \\
25,005 \\
25,245 \\
25,685 \\
25,770 \\
25,945 \\
24,047\end{array}$ & $\begin{array}{l}16,130 \\
16,010 \\
15,510 \\
15,487 \\
15,592 \\
15,716 \\
15,916 \\
16,220 \\
16,330 \\
16,670 \\
16,755 \\
16,905 \\
16,104\end{array}$ & $\begin{array}{l}1,585 \\
1,560 \\
1,620 \\
1,535 \\
1,510 \\
1,560 \\
1,630 \\
1,675 \\
1,620 \\
1,665 \\
1,640 \\
1,575 \\
1,508\end{array}$ & $\begin{array}{l}2.675 \\
2,665 \\
2,680 \\
2,680 \\
2,660 \\
2,680 \\
2,680 \\
2,685 \\
2,685 \\
2,655 \\
2,640 \\
2,655 \\
2,688\end{array}$ & $\begin{array}{l}1,920 \\
1,905 \\
1,755 \\
1,836 \\
1,700 \\
1,545 \\
1,780 \\
1,825 \\
1,830 \\
1,930 \\
1,945 \\
1,936 \\
1,825\end{array}$ & $\begin{array}{l}7.381 \\
7.389 \\
7.348 \\
7,293 \\
7.169 \\
7.167 \\
7.131 \\
6.022 \\
7.030 \\
7.126 \\
7.024 \\
7.103 \\
7.171\end{array}$ & $\begin{array}{l}2,830 \\
2,865 \\
2,835 \\
2,855 \\
2,835 \\
2,830 \\
2,825 \\
2,815 \\
2,860 \\
2,875 \\
2,845 \\
2,785 \\
2,838\end{array}$ & $\begin{array}{l}9,115 \\
8,850 \\
8,760 \\
0,025 \\
8,455 \\
8,440 \\
8,365 \\
8,130 \\
7,980 \\
7,965 \\
7,910 \\
7.870 \\
8,380\end{array}$ & $\begin{array}{l}10,821 \\
10,670 \\
10,744 \\
10,838 \\
10,568 \\
10,758 \\
10,818 \\
10,802 \\
10,873 \\
11,017 \\
10,847 \\
11,074 \\
10,820\end{array}$ & $\begin{array}{l}61,407 \\
60,584 \\
50,912 \\
60,266 \\
50,160 \\
50,400 \\
50,860 \\
50,858 \\
60,123 \\
60,918 \\
60,621 \\
60,942 \\
60,258\end{array}$ \\
\hline 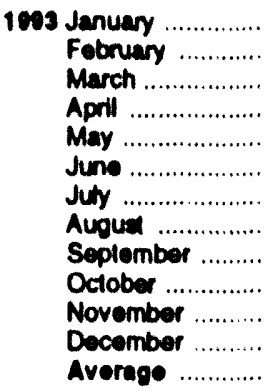 & $\begin{array}{l}26,145 \\
26,250 \\
25,585 \\
25,010 \\
25,238 \\
25,400 \\
25,785 \\
25,775 \\
25,875 \\
25,795 \\
25,495 \\
25,835 \\
25,681\end{array}$ & $\begin{array}{l}17,105 \\
17,325 \\
16,855 \\
16,350 \\
16,548 \\
16,740 \\
17,136 \\
17,045 \\
17,135 \\
17,085 \\
16,795 \\
16,955 \\
18,021\end{array}$ & $\begin{array}{l}1,570 \\
1,610 \\
1,635 \\
1,605 \\
1,630 \\
1,725 \\
1,710 \\
1,770 \\
1,740 \\
1,725 \\
1,675 \\
1,710 \\
1,670\end{array}$ & $\begin{array}{l}2,605 \\
2.610 \\
2.635 \\
2.674 \\
2.673 \\
2.675 \\
2.650 \\
2.650 \\
2.700 \\
2.700 \\
2.730 \\
2.745 \\
2,671\end{array}$ & $\begin{array}{l}1,815 \\
1,925 \\
1.710 \\
1,695 \\
1.745 \\
1,675 \\
1,930 \\
1,940 \\
1,945 \\
2,060 \\
2,195 \\
2,270 \\
1,900\end{array}$ & $\begin{array}{l}7,008 \\
6,957 \\
6,976 \\
6,897 \\
6,803 \\
6,756 \\
6,654 \\
6,732 \\
6,711 \\
6,816 \\
6,888 \\
6,838 \\
6,038\end{array}$ & $\begin{array}{l}2,885 \\
2,875 \\
2,885 \\
2,900 \\
2,025 \\
2,960 \\
2,930 \\
2,855 \\
2,895 \\
2,975 \\
2,945 \\
2,898 \\
2,011\end{array}$ & $\begin{array}{l}7,800 \\
7,786 \\
7,685 \\
7,665 \\
7,495 \\
7,400 \\
7,120 \\
7,025 \\
6,915 \\
6,910 \\
6,915 \\
6,885 \\
7,297\end{array}$ & $\begin{array}{l}10,736 \\
10,877 \\
11,044 \\
11,000 \\
11,048 \\
10,731 \\
11,145 \\
11,021 \\
11,036 \\
11,260 \\
A_{11,440} \\
A_{11,463} \\
A_{11,070}\end{array}$ & 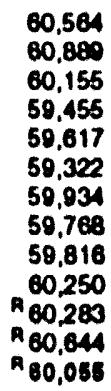 \\
\hline $\begin{array}{l}1004 \text { Jenuary .............. } \\
\text { Fobruay ............ } \\
\text { 2-Mo. Avg. ........ }\end{array}$ & $\begin{array}{r}A_{25,865} \\
25,820 \\
26,844\end{array}$ & $\begin{array}{l}16,895 \\
16,850 \\
16,074\end{array}$ & $\begin{array}{r}1,700 \\
1,700 \\
1,700\end{array}$ & $\begin{array}{l}2.745 \\
2.750 \\
2.747\end{array}$ & $\begin{array}{r}A_{2,295} \\
2,280 \\
2,200\end{array}$ & $\begin{array}{l}6,777 \\
6,745 \\
6,702\end{array}$ & $\begin{array}{l}2,900 \\
2,920 \\
2,900\end{array}$ & $\begin{array}{r}{ }^{A_{6,860}} \\
6,650 \\
6,760\end{array}$ & $\begin{array}{r}11,343 \\
11,431 \\
11,385\end{array}$ & $\begin{array}{r}A_{60,485} \\
60,298 \\
00,308\end{array}$ \\
\hline $\begin{array}{l}1003 \text { 2-Mo. Avg. ....... } \\
1002 \text { 2-Mo. Avg. ....... }\end{array}$ & $\begin{array}{l}26,108 \\
24,004\end{array}$ & $\begin{array}{l}17,200 \\
16,072\end{array}$ & $\begin{array}{l}1,589 \\
1,573\end{array}$ & $\begin{array}{l}2,607 \\
2,670\end{array}$ & $\begin{array}{l}1,867 \\
1,013\end{array}$ & $\begin{array}{r}6,084 \\
7,374\end{array}$ & $\begin{array}{l}2,080 \\
2,047\end{array}$ & $\begin{array}{l}7,703 \\
0,090\end{array}$ & $\begin{array}{l}10,003 \\
10,740\end{array}$ & $\begin{array}{l}0,710 \\
01,000\end{array}$ \\
\hline
\end{tabular}

- 'Tolal OPEC' consists of Algerta, Gabon, Indonesla, Iran, Iraq, Kuwat, Lbya. Nigeria, Oatar, Saud Arabia, the United Arab Enirales, and Venezuela. Production from the Noural Zone between Kuwatt and Saud Arabla in included in "Tolal OPEC."

- The Peralan Gut Nations are Bahrain, Iran, Iraq, Kuwat, Oatar, Saud Arabia, and the United Arab Emirales. Production from the Neutra! Zone between Kuwah and Saudi Arabia is included in "Persian Gull Nalions."

"Other ts a calculated tolal derived lrom the difference between "World" and the sum of production in 'Tolal OPEC." Canada, Mexico, the United Kingdom, the United States, China, and the tormer U.S.S.R.

Rinevised dala. Ex Estimale.

Noles: - Crude oil includes base condensale bul excludes nalural gas plant liquild. - U.S. geographic coverage is the 50 States and the Disirict of Columbia. - Monthly dala are often preliminary ligures and may not average to the annual lotals because of rounding of because updales to the preliminary monthly data are not avallable.

Sources: - United states: Table 3.12. - Other Countrice: Annual Dato-1073.1070-Energy Inlormation Administration (EU). Internationa Energy Annual 1981, Table 8. 1090-ElA, Intemational Energy Annual 1080, Table 1. 1981-EL, Intemational Energy Annual 1990, Tablo 1. 1992-EU Intermational Enorgy Annual 1991, Tablo 1. 1083-1002-ElA, Intermationa Enorgy Annual 1992, Table 1. 1003-Average of monthy data. Monthly dats-Petroleum Intelligence Weekty, the Oil and Gas loumal and other indusiny sources. - World: Annual data-1073-1970-EU, intematione Energy Annual 1981, Table 8. 1980-ElA. Intermational Energy Annual 1089, Table 1. 1981-EL, Intemational Energy Annual 1990, Table 1. 1082-EU International Energy Annual 1991, Table 1. 1083-1092-ElA, Intematione Energy Annual 1992, Table 1. 1003-Average of monthly data. Monthly dato-ELA, International Polroloum Statistice Report, eum of all countriea' monthly dala. 
Figure 10.1 Crude Oll Production

(Million Barrels per Day)

World Production, 1973-1993

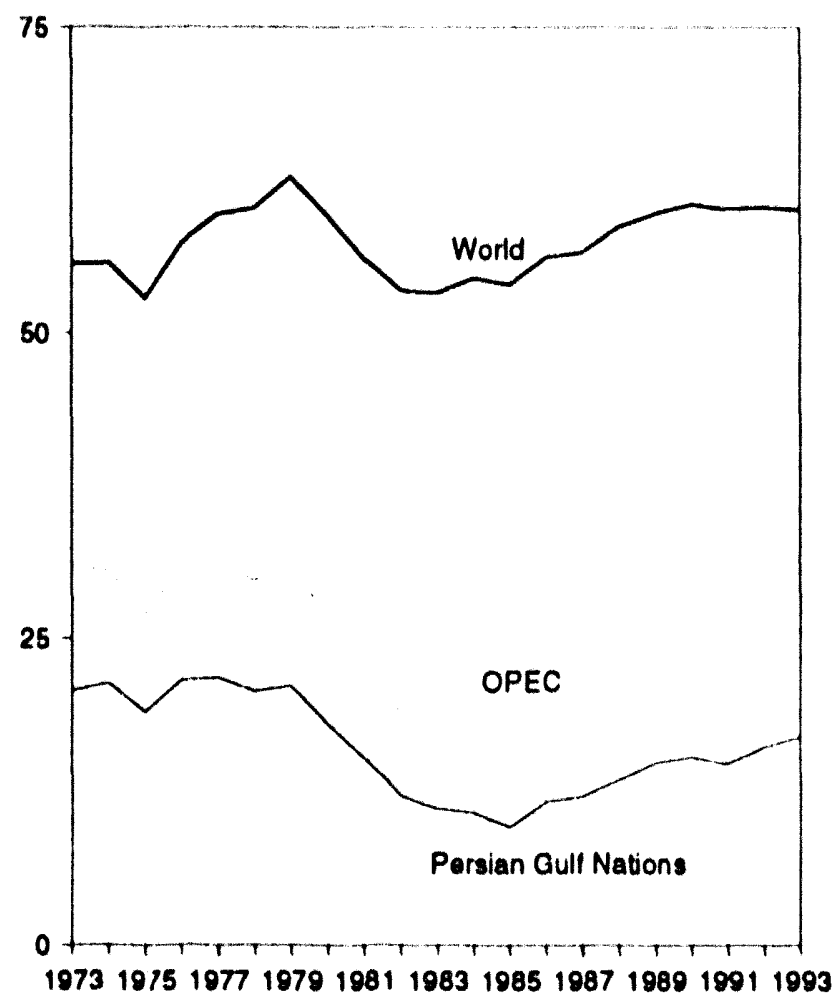

Leading Producers, 1973-1993

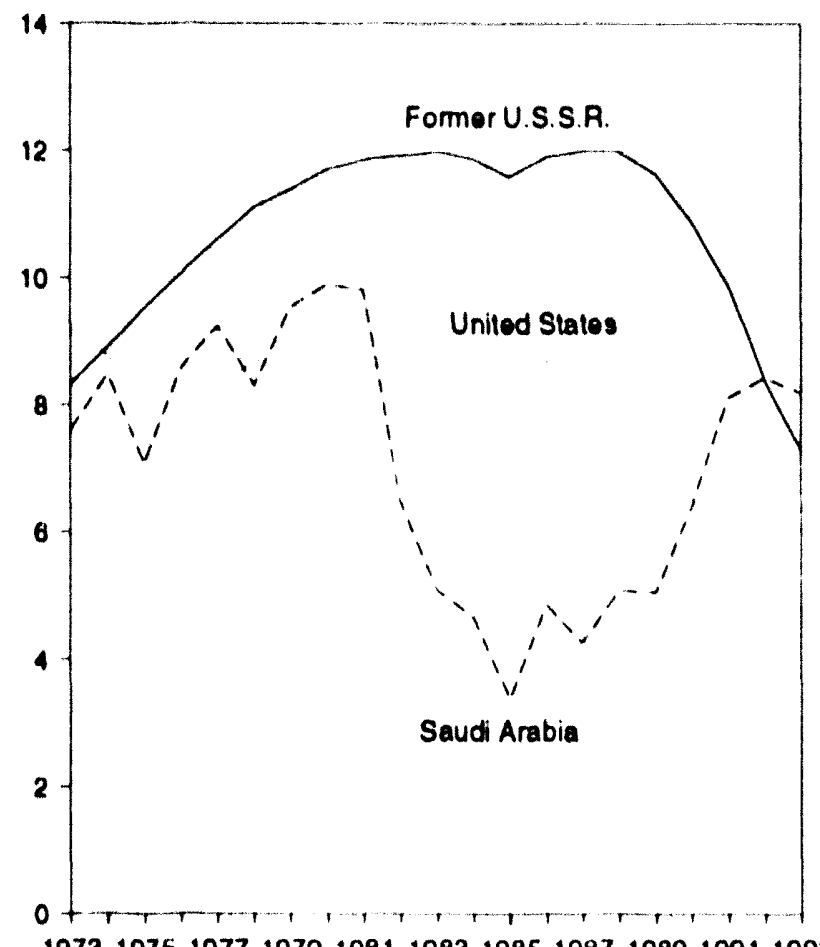

World Production, Monthly

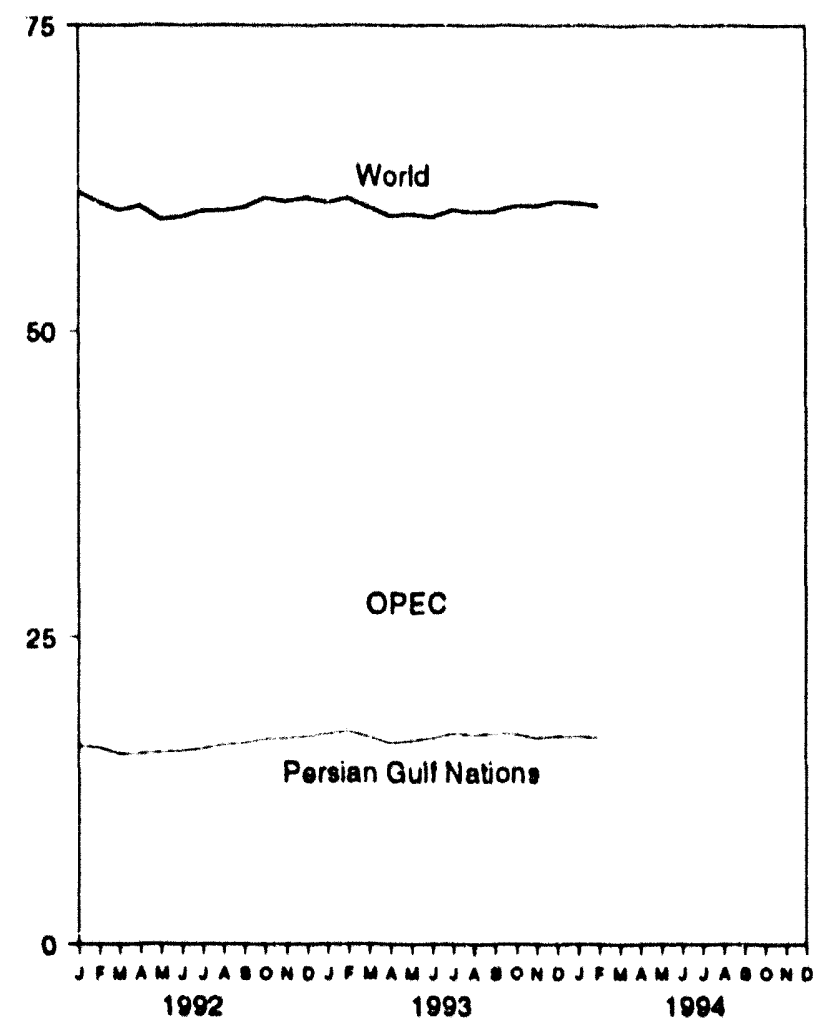

Leading Producers, Monthly

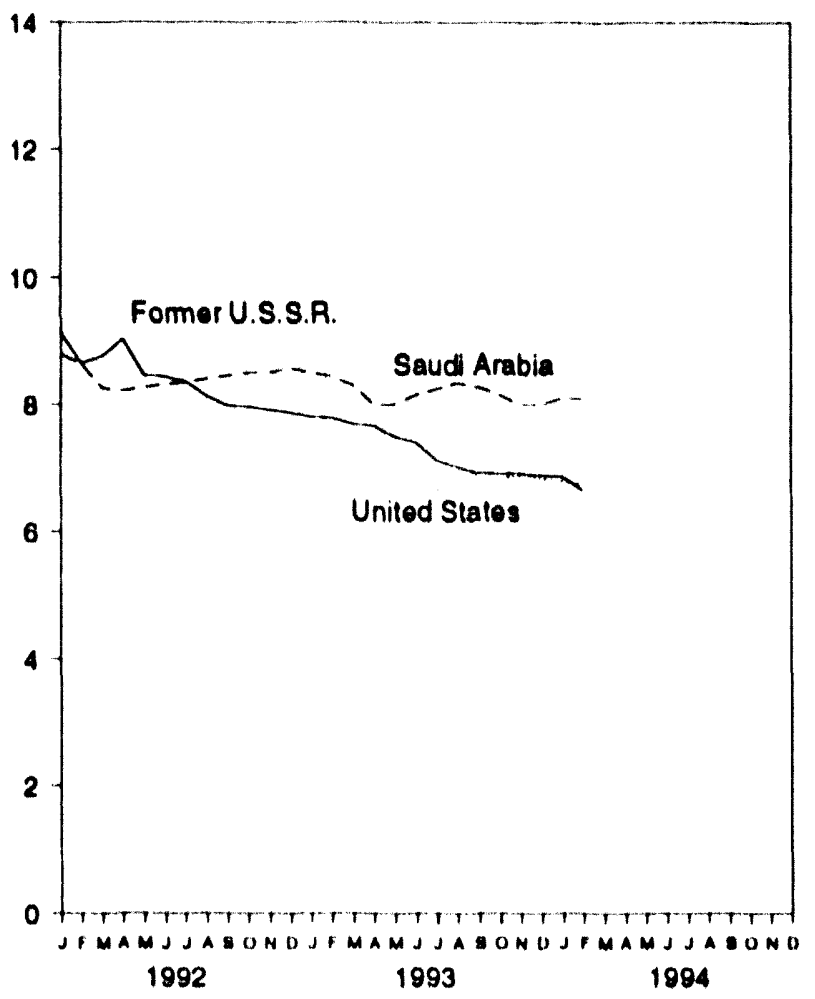

Note: OPEC is the Organization of Petroleum Exporting Counifies. Sources: Tables $10.1 \mathrm{a}$ and $10.1 \mathrm{~b}$ 
Figure 10.2 Crude Oll Production by Selected Country

(Million Barrels per Day)
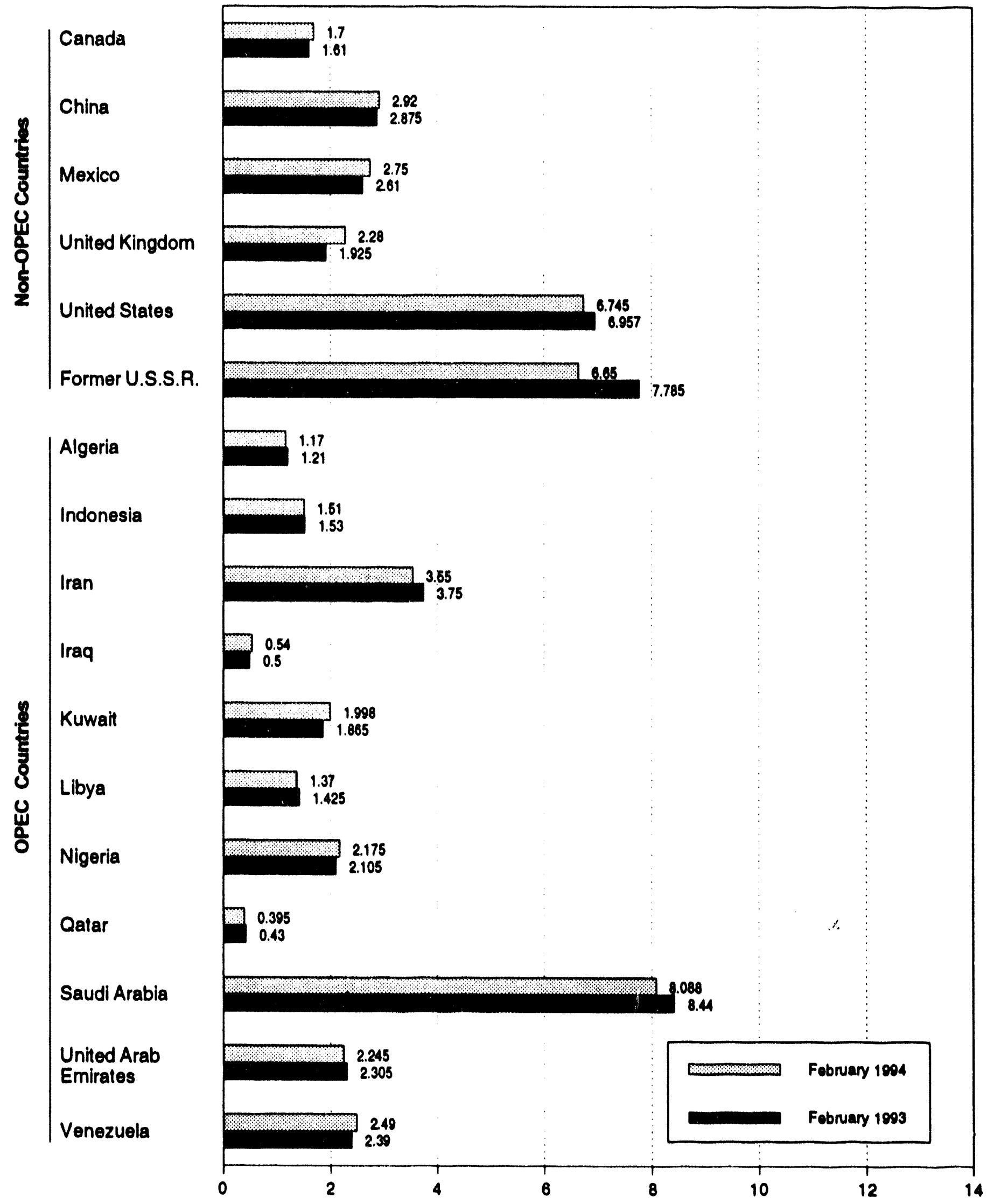

Nole: OPEC is the Organization of Petroleum Exporting Countries. 
Figure 10.3 Petroleum Consumptlon in OECD Countrles

(Million Barrels per Day)

OECD Consumption, 1973-1993

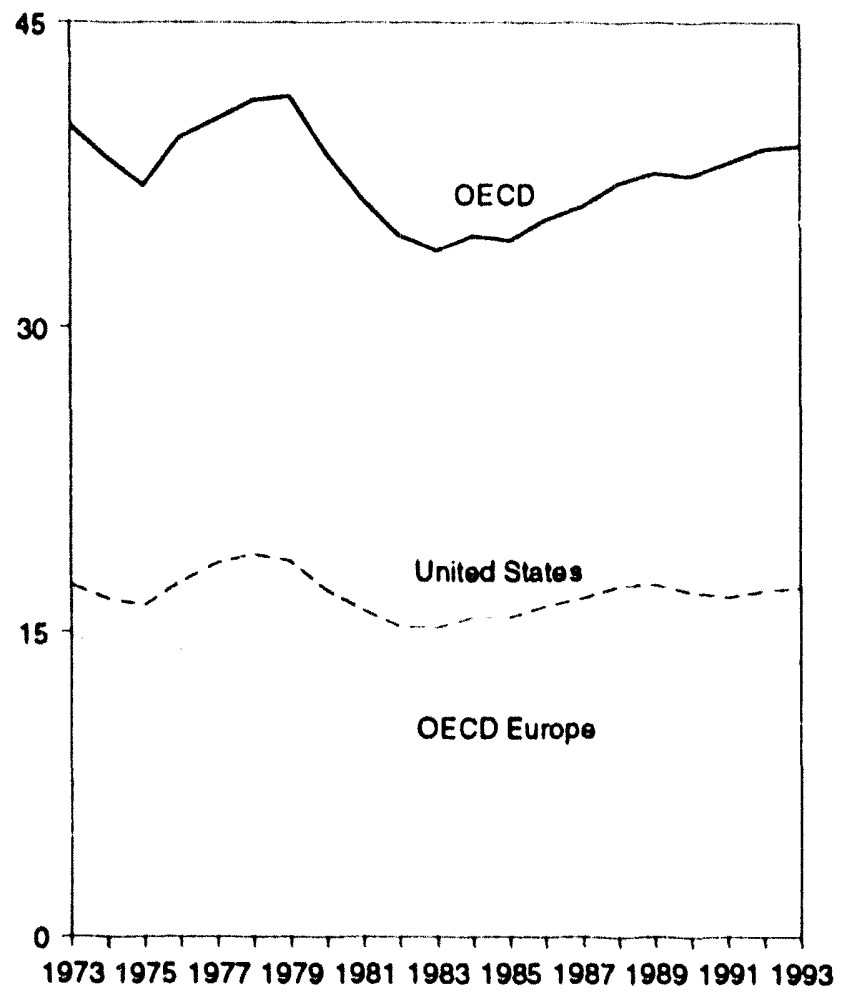

OECD Consumption

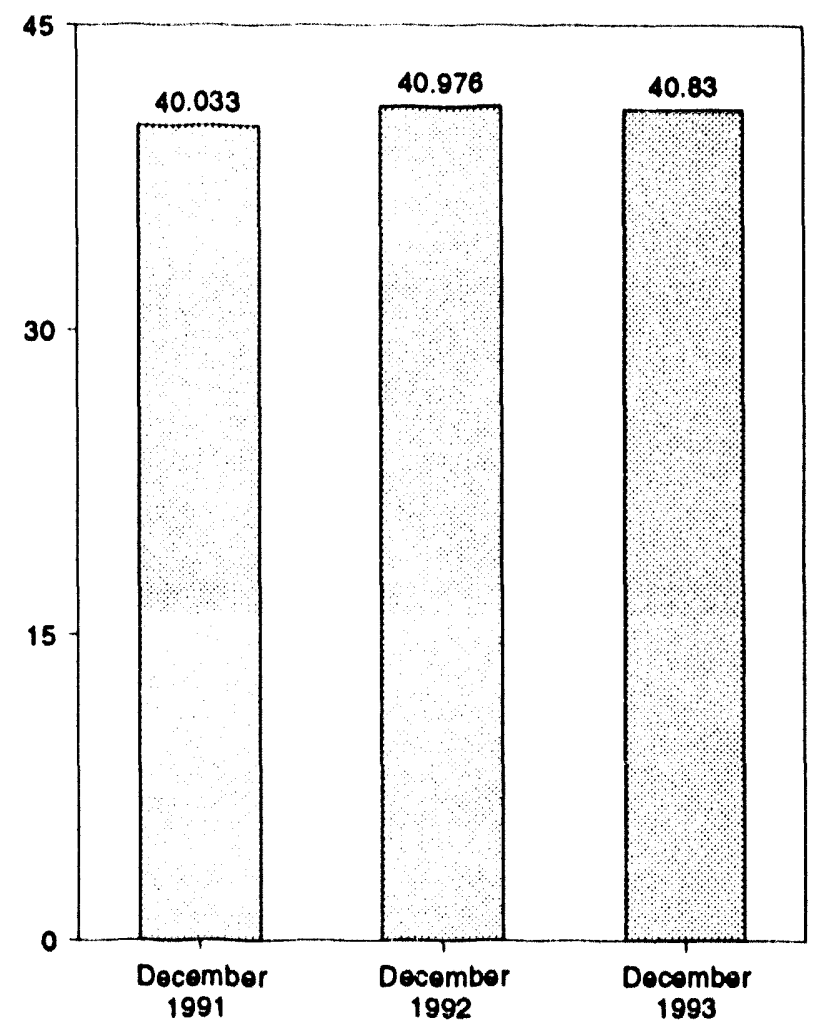

\section{Consumption by Selected OECD Country}

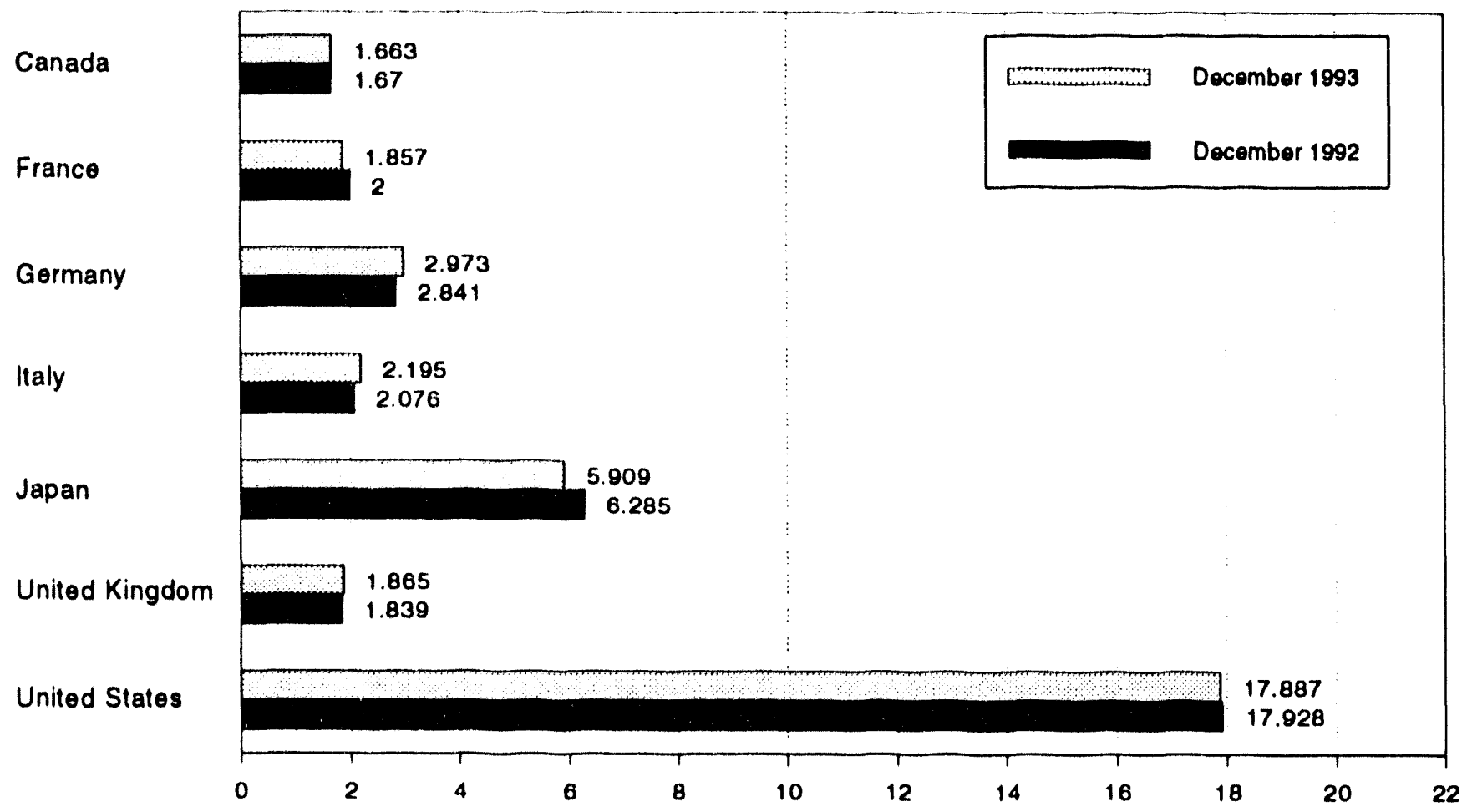

Note: OECD is the Organlzation for Economic Cooperation and Development. Source: Table 10.2 . 


\begin{tabular}{|c|c|c|c|c|c|c|c|c|c|c|}
\hline & Canada & France & Germanya & Italy & Japan & $\begin{array}{l}\text { United } \\
\text { Kingdom }\end{array}$ & $\begin{array}{l}\text { United } \\
\text { States }\end{array}$ & $\begin{array}{c}\text { OECD } \\
\text { Europob }\end{array}$ & $\begin{array}{l}\text { Other } \\
\text { OECDC }\end{array}$ & $O E C D$ \\
\hline 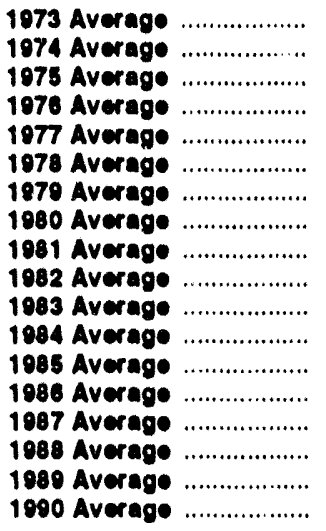 & $\begin{array}{l}1,720 \\
1,779 \\
1,779 \\
1,818 \\
1,850 \\
1,902 \\
1,971 \\
1,873 \\
1,768 \\
1,578 \\
1,448 \\
1,472 \\
1,504 \\
1,508 \\
1,548 \\
1,803 \\
1,733 \\
1,690\end{array}$ & $\begin{array}{l}2,601 \\
2,447 \\
2,252 \\
2,420 \\
2,294 \\
2,408 \\
2,463 \\
2,256 \\
2,023 \\
1,880 \\
1,835 \\
1,754 \\
1,775 \\
1,772 \\
1,789 \\
1,797 \\
1,857 \\
1,818\end{array}$ & $\begin{array}{l}3,055 \\
2,748 \\
2,650 \\
2,877 \\
2,865 \\
2,927 \\
3,003 \\
2,707 \\
2,449 \\
2,372 \\
2,324 \\
2,322 \\
2,338 \\
2,498 \\
2,424 \\
2,422 \\
2,280 \\
2,382\end{array}$ & $\begin{array}{l}2,068 \\
2,004 \\
1,855 \\
1,971 \\
1,897 \\
1,052 \\
2,030 \\
1,934 \\
1,874 \\
1,781 \\
1,750 \\
1,646 \\
1,717 \\
1,738 \\
1,855 \\
1,836 \\
1,930 \\
1,872\end{array}$ & $\begin{array}{l}4,940 \\
4,864 \\
4,621 \\
4,837 \\
4,880 \\
4,945 \\
5,050 \\
4,960 \\
4,848 \\
4,582 \\
4,395 \\
4,578 \\
4,384 \\
4,439 \\
4,484 \\
4,752 \\
4,983 \\
5,140\end{array}$ & $\begin{array}{l}2,341 \\
2,210 \\
1,911 \\
1,892 \\
1,905 \\
1,938 \\
1,971 \\
1,725 \\
1,590 \\
1,590 \\
1,531 \\
1,849 \\
1,634 \\
1,649 \\
1,603 \\
1,697 \\
1,738 \\
1,752\end{array}$ & $\begin{array}{l}17,308 \\
16,653 \\
16,322 \\
17,461 \\
16,431 \\
18,847 \\
18,513 \\
17,056 \\
16,058 \\
15,296 \\
15,231 \\
15,726 \\
15,728 \\
16,281 \\
16,685 \\
17,283 \\
17,325 \\
16,888\end{array}$ & $\begin{array}{l}14,025 \\
13,088 \\
13,217 \\
14,124 \\
13,916 \\
14,290 \\
14,687 \\
13,034 \\
12,515 \\
12,053 \\
11,765 \\
11,736 \\
11,681 \\
12,102 \\
12,255 \\
12,427 \\
12,531 \\
12,620\end{array}$ & $\begin{array}{r}988 \\
1,098 \\
1,041 \\
1,119 \\
1,160 \\
1,204 \\
1,178 \\
1,072 \\
1,080 \\
1,008 \\
954 \\
989 \\
978 \\
981 \\
988 \\
939 \\
908 \\
1,027\end{array}$ & $\begin{array}{l}39,900 \\
38,370 \\
38,080 \\
39,358 \\
40,237 \\
41,187 \\
41,370 \\
38,508 \\
36,289 \\
34,517 \\
33,703 \\
34,500 \\
34,271 \\
35,270 \\
35,011 \\
37,003 \\
37,570 \\
37,478\end{array}$ \\
\hline 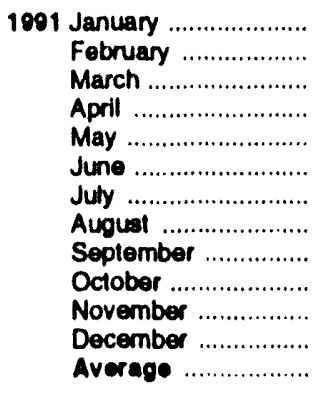 & $\begin{array}{l}1,599 \\
1,613 \\
1,484 \\
1,595 \\
1,637 \\
1,589 \\
1,707 \\
1,693 \\
1,583 \\
1,693 \\
1,602 \\
1,682 \\
1,622\end{array}$ & $\begin{array}{l}2,294 \\
2,009 \\
1,759 \\
1,808 \\
1,773 \\
1,807 \\
1,989 \\
1,795 \\
1,824 \\
2,075 \\
1,953 \\
2,132 \\
1,835\end{array}$ & $\begin{array}{l}2,998 \\
2,783 \\
2,858 \\
2,953 \\
2,912 \\
3,269 \\
2,272 \\
2,609 \\
2,679 \\
2,919 \\
2,860 \\
2,829 \\
2,828\end{array}$ & $\begin{array}{l}2,185 \\
2,025 \\
1,660 \\
1,813 \\
1,722 \\
1,535 \\
1,665 \\
1,546 \\
1,824 \\
2,126 \\
2,031 \\
2.231 \\
1,863\end{array}$ & $\begin{array}{l}5,852 \\
6,155 \\
5,789 \\
5,025 \\
4,880 \\
4,765 \\
5,000 \\
4,888 \\
4,724 \\
4,848 \\
5,581 \\
5,952 \\
5,284\end{array}$ & $\begin{array}{l}1,819 \\
1,837 \\
1,725 \\
1,793 \\
1,799 \\
1,769 \\
1,853 \\
1,812 \\
1,753 \\
1,864 \\
1,829 \\
1,765 \\
1,801\end{array}$ & $\begin{array}{l}16,893 \\
16,339 \\
16,212 \\
16,139 \\
16,189 \\
16,878 \\
16,971 \\
17,183 \\
16,848 \\
16,996 \\
16,730 \\
17,145 \\
16,714\end{array}$ & $\begin{array}{l}14,564 \\
13,804 \\
12,609 \\
13,073 \\
12,965 \\
13,184 \\
12,648 \\
12,727 \\
12,999 \\
14,178 \\
13,736 \\
14,228 \\
13,391\end{array}$ & $\begin{array}{r}1,067 \\
1,043 \\
1,096 \\
1,087 \\
1,108 \\
950 \\
1,005 \\
992 \\
1,028 \\
1,117 \\
1,132 \\
1,047 \\
1,058\end{array}$ & $\begin{array}{l}39,975 \\
38,953 \\
37,190 \\
36,918 \\
36,780 \\
37,366 \\
37,330 \\
37,483 \\
37,182 \\
38,831 \\
38,782 \\
40,033 \\
38,067\end{array}$ \\
\hline 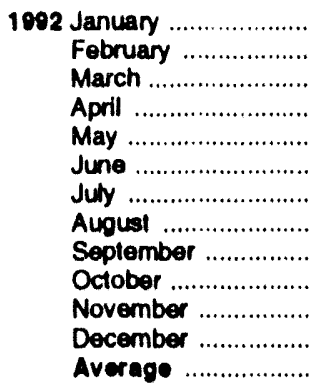 & $\begin{array}{l}1,627 \\
1,623 \\
1,595 \\
1,581 \\
1,589 \\
1,647 \\
1,642 \\
1,676 \\
1,655 \\
1,705 \\
1,714 \\
1,670 \\
1,644\end{array}$ & $\begin{array}{l}2,213 \\
2,108 \\
1,939 \\
1,993 \\
1,632 \\
1,817 \\
1,929 \\
1,735 \\
1,956 \\
1,942 \\
1,890 \\
2,000 \\
1,920\end{array}$ & $\begin{array}{l}2,968 \\
2,814 \\
2,809 \\
2,893 \\
2,588 \\
2,699 \\
3,029 \\
2,829 \\
3,072 \\
2,752 \\
2,823 \\
2,841 \\
2,843\end{array}$ & $\begin{array}{l}2,237 \\
2,149 \\
1,886 \\
1,891 \\
1,671 \\
1,801 \\
1,900 \\
1,655 \\
2,003 \\
1,930 \\
2,053 \\
2,076 \\
1,936\end{array}$ & $\begin{array}{l}5,776 \\
6,347 \\
5,873 \\
5,212 \\
4,845 \\
4,949 \\
5,124 \\
4,964 \\
5,147 \\
5,310 \\
5,644 \\
6,285 \\
5,454\end{array}$ & $\begin{array}{l}1,832 \\
1,819 \\
1,818 \\
1,858 \\
1,694 \\
1,725 \\
1,804 \\
1,699 \\
1,870 \\
1,825 \\
1,852 \\
1,839 \\
1,803\end{array}$ & $\begin{array}{l}17,012 \\
16,893 \\
16,825 \\
16,764 \\
16,485 \\
16,978 \\
17,143 \\
16,929 \\
16,876 \\
17,448 \\
17,091 \\
17,928 \\
17,033\end{array}$ & $\begin{array}{l}14,459 \\
14,052 \\
13,682 \\
13,667 \\
12,347 \\
13,036 \\
13,662 \\
12,909 \\
14,224 \\
13,475 \\
13,806 \\
13,991 \\
13,606\end{array}$ & $\begin{array}{r}1,014 \\
1,045 \\
1,054 \\
1,042 \\
1,002 \\
1,086 \\
1,027 \\
946 \\
1,046 \\
1,014 \\
1,049 \\
1,103 \\
1,035\end{array}$ & $\begin{array}{l}39,888 \\
39,958 \\
39,029 \\
38,267 \\
36,269 \\
37,696 \\
38,590 \\
37,423 \\
38,947 \\
38,953 \\
39,304 \\
40,976 \\
38,772\end{array}$ \\
\hline 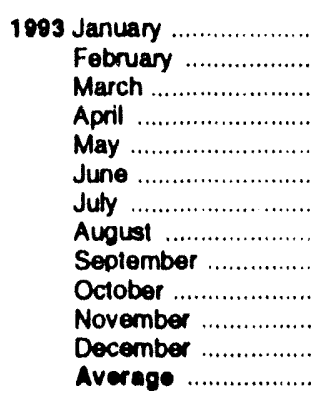 & $\begin{array}{l}A_{1,550} \\
A_{1,683} \\
A_{1,655} \\
R_{1,572} \\
A_{1,583} \\
A_{1,671} \\
A_{1,700} \\
A_{1,716} \\
A_{1,712} \\
R_{1,704} \\
A_{1,653} \\
1,663 \\
1,665\end{array}$ & $\begin{array}{l}A_{1,955} \\
A_{2,145} \\
A_{2,015} \\
A_{1,936} \\
A_{1,703} \\
A_{1,968} \\
A_{1,861} \\
A_{1,663} \\
A_{1,800} \\
A_{1,941} \\
A_{2,122} \\
1,857 \\
1,011\end{array}$ & $\begin{array}{l}R_{2,531} \\
{ }_{2} 2,897 \\
A_{2,934} \\
A_{2,821} \\
A_{2,589} \\
A_{3,046} \\
A_{2,969} \\
A_{2,897} \\
3,167 \\
A_{2,861} \\
A_{3,176} \\
2,973 \\
2,904\end{array}$ & $\begin{array}{l}A_{1,858} \\
A_{1,969} \\
A_{1,946} \\
A_{1,792} \\
A_{1,688} \\
A_{1,735} \\
A_{1,799} \\
A_{1,718} \\
A_{1,921} \\
A_{1,977} \\
\text { A }_{2,042} \\
2,195 \\
1,886\end{array}$ & $\begin{array}{l}A_{5,933} \\
A_{6,281} \\
R_{6,235} \\
A_{5,443} \\
A_{4,755} \\
A_{4,951} \\
A_{4,853} \\
A_{4,780} \\
A_{4,762} \\
A_{5,307} \\
A_{5,573} \\
5,909 \\
5,394\end{array}$ & $\begin{array}{l}A_{1,714} \\
A_{1,864} \\
A_{1,874} \\
A_{1}, 719 \\
A_{1,864} \\
A_{1,795} \\
A_{1}, 794 \\
A_{1}, 777 \\
A_{1,833} \\
A_{1,868} \\
A_{1,854} \\
1,865 \\
1,801\end{array}$ & $\begin{array}{l}16,320 \\
17,397 \\
17,688 \\
16,673 \\
16,340 \\
17,032 \\
17,208 \\
17,176 \\
17,709 \\
17,230 \\
17,688 \\
17,887 \\
17,103\end{array}$ & $\begin{array}{l}A_{12,834} \\
A_{14,043} \\
A_{13,976} \\
A_{13,143} \\
A_{12,065} \\
A_{13,626} \\
A_{13,659} \\
A_{13,101} \\
A_{14,078} \\
A_{13,613} \\
A_{14,644} \\
14,112 \\
13,567\end{array}$ & $\begin{array}{r}A_{968} \\
A_{1,133} \\
A_{1} 1,169 \\
A_{1,125} \\
A_{1,136} \\
A_{1,117} \\
A_{1,054} \\
A_{1,121} \\
A_{1,092} \\
A_{1,111} \\
A_{1,183} \\
1,259 \\
1,122\end{array}$ & 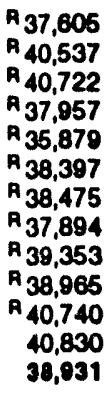 \\
\hline
\end{tabular}

- Through December 1990, the data for Germany are lor the former West Germany only. Beginning with January 1991, the data for Germany are for the unitied Germany, i.e., the lormer East Germany and West Germany.

-OECD Europe" consists of Austria, Belgium, Denmark, Finland, France, Germany, Greoce, Iceland, Ireland, Haly, Luxembourg. the Netherlands. Nonway, Portugal, Spain, Sweden, Swizertand. Turkey, and the Uniled Kingdom.

"Olher OECD" consists of Australia, New Zealand, and the U.S. Terrtionies.

$R=$ Revised data.
Notes: - The Organization for Economic Cooperalion and Developmen (OECD) consists of Canada, Japan, and the United Stales, as well as "OECD Europe" and "Olher OECD." - U.S. geographic coverage th the 50 states and the District of Columbia. - Totals may not equal sum of components due to independent rounding. - Data through 1991 are final. Subeequent data are preliminary.

Sources: - United States: Table 3.18. - All Other Dnta: 1973-1979-International Energy Agency (IEA). Annual Oll and Gas Statstico of OECD Countries. 1080 forward-IEA, quarterty and monthly computer lapes supporting Quarterty Oil Statistics and Energy Balances. 
Figure 10.4 Petroleum Stocks in OECD Countries

(Billion Barrels)

OECD Stocks, End of Year, 1973-1993

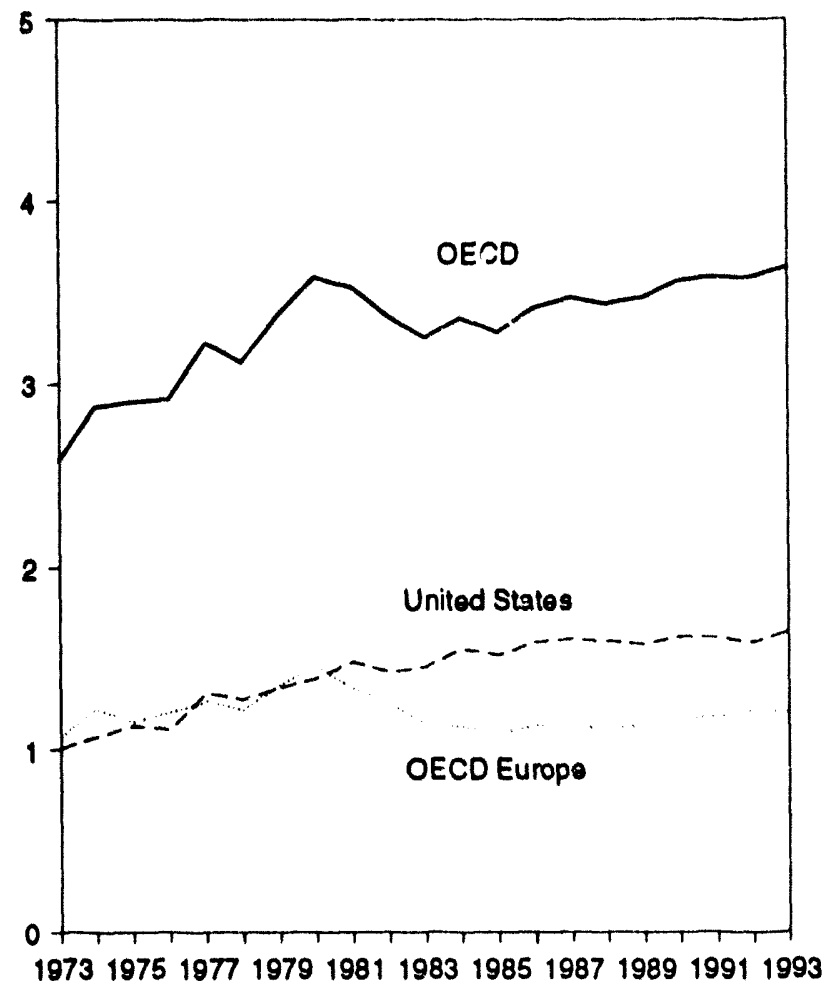

OECD Stocks, End of Month

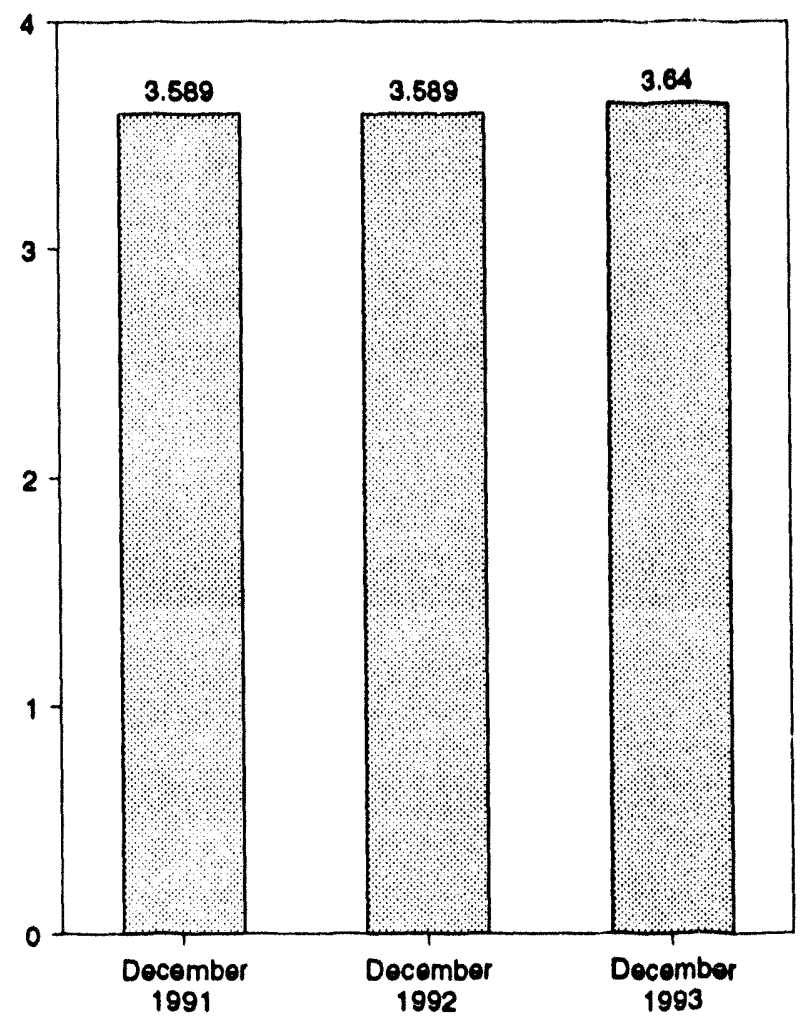

Stocks by Selected Country, End of Month

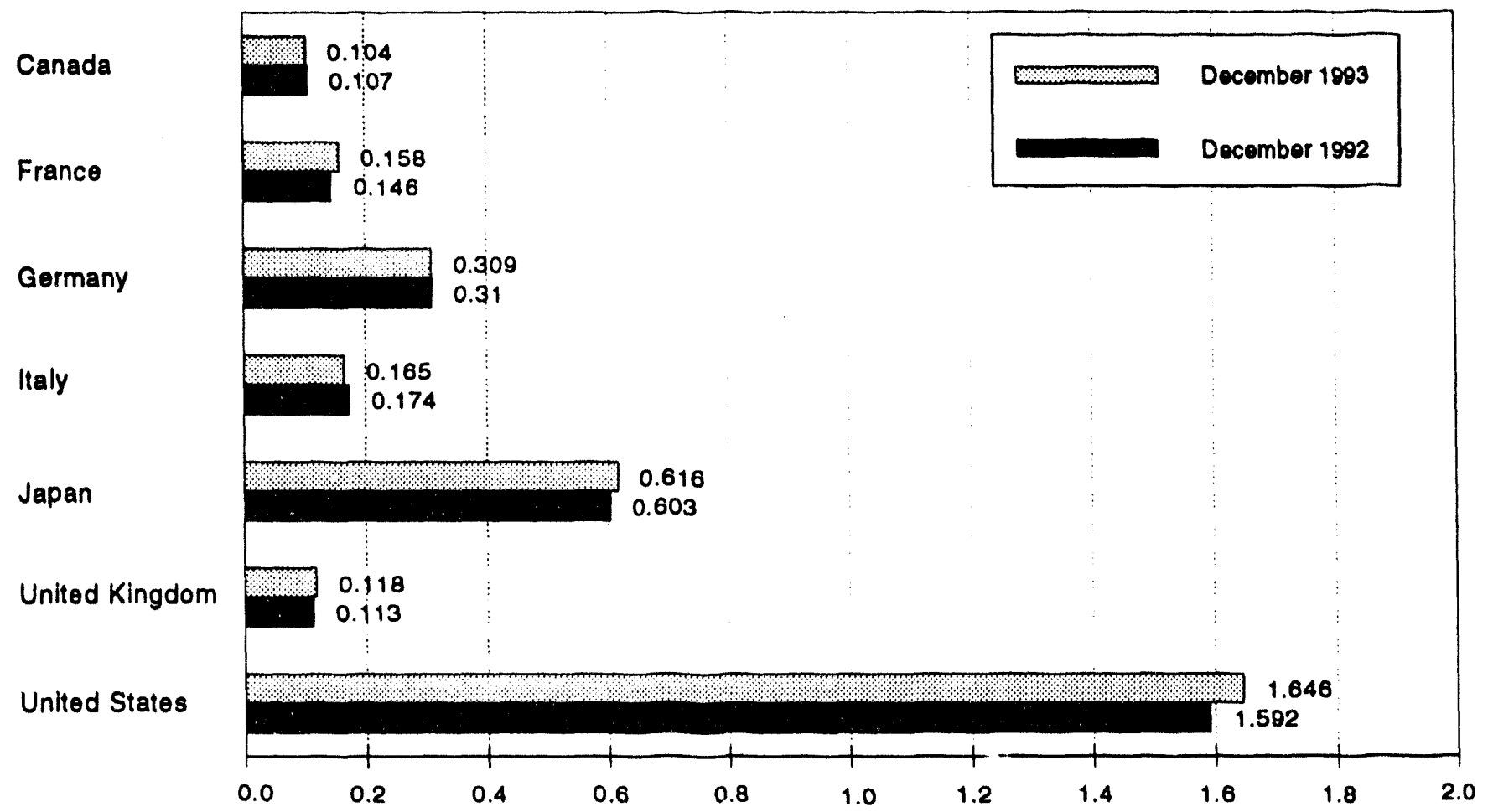

Nole: OECD is the Organization for Economic Cooperation and Development.

Source: Table 10.3. 
Table 10.3 Petroleum Stocks in OECD Countries, End of Period (Million Barrels)

\begin{tabular}{|c|c|c|c|c|c|c|c|c|c|c|}
\hline & Canada & France & Gormany ${ }^{a}$ & Italy & Japan & $\begin{array}{l}\text { United } \\
\text { Kingdom }\end{array}$ & $\begin{array}{l}\text { United } \\
\text { States }\end{array}$ & $\begin{array}{l}\text { OECD } \\
\text { Europeb }^{b}\end{array}$ & $\begin{array}{l}\text { Other } \\
\text { OECDC }\end{array}$ & OECD \\
\hline 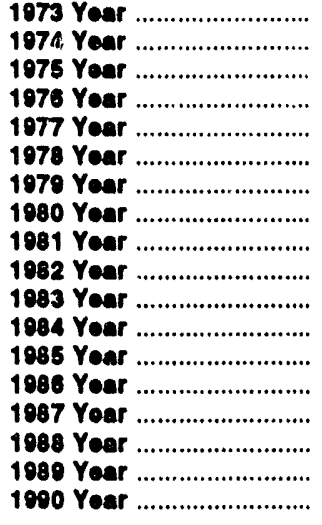 & $\begin{array}{l}140 \\
145 \\
174 \\
153 \\
167 \\
144 \\
150 \\
184 \\
161 \\
136 \\
121 \\
128 \\
113 \\
111 \\
126 \\
116 \\
114 \\
121\end{array}$ & $\begin{array}{l}201 \\
249 \\
225 \\
234 \\
230 \\
201 \\
226 \\
243 \\
214 \\
193 \\
153 \\
152 \\
139 \\
127 \\
127 \\
140 \\
138 \\
140\end{array}$ & $\begin{array}{l}181 \\
213 \\
187 \\
208 \\
225 \\
238 \\
272 \\
319 \\
297 \\
272 \\
249 \\
239 \\
233 \\
252 \\
259 \\
266 \\
271 \\
265\end{array}$ & $\begin{array}{l}152 \\
167 \\
143 \\
143 \\
161 \\
154 \\
163 \\
170 \\
167 \\
179 \\
149 \\
159 \\
157 \\
155 \\
169 \\
155 \\
164 \\
172\end{array}$ & $\begin{array}{l}303 \\
370 \\
375 \\
380 \\
409 \\
413 \\
460 \\
495 \\
482 \\
484 \\
470 \\
470 \\
494 \\
509 \\
540 \\
538 \\
577 \\
590\end{array}$ & $\begin{array}{l}156 \\
101 \\
165 \\
165 \\
148 \\
157 \\
169 \\
168 \\
143 \\
125 \\
118 \\
112 \\
123 \\
124 \\
121 \\
112 \\
118 \\
112\end{array}$ & $\begin{array}{l}1,008 \\
1,074 \\
1,133 \\
1,112 \\
1,312 \\
1,278 \\
1,341 \\
1,392 \\
1,484 \\
1,430 \\
1,454 \\
1,556 \\
1,519 \\
1,593 \\
1,607 \\
1,597 \\
1,581 \\
1,621\end{array}$ & $\begin{array}{l}1,070 \\
1,227 \\
1,154 \\
1,205 \\
1,268 \\
1,210 \\
1,353 \\
1,464 \\
1,337 \\
1,258 \\
1,142 \\
1,130 \\
1,092 \\
1,133 \\
1,130 \\
1,118 \\
1,133 \\
1,163\end{array}$ & $\begin{array}{l}67 \\
64 \\
67 \\
68 \\
68 \\
68 \\
75 \\
72 \\
67 \\
68 \\
68 \\
60 \\
66 \\
72 \\
72 \\
71 \\
71 \\
73\end{array}$ & $\begin{array}{l}2,588 \\
2,880 \\
2,903 \\
2,918 \\
3,224 \\
3,122 \\
3,370 \\
3,587 \\
3,531 \\
3,378 \\
3,255 \\
3,362 \\
3,284 \\
3,418 \\
3,474 \\
3,440 \\
3,476 \\
3,568\end{array}$ \\
\hline 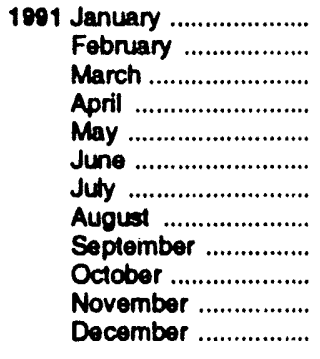 & $\begin{array}{l}116 \\
114 \\
117 \\
110 \\
107 \\
107 \\
118 \\
116 \\
117 \\
118 \\
122 \\
110\end{array}$ & $\begin{array}{l}133 \\
137 \\
142 \\
138 \\
138 \\
144 \\
145 \\
152 \\
150 \\
148 \\
152 \\
153\end{array}$ & $\begin{array}{l}278 \\
278 \\
280 \\
277 \\
279 \\
274 \\
285 \\
284 \\
287 \\
286 \\
289 \\
288\end{array}$ & $\begin{array}{l}174 \\
169 \\
178 \\
177 \\
174 \\
173 \\
169 \\
171 \\
170 \\
165 \\
163 \\
160\end{array}$ & $\begin{array}{l}591 \\
572 \\
593 \\
585 \\
586 \\
590 \\
594 \\
610 \\
622 \\
625 \\
607 \\
607\end{array}$ & $\begin{array}{l}116 \\
119 \\
124 \\
119 \\
113 \\
118 \\
113 \\
118 \\
120 \\
119 \\
120 \\
119\end{array}$ & $\begin{array}{l}1,587 \\
1,573 \\
1,558 \\
1,578 \\
1,626 \\
1,634 \\
1,635 \\
1,648 \\
1,663 \\
1,644 \\
1,647 \\
1,617\end{array}$ & $\begin{array}{l}1,164 \\
1,162 \\
1,178 \\
1,161 \\
1,157 \\
1,161 \\
1,170 \\
1,186 \\
1,195 \\
1,190 \\
1,198 \\
1,182\end{array}$ & $\begin{array}{l}73 \\
72 \\
75 \\
75 \\
75 \\
72 \\
73 \\
76 \\
74 \\
71 \\
70 \\
65\end{array}$ & $\begin{array}{l}3,531 \\
3,493 \\
3,521 \\
3,509 \\
3,551 \\
3,564 \\
3,590 \\
3,638 \\
3,671 \\
3,649 \\
3,643 \\
, 589\end{array}$ \\
\hline 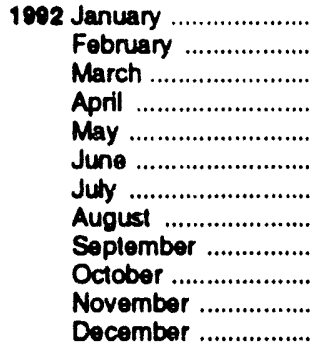 & $\begin{array}{l}117 \\
111 \\
111 \\
111 \\
108 \\
112 \\
110 \\
113 \\
110 \\
108 \\
110 \\
107\end{array}$ & $\begin{array}{l}149 \\
145 \\
142 \\
140 \\
147 \\
148 \\
146 \\
150 \\
148 \\
148 \\
149 \\
148\end{array}$ & $\begin{array}{l}293 \\
303 \\
303 \\
307 \\
311 \\
307 \\
299 \\
303 \\
299 \\
302 \\
306 \\
310\end{array}$ & $\begin{array}{l}167 \\
172 \\
169 \\
165 \\
171 \\
166 \\
166 \\
169 \\
165 \\
166 \\
172 \\
174\end{array}$ & $\begin{array}{l}601 \\
596 \\
586 \\
578 \\
588 \\
583 \\
586 \\
604 \\
608 \\
613 \\
611 \\
603\end{array}$ & $\begin{array}{l}116 \\
118 \\
115 \\
115 \\
115 \\
114 \\
120 \\
117 \\
112 \\
113 \\
116 \\
113\end{array}$ & $\begin{array}{l}1,610 \\
1,588 \\
1,571 \\
1,583 \\
1,602 \\
1,603 \\
1,620 \\
1,621 \\
1,636 \\
1,640 \\
1,636 \\
1,592\end{array}$ & $\begin{array}{l}1,168 \\
1,181 \\
1,162 \\
1,172 \\
1,189 \\
1,190 \\
1,182 \\
1,211 \\
1,194 \\
1,201 \\
1,207 \\
1,210\end{array}$ & $\begin{array}{l}68 \\
66 \\
66 \\
62 \\
63 \\
69 \\
67 \\
69 \\
69 \\
69 \\
71 \\
67\end{array}$ & $\begin{array}{l}3,564 \\
3,542 \\
3,495 \\
3,505 \\
3,551 \\
3,557 \\
3,565 \\
3,617 \\
3,616 \\
3,631 \\
3,634 \\
3,580\end{array}$ \\
\hline 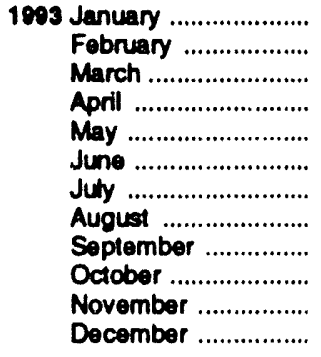 & $\begin{array}{r}110 \\
106 \\
107 \\
110 \\
106 \\
107 \\
112 \\
112 \\
\text { P } 107 \\
105 \\
107 \\
104\end{array}$ & $\begin{array}{r}148 \\
142 \\
138 \\
139 \\
145 \\
139 \\
141 \\
150 \\
149 \\
160 \\
R 157 \\
158\end{array}$ & $\begin{array}{r}319 \\
317 \\
311 \\
311 \\
320 \\
P_{309} \\
312 \\
P_{315} \\
{ }_{311} \\
317 \\
310 \\
308\end{array}$ & $\begin{array}{l}173 \\
168 \\
165 \\
166 \\
172 \\
167 \\
169 \\
170 \\
162 \\
162 \\
165 \\
165\end{array}$ & $\begin{array}{r}614 \\
606 \\
593 \\
584 \\
592 \\
602 \\
617 \\
634 \\
R 648 \\
653 \\
R 643 \\
616\end{array}$ & $\begin{array}{r}120 \\
120 \\
120 \\
116 \\
117 \\
119 \\
115 \\
117 \\
115 \\
111 \\
1116 \\
118\end{array}$ & $\begin{array}{l}1,611 \\
1,595 \\
1,584 \\
1,611 \\
1,643 \\
1,660 \\
1,678 \\
1,674 \\
1,661 \\
1,685 \\
1,684 \\
1,648\end{array}$ & $\begin{array}{r}1,232 \\
1,218 \\
1,201 \\
R_{1}, 195 \\
R_{1,207} \\
R_{1} 1,187 \\
R_{1,189} \\
R_{1,220} \\
R_{1,208} \\
R_{1,222} \\
R_{1,215} \\
1,206\end{array}$ & $\begin{array}{l}68 \\
68 \\
68 \\
73 \\
69 \\
70 \\
70 \\
70 \\
77 \\
78 \\
78 \\
68\end{array}$ & 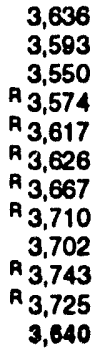 \\
\hline
\end{tabular}

- Through December 1900, the data for Germany are for the former West Germany only. Beginning with January 1991, the data for Germany are for the unified Germany, 1.e., the former East Germany and West Germany.

-OECD Europo" consists of Austria, Bolgium, Donmark, Finland, Franco, Germany, Greece, Kcoland, Ireland, Maly, Luxembourg, the Netherlands Nonway, Pontugal. Spain, Sweden, Swizertand, Turkey, and the United Kingdom.

"Other OECD" consists of Australia, New Zealand, and the U.S. Territories.

R=Revised data.

Notes: - Petroleum stocks include crude oil (including stralegic reserves). untinished olls, natural gas plant liquids, and relined products. Petroleum stocks include all nonmilkany petroleum held for storage, regardless of ownership, within each country in bulk terminals, relinery tanks, pipeline tankage, intercoastal tankers, tankers in port, and inland ship bunkers. Data exclude oll held in pipelines (except for those in the United States), rall and truck cars, sea-going ships' bunkers, service stations, retail stores, and tankers at sea. - The Organization lor Economic Cooperation and Development (OECD) consists of Canada, Japan, and the United States, as well as "OECD Europe" and "Other OECD." - U.S. geographic coverage is the 50 States and the District of Columbia. - Totals may not equal sum of components due to independent rounding. - In the United States in January 1975, 1981, and 1983, numerous respondents were added to bulk terminal and pipeline surveys, thereby affecting subsequent stocks reported. Newbasis end-ot-year U.S. stocks, in million barrels, would have been 1,121 in 1974, 1,425 in 1980, and 1,461 in 1982. Data through 1991 are final. Subsequent data are preliminary.

Sources: - United States: Table 3.1a. - All Other Data: Intemational Energy Agency, quarterly and monthly computer tapes supporting Quarterty Oil Slatistics and Energy Balances. 
Figure 10.5 Nuclear Electricity Gross Generation

(Billion Kilowatthours)

U.S. and World Generation, 1973-1993

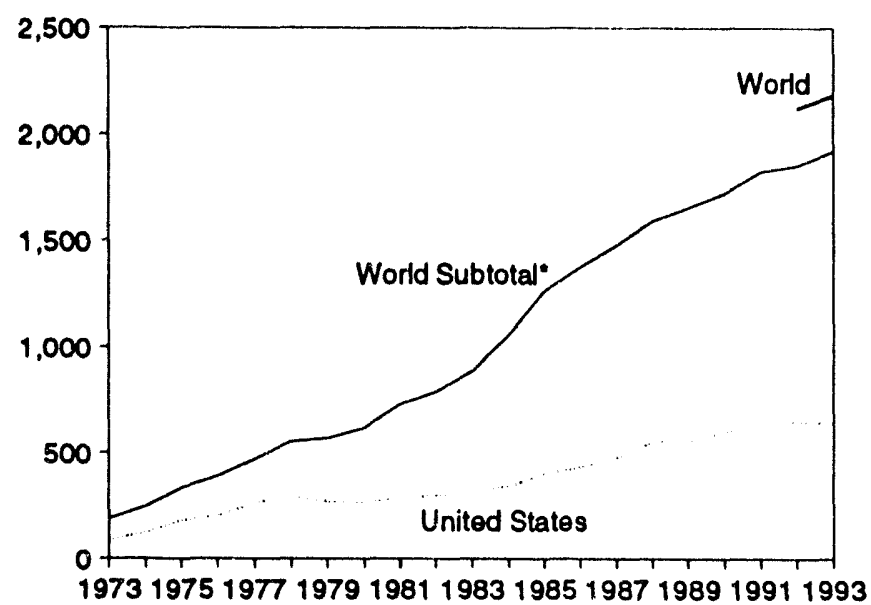

World excluding Eastem Europe.
Generation by Region, February 1994

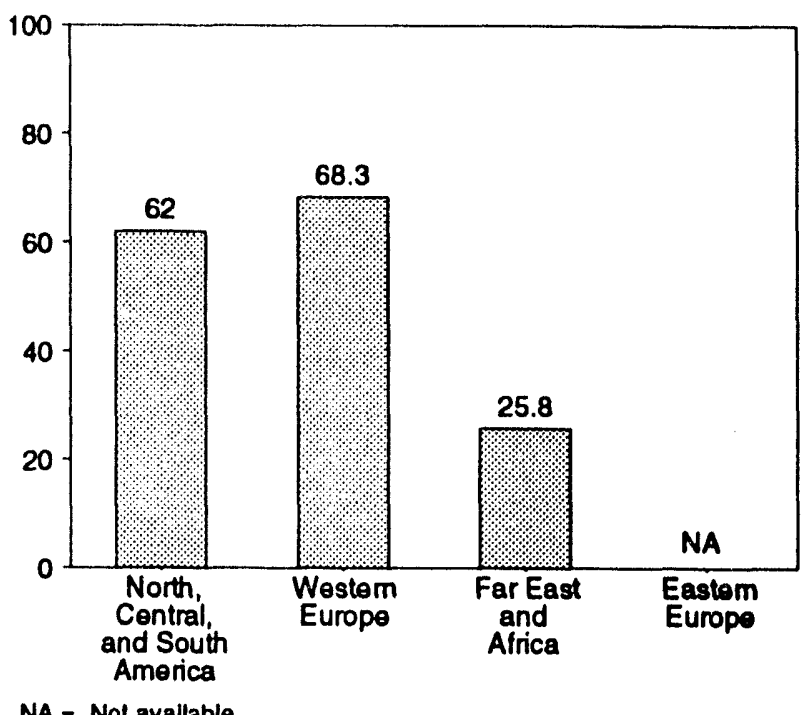

Generation by Selected Country, February 1994

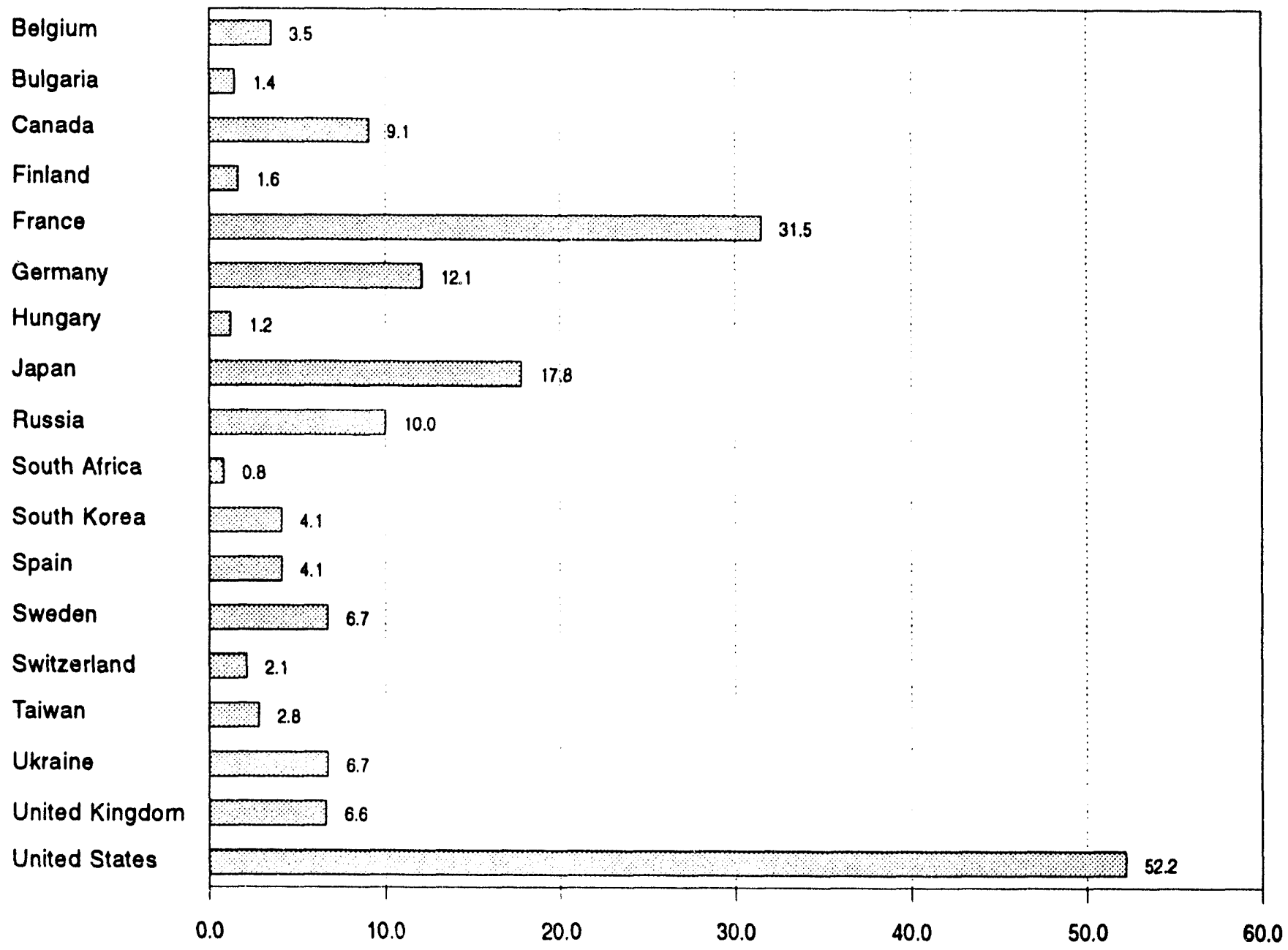

Note: Because vertical scales dufer, graphs should not be compared. Sources: Tables 10.48-10.4e. 
Table 10.4a Nuclear Electricity Gross Generation: Regions and World (Billion Kilowatthours)

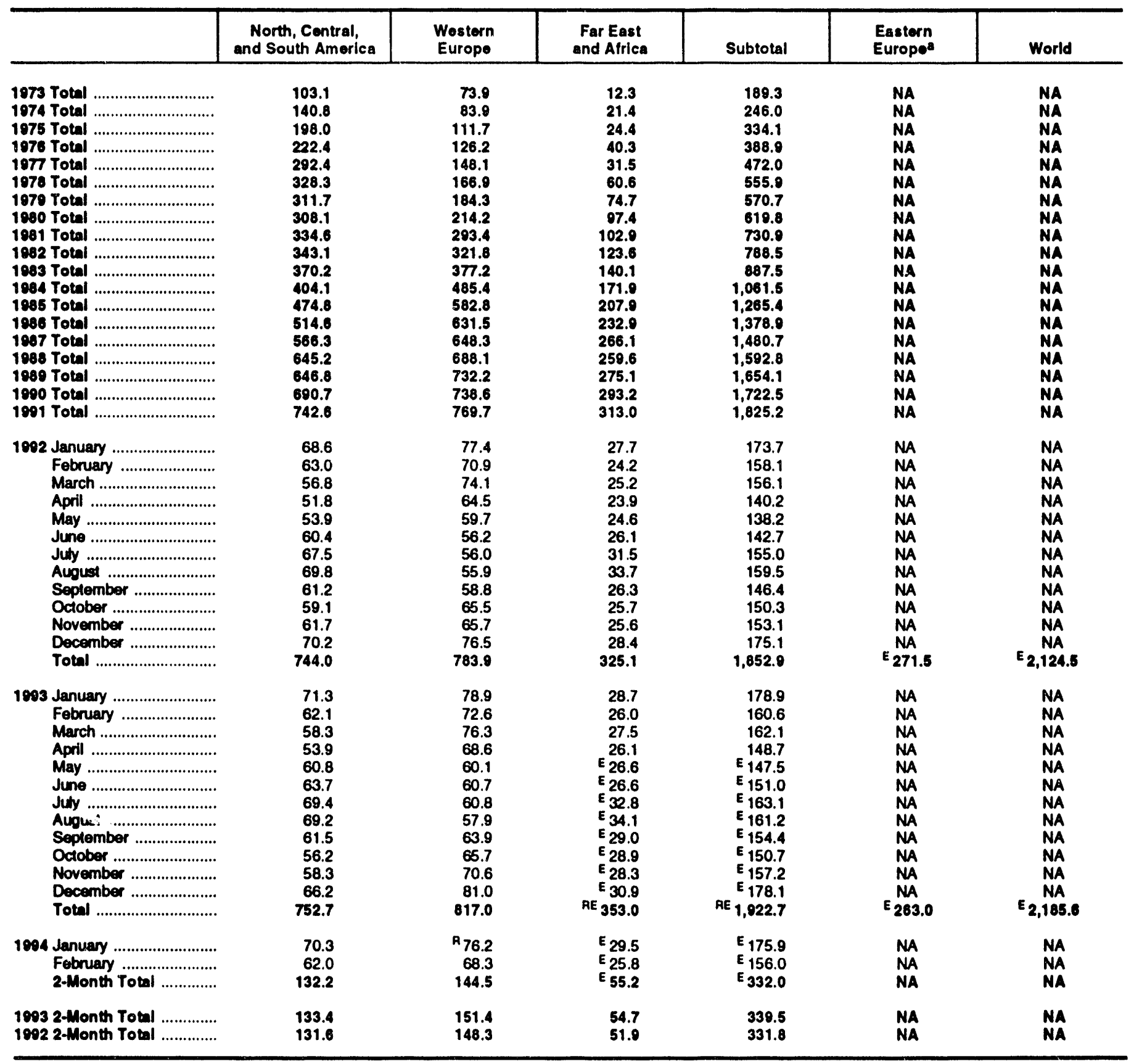

- See Table 10.40 for country-specilic estimated annual generation in 1992 and 1993, and avallable monthly generation in 1993, for Easlem Europe.

$R=$ Revised data. $N A=N a$ available. E=Estimate.

Notes: - Net figures are generally less than gross figures by about 5 percent, the difierence being the energy consumed by the generating plants themselves. - Monthly data may not sum to annual totals due to independent rounding and because precommercial generation is included in some annual totals but not in the monthly data. - Data for countries may not sum to totals due to independent rounding.

Source: McGraw-Hill Publishing Company, Nucleonics Woek. 
Table 10.4b Nuclear Electricity Gross Generation: North, Central, and South America (Billion Kilowatthours)

\begin{tabular}{|c|c|c|c|c|c|c|}
\hline & Argentina & Brazll & Canada & Moxico & United States & $\begin{array}{l}\text { North, Contral, } \\
\text { and South Amorice }\end{array}$ \\
\hline 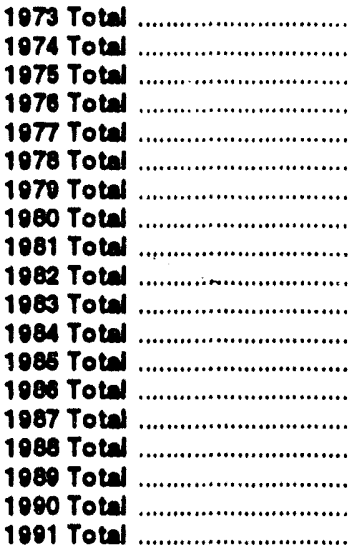 & $\begin{array}{l}- \\
1.0 \\
2.5 \\
2.6 \\
1.6 \\
2.9 \\
2.7 \\
2.3 \\
2.8 \\
1.0 \\
3.4 \\
4.5 \\
5.8 \\
5.7 \\
5.2 \\
5.1 \\
5.0 \\
7.4 \\
7.7\end{array}$ & $\begin{array}{r}- \\
- \\
- \\
- \\
- \\
- \\
- \\
- \\
0.1 \\
.2 \\
2.1 \\
3.4 \\
.1 \\
1.0 \\
.3 \\
1.6 \\
2.0 \\
1.4\end{array}$ & $\begin{array}{l}15.3 \\
15.4 \\
13.2 \\
18.0 \\
28.6 \\
33.0 \\
38.4 \\
40.4 \\
43.3 \\
42.6 \\
53.0 \\
53.8 \\
62.9 \\
74.6 \\
80.6 \\
85.6 \\
83.2 \\
75.8 \\
86.1\end{array}$ & $\begin{array}{l}- \\
- \\
- \\
- \\
- \\
- \\
- \\
- \\
- \\
- \\
- \\
- \\
- \\
- \\
- \\
2.1 \\
4.2\end{array}$ & $\begin{array}{r}87.8 \\
124.3 \\
182.3 \\
201.8 \\
264.2 \\
292.4 \\
270.6 \\
265.4 \\
288.6 \\
208.6 \\
313.6 \\
343.8 \\
402.7 \\
434.1 \\
479.5 \\
554.1 \\
557.0 \\
603.4 \\
643.0\end{array}$ & $\begin{array}{l}103.1 \\
140.8 \\
108.0 \\
222.4 \\
202.4 \\
328.3 \\
311.7 \\
308.1 \\
334.8 \\
343.1 \\
370.2 \\
404.1 \\
474.8 \\
814.8 \\
888.3 \\
845.2 \\
848.8 \\
800.7 \\
742.8\end{array}$ \\
\hline 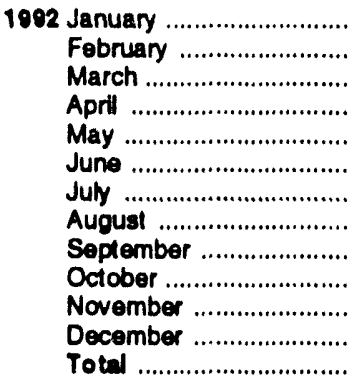 & $\begin{array}{l}.6 \\
.7 \\
.6 \\
.6 \\
.5 \\
.6 \\
.7 \\
.7 \\
.7 \\
.3 \\
.4 \\
.6 \\
7.1\end{array}$ & $\begin{array}{l}.0 \\
.0 \\
.0 \\
.0 \\
.0 \\
.1 \\
.3 \\
.4 \\
.3 \\
.1 \\
.3 \\
.1 \\
1.8\end{array}$ & $\begin{array}{r}6.9 \\
6.4 \\
7.4 \\
6.4 \\
4.8 \\
5.6 \\
7.2 \\
6.9 \\
6.9 \\
7.2 \\
7.4 \\
8.0 \\
81.3\end{array}$ & $\begin{array}{l}.5 \\
.4 \\
.5 \\
.5 \\
.5 \\
.3 \\
.3 \\
.2 \\
.0 \\
(3) \\
.4 \\
.4 \\
3.0\end{array}$ & $\begin{array}{l}60.6 \\
55.4 \\
48.3 \\
44.3 \\
48.1 \\
53.7 \\
59.0 \\
61.6 \\
53.2 \\
51.6 \\
53.2 \\
61.0 \\
650.0\end{array}$ & $\begin{array}{r}68.6 \\
63.0 \\
56.8 \\
51.8 \\
53.9 \\
60.4 \\
67.5 \\
69.8 \\
61.2 \\
59.1 \\
61.7 \\
70.2 \\
744.0\end{array}$ \\
\hline 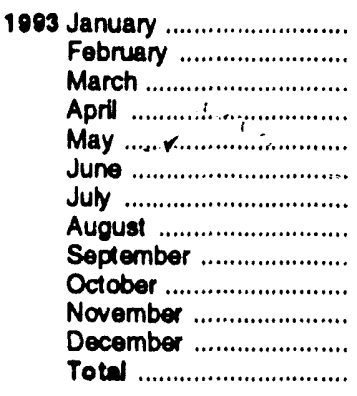 & $\begin{array}{l}.6 \\
.4 \\
.6 \\
.7 \\
.7 \\
.7 \\
.7 \\
.7 \\
.7 \\
.4 \\
.6 \\
.7 \\
7.7\end{array}$ & $\begin{array}{l}.2 \\
.2 \\
(\mathbf{s}) \\
.0 \\
.0 \\
.0 \\
.0 \\
.0 \\
.0 \\
.0 \\
.0 \\
.0 \\
.4\end{array}$ & $\begin{array}{r}8.2 \\
7.4 \\
7.8 \\
7.3 \\
6.7 \\
7.1 \\
9.3 \\
9.1 \\
7.9 \\
8.5 \\
8.2 \\
9.2 \\
97.6\end{array}$ & $\begin{array}{l}.5 \\
.3 \\
.1 \\
.5 \\
.5 \\
.5 \\
.5 \\
.5 \\
.5 \\
.4 \\
.4 \\
.4 \\
4.0\end{array}$ & $\begin{array}{r}61.8 \\
53.7 \\
49.8 \\
45.4 \\
52.8 \\
55.4 \\
68.9 \\
58.9 \\
52.5 \\
46.9 \\
49.1 \\
55.9 \\
642.0\end{array}$ & $\begin{array}{r}71.3 \\
62.1 \\
58.3 \\
53.9 \\
60.8 \\
63.7 \\
69.4 \\
69.2 \\
61.5 \\
56.2 \\
58.3 \\
66.2 \\
762.7\end{array}$ \\
\hline $\begin{array}{l}1094 \text { January .......................... } \\
\text { Fobnuary ..................... } \\
\text { 2.Month Total ............... }\end{array}$ & $\begin{array}{r}.7 \\
.7 \\
1.4\end{array}$ & $\begin{array}{l}.0 \\
.0 \\
.0\end{array}$ & $\begin{array}{r}9.7 \\
9.1 \\
18.8\end{array}$ & $\begin{array}{l}.2 \\
.0 \\
.2\end{array}$ & $\begin{array}{r}59.6 \\
52.2 \\
111.8\end{array}$ & $\begin{array}{r}70.3 \\
62.0 \\
132.2\end{array}$ \\
\hline $\begin{array}{l}1003 \text { 2-Month Total ................ } \\
1092 \text { 2-Month Total ............... }\end{array}$ & $\begin{array}{l}1.0 \\
1.3\end{array}$ & $\begin{array}{l}.4 \\
.0\end{array}$ & $\begin{array}{l}15.6 \\
13.3\end{array}$ & .8 & $\begin{array}{l}115.5 \\
116.0\end{array}$ & $\begin{array}{l}133.4 \\
131.8\end{array}$ \\
\hline
\end{tabular}

- $=$ Nol applicable. (s)=Less than 0.05 billion Wlowatthours.

Notes: - Nel ilgures are generally less than gross figures by about 5 percent, the difference being the energy consumed by the generating plants themselves. - U.S. geographic coverage is the 50 States and the District of Columbia. - Monthly data may not sum to annual tolals due to independent rounding and because precommercial generation is included in some annual totals but not in the monthly data. - Data for countries may not sum to totato due to independent rounding.

Source: McGraw-Hill Publishing Company, Nucleonics Weok. 
Table 10.4c Nuclear Electricity Gross Generation: Western Europe (Billion Kilowatthours)

\begin{tabular}{|c|c|c|c|c|c|c|c|c|c|c|c|}
\hline & Belglum & Finland & France & Germany & Italy & Netherlands & Spain & Sweden & Switzerland & $\begin{array}{c}\text { United } \\
\text { Kingdomb }\end{array}$ & $\begin{array}{l}\text { Wostorn } \\
\text { Europo }\end{array}$ \\
\hline 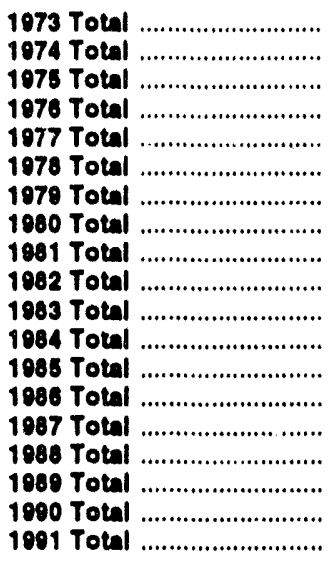 & $\begin{array}{r}0.0 \\
.1 \\
6.8 \\
10.0 \\
11.0 \\
12.5 \\
11.4 \\
12.5 \\
12.8 \\
15.6 \\
24.1 \\
27.7 \\
34.5 \\
38.6 \\
41.9 \\
43.1 \\
41.2 \\
42.7 \\
42.0\end{array}$ & $\begin{array}{c}- \\
- \\
- \\
- \\
2.7 \\
3.3 \\
6.7 \\
7.0 \\
14.5 \\
18.5 \\
17.4 \\
18.5 \\
18.8 \\
18.8 \\
19.4 \\
10.3 \\
18.8 \\
18.0 \\
19.2\end{array}$ & $\begin{array}{l}14.7 \\
14.7 \\
18.3 \\
15.8 \\
17.9 \\
30.6 \\
39.9 \\
61.2 \\
105.2 \\
108.0 \\
144.2 \\
191.2 \\
224.0 \\
254.3 \\
265.5 \\
274.9 \\
302.5 \\
314.1 \\
331.4\end{array}$ & $\begin{array}{r}11.0 \\
12.0 \\
21.7 \\
24.5 \\
36.0 \\
35.7 \\
42.2 \\
43.7 \\
53.4 \\
63.4 \\
65.8 \\
92.6 \\
125.8 \\
118.8 \\
130.2 \\
145.2 \\
149.6 \\
147.2 \\
147.3\end{array}$ & $\begin{array}{l}3.1 \\
3.4 \\
3.8 \\
3.8 \\
3.4 \\
4.5 \\
2.6 \\
2.2 \\
2.7 \\
6.8 \\
5.8 \\
6.9 \\
7.0 \\
8.7 \\
.2 \\
.0 \\
.0 \\
.0 \\
.0\end{array}$ & $\begin{array}{l}1.1 \\
3.3 \\
3.3 \\
3.9 \\
3.7 \\
4.1 \\
3.5 \\
4.2 \\
3.7 \\
3.0 \\
3.8 \\
3.8 \\
3.0 \\
4.2 \\
3.6 \\
3.7 \\
4.0 \\
3.4 \\
3.3\end{array}$ & $\begin{array}{r}6.5 \\
7.2 \\
7.5 \\
7.6 \\
6.5 \\
7.6 \\
6.7 \\
5.2 \\
0.4 \\
8.8 \\
10.7 \\
23.1 \\
28.0 \\
37.5 \\
41.2 \\
50.4 \\
56.1 \\
54.3 \\
55.6\end{array}$ & $\begin{array}{r}2.1 \\
2.3 \\
12.0 \\
16.0 \\
19.9 \\
23.8 \\
21.0 \\
26.7 \\
37.7 \\
38.8 \\
40.4 \\
51.3 \\
58.6 \\
69.0 \\
67.2 \\
69.4 \\
65.6 \\
68.2 \\
76.8\end{array}$ & $\begin{array}{r}6.2 \\
7.0 \\
7.7 \\
7.0 \\
8.1 \\
8.3 \\
11.8 \\
14.3 \\
15.2 \\
15.0 \\
15.5 \\
18.3 \\
22.4 \\
22.5 \\
23.0 \\
22.7 \\
22.0 \\
23.0 \\
22.0\end{array}$ & $\begin{array}{l}28.2 \\
33.8 \\
30.8 \\
38.8 \\
38.1 \\
36.8 \\
38.8 \\
37.2 \\
38.0 \\
44.1 \\
49.6 \\
54.1 \\
\mathbf{5 0 . 7} \\
\mathbf{5 8 . 2} \\
\mathbf{5 6 . 2} \\
\mathbf{5 0 . 4} \\
\mathbf{7 1 . 6} \\
\mathbf{6 8 . 1} \\
\mathbf{7 0 . 4}\end{array}$ & $\begin{array}{r}73.0 \\
83.0 \\
111.7 \\
128.2 \\
148.1 \\
186.9 \\
184.3 \\
214.2 \\
203.4 \\
321.8 \\
377.2 \\
485.4 \\
582.0 \\
831.6 \\
848.3 \\
880.1 \\
732.2 \\
738.0 \\
780.7\end{array}$ \\
\hline 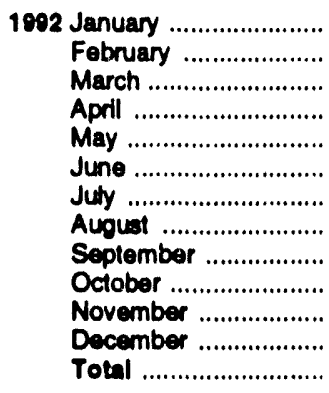 & $\begin{array}{l}4.3 \\
4.0 \\
4.0 \\
3.4 \\
3.8 \\
3.6 \\
3.1 \\
3.4 \\
3.1 \\
3.6 \\
3.3 \\
3.9 \\
43.6\end{array}$ & $\begin{array}{r}1.8 \\
1.7 \\
1.8 \\
1.7 \\
1.3 \\
1.4 \\
1.6 \\
1.4 \\
1.3 \\
1.6 \\
1.7 \\
1.8 \\
19.0\end{array}$ & $\begin{array}{r}33.5 \\
29.8 \\
30.7 \\
28.0 \\
25.6 \\
22.4 \\
23.7 \\
24.6 \\
25.6 \\
28.5 \\
29.5 \\
33.1 \\
337.6\end{array}$ & $\begin{array}{r}15.6 \\
15.2 \\
15.8 \\
14.1 \\
11.8 \\
11.8 \\
12.0 \\
10.9 \\
11.6 \\
13.2 \\
13.0 \\
13.8 \\
158.8\end{array}$ & $\begin{array}{l}.0 \\
.0 \\
.0 \\
.0 \\
.0 \\
.0 \\
.0 \\
.0 \\
.0 \\
.0 \\
.0 \\
.0 \\
.0\end{array}$ & $\begin{array}{r}.4 \\
.3 \\
.1 \\
.1 \\
.3 \\
.3 \\
.4 \\
.4 \\
.4 \\
.4 \\
.4 \\
.4 \\
3.8\end{array}$ & $\begin{array}{r}5.4 \\
4.6 \\
4.2 \\
3.6 \\
4.3 \\
4.5 \\
5.0 \\
5.2 \\
4.2 \\
5.0 \\
4.4 \\
5.4 \\
55.8\end{array}$ & $\begin{array}{r}7.6 \\
6.8 \\
7.1 \\
6.7 \\
4.7 \\
3.9 \\
3.6 \\
3.5 \\
3.9 \\
5.2 \\
5.2 \\
5.4 \\
63.5\end{array}$ & $\begin{array}{r}2.3 \\
2.1 \\
2.2 \\
1.9 \\
1.9 \\
1.3 \\
1.7 \\
1.1 \\
2.0 \\
2.3 \\
2.2 \\
2.3 \\
23.4\end{array}$ & $\begin{array}{r}6.5 \\
6.3 \\
8.3 \\
5.0 \\
6.0 \\
7.0 \\
4.9 \\
5.5 \\
6.9 \\
5.7 \\
6.1 \\
10.4 \\
78.6\end{array}$ & $\begin{array}{r}77.4 \\
70.0 \\
74.1 \\
64.5 \\
59.7 \\
56.2 \\
56.0 \\
55.0 \\
58.8 \\
65.5 \\
65.7 \\
76.5 \\
703.8\end{array}$ \\
\hline 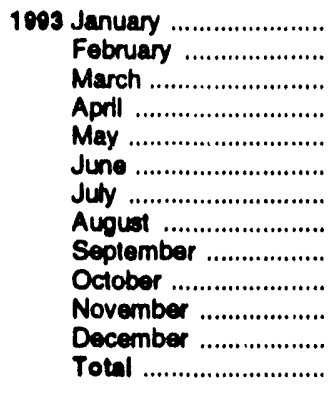 & $\begin{array}{l}4.3 \\
3.7 \\
3.4 \\
3.3 \\
3.1 \\
3.0 \\
3.2 \\
3.4 \\
3.4 \\
3.2 \\
3.7 \\
4.3 \\
41.0\end{array}$ & $\begin{array}{r}1.8 \\
1.6 \\
1.8 \\
1.7 \\
1.3 \\
1.6 \\
1.8 \\
1.5 \\
1.3 \\
1.8 \\
1.7 \\
1.8 \\
19.8\end{array}$ & $\begin{array}{r}36.3 \\
32.7 \\
34.3 \\
30.5 \\
26.9 \\
25.4 \\
26.9 \\
25.9 \\
28.8 \\
29.1 \\
33.7 \\
36.2 \\
366.7\end{array}$ & $\begin{array}{r}15.1 \\
13.9 \\
14.2 \\
12.4 \\
11.8 \\
12.0 \\
12.3 \\
11.1 \\
11.2 \\
12.6 \\
12.6 \\
14.3 \\
153.5\end{array}$ & $\begin{array}{l}.0 \\
.0 \\
.0 \\
.0 \\
.0 \\
.0 \\
.0 \\
.0 \\
.0 \\
.0 \\
.0 \\
.0 \\
.0\end{array}$ & $\begin{array}{r}.4 \\
.3 \\
.1 \\
.1 \\
.4 \\
.4 \\
.4 \\
.4 \\
.4 \\
.4 \\
.4 \\
.4 \\
3.0\end{array}$ & $\begin{array}{r}5.4 \\
4.3 \\
4.9 \\
4.2 \\
4.1 \\
4.4 \\
5.0 \\
5.1 \\
4.6 \\
4.7 \\
4.2 \\
5.2 \\
56.1\end{array}$ & $\begin{array}{r}5.8 \\
5.8 \\
7.1 \\
6.6 \\
4.6 \\
4.7 \\
3.1 \\
3.2 \\
4.1 \\
4.7 \\
5.3 \\
6.3 \\
61.4\end{array}$ & $\begin{array}{r}2.3 \\
2.1 \\
2.3 \\
2.0 \\
1.0 \\
1.2 \\
1.8 \\
1.1 \\
1.7 \\
2.2 \\
2.3 \\
2.4 \\
23.3\end{array}$ & $\begin{array}{r}7.6 \\
7.0 \\
8.3 \\
7.7 \\
6.0 \\
8.2 \\
6.4 \\
6.1 \\
8.4 \\
6.9 \\
6.7 \\
10.2 \\
90.4\end{array}$ & $\begin{array}{r}78.9 \\
72.6 \\
78.3 \\
68.6 \\
60.1 \\
60.7 \\
60.8 \\
67.9 \\
63.0 \\
65.7 \\
70.6 \\
81.0 \\
817.0\end{array}$ \\
\hline 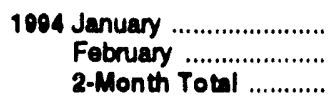 & $\begin{array}{l}4.3 \\
3.5 \\
7.8\end{array}$ & $\begin{array}{l}1.8 \\
1.6 \\
3.4\end{array}$ & $\begin{array}{r}A_{34.1} \\
31.5 \\
65.6\end{array}$ & $\begin{array}{l}13.8 \\
12.1 \\
25.8\end{array}$ & $\begin{array}{l}.0 \\
.0 \\
.0\end{array}$ & $\begin{array}{l}.4 \\
.1 \\
.4\end{array}$ & $\begin{array}{l}5.1 \\
4.1 \\
9.2\end{array}$ & $\begin{array}{r}6.9 \\
6.7 \\
13.6\end{array}$ & $\begin{array}{l}2.4 \\
2.1 \\
4.4\end{array}$ & $\begin{array}{r}7.6 \\
6.6 \\
14.2\end{array}$ & $\begin{array}{r}R_{76.2} \\
88.3 \\
144.6\end{array}$ \\
\hline $\begin{array}{l}1003 \text { 2-Month Total ........... } \\
1002 \text { 2-Month Total .......... }\end{array}$ & $\begin{array}{l}8.0 \\
8.3\end{array}$ & $\begin{array}{l}3.4 \\
3.5\end{array}$ & $\begin{array}{l}69.0 \\
83.3\end{array}$ & $\begin{array}{l}20.0 \\
30.8\end{array}$ & $\begin{array}{l}.0 \\
.0\end{array}$ & .7 & $\begin{array}{r}0.0 \\
10.0\end{array}$ & $\begin{array}{l}11.7 \\
14.4\end{array}$ & 4.4 & $\begin{array}{l}18.8 \\
12.8\end{array}$ & $\begin{array}{l}181.4 \\
148.3\end{array}$ \\
\hline
\end{tabular}

- Through December 1990, the data for Germany are for the former West Germany only. Beginning with January 1991, the data for Germany are for the unifled Germany, I.e., the former East Germany and West Germany.

Monthly data for the Unlied KIngdom are totab for 4- or 5-week reporting periods, not calendar months.

R=Revised dala. - Not applicable. (s) $=$ Less than 0.05 billion kilowatthours.
Notes: - Nef floures are generally less than grose flgures by about 5 percent, the difference being the energy consumed by the generaling plants themselves. - Monthly data may not sum lo annual totalo due to independent rounding and because precommerclal generation b included in some annual totals but not in the monthly data. - Data lor counties may not sum to totals due to independent rounding.

Source: McGraw-Hill Publishing Company, Nucleonics Weak. 
Table 10.4d Nuclear Electricity Gross Generation: Far East and Africa (Billion Kllowatthours)

\begin{tabular}{|c|c|c|c|c|c|c|c|c|}
\hline & ChIna & Indla & Japan & Paklstan & $\begin{array}{l}\text { South } \\
\text { Africe }\end{array}$ & $\begin{array}{l}\text { South } \\
\text { Korea }\end{array}$ & Talwan & $\begin{array}{l}\text { Far East } \\
\text { and Africa }\end{array}$ \\
\hline 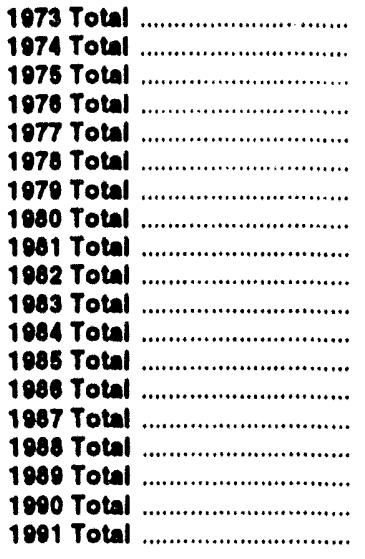 & $\begin{array}{l}- \\
- \\
- \\
- \\
- \\
- \\
- \\
- \\
- \\
- \\
- \\
-\end{array}$ & $\begin{array}{l}2.5 \\
1.9 \\
2.5 \\
3.2 \\
2.8 \\
2.3 \\
3.2 \\
2.9 \\
3.1 \\
2.2 \\
2.0 \\
4.1 \\
4.5 \\
5.1 \\
5.5 \\
6.1 \\
4.0 \\
6.3 \\
5.4\end{array}$ & $\begin{array}{r}0.4 \\
18.9 \\
21.3 \\
36.6 \\
28.2 \\
53.1 \\
62.0 \\
82.8 \\
86.0 \\
104.5 \\
109.1 \\
127.2 \\
152.0 \\
164.8 \\
182.8 \\
173.8 \\
183.7 \\
101.8 \\
205.8\end{array}$ & $\begin{array}{r}0.5 \\
.6 \\
.5 \\
.5 \\
.3 \\
.2 \\
(.6) \\
.1 \\
.2 \\
.1 \\
.2 \\
.3 \\
.3 \\
.5 \\
.3 \\
.2 \\
.1 \\
.4 \\
.4\end{array}$ & $\begin{array}{c}- \\
- \\
- \\
- \\
- \\
- \\
- \\
- \\
- \\
- \\
\overline{-} \\
4.2 \\
5.0 \\
9.3 \\
0.8 \\
11.1 \\
11.7 \\
0.0 \\
0.7\end{array}$ & $\begin{array}{c}- \\
- \\
- \\
- \\
0.1 \\
2.3 \\
3.2 \\
3.5 \\
2.0 \\
3.8 \\
9.0 \\
11.8 \\
16.5 \\
26.1 \\
37.8 \\
38.7 \\
47.2 \\
52.8 \\
56.3\end{array}$ & $\begin{array}{c}- \\
- \\
- \\
- \\
0.1 \\
2.7 \\
6.3 \\
8.2 \\
10.7 \\
13.1 \\
18.0 \\
24.3 \\
28.7 \\
28.0 \\
33.1 \\
20.9 \\
28.3 \\
32.0 \\
35.3\end{array}$ & $\begin{array}{c}12.3 \\
21.4 \\
24.4 \\
40.3 \\
31.6 \\
80.0 \\
74.7 \\
07.4 \\
102.0 \\
123.6 \\
140.1 \\
171.0 \\
207.0 \\
232.0 \\
208.1 \\
259.6 \\
275.1 \\
203.2 \\
313.0\end{array}$ \\
\hline 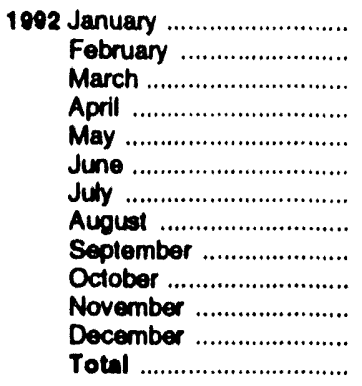 & $\begin{array}{l}- \\
- \\
- \\
- \\
- \\
- \\
- \\
- \\
- \\
-\end{array}$ & $\begin{array}{l}.5 \\
.5 \\
.5 \\
.4 \\
.4 \\
.3 \\
.4 \\
.5 \\
.5 \\
.6 \\
.7 \\
.8 \\
6.3\end{array}$ & $\begin{array}{r}18.5 \\
17.1 \\
17.9 \\
16.0 \\
16.3 \\
17.1 \\
21.1 \\
23.1 \\
17.2 \\
16.2 \\
16.3 \\
19.1 \\
218.0\end{array}$ & $\begin{array}{l}\text { (s) } \\
.0 \\
(9) \\
(s) \\
(s) \\
.1 \\
.1 \\
.1 \\
.1 \\
.1 \\
.1 \\
.1 \\
.6\end{array}$ & $\begin{array}{r}.9 \\
.4 \\
.4 \\
.4 \\
.7 \\
1.2 \\
1.3 \\
1.0 \\
1.1 \\
1.0 \\
.6 \\
.8 \\
0.0\end{array}$ & $\begin{array}{r}4.6 \\
4.0 \\
4.2 \\
4.5 \\
4.5 \\
4.5 \\
5.3 \\
5.4 \\
4.6 \\
4.9 \\
4.7 \\
5.1 \\
56.4\end{array}$ & $\begin{array}{r}3.1 \\
2.2 \\
2.2 \\
2.6 \\
2.6 \\
2.9 \\
3.3 \\
3.6 \\
2.8 \\
2.9 \\
3.2 \\
2.6 \\
33.6\end{array}$ & $\begin{array}{r}27.7 \\
24.2 \\
25.2 \\
23.9 \\
24.6 \\
26.1 \\
31.5 \\
33.7 \\
26.3 \\
25.7 \\
25.6 \\
28.4 \\
325.1\end{array}$ \\
\hline 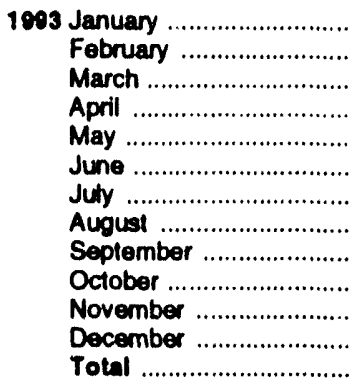 & $\begin{array}{l}- \\
- \\
- \\
- \\
\text { NA } \\
\text { NA } \\
\text { NA } \\
\text { NA } \\
\text { NA } \\
\text { NA } \\
\text { NA } \\
\text { NA } \\
\text { E.6 }\end{array}$ & $\begin{array}{l}.7 \\
.6 \\
.6 \\
.2 \\
.4 \\
.5 \\
.7 \\
.5 \\
.4 \\
.5 \\
.5 \\
.6 \\
6.2\end{array}$ & $\begin{array}{r}19.5 \\
17.4 \\
18.9 \\
17.6 \\
17.4 \\
17.9 \\
22.3 \\
24.2 \\
20.5 \\
20.6 \\
20.9 \\
21.5 \\
243.5\end{array}$ & $\begin{array}{l}\text { (s) } \\
.1 \\
.1 \\
.1 \\
\text { (s) } \\
(s) \\
.1 \\
\text { (s) } \\
.1 \\
\text { (s) } \\
.0 \\
\text { (s) } \\
.4\end{array}$ & $\begin{array}{r}.6 \\
.6 \\
.5 \\
.6 \\
.8 \\
.5 \\
1.0 \\
.9 \\
.5 \\
.4 \\
.4 \\
.8 \\
7.7\end{array}$ & $\begin{array}{l}4.8 \\
4.5 \\
4.6 \\
4.8 \\
5.3 \\
5.1 \\
5.5 \\
4.9 \\
4.6 \\
4.6 \\
4.2 \\
5.1 \\
58.1\end{array}$ & $\begin{array}{l}3.0 \\
2.7 \\
2.8 \\
2.8 \\
2.7 \\
2.6 \\
3.4 \\
3.6 \\
2.9 \\
2.8 \\
2.3 \\
2.8 \\
34.3\end{array}$ & $\begin{array}{r}28.7 \\
26.0 \\
27.5 \\
26.1 \\
E_{26.6} \\
E_{26.6} \\
E_{32.8} \\
E_{34.1} \\
E_{29.0} \\
E_{28.0} \\
E_{28.3} \\
E_{30.9} \\
\text { AE } 353.0\end{array}$ \\
\hline 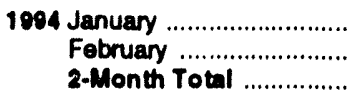 & $\begin{array}{l}\text { NA } \\
\text { NA } \\
\text { NA }\end{array}$ & $\begin{array}{l}.4 \\
.3 \\
.7\end{array}$ & $\begin{array}{l}20.5 \\
17.8 \\
38.3\end{array}$ & $\begin{array}{l}1 \\
(3) \\
.1\end{array}$ & $\begin{array}{r}.9 \\
.8 \\
1.6\end{array}$ & $\begin{array}{l}5.0 \\
4.1 \\
9.1\end{array}$ & $\begin{array}{l}2.6 \\
2.8 \\
6.3\end{array}$ & $\begin{array}{l}\text { E } 29.5 \\
\text { E } 25.8 \\
\text { E } 5.2\end{array}$ \\
\hline $\begin{array}{l}1903 \text { 2-Month Total ............... } \\
1992 \text { 2-Month Total ............... }\end{array}$ & - & $\begin{array}{l}1.4 \\
1.1\end{array}$ & $\begin{array}{l}37.0 \\
35.5\end{array}$ & .1 & $\begin{array}{l}1.3 \\
1.3\end{array}$ & $\begin{array}{l}0.3 \\
8.7\end{array}$ & $\begin{array}{l}5.7 \\
5.3\end{array}$ & $\begin{array}{l}54.7 \\
51.0\end{array}$ \\
\hline
\end{tabular}

- The total gross generation estimate for 1993 for China is calculated as 5 percent more than the annual nel nuclear generation reported by the Intemational Atomic Energy Agency (IAEA) and is published in Nuclear Power Reactors in the Wortd, April 1994.

R=Revised data. $N A=N o t$ available. $-=N o t$ applicable. E=Estimate. (s) =Less than 0.05 billion kllowatthours.

Notes: - Net figures are generally less than gross figures by about 5 percent, the difference being the energy consumed by the generaling plants themselves. - Monthly data may not sum to annual totals due to independent rounding and because precommercial generation b included in some annual tolals but not in the monthly data. - Data for countries may not sum to tolals due to independent rounding.

Source: McGraw-Hill Publishing Company, Nucleonics Week. 
Table 10.4e Nuclear Electricity Gross Generation: Eastern Europe

(Billion Kilowatthours)

\begin{tabular}{|c|c|c|c|c|c|c|c|c|c|c|c|}
\hline & Bulgarla & $\begin{array}{c}\text { Czech } \\
\text { Republle: }\end{array}$ & Hungary & Kazakhstan & Lithuanian & Aomanlab & Ausela & Slovakia & Slovenla & Ukralne & $\begin{array}{l}\text { Eestern } \\
\text { Europes }\end{array}$ \\
\hline 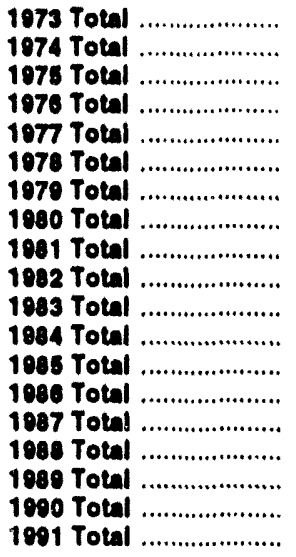 & $\begin{array}{l}\text { NA } \\
\text { NA } \\
\text { NA } \\
\text { NA } \\
\text { NA } \\
\text { NA } \\
\text { NA } \\
\text { NA } \\
\text { NA } \\
\text { NA } \\
\text { NA } \\
\text { NA } \\
\text { NA } \\
\text { NA } \\
\text { NA } \\
\text { NA } \\
\text { NA } \\
\text { NA }\end{array}$ & $\begin{array}{l}- \\
- \\
\overline{-} \\
- \\
- \\
- \\
- \\
- \\
- \\
- \\
- \\
\text { NA } \\
\text { NA } \\
\text { NA } \\
\text { NA } \\
\text { NA } \\
\text { NA } \\
\text { NA }\end{array}$ & $\begin{array}{l}- \\
- \\
- \\
- \\
- \\
- \\
- \\
- \\
- \\
- \\
\text { NA } \\
\text { NA } \\
\text { NA } \\
\text { NA } \\
\text { NA } \\
\text { NA } \\
\text { NA } \\
\text { NA } \\
\text { NA }\end{array}$ & $\begin{array}{l}\text { NA } \\
\text { NA } \\
\text { NA } \\
\text { NA } \\
\text { NA } \\
\text { NA } \\
\text { NA } \\
\text { NA } \\
\text { NA } \\
\text { NA } \\
\text { NA } \\
\text { NA } \\
\text { NA } \\
\text { NA } \\
\text { NA } \\
\text { NA } \\
\text { NA } \\
\text { NA } \\
\text { NA }\end{array}$ & $\begin{array}{l}- \\
- \\
- \\
- \\
- \\
- \\
- \\
- \\
- \\
- \\
- \\
- \\
\text { NA } \\
\text { NA } \\
\text { NA } \\
\text { NA } \\
\text { NA } \\
\text { NA } \\
\text { NA }\end{array}$ & $\begin{array}{l}- \\
- \\
- \\
- \\
- \\
- \\
- \\
- \\
- \\
- \\
- \\
- \\
- \\
- \\
- \\
-\end{array}$ & $\begin{array}{l}\text { NA } \\
\text { NA } \\
\text { NA } \\
\text { NA } \\
\text { NA } \\
\text { NA } \\
\text { NA } \\
\text { NA } \\
\text { NA } \\
\text { NA } \\
\text { NA } \\
\text { NA } \\
\text { NA } \\
\text { NA } \\
\text { NA } \\
\text { NA } \\
\text { NA } \\
\text { NA } \\
\text { NA }\end{array}$ & $\begin{array}{l}\text { NA } \\
\text { NA } \\
\text { NA } \\
\text { NA } \\
\text { NA } \\
\text { NA } \\
\text { NA } \\
\text { NA } \\
\text { NA } \\
\text { NA } \\
\text { NA } \\
\text { NA } \\
\text { NA } \\
\text { NA } \\
\text { NA } \\
\text { NA } \\
\text { NA } \\
\text { NA } \\
\text { NA }\end{array}$ & $\begin{array}{l}- \\
- \\
- \\
- \\
- \\
- \\
- \\
- \\
- \\
\bar{y} \\
\text { NA } \\
\text { NA } \\
\text { NA } \\
\text { NA } \\
\text { NA } \\
\text { NA } \\
\text { NA } \\
\text { NA } \\
\text { NA }\end{array}$ & $\begin{array}{l}- \\
- \\
- \\
- \\
- \\
\text { NA } \\
\text { NA } \\
\text { NA } \\
\text { NA } \\
\text { NA } \\
\text { NA } \\
\text { NA } \\
\text { NA } \\
\text { NA } \\
\text { NA } \\
\text { NA } \\
\text { NA } \\
\text { NA } \\
\text { NA }\end{array}$ & $\begin{array}{l}\text { NA } \\
\text { NA } \\
\text { NA } \\
\text { NA } \\
\text { NA } \\
\text { NA } \\
\text { NA } \\
\text { NA } \\
\text { NA } \\
\text { NA } \\
\text { NA } \\
\text { NA } \\
\text { NA } \\
\text { NA } \\
\text { NA } \\
\text { NA } \\
\text { NA } \\
\text { NA } \\
\text { NA }\end{array}$ \\
\hline 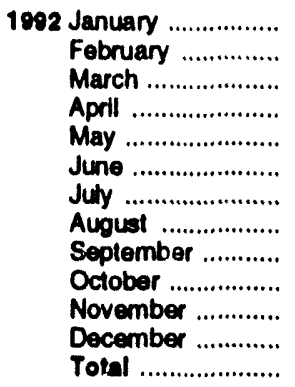 & $\begin{array}{l}\text { NA } \\
\text { NA } \\
\text { NA } \\
\text { NA } \\
\text { NA } \\
\text { NA } \\
\text { NA } \\
\text { NA } \\
\text { NA } \\
\text { NA } \\
\text { NA } \\
\text { NA } \\
\text { 12.2 }\end{array}$ & $\begin{array}{l}\text { NA } \\
\text { NA } \\
\text { NA } \\
\text { NA } \\
\text { NA } \\
\text { NA } \\
\text { NA } \\
\text { NA } \\
\text { NA } \\
\text { NA } \\
\text { NA } \\
\text { NA } \\
\text { 12.0 }\end{array}$ & $\begin{array}{l}\text { NA } \\
\text { NA } \\
\text { NA } \\
\text { NA } \\
\text { NA } \\
\text { NA } \\
\text { NA } \\
\text { NA } \\
\text { NA } \\
\text { NA } \\
\text { NA } \\
\text { NA } \\
\text { E 13.8 }\end{array}$ & $\begin{array}{l}\text { NA } \\
\text { NA } \\
\text { NA } \\
\text { NA } \\
\text { NA } \\
\text { NA } \\
\text { NA } \\
\text { NA } \\
\text { NA } \\
\text { NA } \\
\text { NA } \\
\text { NA } \\
\text { E.5 }\end{array}$ & $\begin{array}{l}\text { NA } \\
\text { NA } \\
\text { NA } \\
\text { NA } \\
\text { NA } \\
\text { NA } \\
\text { NA } \\
\text { NA } \\
\text { NA } \\
\text { NA } \\
\text { NA } \\
\text { NA } \\
\text { 16.4 }\end{array}$ & $\begin{array}{l}- \\
- \\
- \\
- \\
- \\
- \\
- \\
- \\
- \\
-\end{array}$ & $\begin{array}{c}\text { NA } \\
\text { NA } \\
\text { NA } \\
\text { NA } \\
\text { NA } \\
\text { NA } \\
\text { NA } \\
\text { NA } \\
\text { NA } \\
\text { NA } \\
\text { NA } \\
\text { NA } \\
\text { E } 125.6\end{array}$ & $\begin{array}{l}\text { NA } \\
\text { NA } \\
\text { NA } \\
\text { NA } \\
\text { NA } \\
\text { NA } \\
\text { NA } \\
\text { NA } \\
\text { NA } \\
\text { NA } \\
\text { NA } \\
\text { NA } \\
\text { 11.7 }\end{array}$ & $\begin{array}{l}\text { NA } \\
\text { NA } \\
\text { NA } \\
\text { NA } \\
\text { NA } \\
\text { NA } \\
\text { NA } \\
\text { NA } \\
\text { NA } \\
\text { NA } \\
\text { NA } \\
\text { NA } \\
\text { E.0 }\end{array}$ & $\begin{array}{l}\text { NA } \\
\text { NA } \\
N A \\
N A \\
N A \\
N A \\
N A \\
N A \\
N A \\
N A \\
N A \\
N A \\
\text { E4.6 }\end{array}$ & 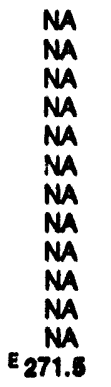 \\
\hline 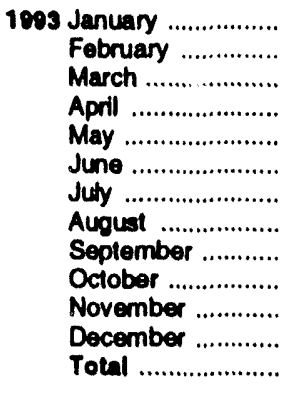 & $\begin{array}{r}E \\
E \\
1.5 \\
E \\
1.5 \\
1.5 \\
1.2 \\
.8 \\
.0 \\
.0 \\
1.1 \\
.6 \\
.9 \\
1.6 \\
14.0\end{array}$ & $\begin{array}{r}\text { NA } \\
N A \\
N A \\
N A \\
N A \\
N A \\
N A \\
N A \\
.9 \\
.9 \\
1.0 \\
.9 \\
\text { E } 13.2\end{array}$ & $\begin{array}{l}1.4 \\
1.2 \\
1.2 \\
1.0 \\
1.0 \\
1.0 \\
1.0 \\
1.0 \\
1.0 \\
1.2 \\
1.3 \\
1.4 \\
13.8\end{array}$ & $\begin{array}{l}\text { NA } \\
\text { NA } \\
\text { NA } \\
\text { NA } \\
\text { NA } \\
\text { NA } \\
\text { NA } \\
\text { NA } \\
\text { NA } \\
\text { NA } \\
\text { NA } \\
\text { NA } \\
E .4\end{array}$ & $\begin{array}{c}\text { NA } \\
\text { NA } \\
\text { NA } \\
\text { NA } \\
\text { NA } \\
\text { NA } \\
\text { NA } \\
\text { NA } \\
\text { NA } \\
\text { NA } \\
\text { NA } \\
\text { NA } \\
\text { E } 12.0\end{array}$ & $\begin{array}{l}- \\
- \\
- \\
- \\
- \\
- \\
- \\
- \\
- \\
- \\
-\end{array}$ & $\begin{array}{r}11.0 \\
9.8 \\
10.6 \\
10.3 \\
9.6 \\
10.1 \\
8.4 \\
9.5 \\
9.3 \\
9.7 \\
10.4 \\
11.9 \\
120.4\end{array}$ & $\begin{array}{l}\text { NA } \\
\text { NA } \\
\text { NA } \\
\text { NA } \\
\text { NA } \\
\text { NA } \\
\text { NA } \\
\text { NA } \\
\text { NA } \\
\text { NA } \\
\text { NA } \\
\text { NA } \\
\text { E 11.6 }\end{array}$ & $\begin{array}{c}.5 \\
.4 \\
.4 \\
.5 \\
.2 \\
.0 \\
(8) \\
.4 \\
.5 \\
.5 \\
.4 \\
.3 \\
.0\end{array}$ & $\begin{array}{r}E_{7.8} \\
\text { E } 7.8 \\
7.8 \\
5.5 \\
5.1 \\
5.0 \\
5.6 \\
6.0 \\
5.1 \\
5.3 \\
5.3 \\
6.3 \\
\text { E } 72.7\end{array}$ & $\begin{array}{c}N A \\
N A \\
N A \\
N A \\
N A \\
N A \\
N A \\
N A \\
N A \\
N A \\
N A \\
N A \\
203.0\end{array}$ \\
\hline $\begin{array}{l}1004 \text { January ................ } \\
\text { February .............. } \\
\text { 2-Month Total ..... }\end{array}$ & $\begin{array}{l}1.6 \\
1.4 \\
3.0\end{array}$ & $\begin{array}{r}\text { A }_{1.2} \\
1.2 \\
2.4\end{array}$ & $\begin{array}{l}1.4 \\
1.2 \\
2.6\end{array}$ & $\begin{array}{l}\text { NA } \\
\text { NA } \\
\text { NA }\end{array}$ & $\begin{array}{l}\text { NA } \\
\text { NA } \\
\text { NA }\end{array}$ & $\begin{array}{l}- \\
-\end{array}$ & $\begin{array}{l}11.0 \\
10.0 \\
21.0\end{array}$ & $\begin{array}{l}\text { NA } \\
\text { NA } \\
\text { NA }\end{array}$ & $\begin{array}{l}.3 \\
.4 \\
.7\end{array}$ & $\begin{array}{r}7.6 \\
6.7 \\
14.4\end{array}$ & $\begin{array}{l}\mathbf{N A} \\
\mathbf{N A}\end{array}$ \\
\hline $\begin{array}{l}1003 \text { 2-Month Total ..... } \\
1002 \text { 2-Month Total ..... }\end{array}$ & $\begin{array}{l}3.0 \\
\text { NA }\end{array}$ & $\begin{array}{l}\text { NA } \\
\text { NA }\end{array}$ & $\begin{array}{l}2.6 \\
\text { NA }\end{array}$ & $\begin{array}{l}\text { NA } \\
\text { NA }\end{array}$ & $\begin{array}{l}\text { NA } \\
\text { NA }\end{array}$ & - & $\begin{array}{l}20.9 \\
\mathrm{NA}\end{array}$ & $\begin{array}{l}\text { NA } \\
\text { NA }\end{array}$ & NA & $\begin{array}{l}15.7 \\
\text { NA }\end{array}$ & $\begin{array}{l}\mathbf{N A} \\
\mathbf{N A}\end{array}$ \\
\hline
\end{tabular}

- The lotal gross generalion estimale for 1993 for Czech Republic, Kazakhstan, Lithuania, and Slovakia is calculaled as 5 percent more than the annual nel nuclear generation reported by the International Alomic Energy Agency (LEA) and is published in Nuclear Power Reactors in the World, April 1994.

Romania has a nucleas generating unit under construction. Its eartiest Initial operation is projected to be in 1995

c The total gross generation estimale for 1992 for Eastern European counitiee are calculated as 5 percert more than the annual net nuclear generation reported by the LAEA and published in the Energy Informalion Administration annual repon. World Nuctaar Capacity and Fuel Cyclo
Requirements 1993, November 1893, Table 10.

$R=$ Revised data. $N A=$ Not available. $-=N o t$ applicable. ExEstimate. Noles: - Armenia has two nuclear generaling units under conetruction. The earliest initial commercial operation for one unit is projected to be in 1995. - Nel figures are generally less than gross llgures by aboul 5 percent, the difference being the energy consumed by the generaling plants themsolves. - Monthly data may not sum to annual tolats due to independent rounding and because precommerclal generation is included in some annual tolas but not in the monthly data. - Data for countries may not sum to totals due to independent rounding.

Source: McGraw-Hill Publishing Company, Nucleonics Weok. 


\section{Appendix A. Thermal Conversion Factors}

The thermal conversion factors presented in the following eight tables can be used to estimate the heat content in British thermal units (Btu) of a given amount of energy measured in physical units, such as barrels or cubic feet. For example, 10 barrels of asphalt have a heat content of approximately 66.36 million Btu (10 barrels $\times 6.636$ million Btu/barrel $=$ 66.36 million $\mathrm{Btu})$.

Thermal conversion factors for hydrocarbon mixes (Table $A 1$ ) are weighted averages of the thermal conversion factors for each bydrocarbon included in the mix. For example, in calculating the thermal conversion factor for a $60-40$ butane-propane mixture, the thermal conversion factor for butane is weighted 1.5 times more heavily than the thermal conversion factor for propane.

In general, the annual thermal conversion factors presented in Tables Al through A8 are computed from final annual data. However, if the current year's final data are not avallable in time for publication, thermal conversion factors for the current year are computed from the best avallable data and are labeled "preliminary." The source of each factor is described in the section entitled "Thermal Conversion Factor Source Documentation," which follows Table A8 in this appendix.

\section{Table A1. Approximate Heat Content of Petroleum Products (Million Btu per Barrel)}

\begin{tabular}{|c|c|c|c|}
\hline Petroleum Product & Heat Content & Petroleum Product & t Content \\
\hline 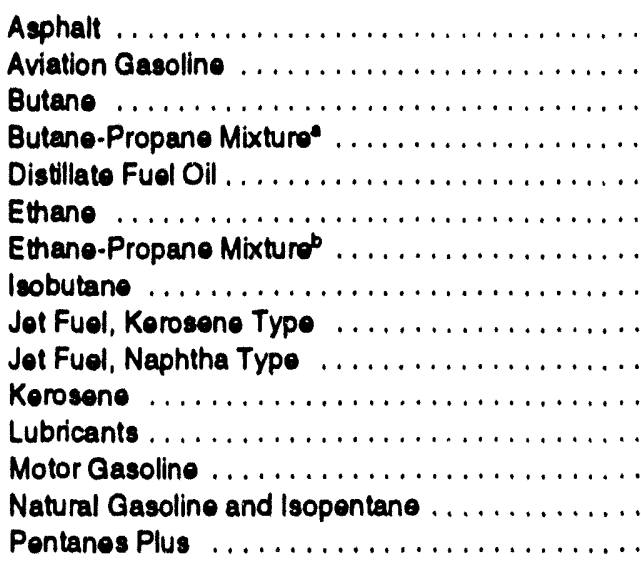 & $\begin{array}{l}6.636 \\
5.048 \\
4.326 \\
4.130 \\
5.825 \\
3.082 \\
3.308 \\
3.974 \\
5.670 \\
5.355 \\
5.670 \\
6.065 \\
5.253 \\
4.620 \\
4.620\end{array}$ & 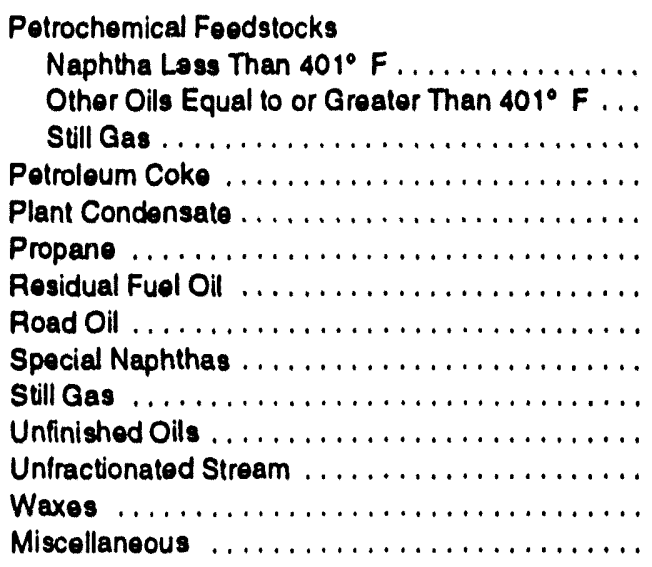 & $\begin{array}{l}5.248 \\
5.825 \\
6.000 \\
6.024 \\
5.418 \\
3.836 \\
6.287 \\
6.636 \\
6.248 \\
6.000 \\
5.825 \\
5.418 \\
5.537 \\
5.796\end{array}$ \\
\hline
\end{tabular}

\footnotetext{
- 60 percent butane and 40 percent propane.

- 70 percent ethane and 30 percent propane.

Source: See "Thermal Conversion Factor Source Documentallon," which lollows Table AB.
} 
Table A2. Approximate Heat Content of Crude Oll, Crude Oll and Products, and Natural Gas Plant Llquids

(Million Btu per Barrel)

\begin{tabular}{|c|c|c|c|c|c|c|}
\hline & \multicolumn{3}{|c|}{ Crude oil } & \multicolumn{2}{|c|}{ Crude Oil and Products } & \multirow{2}{*}{$\begin{array}{l}\text { Nolurel Ces } \\
\text { Plent Llquido } \\
\text { Production }\end{array}$} \\
\hline & Production & Importe & Exports & Imports & Exports & \\
\hline 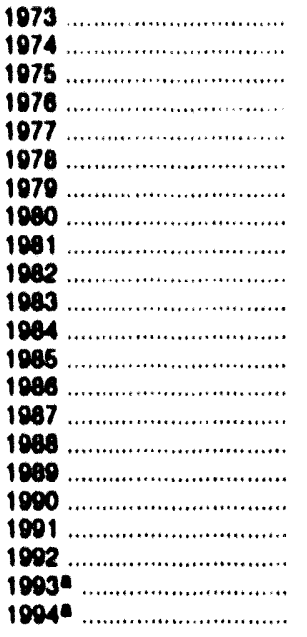 & $\begin{array}{l}5.800 \\
5.800 \\
5.800 \\
5.800 \\
5.800 \\
5.800 \\
5.800 \\
5.800 \\
5.800 \\
5.800 \\
5.800 \\
5.800 \\
5.800 \\
5.800 \\
5.800 \\
5.800 \\
5.800 \\
5.800 \\
5.800 \\
5.800 \\
5.800 \\
5.800\end{array}$ & $\begin{array}{l}5.817 \\
5.827 \\
5.821 \\
5.808 \\
5.810 \\
5.802 \\
5.810 \\
5.812 \\
5.818 \\
5.826 \\
5.825 \\
5.823 \\
5.832 \\
5.903 \\
5.901 \\
5.900 \\
5.006 \\
5.934 \\
5.948 \\
5.953 \\
5.954 \\
5.954\end{array}$ & $\begin{array}{l}5.800 \\
5.800 \\
5.800 \\
5.800 \\
5.800 \\
5.800 \\
5.800 \\
5.800 \\
5.800 \\
5.800 \\
5.800 \\
5.800 \\
5.800 \\
5.800 \\
5.800 \\
5.800 \\
5.800 \\
5.800 \\
5.800 \\
5.800 \\
5.800 \\
5.800\end{array}$ & $\begin{array}{l}5.897 \\
5.884 \\
5.858 \\
5.856 \\
5.834 \\
5.839 \\
5.810 \\
5.706 \\
6.775 \\
5.775 \\
5.774 \\
5.745 \\
5.736 \\
5.808 \\
5.820 \\
5.820 \\
5.835 \\
5.849 \\
5.873 \\
5.877 \\
5.879 \\
5.879\end{array}$ & $\begin{array}{l}5.752 \\
5.774 \\
5.748 \\
5.745 \\
5.797 \\
6.808 \\
5.832 \\
5.820 \\
5.821 \\
6.820 \\
5.800 \\
5.850 \\
5.814 \\
5.832 \\
5.858 \\
6.840 \\
6.857 \\
5.833 \\
6.823 \\
5.777 \\
5.761 \\
5.761\end{array}$ & $\begin{array}{l}4.040 \\
4.011 \\
3.084 \\
3.084 \\
3.041 \\
3.926 \\
3.965 \\
3.914 \\
3.930 \\
3.872 \\
3.830 \\
3.812 \\
3.816 \\
3.707 \\
3.804 \\
3.800 \\
3.820 \\
3.820 \\
3.807 \\
3.804 \\
3.800 \\
3.800\end{array}$ \\
\hline
\end{tabular}

- Preliminary.

Note: Crude oll includes lease condensate

Source: Soe "Thermal Converion Factor Source Documentalion," which lollows Table A8.

Table A3. Approximate Heat Content of Petroleum Product Weighted Averages (Million Btu per Barrel)

\begin{tabular}{|c|c|c|c|c|c|c|c|c|}
\hline & \multicolumn{5}{|c|}{ Coneumption } & \multirow[b]{2}{*}{ Imports } & \multirow[b]{2}{*}{ Exports } & \multirow[b]{2}{*}{$\begin{array}{c}\text { LPQ } \\
\text { Consumption }\end{array}$} \\
\hline & $\begin{array}{l}\text { Realdenila } \\
\text { and } \\
\text { Commercial }\end{array}$ & Induetrial & Traneportation & $\begin{array}{l}\text { Electric } \\
\text { Utilities }\end{array}$ & Total & & & \\
\hline 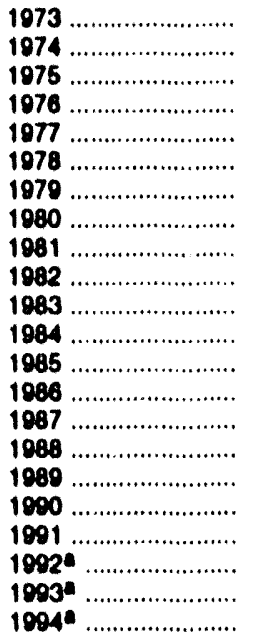 & $\begin{array}{l}5.387 \\
5.377 \\
5.358 \\
5.383 \\
5.389 \\
5.382 \\
5.471 \\
5.468 \\
5.400 \\
5.392 \\
5.288 \\
5.384 \\
5.326 \\
5.357 \\
5.316 \\
5.320 \\
5.257 \\
5.208 \\
5.163 \\
5.169 \\
5.176 \\
5.176\end{array}$ & $\begin{array}{l}5.568 \\
5.538 \\
5.528 \\
5.538 \\
5.555 \\
5.553 \\
5.418 \\
5.378 \\
5.313 \\
5.263 \\
5.273 \\
5.223 \\
5.221 \\
5.286 \\
5.253 \\
5.248 \\
5.233 \\
5.272 \\
5.192 \\
5.188 \\
5.104 \\
5.194\end{array}$ & $\begin{array}{l}5.395 \\
5.394 \\
5.392 \\
5.395 \\
5.400 \\
5.404 \\
5.428 \\
5.440 \\
5.432 \\
5.422 \\
5.415 \\
5.422 \\
5.423 \\
5.427 \\
5.430 \\
5.434 \\
5.440 \\
5.445 \\
5.442 \\
5.445 \\
5.441 \\
5.441\end{array}$ & $\begin{array}{l}6.245 \\
6.238 \\
6.250 \\
6.251 \\
6.249 \\
6.251 \\
6.258 \\
6.254 \\
6.258 \\
6.258 \\
6.255 \\
6.251 \\
6.247 \\
6.257 \\
6.249 \\
6.250 \\
6.241 \\
6.247 \\
6.248 \\
6.243 \\
6.241 \\
6.241\end{array}$ & $\begin{array}{l}5.515 \\
5.504 \\
5.494 \\
5.504 \\
5.518 \\
5.510 \\
5.494 \\
5.479 \\
5.448 \\
5.415 \\
5.408 \\
5.395 \\
5.387 \\
5.418 \\
5.403 \\
5.410 \\
5.410 \\
5.411 \\
5.384 \\
5.378 \\
5.381 \\
5.381\end{array}$ & $\begin{array}{l}5.983 \\
5.950 \\
5.935 \\
5.980 \\
5.908 \\
5.955 \\
5.811 \\
5.748 \\
5.658 \\
5.664 \\
5.677 \\
5.613 \\
5.572 \\
5.624 \\
5.599 \\
5.618 \\
5.641 \\
5.614 \\
5.636 \\
6.623 \\
5.602 \\
5.602\end{array}$ & $\begin{array}{l}5.752 \\
6.773 \\
5.747 \\
5.743 \\
5.706 \\
5.814 \\
5.864 \\
5.841 \\
5.837 \\
5.829 \\
5.800 \\
5.867 \\
5.819 \\
5.830 \\
5.860 \\
5.842 \\
5.869 \\
5.838 \\
5.827 \\
5.774 \\
5.757 \\
5.757\end{array}$ & $\begin{array}{l}3.746 \\
3.730 \\
3.716 \\
3.711 \\
3.677 \\
3.689 \\
3.680 \\
3.674 \\
3.643 \\
3.616 \\
3.614 \\
3.590 \\
3.603 \\
3.640 \\
3.659 \\
3.662 \\
3.683 \\
3.625 \\
3.614 \\
3.624 \\
3.603 \\
3.603\end{array}$ \\
\hline
\end{tabular}

- Preliminary.

Nole: Welghted everages of the products included in each calegory are calculated by using heal content values shown in Table A1.

Source: See "Thermal Conversion Factor Source Documentation." which follows Table AB. 
Table A4. Approximate Heat Content of Natural Gas (Btu per Cubic Foot)

\begin{tabular}{|c|c|c|c|c|c|c|c|}
\hline & \multicolumn{2}{|c|}{ Production } & \multicolumn{3}{|c|}{ Consumption } & \multirow[b]{2}{*}{ Importe } & \multirow[b]{2}{*}{ Expons } \\
\hline & Dry & $\begin{array}{l}\text { Mectroled } \\
\text { WoI) }\end{array}$ & $\begin{array}{c}\text { Then Electirte } \\
\text { Unilliles }\end{array}$ & $\begin{array}{l}\text { Eleotrle } \\
\text { Villities }\end{array}$ & Total & & \\
\hline 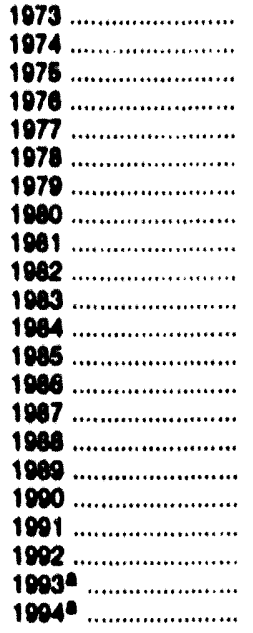 & 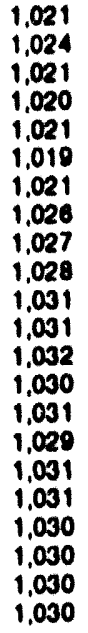 & $\begin{array}{l}1.093 \\
1,097 \\
1,096 \\
1.093 \\
1,093 \\
1,088 \\
1,092 \\
1,098 \\
1.103 \\
1,107 \\
1.116 \\
1.109 \\
1.112 \\
1.110 \\
1.112 \\
1.100 \\
1.107 \\
1.105 \\
1.108 \\
1.110 \\
1.110 \\
1.110\end{array}$ & $\begin{array}{l}1,020 \\
1,024 \\
1,020 \\
1,019 \\
1,019 \\
1,016 \\
1.018 \\
1.024 \\
1,025 \\
1,026 \\
1.031 \\
1,030 \\
1,031 \\
1.029 \\
1.031 \\
1,029 \\
1,031 \\
1.030 \\
1.031 \\
1.031 \\
1.031 \\
1.031\end{array}$ & $\begin{array}{l}1.024 \\
1.022 \\
1,026 \\
1.023 \\
1.029 \\
1.034 \\
1.035 \\
1.036 \\
1,035 \\
1.036 \\
1.030 \\
1,035 \\
1.038 \\
1.034 \\
1.032 \\
1.028 \\
1.030 \\
1.034 \\
1.024 \\
1.022 \\
1.022 \\
1.022\end{array}$ & $\begin{array}{l}1.021 \\
1.024 \\
1.021 \\
1.020 \\
1.021 \\
1,010 \\
1,021 \\
1.026 \\
1,027 \\
1,028 \\
1.031 \\
1.031 \\
1.032 \\
1.030 \\
1.031 \\
1.020 \\
1.031 \\
1.031 \\
1.030 \\
1.030 \\
1.030 \\
1.030\end{array}$ & $\begin{array}{l}1,026 \\
1,027 \\
1,026 \\
1,026 \\
1,026 \\
1,030 \\
1,037 \\
1,022 \\
1,014 \\
1,010 \\
1,024 \\
1,006 \\
1,002 \\
007 \\
009 \\
1,002 \\
1,004 \\
1,012 \\
1,014 \\
1,011 \\
1,011 \\
1,011\end{array}$ & $\begin{array}{l}1,003 \\
1,018 \\
1,014 \\
1,013 \\
1,013 \\
1,013 \\
1,013 \\
1,013 \\
1,011 \\
1,011 \\
1,010 \\
1,010 \\
1,011 \\
1,003 \\
1,011 \\
1,018 \\
1,010 \\
1,018 \\
1,022 \\
1,018 \\
1,018 \\
1,018\end{array}$ \\
\hline
\end{tabular}

- Prollminary.

Source: See "Thermal Conversion Factor Source Documentation," which lollows Table AB.

Table A5. Approximate Heat Content of Coal (Million Btu per Short Ton)

\begin{tabular}{|c|c|c|c|c|c|c|c|c|}
\hline & \multirow[b]{2}{*}{ Production } & \multicolumn{5}{|c|}{ Coneumption } & \multirow[b]{2}{*}{ Importe } & \multirow[b]{2}{*}{ Exporto } \\
\hline & & $\begin{array}{l}\text { Hosidenilal } \\
\text { and } \\
\text { Commercial }\end{array}$ & $\begin{array}{l}\text { Coke } \\
\text { Plants }\end{array}$ & $\begin{array}{c}\text { Other } \\
\text { Industrial* }\end{array}$ & $\begin{array}{l}\text { Electrie } \\
\text { Utilitiosb }\end{array}$ & Total & & \\
\hline 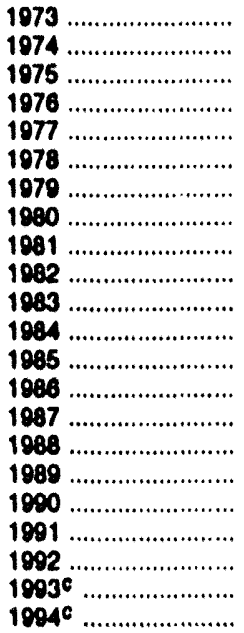 & $\begin{array}{l}23.378 \\
23.072 \\
22.897 \\
22.855 \\
22.597 \\
22.248 \\
22.454 \\
22.415 \\
22.308 \\
22.239 \\
22.052 \\
22.010 \\
21.870 \\
21.913 \\
21.022 \\
21.823 \\
21.765 \\
21.822 \\
21.681 \\
21.646 \\
21.397 \\
\text { ค } 21.397\end{array}$ & $\begin{array}{l}22.831 \\
22.479 \\
22.261 \\
22.774 \\
22.919 \\
22.466 \\
22.242 \\
22.543 \\
22.474 \\
22.695 \\
22.775 \\
22.844 \\
22.646 \\
22.947 \\
23.404 \\
23.571 \\
23.650 \\
23.137 \\
23.114 \\
23.105 \\
\text { ค } 23.124 \\
23.124\end{array}$ & $\begin{array}{l}26.780 \\
26.778 \\
26.782 \\
26.781 \\
26.787 \\
26.789 \\
26.788 \\
26.790 \\
26.794 \\
26.797 \\
26.798 \\
26.799 \\
26.798 \\
26.798 \\
26.799 \\
26.799 \\
26.800 \\
26.799 \\
26.799 \\
26.799 \\
\text { P } 26.800 \\
\text { ค } 26.800\end{array}$ & $\begin{array}{l}22.586 \\
22.419 \\
22.436 \\
22.530 \\
22.322 \\
22.207 \\
22.452 \\
22.690 \\
22.585 \\
22.712 \\
22.691 \\
22.543 \\
22.020 \\
22.198 \\
22.381 \\
22.360 \\
22.347 \\
22.457 \\
22.460 \\
22.250 \\
\text { ค } 22.195 \\
\text { ค } 22.195\end{array}$ & $\begin{array}{r}22.246 \\
21.781 \\
21.642 \\
21.679 \\
21.508 \\
21.275 \\
21.384 \\
21.295 \\
21.085 \\
21.194 \\
21.133 \\
21.101 \\
20.959 \\
21.084 \\
21.136 \\
20.900 \\
20.848 \\
20.929 \\
20.755 \\
20.787 \\
\text { ค } 20.039 \\
\text { ค } 20.630\end{array}$ & $\begin{array}{r}23.057 \\
22.677 \\
22.506 \\
22.498 \\
22.265 \\
22.017 \\
22.100 \\
21.947 \\
21.713 \\
21.674 \\
21.576 \\
21.573 \\
21.366 \\
21.462 \\
21.517 \\
21.328 \\
21.272 \\
21.331 \\
21.146 \\
21.143 \\
\text { A } 20.993 \\
\text { ค } 20.993\end{array}$ & $\begin{array}{l}25.000 \\
25.000 \\
25.000 \\
25.000 \\
25.000 \\
25.000 \\
25.000 \\
25.000 \\
25.000 \\
25.000 \\
25.000 \\
25.000 \\
25.000 \\
25.000 \\
25.000 \\
25.000 \\
25.000 \\
25.000 \\
25.000 \\
25.000 \\
25.000 \\
25.000\end{array}$ & $\begin{array}{r}26.690 \\
26.700 \\
26.582 \\
26.601 \\
26.544 \\
26.478 \\
26.549 \\
26.384 \\
26.160 \\
26.223 \\
26.291 \\
26.402 \\
26.307 \\
26.202 \\
26.291 \\
26.290 \\
26.160 \\
26.202 \\
26.188 \\
26.161 \\
\text { ค26.336 } \\
\text { ค } 26.336\end{array}$ \\
\hline
\end{tabular}

- Inctudes transpontation.

bata shown in this column are nol the same as those shown in the Electric Power Monthy (EPM). The EPM data report coal recelpta; the data shown here represent coal consumption.

- Preliminary.

R=mevised dala.

Source: See "Thermal Conversion Factor Source Documentalion," which lollows Table AB. 
Table A6. Approximate Heat Content of Bltuminous Coal and Lignite (Million Btu per Short Ton)

\begin{tabular}{|c|c|c|c|c|c|c|c|c|}
\hline & \multirow[b]{2}{*}{ Production } & \multicolumn{5}{|c|}{ Coneumption } & \multirow[b]{2}{*}{ Imports } & \multirow[b]{2}{*}{ Exponte } \\
\hline & & Commerold & $\begin{array}{l}\text { Coke } \\
\text { Plante }\end{array}$ & $\begin{array}{c}\text { Othor } \\
\text { Industriste }\end{array}$ & $\begin{array}{l}\text { Electrio } \\
\text { Utilitice }\end{array}$ & Total & & \\
\hline 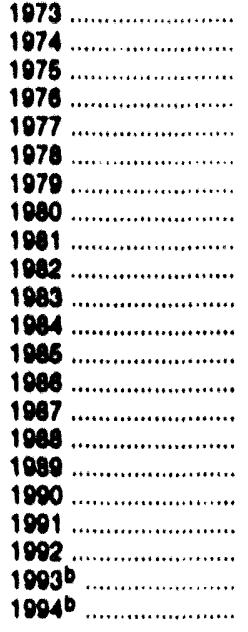 & $\begin{array}{l}23.301 \\
23.087 \\
22.010 \\
22.863 \\
22.607 \\
22.242 \\
22.440 \\
22.411 \\
22.301 \\
22.230 \\
22.049 \\
22.006 \\
21.807 \\
21.006 \\
21.018 \\
21.817 \\
21.750 \\
21.019 \\
21.678 \\
21.643 \\
21.393 \\
21.393\end{array}$ & 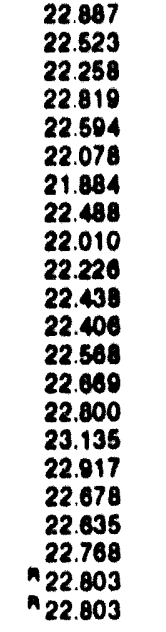 & $\begin{array}{l}26.800 \\
26.800 \\
26.800 \\
26.800 \\
26.800 \\
26.800 \\
26.800 \\
26.800 \\
26.800 \\
26.800 \\
26.800 \\
26.800 \\
26.800 \\
26.800 \\
26.800 \\
26.800 \\
26.800 \\
26.800 \\
26.800 \\
26.800 \\
26.800 \\
28.800\end{array}$ & $\begin{array}{l}22.585 \\
22.420 \\
22.430 \\
22.528 \\
22.290 \\
22.176 \\
22.436 \\
22.690 \\
22.572 \\
22.695 \\
22.680 \\
22.525 \\
22.013 \\
22.185 \\
22.380 \\
22.341 \\
22.324 \\
22.444 \\
22.448 \\
22.242 \\
22.183 \\
22.183\end{array}$ & $\begin{array}{r}22.282 \\
21.709 \\
21.859 \\
21.692 \\
21.521 \\
21.284 \\
21.372 \\
21.301 \\
21.091 \\
21.200 \\
21.141 \\
21.108 \\
20.085 \\
21.001 \\
21.143 \\
20.005 \\
20.854 \\
20.035 \\
20.761 \\
20.702 \\
n_{20.644} \\
n_{20.644}\end{array}$ & $\begin{array}{r}23.073 \\
22.604 \\
22.522 \\
22.509 \\
22.268 \\
22.014 \\
22.100 \\
21.050 \\
21.710 \\
21.670 \\
21.570 \\
21.570 \\
21.368 \\
21.462 \\
21.514 \\
21.324 \\
21.268 \\
21.330 \\
21.146 \\
21.142 \\
220.092 \\
\text { A } 20.992\end{array}$ & $\begin{array}{l}25.000 \\
25.000 \\
25.000 \\
25.000 \\
25.000 \\
25.000 \\
25.000 \\
25.000 \\
25.000 \\
25.000 \\
25.000 \\
25.000 \\
25.000 \\
25.000 \\
25.000 \\
25.000 \\
25.000 \\
25.000 \\
25.000 \\
25.000 \\
25.000 \\
25.000\end{array}$ & 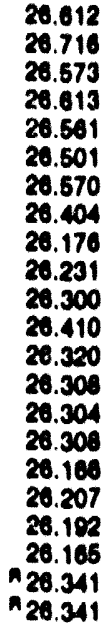 \\
\hline
\end{tabular}

- Inctudec transportation.

- Proliminary.

RaRevieed data.

Source: Ses "Thermal Conversion Factor Source Documentation," which follows Table A8.

Table A7. Approximate Heat Content of Anthracite and Coal Coke (Million Btu per Short Ton)

\begin{tabular}{|c|c|c|c|c|c|c|}
\hline & \multicolumn{5}{|c|}{ Anthrache } & \multirow[b]{3}{*}{$\begin{array}{l}\text { Cod Coke } \\
\text { Imports } \\
\text { and } \\
\text { Exports }\end{array}$} \\
\hline & \multirow[b]{2}{*}{ Production } & \multicolumn{3}{|c|}{ Consumption } & \multirow[b]{2}{*}{$\begin{array}{l}\text { Import } \\
\text { end } \\
\text { Exports }\end{array}$} & \\
\hline & & $\begin{array}{c}\text { Sectore Other } \\
\text { Then Electrie } \\
\text { Utillitos }\end{array}$ & Electric Utilitios & Total & & \\
\hline 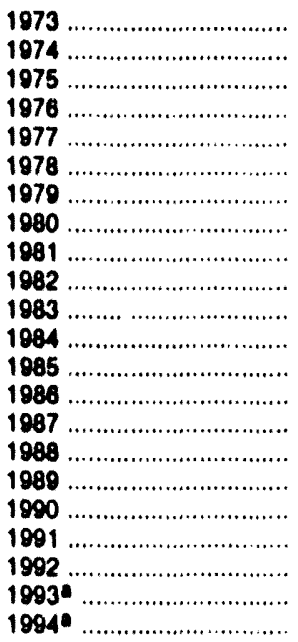 & $\begin{array}{l}22.132 \\
21.711 \\
21.582 \\
22.045 \\
22.661 \\
23.079 \\
23.170 \\
22.869 \\
23.291 \\
23.289 \\
22.734 \\
23.107 \\
22.428 \\
23.084 \\
23.108 \\
23.268 \\
23.385 \\
22.574 \\
22.573 \\
22.572 \\
22.573 \\
\text { ค } 22.573\end{array}$ & $\begin{array}{l}22.674 \\
22.330 \\
22.272 \\
22.618 \\
24.101 \\
24.388 \\
24.272 \\
22.719 \\
23.749 \\
24.578 \\
24.536 \\
25.128 \\
23.031 \\
24.399 \\
26.293 \\
26.021 \\
27.196 \\
25.199 \\
25.268 \\
24.617 \\
\text { R } 24.566 \\
\text { P } 24.566\end{array}$ & $\begin{array}{l}17.920 \\
17.200 \\
17.064 \\
17.526 \\
17.244 \\
17.104 \\
17.454 \\
17.652 \\
18.168 \\
18.160 \\
16.516 \\
17.018 \\
16.784 \\
15.578 \\
15.062 \\
17.312 \\
16.310 \\
16.140 \\
15.858 \\
16.844 \\
16.534 \\
16.534\end{array}$ & $\begin{array}{r}21.464 \\
20.919 \\
20.762 \\
21.254 \\
22.066 \\
22.398 \\
22.069 \\
21.405 \\
22.080 \\
22.518 \\
21.583 \\
22.322 \\
20.817 \\
21.512 \\
22.435 \\
22.423 \\
22.623 \\
21.668 \\
21.410 \\
21.423 \\
\text { A } 21.492 \\
\text { ค } 21.492\end{array}$ & $\begin{array}{l}25.400 \\
25.400 \\
25.400 \\
25.400 \\
25.400 \\
25.400 \\
25.400 \\
25.400 \\
25.400 \\
25.400 \\
25.400 \\
25.400 \\
25.400 \\
25.400 \\
25.400 \\
25.400 \\
25.400 \\
25.400 \\
25.400 \\
25.400 \\
25.400 \\
25.400\end{array}$ & $\begin{array}{l}24.800 \\
24.800 \\
24.800 \\
24.800 \\
24.800 \\
24.800 \\
24.800 \\
24.800 \\
24.800 \\
24.800 \\
24.800 \\
24.800 \\
24.800 \\
24.800 \\
24.800 \\
24.800 \\
24.800 \\
24.800 \\
24.800 \\
24.800 \\
24.800 \\
24.800\end{array}$ \\
\hline
\end{tabular}

- Proliminary.

R=Revied dala.

Source: See "Thermal Conversion Factor Source Documentation," which lollows Table A8. 


\begin{tabular}{|c|c|c|c|c|}
\hline & \multicolumn{3}{|c|}{ Eleotriehy cenerction } & \multirow[b]{2}{*}{$\begin{array}{l}\text { Electrlaily } \\
\text { Consumption }\end{array}$} \\
\hline & $\begin{array}{c}\text { Foesll-Fuded } \\
\text { stesm-Eleotrle } \\
\text { Plente }\end{array}$ & $\begin{array}{l}\text { Nuelest } \\
\text { stonm.Eleotris } \\
\text { Plante }\end{array}$ & $\begin{array}{l}\text { Qeothormal } \\
\text { Enorgy } \\
\text { Plante }\end{array}$ & \\
\hline 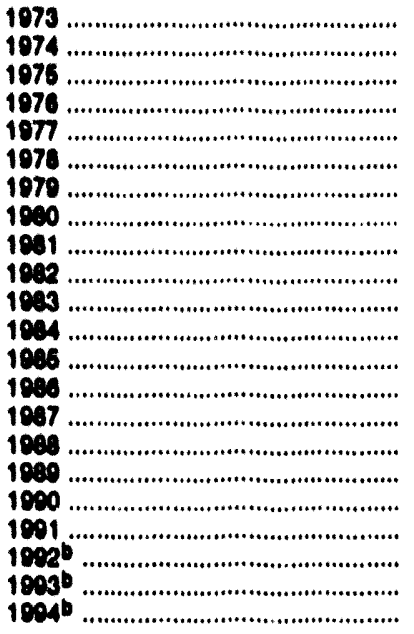 & $\begin{array}{l}10,399 \\
10,412 \\
10,400 \\
10,373 \\
10,435 \\
10,301 \\
10,363 \\
10,388 \\
10,453 \\
10,454 \\
10,520 \\
10,440 \\
10,447 \\
10,446 \\
10,410 \\
10,324 \\
10,317 \\
10,335 \\
10,352 \\
10,302 \\
10,302 \\
10,302\end{array}$ & $\begin{array}{l}10,803 \\
11,161 \\
11,013 \\
11,047 \\
10,789 \\
10,941 \\
10,879 \\
10,908 \\
11,030 \\
11,073 \\
10,005 \\
10,843 \\
10,813 \\
10,790 \\
10,778 \\
10,743 \\
10,724 \\
10,680 \\
10,740 \\
10,678 \\
10,078 \\
10,678\end{array}$ & 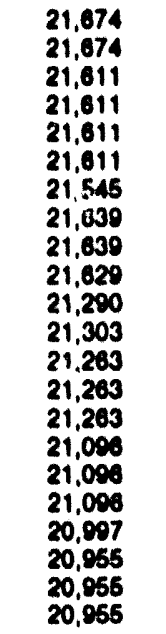 & $\begin{array}{l}3,412 \\
3,412 \\
3,412 \\
3,412 \\
3,412 \\
3,412 \\
3,412 \\
3,412 \\
3,412 \\
3,412 \\
3,412 \\
3,412 \\
3,412 \\
3,412 \\
3,412 \\
3,412 \\
3,412 \\
3,412 \\
3,412 \\
3,412 \\
3,412 \\
3,412\end{array}$ \\
\hline
\end{tabular}

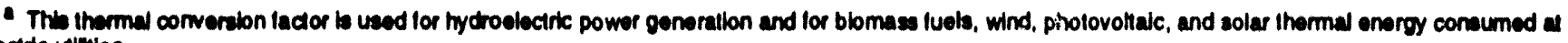
olectile villitios.

Prellininary.

Source: See "Thermal Conversion Factor Source Documentation." which tollows this table.

\section{Thermal Conversion Factor Source Documentation}

\section{Approximate Heat Content of Petroleum and Natural Gas Plant Llquids}

Asphalt. The Energy Information Administration (EIA) adopted the thermal conversion factor of 6.636 million British thermal units (Btu) per barrel as estimated by the Bureau of Mines and first published in the Petroleum Statement, Annual, 1956.

Avlation Gasoline. EIA adopted the Bureau of Mines thermal conversion factor of 5.048 million Btu per barrel for "Gasoline, Aviation" as published by the Texas Eastern Transmission Corporation in Appendix $\mathrm{V}$ of Competition and Growth in American Energy Markets 1947-1985, a 1968 release of historical and projected statistics.

Butane. EIA adopted the Bureau of Mines thermal conversion factor of 4.326 million Btu per barrel in the Califormia Oil World and Petroleum Industry. First Issue, April 1942.

Butane-Propane Mixture. ElA adopted the Bureau of Mines calculation of 4.130 million Btu per barrel based on an assumed mixture of 60 percent butane and 40 percent propane. See Butane and Propane.

Cirude Oll, Exports. Assumed by EIA to be 5.800 million Btu per barrel or equal to the thermal conversion factor for crude oil produced in the United States. See Crude OII and Lease Condensate, Production.

Crude OII, Imports. Calculated annually by EIA by weighting the thermal conversion factor of each type of crude oil imported by the quantity imported. Thermal conversion factors for each type were calculated on a foreign country basis, by determining the average American Petroleum Institute (API) gravity of crude imported from each foreign country from Form ERA-60 in 1977 and converting average API gravity to average Btu content by using National Bureau of Standards. Miscellaneous Publication No. 97. Thermal Properties of Petroleum Products, 1933.

Crude Oll and Lease Condensate, Production. EIA adopted the thermal conversion factor of 5.800 million Btu per barrel as reported in a Bureau of Mines internal memorandum, "Bureau of Mines Standard Average Heating Values of Various Fuels, Adopted January 3, 1950."

Crude OII and Petroleum Products, Exports. Calculated annually by EIA as the average of the thermal conversion factors for each petroleum product 
exported and crude oil exported weighted by the quantity of each petroleum product and crude oil exported. See Crude OII, Exports and Petroleum Products, Exports.

Crude OII and Petroleum Products, Imports. Calculated annually by EIA as the average of the thermal conversion factors for each petroleum product and each type of crude oil imported weighted by the quantity of each petroleum product and each type of crude oil imported. See Crude Oil, Imports and Petroleum Products, Imports.

Distillate Fuel Oll. EIA adopted the Bureau of Mines thermal conversion factor of 5.825 million Btu per barrel as reported in a Bureau of Mines internal memorandum, "Bureau of Mines Standard Average Heating Value of Various Fuels, Adopted January 3, 1950."

Ethane. EIA adopted the Bureau of Mines thermal conversion factor of 3.082 million Bu per barrel in the California Oil World and Petroleum Industry, First Issue, April 1942.

Ethane-Propane Mixture. ElA calculated 3.308 million Btu per barrel based on an assumed mixture of 70 percent ethane and 30 percent propane. See Ethane and Propane.

Isobutane. EIA adopted the Bureau of Mines thermal conversion factor of 3.974 million Btu per barrel in the California Oil World and Petroleum Industry, First Issue, April 1942.

Jet Fuel, Kerosene Type. EIA adopted the Bureau of Mines thermal conversion factor of 5.670 million Btu per barrel for "Jet Fuel, Commercial" as published by the Texas Eastern Transmission Corporation in Appendix $\mathrm{V}$ of Competition and Growth in American Energy Markets 1947-1985, a 1968 release of historical and projected statistics.

Jet Fuel, Naphtha Type. EIA adopted the Bureau of Mines thermal conversion factor of 5.355 million Btu per barrel for "Jet Fuel, Military" as published by the Texas Eastern Transmission Corporation in Appendix $V$ of Competition and Growth in American Energy Markets 1947.1985, a 1968 release of historical and projected statistics.

Kerosene. ElA adopted the Bureau of Mines thermal conversion factor of 5.670 million Btu per barrel as reported in a Bureau of Mines internal memorandum, "Bureau of Mines Standard Average Heating Values of Various Fuels, Adopted January 3, 1950."

Liquened Petroleum Gases (L.PG) Consumption. Calculated annually by EIA as the average of the thermal conversion factors of each liquefied petroleum gas consumed, weighted by the quantity of each liquefied petroleum gas consumed.
Lubricants. EIA adopted the thermal conversion factor of 6.065 million Btu per barrel as estimated by the Bureau of Mines and first published in the Petroleum Statement, Annual, 1956.

Miscellaneous Products. EIA adopted the thermal conversion factor of 5.795 million Btu per barrel as estimated by the Bureau of Mines and first published in the Petroleum Statement, Annual, 1956.

Motor Gasoline. EIA adopted the Bureau of Mines thermal conversion factor of 5.253 million Btu per barrel for "Gasoline, Motor Fuel" by the Texas Eastem Transmission Corporation in Appendix $V$ of Competition and Growth in American Energy Markets 1947-1985, a 1968 release of historical and projected statistics.

Natural Gas Plant Llquids, Production. Calculated annually by EIA as the average of the thermal conversion factors of each natural gas plant liquid produced weighted by the quantity of each natural gas plant liquid produced.

Natural Gasoline. ElA adopted the thermal conversion factor of 4.620 million Btu per barrel as estimated by the Bureau of Mines and first published in the Petroleum Statement, Annual, 1956.

Pentanes Plus. EIA assumed the thermal conversion factor to be 4.620 million Btu per barrel or equal to that for natural gasoline. See Natural Gasoline.

Petrochemical Feedstocks, Naphtha Less Than 401 Degrees Fahrenheit. Assumed by EIA to be 5.248 million Btu per barrel, equal to the thermal conversion factor for special naphtha. See Speclal Naphtha.

Petrochemical Feedstocks, Oils Equal to or Greater Than 401 Degrees Fahrenheit. Assumed by EIA to be 5.825 million Btu per barrel, equal to the thermal conversion factor for distillate fuel oil. See Distillate Fuel Oil.

Petrochemical Feedstocks, Still Gas. Assumed by EIA to be 6.000 million Btu per barrel, equal to the thermal conversion factor for still gas. See Still Gas.

Petroleum Coke. EIA adopled the thermal conversion factor of 6.024 million Btu per barrel as reported in Btu per short ton in the Bureau of Mines internal memorandum, "Bureau of Mines Standard Average Heating Value of Various Fuels, Adopted January 3, 1950." The Bureau of Mines calculated this factor by dividing $30,120,000$ Btu per short ton, as given in the referenced Bureau of Mines internal memorandum, by 5.0 barrels per short ton, as given in the Bureau of Mines Form 6-1300-M and successor EIA forms.

Petroleum Products, Total Consumption. Calculated annually by EIA as the average of the 
thermal conversion factors for all petroleum products consumed, weighted by the quantity of each petroleum product consumed.

Petroleum Products, Consumption by Electric Utilities. Calculated annually by EIA as the average of the thermal conversion factors for all petroleum products consumed at electric utilities, weighted by the quantity of each petroleum product consumed at electric utilities. The quantity of petroleum consumed is estimated in the State Energy Data System as documented in the State Energy Data Report.

Petroleum Products, Consumption by Industria: Users. Calculated annually by EIA as the average of the thermal conversion factors for all petroleum products consumed in the industrial sector, weighted by the estimated quantity of each petroleum product consumed in the industrial sector. The quantity of petroleum products consumed is estimated in the State Energy Data System as documented in the State Energy Data Report.

Petroleum Products, Consumption by Residential and Commercial Users. Calculated annually by EIA as the average of the thermal conversion factors for all petroleum products consumed by the residential and commercial sector, weighted by the estimated quantity of each petroleum product consumed in the residential and commercial sector. The quantity of petroleum products consumed is estimated in the State Energy Data System as documented in the State Energy Data Report.

Petroleum Products, Consumption by Transportation Users. Calculated annually by EIA as the average of the thermal conversion factor for all petroleum products consumed in the transportation sector, weighted by the estimated quantity of each petroleum product consumed in the transportation sector. The quantity of petroleum products consumed is estimated in the State Energy Data System as documented in the State Energy Data Report.

Petroleum Products, Exports. Calculated annually by EIA as the average of the thermal conversion factors for each petroleum product, weighted by the quantity of each petroleum product exported.

Petroleum Products, Imports. Calculated annually by EIA as the average of the thermal conversion factors for each petroleum product imported, weighted by the quantity of each petroleum product imported.

Plant Condensate. Estimated to be 5.418 million Btu per barrel by EIA from data provided by McClanahan Consultants, Inc., Houston, Texas.

Propane. ElA adopted the Bureau of Mines thermal conversion factor of 3.836 million Blu per barrel in the California Oil World and Petroleum Industry. First Issue, April 1942.
Residual Fuel Oll. ElA adopted the thermal conversion factor of 6.287 million Btu per barrel as reported in the Bureau of Mines internal memorandum, "Bureau of Mines Standard Average Heating Values of Various Fuels, Adopted January 3, 1950."

Royd OII. ElA adopted the Bureau of Mines thermal conversion factor of 6.636 million Btu per barrel, which was assumed to be equal to that of asphatt (see Asphalt) and was first published by the Bureau of Mines in the Petroleum Statement, Annual, 1970.

Special Naphtha. EIA adopted the Bureau of Mines thermal conversion factor of 5.248 million Btu per barrel, which was assumed to be equal to that of total gasoline (aviation and motor) factor and was nirst published in the Petroleum Statement, Annual, 1970.

Still Gas. ElA adopted the Bureau of Mines estimated thermal conversion factor of 6.000 million Btu per barrel and first published in the Petroleum Statement, Annual, 1970.

Unfinished Oil. EIA assumed the thermal conversion factor to be 5.825 million Btu per barrel or equal to that for distillate fuel oil (see Distillate Fuel Oil) and first published in the Annual Report to Congress, Volume 3, 1977.

Unfractionated Stream. EIA assumed the thermal conversion factor to be 5.418 million Btu per barrel or equal to that for plant condensate (see Plant Condensate) and first published in the Annual Report 10 Congress, Volume 2, 1981.

Waxes. ElA adopted the thermal conversion factor of 5.537 million Btu per barrel as estimated by the Bureau of Mines and first published in the Petroleum Statement, Annual, 1956.

\section{Approximate Heat Content of Natural Gas}

Natural Gas, Total Consumption. 1973-1979: ElA adopted the thermal conversion factor calculated annually by the American Gas Association (AGA) and published in Gas Facts, an AGA annual publication. 1980 forward: Calculated annually by EIA by dividing the total heat content of natural gas consumed by the total quantity of natural gas consumed. The heat content and quantity consumed are from Form EIA-176. Published sources are: 1980-1989: EIA, Natural Gas Annual 1992, Volume 2, Table 15. 1990-1992: EIA, Natural Gas Annual 1992, Volume 2. Table 16. 1993 forward: 1992 value used as an estimate.

Natural Gas, Consumption by Electric Utilities. Calculated annually by EIA by dividing the total heat content of natural gas received at electric utilities by the total quantity received at electric utilities. The 
beat contents and receipls are from Form FERC-423 and predecessor forms.

Natural Gas, Consumption by Sectors Other Than Electric Utilities. Calculated annually by EIA by dividing the heat content of all natural gas consumed less the heat content of natural gas consumed at electric utilities by the quantity of all natural gas consumed less the quantity of natural gas consumed at electric utilities. Data are from Forms EIA-176, FERC-423, EIA-759, and predecessor forms.

Natural Gas, Exports. Calculated annually by EIA by dividing the heat content of exported natural gas by the quantity of natural gas exported, both reported on Form FPC-14.

Natural Gas, Imports. Calculated annually by EIA by dividing the beat content of imported natural gas by the quantity of natural gas imported, both reported on Form FPC-14.

Natural Gas Production, Dry. Assumed by EIA to be equal to the thermal conversion factor for the consumption of dry natural gas. See Natural Gas Total Consumption.

Natural Gas Production, Marketed (Wet). Calculated annually by EIA by adding the beat content of dry natural gas production and the total heat content of natural gas plant liquids production and dividing this sum by the total quantity of marketed (wet) natural gas production.

\section{Approximate Heat Content of Coal and Coal Coke}

Anthracite, Total Consumption. Calculated annually by EIA by dividing the sum of the heat content of anthracite consumed by electric utilities and all other sectors combined by the total quantity of anthracite consumed.

Anthracite, Consumption by Electric Utilities. Calculated annually by EIA by dividing the heat content of anthracite receipts at electric utilities by the quantity of anthracite received at electric utilities. Heat contents and receipts are from Form FERC-423 and predecessor forms.

Anthracite, Consumption by Sectors Orner Than Electric Utilities. Calculated annually by EIA by dividing the heat content of anthracite production less the heat content of the anthracite consumed at electric utilities, net exports, and shipments to U.S. Armed Forces overseas by the quantity of anthracite consumed by sectors other than electric utilities less the quantity of anthracite stock changes, losses, and "unaccounted for."

Anthracite, Imports and Exports. EIA assumed the anthracite imports and exports to be freshly mined anthracite having an estimated heat content of $\mathbf{2 5 . 4 0}$ million Btu per short ton.

Anthracite, Production. Calculated annually by EIA by dividing the sum of the heat content of freshly mined anthracite (estimated to have an average heat content of 25.400 million Btu per short ton) and the heat content of anthracite recovered from culm banks and river dredging (estimated to have a heat content of 17.500 million Btu per short ton) by the total quantity of anthracite production.

Bituminous Coal and Lignite, Total Consumption. Calculated annually by EIA by dividing the sum of the heat content of bituminous coal and lignite consumed by electric utilities, coal coke plants, other industrial plants, the residential and commercial sector, and the transportation sector by the sum of their respective tonnages.

Bituminous Coal and Lignite, Consumption by Coke Plants. Estimated by EIA to be 26.800 million Btu per short ton on the basis of an input/output analysis of coal carbonization.

Bituminous Coal and Lignite, Consumption by Electric Utilities. Calculated annually by EIA by dividing the total heat content of bituminous coal and lignite received at electric utilities by the total quantity received at electric utilities. Heat contents and receipts are from Form FERC-423 and predecessor forms.

Bituminous Coal and Lignite, Consumption by Other Industrial and Transportation Users. 1973: Calculated by EIA through regression analysis measuring the difference between the average Btu value of coal consumed by other industrial users and that of coal consumed at electric utilities in the 1974-1982 period. 1974 forward: Calculated annually by EIA by assuming that the bituminous coal and lignite delivered to other industrial users from each coal-producing area (reported on Form EIA-6 and predecessor Bureau of Mines Form 6-1419-Q) contained a heat value equal to that of bituminous coal and lignite received at electric utilities from each of the same coal-producing areas (reported on Form FERC-423). The average Btu value of coal by coal-producing area was applied to the volume of deliveries to other industrial users from each coal-producing area, and the sum total of the heat content was divided by the total volume of deliveries. Coal-producing areas are the Bureau of Mines coal-producing districts for 1974 through 1989 and coal-producing States for 1990 forward.

Bituminous Coal and Lignite, Consumption by Residential and Commercial Users. 1973: Calculated by EIA through regression analysis measuring the difference between the average Bitu value of coal consumed by residential and commercial users and that of coal consumed by electric utilities 
in the 1974-1982 period. 1974 forward: Calculated annually by EIA by assuming that the bituminous coal and lignite delivered to residential and commercial users from each coal-producing area (reported on Form EIA-6 and predecessor Bureau of Mines Form $6-1419-Q$ ) contained a heat value equal to that of bituminous coal and lignite received at electric utilities from each of the same coal-producing areas (reported on Form FERC-423). The average Btu value of coal by coal-producing area was applied to the volume of deliveries to residential and commercial users from each coal-producing area, and the total of the heat value was divided by the total volume of deliveries. Coal-producing areas are the Bureau of Mines coal-producing districts for 1974 through 1989 and coal-producing States for 1990 forward.

Bituminous Coal and Lignite, Exports. Calculated annually by EIA by dividing the sum of the heat content of exported metallurgical coal (estimated to average 27.000 million Btu pei short ton) and the heat content of exported steam coal (estimated to have an average thermal content of 25.000 million Btu per short ton) by the total quantity of bituminous coal and lignite exported.

Bituminous Coal and Lignite, Imports. EIA estimated the average thermal conversion factor to be 25.000 million Btu per short ton.

Bituminous Coal and Lignite, Production. Calculated annually by EIA by dividing the sum of the heat content of bituminous coal and lignite consumption, net exports, stock changes, and unaccounted for by the sum of their respective tonnages. Consumers' stock changes by sectors were assumed to have the same conversion factor as that of the consumption sector. Producers' stock changes and unaccounted for were assumed to have the same conversion factor as that for consumption by all users.

Coal, Consumption. Calculated annually by EIA by dividing the sum of the heat content of bituminous coal and lignite and anthracite consumption by the sum of their respective tonnages.

Coal, Consumption by Electric Utilities. Calculated annually $b_{j}$ EIA by dividing the sum of the heat content of bituminous coal and lignite and anthracite received at electric utilities by the sum of their respective tonnages received.

Coal, Consumption by Sectors Other Than Electric Utilities. Calculated annually by EIA by dividing the sum of the beat content of bituminous coal and lignite and anthracite consumed by sectors other than electric utilities by the sum of their respective tonnages.

Coal, Exports. Calculated annually by EIA by dividing the sum of the heat content of bituminous coal and lignite and anthracite exported by the sum of their respective tonnages.
Coal, Imports. Calculated annually by EIA by dividing the sum of the heat content of bituminous coal and lignite and anthracite imported by the sum of their respective tonnages.

Coal, Production. Calculated annually by EIA by dividing the sum of the total heat content of bituminous coal and lignite and anthracite production by the sum of their respective tonnages.

Coal Coke, Imports and Exports. EIA adopted the Bureau of Mines estimate of 24.800 million Btu per short ton.

\section{Approximate Heat Rates for Electricity}

Fossil-Fueled Steam-Electric Plant Generation. There is no generally accepted practice for measuring the thermal conversion rates for power plants that generate electricity from hydroelectric, wood and waste, wind, photovoltaic, or solar thermal energy sources. Therefore, EIA uses data from Form EIA-767 to calculate a rate factor that is equal to the prevailing annual average heat rate factor for fossil-fueled steam-electric power plants in the United States. By using that factor, it is possible to evaluate fossil fuel requirements for replacing those sources during periods of interruption such as droughts. The heat content of a kilowatthour of electricity produced, regardless of the generation process, is 3,412 Btu per kilowatthour. 1973-1991: The weighted annual average heat rate for fossil-fueled steam-electric power plants in the United States, as publsibed by EIA in Electric Plant Cost and Power Production Expenses 1991, Table 9. 1992 forward: Unpublished factors calculated on the basis of data from Form EIA-767.

Geothermal Energy Plant Generation. 1973-1981: Calculated annually by EIA by weighting the annual average beat rates of operating geothermal units by the installed nameplate capacities as reported on Form FPC-12. 1982 forward: Estimated annually by EIA on the basis of an informal survey of relevant plants.

Nuclear Steam-Electric Plant Generation. 1973-1991: Calculated annually by EIA by dividing the total heat content consumed in nuclear generating units by the total (net) electricity generated by nuclear generating units. The heat content and electricity generation are reported on Form FERC-1, Form EIA-41\%, and predecessor forms. The factors, beginning with 1982 data, are published in the following EIA reports-1982: Historical Plant Cost and Annual Production Expenses for Selected Electric Plants 1982, page 215. 1983-1991: Electric Plant Cost and Power Production Expenses 1991, Table 13. 1992 forward: Calculated annually by EIA by dividing the total heat content of the steam leaving the nuclear generating units to generate electricity by the total (net) electricity generated by nuclear generating units. The heat content and electricity generation data are reported in Nuclear Regulatory 


\section{Appendix B. Metric and Other Physical Conversion Factors}

Data presented in the Monthly Energy Review and in other Energy Information Administration publications are expressed predominately in units that historically have been used in the United States, such as British thermal units, barrels, cubic feet, and short tons. However, because U.S. commerce involves other nations, most of which use metric units of measure, the U.S. Government is committed to the transition to the metric system, as stated in the Metric Conversion Act of 1975 (Public Law 94-168), amended by the Omnibus Trade and Competitiveness Act of 1988 (Public Law 100-418), and Executive Order 12770 of July 25, 1991.

The metric conversion factors presented in Table Bl can be used to calculate the metric-unit equivalents of values expressed in U.S. customary units. For example, 500 short tons are the equivalent of 453.6 metric tons ( 500 short tons $x 0.9071847$ metric tons $/$ short ton $=\mathbf{4 5 3 . 6}$ metric tons).

In the metric system of weights and measures, the names of multiples and subdivisions of any unit may be derived by combining the name of the unit with prefixes, such as deka, hecto, and kilo, meaning, respectively, 10, 100, 1,000 , and deci, centi, and milli, meaning, respectively, one-tenth, one-hundredth, and one-thousandth. Common metric prefixes can be found in Table B2.

The conversion factors presented in Table B3 can be used to calculate equivalents in various physical units commonly used in energy analyses. For example, 10 barrels are the equivalent of 420 U.S. gallons (10 barrels $\times 42$ gallons/barrel $=\mathbf{4 2 0}$ gallons) . 


\begin{tabular}{|c|c|c|c|c|c|}
\hline Type of Unit & U.S. Unit & $\begin{array}{c}\text { multiplied } \\
\text { by }\end{array}$ & $\begin{array}{c}\text { Conversion } \\
\text { Factor }\end{array}$ & equals & Motric Unit \\
\hline Maes & $\begin{array}{l}\text { short tons }(2,000 \mathrm{lb}) \\
\text { long tons } \\
\text { pounds (lb) } \\
\text { pounds uranium oxide }\left(\mathrm{lb}_{3} \mathrm{O}_{8} \text { ) }\right. \\
\text { ounces, avoirdupois (avdp oz) }\end{array}$ & $\begin{array}{l}x \\
x \\
x \\
x \\
x\end{array}$ & $\begin{array}{l}0.9071847 \\
1.016047 \\
0.45359237^{b} \\
0.384647^{\mathrm{a}} \\
28.34952\end{array}$ & $\begin{array}{l}= \\
= \\
= \\
= \\
=\end{array}$ & $\begin{array}{l}\text { metric tons }(t) \\
\text { metric tons }(t) \\
\text { kilograms }(\mathrm{kg}) \\
\text { kilograms uranium }(\mathrm{kgU}) \\
\text { grams }(\mathrm{g})\end{array}$ \\
\hline Volume & $\begin{array}{l}\text { barrels of oil (bbl) } \\
\text { cubic yards }\left(\mathrm{y}^{3}\right) \\
\text { cubic feet }\left(\mathrm{ft}^{3}\right) \\
\text { U.S. gallons (gal) } \\
\text { ounces, fluid (fl oz) } \\
\left.\text { cubic inches (in }{ }^{3}\right)\end{array}$ & $\begin{array}{l}x \\
x \\
x \\
x \\
x \\
x\end{array}$ & $\begin{array}{l}0.1589873 \\
0.764555 \\
0.02831685 \\
3.785412 \\
29.57353 \\
16.38706\end{array}$ & $\begin{array}{l}= \\
= \\
= \\
= \\
= \\
=\end{array}$ & $\begin{array}{l}\text { cubic meters }\left(\mathrm{m}^{3}\right) \\
\text { cubic meters }\left(\mathrm{m}^{3}\right) \\
\text { cubic meters }\left(\mathrm{m}^{3}\right) \\
\text { liters }(\mathrm{L}) \\
\text { milliliters }(\mathrm{mL}) \\
\text { milliliters }(\mathrm{mL})\end{array}$ \\
\hline Length & $\begin{array}{l}\text { miles (mi) } \\
\text { yards (yd) } \\
\text { feet (tt) } \\
\text { inches (in) }\end{array}$ & $\begin{array}{l}x \\
x \\
x \\
x\end{array}$ & $\begin{array}{l}1.609344^{b} \\
0.9144^{b} \\
0.3048^{b} \\
2.54^{b}\end{array}$ & $\begin{array}{l}= \\
= \\
= \\
=\end{array}$ & $\begin{array}{l}\text { kilometers }(\mathrm{km}) \\
\text { meters }(\mathrm{m}) \\
\text { meters }(\mathrm{m}) \\
\text { centimeters }(\mathrm{cm})\end{array}$ \\
\hline Area & $\begin{array}{l}\text { acres } \\
\text { square miles }\left(\mathrm{mi}^{2}\right) \\
\text { square yards }\left(\mathrm{yo}^{2}\right) \\
\text { square feet }\left(\mathrm{ft}^{2}\right) \\
\text { square inches }\left(\mathrm{in}^{2}\right)\end{array}$ & $\begin{array}{l}x \\
x \\
x \\
x \\
x\end{array}$ & $\begin{array}{l}0.40469 \\
2.589988 \\
0.8361274 \\
0.09290304^{b} \\
6.4516^{b}\end{array}$ & $\begin{array}{l}= \\
= \\
= \\
=\end{array}$ & $\begin{array}{l}\text { hectares (ha) } \\
\text { square kilometers }\left(\mathrm{km}^{2}\right) \\
\text { square meters }\left(\mathrm{m}^{2}\right) \\
\text { square meters }\left(\mathrm{m}^{2}\right) \\
\text { square centimeters }\left(\mathrm{cm}^{2}\right)\end{array}$ \\
\hline Temperature & degrees Fahrenheit $\left({ }^{\circ} F\right)$ & $x$ & $5 / 9{\text { (after subtracting } 32)^{b, c}}^{b}$ & $=$ & degrees Celsius $\left({ }^{\circ} \mathrm{C}\right)$ \\
\hline Energy & $\begin{array}{l}\text { British thermal units (Btu) } \\
\text { calories (cal) } \\
\text { kilowatthours (kWh) }\end{array}$ & $\begin{array}{l}x \\
x \\
x\end{array}$ & $\begin{array}{c}1,055.05585262^{b, d} \\
4.1868^{b} \\
3.6^{b}\end{array}$ & $\begin{array}{l}= \\
= \\
=\end{array}$ & $\begin{array}{l}\text { joules }(J) \\
\text { joules }(J) \\
\text { megajoules (MJ) }\end{array}$ \\
\hline
\end{tabular}

Calculated by the Energy Intormation Administration.

bxact comversion.

${ }^{\circ}$ To convert degrees Celsius $\left({ }^{\circ} \mathrm{C}\right)$ to degrees Fahrenhelt $\left({ }^{\circ} \mathrm{F}\right)$ exactly, mullply by $9 / 5$, then add 32.

The Btu used in this table is the International Table Btu adopted by the Fith International Conference on Propentes of Steam, London, 1956.

Noles: - Spaces have been inserted after every third digit to the right of the decimal for ease of reading. - Most metric units belong tc the Internallonal System of Units (SI), and the liter, hectare, and metric ton are accepted for use with the SI units. For more intormation about the SI units, contact Dr. Barry Taylor at Bullding 221, Room 8610, Natlonal Instltute of Standards and Technology, Galthersburg. MD 20899, or on telephone number 301-975-4220.

Sources: - General Services Administration, Federal Standard 376B. Preferred Metric Units for General Use by the Federal Government Washington, DC, January 27, 1993), pp. 2-11, 13, and 16. - National Institute of Standards and Technology, Special Publicallons 330, 811, and 814. - American Nallonal Standards instilute/linstitute of Electrical and Electronic Engineers, ANSI/IEEE Std 268-1992, pp. 28 and 29. 
Table B2. Metric Preflxes

\begin{tabular}{|c|c|c|c|c|c|}
\hline $\begin{array}{l}\text { Unit } \\
\text { Muftiple }\end{array}$ & Prefix & Symbol & $\begin{array}{l}\text { Unit } \\
\text { Subdivision }\end{array}$ & Preflx & Symbol \\
\hline $\begin{array}{l}10^{1} \\
10^{2} \\
10^{3} \\
10^{6} \\
10^{0} \\
10^{12} \\
10^{16} \\
10^{18} \\
10^{21} \\
10^{24}\end{array}$ & $\begin{array}{l}\text { deka } \\
\text { hecto } \\
\text { kilo } \\
\text { mega } \\
\text { giga } \\
\text { tera } \\
\text { peta } \\
\text { exa } \\
\text { zetta } \\
\text { yotta }\end{array}$ & $\begin{array}{l}d a \\
h \\
k \\
M \\
G \\
T \\
P \\
E \\
Z \\
Y\end{array}$ & $\begin{array}{l}10^{-1} \\
10^{-2} \\
10^{-3} \\
10^{-6} \\
10^{-9} \\
10^{-12} \\
10^{-16} \\
10^{-18} \\
10^{-21} \\
10^{-24}\end{array}$ & $\begin{array}{l}\text { decl } \\
\text { centl } \\
\text { milli } \\
\text { micro } \\
\text { nano } \\
\text { pico } \\
\text { femto } \\
\text { atto } \\
\text { zepto } \\
\text { yocto }\end{array}$ & $\begin{array}{l}d \\
c \\
m \\
\mu \\
n \\
p \\
1 \\
a \\
z \\
y\end{array}$ \\
\hline
\end{tabular}

Source: U.S. Department of Commerce, Natlonal Insthute of Standards and Technology, The Intermational System of Units (SI), NIST Special Publicallon 330, 1981 Edition (Washington, DC, August 1991), p. 10.

Table B3. Other Physical Conversion Factors

\begin{tabular}{|c|c|c|c|c|c|}
\hline Energy Source & Original Unit & $\begin{array}{c}\text { multiplied } \\
\text { by }\end{array}$ & $\begin{array}{c}\text { Conversion } \\
\text { Factor }\end{array}$ & equals & Final Unit \\
\hline Petroleum & barrels (bbl) & $x$ & $42^{a}$ & $=$ & U.S. gallons (gal) \\
\hline Coal & $\begin{array}{l}\text { short tons } \\
\text { long tons } \\
\text { metric tons ( } t)\end{array}$ & $\begin{array}{l}x \\
x \\
x\end{array}$ & $\begin{array}{l}2,000^{a} \\
2,240^{a} \\
1,000^{a}\end{array}$ & $\begin{array}{l}= \\
= \\
=\end{array}$ & $\begin{array}{l}\text { pounds (lb) } \\
\text { pounds (lb) } \\
\text { kllograms (kg) }\end{array}$ \\
\hline Wood & $\begin{array}{l}\text { cords (cd) } \\
\text { cords (cd) }\end{array}$ & $\begin{array}{l}x \\
x\end{array}$ & $128^{\mathrm{a} 5^{\mathrm{b}}}$ & $\begin{array}{l}= \\
=\end{array}$ & $\begin{array}{l}\text { short tons } \\
\text { cubic foet }\left(\left(t^{3}\right)\right.\end{array}$ \\
\hline
\end{tabular}

Exact converslon.

${ }^{b}$ Calculated by the Energy Intormation Administratlon.

Source: U.S. Department of Commerce, National Instilute of Standards and Technology, Specifications, Tolerances, and Other Technical Requiremente for Weighing and Measuring Devices, NIST Handbook 44, 1994 Edilion (Washington, DC, October 1993), PP. B-10, C-17 and C-21. 


\section{Appendix C. List of Features}

The following is a complete list of features that have appeared in the Monhly Energy Review since the first issue was published in October 1974. There are four categories of features on the list. "Articles" cover a wide range of energyrelated subjects in depth. "Highlights" summarize the most important information presented in the subject Energy Information Administration (EIA) report. "Energy Previews" provide brief overviews of ELA preliminary energy data on a given topic. "EIA Data News" items present information on recent changes in the scope, design, methodology, and findings of ELA's energy surveys and databases. Questions and comments about features may be directed to Barbara $T$. Fichman by telephone on 202-586-5737 or by fax on 202 $586-0018$.

\section{Feature}

\section{Cover Date}

\section{4}

Energy Preview: Commercial Buildings Energy Consumption Survey,

Preliminary Estimates, 1992

Highlights: Household Vehicles Energy Consumption 1991

Highlights: Energy Use and Carbon Emissions: Some International Comparisons

January 1994

February 1994

April 1994

\section{3}

Energy Preview: Residential Transportation Energy Consumption Survey,

Proliminary Estimates, 1991

EIA Data News: Natural Gas Transported for the Account of Others

Highlights: Federal Energy Subsidies: Direct and Indirect Interventions in Energy Markets ...... Highlights: Household Energy Consumption and Expenditures 1990

Article: Demand, Supply, and Price Outlook for Low-Sulfur Diesel Fuel . . . . . . . . . . . . . . . Energy Preview: Manufacturing Energy Consumption Survey, Preliminary Estimates, 1991 . . . . Highlights: Natural Gas 1992: Issues and Trends . . . . . . . . . . . . . . . . . . . Highlights: International Energy Outlook 1993

Highlights: The Changing Structure of the U.S. Coal Industry: An Update ............... Highlights: Emissions of Greenhouse Gases in the United States 1985-1990 . . . . . . . . . . . Highlights: Assessment of Energy Use in Multibuilding Facilities

\section{2}

Energy Preview: Residential Energy Consumption and Expenditures

Preliminary Estimates, 1990

EIA Data News: Oxygenate Data Collection Begins

Highlights: Lighting in Commercial Buildings

Arlcle: Demand, Supply, and Price Outlook for Oxgenated Gasoline, Winter 1992-1993 .......

EIA Data News: ElA Statistics on Electric Utility Demand-Side Management $\ldots \ldots \ldots \ldots \ldots \ldots$

EIA Data News: ElA Statistics on Nonutility Power Producers . . . . . . . . . . . . . . . . . . Highlights: Derived Annual Estimates of Manufacturing Energy Consumption, 1974-1988 ...... Anticle: Energy Efficiency in the Manufacturing Sector

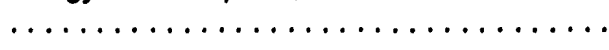

\section{1}

Highlights: U.S. Energy Industry Financial Developments, 1990 Fourth Quarter . . . . . . . . . . . Article: U.S. Wholesale Electricity Transactions

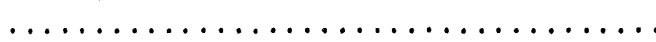

1990

Article: Refining Results Highlight Energy Companies' First-Half Profit Performance $\ldots \ldots \ldots$ Highlights: U.S. Oil and Gas Reserves by Year of Field Discovery
January 1993

February 1993

July 1993

August 1993

August 1993

September 1993

September 1993

October 1993

November 1993

December 1993

December 1993

April 1992

May 1992

June 1992

August 1992

September 1992

October 1992

November 1992

December 1992

March 1991

April 1991

June 1990

August 1990 
Anticle: A Review of Valdez Oil Spill Market Impacts

Article: Monthly U.S. Crude Oil Production Estimates

Article: Superconductivity and Energy Production and Consumption

Highlights: Commercial Buildings Consumption and Expenditures 1986

Article: Higher Prices Yield Improved Energy Industry Financial Results

in the First Halt of 1989

Article: The Future Structure of the U.S. Commerclal Nuclear Power Equlpment

Manufacturing Industry

Highlights: Potential Costs of Restricting Chlorofluorocarbon Use . . . . . . . . . . . . . . . . . . . .

Highlights: Manufacturing Energy Consumption Suvey: Changes in Energy Efficiency, 1980-1985

Highlights: Household Energy Consumption and Expenditures 1987, Part 1: National Data ......

Anticle: Improved Energy Profits Offset by Refining Results in 1989

\section{8}

Article: Measures of Energy Consumption, Expenditures, and Prices

Highllghts: Characteristics of Commercial Buildings 1986

Anticle: The US. Energy Industry's Financial Recovery Continued in the First Halt of $1988 \ldots \ldots . . . .$.

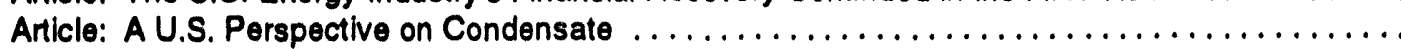

Atticle: State Energy Severance Taxes, $1972-1987 \ldots \ldots \ldots \ldots \ldots \ldots \ldots \ldots \ldots \ldots \ldots \ldots$

Highlights: Manufacturing Energy Consumption Survey: Consumption of Energy, $1985 \ldots \ldots \ldots$

Highlights: Profiles of Foreign Direct Investment in U.S. Energy $1987 \ldots \ldots \ldots \ldots \ldots \ldots$

Highlights: Manufacturing Energy Consumption Survey: Fuel Switching, 1985 . . . . . . . . . . .

Article: Increased Retining Income Led U.S. Energy Industry Financial Recovery in $1988 \ldots \ldots .$.

\section{7}

Article: Manufacturing Sector Energy Consumption, 1985 Provisional Estimates

Highlights: Consumption and Expenditures, April 1984 Through March 1985,

Part 1: National Data

Highlights: Consumption and Expenditures, April 1984 Through March 1985,

Part 2: Regional Data .

Article: U.S. Energy Industry Financial Developments, 1987 Second Quarter . . . . . . . . . . .

Anticle: End-Use Consumption of Residential Energy $\ldots \ldots \ldots \ldots \ldots \ldots \ldots \ldots \ldots \ldots \ldots$

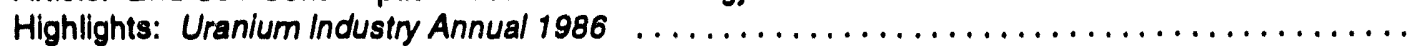

Highlights: Potential Oil Production from ANWR $\ldots \ldots \ldots \ldots \ldots \ldots \ldots \ldots \ldots \ldots \ldots$

Highlights: Profiles of Foreign Direct Investment in U.S. Energy $1986 \ldots \ldots \ldots \ldots \ldots$

Article: The U.S. Energy Industry in 1987: A Slow Recovery

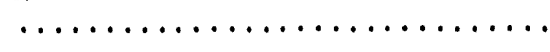

\section{6}

Article: State Motor Gasoline Taxes, 1960-1985

Article: The Impact of Low Oil Prices on Electric Utility Fuel Choice $\ldots \ldots \ldots \ldots \ldots \ldots \ldots \ldots$

Article: U.S. Energy Industry Financial Developments, 1986 Second Quarter . . . . . . . . . . . . Highlights: International Energy Annual 1985

Article: U.S. Energy Industry Financial Developments, 1986

\section{5}

Highlights: Annual Energy Roviow 1984

Highlights: Performance Profiles of Major Energy Producers 1983

Article: Estimating Well Completions

Highlights: State Energy Price and Expenditure Report 1970-1982 . . . . . . . . . . . . . . . . . .

Highlights: State Energy Data Report, Consumption Estimates, $1960-1983 \ldots \ldots \ldots \ldots \ldots$

Highlights: Annual Outlook for U.S. Electric Power $1985 \ldots \ldots \ldots \ldots \ldots \ldots \ldots \ldots \ldots$

Highlights: Short-Term Energy Outlook, Volume 1, October 1985

Highlights: Analysis of Growth in Electricity Demand, 1980-1984

Highlights: Profiles of Foreign Direct Investment in U.S. Energy 1984

Highlights: Performance Profiles of Major Energy Producers 1984
March 1989

March 1989

May 1989

May 1989

June 1989

July 1989

September 1989

October 1989

November 1989

December 1989

May 1988

June 1988

June 1988

June 1988

July 1988

September 1988

October 1988

November 1988

December 1988

January 1987

April 1987

May 1987

June 1987

July 1987

September 1987

October 1987

November 1987

December 1987

March 1986

June 1986

June 1986

September 1986

December 1986

January 1985

Fobruary 1985

March 1985

March 1985

April 1985

June 1985

August 1985

August 1985

November 1985

December 1985 
Highlights: State Energy Data Report, Consumption Estimates, 1960-1982

March 1984

Highlights: State Energy Price and Expenditure Roport 14

Highlights: Solar Colloctor Manufacturing Activ

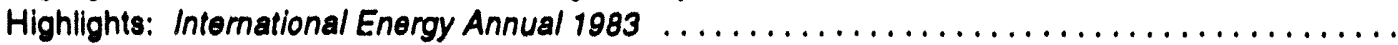

Highlights: Estimates of U.S. Wood Energy Consumption, 1980-1983 ...................

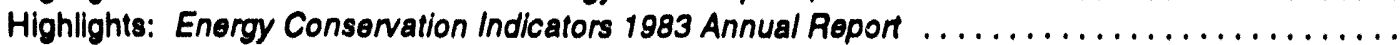

Highlights: Annual Energy Outlook 1984

May 1984

June 1984

September 1984

September 1984

November 1984

December 1884

\section{3}

Highlights: Residential Energy Consumption Survey: Consumption and Expenditures ........... Highlights: Residential Energy Consumption Survey: Housing Characteristics .................

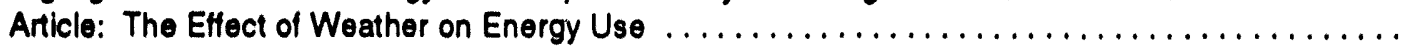

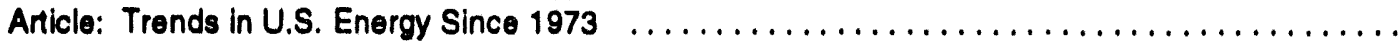

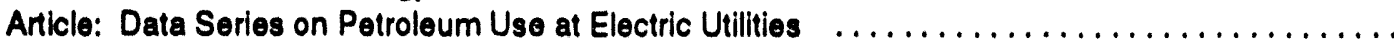

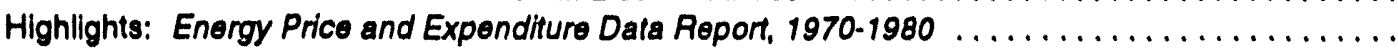

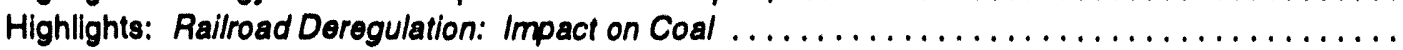

Highlights: Port Deopening and User Foes: Impact on U.S. Coal Exports

Highlights: U.S. Crudo Oil, Natural Gas, and Natural Gas Liquids Reserves,

1982 Annual Report

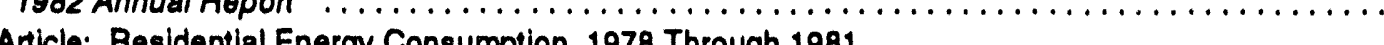

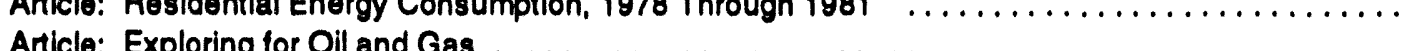

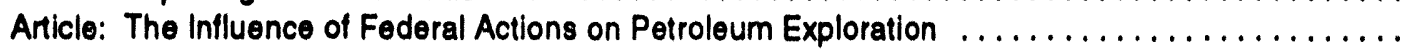

Article: Aggregate Statistics: Accurate or Misieading?

January 1983

February 1983

April 1983

May 1983

July 1983

July 1983

August 1983

August 1983

September 1983

September 1983

November 1983

December 1983[2]

December 1983[3]

January 1982

Fobruary 1982

September 1982

October 1982

November 1982

May 1981

September 1981

December 1981

Article: An Overview of Natural Gas Markets

\section{0}

Article: The Solar Collector Industry and Solar Energy

Article: Trends in the Installation of Energy Using Equipment in New Residential Buildings ........

Article: The Energy Information Administration's Oil and Gas Reserves

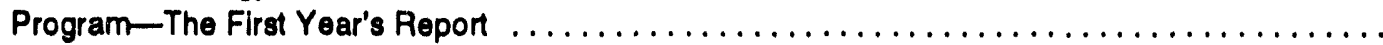

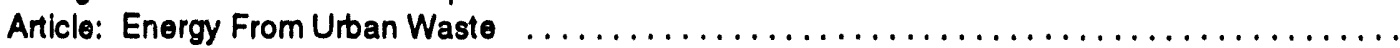

Article: Natural Gas Liquids: Revisions to 1979 Data ................................

Article: EIA Weekly Petroleum Data: Data Collection and Methods of Estimation ..............

Article: The Department of Energy Disclosure Policy for Individually Identifilable

Information Maintained by the Energy Information Administration

February 1980

March 1980

June 1980

August 1980

October 1980

November 1980

December 1980

July 1978

Article: The Energy Requirements of U.S. Agriculture

October 1979

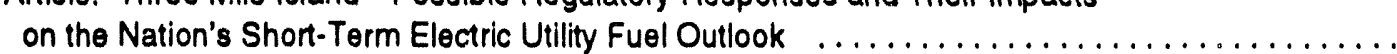

Article: Reduction in Natural Gas Requirements Due to Fuel Switching 
Anticle: Crude Oll Entitlements Program

January 1977

Anticle: Motor Gasoline Supply and Demand July 1977

1976

Article: Curtailments of Natural Gas Service

Anticle: Home Heating Conservation Alternatives and the Solar Collector Industry

January 1976

Article: Trends in United States Petroleum Imports

March 1976

September 1976

\section{5}

Article: Energy Consumption

March 1975

Article: Nuclear Power

Anticle: The Price of Crude Oil

April 1975

Article: U.S. Coal Resources and Reserves

June 1975

Article: Propano-A National Energy Resource

July 1975

Article: Short-Term Energy Supply and Demand Forecasting at FEA 


\section{Glossary}

Anthracite: $A$ hard, black, lustrous coal containing a bigh percentage of fixed carbon and a low percentage of volatile matter. Often referred to as hard coal. It conforms to ASTM Specification D388-84 for anthracite, meta-anthracite, and semianthracite.

Asphalt: A dark-brown-to-black cement-like material containing bitumens as the predominant constituents obtained by petroleum processing. The definition includes crude asphalt as well as the following finished products: cements, fluxes, the asphalt content of emulsions (exclusive of water), and petroleum distillates blended with asphalt to make cutback asphalts.

ASTM: The American Sociely for Testing and Materials.

Aviation Gasoline Blending Components: Naphthas that are used for blending or compounding into finished aviation gasoline (e.g., straight-run gasoline, alkylate, and reformate). Excludes oxygenates (alcohols and ethers), butane, and pentanes plus.

Aviation Gasoline, Finished: All special grades of gasoline for use in aviation reciprocating engines, as given in ASTM Specification D910 and Military Specification MIL-G-5572. Excludes blending components that will be used in blending or compounding into finished aviation gasoline.

Barrel (petroleum): A unit of volume equal to 42 U.S. gallons.

Base (Cushion) Gas: The volume of gas needed as a permanent inventory to maintain adequate underground storage reservoir pressures and deliverability rates throughout the withdrawal season. All native gas is included in the base gas volume.

Bituminous Coal: A dense black coal, often with well-defined bands of bright and dull material, with a moisture content usually less than 20 percent. Often referred to as soft coal. It is the most common coal and is used primarily for generating electricity, making coke, and space heating. It conforms to ASTM Specification D388-84 for bituminous coal. In this report, bituminous coal includes subbituminous coal.

British Thermal Unit (Btu): The quantity of heat needed to raise the temperature of 1 pound of water by $1^{\circ} \mathrm{F}$ at or ness $39.2^{\circ} \mathrm{F}$. See Heat Content of a Quantity of Fuel, Gross and Heat Content of a Quantity of Fuel, Net.
Butane: A normally gaseous straight-chain or branched-chain hydrocarbon $\left(\mathrm{C}_{4} \mathrm{H}_{10}\right)$. It is extracted from natural gas or refinery gas streams. It includes isobutane and normal butane and is designated in ASTM Specification D1835 and Gas Processors Association Specifications for commercial butane.

- Isobutane: A normally gaseous branched-chain hydrocarbon. It is a colorless parafinic gas that boils at a temperature of $10.9^{\circ} \mathrm{F}$. It is extracted from natural gas or refinery gas streams.

- Normal Butane: A normally gaseous straight-chain hydrocarbon. It is a colorless paraffinic gas that boils at a temperature of $31.1^{\circ} \mathrm{F}$. It is extracted from natural gas or refinery gas streams.

Butylene: An olefinic hydrocarbon $\left(\mathrm{C}_{4} \mathrm{H}_{8}\right)$ recovered from refinery processes.

Capacity Factor: The ratio of the electrical energy produced by a generating unit for a given period of time to the electrical energy that could have been produced at continuous full-power operation during the same period.

CIF: See Cost, Insurance, Freight.

City Gate: A point or measuring station at which a distribution gas utility receives gas from a natural gas pipeline company or transmission system.

Coal: A black or brownish-black solid, combustible substance formed by the partial decomposition of vegetable matter without access to air. The rank of coal, which includes anthracite, bituminous coal, subbituminous coal, and lignite, is based on fixed carbon, volatile matter, and heating value. Coal rank indicates the progressive alteration, or coalification, from lignite to anthracite. Lignite contains approximately 9 to 17 million Btu per ton. The beat contents of subbituminous and bituminous coal range from 16 to 24 million Btu per ton, and from 19 to 30 million Btu per ton, respectively. Anthracite contains approximately 22 to 28 million Btu per ton.

Coal Coke: A hard, porous product made from baking bituminous coal in ovens at temperatures as high as $2,000^{\circ} \mathrm{F}$. It is used both as a fuel and as a reducing agent in smelting iron ore in a blast furnace.

Commercial Sector: The commercial sector, as defined economically, consists of business establishments that are not engaged in transportation or in manufacturing or other types of industrial activity (agriculture, mining, or construction). Cornmercial establishments include hotels, motels, 
restaurants, wholesale businesses, retail stores, laundries, and other service enterprises; religious and nonprofit organizations; health, social, and educational institutions; and Federal, State, and local governments. Street lights, pumps, bridges, and public services are also included if the establishment operating them is considered commercial.

Completion: The instaliation of permanent equipment for the production of oil or gas. If a well is equipped to produce only oil or gas from one zone or reservoir. the definition of a weli (classified as an oil well or gas well) and the definition of a completion are identical. However, if a well is equipped to produce oil and/or gas separately from more than one reservoir. a well is not synonymous with a completion.

Conversion Factor: $A$ number that translates units of one system into corresponding values of another system. Conversion factors can be used to translate physical units of measure for various fuels into Btu equivalents.

Cost, Insurance, Froight (CIF): A type of sale in which the buyer of the product agrees to pay a unit price that includes the f.o.b. value of the product at the point of origin plus all costs of insurance and transportation. This type of transaction differs from a "delivered" purchase in that the buyer accepts the quantity as determined at the loading port (as certified by the Bill of Loading and Quality Report) rather than pay on the basis of the quantity and quality ascertained at the unloading port. It is similar to the terms of an f.o.b. sale, except that the seller, as a service for which be is compensated, arranges for transportation and insurance.

Crude Oll l.o.b. Price: The crude oil price actually charged at the oil-producing country's port of loading. Includes deductions for any rebates and discounts or additions of premiums, where applicable. It is the actual price paid with no adjustment for credit terms.

Crude Oll (Including Lease Condensate): A mixture of bydrocarbons that exists in liquid phase in underground reservoirs and remains liquid at atmospheric pressure after passing through surface separating facilities. Included are lease condensate and liquid hydrocarbons produced from tar sands, gilsonite, and oil shale. Drip gases are also included, but topped crude oil (residual oil) and other unfinished oils are excluded. Where identifiable, liquids produced at natural gas processing plants and mixed with crude oil are likewise excluded.

Crude Oll Landed Cost: The price of crude oil at the port of discharge, including charges associated with the purchase, transporting, and insuring of a cargo from the purchase point to the port of discharge. The cost does not include charges incurred at the discharge port (e.8., impon tariffs or fees, wharfage charges, and demurrage).
Crude Oll Refinery Input: The total crude oil put into processing units at refineries.

Crude OII Stocks: Stocks of crude oil and lease condensate held at refineries, in pipelines, at pipeline terminals, and on leases.

Crude Oil Used Directly: Crude oil consumed as fuel by crude oil pipelines and on crude oil leases.

Cuble Foot (natural gas): A unit of volume equal to 1 cubic foot at a pressure base of 14.73 pounds standard per square inch absolute and a temperature base of $60^{\circ} \mathrm{F}$.

Degree-Day Normals: Simple arithmetic averages of monthly or annual degree-days over a long period of time (usually the 30-year period 1961-1990). The averages may be simple degree-day normals or population-weighted degree-day normals.

Degree-Days, Cooling (CDD): The number of degrees per day that the daily average temperature is above $65^{\circ} \mathrm{F}$. The daily average temperature is the mean of the maximum and minimum temperatures for a 24-hour period.

Degree-Days, Heating (HDD): The number of degrees per day that the daily average temperature is below $65^{\circ} \mathrm{F}$. The daily average temperature is the mean of the maximum and minimum temperatures for a 24-hour period.

Degree-Days, Population-Welghted: Heating or cooling degree-days weighted by the population of the area in which the degree-days are recorded. To compute State population-weighted degree-days, each State is divided into from one to nine climatically homogeneous divisions, which are assigned weights based on the ratio of the population of the division to the total population of the State. Degree-day readings for each division are multiplied by the corresponding population weight for each division and those products are then summed to arrive at the State population-weighted degree-day figure. To compute national population-weighted degree-days, the Nation is divided into nine Census regions comprised of from three to eight States, which are assigned weights based on the ratio of the population of the region to the total population of the Nation. Degree-day readings for each region are multiplied by the corresponding population weight for each region and those products are then summed to arrive at the national population-weighted degree-day figure.

Design Electrical Rating, Net: The nominal net electrical output of a nuclear unit as specified by the electric utility for the purpose of plant design.

Development Well: $A$ well drilled within the proved area of an oil or gas reservoir to the depth of a stratigraphic horizon known to be productive. 
Diatillate Fuel Oll: A general classification for one of the petroleum fractions produced in conventional distillation operations. Included are products known as No. 1, No. 2, and No. 4 fuel oils and No. 1, No. 2. and No. 4 diesel fuels. It is used primarily for space beating, on- and off-bighway diesel engine fuel (including rallroad engine fuel and fuel for agricultural machinery), and electric power generation.

Dry Hole: An exploratory or development well found to be incapable of producing either oil or gas in sufficient quantities to justify completion as an oil or gas well.

Dry Natural Gas Production (as a decrement from gas reserves): The volume of natural gas withdrawn from reservoirs during the report year less (1) the volume returned to such reservoirs in cycling. repressuring of oil reservoirs, and conservation operations; (2) strinkage resulting from the removal of lease condensate and plant llquids; and (3) nonbydrocarbon gases, where they occur in sufficient quantity to render the gas unmarketable. Volumes of gas withdrawn from gas storage reservoirs and native gas that has been transferred to the storage category are not considered production. This is not the same as marketed production, since the latter also excludes vented and nared gas but contains liquids.

Dry Natural Gas Production (as an Increment to gas supply): Gross withdrawals from production reservoirs less gas used in reservoir repressuring. amounts vented and flared, nonhydrocarbons removed, and various natural gas constituents, such as ethane. propane, and butane, removed at natural gas processing plants. The parameters for measurement are $60^{\circ} \mathrm{F}$ and 14.73 pounds standard per square inch absolute.

Electrical System Energy Losses: The amount of energy lost during generation, transmission, and distribution of electricity, including plani and unaccounted-for uses.

Electricity Generation: The process of producing electric energy or transforming other forms of energy into electric energy. Also the amount of electric energy produced or expressed in watthours (Wh).

Electricity Generation, Gross: The total amount of electric energy produced by the generating station or stations, measured at the generator terminals.

Electricity Generation, Net: Gross generation less electricity consumed at the generating plant for station use. Electricity required for pumping at pumped-storage plants is regarded as plant use and is deducted from gross generation.

Electricity Production: Net electricity (gross electricity output measured at generator terminals minus power plant use) generated by publicly and privately owned electric utilities. Excludes industrial electricity generation (except autogeneration of hydroelec(ric power).

Electricity Sales: The amount of kilowatthours sold in a given period of time; usually grouped by classes of service. such as residential, commercial, industrial, and other. "Other" sales include sales for public street and highway lighting and otber sales to public authorities, sales to railroads and railways, and interdeparumental sales.

Electrlc Power Plant: A station containing prime movers, electric generators, and auxiliary equipment for converting mechanical, chemical, and/or fission energy into electric energy.

Electric Uttlity: A corporation, person, agency, authority, or other !egal entity or instrumentality that owns and/or operates facilities for the generation, transmission, distribution, or sale of electric energy, primarily for use by the public, and that files forms listed in the Code of Federal Regulations. Tiue 18, Part 141. Facilities that qualify as cogenerators or small power producers under the Public Utility Regulatory Policies Act are not considered electric utilities.

Electric Utility Sector: The electric utility sector consists of privately and publicly owned establishments that generate, transmit, distribute, or sell electricity primarily for use by the public and that meet the definition of an electric utility. Nonutility power producers are not included in the electric utility sector.

End-Use Sectors: The residential, commercial, industrial, and transportation sectors of the economy.

Energy: The capacity for doing work as measured by the capability of doing work (potential energy) or the conversion of this capability 10 motion (kinetic energy). Energy has several forms, some of which are easily convertible and can be changed to another form useful for work. Most of the world's convertible energy comes from fossil fuels that are burned to produce heat that is then used as a transfer medium to mechanical or other means in order to accomplish tasks. Electrical energy is usually measured in kilowatthours, while heat energy is usually measured in British thermal units.

Energy Consumption: The use of energy as a source of heat or power or as an input in the manufacturing process.

Energy Consumption, End.Use: Primary end-use energy consumption is the sum of fossil fuel consumption by the four end-use sectors (residential, commercial, industrial, and transportation) and generation of hydroelectric power by nonelectric utilities. Net end-use energy consumption includes 
electric utility sales to those sectors but excludes electrical system energy losses. Total end-use energy consumption includes both electric utility sales to the four end-use sectors and electrical system energy losses.

Energy Consumption, Total: The sum of fossil fuel consumption by the five sectors (residential, commercial, industrial, transportation, and electric utility) plus bydroelectric power, nuclear electric power, net imports of coal coke, and electricity generated for distribution from wood, waste, geothermal, wind, photovoltaic, and solar thermal energy.

Energy Source: A substance, such as petroleum, natural gas, or coal, that supplies heat or power. In Energy Information Administration reports, electricity and renewable forms of energy, such as biomass, geothermal, wind, and solar, are considered to be energy sources.

Ethane: A normally gaseous straight-chain hydrocarbon $\left(\mathrm{C}_{2} \mathrm{H}_{0}\right)$. It is a colorless, paraffinic gas that boils at a temperature of $-127.48^{\circ} \mathrm{F}$. It is extracted from natural gas and refinery gas streams.

Ethylene: An olefinic hydrocarbon $\left(\mathrm{C}_{2} \mathrm{H}_{4}\right)$ recovered from refinery processes or petrochemical processes.

Exploratory Well: A well drilled to find and produce oil or gas in an unproved area, to find a new reservoir in a field previously found to be productive of oil or gas in another reservoir, or to extend the limit of a known oil or gas reservoir.

Exports: Shipments of goods from the 50 States and the District of Columbia to foreign countries and to Puerto Rico, the Virgin Islands, and other U.S. possessions and territories.

\section{P.a.s.: See Free Alongside Ship.}

Federal Energy Regulatory Commission (FERC): The Federal agency with jurisdiction over interstate electricity sales, wholesale electric rates, hydroelectric licensing, natural gas pricing, oil pipeline rates, and gas pipeline certification. FERC is an independent regulatory agency within the Department of Energy and is the succussor to the Federal Power Commission.

Federal Power Commission (FPC): The predecessor agency of the Federal Energy Regulatory Commission. The Federal Power Commission was created by an Act of Congress under the Federal Water Power Act on June 10,1920 . It was charged originally with regulating the electric power and natural gas industries. It was abolished on September 30, 1977. when the Department of Energy was created. Its functions were divided between the Department of
Energy and the Federal Energy Regulatory Commission, an independent regulatory agency.

First Purchase Price: The marketed first sales price of domestic crude oil, consistent with the removal price defined by the provisions of the Windfall Profits Tax on Domestic Crude Oil (Public Law 96-223, Sec. 4998 (c)).

Flared Natural Gas: Natural gas burned in flares on the base site or at gas processing plants.

\section{P.o.b.: See Free on Board.}

Footuge Urilled: Total footage for wells in various categories, as reported for any specified period, includes (1) the deepest total depth (length of well bores) of all wells drilled from the surface, (2) the total of all bypassed footage drilled in connection with reported wells, and (3) all new footage drilled for directional sidetrack wells. Footage reported for directional sidetrack wells does not include footage in the common bore, which is reported as footage for the original well. In the case of old wells drilled deeper, the reported footage is that which was drilled below the total depth of the old well.

Former U.S.S.R.: Sce U.S.S.R.

Fossil Fuel: Any naturally occurring organic fuel, such as petroleum, coal, and natural gas.

Fossil Fuel Steam-Electric Power Plant: An electricity generation plant in which the prime mover is a turbine rotated by high-pressure steam produced in a boiler by heat from burning fossil fuels.

Free Alongside Ship (f.a.s.): The value of a commodity at the port of exportation, generally including the purchase price, plus all charges incurred in placing the commodity alongside the carrier at the port of exportation.

Free on Board (f.o.b.): A transaction whereby the seller makes the product available within an agreed-on period at a given port at a given price. It is the responsibility of the buyer to arrange for the transportation and insurance.

Fuel Ethanol: An anhydrous, denatured aliphatic alcohol $\left(\mathrm{C}_{2} \mathrm{H}_{3} \mathrm{OH}\right)$ intended for motor gasoline blending. See Oxygenates.

Full-Power Operation: Operation of a nuclear generating unit at 100 percent of its design capacity. Full-power operation precedes commercial operation.

Gasohol: A blend of finished motor gasoline (leaded or unleaded) and alcohol (generally ethanol but sometimes methanol) limited to 10 percent by volume 
of alcobol. Gasohol is included in finished leaded and unleaded motor gasoline.

Gas-Turbine Electric Power Plant: A plant in which the prime mover is a gas turbine. A gas turbine typically consists of an axial-flow air compressor, one or more combustion chambers where liquid or gaseous fuel is bumed and the bot gases expand to drive the generator and then are used to run the compressor.

Gas Well: A well completed for the production of natural gas from one or more gas zones or reservoirs. (Wells producing both crude oil and natural gas are classified as oil wells.)

Geothermal Energy: Energy from the internal heat of the Earth, which may be residual heat, friction heat, or a result of radioactive decay. The heat is found in rocks and fluids at various depths and can be extracted by drilling and/or pumping.

Geothermal Energy (as used at electric utilities): Hot water or steam extracted from geothermal reservoirs in the Earth's crust and supplied to steam turbines at electric utilities that drive generators to produce electricity.

Gross Domestic Product (GDP): The total value of goods and services produced by labor and property located in the United States. As long as the labor and property are located in the United States, the supplier (that is, the workers and, for property, the owners) may be either U.S. residents or residents of foreign countries.

Heat Content of a Quantity of Fuel, Gross: The total amount of beat released when a fuel is burned. Coal, crude oil, and natural gas all include chemical compounds of carbon and hydrogen. When those fuels are burned, the carbon and hydrogen combine with oxygen in the air to produce carbon dioxide and water. Some of the energy released in burning goes into transforming the water into steam and is usually lost. The amount of heat spent in transforming the water into steam is counted as part of gross heat content but is not counted as part of net heat content. Also referred to as the higher heating value. Btu conversion factors typically used in EIA represent gross beat content.

Heat Content of a Quantity of Fuel, Net: The amount of usable heat energy released when a fuel is burned under conditions similar to those in which it is normally used. Also referred to as the lower heating value. Btu conversion factors typically used in EIA represent gross heat content.

Heavy Oil: The fuel oils remaining after the lighter oils have been distilled off during the refining process. Except for start-up and flame stabilization, virtually all petroleum used in steam-electric power plants is heavy oil.
Hydrocarbon: $A n$ organic chemical compound of hydrogen and carbon in the gaseous, liquid, or solid phase. The molecular structure of hydrocarbon compounds varies from the simplest (methane, the primary constituent of natural gas) to the very heavy and very complex.

Hydroelectric Power: The production of electricity from the kinetic energy of falling water.

Hydroelectric Power Plant: A plant in which the turbine generators are driven by falling water.

Imports: Receipts of goods into the 50 States and the District of Columbia from foreign countries and from Puerto Rico, the Virgin Islands, and other U.S. possessions and territories.

Industrial Sector: The industrial sector comprises manufacturing industries, which make up the largest part of the sector, along with mining, construction, agriculture, fisheries, and forestry. Establishments in this sector range from steel mills, to small farms, to companies assembling electronic components.

Internal Combustion Electric Power Plant: A power plant in which the prime mover is an internal combustion engine. Diesel or gas-fired engines are the principal types used in electric power plants. The plant is usually operated during periods of high demand for electricity.

Jet Fuel: The term includes kerosene-type jet fuel and naphtha-type jet fuel. Kerosene-type jet fuel is a kerosene-quality product used primarily for commercial turbojet and turboprop aircraft engines. Naphtha-type jet fuel is a fuel in the heavy naphthas range used primarily for military turbojet and turboprop aircraft engines.

Kerosene: A petroleum distillate that has a maximum distillation temperature of $401^{\circ} \mathrm{F}$ at the 10 -percent recovery point, a final boiling point of $572^{\circ} \mathrm{F}$, and a minimum flash point of $100^{\circ} \mathrm{F}$. Included are the two grades designated in ASTM D3699 (No. 1-K and No. $2-K)$ and all grades of kerosene called range or stove oil. Kerosene is used in space heaters, cook stoves, and water heaters; it is suitable for use as an illuminant when burned in wick lamps.

Lease and Plant Fuel: Natural gas used in well, field, and lease operations (such as gas used in drilling operations, heaters, dehydrators, and field compressors), and as fuel in natural gas processing plants.

Lease Condensate: A natural gas liquid recovered from gas well gas (associated and non-associated) in lease separators or natural gas field facilities. Lease condensate consists primarily of pentanes and heavier hydrocarbons. 
Light Oil: Lighter fuel oils distilled off during the refining process. Virtually all petroleum used in internal combustion and gas-turbine engines is light oil.

Lignite: A brownish-black coal of low rank with a bigh content of moisture and volatile matter. Often referred to as brown coal. It is used almost exclusively for electric power generation. It conforms to ASTM Specification D388-84 for lignite.

Liquefied Natural Gas (LNG): Natural gas (primarily methane) that has been liquefied by reducing its temperature to $-260^{\circ} \mathrm{F}$ at atmospheric pressure.

Liquefied Petroleum Gases (LPG): Ethane, ethylene, propane, propylene, normal butane, butylene, and isobutane produced at refineries or natural gas processing plants, including plants that fractionate new natural gas plant liquids.

Low-Power Testing: The period of time between a nuclear generating unit's initial fuel loading date and the issuance of its operating (full-power) license. The maximum level of operation during that period is 5 percent of the unit's design thermal rating.

Lubricants: Substances used to reduce friction between bearing surfaces or as process materials either incorporated into other materials used as processing aids in the manufacturing of other products or as carriers of other materials. Petroleum lubricants may be produced either from distillates or residues. Other substances may be added to impart or improve certain required properties. Excluded are byproducts of lubricating oil refining, such as aromatic extracts derived from solvent extraction or tars derived from deasphalting. Included are all grades of lubricating oils from spindle oil to cylinder oil and those used in greases. Lubricant categories are paraffinic and naphthenic.

Marketed Production: Gross withdrawals less gas used for repressuring, quantities vented and flared, and nonhydrocarbon gases removed in treating or processing operations. Includes all quantities of gas used in field and processing operations.

Methanol: A light, volatile alcohol $\left(\mathrm{CH}_{3} \mathrm{OH}\right)$ eligible for motor gasoline blending. See Oxygenates.

Miscellaneous Petroleum Products: All finished petroleum products not classified elsewhere-for example, petrolatum, lube refining byproducts (aromatic extracts and tars), absorption oils, ram-jet fuel, petroleum rocket fuels, synthetic natural gas feedstocks, and specialty oils.

Motor Gasoline Blending Components: Naphthas that will be used for blending or compounding into finished motor gasoline (e.g., straight-run gasoline. alkylate, reformate, benzene, toluene, and zylene).
Excluded are oxygenates (alcohols and ethers), butane, and pentanes plus.

Motor Gasoline, Finished: A complex mixture of relatively volatile hydrocarbons, with or without small quantities of additives, that has been blended to form a fuel suitable for use in spark-ignition engines. Motor gasoline, as given in ASTM Specification D439 or Federal Specification VV-G-1690B, includes a range in distillation temperatures from 122 to $158^{\circ} \mathrm{F}$ at the 10-percent recovery point and from 365 to $374^{\circ} \mathrm{F}$ at the 90 -percent recovery point. Motor gasoline includes reformulated motor gasoline, oxygenated motor gasoline, and other finished motor gasoline. Blendstock is excluded until blending bas been completed.

- Reformulated Motor Gasoline: Motor gasoline, formulated for use in motor vehicles, the composition and properties of which are certified as "reformulated motor gasoline" by the Environmental Protection Agency.

- Oxygenated Motor Gasoline: Motor gasoline, formulated for use in motor vehicles, that has an oxygen content of 1.8 percent or higher by weight.

- Other Finished Motor Gasoline: Motor gasoline that is not included in the reformulated or oxygenated categories.

Motor Gasoline, Finished Gasohol: A blend of finished motor gasoline (leaded or unleaded) and alcohol (generally ethanol, but sometimes methanol) in which 10 percent or more of the product is alcohol.

Motor Gasoline, Finished Leaded: Motor gasoline that contains more than 0.05 gram of lead per gallon or more than 0.005 gram of phosphorus per gallon. Premium and regular grades are included, depending on the octane rating. Includes leaded gasohol. Blendstock is excluded until blending has been completed. Alcohol that is to be used in the blending of gasohol is also excluded.

Motor Gasoline, Finished Leaded Premium: Motor gasoline having an antiknock index, calculated as $(R+M) / 2$, greater than 90 and containing more than 0.05 gram of lead per gallon or more than 0.005 gram of phosphorus per gallon.

Motor Gasoline, Finished Leaded Regular: Motor gasoline having an antiknock index, calculated as $(\mathrm{R}+\mathrm{M}) / 2$, greater than or equal to 87 and less than or equal to 90 and containing more than $0.05 \mathrm{gram}$ of lead or 0.005 gram of phosphorus per gallon.

Motor Gasoline, Finished Unleaded: Motor gasoline containing not more than 0.05 gram of lead per gallon and not more than $0.005 \mathrm{gram}$ of phosphorus per gallon. Premium and regular grades are included, depending on the octane rating. Includes unleaded gasohol. Blendstock is excluded until blending has 
been completed. Alcohol that is to be used in the blending of gasobol is also excluded.

Motor Gasoline, Finished Unleaded Midgrade: Motor gasoline having an antiknock index, calculated as $(R+M) / 2$, greater than or equal to 88 and less than or equal to 90 and containing not more than $0.05 \mathrm{gram}$ of phosphorus per gallon.

Motor Gasoline, Finished Unleaded Premium: Motor gasoline having an antiknock index, calculated as $(R+M) / 2$, greater than 90 and containing not more than $0.05 \mathrm{gram}$ of lead or $0.005 \mathrm{gram}$ of phosphorus per gallon.

Motor Gasoline, Finished Unleaded Regular: Motor gasoline having an antiknock index, calculated as $(\mathrm{R}+\mathrm{M}) / 2$, of 87 containing not more than $0.05 \mathrm{gram}$ of lead per gallon and not more than 0.005 gram of phosphorus per gallon.

Motor Gasoline Retail Prices: Motor gasoline prices calculated each month by the Bureau of Labor Statistics (BLS) in conjunction with the construction of the Consumer Price Index (CPI). Those prices are collected in 85 urban areas selected to represent all urban consumers-about 80 percent of the total U.S. population. The service stations are selected initially, and on a replacement basis, in such a way that they represent the purchasing habits of the CPI population. Service stations in the current sample include those providing all types of service (i.e., full-, mini-, and self-service).

Motor Gasoline, Total: Includes finished leaded motor gasoline (premium and regular), finished unleaded motor gasoline (premium, midgrade, and regular), motor gasoline blending components, and gasohol.

MTBE (Methyl Tertiary Butyl Ether): An ether, $\left(\mathrm{CH}_{3}\right)_{3} \mathrm{COCH}_{3}$, intended for motor gasoline blending. See Oxygenates.

Naphtha: A genetic term applied to a petroleum fraction with an approximate boiling range between 122 and $400^{\circ} \mathrm{F}$.

Natural Gas: A mixture of hydrocarbons (principally methane) and small quantities of various nonhydrocarbons existing in the gaseous phase or in solution with crude oil in underground reservoirs.

Natural Gas, Dry: The marketable portion of natural gas production, which is obtained by subtracting extraction losses, including natural gas liquids removed at natural gas processing plants, from total production.

Natural Gas Marketed Production: Gross withdrawals of natural gas from production reservoirs, less gas used for reservoir repressuring; nonhydrocarbon gases removed in treating and processing operations; and quantities vented and flared.

Natural Gas Plant Liquids (NGPL): Natural gas liquids recovered from natural gas in processing plants and, in some situations, from natural gas field facilities, as well as those extracted by fractionators. Natural gas plant liquids are defined according to the published specifications of the Gas Processors Association and the American Society for Testing and Materials as follows: ethane, propane, normal butane, isobutane, pentanes plus, and other products from natural gas processing plants (i.e., products meeting the standards for finished petroleum products produced at natural gas processing plants, such as finished motor gasoline, finished aviation gasoline, special naphthas, kerosene, distillate fuel oil, and miscellaneous products).

Natural Gas Wellhead Price: The wellhead price of natural gas is calculated by dividing the total reported value at the wellhead by the total quantity produced as reported by the appropriate agencies of individual producing States and the U.S. Minerals Management Service. The price includes all costs prior to shipment from the lease, including gathering and compression costs, in addition to State production, severance, and similar charges.

Natural Gas, Wet: Natural gas prior to the extraction of liquids and other miscellaneous products.

Net Consumption: See Energy Consumption, End-Use.

Nonhydrocarbon Gases: Typical nonhydrocarbon gases that may be present in reservoir natural gas are carbon dioxide, helium, hydrogen sulfide, and nitrogen.

Nuclear Electric Power: Electricity generated by an electric power plant whose turbines are driven by steam generated in a reactor by beat from the fissioning of nuclear fuel.

Nuclear Electric Power Plant: A single-unit or multiunit facility in which heat produced in one or more reactors by the fissioning of nuclear fuel is used to drive one or more steam turbines.

Nuclear Reactor: An apparatus in which the nuclear fission chain can be initiated, maintained, and controlled so that energy is released at a specific rate. The reactor includes fissionable material (fuel), such as uranium or plutonium; fertile material; moderating material (unless it is a fast reactor); a heavy-walled pressure vessel; shielding to protect personnel; provision for heat removal; and control elements and instrumentation. 
Offshore: That geographic area that lies seaward of the coastline. In general, the coastline is the line of ordinary low water along with that portion of the coast that is in direct contact with the open sea or the line marking the seaward limit of inland water.

\section{Oil: See Crude Oil (Including Lease Condensate).}

Oil Well: A well completed for the production of crude oil from one or more oil zones or reservoirs. Wells producing both crude oil and natural gas are classified as oil wells.

Operable (nuclear): A U.S. nuclear generating unit is considered operable after it completes low-power testing and is issued a full-power operating license by the Nuclear Regulatory Commission. A foreign nuclear generating unit is considered operable once it has generated electricity to the grid.

Organization for Economic Cooperation and Development (OECD): Current members are Australia, Austria, Belgium, Canada, Denmark, Finland, France, Greece, Iceland, Ireland, Italy, Japan, Luxembourg, the Netherlands, New Zealand, Norway, Portugal, Spain, Sweden, Switzerland, Turkey, the United Kingdom, the United States and its territories (Guam, Puerto Rico, and the Virgin Islands), and Germany.

Organization of Petroleum Exporting Countries (OPEC): Countries that have organized for the purpose of negotiating with oil companies on matters of oil production, prices, and future concession rights. Current members are Algeria, Gabon, Indonesia, Iran, Iraq, Kuwait, Libya, Nigeria, Qatar, Saudi Arabia, the United Arab Emirates, and Venezuela.

Oxygenated Motor Gasoline: See Motor Gasoline, Finished.

Oxygenates: Any substance which, when added to motor gasoline, increases the amount of oxygen in that motor gasoline blend. Through a series of waivers and interpretive rules, the Environmental Protection Agency (EPA) has determined the allowable limits for oxygenates in unleaded gasoline. The "Substantially Similar" Interpretive Rules (56 FR [February 11, 1991]) allows blends of aliphatic alcohols other than methanol and aliphatic ethers, provided the oxygen content does not exceed 2.7 percent by weight. The "Substantially Similar" Interpretive Rules also provide for blends of methanol up to 0.3 percent by volume exclusive of other oxygenates, and butanol or alcohols of a higher molecular weight up to 2.75 percent by weight. Individual waivers pertaining to the use of oxygenates in unleaded motor gasoline have been issued by the EPA. They include:

- Fuel Ethanol. Blends of up to 10 percent by volume anhydrous ethanol (200 proof).

- Methanol. Blends of methanol and gasoline-grade tertiary butyl alcohol (GTBA) such that the total oxygen content does not exceed 3.5 percent by weight and the ratio of methanol to GTBA is less than or equal to 1 . It is also specified that this blended fuel must meet ASTM volatility specifications.

Blends of up to 5.0 percent by volume methanol with a minimum of 2.5 percent by volume cosolvent alcohols having carbon number of 4 or less (i.e., ethanol, propanol, butanol, ano'/or GTBA). The total oxygen must not exceed 3.7 percent by weight, and the blend must meet ASTM volatility specifications as well as phase separation and alcohol purity specifications.

- MTBE (Methyl tertiary butyl ether). Blends up to 15.0 percent by volume MTBE that must meet the ASTM D4814 specifications. Blenders must take precautions that the blends are not used as base gasolines for other oxygenated blends.

Pentanes Plus: A mixture of hydrocarbous, mostly pentanes and heavier, extracted from natural gas. Includes isopentane, natural gasoline, and plant condensate.

Petrochemical Feedstocks: Chemical feedstocks derived from petroleum principally for the manufacture of chemicals, synthutic rubber, and a variety of plastics. The categories reported are naphthas less than $401^{\circ} \mathrm{F}$ endpoint and other oils equal to or greater than $401^{\circ} \mathrm{F}$ endpoint.

Petroleum: A generic term applied to oil and oil products in all forms, such as crude oil, lease condensate, unfinished oils, petroleum products, natural gas plant liquids, and nonhydrocarbon compounds blended into finished petroleum products.

Petroleum Coke: A residue that is the final product of the condensation process in cracking. The product is either marketable petroleum coke or catalyst petroleum coke.

Petroleum Coke, Catalyst: The carbonaceous residue that is deposited on and deactivates the catalyst used in many catalytic operations (e.g., catalytic cracking). Carbon is deposited on the catalyst, thus deactivating the catalyst. The catalyst is reactivated by burning off the carbon, which is used as a fuel in the refining process. That carbon or coke is not recoverable in a concentrated form.

Petroleum Coke, Marketable: Those grades of coke produced in delayed or fluid cokers that may be recovered as relatively pure carbon. Marketable petroleum coke may be sold as is or further purified by calcining.

Petroleum Consumption: The sum of all refined petroleum products supplied. For each refined petroleum product, the amount supplied is calculated by adding production and imports, then subtracting 
changes in primary stocks (net withdrawals are a plus quantity and net additions are a minus quantity) and exports.

Petroleum Imports: Imports of petroleum into the $\mathbf{5 0}$ States and the District of Columbia from foreign countries and from Puerto Rico, the Virgin Islands, and other U.S. territories and possessions. Included are imports for the Strategic Petroleum Reserve and withdrawals from bonded warehouses for onshore consumption, offshore bunker use, and military use. Excluded are receipts of foreign petroleum into bonded warehouses and into U.S. territories and U.S. Foreign Trade Zones.

Petroleum Products: Products obtained from the processing of crude oil (including lease condensate), natural gas, and other hydrocarbon compounds. Petroleum products include unfinished oils, liquefied petroleum gases, pentanes plus, aviation gasoline, motor gasoline, naphtha-type jet fuel, kerosene-type jet fuel, kerosene, distillate fuel oil, residual fuel oil, petrochemical feedstocks, special naphthas, lubricants, waxes, petroleum coke, asphalt, road oil, still gas, and miscellaneous products.

Petroleum Products Supplied: Se e Petroleum Consumption.

Petroleum Stocks, Primary: For individual products, quantities that are held at refineries, in pipelines, and at bulk terminals that have a capacity of 50,000 barrels or more, or that are in transit thereto. Stocks held by product retailers and resellers, as well as tertiary stocks beld at the point of consumption, are excluded. Stocks of individual products held at gas processing plants are excluded from individual product estimates but are included in other oils estimates and total.

Photovoltaic and Solar Thermal Energy (as used at eleciric utilities): Energy radiated by the sun as electromagnetic waves (electromagnetic radiation) that is converted at electric utilities into electricity by means of solar (photovoltaic) cells or concentrating (focusing) collectors.

Pipeline Fuel: Gas consumed in the operation of pipelines, primarily in compressors.

Primary Consumption: See Energy Consumption, End-Use.

Propane: A normally gaseous straight-chain hydrocarbon $\left(\mathrm{C}_{3} \mathrm{H}_{8}\right)$. It is a colorless paraffinic gas that boils at a temperature of $-43.67^{\circ} \mathrm{F}$. It is extracted from natural gas or refinery gas streams. It includes all products designated in ASTM Specification D1835 and Gas Processors Association Specifications for commercial propane and HD-5 propane.

Propylene: An olefinic hydrocarbon $\left(\mathrm{C}_{3} \mathrm{H}_{6}\right)$ recovered from refinery or petrochemical processes.
Refiner Acquisition Cost of Crude Oil: The cost of crude oil to the refiner, including transportation and fees. The composite cost is the weighted average of domestic and imported crude oil costs.

Refinery (petroleum): An installation that manufactures finished petroleum products from crude oil, unfinished oils, natural gas liquids, other hydrocarbons, and alcohol.

Renewable Energy: Energy obtained from sources that are essentially inexhaustible (unlike, for example, the fossil fuels, of which there is a finite supply). Renewable sources of energy include wood, waste, photovoltaic, and solar thermal energy.

Repressuring: The injection of a pressurized fluid (such as air, gas, or water) into oil and gas reservoir formations to effect greater ultimate recovery.

Residential Sector: The residential sector is considered to consist of all private residences, whether occupied or vacant, owned or rented, including single-family homes, multifamily housing units, and mobile homes. Secondary homes, such as summer homes, are also included. Institutional housing, such as school dormitories, hospitals, and military barracks, generally are not included in the residential sector; they are included in the commercial sector.

Residual Fuel Oil: The heavier oils that remain after the distillate fuel oils and lighter hydrocarbons are distilled away in refinery operations and that conform to ASTM Specifications D396 and 975 . Included are No. 5, a residual fuel oil of medium viscosity; Navy Special, for use in steam-powered vessels in government service and in shore power plants; and No. 6, which includes Bunker $C$ fuel oil and is used for commercial and industrial heating, electricity generation, and to power ships. Imports of residual fuel oil include imported crude oil burned as fuel.

Road Oil: Any heavy petroleum oil, including residual asphaltic oil used as a dust palliative and surface treatment on roads and highways. It is generally produced in six grades, from 0 , the most liquid, to 5 , the most viscous.

Rotary Rig: A inachine used for drilling wells that employs a rotating tube attached to a bit for boring holes through rock.

Short Ton (coal): A unit of weight equal to 2,000 pounds.

SIC: See Standard Industrial Classification.

Solar Energy: The radiant energy of the sun, which can be converted into other forms of energy, such as heat or electricity. 
Standard Industrial Classification (SIC): A set of codes developed by the Office of Management and Budget which categorizes industries into groups with similar economic activities.

Startup Test Phase of Nuclear Power Plant: A nuclear power plant that has been licensed by the Nuclear Regulatory Commission to operate but is still in the initial testing phase, during which the production of electricity may not be continuous. In general, when the electric utility is satisfied with the plant's performance, it formally accepts the plant from the manufacturer and places it in commercial operation status. A request is then submitted to the appropriate utility rate commission to include the power plant in the rate base calculation.

Steam-Electric Power Plant: A plant in which the prime mover is a steam turbine. The steam used to drive the turbine is produced in a boiler where fossil fuels are bumed.

Strategic Petroleum Reserve (SPR): Petroleum stocks maintained by the Federal Government for use during periods of major supply interruption.

Supplemental Gaseous Fuels: Any gaseous substance that, introduced into or commingled with natural gas, increases the volume available for disposition. Such substances include, but are not limited to, propane-air, refinery gas, coke oven gas, still gas, manufactured gas, biomass gas, or air or inert gases added for Btu stabilization.

Synthetic Natural Gas (SNG): A manufactured product chemically similar in most respects to natural gas, resulting from the conversion or reforming of petroleum hydrocarbons. It may easily be substituted for, or interchanged with, pipeline quality natural gas. Also referred to as substitute natural gas.

Total Consumption: See Energy Consumption, End-Use.

Transportation Sector: The transporation sector consists of private and public vehicles that move people and commodities. Included are automobiles, trucks, buses, motorcycles, railroads and railways (including streetcars), aircraft, ships, barges, and natural gas pipelines.

Unaccounted-for Crude Oil: Arithmetic difference between the calculated supply and the calculated disposition of crude oil. The calculated supply is the sum of crude oil production and imports, less changes in crude oil stocks. The calculated disposition of crude oil is the sum of crude oil input to refineries, crude oil exports, crude oil burned as fuel, and crude oil losses.
Underground Storage: The storage of natural gas in underground reservoirs at a different location from which it was produced.

United States: Unless otherwise noted, "United States" in this publication means the 50 States and the District of Columbia. U.S. exports include shipments to U.S. territories, and imports include receipts from U.S. territories.

U.S.S.R.: The Union of Soviet Socialist Republics consisted of 15 constituent republics: Armenia, Azerbaijan, Belorussia, Estonia, Georgia, Kazakhstan, Kirghizia, Latvia, Lithuania, Moldavia, Russia, Tadzhikistan, Turkmenistan, Ukraine, and Uzbekistan. As a political entity, the U.S.S.R. ceased to exist as of December 31, 1991.

Vented Natural Gas: Gas released into the air on the base site or at processing plants.

Wellhead Price: The value of crude oil or natural gas at the mouth of the well.

Well Servicing Unit: Truck-mounted equipment generally used for downhole services after a well is drilled. Services include well completions and recompletions, maintenance, repairs, workovers, and well plugging and abandonments. Jobs range from minor operations, such as pulling the rods and rod pumps out of an oil well, replacing the pump and rerunning the assemblage into the well, to major workovers, such as milling out and repairing collapsed casing. Well depth and characteristics determine the type of equipment used.

Wind Energy (as used at electric utilities): The kinetic energy of wind converted at electric utilities into mechanical energy by wind turbines (i.e., blades rotating from a bub) that drive generators to produce electricity for distribution.

Wood and Waste (as used at electric utilities): Wood energy, garbage, bagasse, sewerage gas, and other industrial, agricultural, and urban refuse used to generate electricity for distribution.

Wood Energy: Wood and wood products used as fuel, including round wood (cord wood), limb wood, wood chips, bark, sawdust, forest residues, charcoal, pulp waste, and spent pulping liquor.

Working Gas: The gas in a reservoir that is in addition to the base (cushion) gas. It may or may not be completely withdrawn during any particular withdrawal season. Conditions permitting, the total working capacity could be used more than once during any given season. 


\title{
Publication Order Form \\ State Energy Data Report 1992, Consumption Estimates
}

\author{
Published: May 1994 \\ Energy Information Administration \\ GPO Stock No. 061-003-00854-2 \\ Price per copy: $\$ 33.00$
}

Company or Personal Name:

Additional Address/Attention Line:

Street Address:

City, State, Zip Code:

Daytime Phone Number (area code first):

Purchase Order No:

May we make your name and address available to other mailers?

The State Energy Data Report 1992, Consumption Estimates, presents annual energy consumption estimates for the 50 States, the District of Columbia, and the United States. The estimates are provided by type of energy (refined petroleum, natural gas, coal, and electricity) and by major consuming sectors (residential, commercial, industrial, transportation, and electric utilities) in physical units and in British thermal units for 1960, 1965, and 1970 through 1992. The 522-page report includes technical documentation describing the data sources and estimation procedures used.

\section{Please include payment with this order form. Allow a minimum of 4 weoks for domestic dellvery and an additional 6 weeks for internatlonal dellvery.}

Quantity $x \$ 33.00=\$$ (total due). (International customers add 25\%.)

$\square$ Check payable to Superintendent of Documents GPO Deposit Account No. VISA or MasterCard Account Authorizing Signature

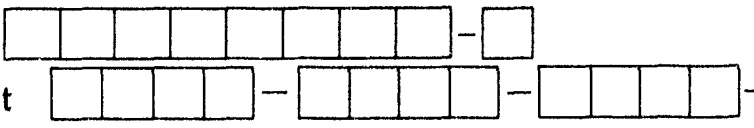
Credit Card Expiration Date
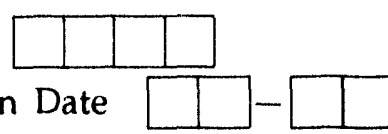

Note: Price includes regular domestic postage and handling. It is subject to change.

Mail order form to: U.S. Government Printing Office

P.O. Box 371954

Pittsburgh, PA 15250-7954

Or fax order form to: $202-512-2250$ 

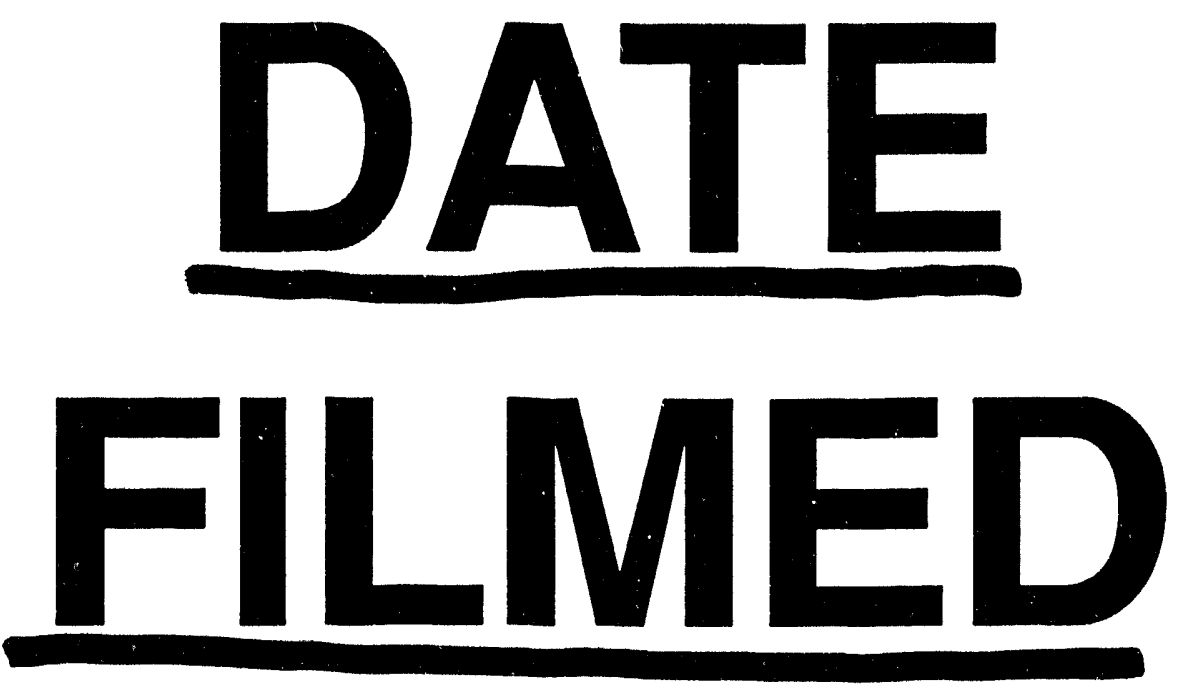

$6 / 22 / 94$
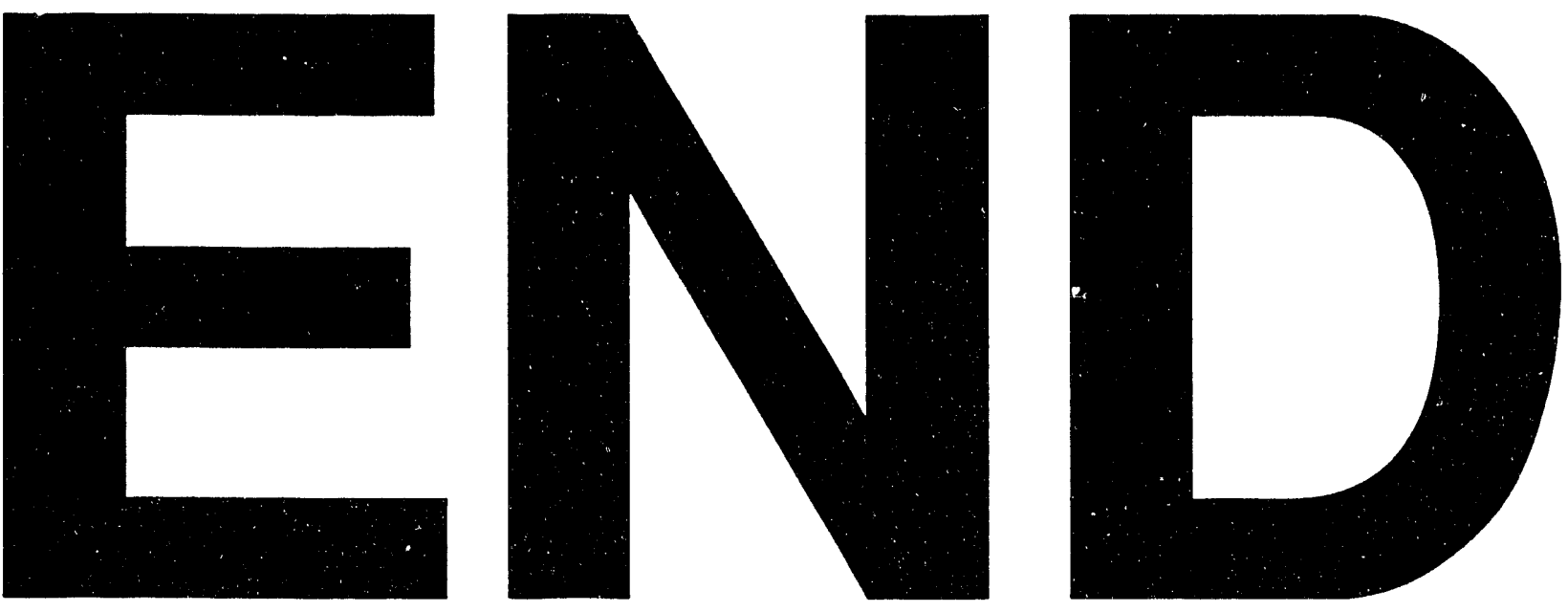
